\title{
RELIABILITY IN PAVEMENT DESIGN
}

\author{
PAOLA DALLA VALLE, BEng
}

Thesis submitted to the University of Nottingham for the degree of Doctor of Philosophy

\section{JUNE 2015}


To Dermott \& Finn, and all my fabulous family in Italy 


\section{ABSTRACT}

This research presents a methodology that accounts for variability of key pavement design input variables and variations due to lack-of-fit of the design models and assesses effects on pavement performance (fatigue and deformation life). Variability is described by statistical terms such as mean and standard deviation and by its probability density distribution.

The subject of reliability in pavement design has pushed many highway organisations around the world to review their design methodologies to evaluate the effect of variations in materials on pavement performance. This research has reinforced this need for considering the variability of design parameters in the design procedure and to conceive a pavement system in a probabilistic way, similar to structural designs.

This study has only considered flexible pavements. The sites considered for the analysis, all in the UK (including Northern Ireland), were mainly motorways or major trunk roads. Pavement survey data analysed were for Lane 1, the most heavily trafficked lane. Sections $1 \mathrm{~km}$ long were considered wherever possible.

Statistical characterisation of the variation of layer thickness, asphalt stiffness and subgrade stiffness input parameters is addressed. A model is then proposed which represents an improvement on the Method of Equivalent Thickness for the calculation of strains and life for flexible pavements. The output is a statistical assessment of the estimated pavement performance. The proposed model to calculate the fatigue and deformation life is very fast and simple, and is well suited to use in a pavement management system where stresses and strains must be calculated millions of times.

The research shows that the parameters with the greatest influence on the variability of predicted fatigue performance are the asphalt stiffness modulus and thickness. The parameters with the greatest influence on the variability of predicted deformation performance are the granular subbase thickness, the asphalt thickness and the subgrade stiffness. 


\section{ACKNOWLEDGEMENTS}

The research described in this thesis was carried out on a part-time basis between January 2006 and September 2014, with one year off for maternity.

The project initially started as an MPhil study on layer thickness variability. However, the scope of the research increased through the course of the years and the study successfully progressed to a PhD degree in 2008.

I gratefully acknowledge the sponsorship from Mr John Williams (now retired) and Mrs Donna James - members of the Pavement Engineering Team of the Highways Agency, UK - and from my company, Ove Arup \& Partners (Mr Nigel Hailey, Mrs Kate Hall and Mr Geoffrey Griffiths). I also express immense gratitude to Prof Andrew Collop (De Montfort University) and Dr Nick Thom, from the NTEC department of Nottingham University, for their invaluable contribution to the development of this project, and to Dr Mike Nunn and Dr Michael Gordon for their technical support. I would like to thank Dr John Lambert and Mr Dermott Doyle, from Aecom, for providing site data.

Finally, let me thank my husband, and my family in Italy for providing encouragement and support to finish this degree.

This dissertation is the result of my own original work, except where specific reference has been made to the work of others. 


\section{LIST OF CONTENTS}

1 Introduction 1

1.1 Aim of the research 2

1.2 Report layout 3

2 Variability in pavement design 5

$\begin{array}{lll}2.1 & \text { Parameters affecting pavement performance }\end{array}$

$\begin{array}{lll}2.2 & \text { Pavement design approaches } & 6\end{array}$

$\begin{array}{lll}\text { 2.2.1 Probabilistic analysis } & 7\end{array}$

$\begin{array}{lll}\text { 2.2.2 } & \text { Performance prediction models } & 8\end{array}$

2.2.2.1 Mechanistic 8

2.2.2.2 Empirical 9

2.2.2.3 Mechanistic-empirical 9

$\begin{array}{lll}2.3 & \text { Definition of failure } & 10\end{array}$

$\begin{array}{lll}\text { 2.3.1 Stress calculation } & 10\end{array}$

$\begin{array}{lll}2.3 .2 & \text { Transfer functions } & 10\end{array}$

$2.4 \quad$ Performance of pavement designs 11

2.5 Impact of variability on pavement performance 12

$\begin{array}{lll}2.6 & \text { Uncertainties of design parameters } & 13\end{array}$

2.6.1 Variations within a design length 13

2.6.1.1 Variations in pavement/subgrade stiffness 13

2.6.1.2 Variations in pavement layer thickness 14

2.6.1.3 Variations in smoothness 14

2.6.2 Variations between design and actual values 14

2.6.2.1 Pavement and subgrade stiffness 14

2.6.2.2 Layer thickness 15

2.6.2.3 Climatic variations 16

2.6.2.4 Pavement smoothness 17

2.6.2.5 Traffic load forecasting variations 17

2.6.3 Variability due to the imprecision in quantifying the parameters 18

2.6.3.1 GPR (Ground Penetrating Radar) accuracy 18

2.6.3.2 FWD accuracy 20

2.6.3.3 Weigh-in-motion (WIM) systems accuracy 20

$\begin{array}{lll}2.7 & \text { Systematic errors } & 21\end{array}$

2.7.1 Variations due to model bias 21

2.7.2 Variations due to statistical error 22 
$2.8 \quad$ Summary of variability of design input parameters 24

2.9 Summary of sensitivity of design input on pavement performance 26

$\begin{array}{lll}2.9 .1 & \text { Critical input parameters } & 26\end{array}$

$\begin{array}{lll}\text { 2.9.2 Characteristic distribution } & 27\end{array}$

3 Literature review on reliability in pavement design 28

$\begin{array}{ll}3.1 & \text { What is reliability? } \\ & 28\end{array}$

$\begin{array}{lll}3.1 .1 & \text { Measures of reliability } & 29\end{array}$

3.1.1.1 The conventional factor of safety 30

3.1.1.2 The central factor of safety (CFS) 31

3.1.1.3 The safety margin $S$

3.1.1.4 The reliability index $\beta \quad 32$

3.1.2 Reliability analysis 32

3.1.2.1 Simulation Techniques $\quad 35$

3.1.2.2 First-Order Reliability Methods (FORM) 36

3.1.2.3 Point Estimate Method (PEM) 40

3.1.2.4 Distribution of residuals 44

3.1.3 System reliability for multiple performance criteria 45

3.1.4 Reliability and the effect of time 45

3.2 The engineering problem 45

$\begin{array}{lll}3.3 & \text { Deterministic versus probabilistic design } & 47\end{array}$

3.4 Application of reliability analysis to pavement design 48

3.4.1 United Kingdom 48

$\begin{array}{lll}3.4 .2 & \text { United States } & 49\end{array}$

3.4.2.1 Design standards 49

3.4.2.2 Reliability research results to date 52

3.4.3 France 55

$\begin{array}{lll}3.4 .4 & \text { Australia } & 56\end{array}$

$\begin{array}{lll}3.5 & \text { Discussion } & 56\end{array}$

4 Methodology 61

$\begin{array}{lll}4.1 & \text { Data collection techniques } & 61\end{array}$

$\begin{array}{lll}4.2 & \text { Standards } & 64\end{array}$

4.3 Research challenges and methodologies 64

4.4 Generation of random numbers 65

4.4.1 Power Spectrum Analysis $\quad 65$

$\begin{array}{lll}4.5 & \text { Calculation of stresses and strains } & 67\end{array}$

$\begin{array}{lll}\text { 4.5.1 Response models } & 68\end{array}$ 
4.5.1.1 Semi-infinite half space 68

4.5.1.2 Layered systems 70

4.5.2 Literature review on layered systems response models 73

4.5.2.1 Current use of LET \& FEM 73

4.5.2.2 Limitations of layered elastic theory 75

4.5.2.3 Advantages of layered elastic theory 76

4.5.2.4 Advantages of MET 77

4.5.2.5 Discussion 79

$\begin{array}{lll}\text { 4.5.3 Performance models } & 80\end{array}$

$\begin{array}{lll}4.6 & \text { Conclusions } & 81\end{array}$

5 Statistical characterisation of layer thickness variability 82

$\begin{array}{lll}5.1 & \text { Design vs. constructed thickness }\end{array}$

5.2 Construction tolerances for analytical designs 83

5.3 Importance of layer thickness for rehabilitation design 84

$5.4 \quad$ Evaluation of pavement layer thickness variability 85

5.4.1 Literature research results 85

5.4.2 Statistical results for sites M01 to M08 (mean, standard deviation, and probability distribution) $\quad 86$

5.4.3 Correlation between asphalt and sub-base layer thickness 93

5.4.4 Construction tolerances 94

$\begin{array}{lll}5.5 & \text { Generation of random signals } & 99\end{array}$

$\begin{array}{lll}5.6 & \text { Conclusions } & 105\end{array}$

6 Statistical characterisation of asphalt stiffness modulus variability 107

6.1 Background to the estimation of asphalt stiffness in the UK 107

6.1.1 Determination the indirect tensile stiffness modulus (ITSM) 107

6.1.2 Determination of asphalt stiffness through FWD back analysis 109

6.1.3 Comparing FWD Stiffnesses with ITSM (HD 30, DMRB Volume 7) 111

6.2 Accuracy in back-calculation of pavement layer moduli 112

6.2.1 Effect of pavement thickness errors on back-calculated moduli 112

6.2.1.1 Author's revision of equation 10 in Collop (2000) 114

6.2.2 Effect of random errors from deflection measurements on backcalculated moduli 116

6.2.2.1 Sources of error in deflection measurement 117

6.2.2.2 Methodology used for the analysis and results 119

6.2.3 Conclusions from analysis of random deflection measurement error 129

6.2.4 Effect of limited number of data points on back-calculated moduli 131 
$\begin{array}{lll}6.4 & \text { Conclusions } & 135\end{array}$

$\begin{array}{lll}7 & \text { Statistical characterisation of subgrade stiffness variability } & 137\end{array}$

$\begin{array}{lll}7.1 & \text { Subgrade modulus for foundation design } & 137\end{array}$

7.2 Methods for estimating stiffness of subgrade 140

$\begin{array}{ll}\text { 7.2.1 California Bearing Ratio (CBR) } & 141\end{array}$

7.2.2 Dynamic Cone Penetrometer (DCP) 142

$\begin{array}{lll}\text { 7.2.3 Plate bearing tests } & 143\end{array}$

$\begin{array}{lll}\text { 7.2.4 Dynamic Plate testing } & 144\end{array}$

$\begin{array}{lll}7.3 & \text { Spatial and seasonal variation of subgrade stiffness } & 145\end{array}$

7.4 Research methodology to assess subgrade stiffness variability 149

7.5 Sensitivity analysis 152

$\begin{array}{lll}7.6 & \text { Conclusions } & 161\end{array}$

$8 \quad$ Proposed model to predict the pavement design life 163

$\begin{array}{lll}8.1 & \text { Sensitivity Analysis } & 164\end{array}$

8.1.1 Regression analysis - 3-layer pavement structure 166

8.1.1.1 Model for asphalt and subgrade strains 167

8.1.1.2 Model for performance prediction 169

8.1.2 Regression analysis for alternative fatigue model - 2-layer pavement structure 172

8.1.2.1 Model for asphalt strains 177

8.1.2.2 Model for performance prediction 178

8.1.3 Regression analysis for alternative deformation model - 3-layer pavement structure 179

8.1.4 Model correction 182

8.2 Assessment of pavement structure modelling in backanalysis procedures 187

8.3 Summary and Conclusions 192

$9 \quad$ Monte Carlo simulation and design life probability distributions 194

9.1 Steps of Monte Carlo simulation for pavement performance analysis 195

9.1.1 Artificial adjustments to subbase stiffness $\left(E_{s b}\right) \quad 198$

9.2 Results of MCS for homogeneous sections 200

9.3 Results of MM for homogeneous sections 202

9.4 Effect of correlation between variables 210

$\begin{array}{lll}9.5 & \text { Conclusions } & 213\end{array}$

10 Conclusions and Recommendations 214

10.1 Examples of risk application of proposed research 216

10.2 Summary of main conclusions 218

10.2.1 Variability in pavement design (chapter 2) 218 
10.2.2 Literature review on reliability in pavement design (chapter 3) 219

10.2.3 Methodology (chapter 4) 220

10.2.4 Statistical characterisation of layer thickness variability (chapter 5) 221

10.2.5 Statistical characterisation of asphalt stiffness modulus variability (chapter 6)

10.2.6 Statistical characterisation of subgrade stiffness modulus variability (chapter 7)

10.2.7 Proposed model to predict the pavement design life (chapter 8) 223

10.2.8 Monte Carlo simulation and design life probability distributions (chapter 9)

10.3 Recommendations for further work

10.3.1 Investigation on how design relates to in-service performance 225

10.3.2 Include variability in life cycle cost analysis 226

10.3.3 Apply research to a larger number of real sites 226

10.3.4 Variability to include more material properties 226

11 References 228

A1 Mean, standard deviation and coefficient of variation for all sites 237

A1.1 Asphalt mean, standard deviation and coefficient of variation 237

A1.2 Sub-base mean, standard deviation and coefficient of variation 238

A1.3 Ratio of standard deviation over mean for asphalt and sub-base layer thickness 239

A2 Layer thickness distribution from GPR surveys 240

$\begin{array}{lll}\text { A2.1 M01 } & 240\end{array}$

$\begin{array}{lll}\text { A2.2 M02 } & 247\end{array}$

A2.3 M03 248

A2.4 M04 250

A2.5 M05 252

A2.6 M06 258

$\begin{array}{lll}\text { A2.7 } & \text { M07 } & 261\end{array}$

$\begin{array}{lll}\text { A2.8 } & \text { M08 } & 263\end{array}$

A3 Construction tolerance 265

$\begin{array}{lll}\text { A3.1 M01 } & 265\end{array}$

A3.2 M02 272

A3.3 M03 273

$\begin{array}{lll}\text { A3.4 M04 } & 275\end{array}$

A3.5 M05 277

A3.6 M06 283 
A4 Monte Carlo Simulation results

A4.1 M01

286

A4.1.1 Section 3

286

A4.1.2 Section 16

290

A4.2 M04

293

A4.2.1 Section 1

293

A4.2.2 Section 10

296

A4.2.3 Section 20

299

A4.2.4 Section 30

302

A4.2.5 Section 37

305

A4.3 M05 ACW

308

A4.3.1 Section 10

308

A4.3.2 Section 11

311

A4.4 M05 CW

314

A4.4.1 Section 4

314

A4.4.2 Section 42

317

A4.5 M07 NB

320

A4.5.1 Section 2

320

A4.5.2 Section 3

323

A4.5.3 Section 4

326

A4.5.4 Section 5

329

A4.5.5 Section 6

332

A4.6 M07 SB

335

A4.6.1 Section 3

335

A4.6.2 Section 7

338

A4.6.3 Section 8

341

A4.6.4 Section 12

344

A4.7 M08 EB 347

A4.7.1 Section 4 347

A4.7.2 Section 6 350

A4.8 M08 WB 353

A4.8.1 Section 2

353

A4.8.2 Section 5

356 


\section{LIST OF FIGURES}

Figure 2.1 Factors influencing pavement performance (from Tighe et al., 2001) ................. 6

Figure 2.2 Performance of existing pavement designs (from Transportation Research

Circular E-C118, 2007)....

Figure 2.3 Illustration of in situ stiffness coefficients for various pavement materials (Figure

4.12 from Darter et al. 1973) ................................................................. 15

Figure 2.4 Effect of Weigh-in-Motion scale calibration drift on the accuracy of ESAL calculations (from Shekharan et al. 2002)

Figure 2.5 Total cost versus reliability for specific pavement design problem (from Darter et

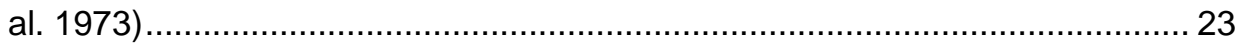

Figure 3.1 Model of the civil engineering reliability (from Harr, 1987) ............................... 28

Figure 3.2 Fundamentals of risk evaluation (from Haldar et al., 2000) .............................. 31

Figure 3.3 Hasofer-Lind reliability index for linear limit state function (adapted from Haldar et al., 2000).....

Figure 3.4 Hasofer-Lind reliability Index for nonlinear performance functions (from Haldar et al., 2000).

Figure 3.5 Obtaining $\mathrm{T}-1$ (ui) ( $\mathrm{x}$ in the table) from ui ( $\mathrm{n}$ in the table), based on $\mathrm{F}(\mathrm{ui})=\Phi(\mathrm{ui})$

$(F(x)=\Phi(n)$ in the table) (Low and Tang 2007)

Figure 3.6 Modern probabilistic design method .......................................................... 47

Figure 3.7 Design Reliability Concept for Smoothness (IRI) (from 2008 MEPDG) .............. 51

Figure 3.8 Comparison of failure probabilities using methods of moments and MCS (from

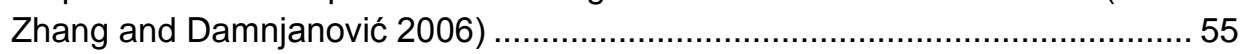

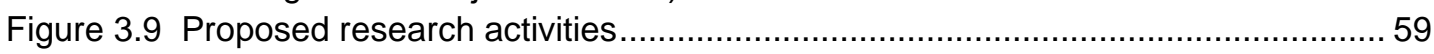

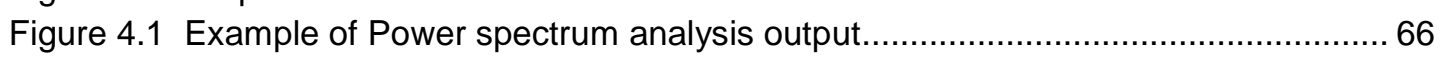

Figure 4.2 Odemark's transformation of a four-layer system....................................... 70

Figure 5.1 GPR thickness values for total asphalt and sub-base thickness, relating to $1 \mathrm{~km}$

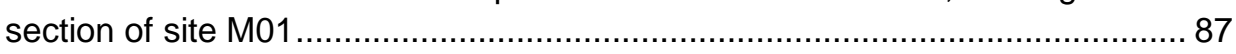

Figure 5.2 Histogram representation for total asphalt layer thickness distribution, relating to

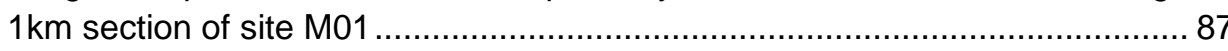

Figure 5.3 Histogram representation for sub-base layer thickness distribution, relating to

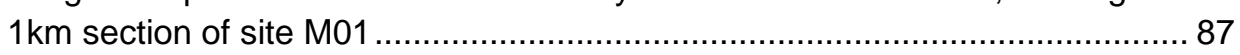

Figure 5.4 Asphalt versus sub-base thickness for $1 \mathrm{~km}$ section (hardshoulder) of M01 ....... 93

Figure 5.5 Lower tolerance range for total asphalt layer thickness ................................... 96

Figure 5.6 Upper and lower tolerance range for sub-base layer thickness ....................... 97

Figure 5.7 Schematic explanation of random signals generation .................................. 101

Figure 5.8 Random generated profiles ................................................................... 101

Figure 5.9 Spectral density of generated profiles and real surface profile ........................ 102

Figure 5.10 Comparison between real (from GPR data) and artificially generated (from profile) layer thicknesses....................................................................... 102

Figure 5.11 Match between the real and the artificial probability distributions of the asphalt

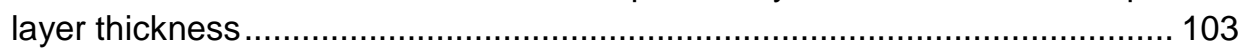

Figure 5.12 Match between the real and the artificial probability distributions of the sub-base

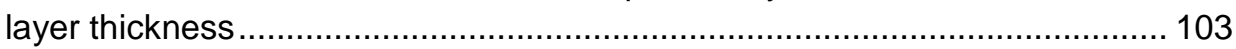

Figure 5.13 Spectral density of real and artificial layer thicknesses ................................ 104

Figure 5.14 Asphalt thickness versus sub-base thickness for both real and artificial data 104

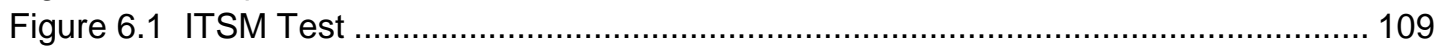

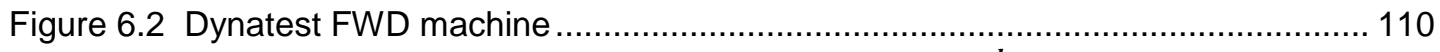

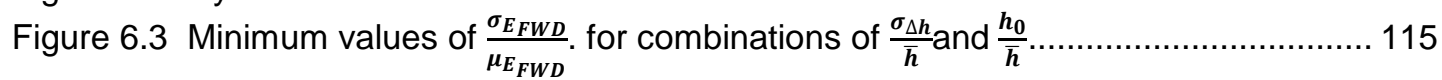

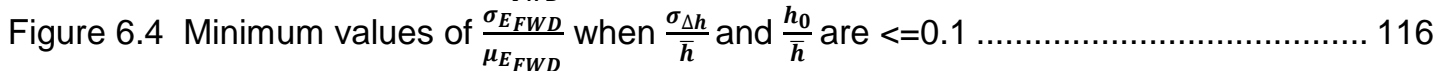


Figure 6.5 Random and systematic deflection measurements errors (figure taken from Irwin, 2009)

Figure 6.6 ITSM stiffness data for motorway M01 …................................................ 133

Figure 7.1 Foundation layers (from Figure 2.1 in IAN 73/09 Rev 1) .............................. 138

Figure 7.2 Dynamic Cone Penetrometer (DCP) …….............................................. 142

Figure 7.3 Plate bearing test (from M\&H testing Ltd website) ....................................... 143

Figure 7.4 Dynamic Plate tests (LWD on the left, FWD on the right) .............................. 144

Figure 7.5 Estimated pavement life (in ESALs) due to fatigue and rutting failures due to seasonal variations (from Bayomy and Salem 2005) .................................. 148

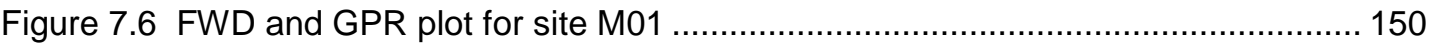

Figure 7.7 Example of Cusum analysis for site M01 ................................................. 150

Figure 7.8 Summary of Ratio of fatigue (Nf) and deformation life $\left(\mathrm{N}_{\mathrm{d}}\right)$ over base case based on average results (graphical format) ...................................................... 160

Figure 8.1 Model for 3-layer pavement structure .................................................... 166

Figure 8.2 Linear regression analysis for the asphalt strain .......................................... 168

Figure 8.3 Linear regression analysis for the subgrade strain ...................................... 168

Figure 8.4 Linear regression analysis for the fatigue life model (with eq 4.13) from LR1132

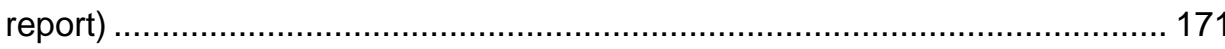

Figure 8.5 Linear regression analysis for the deformation life model (with eq. 4.14 from

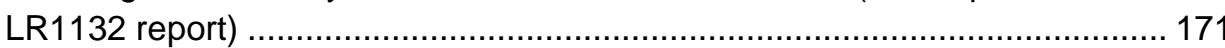

Figure 8.6 Model for 3-layer pavement structure (left) and 2-layer pavement structure (right) used in the alternative model ..................................................................... 172

Figure 8.7 Schematic diagram representing the process of finding EFM_actual ............... 173

Figure 8.8 EFM calculated with eq. (8.4) versus actual EFM (calculated with Matlab) ...... 176

Figure 8.9 EFM calculated with (8.5) versus actual EFM (calculated with Matlab) ............ 176

Figure 8.10 Linear regression analysis for the asphalt strain with proposed model ........... 177

Figure 8.11 Linear regression analysis for the fatigue life with proposed model................ 179

Figure 8.12 Linear regression analysis for the subgrade strain with alternative model...... 181

Figure 8.13 Linear regression analysis for the deformation life with alternative model...... 181

Figure 9.1 Steps of Monte Carlo simulation for pavement performance analysis .............. 197

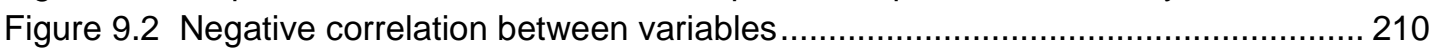

Figure 9.3 Fatigue life for Section 3 of site M01 - uncorrelated variables.......................... 211

Figure 9.4 Deformation life for Section 3 of site M01 - uncorrelated variables .................. 212

Figure 9.5 Fatigue life for Section 3 of site M01 -correlated variables............................. 212

Figure 9.6 Deformation life for Section 3 of site M01 - correlated variables ..................... 213

Figure 10.1 Practical application of the research activities shown in Figure 3.9 ............... 216 


\section{LIST OF TABLES}

Table 2.1 Summary of pavement material COVs from available literature (for the Mechanistic-Empirical pavement design approach) .................................... 25

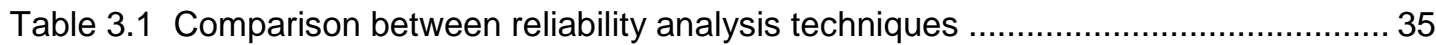

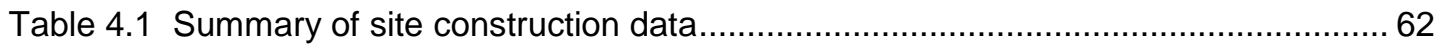

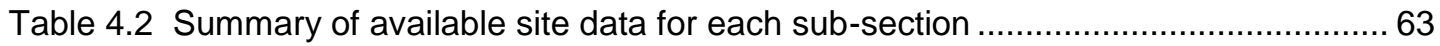

Table 5.1 Statistical analysis of pavement layer thickness for available sites ...................... 89

Table 5.2 Probability distribution for pavement layer thickness for available sites............... 90

Table 5.3 Comparison between sites' coefficient of variation for the pavement layer thickness and literature review values......................................................... 91

Table 5.4 Tolerances in surface levels of pavement courses (SHW, Series 700) ................ 94

Table 5.5 Review of construction tolerances for site M01, assuming $95 \%$ confidence ........ 98

Table 5.6 Summary of IRI values calculated for each section of motorway M01 ............... 105

Table 6.1 Application of equation (9) (Collop, 2000) to two sections of motorway M01 .... 114

Table 6.2 Application of equation (10) (Collop, 2000) to two sections of motorway M01 ... 114

Table 6.3 Pavement structure 1 (controlled sections) …………..................................... 119

Table 6.4 Pavement structure 2 (2-layer structure) ………........................................ 119

Table 6.5 Pavement structure 3 (3-layer structure) …................................................ 120

Table 6.6 Pavement structure 1, Deflection bowl calculated with BISAR .......................... 120

Table 6.7 Pavement structures 2 \& 3, real deflection bowl............................................. 120

Table 6.8 Effect of random deflection measurement error on back-calculated asphalt modulus on pavement structure 1 - single drop per case ............................ 122

Table 6.9 Effect of random deflection measurement error on back-calculated asphalt modulus on pavement structure 2 - single drop per case ............................ 122

Table 6.10 Effect of random deflection measurement error on back-calculated asphalt modulus on pavement structure 3 - single drop per case

Table 6.11 Effect of random deflection measurement error on back-calculated Equivalent Foundation Modulus (EFM) on pavement structure 1 - single drop per case 123

Table 6.12 Effect of random deflection measurement error on back-calculated EFM on pavement structure 2 - single drop per case

Table 6.13 Effect of random deflection measurement error on back-calculated sub-base modulus on pavement structure 3 - single drop per case

Table 6.14 Effect of random deflection measurement error on back-calculated subgrade modulus on pavement structure 3 - single drop per case

Table 6.15 Effect of random deflection measurement error on back-calculated asphalt modulus on pavement structure 1 - average of three drops per case.

Table 6.16 Effect of random deflection measurement error on back-calculated asphalt modulus on pavement structure 2 - average of three drops per case.

Table 6.17 Effect of random deflection measurement error on back-calculated asphalt modulus on pavement structure 3 - average of three drops per case.

Table 6.18 Effect of random deflection measurement error on back-calculated Equivalent Foundation Modulus (EFM) on pavement structure 1 - average of three drops per case......

Table 6.19 Effect of random deflection measurement error on back-calculated Equivalent Foundation Modulus (EFM) on pavement structure 2 - average of three drops per case.

Table 6.20 Effect of random deflection measurement error on back-calculated sub-base modulus on pavement structure 3 - average of three drops per case

Table 6.21 Effect of random deflection measurement error on back-calculated subgrade modulus on pavement structure 3 - average of three drops per case 


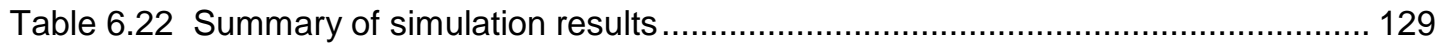

Table 6.23 Summary of $1^{\text {st }}$ simulation results from Irwin et al. (1989) .............................. 130

Table 6.24 Summary of 2nd simulation results from Irwin et al. (1989) ............................ 130

Table 6.25 Statistical properties of ITSM data from motorway M01 .............................. 133

Table 6.26 M01 Clockwise: ITSM tests for base layer.................................................. 134

Table 6.27 M01 Anti-Clockwise: ITSM tests for base layer ........................................... 134

Table 6.28 M01 Clockwise: ITSM tests for binder course layer....................................... 134

Table 6.29 M01 Anti-Clockwise: ITSM tests for binder course layer ................................ 134

Table 6.30 Comparison between standard deviations of the asphalt stiffness modulus .... 134

Table 7.1 Equilibrium suction-index CBR values (from Table C1 in Appendix C in LR 1132,

Powell et al. 1984)

Table 7.2 Subgrade stiffness variability from FWD back-analysis for all sites (for both threeand two-layer pavement structure model) ................................................... 151

Table 7. 3 Mean \& standard deviation, 15th \& 85th percentiles of the design input parameters for all homogeneous sections

Table 7.4 Asphalt stiffness $15^{\text {th }}$ and $85^{\text {th }}$ percentiles - Ratio of fatigue $\left(\mathrm{N}_{\mathrm{f}}\right)$ and deformation

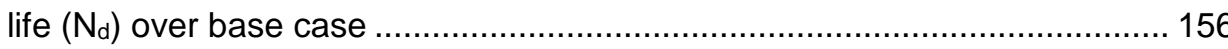

Table 7.5 Asphalt thickness $15^{\text {th }}$ and $85^{\text {th }}$ percentiles - Ratio of fatigue $\left(\mathrm{N}_{\mathrm{f}}\right)$ and deformation life $\left(\mathrm{N}_{\mathrm{d}}\right)$ over base case ........................................................................ 157

Table 7.6 Subbase stiffness 15th and 85 th percentiles - Ratio of fatigue $\left(\mathrm{N}_{\mathrm{f}}\right)$ and deformation life $\left(\mathrm{N}_{\mathrm{d}}\right)$ over base case .......................................................... 157

Table 7.7 Subbase thickness 15th and 85th percentiles - Ratio of fatigue $\left(\mathrm{N}_{\mathrm{f}}\right)$ and deformation life $\left(\mathrm{N}_{\mathrm{d}}\right)$ over base case ....................................................... 158

Table 7.8 Subgrade stiffness 15 th and 85 th percentiles - Ratio of fatigue $\left(\mathrm{N}_{\mathrm{f}}\right)$ and deformation life $\left(\mathrm{N}_{\mathrm{d}}\right)$ over base case

Table 7.9 Summary of Ratio of fatigue $\left(\mathrm{N}_{\mathrm{f}}\right)$ and deformation life $\left(\mathrm{N}_{\mathrm{d}}\right)$ over base case based on average results (tabular format) ........................................................... 159

Table 8.1 Pavement structures considered in the analysis ............................................ 165

Table 8.2 Regression coefficients of eq. (8.5) for each asphalt thickness group ............... 175

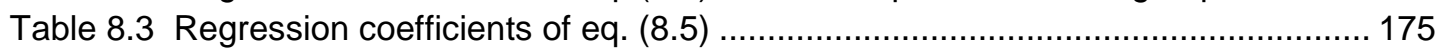

Table 8.4 Ratio of fatigue life calculated with model over fatigue life calculated with BISAR

Table 8.5 Ratio of deformation life calculated with model over deformation life calculated

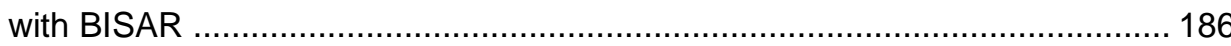

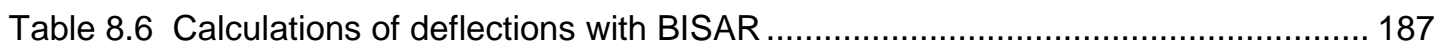

Table 8.7 Layer stiffness range used in back-calculation analysis with MODULUS .......... 188

Table 8.8 Example of back-calculation results for 3-layer and 2-layer model structures with MODULUS and location of asphalt and subgrade strains in subsequent forward

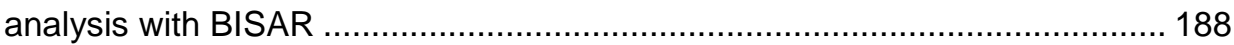

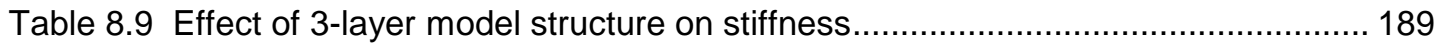

Table 8.10 Effect of 2-layer model structure on stiffness............................................... 189

Table 8.11 Effect of 3-layer model structure on fatigue and deformation life with LR1132 equations

Table 8.12 Effect of 2-layer model structure on fatigue and deformation life with LR1132 equations

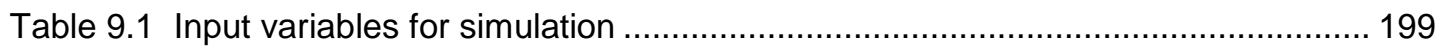

Table 9.2 Input variables for simulation - adjustments to subbase stiffness ..................... 200

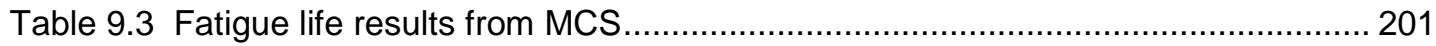

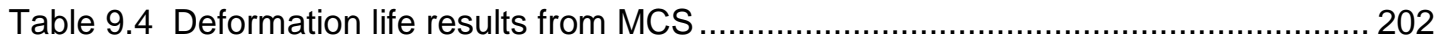

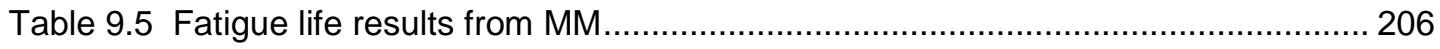

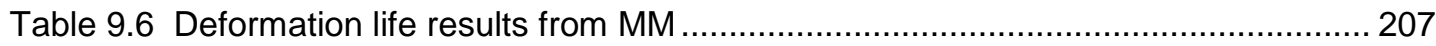

Table 9.7 Fatigue and deformation lives from average values of input variables............... 208 
Table 9.8 Fatigue and deformation life results for Section 3 of site M01 - effect of correlation between asphalt thickness and asphalt stiffness 211

Table 10.1 Parameters of performance life for hypothetical section, for example Section 4 of site M05 CW and Section 6 of site M07 NB 217

Table 10.2 Example of statistical analysis of fatigue and deformation life results for risk analysis. 


\section{Introduction}

Achieving durability of pavement structures is crucial not only for the Highways Agency in the UK but for any other highway authority in the world. Funding is getting tighter for both new build and maintenance of existing assets with consequent pressure on highway designers to construct durable pavements which offer at the same time good value for money to the client, minimum disruption to road users, and minimal impact on the environment.

However, it is difficult to accurately design and predict the life and form of distress of a pavement structure because all of the variables involved, such as traffic prediction, material properties and layer thickness, are random/uncertain/stochastic in nature. This means that these variables vary in space and time with resulting variability in the performance of a pavement. The recognition of the stochastic or random nature of material properties has brought more attention to the explicit use of reliability concepts within the field of pavement design (i.e., structural thickness) and maintenance (i.e., overlay thickness) around the world. Reliability is defined as the probability that the pavement system will perform its intended function over its design life and under certain conditions (i.e., environmental and operating).

Most pavement engineers know that pavement materials, environment, loading and construction affect the performance of a pavement and the variability observed in each of these parameters introduces a certain level of risk. The recognised need to account for these variabilities in the design process is pushing many highway authorities in the world to move from a traditional deterministic approach, based on a single input/output value, towards a probabilistic design, which includes a mean, variance and probability distribution. The probabilistic approach offers a way of incorporating risk assessment considerations which are vital for whole-life cycle economic analysis and decisions.

Consequently, variability of the pavement design input parameters and appropriate methods to account for this variability to estimate correctly the probability of failure have to be considered and developed. Accurate and 
reliable pavement performance predictions during a pavement's design life are of vital importance to minimise the risk of premature failure.

\subsection{Aim of the research}

Premature failure of pavement structures is often due to the inability to incorporate variations in materials and construction into the design procedure. Among the various parameters responsible for a successful performance of a highway system is the layer thickness. The research starts by looking at the layer thickness variation in detail, to quantify its variability on a number of case studies in the UK and to develop a methodology to account for this variability on the performance life (i.e., fatigue cracking and permanent deformation or rutting). The importance of layer thickness variability is principally for:

- Impact on expected pavement life.

- Back-calculation analysis hence overlay design.

- More reliable maintenance strategy decisions.

Once the assessment of layer thickness variation is satisfied, the research will concentrate on the variability of the other important factors involved in the pavement design such the subgrade strength and the asphalt fatigue properties.

The subject of reliability in pavement design has pushed many highway organisations around the world to review their design methodologies, mainly empirical, to move towards mechanistic-empirical analysis and design which provide the tools for the designer to evaluate the effect of variations in materials on pavement performance. It is the final aim of this research to reenforce this need for considering the variability of design parameters in the design procedure and to conceive a pavement system in a probabilistic way similarly to structural designs. It is also intended to assess the variability of input (and output) parameters by using simple relationships for fatigue and permanent deformation (rutting) prediction. This because the complexity of the predictive equation has no direct influence on variability of prediction, and so it is not relevant for this work. 
The scope of the study is to consider flexible pavements only and to consider mainly thickness data from non-destructive radar surveys. The sites considered for the analysis, all in the UK (including Northern Ireland), are mainly motorways or major trunk roads. The exact location of some of the sites cannot be revealed due to confidentiality of information. The focus of the analysis remains on Lane 1 , the most heavily trafficked lane, and sections $1 \mathrm{~km}$ long have been considered wherever possible. A total of eight sites have been considered in the research. Two thin pavement structures have also been included in the assessment.

\section{$1.2 \quad$ Report layout}

The report has been organised with the following structure:

- Chapter 2 reviews the variability in pavement design by looking at the uncertainties of the main design input parameters.

- Chapter 3 presents a literature review on reliability in pavement design. Reliability theory and the most common reliability methodologies are introduced followed by an overview of how reliability concepts are addressed around the world. Gaps in the research are identified and a research methodology is proposed at the end of the chapter.

- Chapter 4 discusses the methodology used to develop the research objectives. A description of the sites is provided and data collection techniques used are discussed. The theory behind the Power Spectrum analysis and the Method of Equivalent Thickness (MET) is presented.

- Chapter 5 reports all the findings made on the characterisation of the variation of the layer thickness input parameter. The chapter also explains how a Power Spectral Analysis has been used to generate random asphalt and sub-base thickness profiles for the purpose of future simulations.

- In Chapter 6 the statistical characterisation of asphalt stiffness modulus variability is introduced. Then, the impact of three major sources of errors on the back-calculated pavement layer moduli - namely pavement thickness errors, random deflection errors and limited number of data points - are discussed and a methodology is proposed for possible 
inclusion of the above errors in the overall asphalt stiffness variability assessment. Finally, a comparison is made between field and laboratory tests typically used to assess the structural characteristics of a pavement structure.

- In chapter 7 the statistical characterisation of subgrade stiffness variability and the effect of subgrade stiffness variations on expected pavement life are assessed. A sensitivity analysis is also carried out on homogeneous sections identified on each site to assess which design input parameters most greatly affect the resulting pavement life.

- Chapter 8 presents the model used to calculate the pavement life. The chapter starts with the review of the Method of Equivalent Thickness and a comparison is made with another analytical tool (BISAR) to calculate strains and stresses in a pavement structure. Then a model is proposed which represents an improvement on the Method of Equivalent Thickness for the calculation of strains and fatigue life for flexible pavements. The model provides a simple and efficient method for practical purposes, for example in Pavement Management Systems or in simulation of pavement deterioration, where stresses and strains must be calculated a large number of times. Possible model adjustments to the calculated mean and variance are discussed to account for the model bias error. This chapter also explores the difference in back-analysis results when different numbers of layers are used to model a pavement structure.

- Chapter 9 presents examples of the Monte Carlo Simulation (MCS) and the Method of Moments (MM) methods to show how variability of design input parameters can be used and how they affect the design life probability distribution.

- Chapter 10 presents a summary of the research findings and conclusions are made based on the results of the previous observations. Ideas for future related work are then introduced. 


\section{Variability in pavement design}

Variability in the "as-built" properties of pavement materials and uncertainties in traffic loading forecast have been recognised for quite a long time. Darter et al. (1973) used data from the Texas flexible pavement system to illustrate three basic types of variations encountered in flexible pavement designs: variations within a design length (i.e. a section with nominally uniform pavement thickness and materials type); variations between design and actual values; and variations due to lack-of-fit of the design models.

This section introduces 1) what parameters affect the pavement performance, 2) an overview of currently used pavement design approaches, 3) the definition of failure, and 4) an overview of the variability of the factors affecting the pavement performance.

\subsection{Parameters affecting pavement performance}

The main parameters affecting pavement performance can be grouped as follows (see Figure 2.1):

- The properties of the subgrade soil on which the pavement layers will be constructed. This parameter is generally represented by the subgrade stiffness.

- The pavement layer characteristics represented by the layer thicknesses and stiffnesses.

- The traffic characteristics that the pavement will withstand during the design period. This parameter is generally represented by the accumulated number of ESALs (Equivalent Standard Axle Loads).

- The climatic conditions that the pavement will be subjected to during the design period.

- The quality of construction methods and workmanship.

- The timing and appropriate schedule of maintenance treatments. 


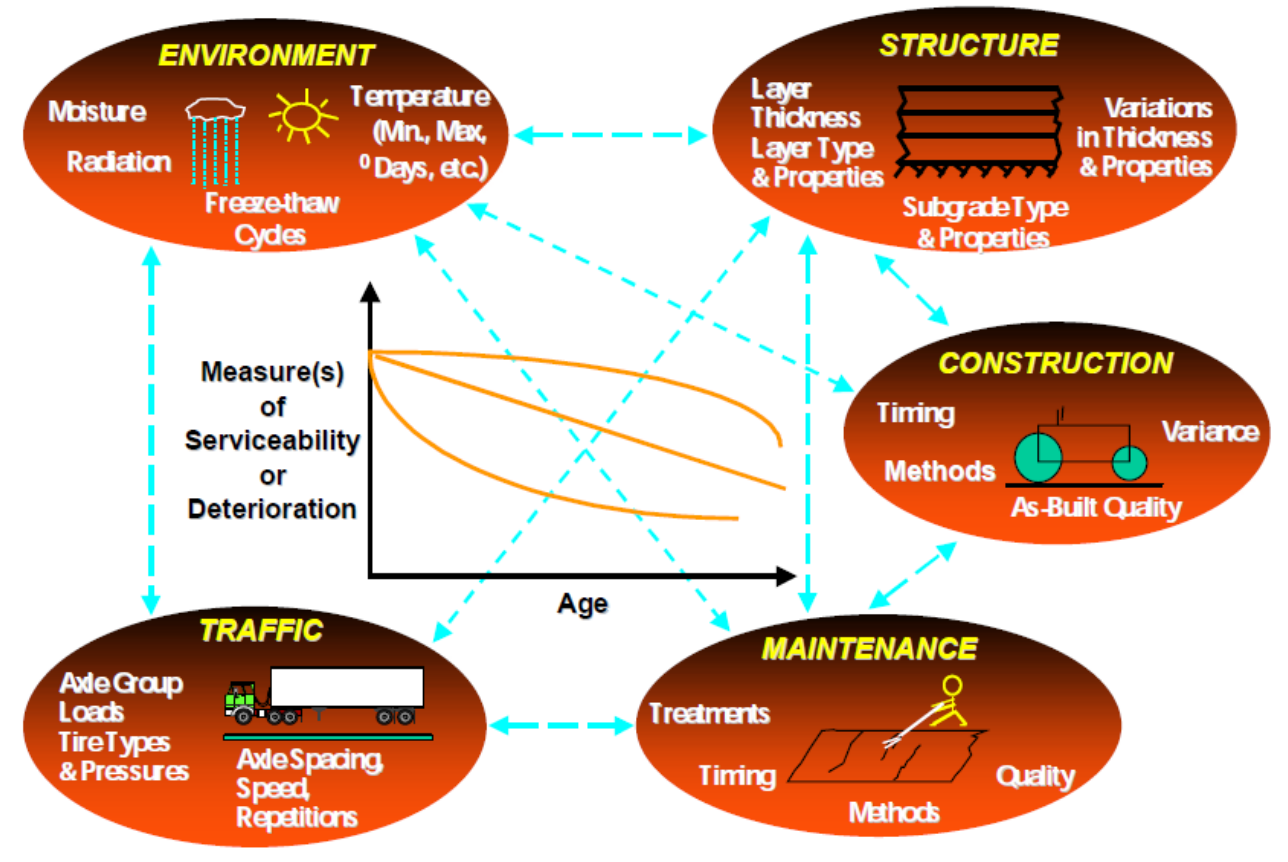

Figure 2.1 Factors influencing pavement performance (from Tighe et al., 2001)

While construction and maintenance activities clearly affect the pavement performance, these factors cannot be directly accounted for in the design process. Much emphasis is, therefore, given to the other parameters used in the design: namely traffic, the environmental conditions, and structure and materials.

\subsection{Pavement design approaches}

The major components of pavement design are the input system, prediction models, transfer functions and an output system that consists of predicted pavement performance. Current pavement design approaches (Jiménez and Mrawira, 2012) can be divided into two categories: deterministic and probabilistic. In a deterministic approach single values are adopted for all input parameters and the prediction results are single numbers of pavement performance. In contrast, probabilistic analysis treats all input parameters as variables that change according to an assigned probability distribution function. Probabilistic analysis also yields a more comprehensive estimate than deterministic analysis by providing a range of likely outputs. 


\subsubsection{Probabilistic analysis}

Probabilistic analysis, first introduced by Darter and Hudson in 1973, has received considerable attention in pavement management. Many examples exist in the literature where transformation of pavement performance prediction from a deterministic to a probabilistic approach has been developed. Generally, probabilistic analysis represents a reliability-based approach to pavement design to address uncertainties in predicting performance of a pavement during its intended life.

In the literature often probabilistic assessments of pavement performance were carried out by 1) ignoring traffic uncertainties and/or environmental actions, i.e., the pavement performance is a function of a set of random variables that do not vary with time (Alsherri and George 1988); or 2) assuming that the pavement deterioration can be modelled as a Markov process (Wang et al. 1994, Li et al. 1996, Lemer and Moavenzadeh 1971).

Probabilistic analysis based on the assumption given in 1) can be carried out by using, for example, the first order second moment method (Basma and AlBalbissi 1989) or the first order reliability method (FORM). The use of FORM has been considered by Chua et al. (1992) for predicting pavement distress and Kim and Buch (2003) for fatigue design of a pavement. Simple simulation techniques are also often used to assess the pavement performance (Lemer and Moavenzadeh 1971, Timm et al. 2000, Hong and Somo 2001). All of these techniques basically attempt to evaluate the multidimensional probability integral that defines the structural failure.

The Markov approach has been used to address the stochastic variation of the traffic load and environmental effects. The Markov chain based models for pavement performance prediction consider that if one can predict the pavement performance at time $\mathrm{t} 1$, the pavement performance at time $\mathrm{t} 2, \mathrm{t} 2>\mathrm{t} 1$, is not known exactly except in probabilistic terms. The Markov Chain is perhaps the most popular probabilistic analysis technique for network level analysis. Probabilistic methods involve the development of a Transition Probabilities Matrix (TPM). Li et al. (1996) suggested constructing the transition matrix through Monte Carlo simulation (i.e., by treating data input of 
pavement design parameters as random variables) and using the constructed transition matrix to predict the pavement performance. However, according to Paramapathy and Pandey 2000 (as cited in Hong and Somo 2001), one may directly evaluate the pavement performance using simple simulation techniques instead of constructing such a matrix.

Whether the possible temporal correlation between the traffic load and the environmental effects can be ignored for reliability analysis was investigated by Wang and Hong (2004). They concluded that the pavement performance variability is relatively insensitive to uncertainties in traffic load and environmental actions. Pavement performance variability is instead more sensitive to uncertainties in material properties and geometry variables (subgrade stiffness and total pavement thickness).

\subsubsection{Performance prediction models}

Performance prediction models can be divided into purely mechanistic, empirical (regression) models, and mechanistic-empirical.

\subsubsection{Mechanistic}

Mechanistic models require knowledge of the mechanical behaviour of the various materials - specifically their stress-strain responses. However, the materials used in pavements do not have linear elastic behaviour and using linear elastic models is a large simplification. For example, granular materials have a nonlinear elastic response, which depends on the confinement of the material and the degree of compaction and saturation. Asphalt concrete has a visco-plastic response to loading, dependent on the compaction of the material, the time of loading, and the temperature. Predictions from any deterministic or mechanistic model only provide the mean value, and are not capable of modelling the dispersion. The use of probabilistic approaches to address such limitations on performance modelling has been suggested elsewhere (Li et al., 1996). 


\subsubsection{Empirical}

Empirical procedures are those that rely on models developed from experience or observations of past performance. Traditional regression modelling employs either: the least square or the maximum likelihood approach, to find the values of the coefficients of a given functional form (linear, exponential, potential, logarithmic or polynomial). An example of empirical models is the 1993 AASHTO guide which is based on empirical models drawn from field performance data measured at the AASHO road test in the late 1950's. The main limitations of empirical models are:

- Developed for use under particular conditions - difficult to use under different conditions.

- Most of them do not contain material properties.

- They are not comprehensive (do not consider all influencing factors).

The UK pavement design method described in HD 26 (Design Manual for Roads and Bridges, Volume 7) is semi-empirical (analytical alternatives are allowed).

\subsubsection{Mechanistic-empirical}

Mechanistic-empirical models combine some mechanical modelling with an empirically calibrated transfer function, relating the distress prediction to some calculated critical stress/strain in the pavement. They are particularly useful at the project level - for design of the pavement structure. An example of mechanistic-empirical models is that provided in the $2008 \mathrm{M}-\mathrm{E}$ Design Guide (AASHTO, 2008). The main limitations of M-E models are:

- Accuracy depends on both the response models and the performance models.

- Response models contain several simplifications (eg. Material behaviour).

- More detailed data input is required. 
A pavement is designed to withstand the design traffic during its design life. A pavement failure is characterised by the development of a particular type of distress (such as fatigue cracking and rutting on flexible pavements) of sufficient severity and extent at different points within a pavement section. Despite a pavement section being designed and constructed the same way, random variations in material properties and as-built characteristics cause localised deficiencies.

\subsubsection{Stress calculation}

A number of different analytical models can be used to predict the stress, strain and deformation in a pavement under simulated wheel and environmental loading conditions. The main models are based on multilayer elastic theory and Finite Element analysis. The multilayer elastic models are relatively easy to operate and widely used. However, they cannot address issues related to environmental loading (i.e., that due to daily temperature changes, moisture variations etc.). FE models are capable of addressing both wheel and environmental loading but are very complicated to operate and timeconsuming.

\subsubsection{Transfer functions}

Transfer functions are relationships developed to relate the state of stress in a pavement to its overall performance. In current M-E design procedures for flexible pavements - despite the multitude of relationships available - the primary transfer functions are those that relate 1) wheel load tensile strain at the bottom of the asphalt layers to eventual fatigue cracking and 2) wheel load compressive strain (or stress) at the top of the subgrade to permanent deformation. These models are generally derived from extensive research on correlations between pavement response and observed performance of laboratory test specimens, full-scale road test experiments (i.e., AASHTO), or by both methods. 


\subsection{Performance of pavement designs}

All variations in materials and construction, if not properly incorporated into the design procedure, may contribute to premature failure of a pavement structure. The author's experience has shown that some recently built motorways in the UK have suffered from premature failure because of the ignoring of these variabilities. In addition, the design equations are often based on observations of the performance of sections of roads built in the past where different material properties and construction techniques were employed compared to those of today. This issue probably justifies the large difference sometimes observed between the performance life and the intended design life.

These considerations are expressed in Figure 2.2. For example, although a pavement is designed for a 20 year design period, with reconstruction or rehabilitation expected at the end of the design life, the actual pavement may exhibit sections that fail prematurely due to, for example, errors in design, in construction, in materials and in maintenance.

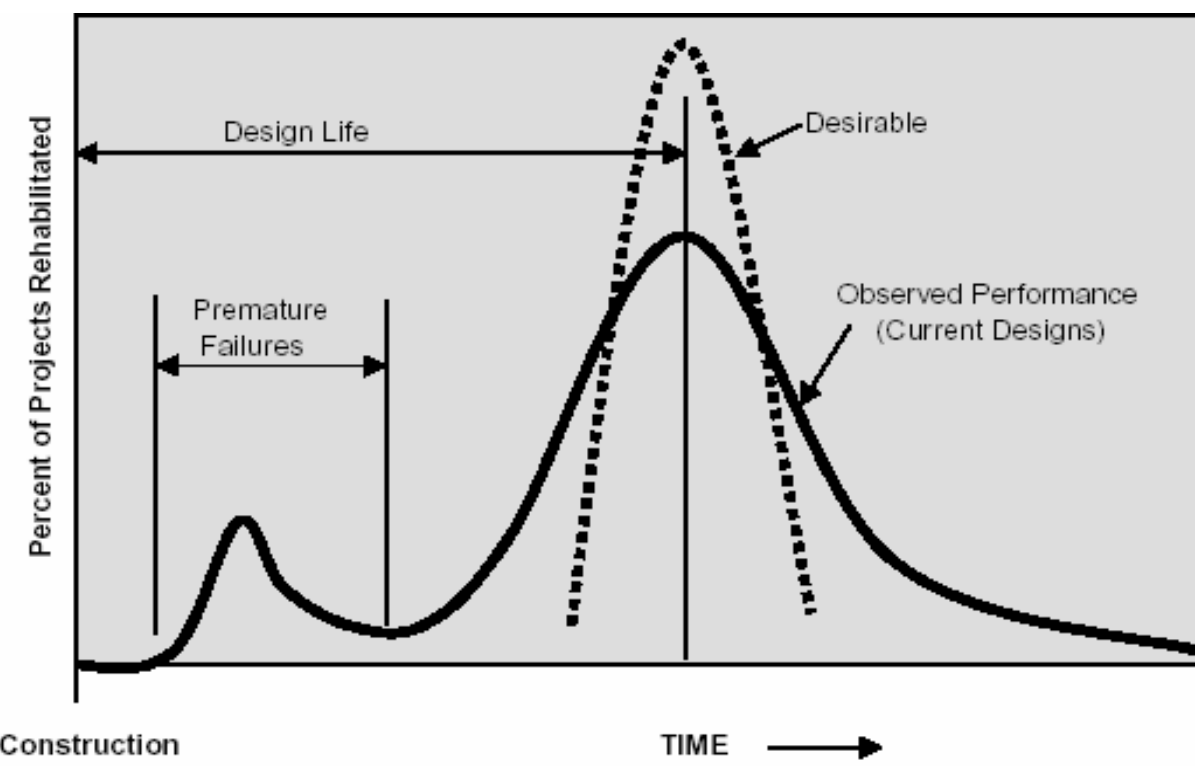

Figure 2.2 Performance of existing pavement designs (from Transportation Research Circular E-C118, 2007)

If the material variability were known and included in the design procedure the difference between the design and in-service life should be reduced. This would also allow better and more robust decisions to be taken in terms of whole life cost analysis. 
Material variability can be described by statistical terms such as mean and standard deviation (assuming a normal distribution) or by its probability density distribution. A useful dimensionless way of expressing the variability of a material's property is to use the ratio of the standard deviation over the mean, known as coefficient of variation (COV). Knowledge of the coefficient of variation of each design input is extremely important to more accurately estimate their influence on the predicted pavement life.

\subsection{Impact of variability on pavement performance}

Many pavement design procedures are based around single values of the pavement and traffic characteristics which represent average conditions average values, sometimes with a margin of safety, that do not account for variability in the pavement and traffic loads. Variability exists in pavements due to construction practices, quality control, environmental conditions, material characteristics and traffic conditions and this variability has been known for quite a while (Darter et al., 1973).

Commonly observed variability in pavement characteristics have been quantified by Darter et al. (1973), Noureldin et al. (1994), Hughes (1996), Kim et al. (2003), Huang (2004). The variability associated with traffic and pavement parameters has a significant impact on the performance of pavements (and reliability of pavement design) and should, therefore, be addressed and quantified when modelling pavement performance and when developing maintenance programmes. Therefore, the major design input parameters for pavement design such as moduli of layers, thickness of layers, traffic volume etc. should each be defined as a random variable with its mean and standard deviation (assuming a normal distribution) or its complete probability distribution. The pavement performance function can subsequently also be characterised in statistical terms. In other words, because the values used to calculate the performance life of a pavement structure (e.g. fatigue life $\mathrm{N}_{f}$ ) are not exact values but are distributed over a range, for each pavement there is an expected value of $\mathrm{N}_{\mathrm{f}}$ and associated variance that describes the distribution $\mathrm{N}_{\mathrm{f}}$ will follow. George and Husain (1986) and later Prozzi and Guo 
(2007) have supported previous significant experimental evidence that the distribution of fatigue lives at a particular stress level is lognormal.

Quantifying and analysing variability of pavement materials and design inputs are, therefore, fundamental in developing a probabilistic-based design that evaluates reliability.

Kim et al. (2003) grouped the main sources of uncertainty in the AASHTO ME flexible pavement design into two categories:

- Uncertainties of design parameters and

- Systematic errors

The first group represents the spatial variability and inconsistent estimation of the parameters (e.g. random measurement error in determining the strength of subgrade soil) while the second group is related to the model bias in predicting pavement performance.

\subsection{Uncertainties of design parameters}

\subsubsection{Variations within a design length}

Spatial variability includes the differences in the basic properties of materials from one point to another in what are assumed to be homogeneous layers within a design length as well as differences in the material and cross-sectional properties due to construction quality. This is also referred as inherent variability or randomness.

\subsubsection{Variations in pavement/subgrade stiffness}

Darter et al. (1973) reported the results of pavement and subgrade stiffness variability related to 153 in-service pavements in Texas as obtained by FWD (Falling Weight Deflectometer) measurements:

- The pavement and subgrade stiffness coefficients come from approximately normal distributions, with a tendency to skew toward the lower values. 
- The in-situ coefficient of variation for the various materials considered ranged from 9 to 24 percent.

- The average coefficient of variation of the subgrade stiffness coefficient was $10 \%$.

\subsubsection{Variations in pavement layer thickness}

Darter et al. (1973) reported an average coefficient of variation of about $10 \%$ for the pavement thickness (from core measurements) with an approximately normal distribution type.

\subsubsection{Variations in smoothness}

Roughness is related to the riding quality property of a pavement and affects the initial serviceability index in the AASHTO design manual. Darter et al. (1973) showed that the serviceability index calculated from a Surface Dynamics Profilometer followed a normal distribution.

\subsubsection{Variations between design and actual values}

These variations result mainly from pavement construction conditions and variability can be minimised through standard specifications and quality control and assurance processes. Variations are for pavement and subgrade stiffness, layer thickness, climatic variations, pavement smoothness and traffic load forecasting variations.

\subsubsection{Pavement and subgrade stiffness}

Darter et al. (1973) reported that these variations can be quite large. Figure 2.3 shows the wide range in stiffness, especially for the treated base materials, that exists in-situ in highway pavements for the same material type. 


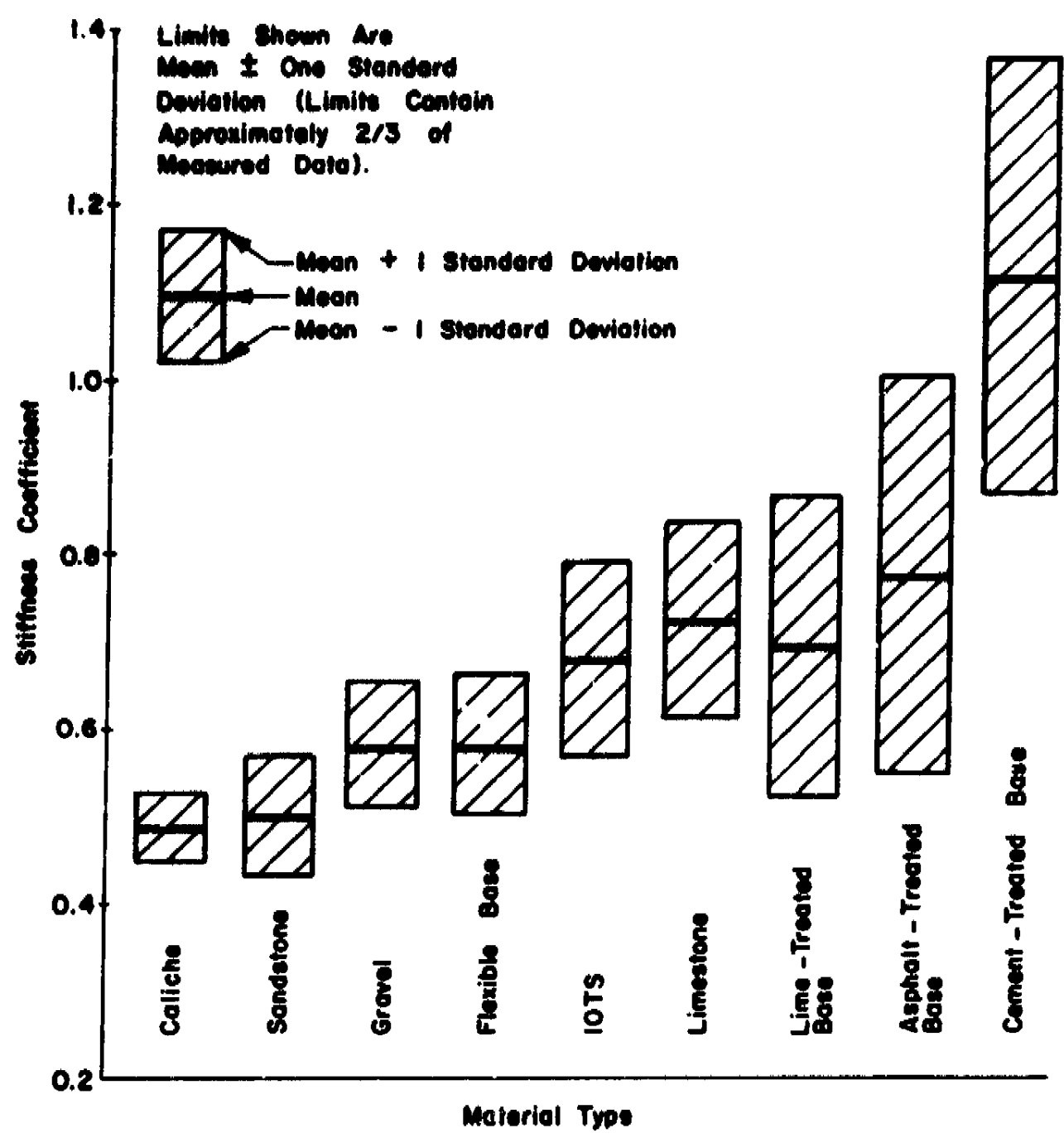

Figure 2.3 Illustration of in situ stiffness coefficients for various pavement materials (Figure 4.12 from Darter et al. 1973)

\subsubsection{Layer thickness}

Selezneva et al. (2003) studied layer thickness variability by analysing thickness data (elevation and core measurements) from a large number of newly constructed flexible and rigid pavement sections taken from the Long Term Pavement Performance (LTPP) database. The study - which focussed on the comparison between the as-constructed and as-designed flexible pavement layer thicknesses - concluded that:

- The mean deviations between as-designed and as-constructed layer thicknesses for layers with the same material type and design thickness combination follow a normal distribution. 
- Despite different computed description statistics between elevation and core measurement data, the variances for core and elevation measurements can be assumed "equal" for the majority of the layers, while the means are tested "equal" for $46 \%$ of the layers.

- The mean constructed layer thicknesses tend to be above the designed value for the thinner layers and below the design value for the thicker layers for the same layer and material type.

A study from Aguiar-Moya et al. (2009) on Long Term Pavement Performance (LTPP) SPS-1 sections located in the state of Texas concluded that $86.1 \%$ of the analysed pavement layers - hot-mix asphalt (HMA) surface layer, the HMA binder course, and the granular base layer, as determined by Ground Penetrating Radar (GPR) - have normally distributed thicknesses.

Maser et al. (2013) have demonstrated the use of non-destructive evaluation (NDE) methods - represented by GPR surveys - for rapidly determining the average pavement thickness on a newly constructed section to be within $2.5 \mathrm{~mm}$ of the true value (accuracy requirement for quality assurance of new construction, according to Deacon et al. 1997 as cited in Maser et al. 2013), without extensive reliance on cores.

A 'pegless paving control' is not a dream anymore. In an article to the NCE magazine Hayward (2010) describes the incredible accuracy that is reached nowadays with 3D paving technology. A $5 \mathrm{~mm}$ level accuracy was achieved on the Irish motorway contact mentioned in the article without a site-worn ruler or sagging fish-gut stringline to worry about. Accuracy to as low as $1 \mathrm{~mm}$ level is claimed by the manufacturer, Trimble PCS900 Paving Control System.

\subsubsection{Climatic variations}

Environmental variations have an impact on the pavement and subgrade stiffness values. Unbound materials are affected by moisture change and by freeze-thaw cycles during winter and spring seasons. Asphalt concrete responds to temperature variations, which affects directly the dynamic modulus of the mixture. 


\subsubsection{Pavement smoothness}

An estimate of the difference between the initial design serviceability of a newly constructed pavement and the actual as-built serviceability was reported by Darter et al. (1973) - based on serviceability measurements made in Utah and Minnesota - to be negligible.

In a more recent extensive study carried out by AASHTO in 2008, data collected from 32 states showed that network-level IRI (International Roughness Index) data from different states contain baseline measurement error of the order of 15 percent due to differences in equipment, calibration practices, and variations across operators.

\subsubsection{Traffic load forecasting variations}

Proper vehicle classification, accurate estimate of average daily traffic, and percentage of different vehicle types in a given lane are particularly important in the calculation of the cumulative design traffic that the pavement will carry. In the case of new roads this forecast is particularly difficult as the traffic volume and type are not known.

Darter et al. (1973) reported that there are many uncertainties associated with the design load that a pavement will carry. They reported the following coefficients of variations for the main types of uncertainties:

- Design AADTs (i.e. conversion of mix traffic to 8t equivalent axle loads): Existing highways COV from $10 \%$ to $20 \%$; New highways COV from $15 \%$ to $30 \%$.

- Axle factor: COV from $5 \%$ to $15 \%$.

- Percent trucks: COV from $10 \%$ to $15 \%$.

Shekharan and al. (2002) investigated the variation in reliability with traffic loads and they concluded that:

- The reliability decreases with an increase of Class 9 vehicles (i.e., five-axle semitrailer trucks with a steering axle, tandem drive axles, and rear tandem axles). 
- The variation in reliability of pavements that are thicker is less. In other words, in thinner sections (lower structural number or thinner slab) reliability changes rapidly with an increase in the number of ESALs or the percentage of heavier trucks.

\subsubsection{Variability due to the imprecision in quantifying the parameters}

The imprecision in quantifying the parameters is related to the random measurement error of the pavement material properties. This variability is usually minimised through standard testing procedures and annual correlation trials. Although a level of uncertainty is always inherent to any measurement process, it must also be appropriately quantified or assessed.

\subsubsection{GPR (Ground Penetrating Radar) accuracy}

GPR surveys are normally calibrated against cores to increase their reliability. In the USA, Maser and Vandre (2006) defined the accuracy of GPR data as the deviation between a core thickness and the GPR thickness calculated at the core location and reported the expected accuracy to be from 5 to $10 \%$ of the total thickness for project level evaluations. According to Loizos and Plati (2007), at network level GPR surveys are sufficient and effective in estimating the AC (Asphalt Concrete) layer thickness; additional cores to support GPR surveys are recommended for detailed investigation.

In the UK, annual accreditation trials take place for all the major condition survey techniques used on the network, including the GPR equipment. Clause 6.20 in HD 29/08 states that "GPR trials have shown that at slow speed $(<25 \mathrm{~km} / \mathrm{h})$, GPR could determine the combined bound layer thickness with an accuracy of approximately 5 per cent of the real thickness. However, at traffic speed $(70 \mathrm{~km} / \mathrm{h})$ the error could increase to approximately 9 per cent. For underlying layers of hydraulically bound and unbound material, the expected accuracies are approximately \pm 15 per cent and \pm 30 per cent, respectively, of the real thickness." As for core survey accuracy, Clause 6B.9 in HD 29/08 states that "Typically, the accuracy with which the actual thicknesses may be measured from a core is $\pm 5 \mathrm{~mm}$ if the core is fully extracted and not damaged." 
The impact of GPR layer thickness errors on data analysis, such as in FWD backanalysis, has been found in several studies (Briggs et al. 1992, Collop 2000, Lagarde-Forest et al. 2008). The main conclusions from their investigations are:

- Variations in layer thicknesses did not appreciably affect the backcalculated modulus of the subgrade.

- Variations in layer thicknesses affect noticeably the backcalculated modulus of the asphalt surfacing and unbound base materials.

- According to Collop (2000), incorrect assumptions regarding the mean asphalt layer thickness primarily influence the mean value of the backcalculated asphaltic material stiffness modulus. For example, incorrect assumptions regarding the correct asphalt layer thickness in the range of $\pm 15 \%$ result in a FWD stiffness modulus mean error of between $~-30 \%$ and $+40 \%$.

- According to Collop (2000), both incorrect assumptions regarding the mean asphalt layer thickness and variations in the thickness of the asphalt layer around the mean value influence the standard deviation of the backcalculated asphaltic material stiffness modulus. For example, random asphalt thickness variations in the range 0 to $15 \%$ result in a FWD stiffness modulus standard deviation error of between $\sim-30 \%$ and $+340 \%$.

Clause 5.28 in HD 29/08 gives a flavour of the impact of inaccurate layer thickness information on backcalculated moduli, which is "For example, a 15 per cent underestimate of the thickness of a bound layer can result in a fifty per cent overestimate of the stiffness of that layer."

To improve the reliability of thickness data obtained by GPR surveys LagardeForest et al. (2008) proposed a unified Quality Assurance approach which includes operator training, equipment accreditation, as well as internal and external audit requirements. 


\subsubsection{FWD accuracy}

The accuracy of FWD measurements is important primarily for backanalysis calculation of pavement layer moduli.

Typical FWD specifications state that "Deflections shall be accurate to \pm 2 percent or \pm 2 microns, whichever is larger."

- The \pm 2 micron (standard deviation) error is a random error, independent of the magnitude of the deflection;

- The \pm 2 percent error is a systematic error.

Typically, the root mean square error is used to quantify the goodness of fit of the entire set of deflections and, therefore, to assess the accuracy of the backcalculated moduli. The solution produced by computer programs for the backcalculated pavement moduli at each measured point is considered satisfactory if the error between the measured and the computed deflection is minimised to a specified threshold. However, this criterion does not necessarily give reasonable values, especially because the iterative process during backcalculation depends heavily on the initial seed value provided by the user. Also, other important aspects such as the presence of damaged layers, thickness variation, and temperature changes are known to significantly affect the results.

Irwin et al (1989) simulated the effect of random deflection measurement errors combined with random variability of pavement layer thickness. They concluded that while the combined variability did not noticeably affect the backcalculated modulus of the subgrade a high degree of variability was observed in the backcalculated moduli of the surface and (unbound) base course layers.

\subsubsection{Weigh-in-motion (WIM) systems accuracy}

WIM systems are used to automatically collect traffic data information for pavement design or for monitoring performance in the case of existing pavements. One source of variation is the calibration drift. Figure 1 in Shekharan et al. (2002)'s paper (see Figure 2.4) shows the effects of scale 
calibration error on the computation of ESAL values. For example, a 5\% error in the determination of actual load values can result in about a $21 \%$ difference in the ESAL values.

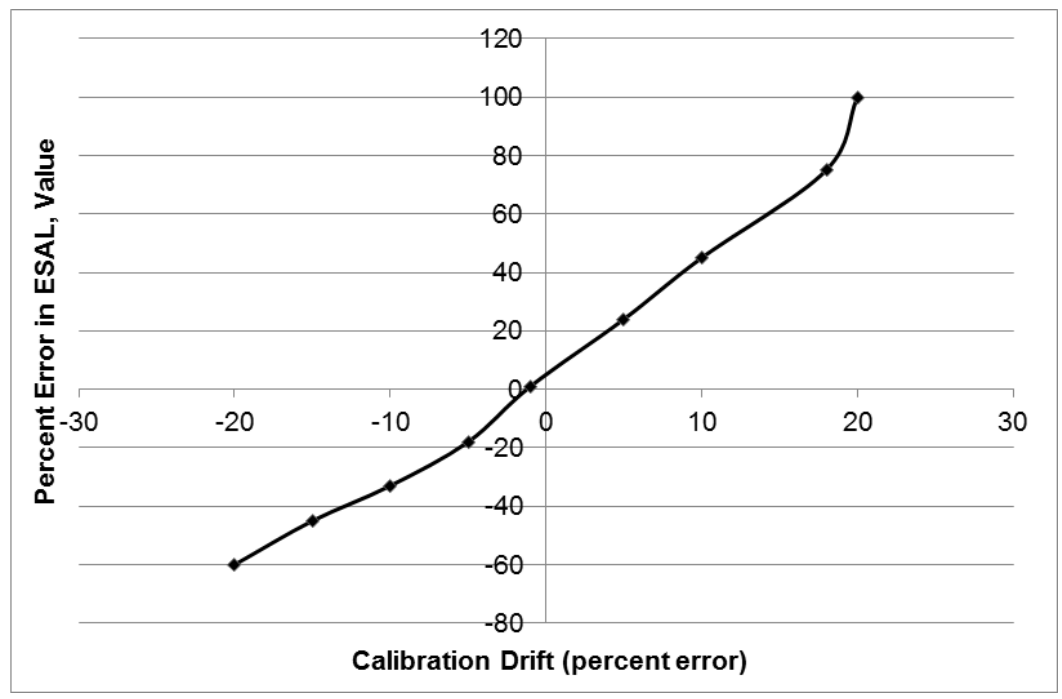

Figure 2.4 Effect of Weigh-in-Motion scale calibration drift on the accuracy of ESAL calculations (from Shekharan et al. 2002)

\subsection{Systematic errors}

\subsubsection{Variations due to model bias}

Model bias is related to the assumptions made and to the simplification of a complex pavement performance model to a simple mathematical expression. Theyse (2006) demonstrated how important it is that realistic and accurate stress/strain and damage models are used in the design method. He showed that any error in the engineering models is reflected out of proportion in the structural capacity estimate because of the mostly logarithmic expressions used in the engineering models.

For example, in mechanistic pavement designs the prediction of pavement fatigue life is related to the estimates of fatigue cracking which is assumed to be related to the tensile strain at the bottom of the asphalt layer, calculated using a layered elastic analysis, at a single temperature and using the stiffness modulus of the bituminous mixture, measured from frequency sweep tests (Pais et al. 2002). The fatigue life is usually obtained by fatigue testing conducted at relatively large strain levels, and using few strain levels to 
minimise the required laboratory testing time. Then, assuming logarithmic linearity in the fatigue life, a regression line is typically fitted to the data and extrapolation is applied to the design strain level. The accuracy of this extrapolation depends on the number of laboratory fatigue tests used to obtain the fatigue life. The fatigue law, which represents the best fit of all fatigue test results, is obtained testing specimens at two or more strain levels and some replicates for each strain level. However, an increased accuracy in the fatigue life by increasing the number of test strain levels and number of replicates for each strain level needs to be balanced with the increased costs of material characterisation. Pais et al. (2002) carried out a statistical analysis and concluded that the variability of fatigue life can be reduced by using 3 strain levels and at least 3 test replicates for each strain level.

\subsubsection{Variations due to statistical error}

Statistical error derives from the lack of fit of the regression equation of the design models as well as the sample size. The lack-of-fit deviation represents one or more deficiencies in the design equation and the difference may be either systematic or random (Shekharan et al., 2002). Changing mathematical models to refit the original data and changing fitting procedures may alleviate the design equation deficiencies but a certain degree of lack of fit will always exist.

This type of variation is due to the poor estimate of exact results when actual average values of all design parameters are known. In other words it is due to the lack of fit between the model predictions and actual results. Darter et al. (1973) performed a quantitative study about the effect of variations of the design factors on pavement costs and for different levels of reliability. The study (see Figure 2.5) shows that among all variations, the variations due to lack-of-fit associated with the design models and the variations associated with the pavement/subgrade are the most influential in increasing the costs of a pavement design. The costs also were shown to increase with the increase in the level of reliability. 


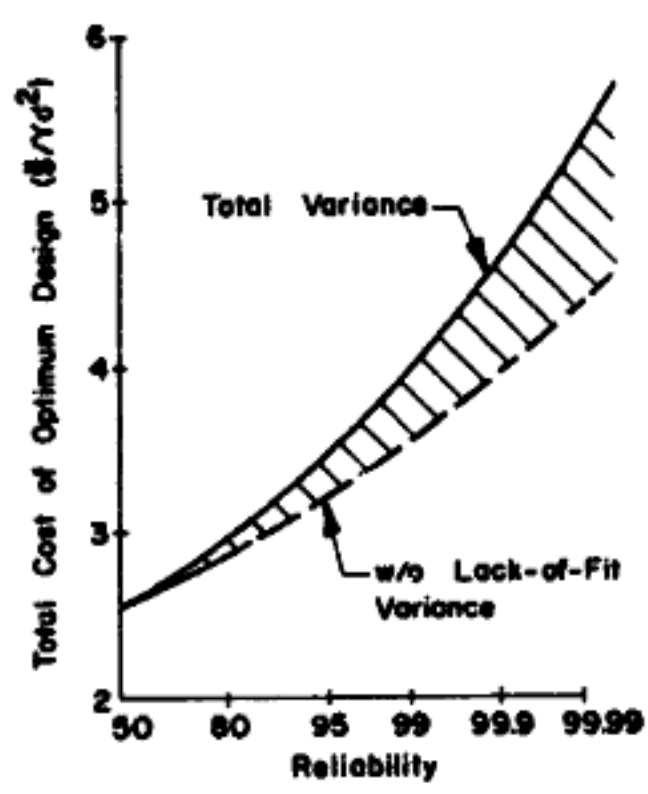

(a) Lack-of-fit.

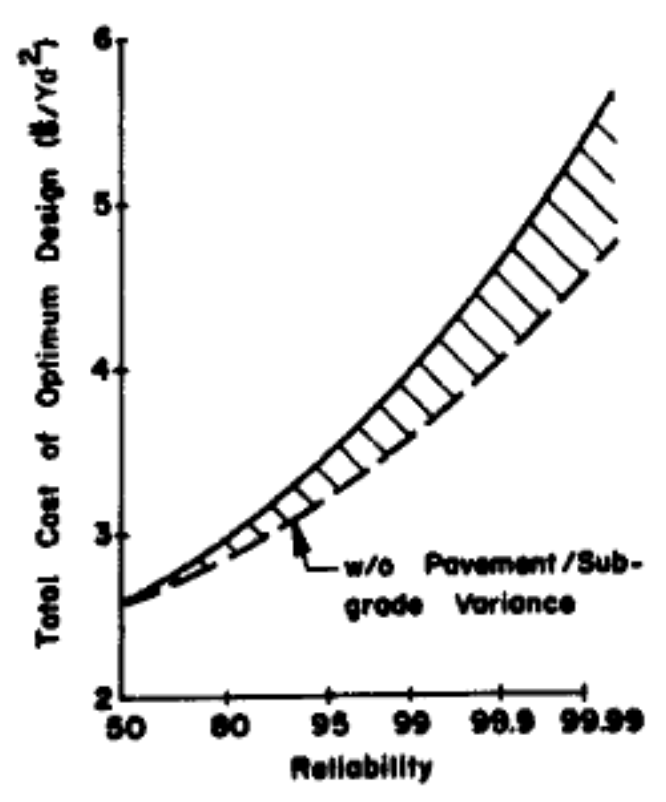

(c) Paveoat/ouberade.

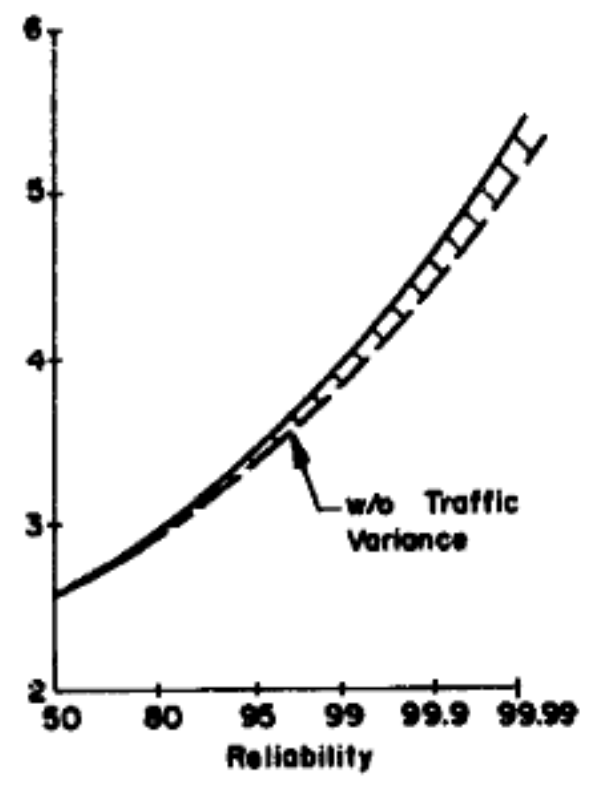

(b) Traffic forecasting.

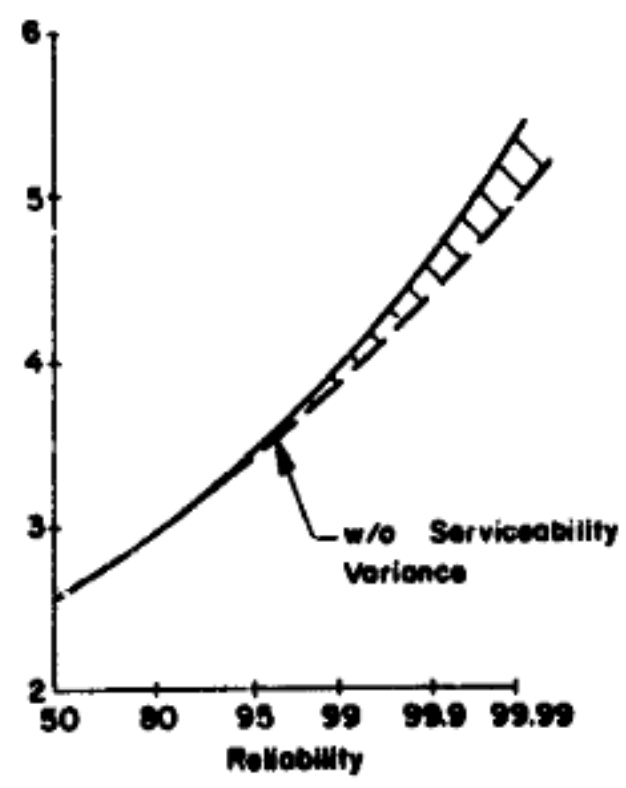

(d) Inft1al serviceability.

Figure 2.5 Total cost versus reliability for specific pavement design problem (from Darter et al. 1973)

- Prozzi et al. (2005) also confirmed that the surface thickness and the model error have the largest influence on pavement performance while the base and subbase thicknesses have the smallest effect on performance. They estimated that if the surface thickness (which in the UK generally includes the binder course too) is sampled one standard deviation below its mean it will, on average, cause a reduction in pavement life of approximately 3 
years. If the model error (modelled as normal random variables with a zero mean and certain standard deviation) is sampled one standard deviation below its mean it will, on average, cause a reduction in pavement life of approximately 5 years. If the base and subbases have values that are one standard deviation below their means the pavement life is, on average, reduced by one and a half years. They also emphasised that to increase pavement life - since model error is inherent to the modelling process and can not be avoided - placing stricter quality control on the upper layers is far more important than controlling the base and subbase thicknesses.

- Prozzi et al. (2005) and Darter et al. (1973) concluded that model error can not be ignored when considering overall pavement performance variability.

The variability from the modelling process can be minimised by considering and refining all design parameters that affect pavement performance (Harr, 1987).

\subsection{Summary of variability of design input parameters}

A summary of the variability of design input parameters from published sources - for the Mechanistic-Empirical pavement design approach - is depicted in Table 2.1. 
Table 2.1 Summary of pavement material COVs from available literature (for the Mechanistic-Empirical pavement design approach)

\begin{tabular}{|c|c|c|c|c|c|}
\hline \multirow[t]{2}{*}{ Property } & \multirow[t]{2}{*}{ Description } & \multicolumn{4}{|c|}{ Previous Investigation } \\
\hline & & $\begin{array}{l}\text { Range of } \\
\text { COV (\%) }\end{array}$ & $\begin{array}{l}\text { Typical COV } \\
(\%)\end{array}$ & $\begin{array}{l}\text { Type of } \\
\text { distribution }\end{array}$ & Reference \\
\hline \multirow[t]{8}{*}{$\begin{array}{l}\text { Layer } \\
\text { Thickness }\end{array}$} & \multirow[t]{2}{*}{$\begin{array}{l}\text { Bituminous } \\
\text { surface }\end{array}$} & $3-12$ & 7 & Normal & $\begin{array}{l}\text { Timm et al. (2000), } \\
\text { Noureldin et al. (1994) }\end{array}$ \\
\hline & & $3.2-18.4$ & 7.2 & Normal & $\begin{array}{l}\text { Aguiar-Moya et al. } \\
(2009)\end{array}$ \\
\hline & \multirow[t]{2}{*}{$\begin{array}{l}\text { Bituminous } \\
\text { binder course }\end{array}$} & $11.7-16.0$ & 13.8 & Normal & $\begin{array}{l}\text { Aguiar-Moya et al. } \\
(2009)\end{array}$ \\
\hline & & $5-15$ & 10 & Normal & Noureldin et al. (1994) \\
\hline & \multirow[t]{2}{*}{ Granular base } & $10-15$ & 12 & Normal & Noureldin et al. (1994) \\
\hline & & $6.0-17.2$ & 10.3 & Normal & Noureldin et al. (1994) \\
\hline & $\begin{array}{l}\text { Granular } \\
\text { subbase }\end{array}$ & $10-20$ & 15 & Normal & Noureldin et al. (1994) \\
\hline & $\begin{array}{l}\text { Overlay } \\
\text { thickness }\end{array}$ & & & Lognormal & Tighe (2001) \\
\hline \multirow{8}{*}{$\begin{array}{l}\text { Elastic } \\
\text { Modulus }\end{array}$} & \multirow{2}{*}{$\begin{array}{l}\text { Bituminous } \\
\text { Layers }\end{array}$} & $10-20$ & 15 & Normal & Noureldin et al. (1994) \\
\hline & & $10-40$ & & Lognormal & Timm et al. (2000) \\
\hline & \multirow[t]{2}{*}{ Granular base } & $10-30$ & 20 & Normal & Noureldin et al. (1994) \\
\hline & & $5-60$ & & Lognormal & Timm et al. (2000) \\
\hline & \multirow{2}{*}{$\begin{array}{l}\text { Granular } \\
\text { subbase }\end{array}$} & $10-30$ & 20 & Normal & Noureldin et al. (1994) \\
\hline & & $5-60$ & & Lognormal & Timm et al. (2000) \\
\hline & \multirow[t]{2}{*}{ Subgrade } & $10-30$ & 20 & Normal & Noureldin et al. (1994) \\
\hline & & $20-45$ & & Lognormal & Timm et al. (2000) \\
\hline $\begin{array}{l}\text { Marshall } \\
\text { Stability }\end{array}$ & & $10-20$ & 15 & Normal & Noureldin et al. (1994) \\
\hline \multirow[t]{3}{*}{ CBR } & Base & $10-30$ & 20 & Normal & Noureldin et al. (1994) \\
\hline & Subbase & $10-30$ & 20 & Normal & Noureldin et al. (1994) \\
\hline & Subgrade & $10-30$ & 20 & Normal & Noureldin et al. (1994) \\
\hline \multirow{4}{*}{$\begin{array}{l}\text { Percent } \\
\text { Compaction }\end{array}$} & Surface & $1-2$ & 1.5 & Normal & Noureldin et al. (1994) \\
\hline & Binder course & $2-3$ & 2.5 & Normal & Noureldin et al. (1994) \\
\hline & Subbase & $2-3$ & 2.5 & Normal & Noureldin et al. (1994) \\
\hline & Subgrade & $2-3$ & 2.5 & Normal & Noureldin et al. (1994) \\
\hline$\frac{\text { Maximum }}{\text { deflection }}$ & & $10-30$ & 20 & Normal & Noureldin et al. (1994) \\
\hline \multirow[t]{3}{*}{$\overline{\text { Traffic }}$} & & 42 & & Lognormal & $\begin{array}{l}\text { NCHRP Project 20-7/24 } \\
\text { (1985) }\end{array}$ \\
\hline & & - & & $\begin{array}{l}\text { Extreme Value } \\
\text { Type I }\end{array}$ & Timm et al. (2000) \\
\hline & & - & & $\begin{array}{l}\text { Normal, } \\
\text { Lognormal } \\
\text { and Poisson }\end{array}$ & $\begin{array}{l}\text { Zollinger and } \\
\text { McCullough (1994) }\end{array}$ \\
\hline
\end{tabular}




\subsection{Summary of sensitivity of design input on pavement performance}

The results of various sensitivity analyses carried out in the past to assess the variability of each design parameter and to measure its effect on the design are summarised below. They refer to a typical 3-layer flexible pavement structure consisting of $A C$ (asphalt) surface (equivalent to surface course and binder course in the UK), on top of a granular aggregate base and subgrade.

\subsubsection{Critical input parameters}

- As first shown by Darter et al. (1973), Li et al. (2002) quantified the effect of variability associated with layer materials and thicknesses, traffic (ESALs), and lack-of-fit error in asphalt pavements on reliability. The conclusions of their sensitivity analysis are that the most influential design inputs on reliability are layer properties and thickness, followed by traffic and lack-of-fit error. For example, for a COV of $15 \%$ in the above variables the estimated reliability after 15 years - from an initial value of 1 at construction - is expected to decrease to $\sim 0.915,0.975$ and 0.968 due to variability in layer properties and thickness, traffic and lack-of-fit error respectively. The reliability is defined as the probability that the pavement's traffic load capacity exceeds the cumulative traffic loading on the pavement design during a selected design life.

- The parameters with the greatest influence on the variability of predicted fatigue performance, without considering variable loads, are AC (asphalt) modulus and thickness (Timm et al. 2000, Maji and Das 2008).

- Fatigue cracking is affected by changes in the asphalt layer thickness while it is unaffected by changes in the granular base layer thickness (AguiarMoya et al. 2009).

- The parameters with the greatest influence on the variability of predicted deformation (rutting) performance, without considering variable loads, are the granular base thickness, AC (asphalt) thickness, and stiffness of the subgrade (Timm et al. 2000, Maji and Das 2008). 
- If the traffic axle weight variability is added the output variability for fatigue and deformation performance is significantly changed (i.e., more than doubled). Therefore, the traffic weight is the single most important input parameter (Timm et al. 2000, Shekran et al. 2002, Cunagin and Kent 1998).

\subsubsection{Characteristic distribution}

- Timm et al. (2000) found that the output distributions of both fatigue and deformation performance, obtained from Monte Carlo simulation by using the input distributions, may well be approximated by an extreme-value Type I distribution (sometimes referred to as Gumbel distribution).

- Theoretically, all of the variables associated with pavement structure vary from zero to infinity, and the lognormal distribution may describe their variations best. However, the normal distribution is probably the most popular model because of its simplicity. Li et al. (2002) showed that the difference between the results from assumed normal and lognormal distributions in simulation exercises is negligible. When the variation in a design variable is large, resulting in some cases in negative values when generated by a normal distribution, a log normal distribution is preferred. 


\section{$3 \quad$ Literature review on reliability in pavement design}

The first section of this chapter aims to briefly summarise the fundamental aspects of the classic theory of structural reliability, developed primarily in the US where this approach has been applied to pavement systems (Harr, 1987). The second part summarises worldwide applications of reliability concepts in pavement design. The third part discusses the current gaps in the active area of research in probabilistic pavement design and describes how the study will address this challenge.

\subsection{What is reliability?}

The aim of every engineering system, whether it is a bridge or a dam or a highway, is to provide good value for money at a specified level of safety and in respect of the environment. To achieve these objectives it is necessary to predict the performance of a system for which little or no previous experience exists. The model elements of the civil engineering design process can be thought of as links of a chain (Harr, 1987), see Figure 3.1.

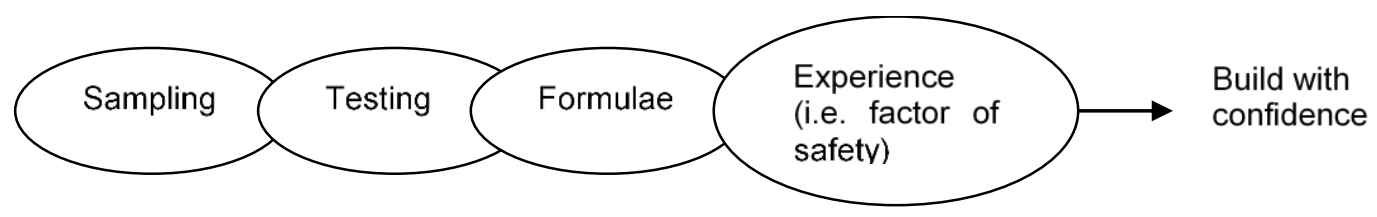

Figure 3.1 Model of the civil engineering reliability (from Harr, 1987)

However, civil engineering systems fail, therefore considerable differences exist between hypothetical and actual systems. All elements of the model, such as properties of materials, induced loadings, and developed design procedures are far from certain. They are all subject to varying degrees of randomness such as material defects, human errors, etc., therefore satisfactory performance cannot be absolutely guaranteed. Instead, assurance can only be given in terms of the probability of success in satisfying some performance criterion, which is referred to as reliability. A common engineering definition of reliability is as follows (Harr, 1987): Reliability is the probability of an object (item or system) performing its required function 
adequately for a specified period of time under stated conditions. The antonym of reliability is failure, which is the inability of a system to perform its intended function adequately on demand over a period of time and under specified conditions.

The failure of a highway is not considered catastrophic as is, for example, the collapse of a bridge. Nevertheless, the economic and social implications of the failure of a highway, in the form of rapid deterioration, cannot be neglected. Reliability can be defined and quantified by using the methods of statistics and probability. Through the statistics (for example mean and standard deviation of a variable) the uncertainty is mathematically quantified; through the probability theory the information from statistics is used to compute the likelihood of specific events (Haldar et al., 2000).

Reliability is indicated by a number between 1 and 0 , with 1 indicating that a structure (for example, a pavement) will certainly perform satisfactorily for the conditions for which it was designed, and 0 indicating that the structure will fail.

\subsubsection{Measures of reliability}

Reliability is often referred to as the probability that the capacity C (i.e., strength, resistance) should at least satisfy the demand D (i.e., stress, load), see Figure 3.2. For flexible pavements, strength is generally defined with distress models, often called design equations or transfer functions. Transfer functions establish the relationship between the structural responses and the permissible number of load repetitions for defined failure criteria. A pavement structure is considered to fail when the level of stress exceeds the level of strength (i.e., excessive cracking and/or rutting). The probability of successful performance, alias reliability, is generally expressed as a decimal, such as 0.99 or as a percentage, such as $99 \%$. The corresponding probability of failure or risk is 0.01 or $1 \%$. Many different terms are used to describe the reliability of an engineering system. Four measures of reliability are commonly used (Harr, 1987):

- The conventional factor of safety FS

- The central factor of safety CFS 
- The safety margin S; and

- The reliability index $\beta$

Variability of the layer materials and construction practices will primarily affect the probability distribution function of the capacity $\mathrm{C}$, whereas the uncertainties associated with vehicle loadings and environmental effects will mainly influence the demand D.

\subsubsection{The conventional factor of safety}

Engineers in the past have accounted for the uncertainties involved in the analysis and design of engineering systems, and therefore assessed the risk of failure by using empirical safety factors, learned from previous experience. The design safety is ensured in a deterministic approach (i.e., using nominal values) by assuring that the capacity is greater than the demand with a specified factor of safety:

$$
\text { Nominal } \quad F_{S}=\frac{C_{N}}{D_{N}}
$$

Where $F_{S}$ is the safety factor, $C_{N}$ and $D_{N}$ are the deterministic (nominal) values of the capacity and demand parameters respectively.

If the calculated factor of safety is greater than a prescribed minimum value learned from experience with a particular design, then the design is considered satisfactory. But safety factors do not absolutely guarantee safety or satisfactory performance.

Also, they do not provide any information on how different parameters of the system influence safety.

In practice both the capacity and demand functions are random variables, the values of which are scattered about their respective expected (or mean) values. Their randomness is characterised by their means $\mu \mathrm{D}$ and $\mu \mathrm{c}$, standard deviations $\sigma_{D}$ and $\sigma \mathrm{C}$, and corresponding probability functions as shown in Figure 3.2 . 
This results in an overlap in the distributed values of capacity and demand that might lead to the failure of the system. Therefore, the safety factor defined as the ratio $C / D$ is itself a random variable.

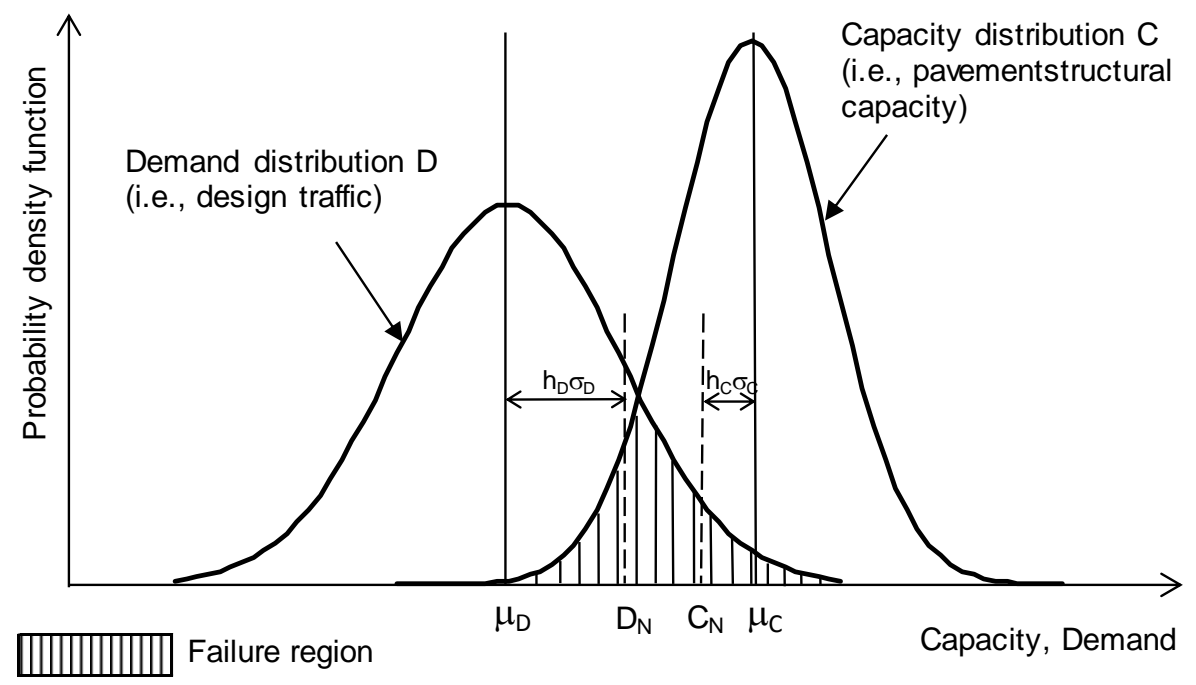

Figure 3.2 Fundamentals of risk evaluation (from Haldar et al., 2000)

\subsubsection{The central factor of safety (CFS)}

This represents a probabilistic version of the safety factor expressed as:

$$
C F S=\frac{E[C]}{E[D]}=\frac{\mu_{C}}{\mu_{D}}
$$

where $\mathrm{E}[\mathrm{]}$ indicates the expected value.

Common practice would assign a nominal value of capacity less than that of its expected value and a nominal value of demand greater than that of its expected value:

$$
\begin{aligned}
& C_{N}=\mu_{C}-h_{C} \sigma_{C} \\
& D_{N}=\mu_{D}+h_{D} \sigma_{D}
\end{aligned}
$$

where, $h_{C}$ and $h_{D}$ are $h$ sigma units (the number of standard deviations $\sigma$ ) of their respective functions.

Therefore, equation (3.1) can be re-written as:

$$
F S=\frac{\mu_{C}-h_{C} \sigma_{C}}{\mu_{D}+h_{D} \sigma_{D}}<C F S
$$




\subsubsection{The safety margin $S$}

As shown in Figure 3.2, if the demand is greater than the capacity the distributions will overlap and there will be a probability of failure. A common way of assessing this probability is to use the safety margin $S$ (a random variable itself) expressed as:

$$
S=C-D
$$

Since the hatched area in is the probability of failure, this can be expressed as:

$$
p(f)=P(\text { failure })=P[(C-D) \leq 0]=P[S \leq 0]
$$

\subsubsection{The reliability index $\beta$}

The reliability index $\beta$ is defined as the number of $h$ sigma units between the mean value of the safety margin $E[S]$ and $S=0$, which is:

$$
\beta=\frac{E[S]}{\sigma[S]}=\frac{E[C]-E[D]}{\sigma[S]}=\frac{\mu_{C}-\mu_{D}}{\sqrt{\sigma^{2}[C]+\sigma^{2}[D]-2 \rho \sigma[C] \sigma[D]}}
$$

where $\rho$ is the coefficient of correlation between $C$ and $D$. If $C$ and $D$ are not correlated, $\rho=0$ and eq. (3.8) becomes:

$$
\beta=\frac{E[S]}{\sigma[S]}=\frac{E[C]-E[D]}{\sigma[S]}=\frac{\mu_{C}-\mu_{D}}{\sqrt{\sigma^{2}[C]+\sigma^{2}[D]}}
$$

If the capacity and demand functions are independent and normally distributed, it can be demonstrated that the safety margin will also be a normal variate. The probability of failure can be expressed as:

$$
p(f)=1-\Phi\left(\frac{\mu_{C}-\mu_{D}}{\sigma[S]}\right)=1-\Phi(\beta)
$$

where $\Phi$ is the cumulative distribution function of the standard normal variate.

\subsubsection{Reliability analysis}

The demand-capacity inference model described in the previous section shows that if both demand (i.e., stress) and capacity (i.e., strength) are 
independent random variables (i.e., $\rho=0$ ) with normal distributions, then there is a simple analytical solution to the failure probability. When the stress and capacity probability distributions are not normal, a solution to the failure probability is not easily obtainable and requires the application of transformation techniques. Also, when the strength itself is a function of other random variables it is more appropriate to use their distribution rather than assuming a specific distribution for the strength (Zhang et al., 2006).

The first step in evaluating the reliability or probability of failure of a structure is to decide on specific performance criteria and the relevant stress and strength parameters, called the basic variables $X_{i}$, and the functional relationships among them corresponding to each performance criterion (Haldar et al., 2000). The performance function can be described as:

$$
G=g\left(X_{1}, X_{2}, \ldots, X_{n}\right)
$$

The failure surface or the limit state of interest can be defined as $G=0$. Conceptually, the generalised function defines a (critical) hypersurface in $n$ dimensional space wherein $G>0$ is the safe state and $G<0$ is the failure state (see Figure 3.3 for the case of two random variables). The probability of failure, $p(f)$, is mathematically given by the $n$-dimensional probability integral:

$$
p(f)=P[G \leq 0]=\int \ldots \int_{g 0<0} f_{X}\left(x_{1}, x_{2}, \ldots, x_{n}\right) d x_{1} d x_{2} \ldots d x_{n}
$$

in which $\mathrm{fx}_{\mathrm{x}}\left(\mathrm{x}_{1}, \mathrm{x}_{2}, \ldots, \mathrm{x}_{n}\right)$ is the joint probability density function for the basic random variables $X_{1}, X_{2}, \ldots, X_{n}$ and the integration is performed over the failure region, that is $g()<0$. 


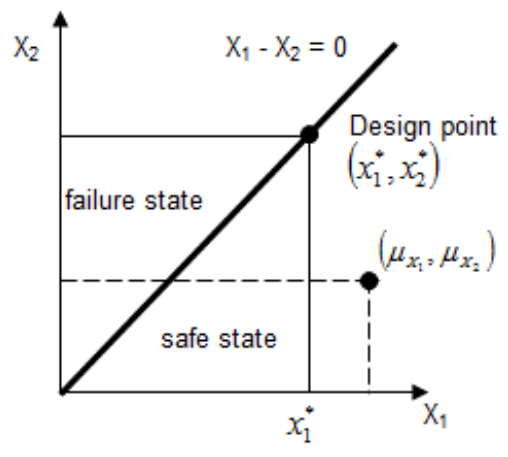

(a) Original coordinates

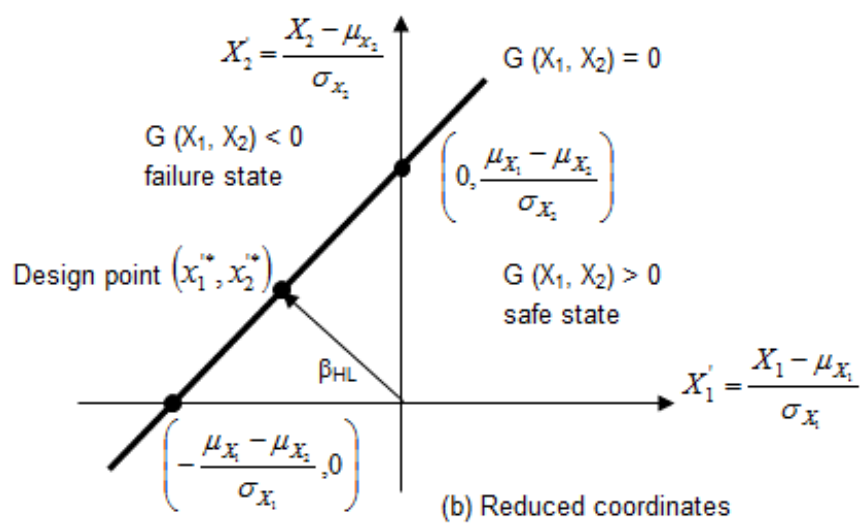

(b) Reduced coordinates

Figure 3.3 Hasofer-Lind reliability index for linear limit state function (adapted from Haldar et al., 2000)

The minimum distance from the critical hypersurface (in reduced coordinate space) to the origin is taken as the measure of the reliability index. The test of adequacy for the reliability index concepts generally rests on comparing computed reliability indexes $\beta$ with recommended values or simply to assume $\beta$ to be a normal variate (eq. 3.10).

The computation of the n-dimensional probability integral in eq. (3.12) is difficult. Various approximation techniques are available to help determine the probability distribution given a function (linear or nonlinear) of a number or random variables. The methodologies can be grouped into three categories:

- Simulation Techniques (i.e., the Monte Carlo simulation technique)

- First-Order Reliability Methods (FORM)

- Point Estimate Method (PEM)

The following sections aim to summarise the main features of these methods. A summary of the advantages and disadvantages of each technique is depicted in Table 3.1. 


\section{Table 3.1 Comparison between reliability analysis techniques}

\begin{tabular}{|c|c|c|c|}
\hline & Basic Concept & Advantages & Disadvantages \\
\hline $\begin{array}{l}\text { Monte } \\
\text { Carlo } \\
\text { Simulation } \\
\text { Technique }\end{array}$ & $\begin{array}{l}\text { In the Monte Carlo simulation technique, } \\
\text { values of the random variables are } \\
\text { generated in a fashion consistent with their } \\
\text { probability distribution, and the performance } \\
\text { function is calculated for each generated } \\
\text { set. The process is repeated numerous } \\
\text { times, typically thousands, and the } \\
\text { expected value, standard deviation, and } \\
\text { probability distribution of the performance } \\
\text { function are taken to match that of the } \\
\text { calculated values. }\end{array}$ & $\begin{array}{l}\text { - Applicable to wide } \\
\text { range of problems } \\
\text { - The probability } \\
\text { distribution functions of } \\
\text { all components variables } \\
\text { are obtained }\end{array}$ & $\begin{array}{l}\text { - Computationally } \\
\text { intensive } \\
\text { - Cannot be easily } \\
\text { implemented in } \\
\text { mathematical } \\
\text { programming }\end{array}$ \\
\hline FORM & $\begin{array}{l}\text { Analytical method based on linear } \\
\text { approximation of a nonlinear limit state at } \\
\text { the design point. }\end{array}$ & $\begin{array}{l}\text { - Accurate even for } \\
\text { extremely small } \\
\text { probabilities }\end{array}$ & $\begin{array}{l}\text { - Lack of accuracy for } \\
\text { highly nonlinear limit } \\
\text { state functions } \\
\text { - Requires the } \\
\text { computation of } \\
\text { derivatives } \\
\text { - Difficulties involved in } \\
\text { searching the design } \\
\text { point by iteration using } \\
\text { the derivatives of the } \\
\text { performance function }\end{array}$ \\
\hline PEM & $\begin{array}{l}\text { The moments of the performance function } \\
\text { are determined by evaluating it at a set of } \\
\text { combinations of high and low parameter } \\
\text { values, with the results weighted by factors. }\end{array}$ & $\begin{array}{l}\text { - The method, in } \\
\text { general, does not } \\
\text { require knowledge of the } \\
\text { probability distributions } \\
\text { of the basic random } \\
\text { variables involved in the } \\
\text { limit state function but } \\
\text { their statistical moments. }\end{array}$ & $\begin{array}{l}\text { - Approximate solution } \\
\text { in multivariate } \\
\text { problems } \\
\text { - It is limited by the } \\
\text { need to make } 2^{\text {n }} \\
\text { evaluations when } \\
\text { there are } n \text { random } \\
\text { variables }\end{array}$ \\
\hline
\end{tabular}

\subsubsection{Simulation Techniques}

A simulation technique, such as the Monte Carlo analysis, uses computers to study the uncertainty and is probably the most popular method to estimate the probability of failure. The simulation basically consists of the five elements: (1) selection of a range and distribution for each input variable; (2) generation of a sample from the input variables (i.e., $\mathrm{N}$ random numbers for each of the random variables will give $\mathrm{N}$ sets of random numbers, each set representing a realisation of the problem); (3) evaluate the problem deterministically for each set of realisations of all the random variables; (4) extract probabilistic information from $\mathrm{N}$ such realisations; and (5) perform sensitivity analysis to determine the accuracy and efficiency of the simulation. The accuracy of the analysis will increase as the number of simulations $\mathrm{N}$ increases. The probability of failure is given by:

$$
P_{f_{M C}}=E[I(g(X) \leq 0)] \cong \frac{\sum_{i}^{N} I(g(X) \leq 0)}{N}
$$


Where $\mathrm{I}(\mathrm{g}(\mathrm{X}) \leq 0)$ is an indicator function that is equal to 1 if $g(X) \leq 0$ or 0 otherwise. Finally, the simulation is repeated several times to determine the variability of the estimate.

This technique can also be used for reliability analysis of structures with implicit performance functions, as long as an algorithm is available to compute the structure response. The Monte Carlo simulation is computationally intensive, requiring a large number of iterations (in the order of thousands) in order to obtain reliable results.

\subsubsection{First-Order Reliability Methods (FORM)}

FORM methods were historically derived from second-moment methods, which used information on first and second moments of the random variables (i.e., on mean and standard deviation). These are First-Order Second Moment (FOSM) and Advanced First-Order Second Moment (AFOSM) methods. In FOSM methods, the information on the distribution of random variables is ignored; in AFOSM methods, this information is appropriately used.

The FOSM method can be used to evaluate the n-dimensional probability integral in eq. (3.12) when the limit state function is a linear function of uncorrelated (i.e., $\rho=0$ ) normal variables or when the nonlinear state function is represented by a first-order (linear) approximation (i.e., Taylor series) with equivalent normal variables.

The FOSM method is also referred as Mean Value First-Order SecondMoment (MVFOSM) method in the literature. The MVFOSM method is based on a first-order Taylor series approximation of the performance function linearised at the mean values of the random variables, and uses only secondmoment statistics (means and covariances) of the random variables. The first author to develop this method was Cornell in 1969.

It can be demonstrated (Harr, 1987) that for $N$ uncorrelated variables, $F\left(x_{1}, x_{2}\right.$, $\ldots, \mathrm{xN}_{\mathrm{N}}$ ), retaining only linear terms in Taylor series, the expected value and variance of the function $F$ are:

$$
E[F]=F\left(\overline{x_{1}}, \overline{x_{2}}, \ldots, \overline{x_{N}}\right)
$$




$$
V[F]=\sum_{i=1}^{N}\left(\frac{\partial F}{\partial x_{i}}\right)^{2} V\left[x_{i}\right]
$$

Where $\overline{x_{i}}=E\left[x_{i}\right]$ and all derivatives are evaluated at the expected values of all the variables contained within their expressions. The safety index can then be calculated by taking the ratio of the mean and standard deviation of $F$ as in eq. (3.9).

One of the problems of the FOSM method is that the safety index defined by eq. (3.9) fails to be constant under different but mechanically equivalent formulations of the same performance function (e.g., Capacity / Demand $=1$ or Capacity - Demand $=0$ ). This problem was solved by Hasofer and Lind (1974) for normal variables with the so-called Advanced First-Order Second Moment (AFOSM), see Figure 3.3. The Hasofer-Lind reliability index $\beta$ HL is defined as the minimum distance from the origin of the reduced coordinate system to the design point on the limit state function.

The reduced variables are defined as:

$$
X_{i}^{\prime}=\frac{X_{i}-\mu_{X_{i}}}{\sigma_{X_{i}}} \quad(\mathrm{i}=1,2, \ldots, \mathrm{n})
$$

where $X_{i}^{\prime}$ is a random variable with zero mean and unity standard deviation. The $X$ coordinate system is the original coordinate system while the $X^{\prime}$ coordinate system is the reduced coordinate system. Note that if $X_{i}$ is normal, $X_{i}^{\prime}$ is standard normal. For a simple two-dimensional linear limit state function $\left[G(X)=X_{1}-X_{2}\right]$ with normal basic random variables $\left(X_{1}, X_{2}\right)$, the Hasofer-Lind reliability index $\beta_{\mathrm{HL}}$ is determined by an iterative process and it is defined as:

$$
\begin{array}{lr}
\beta_{H L}=\min _{g=0} \sqrt{\left(x^{*}\right)^{t} *\left(x^{* \prime}\right)} & \text { for uncorrelated variables } \\
\beta_{H L}=\min _{g=0} \sqrt{\left(x^{* \prime \prime}\right)^{t} *[R]^{-1}\left(x^{* *}\right)} & \text { for correlated variables }
\end{array}
$$

where $x^{\prime *}$ is the vector of the random variable values at the design point reduced to standard normal space and $[R]$ is the correlation matrix of the variables. An explanation of the method is given below. Consider the linear limit state equation in two variables, see Figure 3.3: 


$$
G=X_{1}-X_{2}=0
$$

A set of reduced variables is introduced as:

$$
\begin{aligned}
& X_{1}^{\prime}=\frac{X_{1}-\mu_{X_{1}}}{\sigma_{X_{1}}} \\
& X_{2}^{\prime}=\frac{X_{2}-\mu_{X_{2}}}{\sigma_{X_{2}}}
\end{aligned}
$$

By substituting these into eq. (3.19), the limit state equation in the reduced coordinate system becomes:

$$
g()=\sigma_{X_{1}} X_{1}^{\prime}-\sigma_{X_{2}} X_{2}^{\prime}+\mu_{X_{1}}-\mu_{X_{2}}=0
$$

The position of the limit state with respect to the origin in the reduced coordinate system represents a measure of reliability. The coordinates of the intercepts of eq. (3.22) on the $X_{1}^{\prime}$ and $X_{2}^{\prime}$ axes are $\left(-\frac{\mu_{X_{1}}-\mu_{X_{2}}}{\sigma_{X_{1}}}, 0\right)$ and $\left(0, \frac{\mu_{X_{1}}-\mu_{X_{2}}}{\sigma_{X_{2}}}\right)$ respectively.

Recalling from analytic geometry that the shortest distance from the origin to a line, say $a x+b y+c=0$ is $c /\left(a^{2}+b^{2}\right)^{1 / 2}$, we find that:

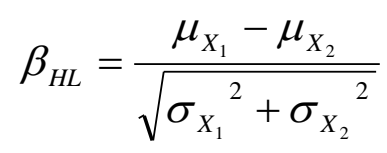

This reliability index or safety index $\beta \mathrm{HL}$ is the same as defined in eq. (3.9) if both variables are normal, independent, and if the limit state is linear but obtained by geometric considerations. For nonlinear limit states and for nonnormal variables algorithms were developed by Rackwitz and Fiessler (1978) and by Chen and Lind (1983) to compute the reliability index and the design point through an iteration-based process (Haldar et al., 2000).

Figure 3.4 shows that the limit state $g\left(X^{\prime}\right)=0$ is a nonlinear function, with $g\left(X^{\prime}\right)>0$ representing the safe state and $g\left(X^{\prime}\right)<0$ representing the failure state. Again the Hasofer-Lind reliability index $\beta \mathrm{HL}$ is defined as the minimum distance from the origin to the design point $\left(x^{\prime *}\right)$ on the limit state in the reduced coordinates and can be expressed by eq. (3.17) and (3.18). For nonlinear limit states, the computation of the minimum distance becomes an optimisation problem: 
Minimise $\mathrm{D}=\sqrt{x^{t} * x^{\prime}}$

Subject to the constraint $g(x)=g\left(x^{\prime}\right)=0$

Where $x^{\prime}$ represents the coordinates of the checking point on the limit state equation in the reduced coordinates to be estimated.

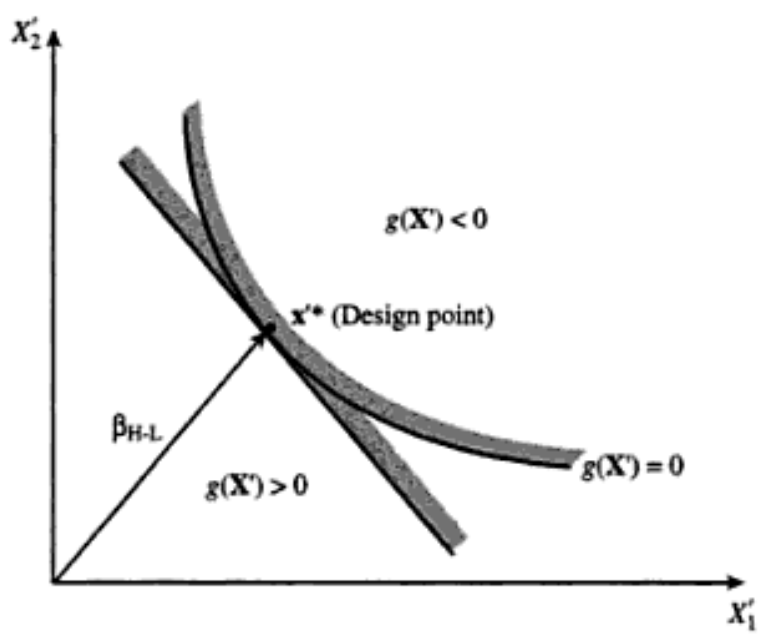

Figure 3.4 Hasofer-Lind reliability Index for nonlinear performance functions (from Haldar et al., 2000)

The Hasofer-Lind reliability index can be used to calculate a first-order approximation of the failure probability as probability of failure is:

$$
p(f)=P[G(X) \leq 0]=\Phi\left(-\beta_{H L}\right)=1-\Phi\left(\beta_{H L}\right)
$$

From both Figure 3.3 and Figure 3.4 it can be seen that the nearer the design point $x^{\prime *}$ is to the origin, the larger is the failure probability.

The advantage of FORM is its accuracy even for extremely small probabilities and its ability to easily deal with complex nonlinear limit state functions without the need to take any derivatives. This characteristic is very important in pavement engineering where the definition of the strength of a pavement often involves complex nonlinear forms of transfer functions. The FORM method can be used even if the limit state function consists of correlated basic random variables. The orthogonal transformation is commonly used to transform correlated into uncorrelated variables (Haldar et al., 2000). 
The main drawbacks are the lack of accuracy for highly nonlinear limit state functions and the difficulty in its iteration-based process of searching for the design point.

Although some of the FORM shortcomings are addressed by considering the Second-Order Reliability Method (SORM), which was first explored by Fiessler et al. in 1979, the First-Order Third-Moment (FOTM) method, and genetic algorithms, inherited problems with nonlinear iteration algorithms in search for the design point which are still difficult to overcome (Zhao and Ono 2001).

\subsubsection{Point Estimate Method (PEM)}

The point estimate method approach, developed by Rosenblueth in 1975 and summarised by Harr (1987), does not use the Taylor series but instead uses measured values of the independent variables at different points to estimate the random dependent variable. The method is capable of estimating a statistical moment of any order of a model output involving several stochastic variables that are correlated or uncorrelated, symmetric, or asymmetric. However, in multivariate problems with more than two stochastic variables involved, the Rosenblueth method is not able to provide a unique solution, rather an approximate solution to indeterminate problems. This is attributed to the fact that the number of unknowns to be solved is larger than the number of governing equations provided.

Zhao and Ono $(2000,2001)$ investigated the method of moments (MM) for structural reliability. The main advantages of this method - over for example Monte Carlo simulation - are the computational simplicity, that it has no shortcomings with respect to the design points, requires neither iteration nor the computation of derivatives, and has a satisfactory level of accuracy even for highly nonlinear limit state functions. The method of moments consists 1) in the estimation of the moments of the limit state functions using the point estimates obtained in standard normal space and 2) after the moments of the limit state functions are obtained, the calculation of the reliability index and the failure probability using the existing standardised functions. With the Method of Moments the reliability can be expressed as a closed-form function of many 
basic random variables defining structural characteristics of a pavement and traffic utilisation.

For a performance function of many variables $G(X)$, assuming that $G(X)$ obeys normal distribution and $\mathrm{X}=\mathrm{X}_{1}, \mathrm{X}_{2}, \ldots, \mathrm{X}_{\mathrm{n}}$ are $\mathrm{n}$ variables, the method of moments can be summarised as the following four steps:

1. Determine the constant $\mathrm{G} \mu$ and the function $\mathrm{G}_{i}$ of only one variable $u_{i}$ using equations (3.26) to (3.30)

$$
\begin{aligned}
G_{\mu} & =G(\mu) \\
G_{i} & =G\left[T^{-1}\left(U_{i}\right)\right]
\end{aligned}
$$

$\mu$ represents the vector in which all the random variables take their mean values, and $U_{i}=\left[u_{\mu 1}, u_{\mu 2}, \ldots, u_{i}, u_{\mu i+1}, \ldots, u_{\mu m}\right]^{\top}$ where $u_{\mu k}, k=1, \ldots, n$ except $i$, is the kth value of $u_{\mu}$, which is the vector in $u$-space corresponding to $\mu$. $G_{\mu}$ is a constant and $G_{i}$ is a function of only $u_{i}$, for a specific $G(X)$. The number of estimating points used in the point estimates for functions of respective single random variables is $\mathrm{m} . \mathrm{T}^{-1}$ is the inverse Rosenblatt transformation. The inverse Rosenblatt transformation is used to transform the point estimates from the standard normal space back to the probability space of the original random variables.

Usually, five or seven points are used as estimating points. For a five-point estimate in standard normal space, the estimating points and corresponding weights are:

$$
\begin{array}{ll}
\mathrm{u}_{0}=0 & \mathrm{P}_{0}=8 / 15 \\
\mathrm{u}_{1+}=-\mathrm{u}_{1-}=1.3556262 & \mathrm{P}_{1}=0.2220759 \\
\mathrm{u}_{2+}=-\mathrm{u}_{2}=2.8569700 & \mathrm{P}_{2}=1.12574^{*} 10^{-2}
\end{array}
$$

For a seven-point estimate in standard normal space, the estimating points and corresponding weights are:

$$
\mathrm{u}_{0}=0 \quad \mathrm{P}_{0}=16 / 35
$$




$$
\begin{array}{ll}
\mathrm{u}_{1+}=-\mathrm{u}_{1-}=1.1544054 & \mathrm{P}_{1}=0.2401233 \\
\mathrm{u}_{2+}=-\mathrm{u}_{2}=2.3667594 & \mathrm{P}_{2}=3.07571^{*} 10^{-2} \\
\mathrm{u}_{3+}=-\mathrm{u}_{3}=3.7504397 & \mathrm{P}_{3}=5.48269^{*} 10^{-4}
\end{array}
$$

The inverse Rosenblatt transformation is expressed as:

$$
\mathrm{T}^{-1}\left(\mathrm{u}_{\mathrm{i}}\right)=\mathrm{F}^{-1}\left[\Phi\left(\mathrm{u}_{\mathrm{i}}\right)\right]
$$

where $\mathrm{F}=$ cumulative distribution function of $\mathrm{G}_{\mathrm{i}}$; and $\Phi=$ standard normal

\begin{tabular}{|c|c|}
\hline Type of $F(x)$ & $x=F^{-1}[\Phi(n)]$ \\
\hline Normal: mean $\mu_{x^{\prime}}$ std. dev. $\sigma_{x}$ & $x=\mu_{x}+n \sigma_{x}$ \\
\hline $\begin{array}{l}\text { Lognormal: mean } \mu_{x^{\prime}} \\
\text { std. dev. } \sigma_{\mathrm{x}}\end{array}$ & $x=\exp [\lambda+\zeta n], \quad \zeta=\sqrt{\ln \left(1+\left(\sigma_{x} / \mu_{x}\right)^{2}\right)}, \quad \lambda=\ln \mu_{x}-0.5 \zeta^{2}$ \\
\hline $\begin{array}{l}\text { Extreme Value (Gumbel): } \\
\text { mean } \mu_{x^{\prime}} \text { std. dev. } \sigma_{\mathrm{x}}\end{array}$ & $x=u-\frac{\ln [-\ln (\Phi(n))]}{\alpha}, \quad \alpha=\frac{1}{\sqrt{6}}\left(\frac{\pi}{\sigma_{x}}\right), \quad u=\mu_{* *}-\frac{0.5772}{\alpha}$ \\
\hline Exponential: mean $=\mu_{x}$ & $x=-\mu_{x} \ln (1-\Phi(n))$ \\
\hline Uniform: $\bigsqcup_{x_{\min }} x_{x_{\max }}$ & $x=x_{\min }+\left(x_{\max }-x_{\min }\right) \times \Phi(n)$ \\
\hline Triangular: & $\begin{array}{l}x=a+\sqrt{\Phi(n) \times(m-a) \times(c-a)}, \quad \text { if } \Phi(n) \leq(m-a) /(c-a) . \\
x=c-\sqrt{(1-\Phi(n)) \times(c-a) \times(c-m)}, \text { otherwise. }\end{array}$ \\
\hline Weibull CDF: $1-\exp \left[-(x / \lambda)^{\alpha}\right]$ & $x=\lambda[-\ln (1-\Phi(n))]^{1 / \alpha}$ \\
\hline $\begin{array}{l}\text { Gamma with PDF: } \\
\qquad f(x)=\frac{x^{\alpha-1} \exp (-x / \alpha)}{\lambda^{\top} \Gamma(\alpha)}\end{array}$ & $\begin{array}{l}\text { Para1 }=\alpha, \text { Para2 }=\lambda \\
\text { Mean: } \mu_{x}=\alpha \times \lambda \\
\text { Newton method used to obtain } \mathrm{x} \text { from } \mathrm{n} \text {, starting from } \mu_{\mathrm{x}}\end{array}$ \\
\hline Beta distribution & $\begin{array}{l}\text { Para } 1=\alpha, \text { Para } 2=\lambda, \text { Para } 3=\min , \text { Para } 4=\max \\
\text { Mean: } \mu_{x}=\min +(\max -\min ) \alpha /(\alpha+\lambda) \\
\text { Newton method used to obtain } \mathrm{x} \text { from } \mathrm{n} \text {, starting from } \mu_{\mathrm{x}}\end{array}$ \\
\hline PERT distribution & $\begin{array}{l}\text { Para1 }=\min , \text { Para } 2=\text { mode, } \text { Para } 3=\max \\
\text { Mean: } \mu_{x}=\left(\min +4^{*} \text { mode }+\max \right) / 6 \\
\text { Newton method used to obtain } \mathrm{x} \text { from } \mathrm{n} \text {, starting from } \mu_{\mathrm{x}} .\end{array}$ \\
\hline
\end{tabular}
probability.

Low and Tang (2007) have tabulated the Rosenblatt transformation for some common probability distribution functions; see Figure 3.5.

Figure 3.5 Obtaining T-1(ui) ( $x$ in the table) from ui ( $n$ in the table), based on $F(u i)=\Phi(u i)(F(x)=\Phi(n)$ in the table) (Low and Tang 2007) 
2. Compute the first four moments of Gi using equations (3.31) to (3.33), with the estimating points and corresponding weights listed in equations (3.28) and(3.29).

$$
\begin{aligned}
& \mu_{G_{i}}=\sum_{k=1}^{m} P_{k} G_{i}\left[T^{-1}\left(u_{k}\right)\right] \\
& \sigma_{G_{i}}^{2}=\sum_{k=1}^{m} P_{k}\left\{G_{i}\left[T^{-1}\left(u_{k}\right)\right]-\mu_{G_{i}}\right\}^{2} \\
& \alpha_{r G_{i}} \sigma_{G_{i}}^{r}=\sum_{k=1}^{m} P_{k}\left\{G_{i}\left[T^{-1}\left(u_{k}\right)\right]-\mu_{G_{i}}\right\}^{r}
\end{aligned}
$$

where $\mu_{G_{i}}, \sigma_{G_{i}}^{2}$, and $\alpha_{r G_{i}} \sigma_{G_{i}}^{r}$ are the mean value, standard deviation and the rth dimensionless central moment of $G_{i}$ respectively; $\mathrm{T}^{-1}$ is the inverse Rosenblatt transformation; and $\mathrm{u}_{1}, \ldots, \mathrm{u}_{\mathrm{m}}$ are the estimating points with $\mathrm{P}_{1}$, $\ldots, P_{m}$ the corresponding weights, see previous step.

3. Compute the first four moments of the performance function using equations (3.34) to (3.38).

$$
\begin{aligned}
& G^{*}=\sum_{i=1}^{n}\left(G_{i}-G_{\mu}\right)+G_{\mu} \\
& \mu_{G^{*}}=\sum_{i=1}^{n}\left(\mu_{G_{i}}-G_{\mu}\right)+G_{\mu} \\
& \sigma_{G^{*}}^{2}=\sum_{i=1}^{n} \sigma_{G_{i}}^{2} \\
& \alpha_{3 G^{*}} \sigma_{G^{*}}^{3}=\sum_{i=1}^{n} \alpha_{3 G_{i}} \sigma_{G_{i}}^{3} \\
& \alpha_{4 G^{*}} \sigma_{G^{*}}^{4}=\sum_{i=1}^{n} \alpha_{4 G_{i}} \sigma_{G_{i}}^{4}+6 \sum_{i=1}^{n-1} \sum_{j>i}^{n} \sigma_{G_{i}}^{2} \sigma_{G_{j}}^{2}
\end{aligned}
$$

where $G^{*}$ represents the approximation of the limit state function $G(X), \mu G^{*}$, $\sigma G^{*}, \alpha_{3 G^{*}}$, and $\alpha_{4 G^{*}}$ are the first dimensionless central moment (mean value), the second dimensionless central moment (standard deviation), the third dimensionless central moment (skewness) and the fourth dimensionless central moment (kurtosis) of $\mathrm{G}_{\mathrm{i}}$ respectively.

4. Compute the $2 \mathrm{M}, 3 \mathrm{M}$, and $4 \mathrm{M}$ reliability indices using equations (3.39) to (3.41). 


$$
\begin{array}{ll}
\beta_{2 M}=\frac{\mu_{G^{*}}}{\sigma_{G^{*}}} & P f_{2 M}=\Phi\left(-\beta_{2 M}\right) \\
\beta_{3 M}=\frac{-\operatorname{sgn}\left(\alpha_{3 G^{*}}\right)}{\sqrt{\ln (A)} \ln \left[\sqrt{A}\left(1+\frac{\beta_{2 M}}{u_{b}}\right)\right]} & P f_{3 M}=\Phi\left(-\beta_{3 M}\right) \\
A=1+\frac{1}{u_{b}^{2}} & \\
u_{b}=(a+b)^{1 / 3}+(a-b)^{1 / 3}-\frac{1}{\alpha_{3 G^{*}}} & \\
a=-\frac{1}{\alpha_{3 G^{*}}}\left(\frac{1}{\alpha_{3 G^{*}}^{2}}+\frac{1}{2}\right) & \\
b=-\frac{1}{2 \alpha_{3 G^{*}}^{2}} \sqrt{\alpha_{3 G^{*}}^{2}+4} & P f_{4 M}=\Phi\left(-\beta_{4 M}\right) \\
\beta_{4 M}=\frac{3\left(\alpha_{4 G^{*}}-1\right) \beta_{2 M}+\alpha_{3 G^{*}}\left(\beta_{2 M}^{2}-1\right)}{\sqrt{\left(9 * \alpha_{4 G^{*}}-5 \alpha_{3 G^{*}}^{2}-9\right)\left(\alpha_{4 G^{*}}-1\right)}} &
\end{array}
$$

where $\beta_{2 \mathrm{M}}, \beta_{3 \mathrm{M}}$, and $\beta_{4 \mathrm{M}}$ are the second-, third- and fourth-moment reliability indices respectively and $\mathrm{Pf}_{2 \mathrm{M}}, \mathrm{Pf}_{3 \mathrm{M}}$ and $\mathrm{Pf}_{4 \mathrm{M}}$ the associated failure probabilities.

\subsubsection{Distribution of residuals}

This method has been used in the American Mechanistic-Empirical Pavement Design Guide which is beginning to replace the 1993 version of the AASHTO guide (see 3.4.2.1). Instead of using simulation methods and other closed form analytical methods, too complex and requiring sophisticated computer programs, the reliability process in this guide is based on the ability of the performance models to predict actual distresses of the sites used for calibration. The transfer functions were calibrated utilising data from the LongTerm Pavement Performance (LTPP) and other field pavement studies. The general concept for reliability uses the standard deviation of the prediction model to determine the appropriate reliability level. Although practical, this method does not allow to evaluate change in performance due to differences in variability of individual factors. 


\subsubsection{System reliability for multiple performance criteria}

So far reliability has been estimated for a single performance criterion or limit state with one of the methods discussed in the previous section. In general, any engineering system has to satisfy more than one performance criterion. The concept used to determine the overall reliability of a system composed of many components is known as system reliability evaluation. A complete reliability analysis includes both component-level and system-level estimates.

For a system that has more than one performance criterion, system failure may occur when any of the criteria are violated. This system is referred as a system in series and the system failure is defined by the union of each performance component failure. Alternatively, a system may fail only when all the components fail and this is known as a parallel system. System failure in this case is defined by the intersection of the individual component failure events. The two models represent extremes. In reality, engineering systems have a combination of components, with some in series and others in parallel. Reliability bounds are available in the literature for the probability of failure of a system (Haldar et al., 2000).

\subsubsection{Reliability and the effect of time}

Failure is the inability of a system to perform its intended function over a specified period of time and under specified operating conditions. A system could become less and less reliable with the passage of time due to fatigue, creep, increased traffic, material degradation, and changeable environmental conditions. Methods for time-variant reliability analysis are still under development.

\subsection{The engineering problem}

As mentioned in the introduction, early failure in pavements occurs often in the UK. Premature failure occurs when a pavement does not perform the intended function which it was designed for, whether it is in terms of reduced structural capacity, poor ride quality, reduced surface skid resistance or any other possible number of unforeseen circumstances. Although failure is not a 
catastrophic event for pavements, as the collapse of a building, it still requires attention as it degrades the overall pavement performance.

The design of a flexible pavement is influenced by the amount and composition of the expected traffic, the sub-grade strength properties, the paving material properties and the environment in which the pavement is to perform. The two major forms of distress in flexible pavements are surface rutting and asphalt layer cracking. The general failure areas in a flexible pavement include tensile stress failure of asphalt pavement layers, shear failures of surface and base courses, and compressive failures of the sub-grade.

The causes of failures, whether they are structural or surface, can be grouped into three main categories:

- Construction-related problems, such as curing conditions during construction, construction under adverse weather conditions, inexperienced and/or inadequate testing and inspection in the field;

- Materials-related problems, such as variability of material properties, errors from different testing results (i.e., laboratory tests and actual field performance);

- Design-related problems, such as poor or insufficient site inspection or investigation prior to design, errors in traffic forecasts, and financial constraints.

It is essential to identify the causes of failures to allow the simplest and most cost effective maintenance treatment to be implemented to solve the problem.

While most pavements do not experience catastrophic failure, some do and sometimes this occurs at a random stage in their life. These pavements generally represent the minority and tend to receive high amounts of maintenance funds until the pavement can be rehabilitated and/or reconstructed within a short period of time.

For the safety and comfort of the travelling public but also for the government maintenance programmes it is essential to identify and assess the consequences of localised early pavement failures. In other words the risk of 
early failure needs to be assessed in order to decide the best engineering solution which will offset the ultimate pavement failure.

\subsection{Deterministic versus probabilistic design}

Most pavement engineers know that pavement materials, environment, loading and construction affect the performance of a pavement and the variability observed in each of these parameters introduces a certain level of risk. The recognised need to account for these variabilities in the design process is pushing many highway authorities in the world to move from a traditional deterministic approach, based on a single input/output value, towards a probabilistic design, which includes a mean, variance and probability distribution. The probabilistic approach offers a way of incorporating risk assessment considerations which are vital for whole-life cycle economic analysis and decisions.

Briefly, the probabilistic approach assesses the uncertainty in the inputs and carries that uncertainty through the computation process to generate a probabilistic distribution of results (a range of possible outcomes along with their likelihood of occurrence), see Figure 3.6.

INPUT

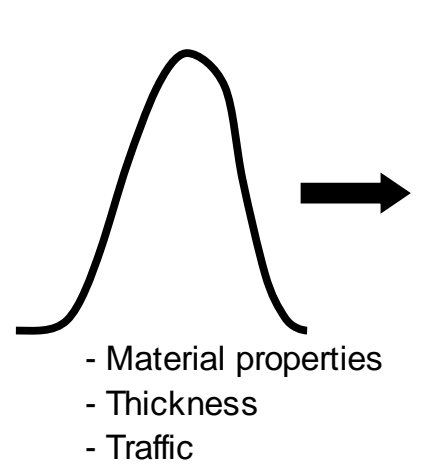

OUTPUT

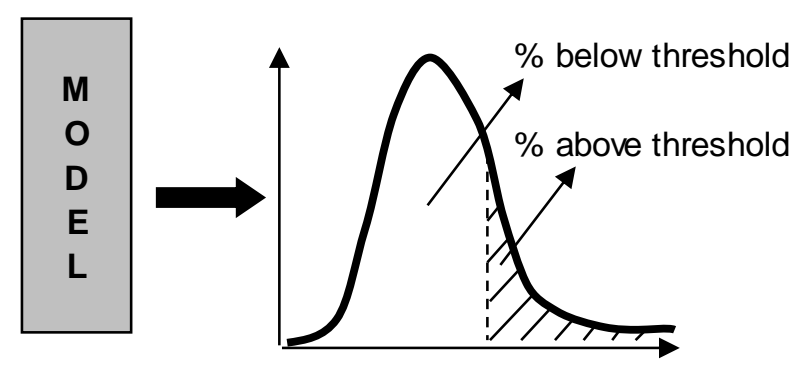

Pavement performance

Figure 3.6 Modern probabilistic design method

For example, if Monte Carlo simulation techniques are used in the design process, random sampling within the distributions of the input parameters can be performed and from this a high number of estimates of performance can be produced. Risk can then be assessed as the probability of premature failure. 
This information can be used to make a decision between different design alternatives.

Some authors (Theyse, 2006) believe that although simulation techniques make the design process more realistic, the accuracy of the design process and the calculation of the design risk are not actually improved. The accuracy of the structural capacity estimation and design risk calculation are governed by the validity and accuracy of the models used in the design method. Although this is a fair point, one should keep in mind that variability needs to be incorporated in the design but without producing an over conservative or too expensive design because of the evaluation of all parameters or extensive investigation into the validity of the design models. The latter clearly involves an extensive use of resources and its economic benefits need to be assessed at policy level.

\subsection{Application of reliability analysis to pavement design}

This section aims to summarise the information available on the application of reliability concepts as experienced in the UK, United States, France and Australia.

\subsubsection{United Kingdom}

For many years the design of flexible pavements in the UK was based on the TRRL Laboratory Report 1132, "The structural design of bituminous roads" (1984). The report made use of the results of full-scale road experiments and used analytical techniques to rationalise and extend the data.

Following the rapid increase in traffic volumes and compositions and the range of new materials available for both asphalt and foundation layers, a new versatile design approach was introduced in 2004 (TRL Report 615) and is currently included in the Highways Agency design standards (DMRB Vol 7). In both documents, the response model to determine the stress or strain at critical locations is represented by a multi-layered, linear elastic model. These criteria have been calibrated using information on pavement performance. 
In the current UK standards reliability is implicitly embraced in the response model such that a pavement has an $85 \%$ probability of achieving the design life.

No material has been found on the subject of reliability or probabilistic pavement design in the UK.

\subsubsection{United States}

\subsubsection{Design standards}

One of the most widely accepted design guides in the Unites States is that offered by the American Association of State Highway and Transportation Officials (AASHTO). This guide, based on the results of the AASHO road test in the late 1950's and early 1960's, was first published in 1961 and revised in 1972, 1981 and 1986. Reliability concepts were formally introduced in the 1986 version of the design guide, where reliability was expressed as the probability that the serviceability level at the end of the design period $(p)$ is greater than the terminal serviceability level $\left(p_{t}\right)$ :

$$
R=P\left[p \geq p_{t}\right]
$$

Another version was published in 1993, where reliability was defined in terms of the number of equivalent single axle loads to terminal serviceability $(n)$ being less than the number of equivalent single axle loads actually applied $(\mathrm{N})$ :

$$
R=P[N>n]
$$

The 1993 model for flexible pavements is based on design variables such as: layer thickness, roadbed modulus (Mr), drainage and climate conditions, and pavement functional factors (terminal PSI). The empirical design equations in the 1993 AASHTO guide contain a "safety factor" $\left(Z_{r} S_{0}\right.$, where $Z r$ is the standard normal deviate and $S_{0}$ the standard error) to account for the uncertainty in the design process. One drawback of the 1993 reliability model is that the reliability analysis basically used a large multiplier on the traffic input resulting in quite conservative design. 
In general, the equations and procedures used in the 1972, 1986 and 1993 AASHTO Guides were empirical in nature, developed using statistical regression models, performance measurements, or observations rather than using fundamental material properties and constitutive mathematical relationships. The significant changes in traffic volumes and truck configurations, materials, new mix design and construction procedures, climatic conditions coupled with the relatively poor performance of the existing roadways led to the need of improving the pavement design process. This was the main goal of the National Cooperative Highway Research Program (NCHRP) initiated project 1-37A to develop a new pavement design guide for new and rehabilitated pavements based on a mechanistic empirical (M-E) approach. A mechanistic-empirical design is characterised by an empirical relationship that links the mechanistic response of the pavement (i.e., from linear elastic theory) to an observed distress. In the current 2008 pavement guide reliability is defined as the probability that each of the key distress types and smoothness will be less than a selected critical level over the design period:

$\mathrm{R}=\mathrm{P}$ [Distress over Design Period $<$ Critical Distress Level]

$\mathrm{R}=\mathrm{P}$ [International Roughness Index (IRI) over Design Period $<$ Critical IRI Level]

In the mechanistic-empirical pavement design guide (MEPDG) reliability analysis of distress types is based on analysis of the predicted versus measured values and estimation of parameters of the corresponding error distribution (assumed normal). 


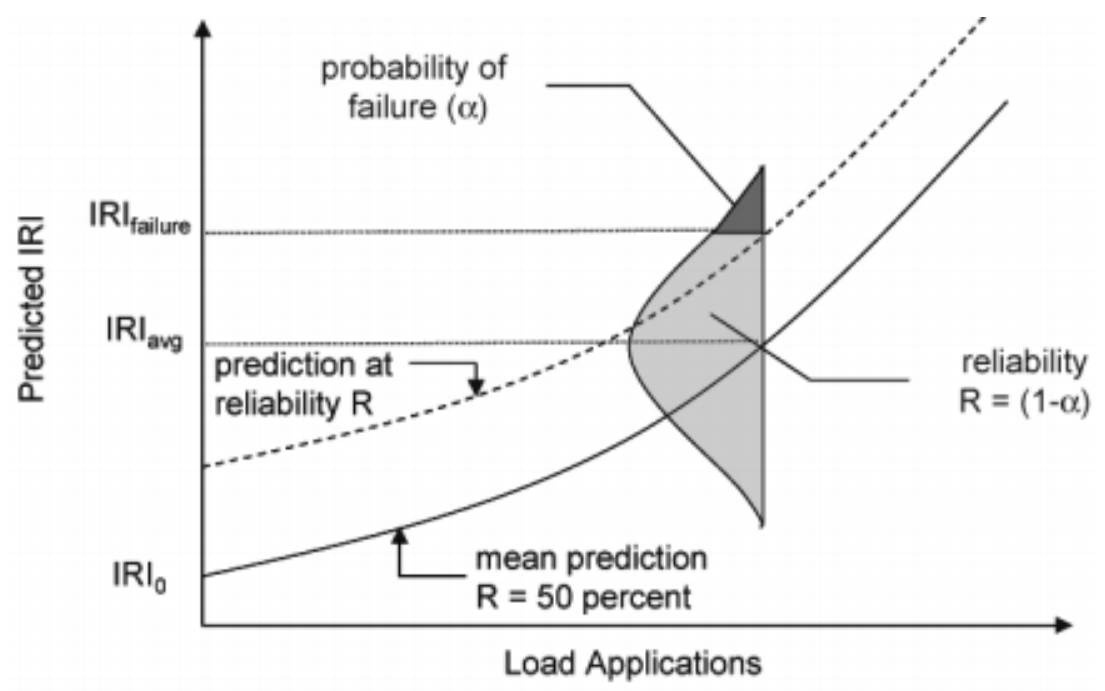

Figure 3.7 Design Reliability Concept for Smoothness (IRI) (from 2008 MEPDG)

In the MEPDG, the design inputs (expressed in terms of mean values) are processed through the mechanistic model (i.e., to evaluate stresses, strains and deflections) and then transfer functions are applied to predict distress. These predicted distress levels represent mean values (50\% reliability level), which means that there is a $50 \%$ possibility that the predicted distress will be higher or lower than this value (see solid line in Figure 3.7). A reliability factor is then applied to this mean value based on the accuracy of the prediction model. In other words, for nearly all projects, the designer will require a reliability higher than 50 percent that the design will meet the performance criteria over the design life (see dashed line in Figure 3.7). Primary transfer functions in current M-E design procedures for flexible pavements are those that relate maximum wheel load tensile strain at the bottom of the asphalt layer to eventual fatigue cracking and wheel load compressive strain (or stress) at the top of the subgrade layer to rutting at the surface. The design process is an iterative procedure that starts with a trial design and ends with predicted distress levels that meet the acceptable limits based on the desired level of reliability.

Common to the 1993 and 2008 design guides is the concept that the more important the project is (and the consequences of failure), the higher the reliability. For example, a $90 \%$ reliability would be the norm for major roads. 
Some issues have already been identified with regard to the way reliability of a pavement design is estimated using the MEPDG. One of these is that the variability of the predicted performance is not necessarily normally distributed (Graves et al., 2007). Further work may be necessary to develop alternative methods to address reliability, such as substituting the assumption of normality with the actual distribution of error/residuals. Furthermore, the implemented approach does not account for the effect of design input variability on the design variability, which could be achieved by the Monte Carlo simulation technique (Darter et al., 2005).

\subsubsection{Reliability research results to date}

The main sources of information have been obtained by the following institutions/journals:

- Transportation Research Board

- Transportation Engineering Journal of ASCE

- Association of Asphalt Pavement Technologists

- Canadian Journal of Civil Engineering

The literature review has found that the two most popular methods employed in the reliability analysis are FORM and the Monte Carlo simulation technique.

The early concepts for reliability inclusion in pavement design have been summarised by Hudson (1975). Prior to 1965 , variation or uncertainty was considered in design by the use of safety factors based on experience. However, the use of safety factors, besides resulting in sometimes uneconomical designs, has also resulted in few failures because of little consideration being given to the uncertainty of design factors. Later, Lemer and Moavenzadeh (1971) developed one of the first models dealing with reliability of pavements. Lemer attempted to apply the Monte Carlo simulation technique - where the input variables were described by appropriate probability distributions - to a complex pavement design method for the Federal Highway Administration (FHWA). However, the computer time associated with the 
Monte Carlo simulation technique proved to be excessive and impractical for practical situations.

The reliability model developed by Darter and Hudson (1973) considers two major factors associated with the failure of a pavement: the allowable load applications, N, and the applied load applications, n. Reliability was defined as the probability that $\mathrm{N}$ will exceed $\mathrm{n}$, see eq. (3.43). The variables $\mathrm{n}$ and $\mathrm{N}$ were believed to be approximately log normally distributed and the condition of the pavement was described by the failure function $D=\log N-\log n$. The pavement was considered to have failed to perform its function when $n$ exceeds $\mathrm{N}$, or $\mathrm{D}<0$. Darter and Hudson used the method called Mean Value First Order Second Moment (MVFOSM) to obtain mean and standard deviation of D. In general, this method (Cornel, 1969; Ditlevesen, 1971) aims at obtaining the mean, $\mu_{z}$, and standard deviation, $\sigma_{z}$, of the performance function $Z=f\left(X_{1}, X_{2}, \ldots, X_{n}\right)$. The safety index, $\beta=\mu_{z} / \sigma_{z}$, is a measure of the reliability system.

Later work in probabilistic design adopted a similar approach in the formulation, differing only in the procedure used for determining the mean and standard deviation of N (Moavenzadeh and Brademeyer, 1977; Riggins et al., 1985; George and Husain, 1986; and Irik at al., 1987). Advanced reliability methods were developed by Hasofer and Lind (1974), who solved the problem of lack of invariance of the MVFOSM method with respect to the formulation of the failure function, first explained by Ditlevsen (1981). The failure surface is approximated to a tangent hyperplane at the design point, which corresponds to the point on the failure surface closest to the origin in a normalised variable space. Chua et al. (1992) applied a reliability-based method to a pavement system that included two distress modes, fatigue cracking and rutting. The model accounts for the variations in the input parameters and predicts the probability of failure for each distress mode and for the system. The analysis considered three reliability models: FORM, which approximates the failure surface to a plane at the design point, SORM, which approximates the failure to a quadratic surface, and the Monte Carlo simulation technique for comparison of results from the previous two methods. The research concluded that the failure surface was not highly nonlinear and that the approximation by 
a plane (FORM) was acceptable for design applications. Retherford and McDonald (2010) also found that the FORM method is well suited for pavement design regarding fatigue cracking and subgrade rutting, with errors (calculated by the root sum of squares method) less than $1 \%$ when compared to the Monte Carlo simulation method. However, the FORM method is computationally intensive when the number of variables increases. The FOSM and the Rosenblueth methods were found to be the simplest processes to implement (i.e., they provided the cheapest computational effort) but were the least accurate. Other recent applications of FORM can be found in Kim (2006), Liu et al. (2005), Tighe et al. (2001), Killingsworth and Zollinger (1995).

In recent years, Zhao and Ono (2001) developed a new point estimate approach called the method of moments (MM) (see 3.1.2.2), where the first four moments of the limit state function are used to evaluate the multidimensional failure probability integral. The moment method, being very simple, has no shortcomings with respect to design points, and requires neither iteration nor the computation of derivatives, and thus is convenient to be applied to structural reliability analysis. Zhang and Damnjanović. (2006) showed an application of this method on the 1993 AASHTO pavement design equation. The method of moments is based on two sequential steps. First, the moments of the limit state function are estimated using the Monte Carlo simulation technique, using the Taylor expansion as applied in FORM or using point estimates. Second, after the moments of the limit state function are obtained, the reliability index and the failure probability are estimated using some existing standardised functions. The result from the comparison of three different methods of moments ( $2 \mathrm{M}, 3 \mathrm{M}$ and $4 \mathrm{M}$ ) with Monte Carlo simulations indicated that the $4 \mathrm{M}$ method gives the most accurate predictions of failure probability. In general, the quality of estimation improves as the order of moments increases. When comparing the second moment reliability estimates to those obtained by Monte Carlo simulations errors were found to be less than $1 \%$, see Figure 3.8 . 


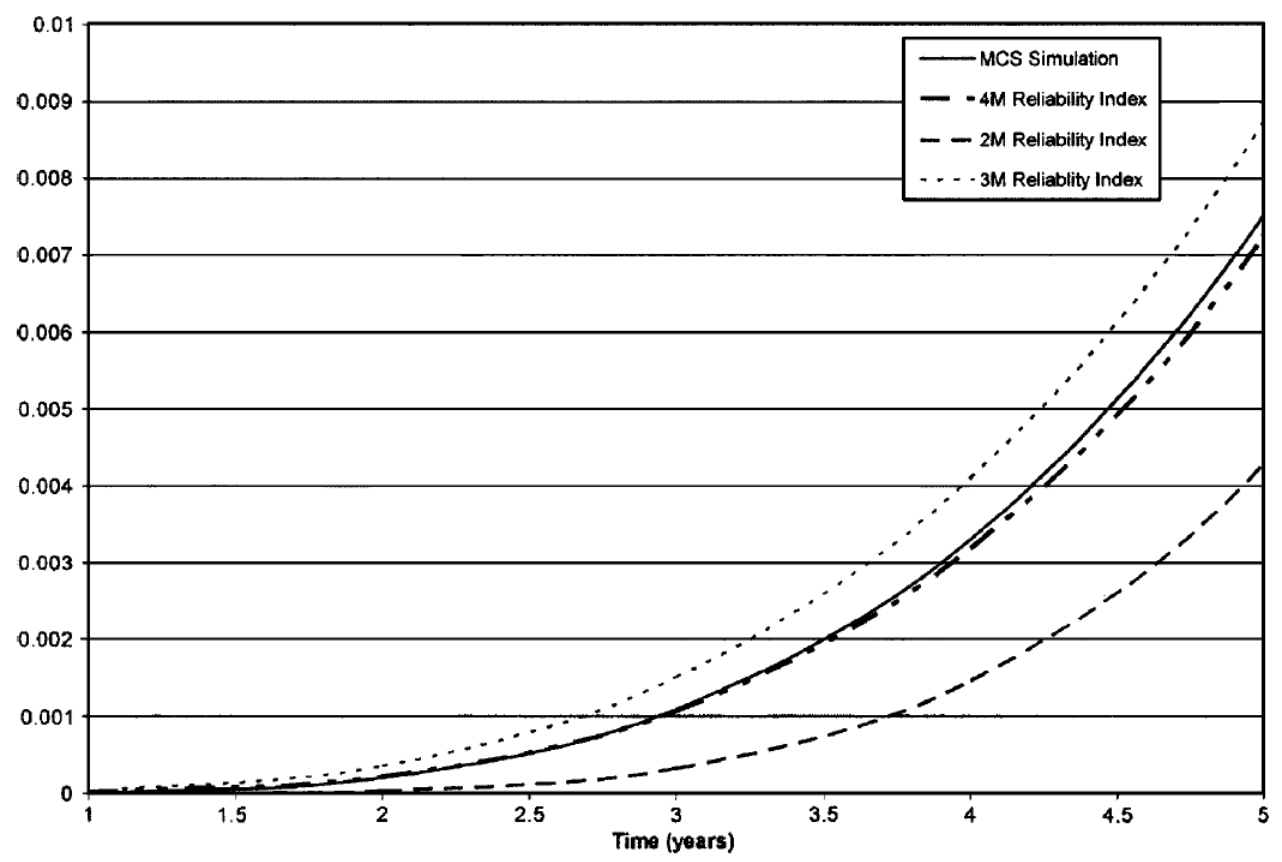

Figure 3.8 Comparison of failure probabilities using methods of moments and MCS (from Zhang and Damnjanović 2006)

In the last few years, especially with the recognised limitations of the 2002 pavement design guide, the use of the Monte Carlo simulation technique to evaluate the variation in predicted performance based on the variability of a set of input parameters for a specified pavement design section is recommended by many authors (Graves and Mahboub, 2007; Darter et al. 2005; Zhang and Piepmeyer, 2005; Timm et al., 2000).

\subsubsection{France}

Before the French Technical Guide was introduced, the design of pavement structures was specified in catalogues of standard structures in which the pavement thickness was fixed according to the traffic category and subgrade strength. Because of the changes in material performance and investment strategies, the catalogues were substituted by a more flexible pavement design method.

The French method adopts a probabilistic approach to the design by recognising all sources of uncertainty and, therefore, allowing the risk of pavement deterioration to be assessed. The concept of design risk is introduced as equivalent to the probability of failure. The success (therefore 
reliability) of a design is such that the probability of structural deterioration over the initial design period is less than a determined value.

The two major factors considered responsible for the variability of the pavement performance are the dispersions (standard deviations) in fatigue test results and layer thicknesses in construction. Both the layer thickness and fatigue test results are considered normally distributed. Relationships that relate working stresses or strains to design risk have been implicitly developed.

\subsubsection{Australia}

In Australia (Austroads 2004) the use of desired project reliability is introduced similarly to the AASHTO guidance. Project reliability is chosen by the designer. Reliability factors, which are similar to a multiplier for the calculated design traffic, are introduced to the performance models, based on the desired project reliability.

Reliability concepts are then explored by Youdale et el. (2003) to assess the risk associated with various uncertainties (uncertainty in traffic loading estimation, variations in in-situ material properties, variations in pavement layer thicknesses, and accuracy of the design method for various pavement types) in the whole-life cost estimation.

The reliability factors, similar to those contemplated in the 1993 version of the AASHTO guidance, may however be seen as resulting in conservative designs.

\subsection{Discussion}

The review of the literature available in the UK, US, France and Australia, has shown that design reliability is becoming increasingly important for consideration in pavement design procedures and whole-life cost analysis. Modern design methods are moving towards a probabilistic approach to design because it is better suited to treat the variety of uncertainty, namely that related to the prediction of traffic loading and prediction of pavement performance. The variability observed in pavement performance is primarily derived from variability in pavement materials, layer thicknesses, climatic effects, and the 
distribution of pavement distress along the length of the pavement may vary as a result.

Regardless of the various design procedure types around the world, inherent variability associated with the design input parameters will produce variable pavement performance predictions. Consequently, for a complete design procedure, the input variability must be addressed. To account for input variability, different techniques are available and probably the Monte Carlo simulation technique is the simplest, albeit time consuming and costly, to be implemented in computer programming.

The US experience has shown a structural reliability approach to pavement design where mainly the Monte Carlo simulation technique and complicated tools such as FORM and SORM are used for the analysis. It is apparent that the latter techniques, which involve very complex mathematical relationships, outside the academic or research environment are not easily accessible by the pavement community. As for the Mechanistic-Empirical Pavement Design Guide, the reliability is related to the distribution of residuals (error) between measured and predicted distress values. The error is assumed to have a normal distribution and this has already been identified by many authors as an area for further improvement.

The French approach to probabilistic design, although relatively simple, only considers two sources of variability (fatigue results and thickness) and assumes normal distribution for them. This assumption, however, may result in misleading pavement performance predictions because the variables could have probability distributions different from normal.

The Australian reliability factors, similar to those contemplated in the 1993 version of the AASHTO guidance, may be seen as resulting in conservative designs (i.e., thicker pavements).

The UK design standards generally assume an implicit $85 \%$ probability of design life survival. One of the limitations of this approach is that it does not allow the designer to choose different design reliability levels, such as for low volume roads or, on the other extreme, for very important highways involving millions of pounds. 
The common aspect to all of the approaches assessed so far can be summarised in the development of a reliability model to account for much of the uncertainty in determining design inputs and predicting pavement performance. The development of probabilistic methods and their use of distributional information about the random variables - as acknowledged by Retherford and McDonald (2010) - for reliability analysis would benefit the highway community, for example:

- They would provide a basis for quantifying the benefits of quality control and quality assurance and provide a robust basis for computation of pay factors to be awarded to contractors for meeting certain quality control standards.

- They would enable the design engineer to account for uncertainty in the design parameters and to design pavement accordingly.

The objective of this research study is to develop a methodology to address the above problem and to validate it with the incorporation of data from selected UK projects. The main aspiration is to contribute to the development of a robust but accessible reliability model which could be used to assess the probability of failure of a pavement.

To overcome the limitations and difficulties of current approaches to the reliability of flexible pavement design, as discussed in this chapter and in this final section, this research study aims to complete the following tasks on a set of existing UK road schemes:

- To assess the variability of the major input design variables (layer thickness, asphalt stiffness and subgrade stiffness) in terms of mean, variance and probability distribution.

- To identify and use simple performance equations that relate the expected fatigue $\left(\mathrm{N}_{\mathrm{f}}\right)$ and deformation life $\left(\mathrm{N}_{\mathrm{d}}\right)$ of a pavement to the major input variables named above. The purpose is to use these simplified relationships to conduct a sensitivity analysis of the input variables on the expected performance life. The analysis will assume that all causes of uncertainty are independent and not variable with time; interdependence between variables will not be considered. Although the limits of simplified 
equations are recognised (i.e., less accurate), for the purpose of this scheme-level research the benefits will be in improved understanding of the effects of the input variability in the output performance. If simple but reasonable relationships are used, some analytical approximations (i.e., Taylor series, see eq. (3.13) and (3.14)) could be employed to quickly assess the reliability of the pavement (in terms of mean and standard deviation) when expected values and coefficients of variation of the input variables are known.

- Related to the above point (i.e., to validate the results of the analytical calculations) is the run of a satisfactory number of different scenarios (i.e., based on the Monte Carlo simulation technique), taken by random sampling of the input variables, and to evaluate the resulting distribution of probability of failure.

The proposed research activities are briefly summarised in Figure 3.9.

Characterise variations in major design input parameters (layer thickness (h), subgrade stiffness (k) and asphalt stiffness (E)

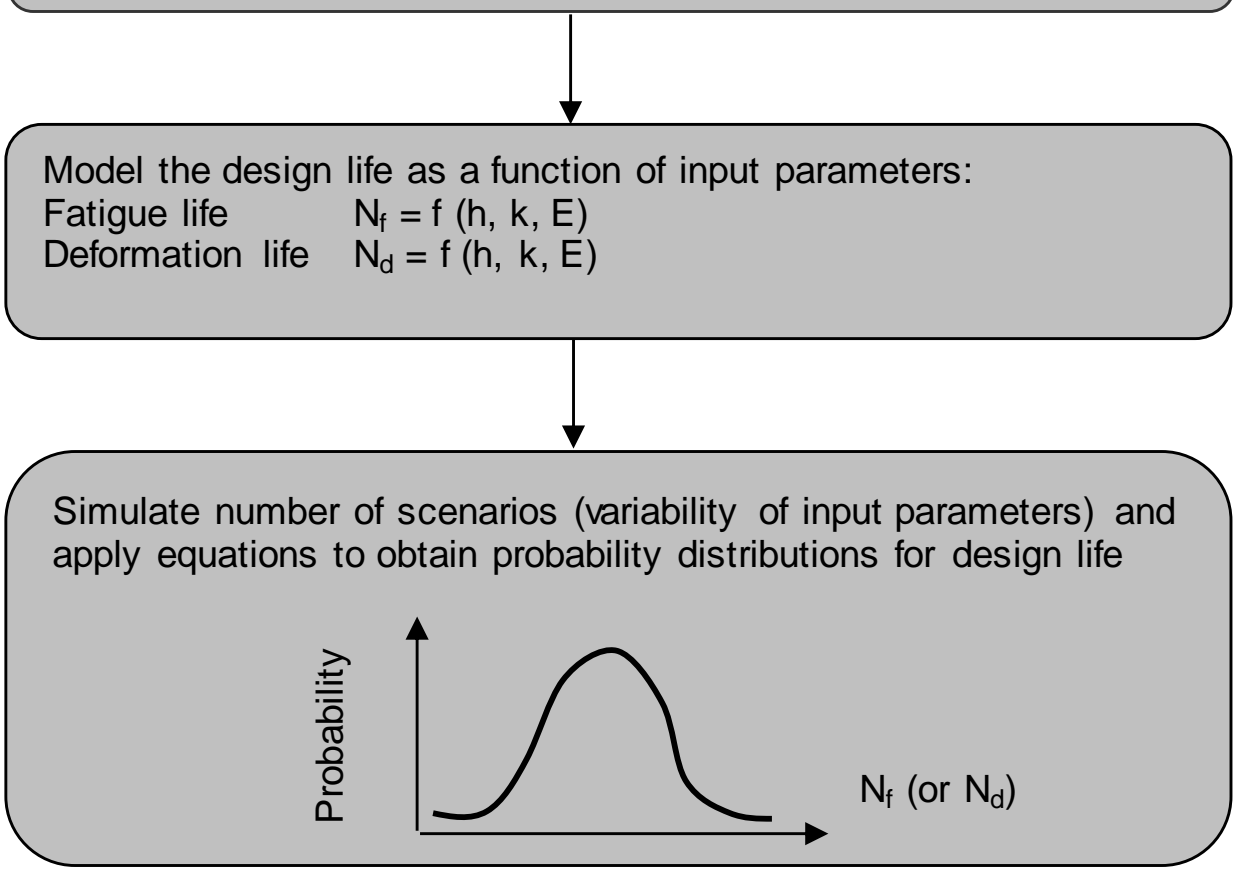

Figure 3.9 Proposed research activities

If the methodology is revealed to be sound, the implications in terms of improved grasp of design risk could be significant and could offer an attractive 
tool for designers and contractors to economically evaluate this risk. Given that some variability is expected with the major input design parameters (i.e., their values are not fixed), it is possible that these variations can greatly decrease the expected performance of the pavement structure being designed. Therefore, understanding and assessing the risk of the pavement performance throughout its design life is of paramount importance for both design and maintenance aspects. 


\section{Methodology}

This chapter provides information on the type of surveys used for the analysis, the type of sites, the standards used in the analysis, and the approaches used in the research. Specialist software packages have been used, namely:

- Matlab (R2010a, MathWorks, Cambridge, UK) for Power Spectrum analysis and Monte Carlo simulation;

- DataFit (Version 9, Oakdale Engineering, Oakdale, USA) for fitting best model to data;

- BISAR (Version 3.0, Shell, UK) for calculation of stresses and strains in the pavement structure;

- MODULUS-HA (Version 5.1, Highways Agency, UK) for Falling Weight Deflectometer back-analysis.

\subsection{Data collection techniques}

The sites used in the research are eight in total and are mainly motorways with two further sites being a thin pavement construction (M07 and M08). All sites have a fully flexible pavement construction. The sites cannot be named so a road numbering system has been employed to protect identity (i.e., M01, M02 etc).

The following survey test results have been collected:

- Ground Penetrating Radar (GPR) for thickness information of the pavement and foundation layers;

- Cores to support the GPR thickness information and for description of material types and condition of the pavement layers;

- Falling Weight Deflectometer (FWD) for assessment of the structural capacity of the pavement and foundation layers (including the subgrade);

- Dynamic Cone Penetrometer (DCP) for thickness and material information (for example CBR value) on foundation layers and subgrade; 
- Laboratory tests (i.e., Indirect Tensile Stiffness Modulus) to support the structural assessment of the pavement layers; and

- Traffic information in terms of Average Annual Daily Traffic (AADT) and percentage of Heavy Good Vehicles (HGVs).

The survey data generally refer to lane 1, the most heavily trafficked lane. The length of each site varies but, wherever possible, a length of $1 \mathrm{~km}$ has been used.

A summary of the available survey data for all sites is presented in Table 4.1 and Table 4.2. Despite a full set of survey data not being available for all sites, the information is considered sufficient to conduct a statistical analysis, particularly for the GPR data available for all sites and for the FWD survey data.

Table 4.1 shows that - except for motorway M01 - the original pavement construction for the various sites is not available. Expect for the M01 and the two thin pavement sites (M07 and M08), the pavement of all other sites has received at least one overlay treatment in the past twenty years (deduced from cores).

\section{Table 4.1 Summary of site construction data}

\begin{tabular}{|c|c|c|c|c|c|c|c|}
\hline \multirow[t]{2}{*}{ Site ID } & \multirow[t]{2}{*}{ Road type } & \multirow{2}{*}{$\begin{array}{l}\text { Year of } \\
\text { construction/last } \\
\text { major maintenance }\end{array}$} & \multicolumn{2}{|c|}{$\begin{array}{l}\text { Design Asphalt material } \\
\text { layers }\end{array}$} & \multicolumn{2}{|c|}{$\begin{array}{l}\text { Design Granular foundation } \\
\text { layers }\end{array}$} & \multirow{2}{*}{$\begin{array}{l}\text { Probable } \\
\text { Design } \\
\text { CBR }\end{array}$} \\
\hline & & & $\begin{array}{l}\text { Total Asphalt } \\
\text { thickness }(\mathrm{m})\end{array}$ & $\begin{array}{l}\text { Asphalt } \\
\text { Material }\end{array}$ & $\begin{array}{l}\text { Sub-base } \\
\text { thickness (m) }\end{array}$ & $\begin{array}{l}\text { Capping } \\
\text { Thickness (m) }\end{array}$ & \\
\hline M01 & Motorw ay & Cons: 2000 & 0.260 & HMB15 & 0.150 & 0.350 to 0.600 & 3 \\
\hline M02 & Motorw ay & Cons: 1962; Maint: 1991 & Unknow n & DBM100 & Unknow $n$ & Unknow n & 5 \\
\hline M03 & Motorw ay & Cons: 1966; Maint: 1995 & Unknow n & DBM100 & Unknow n & Unknow n & 5 \\
\hline M04 & Motorw ay & Cons: 1986 & Unknow $\mathrm{n}$ & DBM100 & Unknow n & Unknow n & 3 \\
\hline M05 & Motorw ay & Unknow n ( 1960) & Unknow $n$ & HRA & Unknow $n$ & Unknow n & 2.5 \\
\hline M06 & Motorw ay & Cons: 1986 & Unknow $n$ & DBM125 & Unknow $n$ & Unknow n & 2.5 \\
\hline M07 & Single c/w ay & Unknow n & Unknow $\mathrm{n}$ & DBM100 & NA & N/A & $\mathrm{N} / \mathrm{A}$ \\
\hline M08 & Single c/w ay & Cons: 1986 & Unknow $n$ & DBM100 & N/A & $\mathrm{N} / \mathrm{A}$ & $\mathrm{N} / \mathrm{A}$ \\
\hline
\end{tabular}

The forecasted future traffic for a 20-year design period (2006 to 2026) is above $80 \mathrm{msa}$ (million standard axles) for the majority of the sites considered. 
Table 4.2 Summary of available site data for each sub-section

\begin{tabular}{|c|c|c|c|c|c|c|c|c|c|c|c|c|c|c|c|c|c|c|}
\hline \begin{tabular}{|l|}
$\begin{array}{l}\text { Site } \\
\text { ID }\end{array}$ \\
\end{tabular} & $\begin{array}{l}\text { Traffic count } \\
\text { (year) }\end{array}$ & $\begin{array}{l}\text { AADT 1-way } \\
\text { (\%HGV) }\end{array}$ & $\begin{array}{l}\text { Past traffic } \\
\text { msa (up to } \\
\text { 2006) }\end{array}$ & $\begin{array}{l}\text { Future } 20 \text { years } \\
\text { design trafific msa } \\
\text { (2006 to 2026) }\end{array}$ & Direction & $\begin{array}{l}\text { Section } \\
\text { Length }(m)\end{array}$ & Chainage & Lane considered & $\begin{array}{l}\text { GPR } \\
\text { spacing (m) }\end{array}$ & $\begin{array}{l}\text { Nrs of points } \\
\text { every } 10 \mathrm{~m}\end{array}$ & & EW & DCP & Cores & ITSM & $\begin{array}{l}\text { Number of } \\
\text { cores }\end{array}$ & \begin{tabular}{|l|} 
Number of \\
ITSM tests
\end{tabular} & Comments \\
\hline M01 & 2006 & $\frac{44700(10)}{36700(13)}$ & $\frac{15.3}{16.2}$ & $\frac{74}{78.2}$ & Clockwise & $\frac{1000}{1000}$ & $\frac{35 / 0 \text { to } 36 / 0}{36 / 0 \text { to } 37 / 0}$ & $\frac{L 1-\operatorname{Sec} A}{L 1-\operatorname{Sec} B}$ & 0.5 & 20 & \begin{tabular}{|l|l}
$r$ \\
$r$
\end{tabular} & \begin{tabular}{|l|l}
$r$ & 1 \\
$r$ &
\end{tabular} & \begin{tabular}{|l|}
$N A$ \\
$r$
\end{tabular} & \begin{tabular}{|l|l}
2 \\
2 \\
\end{tabular} & $\frac{N A}{r}$ & $\frac{2}{16}$ & & \begin{tabular}{|l|} 
Granular sub-base \\
Granular sub-base
\end{tabular} \\
\hline & & $\frac{1070(13)}{36700(13)}$ & $16.2^{162}$ & 78.2 & & 1000 & $37 / 0$ to $38 / 0$ & $\frac{\mid L-\sec B}{L 1-\operatorname{Sec} C}$ & & & \begin{tabular}{|l|l} 
\\
2
\end{tabular} & $\frac{2}{r}$ & NA & NA & NA & & $\frac{111}{10}$ & $\begin{array}{l}\text { Grantiar s sub-base } \\
\text { Piled raft section / CBM sub-base }\end{array}$ \\
\hline & & $36700(13)$ & 16.2 & 78.2 & & 700 & $38 / 3$ to $39 / 0$ & L1-Sec D & & & $v^{2}$ & NA & NA & & $\frac{1}{2}$ & 6 & 6 & Piled raft section / CBM sub-base \\
\hline & & $38800(16)$ & 20.2 & 97.2 & & 1000 & $39 / 0$ to $40 / 0$ & $L 1-\operatorname{Sec} E$ & & & \begin{tabular}{|l|l|l|l|l}
$r$ \\
\end{tabular} & NA & & 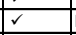 & NA & 13 & o & CBM sub-base \\
\hline & & $38800(16)$ & 20.2 & 97.2 & & 1000 & $40 / 0$ to $41 / 0$ & $\angle 1-\operatorname{Sec} F$ & & & r & NA & NA & $\checkmark$ & $\frac{d}{d}$ & 5 & 5 & CBM sub-base \\
\hline & & 45950 (14) & 20.8 & 100.1 & & 1000 & $41 / 0$ to $42 / 0$ & $L 1-\operatorname{Sec} G$ & & & $\begin{array}{r} \\
\end{array}$ & NA & & $\begin{array}{r}r \\
\end{array}$ & NA & & 0 & CBM sub-base \\
\hline & & 45950 (14) & 20.8 & 100.1 & & 1000 & $42 / 10$ to $43 / 0$ & $L 1-\operatorname{Sec} H$ & & & 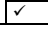 & NA & $v$ & 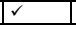 & $\mid r$ & & 3 & CBM sub-base \\
\hline M01 & 2006 & $44700(10)$ & 15.3 & 774 & Anti-Clockw ise & 1000 & $35 / 0$ to $36 / 0$ & LL1-Sec A & 0.5 & 20 & $\begin{array}{rr} \\
\end{array}$ & NA & NA & NA & INA & 0 & 0 & $\begin{array}{l}\text { Granular sub-base } \\
\text { causb-bon }\end{array}$ \\
\hline & & $36700(13)$ & $\frac{16.2}{162}$ & $\frac{78.2}{782}$ & & 1000 & $36 / 1$ to $37 / 0$ & $\angle 1-\operatorname{Sec} B$ & & & \begin{tabular}{|l|l|l}
$r$ \\
\end{tabular} & 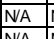 & NA & $\frac{1}{y}$ & $\frac{4}{4}$ & 4 & 3 & $\begin{array}{l}\text { CBM sub-base } \\
\text { Dild roft }\end{array}$ \\
\hline & & $\frac{36700(13)}{36700(13)}$ & 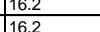 & $\frac{78.2}{78.2}$ & & $\frac{1000}{1000}$ & 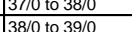 & $\begin{array}{l}\frac{L 1-\operatorname{Sec} C}{\mid L-\operatorname{Sec} D} \\
\end{array}$ & & & \begin{tabular}{|l|l|l}
2 \\
\end{tabular} & $\frac{N A}{N A}$ & 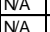 & $\frac{N A}{v}$ & $\frac{N A}{r}$ & $\frac{0}{7}$ & $\frac{0}{8}$ & 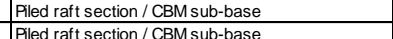 \\
\hline & & $\frac{50100(15)}{38800(16)}$ & $\frac{10.2}{20.2}$ & $\frac{10.2}{97.2}$ & & $\frac{000}{1000}$ & $39 / 0$ to $40 / 0$ & $\frac{L}{L 1-\operatorname{Sec} E}$ & & & $e^{2}$ & NA & NA & NA & NA & trit & $\frac{10}{10}$ & \begin{tabular}{|l} 
Piled ratt section / CBM sub-base \\
CBM subb-base
\end{tabular} \\
\hline & & (38000 (16) & 20.2 & 97.2 & & 1000 & $40 / 0$ to $41 / 10$ & \begin{tabular}{|l}
$L 1-\operatorname{Sec} E$ \\
$L 1-\operatorname{Sec} F$
\end{tabular} & & & \begin{tabular}{|l|l|l|l}
$z$ \\
\end{tabular} & NA & NA & & & $\frac{0}{7}$ & $\frac{0}{1}$ & Granular sub-base \\
\hline & & $45950(14)$ & 20.8 & 100.1 & & 1000 & $41 / 0$ to $42 / 0$ & $L 1-\operatorname{Sec} G$ & & & \begin{tabular}{|l|l}
$r$ \\
\end{tabular} & NA & NA & NA & NA & 10 & 10 & Granular sub-base \\
\hline & & $45950(14)$ & 20.8 & 100.1 & & 1000 & $42 / 10$ to $43 / 0$ & $\angle 1-\operatorname{Sec} H$ & & & $e^{\prime}$ & NA & NA & & a & 4 & 2 & CBM sub-base \\
\hline 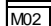 & 2004 (on lane 1) & $28688(12 \%)$ & Untrafficked & $\mid 161.8$ & Northbound & 1000 & |700 to 1700 & Hardshoulder & 1 & $\frac{110}{10}$ & 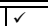 & NA & 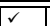 & 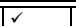 & 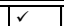 & $\sqrt{3}$ & $\sqrt{3}$ & FWD only on Lane 1; GPR on HS only \\
\hline M02 & 2004 (on lane 1) & $36512(12 \%)$ & Untrafficked & 161.8 & Southbound & 800 & 2400 to 3200 & Hardshoulder & 11 & 10 & er & NA & v & 2 & 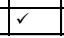 & 3 & 3 & FWD only on Lane 1; GPR on HSS only \\
\hline M03 & 2004 (on lane 1) & $31500(11 \%)$ & Untrafficked & 162.1 & Southbound & 1000 & 10 to 1010 & Hardshoulder - Sec A & 1 & 110 & $\overline{I r}$ & NA & $\overline{\mid l v}$ & $\bar{s}$ & $\overline{l e}$ & & 2 & FWDD only on Lane 1; GPR on HS only \\
\hline M03 & 2004 (on lane 1) & $31500(11 \%)$ & Untrafficked & 62.1 & Southbound & 1000 & 1010 to 2010 & Hardshoulder - Sec B & 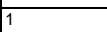 & 10 & $v^{2}$ & NA & $\begin{array}{llllll} & \end{array}$ & $a^{2}$ & 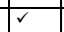 & 2 & 2 & FWD only on Lane 1; GPR on HS only \\
\hline M03 & 2004 (on lane 1) & $31500(11 \%)$ & Untrafficked & 62.1 & Southbound & 1000 & 2010 to 3010 & Hardshoulder - Sec C & 1 & 10 & $a^{2}$ & NA & $\begin{array}{llll} \\
\end{array}$ & 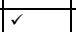 & $v^{2}$ & $t$ & 3 & FWD only on Lane 1; GPR on HS only \\
\hline M03 & 2004 (on lane 1) & $31500(11 \%)$ & Untrafficked & 162.1 & Southbound & 1000 & 3010 to 4010 & Hardshoulder - Sec D & 1 & 10 & r & NA & $r$ & $r$ & r & 2 & 3 & FWD only on Lane 1; GPR on HS only \\
\hline M04 & 2003 & $\begin{array}{lll}88976(11 \%) \\
\end{array}$ & 88.7 & 1148.9 & Southbound & 500 & 500 to 1000 & L1-Sec A & 1 & 110 & 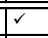 & 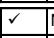 & NA & 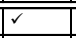 & $\overline{l e}$ & & 3 & 2 layers of gran material is show $n$ on GPR profiles \\
\hline M04 & 2003 & $88976(11 \%)$ & 83.7 & 148.9 & Southbound & 1000 & 1000 to 2000 & $L 1-\operatorname{Sec} B$ & $=$ & 10 & $e^{2}$ & 2 & $\checkmark$ & 2 & a & 2 & 6 & 2 layers of gran material is show $n$ on GPR profiles \\
\hline M04 & 2003 & $88976(11 \%)$ & 83.7 & 148.9 & Southbound & 1000 & 2000 to 3000 & L1-Sec C & $=$ & 10 & r & a & $\checkmark$ & 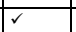 & r & 2 & 3 & 2 layers of gran material is show $n$ on GPR profiles \\
\hline M04 & 2003 & $88976(11 \%)$ & 83.7 & 148.9 & Southbound & 500 & 3000 to 3500 & $L 1-\operatorname{Sec} D$ & 1 & 10 & r & a & NA & NA & NA & 10 & 0 & 2 layers of gran material is show $n$ on GPR profiles \\
\hline M05 & 2006 & Past \& future & 67 & 107 & Clockwise & 1000 & 10 to 1010 & L1-Sec A & 10 & 1 & $\frac{1}{2}$ & 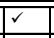 & 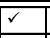 & $\sqrt{2}$ & $\bar{c}$ & & 8 & Crushed rock sub-base \\
\hline M05 & 52006 & Past \& future & 67 & 107 & Clockwise & 1000 & 1010 to 2000 & $L 1-\operatorname{Sec} B$ & 10 & 1 & $\frac{1}{d}$ & 2 & $\begin{array}{llll} \\
\end{array}$ & $\begin{array}{ll} \\
\end{array}$ & $e^{2}$ & & 2 & Crushed rock sub-base \\
\hline M05 & 52006 & Past \& future & 67 & 107 & Clockwise & 500 & 2010 to 2500 & $L 1-\operatorname{Sec} C$ & 10 & 1 & $v^{2}$ & $e^{2}$ & $\frac{a}{a}$ & $e^{2}$ & $e^{\prime}$ & & 2 & Crushed rock sub-base \\
\hline M05 & 52006 & Past \& future & 67 & 107 & Clockwise & 300 & 2710 to 3000 & L1 - Sec D & 10 & 1 & 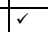 & er & NA & NA & NA & 0 & 10 & Crushed rock sub-base \\
\hline \begin{tabular}{|l|} 
M05 \\
\end{tabular} & 52006 & Past \& future & 67 & 107 & Clockwise & 1000 & 3010 to 4000 & L1 - Sec E & 10 & & $e^{2}$ & a & & Fin & $a^{2}$ & & 2 & Crushed rock sub-base \\
\hline \begin{tabular}{|l|} 
MO5 \\
\end{tabular} & 2006 & Past \& future & 67 & 107 & Clockwise & 1000 & 4010 to 5000 & $L 1-\operatorname{Sec} F$ & 10 & $f$ & $\frac{1}{2}$ & \begin{tabular}{|l} 
\\
\end{tabular} & NA & NA & NA & to & 10 & Crushed rock sub-base \\
\hline \begin{tabular}{|l|} 
MO5 \\
\end{tabular} & 52006 & Past \& future & 67 & 107 & Anti-Clockw ise & 1000 & 10 to 1010 & $L 1-\operatorname{Sec} A$ & 10 & 1 & v & 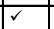 & $\begin{array}{llll} \\
\end{array}$ & S & $e^{2}$ & & & Crushed rock sub-base \\
\hline \begin{tabular}{|l|} 
M05 \\
\end{tabular} & 52006 & Past \& future & 67 & 107 & Anti-Clockw ise & 1000 & 1010 to 2000 & L1 - Sec B & 10 & $T$ & r & \begin{tabular}{|l|l|l|l|l}
$d$ \\
\end{tabular} & $v^{2}$ & $\checkmark$ & $v^{2}$ & & $\frac{1}{4}$ & $\begin{array}{l}\text { Crushed rock sub-base } \\
\end{array}$ \\
\hline M05 & 2006 & Past \& future & 67 & 107 & \begin{tabular}{|l} 
Anti-Clockw ise \\
\end{tabular} & 400 & 2010 to 2400 & $L 1-\operatorname{Sec} C$ & 10 & 1 & r & er & NA & NA & NA & $0_{0}^{2}$ & 0 & Crushed rock sub-base \\
\hline M05 & 2006 & Past \& future & 67 & 107 & Anti-Clockw ise & 400 & 2610 to 3000 & $L 1-\operatorname{Sec} D$ & 10 & 1 & r & er & $\begin{array}{llll}r \\
\end{array}$ & + & NA & & 0 & Crushed rock sub-base \\
\hline M05 & 52006 & Past \& future & 67 & 107 & Anti-Clockw ise & 1000 & 3010 to 4000 & L1 - Sec E & 10 & 1 & $a^{2}$ & a & & $\checkmark r$ & $e^{\prime}$ & & & Crushed rock sub-base \\
\hline M05 & 52006 & Past \& future & 67 & 107 & Anti-Clockw ise & 1000 & 4010 to 5000 & $L 1-\operatorname{Sec} F$ & 10 & 1 & $\frac{1}{2}$ & 2 & NA & NA & NA & 10 & 10 & Crushed rock sub-base \\
\hline M06 & 2004 & \begin{tabular}{|l|l|l}
$21700(24 \%)$ \\
\end{tabular} & 35.4 & 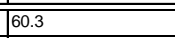 & Southbound & 500 & 29600 to 29100 & L1-LS2 & 1 & 110 & $\overline{T r}$ & $\rho^{\prime}$ & 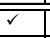 & 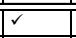 & $\overline{l v}$ & 2 & $I^{2}$ & \\
\hline M06 & 2004 & $21700(24 \%)$ & 8.1 & 13.7 & Southbound & 500 & 29600 to 29100 & L2-LS2 & $=$ & 10 & r & er & $\begin{array}{ll} \\
\end{array}$ & \begin{tabular}{|l} 
\\
\end{tabular} & r & 2 & 4 & \\
\hline M06 & 2004 & $27900(21 \%)$ & 39.1 & 66.4 & Northbound & 600 & 37500 to 38100 & L1-MN7 & 1 & 10 & r & a & NA & NA & NA & 10 & 0 & \\
\hline M06 & 2004 & $25400(24 \%)$ & 40.3 & 68.6 & Southbound & 600 & 33420 to 32820 & L1 - MS11 & 1 & 10 & 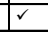 & 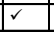 & $\checkmark r$ & $a^{v}$ & NA & 1 & 0 & \\
\hline M06 & 2004 & $28300(21 \%)$ & 39.5 & 67.2 & Southbound & 1000 & 38500 to- 37500 & L1-MS13A & 1 & 10 & $\frac{1}{2}$ & 2 & & 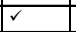 & $\frac{1}{2}$ & 1 & 2 & \\
\hline Mo6 & 2004 & $28300(21 \%)$ & 39.5 & 67.2 & Southbound & 1000 & 37500 to 36500 & L1-MS13B & 1 & 10 & in & \begin{tabular}{|l|l} 
\\
\end{tabular} & NA & NA & NA & po & 10 & \\
\hline M07 & unknown & unknown $n$ & unknown & unknown & Northbound & 1000 & 10 to 1000 & LL1 - Sec A & 0.25 & 140 & $\overline{d r}$ & $\overline{I v}$ & NA & NA & INA & 10 & 10 & Thin Pavement \\
\hline \begin{tabular}{|l|l|} 
M07 \\
\end{tabular} & unknow $n$ & unknow $n$ & unknow $\mathrm{n}$ & unknow $n$ & Northbound & 900 & 1000 to 1900 & $\angle 1-\operatorname{Sec} B$ & 0.25 & 40 & 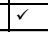 & $r$ & NA & NA & NA & 0 & 0 & Thin Pavement \\
\hline \begin{tabular}{|l|l} 
M07 \\
\end{tabular} & unknow $n$ & unknow $n$ & unknown & unknow $n$ & Southbound & 1000 & 0 to 1000 & $L 1-\operatorname{Sec} A$ & 0.25 & 40 & $\checkmark$ & 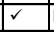 & NA & NA & NA & 0 & 0 & Thin Pavement \\
\hline \begin{tabular}{|l|} 
M07 \\
\end{tabular} & unknown & unknown & unknown & unknown & Southbound & 900 & 1000 to 1900 & $L 1-\operatorname{Sec} B$ & 0.25 & 40 & $e^{2}$ & ir & NA & NA & NA & 10 & 10 & Thin Pavement \\
\hline M08 & 2004 & $77708(7 \%)$ & 4.3 & 7.3 & Eastbound & 1000 & Do to 1000 & $L 1-\operatorname{Sec} A$ & 0.25 & 40 & $\overline{I r}$ & 18 & NA & & INA & 焉 & 10 & Thin Pavement \\
\hline \begin{tabular}{|l|} 
M08 \\
\end{tabular} & \begin{tabular}{|l|l|}
32004 \\
\end{tabular} & $7708(7 \%)$ & 4.3 & 7.3 & Eastbound & 200 & 1290 to 1490 & $L 1-\operatorname{Sec} B$ & 0.25 & 40 & $r$ & a & NA & NA & NA & 0 & 0 & Thin Pavement \\
\hline \begin{tabular}{|l|l} 
M08 \\
\end{tabular} & 2004 & $7708(7 \%)$ & 4.3 & 7.3 & Westbound & 1000 & 0 to 1000 & L1 - Sec A & 0.25 & 40 & v & 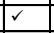 & NA & & $\checkmark r$ & J & 4 & Thin Pavement \\
\hline \begin{tabular}{|l|l} 
M08 \\
\end{tabular} & 32004 & $7708(7 \%)$ & 4.3 & 7.3 & Westbound & 500 & 1000 to 1490 & $\angle 1-\operatorname{Sec} B$ & 0.25 & 40 & d & \begin{tabular}{|l|}
2 \\
\end{tabular} & NA & 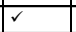 & $\checkmark$ & $T$ & 1 & Thin Pavement \\
\hline
\end{tabular}




\subsection{Standards}

The UK standards considered in the analysis are:

- Report LR1132 (Powell et al., 1984) for the calculations of fatigue and deformation life;

- HD26/01, Pavement Design, (DMRB Volume 7); and

- HD25/94, Foundations (DMRB Volume 7)

It should be noted that the above standards are now superseded. The differences between the old and the new standards do not affect this research as the ultimate goal of the study is not to focus on absolute values of fatigue and deformation life but rather on the variability (spread) of the results.

\subsection{Research challenges and methodologies}

As introduced in the previous thesis chapters, the objective of this research is to produce prediction models of the pavement design life once the variability of the design input parameters - in terms in mean, standard deviation and probability distribution - is known. In order to achieve the research objectives two main challenges need to be overcome:

- To generate artificial data for example for layer thickness and stiffness; and

- To calculate the stresses and strains at critical locations within the pavement structure for a large number of data points.

Both challenges are particularly important for the Monte Carlo simulation analysis, proposed to assess the effect of variability of input parameters on pavement performance (see chapter 3 ). The following paragraphs provide the theoretical background to the techniques used to address the research challenges above. The development and improvement of these techniques is then discussed in future chapters. 
Generation of random numbers for modelling purposes is widely used and well known in the world of science. For example, random numbers from a normal distribution with specific mean $(\mu)$ and variance $\left(\sigma^{2}\right)$ are easily obtained from statistical computer packages. If $Z$ is a standard normal deviate with mean 0 and variance 1 , then $X=Z^{*} \sigma+\mu$ will have a normal distribution with expected value $\mu$ and standard deviation $\sigma$.

Similarly, the random numbers from a lognormal distribution can be generated. The lognormal distribution is one for which the logarithms of the data values are normally distributed. If $\mathrm{W}$ has a normal distribution with mean $\mu$ and variance $\sigma^{2}$, then $\mathrm{X}=\exp (\mathrm{W})$ is a lognormal random variable with mean $m$ and variance $v$, as described by the following equations:

$$
\begin{aligned}
& m=\exp \left(\mu+\frac{\sigma^{2}}{2}\right) \\
& v=\exp \left(2 * \mu+\sigma^{2}\right) *\left(\exp \left(\sigma^{2}\right)-1\right) \\
& \mu=\log \left(\frac{m^{2}}{\sqrt{v+m^{2}}}\right) \\
& \sigma=\sqrt{\log \left(\frac{v}{m^{2}}+1\right)}
\end{aligned}
$$

where all of the logarithms are natural (base e) logarithms.

However, the techniques described above cannot realistically replicate the typical thickness profile data obtained by GPR surveys. To realistically model the real frequency characteristics of the thickness data a power spectrum analysis has been utilised. The practical application of the power spectrum analysis is described in chapter 5 .

\subsubsection{Power Spectrum Analysis}

An attempt was made to represent the layer thickness profile obtained by the GPR survey as a waveform. Any periodic non-sinusoidal waveform can be shown to be composed of a combination of sine waves, which may vary in amplitude, frequency, and phase. A rigorous mathematical technique known as 'Fourier Analysis' was applied which allows a time-domain waveform to be described in terms of both frequency-domain magnitude and phase. Once 
magnitude and phase characteristics are known, the original signal (i.e. layer thickness profile) can be reconstructed. Fourier analysis is used in many of the sciences as an analysis/synthesis method of signals. The Power spectrum density of a non-periodic signal is a function of frequency, and the area under a spectral density curve is equal to the mean square value of the signal (i.e., total signal power). The basics behind this analysis are to analyse the amplitude and the phase characteristics of a signal, by use of the Fast Fourier Transform, and then to reconstruct the original signal.

The first step was to identify uniform sections, $1 \mathrm{~km}$ long, with continuous data information for both the asphalt and granular layers thickness. Various Matlab algorithms have been used to calculate the direct spectrum and to see whether the two signals were in phase or out of phase. Some typical plots are presented in Figure 4.1 which refers to a section of the M01 (Section A, site M01, Northbound direction between MP42/0 and MP43/0).
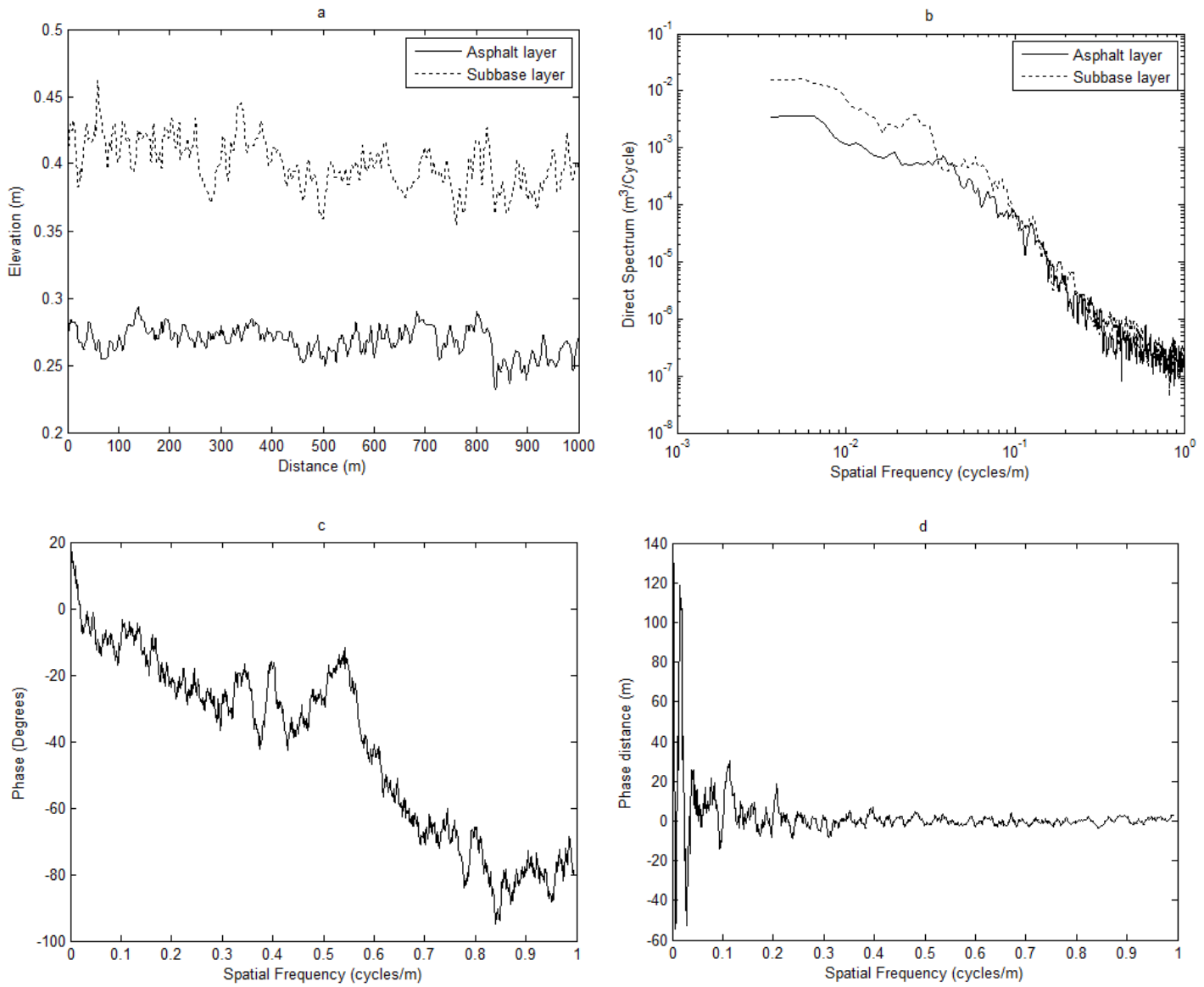

Figure 4.1 Example of Power spectrum analysis output 
Graph a) is simply a plot of the depth from the surface of the asphalt layer and of the granular sub-base layer. Graph b) is the single sided power spectral density and shows that most of the energy is in wavelengths of $2.5 \mathrm{~m}(1 / 0.4)$ or higher. Graph c) indicates, as a function of the frequency, whether the two signals are in phase or out of phase. For example, at 0.5 frequency (cycle $/ \mathrm{m}$ ) there is a difference in phase of about $30^{\circ}$. Graph $\mathrm{d}$ ) is a transformation of the phase shift into distance, always as a function of frequency.

The purpose of this analysis was to get information about the phase and amplitude contents of the various signals in order to reconstruct the original signals as well as create other hypothetical signals which could represent various scenarios of pavement thickness distributions.

\subsection{Calculation of stresses and strains}

The Analytical-Empirical (or Mechanistic-Empirical) approach to design of flexible pavements involves first the calculation of the critical stresses, strains and displacements (response models) in the individual pavement layers using an analytical model and second a comparison to permissible stresses or strains (performance/deterioration models).

Response models are developed to examine the response (deflection, strain, stress) of the pavement under the traffic loads based on a continuum mechanics approach. Responses at critical locations are often used in pavement analysis. For instance, the horizontal tensile strain at the bottom of the asphalt layer can be used to predict fatigue failure in the asphalt. The compressive strain at the top of the subgrade is used to predict deformation (rutting) failure.

In this research, Odemark's Method of Equivalent Thicknesses (MET) (Ullidtz, $1987)$ and Shell's specialist software "BISAR" were used to calculate the stresses and strains for various pavement structures.

BISAR is one amongst many available multi-layer computer programs used to calculate stresses and strains in pavements, but the main limitation is that only a maximum of 10 pavement structures can be analysed at a time and all input parameters need to be inserted manually by the user. The MET methodology 
on the other hand is not a precise method from a mathematical point of view, but it can give an instant prediction of the strains and stresses in pavement layers. It should be considered as a simple and efficient method for practical purposes, for example in Pavement Management Systems or in simulation of pavement deterioration, where stresses and strains must be calculated large numbers of times (Pearson, 2011).

\subsubsection{Response models}

The Amadeus report (2000) groups response models into two categories, namely semi-infinite half space and layered systems. Particular attention is given in this section to the MET and BISAR response models for which a comparison has been completed in the analysis section, see chapter 8 .

\subsubsection{Semi-infinite half space}

In 1885 Boussinesq solved the equations for the response of a semi-infinite elastic solid. His basic assumptions were:

- Static equilibrium.

- Compatibility (continuous solid material).

- Hooke's law.

Based on these assumptions, he established a fourth order differential equation that he solved for a point load perpendicular to the surface and for the center line of a circular load. Boussinesq's closed form solutions are very simple and allow the calculation of stresses, strains, both normal and shear, as well as displacements at any point in the halfspace under a point load or on the center line of a circular load.

For the center line of a uniformly distributed circular load, with radius $a$ and contact stress $\sigma_{o}$, the vertical stress $\sigma_{z}$, the horizontal stress $\sigma_{r}$ or $\sigma_{t}$, the vertical strain $\varepsilon_{z}$, the horizontal strain $\varepsilon_{r}$ and the vertical deflection $d_{z}$, may be calculated from: 


$$
\begin{aligned}
& \sigma_{z}=\sigma_{o}\left(1-\frac{1}{\left(\sqrt{1+\left(\frac{a}{z}\right)^{2}}\right)^{3}}\right) \\
& \sigma_{r}=\sigma_{t}=\sigma_{o}\left[\frac{1+2 v}{2}-\frac{1+v}{\sqrt{1+\left(\frac{a}{z}\right)^{2}}}+\frac{1}{2\left(\sqrt{1+\left(\frac{a}{z}\right)^{2}}\right)^{3}}\right] \\
& \varepsilon_{z}=\frac{(1+v) \sigma_{o}}{E}\left[\frac{\frac{z}{a}}{\left(\sqrt{1+\left(\frac{z}{a}\right)^{2}}\right)^{3}}-(1-2 v)\left(\frac{\frac{z}{a}}{\sqrt{1+\left(\frac{z}{a}\right)^{2}}}-1\right]\right. \\
& \varepsilon_{r}=\frac{(1+v) \sigma_{o}}{2 E}\left[\frac{-\frac{z}{a}}{\left(\sqrt{1+\left(\frac{z}{a}\right)^{2}}\right)^{3}}-(1-2 v)\left(\frac{\frac{z}{a}}{\sqrt{1+\left(\frac{z}{a}\right)^{2}}}-1\right]\right. \\
& d_{z}=\frac{(1+v) \sigma_{o} a}{E}\left[\frac{1}{\left.\sqrt{1+\left(\frac{z}{a}\right)^{2}}+(1-2 v)\right)}\right]
\end{aligned}
$$

The horizontal strain $\varepsilon_{r}$ may also be calculated using Hooke's law:

$$
\varepsilon_{r}=\varepsilon_{t}=\frac{\frac{1-v}{2 v}\left(\sigma_{z}-E \varepsilon_{z}\right)-v \sigma_{z}}{E}
$$

where $v$ is the Poisson's ratio, $E$ is the modulus of a homogeneous, isotropic, linear elastic semi-infinite space and $z$ is the depth.

A review made in the Amadeus report (2000) citing Fröhlich (1934), Kögler \& Scheidig (1938), and Boussinesq (1876) showed that the theory of elasticity was not satisfactory, particularly for granular materials, and alternative models were proposed. A large number of models have been developed since in order to achieve a better description of the stresses and strains in real materials. All of these models require supplementary input parameters in addition to the elastic parameters, and these supplementary input parameters are normally very difficult to measure. 


\subsubsection{Layered systems}

No closed form solutions, like Boussinesq's equations, exist for a layered system, like a pavement. The different approaches used to deal with layered elastic systems may be divided into: the Method of Equivalent Thicknesses (MET), Layered Analytical Models (LAM) (also referred as to Layered Elastic Theory, LET) and Finite Element Models (FEM).

\section{Method of Equivalent Thicknesses (MET)}

In 1949 Odemark presented a simplified method for dealing with a layered system. Odemark transformed the layered system into semi-infinite halfspaces, on which Boussinesq's closed form solutions could be used, see eq. (4.5) to eq. (4.10). The transformation is done by calculating the "equivalent thickness" in such a way that the stiffness of each layer is maintained (see Figure 4.2). Odemark's method is based on the assumption that the stresses and strains below a layer depend on the bending stiffness of that layer only. If the thickness, modulus and Poisson's ratio of a layer are changed, but the bending stiffness remains unchanged, the stresses and strains below the layer should also remain (relatively) unchanged (Ullidtz, 1998). The bending stiffness of a layer is proportional to:

$$
\frac{h^{3} E}{1-v^{2}}
$$

Where $h$ is the thickness of the layer, $E$ is the elastic modulus and $v$ is the Poisson's ratio. This very simple method can be used iteratively to simulate a multilayer system.

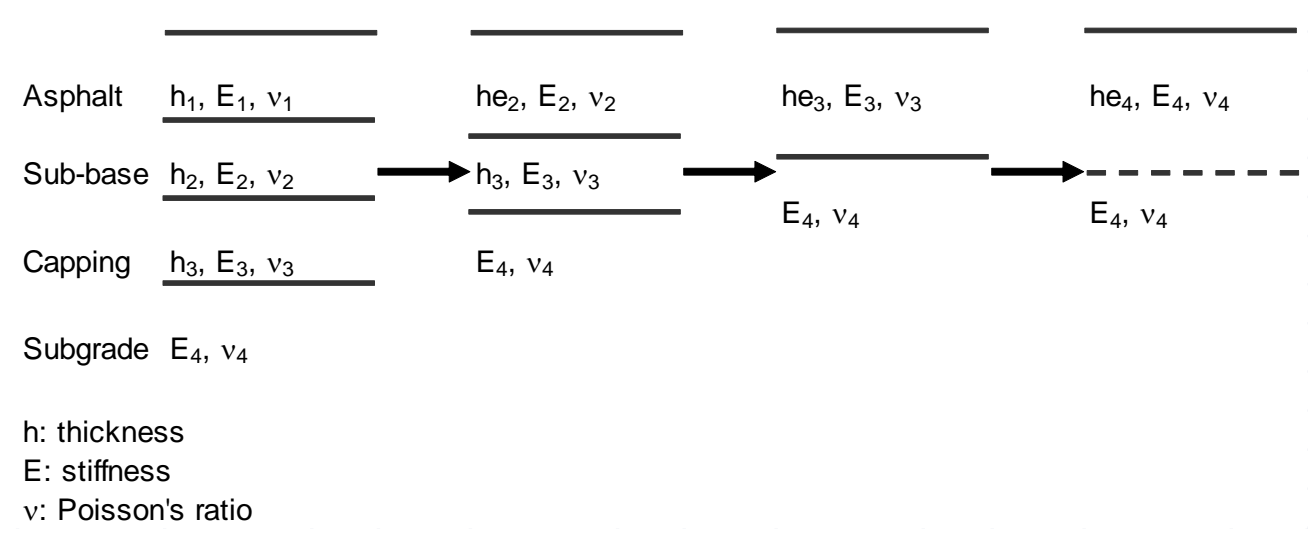

Figure 4.2 Odemark's transformation of a four-layer system 
For a multi-layer system the equivalent thickness of the upper $n-1$ layers with respect to the modulus of layer $n$ - assuming the Poisson's ratio to be the same for all layers - may be calculated from (Ullidtz, 1998 and 1987):

$$
h_{e, n}=f^{*} \sum_{i=1}^{n-1} h_{i} \sqrt[3]{\frac{E_{i}}{E_{n}}}
$$

where $h_{e, n}$ is the equivalent thickness of the layer of interest (layer n), $f$ is a correction factor used to obtain results close to the theory of elasticity, $h_{i}$ is the thickness of the layer $\mathrm{i}, E_{i}$ and $E_{n}$ are the elastic moduli of layers $i$ and $n$ respectively. Reasonably good agreement with the theory of elasticity is obtained with a correction factor of 0.8 , except for the first interface where a factor of 0.9 is used for a two-layer system and 1.0 for a multi-layer system. Odemark's method will give answers reasonably close to the theory of elasticity provided that:

- Moduli are decreasing with depth $\left(E_{i} / E_{i+1}>2\right)$, and

- The equivalent thickness of each layer is larger than the radius of the loaded area.

A limitation of the MET method for a 3 or more layer modelled pavement structure is that the asphalt strain is independent of the characteristics of the formation layers (i.e., subbase thickness and subgrade stiffness for a 3-layer pavement structure; subbase thickness, capping thickness, capping stiffness and subgrade stiffness for a 4-layer pavement structure).

\section{Layered Analytical Models}

Layered Analytical Models are generally based on the work of Burmister (1943). They are often referred to as mathematically exact solutions, where the fourth order differential equation is solved for the given boundary conditions using numerical integration. These models give the response (stresses and strains) at any point of the pavement structure induced by a wheel load, in a multi-layered, linear elastic pavement in which the layers are treated as being horizontally infinite and resting on a semi-infinite subgrade. Originally, layered analytical models of this type only considered linear elastic isotropic layers, uniform circular loading and full bond at the layer interfaces, 
but now some models can consider complex interactions between the road and tyre, multiple wheel loads and complex material behaviour (cross anisotropy, viscoelasticity). Some of the more well-known computer programs developed for calculating stresses, strains and displacements in layered elastic systems are Elsym5 (University of California), WESLEA (Waterways Experiment Station), Circly (Commonwealth Scientific and Industrial Research Organisation) and BISAR.

BISAR 3.0 (Bltumen Stress Analysis in Roads) is a linear elastic response model developed by Shell. Loading is defined for up to 10 circular areas by specifying the uniformly distributed vertical load and also a unidirectional horizontal shear. Conventional layer parameters (layer stiffness $E$ in $\mathrm{MPa}$, layer thickness $T$ in $\mathrm{m}$ and Poisson's ratio $v$ ) are needed to define the material and also slip can be defined between each layer. Input is provided via a user friendly interface. This interface allows up to ten different cases to be defined and calculated simultaneously. Facilities for duplicating the cases are included allowing for easy and rapid variation of input parameters. Included in the user interface is the facility to save all three components of a BISAR run (loads, structure, output positions) independently. This in some way is a database whereby standard loads and structures can be recalled for use in other analyses. Output is offered in two styles (a report suitable for printing or a table suitable for importing into a spreadsheet) and in two forms (block summary or detailed results). The programme is also suited to analyse stress/strain profiles in more complex designs and loading patterns, e.g. in airfields.

A limitation of BISAR is that only a maximum of 10 pavement structures can be analysed at a time and all input parameters need to be inserted manually by the user. Consequently, the software is not suitable for obtaining stresses and strains for a large number of pavement structures.

\section{Finite Element Models}

Finite Element Models are those based on the method of finite elements. This method assumes that a continuum can be divided into smaller more manageable elements. These finite elements, as the name suggests, are finite 
in size and together form a finite element mesh. Each element has its material behaviour defined. The behaviour of each element can be analysed separately and the cumulative deformations of the elements brought together to give a resultant deformation for the whole structure. These models can deal with non-linear material behaviour, complex tyre contact stresses and virtually any geometric condition, including pavement discontinuities. On the basis of the nodal displacements, stresses and strains can be computed at any location of the structure. A distinction should be made between plane stress/strain twodimensional methods (2D), axi-symmetric methods and three-dimensional methods (3D).

When moving from MET, over simple layered analytical models, via more advanced analytical models, to axi-symmetric and 3D FEM, the complexity increases, not only of the models but normally also of the input parameters required and the operation of the program. This increased complexity can only be justified if the response predicted by the more complex models is significantly closer to the actual pavement response. If the more complex models do not improve the agreement with the actual pavement response, then the simpler models are to be preferred (AMADEUS report, 2000).

\subsubsection{Literature review on layered systems response models}

\subsubsection{Current use of LET \& FEM}

Layered elastic theory (LET) - also known as Layered Analytical Models (LAM) - has been used successfully in the design and analysis of asphalt pavements for more than 50 years (Khazanovich \& Wang, 2007). LET was introduced by Burmister, who developed analytical solutions for a two-layered system and later extended them to a three-layered system. Over the years, the theory was extended for an arbitrary number of layers.

During the last thirty years, many computer codes have been developed based on the layered elastic theory. BISAR (Shell), Elsym5 (University of California), Circly (Commonwealth Scientific and Industrial Research Organisation), KENLAYER (University of Kentucky), WESLEA (Waterways Experiment 
Station), JULEA (AASHTO), and LEAF(Federal Aviation Administration) can be mentioned among others. More information on comparison of response models to measured response can be found in the AMADEUS report (2000). LET programs have been widely used in flexible pavement analysis and design procedures. For example, WESLEA is incorporated into the Minnesota Department of Transportation design program MnPAVE, and LEAF is embedded into the Federal Aviation Administration design program LEDFAA [now FAAFIELD]. BISAR is widely used in the UK for analytical design procedures and in forward analysis while MODULUS, also based on multilayer elastic theory, is recommended by the Highways Agency for FWD backanalysis.

A new mechanistic-based design procedure (MEPDG, AASHTO 2008) has been developed by the National Cooperative Highway Research Program (NCHRP) under AASHTO sponsorship. The models for the design of flexible pavements in the MEPDG guide are based on the response computed from:

- the multi-layer elastic program JULEA (Jacob Uzan Linear Elastic Analysis); or

- the 2-D Finite Element Analysis (FEA) program, Disturbed State Concept DSC2D (for non-linear analysis). The FEA program is used only when the designer chooses to use the Level 1 input to characterize the non-linear response of unbound layer materials (such as bases, sub-bases and/or sub-grades) while all bituminous and stabilised layers are always treated as linear.

MEPDG usually uses the LET program JULEA to calculate the structural responses (stresses, strains, and displacements) of flexible pavement and uses these responses to predict the type and extent of damage at various times using mechanistic or empirical models. The procedure requires performing layered elastic analysis more than 1000 times for a single design simulation of a flexible (asphalt) pavement. This makes the flexible design process very time consuming but manageable with LET analysis. The reason for not using the FEA program for regular linear analysis is because of the computational time needed for FEA. 


\subsubsection{Limitations of layered elastic theory}

As discussed in Pearson (2011), 'exact' solutions offered by computer programs are only close to 'exact' in a mathematical sense related to the numerical integration procedures. The assumptions made for static equilibrium, compatibility (or continuity) and constitutive equations (Hooks' law) are not correct for pavement structures:

- Loads are mostly dynamic, not static and are not usually circular or uniformly distributed.

- The materials are not continuous - some are even particulate (granular).

- Deformations are not only elastic but also plastic, viscous and viscoelastic and they are mostly non-linear and anisotropic.

- Precise information on the elastic parameters of granular materials and subgrades is in most cases very limited.

- When elastic equations are applied, unreal values of stress are generated in the granular layers (statically impossible). This happens especially on pavements with thin asphalt layers (Rondón et al., 2012).

Therefore, in a physical sense all solutions are far from being 'exact', they are only approximate.

Loulizi and Al-Qadi (2004) as cited in Yin (2012) - based on a study on Virginia Smart Road - identified the inability of two-dimensional (2D) layered elastic analysis LEA to predict pavement response under complex loading and environmental conditions. The authors demonstrated that the limitations of LEA can be easily managed in three-dimensional finite element analysis (3D FEA). The 3D FEA model offered a reasonable accuracy in the prediction of tensile strains when compared to field measurements.

Ozawa et al. (2009) demonstrated that software widely used for pavement structural analysis, like BISAR and GAMES, which were developed for circular loads based on multi-layer linear elastic theory, are unable to evaluate nonuniformly distributed loads (e.g. rectangular area) derived from field tyre foot prints measured by systems such as Stress-In-Motion (SIM) technology. 


\subsubsection{Advantages of layered elastic theory}

Despite the limitations of linear-elastic analysis because just a few pavement materials have a constant modulus of elasticity, Monismith (1987) as cited in Fernandes $\mathrm{Jr}$ et al. (2006) validated their use for estimation of the structural behaviour of pavements under traffic loading. Besides that, linear-elastic analysis allows a fast determination of structural responses and needs just a few parameters.

It is generally accepted that the BISAR program can be taken as the reference to which all other LET programs can be compared (Molenaar, 2007). This is because of the high mathematical stability of the BISAR program. Khazanovich \& Wang (2007) also reported BISAR as being widely recognised as the most robust LET program.

Despite the significant simplification used by multilayer linear elastic theory, a number of studies - Hildebrand (2002), Mateos et al. (2008), Mateos \& Snyder (2002) as cited in Mateos et al. (2013) - show that a reasonable degree of accuracy can be achieved by using linear elasticity (BISAR software) in order to predict pavement structural response, as soon as appropriate conditions are selected for characterisation of pavement materials. In particular, asphalt modulus should be selected for a frequency that is representative of the vehicle load pulse, and soil modulus should be determined for a load level that adequately represents the stress state developed under the vehicle loads. In a recent study conducted by Johanneck and Dai (2013) on three full-depth reclamation (FDR) sections constructed at the Minnesota Road Research Facility in 2008, BISAR simulations were performed as a means to validate sensor responses qualitatively and to examine differences in the strain profile through the depth of the pavement. The researchers found that despite the non-perfect agreement between modelled (with BISAR) and measured strain responses (i.e. in their absolute values), qualitatively, BISAR confirmed the measured observations.

Park et al. (2005) investigated the influence of 3D tyre contact stresses on predicted pavement life by comparing the results of the 3D FE model with corresponding results obtained using the layered linear elastic program, 
BISAR. Three different analysis methods were used: 3D FE with measured tyre contact stresses; BISAR using measured tyre contact areas to determine equivalent uniform circular pressure distributions (BM analysis); and BISAR using the conventional procedure of calculating the equivalent circular loaded area by dividing the tyre load by the tyre inflation pressure ( $B C$ analysis). Based on the results from comparisons of the 3D FE, BM and BC methods, the authors recommend the application of the BM method in layered elastic analyses of pavement response, for accurate predictions of both fatigue and rutting life. The results of Chen et al. (2012) also showed that the BISAR program with a single-line load model could be used to evaluate the potential for pavement failure as well as to predict pavement performance under super heavy loads. They found that BISAR gave similar results to those provided by finite element software.

\subsubsection{Advantages of MET}

Odemark's method of equivalent layer thickness (MET) has been widely used for pavement response analyses (Ullidtz, 1987) and FWD backcalculation (Ullidtz et al., 2006). Ullidtz (1987) showed that the pavement responses (stresses, strains and deflections) calculated by the method of equivalent thickness utilising Boussinesq's equations were comparable to those calculated for the same structure with the CHEVRON (Elsym 5) computer program. The results obtained with this method varied between $89 \%$ and $92 \%$ of the values obtained from the theory of elasticity.

In a recent and extensive study - based on a two-layer system with the first layer thickness varying from 2 in $(50 \mathrm{~mm})$ to $15 \mathrm{in}(380 \mathrm{~mm})$, with 5 different modular ratios of $E_{1} / E_{2}$ ranging from $\sim 3$ to $\sim 70$ and with a Poisson's ratio of 0.35 for all layers - El-Badawy \& Kamel (2011) concluded that:

- A good agreement existed between the vertical stresses at the interface between the two layers, in a two-layer system, calculated using the theory of elasticity and Odemark's concept when using a correction factor $(f)$ in the range of 0.8 to 0.9 which agrees with the other literature studies. 
- However, at any other depth within each layer, this correction factor is not a constant value but was found to be a function of the layer thickness, depth and modular ratio.

Lu et al. (2008) investigated the relative accuracy of backcalculated moduli using three response models (i.e., to calculate deflections) - Odemark, the linear elastic program WESLEA developed by the U.S. Army Corps of Engineers Waterways Experiment Station, and a layered elastic program called LEAP developed by Symplectic Engineering, Inc. for the University of California - on a three-layer pavement structure consisting of a hot-mix asphalt layer over aggregate base and subgrade $(\mathrm{AC} / \mathrm{AB} / \mathrm{SG})$ with various known moduli assumed. Results of their analysis showed that the backcalculated moduli from the three models are all within 7 percent of the assumed values. Calculated deflections with MET, from 30 tests sites, were found generally to be within $10 \%$ of the measured (from FWD) centreline deflections (Pologruto, 2001).

As reported in Ullidtz and Zhang (2002), strains under the FWD load predicted by using three methods (Odemark method (MET), the linear elastic theory (LET) and the finite element method (FEM)) reproduce with reasonable accuracy real strains measured in pavements, even if in most cases the best agreement (and the most reasonable values for layer moduli) was found using the method of equivalent thicknesses. Similar conclusions were drawn by Zhang \& Macdonald (2000). For the horizontal strains at the bottom of the asphalt layer, the calculated values with all three methods could be seen to match the measured values. The calculated vertical strains with MET were within the range of the measured values. Similarly, analysis of the backcalculation results from five $200 \mathrm{~m}$ long experimental sites in Tuscany (Italy) demonstrated that a good correlation could be established between the modulus and strain values obtained with the different calculation methods adopted (MET, LET and FEM) (Losa et al., 2008). They noted that all three calculation methods provided fairly similar results (with variations of about $10 \%$ ) for the horizontal strain at the bottom of the asphalt concrete layer while FEM and LET underestimate by an average of $14 \%$ the vertical strain on top of subgrade when compared to the value obtained by the MET approach. 


\subsubsection{Discussion}

This literature review shows that elastic multi-layer theory can be used to obtain horizontal strains at the bottom of the asphalt layer. However linear elastic models cannot correctly predict the vertical compressive strain at the top of the subgrade. To overcome this problem two main options are proposed, as indicated in the AMADEUS report (2000):

- One is to make use of a very simple model relying on Boussinesq's equations, modified for non-linear material behaviour, and Odemark's transformation of a layered system. Because of its simplicity, this method lends itself quite well to an incremental procedure.

- The other option is to make use of the Finite Element Method (FEM) which could treat material non-linearity mathematically exactly and which also allowed visco-elastic, elastoplastic and anisotropic materials. The main drawback of this method is that it is reported as being very computer intensive, particularly if 3D problems had to be treated.

Although MET (Method of Equivalent Thickness) is not a precise method from a mathematical point of view, it can predict the strains and stresses in pavement layers reasonably well. This should be considered as a simple and efficient method for practical purposes (AMADEUS, 2000). Pearson (2011) also states clear advantages with the use of Odemark's method:

- It is very fast and simple, can be included in a spreadsheet or used in a pavement management system where stresses and strains must be calculated millions of times. Therefore, this method makes structural analysis of pavements a more practical and convenient tool for engineers.

- For most practical purposes, the accuracy of approximate methods such as the MET should be quite sufficient.

- A non-linear elastic subgrade may be easily included.

Based on the results of the literature review and on the wide use of the BISAR program in the UK for analytical design it was decided to make a comparison between the MET and the BISAR approaches, see chapter 8 . The main focus of this research is not in the absolute accuracy in the calculations of stresses 
and strains in pavements (and consequent pavement lives). The main objective is rather the development of a practical approach to pavement design where the effect of variations in material properties on pavement performance can be easily assessed and statistically expressed. More precise but more complicated design formulae would necessitate sophisticated computer programs or laborious calculations which are outside the scope of this work.

\subsubsection{Performance models}

Pavement performance models form a key element in a pavement management system (PMS). At the network level, performance models are used to predict the future condition of roads. This information forms the basis for assessing the short-term and long-term rehabilitation needs of the road network. At the project level, performance models are used to carry out economic evaluation of alternative pavement design strategies (i.e. reconstruction, rehabilitation, maintenance) in order to find the most costeffective solution for the road. The review of design methods carried out by Nunn and Merrill (1997) showed that $60 \%$ of the European countries surveyed had developed an analytical design method and that these methods are very similar in concept. These methods deal with only 2 forms of deterioration, fatigue and structural deformation, in a fairly simplistic manner.

The performance prediction models used in the UK and adopted in this paper are (Powell et al., 1984):

- Structural cracking: the number of traffic loads to fatigue failure $\left(N_{t}\right)$ of asphalt layers is determined on the basis of horizontal tensile strain at the bottom of the asphalt layer $\left(\varepsilon_{r}\right)$ :

$$
\log N_{f}=-9.38-4.16 \times \log \varepsilon_{r}
$$

- Structural deformation: the number of traffic loads to deformation (rutting) failure $\left(N_{d}\right)$ is determined on the basis of vertical compressive strain at the top of the subgrade $\left(\varepsilon_{z}\right)$ :

$\log N_{d}=-7.21-3.95 \times \log \varepsilon_{z}$ 


\subsection{Conclusions}

This chapter has summarised the available survey data for all sites included in the analysis and has introduced the theory behind Fourier analysis and the Method of Equivalent Thickness (MET).

Fourier analysis has been explored to obtain information about the frequency characteristics of the GPR profile data for future artificial generation of layer thickness in Monte Carlo simulation.

The MET technique will be utilised because it is very powerful when calculating stresses and strains for a large number of pavement structures, and for Monte Carlo simulation.

The development of the above research methodologies will be discussed in chapter 5 and 8 respectively. 


\section{Statistical characterisation of layer thickness variability}

This chapter focusses on the characterisation of the variation of the layer thickness input parameter. Layer thickness information is generally obtained from as-built records, from coring or from Ground Penetrating Radar (GPR) surveys. As-built information is often difficult to find and only general layer thickness information is obtained from records. Cores/bores give more accurate layer thickness information but the survey is hazardous, destructive, time consuming and information obtained is point-specific. On the other hand, GPR surveys are rapid, cost effective, and non-destructive. However, particularly at scheme level, GPR surveys need to be calibrated against cores to increase the level of confidence in the results.

Thickness information from radar surveys has mainly been used in this research analysis given the limited amount of coring information generally available for a road scheme. To perform a statistically valid comparison between GPR and coring thickness a large number of cores would be required. Where appropriate, relevant research assumptions are summarised in italics at the end of the section.

\subsection{Design vs. constructed thickness}

Flexible pavement design in the UK comprises the following steps (input parameters):

- Calculation of the design traffic in terms of millions of standard axles (msa);

- Estimation of subgrade strength in terms of CBR value;

- Selection of bituminous material based on site characteristics and experience.

All input values are generally expressed by a single figure. The outputs of the design process are a certain thickness for the foundation and a certain thickness for the pavement layers, both characterised by a certain stiffness value. The thickness is derived directly from charts (HD 26, Design Manual 
for Roads and Bridges, Volume 7) or it can be the result of an analytical design. For major UK motorways, an $85 \%$ probability design reliability is assumed in the design equations which means that $85 \%$ of the pavement will exceed the design life.

However, the premature failure of some recently built motorways and the use of automated surveys such the Ground Penetrating Radar (GPR) have shown that the quality of construction is sometimes poor and significant variances may occur between the pavement design thickness specified and that actually constructed.

\subsection{Construction tolerances for analytical designs}

Quality of construction is controlled by the use of specifications. Construction tolerances are used to control the pavement construction process to ensure that the produced layers are within the allowed thickness tolerance. Most of the specifications now, as in the UK and USA, are performance related. They account for variability on highway pavement construction by establishing upper and lower limits for the specific material properties. As for the thickness, tolerances apply to the constructed surface of the pavement courses, as described in the Highways Agency Manual of Contract Documents for Highway Works (MCHW) Series 700. The standard clauses contain a number of tolerances which allow the contractor to complete the construction of the pavement within a variation in thickness, see also section 5.4.4.

The design thickness charts mentioned in HD26 (Design Manual for Roads and Bridges, DMRB Vol 7) have these tolerances built in. However, the tolerances currently in $\mathrm{MCHW}$ are not applicable to analytical design derived thicknesses. Experience on current projects suggests that thin bituminous pavements may require the addition of construction variability to the design to allow the successful completion of the works. The final pavement thickness must never fall below the design thickness obtained from the pavement calculation. The construction specification tolerances and the thickness of the existing bituminous platform must be combined to produce the completed pavement thickness. 
The same concept applies to the overlay maintenance scenario: the construction of remedial works must ensure that the Specification thicknesses and tolerances and existing pavement thickness will confidently produce the required minimum pavement design. Zaghloul et al. (2005) also recommended that the variability expressed by the tolerance range should be considered in the backcalculation analysis.

\subsection{Importance of layer thickness for rehabilitation design}

Pavement structural performance and rehabilitation (i.e., overlay) design depend on the in-situ layer thickness and material type and quality. Backcalculation analysis performed on measured surface deflections is used to assess the in-situ pavement structural capacity by estimating the in-situ pavement and subgrade moduli. Minor changes in asphalt layer thickness can produce significant changes in the analytical results of elastic properties of pavements (Attoh-Okine and Roddis, 1994).

The impact of variation of asphalt layer thickness on the back-calculated modulus has been investigated by Irwin (1989), Briggs et al. (1992), and Collop (2000). Attoh-Okine (1994) concluded that continuous thickness data as provided by GPR surveys, combined with deflection data, improves the interpretation and characterisation of the structural integrity of an entire length of pavement. The use of discrete core thickness (or similarly, the use of historical thickness information from a database), which in some cases does not correspond to the location of the measured deflections, will result in an inaccurate interpretation of the structural conditions of the site.

The impact of layer thickness variation on back-analysed asphalt stiffness modulus is discussed in chapter 6 . 


\subsection{Evaluation of pavement layer thickness variability}

\subsubsection{Literature research results}

As mentioned before, the pavement performance can vary significantly due to the variability in pavement layer thickness (see also section 2.6.2.2), which is mainly due to the construction process and quality control procedures in place. Therefore, even though a unique design thickness is specified for a road section (for example, in Appendix 7/1 of Series 700 of the Specification for Highway Works on the Highways Agency network) the actual (as-built) thickness is not constant. The pavement layer thickness is expected to have a certain probability distribution with a higher density around the mean target thickness. Darter et al. (1973) quantified for the first time the variability in the layer thickness as measured through standard deviation values for the various pavement materials considered: $0.41 \mathrm{in}(10.4 \mathrm{~mm})$ for the asphalt concrete, 0.68in $(17.3 \mathrm{~mm})$ for cement-treated bases, $0.79 \mathrm{in}(20.1 \mathrm{~mm})$ for aggregate bases and $1.25 \mathrm{in}(31.8 \mathrm{~mm})$ for aggregate subbases. These values were obtained from measured pavement thicknesses in highway construction in California from 1962 to 1969. Using corresponding thicknesses of these layers, an average coefficient of variation (COV) of about $10 \%$ was obtained. This was later confirmed by Noureldin et al. (1994) who summarised the COVs for the layer thickness (and other properties) of the 1986 AASHTO design guide, see Table 5.3.

More recently, Selezneva et al. (2003) studied layer thickness variability by analysing thickness data (elevation and core measurements) from a large number of newly constructed flexible and rigid pavement sections taken from the Long Term Pavement Performance (LTPP) database. The authors concluded that $86 \%$ of 1034 analysed pavement layers -corresponding to Specific Pavement Studies (SPS) sections - follow a normal distribution, with a mean COV for asphalt layers around $10 \%$. They also found that only $60 \%$ of the pavement layers have a mean thickness within $0.25 \mathrm{in}(6.4 \mathrm{~mm})$ of the required design thickness. The results above refer to a typical section containing approximately five core samples - therefore a very small sample 
size - and around 50 elevation measurements (typically one every $15 \mathrm{~m}$; section about $\sim 800 \mathrm{~m}$ long).

The LTPP database is also populated with an additional set of thickness data collected by GPR surveys for selected SPS sections. Based on the availability of GPR data for the SPS-1 sections in the State of Texas, Aguiar et al. (2009) characterised the type of distribution for the HMA (Hot Mix Asphalt concrete) surface and binder course layers, and the granular base layer thickness. The authors concluded that pavement layer thicknesses followed a normal distribution and the spread of such a distribution, measured by the coefficient of variation, is summarised in Table 5.3. A general observation - confirmed by the results in Table 5.3 - is that the variability in pavement thickness decreases from the subbase to the base layer to the surface layer due to tighter construction tolerances on the upper pavement layers. The results are in agreement with the reported values of COV by Attoh-Okine (1994) which ranged from $7.8 \%$ to $25.6 \%$ for five pavement sites in Kansas.

\subsubsection{Statistical results for sites M01 to M08 (mean, standard deviation, and probability distribution)}

To characterise layer thickness variability for the available sites, the first task was to plot the GPR data and then to group the thickness values into defined interval ranges. This exercise was done purely to obtain a feeling for the dispersion of the data. For example, a $1 \mathrm{~km}$ section of a motorway (Section A, site M01, Northbound direction) is here shown in Figure 5.1 to Figure 5.3. The pavement structure of the M01 motorway consists of $260 \mathrm{~mm}$ of asphalt material $35 \mathrm{~mm}$ of surface course, $90 \mathrm{~mm}$ of HMB15 binder course and $135 \mathrm{~mm}$ of HMB15 base) on top of $150 \mathrm{~mm}$ of granular sub-base and $350 \mathrm{~mm}$ of capping material. From the GPR survey, continuous values for the foundation are only available for the top layer (i.e., sub-base). 


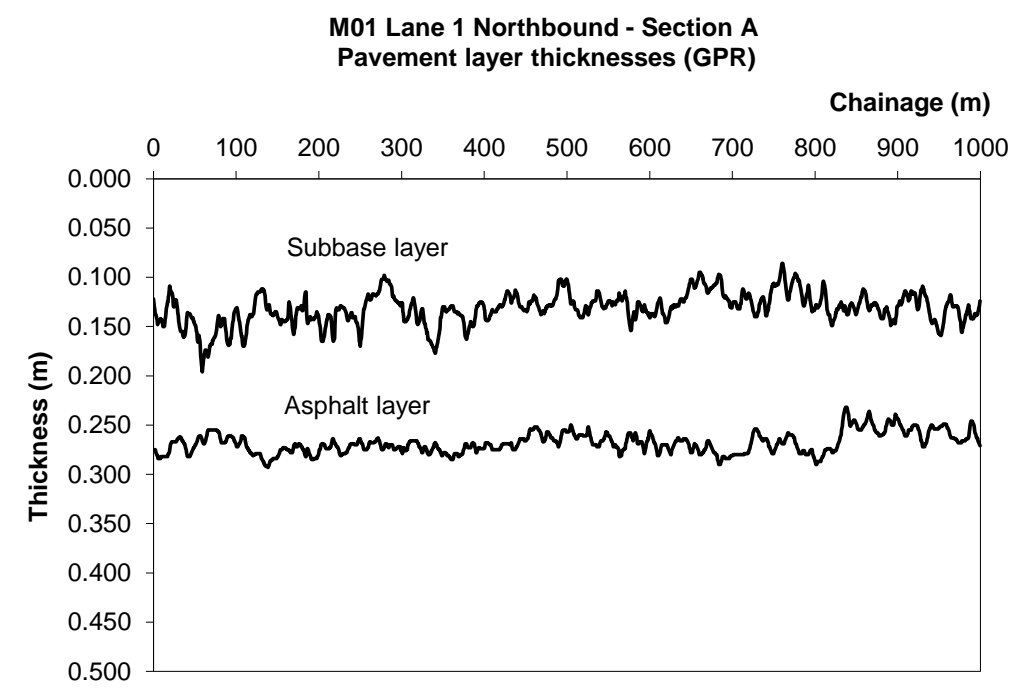

Figure 5.1 GPR thickness values for total asphalt and sub-base thickness, relating to $1 \mathrm{~km}$ section of site M01

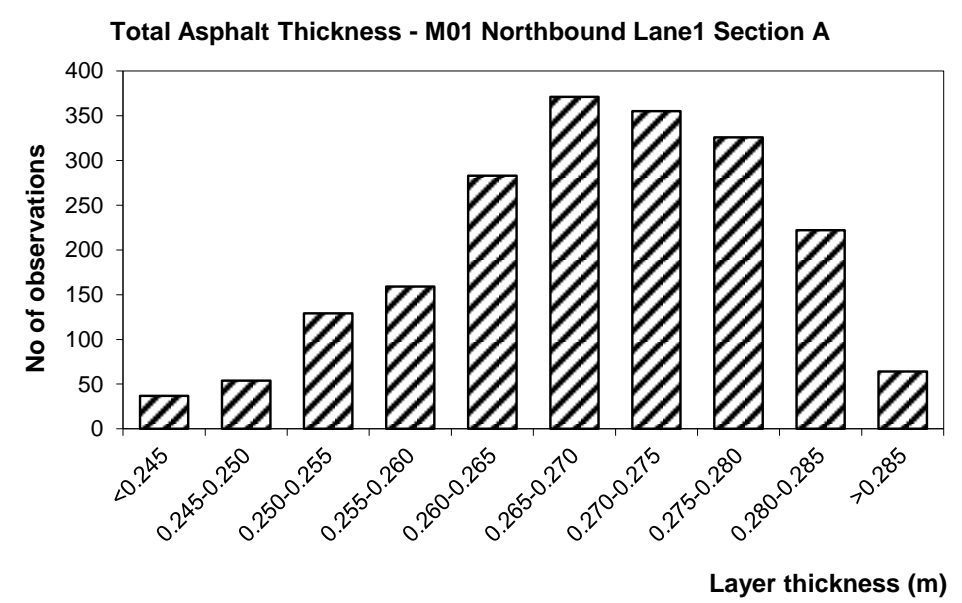

Figure 5.2 Histogram representation for total asphalt layer thickness distribution, relating to $1 \mathrm{~km}$ section of site M01

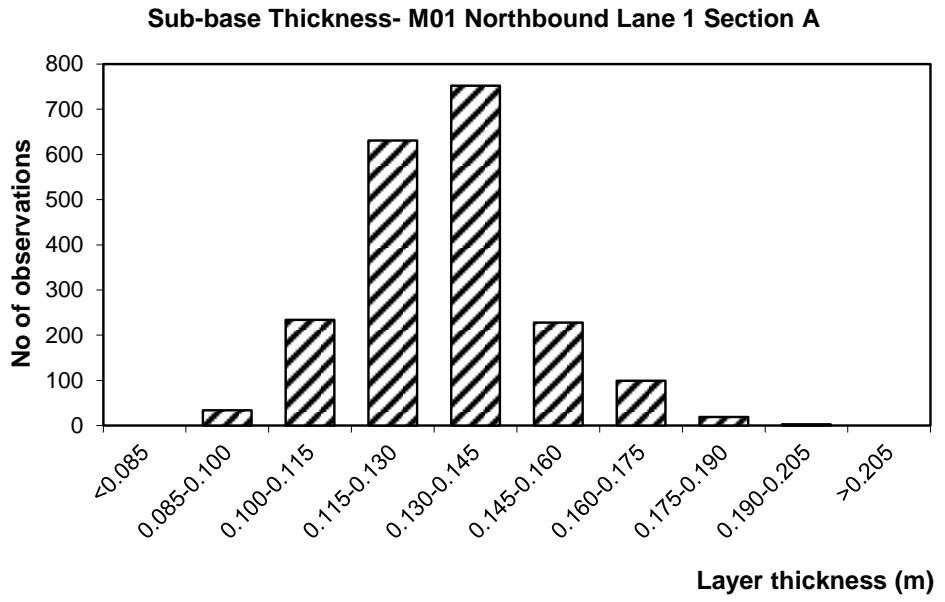

Figure 5.3 Histogram representation for sub-base layer thickness distribution, relating to $1 \mathrm{~km}$ section of site M01 
From the figures above it can be seen how variable the distribution can be around the design mean thickness of $260 \mathrm{~mm}$ and $150 \mathrm{~mm}$, for the asphalt and sub-base layer respectively.

A basic statistical analysis of the GPR data available on all sites has been performed and parameters such as mean thickness, standard deviation, coefficient of variation (COV) and the probability distribution that fits the data best have been calculated for the total asphalt pavement layer and for the subbase layer, for each section length. The results for selected sites are summarised in Table 5.1 and Table 5.2. The measured GPR thickness for the asphalt layer thickness of site M01 is above the design thickness of $0.260 \mathrm{~mm}$ in all sections. The subbase layer thickness is instead more variable and often below the design thickness of $0.150 \mathrm{~mm}$. A summary of the mean, standard deviation and coefficient of variation of the asphalt and sub-base layer thicknesses of all sites is also shown in Appendix A1. See Appendix A2 for plots of GPR layer measurements for all sites. 
Table 5.1 Statistical analysis of pavement layer thickness for available sites

\begin{tabular}{|c|c|c|c|c|c|c|c|c|c|c|c|c|}
\hline \multirow[t]{2}{*}{ Sites ID } & \multirow[t]{2}{*}{ Lane } & \multirow{2}{*}{\begin{tabular}{|l|} 
C/way \\
Direction
\end{tabular}} & \multirow[t]{2}{*}{ Sections } & \multicolumn{3}{|c|}{ Asphalt thickness } & \multicolumn{3}{|c|}{ Sub-base thickness } & \multirow{2}{*}{$\begin{array}{l}\text { Section } \\
\text { Length } \\
(\mathrm{m})\end{array}$} & \multirow{2}{*}{$\begin{array}{l}\text { GPR } \\
\text { recording } \\
\text { interval } \\
(\mathrm{m})\end{array}$} & \multirow{2}{*}{$\begin{array}{l}\text { Nr of } \\
\text { points per } \\
\text { interval }\end{array}$} \\
\hline & & & & mean $(\mathrm{m})$ & stdev (m) & COV (\%) & mean (m) & stdev (m) & Cov (\%) & & & \\
\hline M01 & L1 & CW & A & 0.279 & 0.010 & 3.4 & 0.171 & 0.036 & 20.8 & 1000 & 0.5 & 2000 \\
\hline M01 & L1 & CW & $\mathrm{B}$ & 0.292 & 0.015 & 5.0 & 0.112 & 0.038 & 33.8 & 1000 & 0.5 & 2000 \\
\hline M01 & L1 & CW & $\mathrm{D}$ & 0.268 & 0.010 & 3.5 & 0.133 & 0.015 & 11.6 & 700 & 0.5 & 1400 \\
\hline M01 & L1 & CW & $\mathrm{E}$ & 0.274 & 0.009 & 3.1 & 0.123 & 0.016 & 12.6 & 1000 & 0.5 & 2000 \\
\hline M01 & L1 & CW & $\mathrm{F}$ & 0.274 & 0.008 & 3.0 & 0.130 & 0.016 & 12.0 & 1000 & 0.5 & 2000 \\
\hline M01 & L1 & CW & $G$ & 0.276 & 0.012 & 4.2 & 0.122 & 0.029 & 24.0 & 1000 & 0.5 & 2000 \\
\hline M01 & L1 & CW & $\mathrm{H}$ & 0.266 & 0.016 & 6.1 & 0.133 & 0.020 & 14.9 & 1000 & 0.5 & 2000 \\
\hline M01 & L1 & ACW & A & 0.278 & 0.008 & 2.9 & 0.164 & 0.026 & 16.0 & 1000 & 0.5 & 2000 \\
\hline M01 & L1 & ACW & B & 0.276 & 0.008 & 2.9 & 0.129 & 0.014 & 10.5 & 1000 & 0.5 & 2000 \\
\hline M01 & L1 & ACW & D & 0.280 & 0.012 & 4.3 & 0.157 & 0.025 & 16.0 & 600 & 0.5 & 1200 \\
\hline M01 & L1 & ACW & $E$ & 0.274 & 0.009 & 3.2 & 0.133 & 0.018 & 13.3 & 1000 & 0.5 & 2000 \\
\hline M01 & L1 & ACW & $\mathrm{F}$ & 0.269 & 0.011 & 4.1 & 0.129 & 0.018 & 13.6 & 1000 & 0.5 & 2000 \\
\hline M01 & L1 & ACW & $G$ & 0.271 & 0.011 & 4.1 & 0.146 & 0.017 & 11.8 & 1000 & 0.5 & 2000 \\
\hline M01 & L1 & ACW & $\mathrm{H}$ & 0.268 & 0.011 & 3.9 & 0.132 & 0.016 & 12.2 & 1000 & 0.5 & 2000 \\
\hline$\longdiv { M 0 2 }$ & Hardshoulder & NB & & 0.235 & 0.021 & 8.9 & 0.151 & 0.029 & 19.4 & 1000 & 1 & 1000 \\
\hline $\begin{array}{l}\mathrm{M} 02 \\
\end{array}$ & Hardshoulder & SB & & 0.230 & 0.019 & 8.3 & 0.195 & 0.040 & 20.4 & 800 & 1 & 800 \\
\hline$\longdiv { M 0 3 }$ & Hardshoulder & SB & $A$ & 0.236 & 0.028 & 11.8 & 0.238 & 0.036 & 15.2 & 1000 & 1 & 1000 \\
\hline M03 & Hardshoulder & SB & $\mathrm{B}$ & 0.242 & 0.027 & 11.1 & 0.210 & 0.047 & 22.4 & 1000 & 1 & 1000 \\
\hline M03 & Hardshoulder & SB & C & 0.273 & 0.012 & 4.5 & 0.172 & 0.033 & 19.4 & 1000 & 1 & 1000 \\
\hline M03 & Hardshoulder & SB & $D$ & 0.248 & 0.016 & 6.6 & 0.200 & 0.048 & 24.1 & 1000 & 1 & 1000 \\
\hline M04 & L1 & SB & A & 0.451 & 0.016 & 3.6 & 0.246 & 0.066 & 26.8 & 500 & 1 & 500 \\
\hline M04 & L1 & SB & $B$ & 0.444 & 0.016 & 3.5 & 0.213 & 0.034 & 15.9 & 1000 & 1 & 1000 \\
\hline $\begin{array}{l}\text { M04 } \\
\end{array}$ & L1 & SB & $C$ & 0.446 & 0.021 & 4.7 & 0.199 & 0.056 & 28.0 & 1000 & 1 & 1000 \\
\hline \begin{tabular}{|l} 
M04 \\
\end{tabular} & L1 & SB & $D$ & 0.443 & 0.010 & 2.2 & 0.188 & 0.083 & 44.2 & 500 & 1 & 500 \\
\hline M05 & L1 & CW & $A$ & 0.334 & 0.014 & 4.0 & 0.185 & 0.035 & 18.7 & 1000 & 10 & 100 \\
\hline \begin{tabular}{|l|} 
M05 \\
\end{tabular} & L1 & CW & $B$ & 0.341 & 0.014 & 4.1 & 0.170 & 0.027 & 15.6 & 1000 & 10 & 100 \\
\hline M05 & L1 & CW & $C$ & 0.336 & 0.015 & 4.5 & 0.173 & 0.040 & 23.0 & 500 & 10 & 50 \\
\hline M05 & L1 & CW & $D$ & 0.324 & 0.012 & 3.8 & 0.175 & 0.025 & 14.5 & 300 & 10 & 30 \\
\hline M05 & L1 & CW & $E$ & 0.332 & 0.018 & 5.4 & 0.175 & 0.019 & 10.7 & 1000 & 10 & 100 \\
\hline M05 & L1 & CW & $\mathrm{F}$ & 0.355 & 0.017 & 4.8 & 0.183 & 0.006 & 3.2 & 1000 & 10 & 100 \\
\hline M05 & L1 & ACW & A & 0.315 & 0.017 & 5.5 & 0.204 & 0.028 & 13.7 & 1000 & 10 & 100 \\
\hline M05 & L1 & ACW & $B$ & 0.332 & 0.016 & 4.8 & 0.187 & 0.026 & 13.9 & 1000 & 10 & 100 \\
\hline M05 & L1 & ACW & $C$ & 0.336 & 0.015 & 4.6 & 0.167 & 0.021 & 12.4 & 400 & 10 & 40 \\
\hline M05 & L1 & ACW & $D$ & 0.317 & 0.043 & 13.5 & 0.188 & 0.055 & 29.2 & 400 & 10 & 40 \\
\hline M05 & L1 & ACW & $\mathrm{E}$ & 0.327 & 0.012 & 3.8 & 0.181 & 0.030 & 16.4 & 1000 & 10 & 100 \\
\hline M05 & L1 & ACW & $\mathrm{F}$ & 0.327 & 0.013 & 4.1 & 0.201 & 0.027 & 13.6 & 1000 & 10 & 100 \\
\hline \begin{tabular}{|l|} 
M06 \\
\end{tabular} & L1 & SB & LS2-L1 & 0.347 & 0.020 & 5.7 & 0.187 & 0.046 & 24.7 & 500 & 1 & 500 \\
\hline M06 & L1 & SB & LS2-L2 & 0.479 & 0.015 & 3.2 & 0.175 & 0.030 & 17.0 & 500 & 1 & 500 \\
\hline M06 & L1 & NB & MN7 & 0.380 & 0.065 & 17.0 & 0.163 & 0.038 & 23.1 & 600 & 1 & 600 \\
\hline M06 & L1 & SB & MS11 & 0.374 & 0.101 & 27.0 & 0.167 & 0.048 & 28.6 & 600 & 1 & 600 \\
\hline M06 & L1 & SB & MS13A & 0.384 & 0.051 & 13.4 & 0.245 & 0.046 & 18.8 & 1000 & 1 & 1000 \\
\hline \begin{tabular}{|l|} 
M06 \\
\end{tabular} & L1 & SB & MS13B & 0.393 & 0.056 & 14.1 & 0.210 & 0.049 & 23.4 & 1000 & 1 & 1000 \\
\hline M07 & L1 & NB & A & 0.162 & 0.043 & 26.5 & & & & 1000 & 0.25 & 4000 \\
\hline M07 & L1 & NB & $B$ & 0.230 & 0.030 & 12.9 & & & & 900 & 0.25 & 3600 \\
\hline M07 & L1 & SB & A & 0.157 & 0.036 & 22.9 & & & & 1000 & 0.25 & 4000 \\
\hline \begin{tabular}{|l|} 
M07 \\
\end{tabular} & L1 & SB & $B$ & 0.228 & 0.031 & 13.6 & & & & 900 & 0.25 & 3600 \\
\hline $\begin{array}{l}\text { M08 } \\
\end{array}$ & L1 & $E B$ & A & 0.254 & 0.032 & 12.6 & & & & 1000 & 0.25 & 4000 \\
\hline M08 & L1 & $E B$ & $B$ & 0.252 & 0.026 & 10.3 & & & & 200 & 0.25 & 800 \\
\hline M08 & L1 & WB & $\mathrm{A}$ & 0.225 & 0.036 & 16.1 & & & & 1000 & 0.25 & 4000 \\
\hline $\begin{array}{l}\text { M08 } \\
\end{array}$ & L1 & WB & $B$ & 0.207 & 0.045 & 21.8 & & & & 490 & 0.25 & 1960 \\
\hline
\end{tabular}


Table 5.2 Probability distribution for pavement layer thickness for available sites

\begin{tabular}{|c|c|c|c|c|c|c|c|}
\hline \multirow[t]{2}{*}{ Sites ID } & \multirow[t]{2}{*}{ Lane } & \multirow[t]{2}{*}{$\begin{array}{l}\text { C/way } \\
\text { Direction }\end{array}$} & \multirow[t]{2}{*}{ Sections } & \multicolumn{2}{|c|}{$\begin{array}{l}\text { Probability Distribution } \\
\text { for Asphalt layer } \\
\text { thickness }\end{array}$} & \multicolumn{2}{|c|}{$\begin{array}{l}\text { Probability Distribution } \\
\text { for Sub-base layer } \\
\text { thickness }\end{array}$} \\
\hline & & & & Normal & Lognormal & Normal & Lognormal \\
\hline M01 & L1 & cW & $\mathrm{A}$ & $\checkmark$ & & & $\checkmark$ \\
\hline M01 & L1 & CW & $B$ & $\checkmark$ & & & $\checkmark$ \\
\hline$\overline{M 01}$ & L1 & CW & $\mathrm{D}$ & $\checkmark$ & & $\checkmark$ & \\
\hline$\overline{M 01}$ & L1 & CW & $E$ & $\checkmark$ & & $\checkmark$ & \\
\hline M01 & L1 & CW & $\mathrm{F}$ & $\checkmark$ & & & $\checkmark$ \\
\hline$\overline{M 01}$ & L1 & CW & $G$ & & $\checkmark$ & & $\checkmark$ \\
\hline$\overline{M 01}$ & $\mathrm{~L} 1$ & CW & $\mathrm{H}$ & & $\checkmark$ & $\checkmark$ & \\
\hline$\longdiv { \text { M01 } }$ & L1 & ACW & $A$ & $\checkmark$ & & $\checkmark$ & \\
\hline$\overline{M 01}$ & $\mathrm{~L} 1$ & ACW & $B$ & $\checkmark$ & & & $\checkmark$ \\
\hline$\overline{M 01}$ & L1 & ACW & $\mathrm{D}$ & & $\checkmark$ & $\checkmark$ & \\
\hline M01 & L1 & ACW & $E$ & $\checkmark$ & & & $\checkmark$ \\
\hline$\overline{M 01}$ & $\mathrm{~L} 1$ & ACW & $\mathrm{F}$ & $\checkmark$ & & $\checkmark$ & \\
\hline$\overline{M 01}$ & L1 & ACW & $\mathrm{G}$ & $\checkmark$ & & & $\checkmark$ \\
\hline \begin{tabular}{|l} 
M01 \\
\end{tabular} & L1 & ACW & $\mathrm{H}$ & $\checkmark$ & & & $\checkmark$ \\
\hline$\overline{\mathrm{M} 02}$ & Hardshoulder & $\mathrm{NB}$ & & & $\checkmark$ & & $\checkmark$ \\
\hline \begin{tabular}{|l} 
M02 \\
\end{tabular} & Hardshoulder & SB & & & $\checkmark$ & $\checkmark$ & \\
\hline$\overline{\mathrm{M} 03}$ & Hardshoulder & SB & A & $\checkmark$ & & $\checkmark$ & \\
\hline$\overline{\mathrm{M} 03}$ & Hardshoulder & SB & $\mathrm{B}$ & $\checkmark$ & & $\checkmark$ & \\
\hline$\overline{\mathrm{M} 03}$ & Hardshoulder & SB & $\mathrm{C}$ & & $\checkmark$ & & $\checkmark$ \\
\hline \begin{tabular}{|l}
$\mathrm{M} 03$ \\
\end{tabular} & Hardshoulder & SB & $\mathrm{D}$ & $\checkmark$ & & & $\checkmark$ \\
\hline$\overline{M 04}$ & L1 & SB & A & & $\checkmark$ & $\checkmark$ & \\
\hline$\overline{\mathrm{M} 04}$ & $\mathrm{~L} 1$ & SB & $B$ & & $\checkmark$ & & $\checkmark$ \\
\hline$\longdiv { \mathrm { M04 } }$ & $\mathrm{L} 1$ & SB & $\mathrm{C}$ & & $\checkmark$ & $\checkmark$ & \\
\hline$\overline{M 04}$ & L1 & SB & $\mathrm{D}$ & $\checkmark$ & & & $\checkmark$ \\
\hline$\overline{M 05}$ & $\mathrm{~L} 1$ & CW & $A$ & & $\checkmark$ & & $\checkmark$ \\
\hline$\overline{M 05}$ & L1 & CW & $B$ & . & $\checkmark$ & & $\checkmark$ \\
\hline$\overline{M 05}$ & L1 & CW & $\mathrm{C}$ & $\checkmark$ & & $\checkmark$ & \\
\hline $\begin{array}{l}\text { M05 } \\
\end{array}$ & $\mathrm{L} 1$ & CW & $\mathrm{D}$ & $\checkmark$ & & $\checkmark$ & \\
\hline$\overline{M 05}$ & L1 & CW & $E$ & & $\checkmark$ & $\checkmark$ & \\
\hline M05 & L1 & $\mathrm{CW}$ & $\mathrm{F}$ & $\checkmark$ & & $\checkmark$ & \\
\hline $\begin{array}{l}\text { M05 } \\
\end{array}$ & L1 & ACW & $\mathrm{A}$ & $\checkmark$ & & $\checkmark$ & \\
\hline \begin{tabular}{|l} 
M05 \\
\end{tabular} & L1 & ACW & $\mathrm{B}$ & & $\checkmark$ & $\checkmark$ & \\
\hline $\begin{array}{l}\text { M05 } \\
\end{array}$ & L1 & ACW & $\mathrm{C}$ & & $\checkmark$ & $\checkmark$ & \\
\hline M05 & L1 & ACW & $\mathrm{D}$ & $\checkmark$ & & & $\checkmark$ \\
\hline$\overline{M 05}$ & L1 & ACW & $E$ & $\checkmark$ & & $\checkmark$ & \\
\hline M05 & L1 & ACW & $\mathrm{F}$ & $\checkmark$ & & $\checkmark$ & \\
\hline$\overline{M 06}$ & L1 & SB & LS2-L1 & & $\checkmark$ & $\checkmark$ & \\
\hline $\begin{array}{l}\text { M06 } \\
\end{array}$ & L1 & SB & LS2-L2 & & $\checkmark$ & $\checkmark$ & \\
\hline $\begin{array}{l}\text { M06 } \\
\end{array}$ & L1 & NB & MN7 & & $\checkmark$ & & $\checkmark$ \\
\hline $\begin{array}{l}\text { M06 } \\
\end{array}$ & L1 & SB & MS11 & & $\checkmark$ & & $\checkmark$ \\
\hline $\begin{array}{l}\text { M06 } \\
\end{array}$ & L1 & SB & MS13A & & $\checkmark$ & $\checkmark$ & \\
\hline$\overline{M 06}$ & $\mathrm{~L} 1$ & SB & MS13B & & $\checkmark$ & & $\checkmark$ \\
\hline $\begin{array}{l}\text { M07 } \\
\end{array}$ & L1 & $\mathrm{NB}$ & $\mathrm{A}$ & $\checkmark$ & & $\mathrm{N} / \mathrm{A}$ & $\mathrm{N} / \mathrm{A}$ \\
\hline $\begin{array}{l}\text { M07 } \\
\end{array}$ & L1 & NB & $B$ & $\checkmark$ & & NA & N/A \\
\hline \begin{tabular}{|l} 
M07 \\
\end{tabular} & L1 & SB & $\mathrm{A}$ & $\checkmark$ & & N/A & N/A \\
\hline M07 & L1 & SB & B & $\checkmark$ & & NAA & NA \\
\hline \begin{tabular}{|l} 
M08 \\
\end{tabular} & L1 & $E B$ & $\mathrm{~A}$ & & $\checkmark$ & N/A & $\mathrm{N} / \mathrm{A}$ \\
\hline \begin{tabular}{|l} 
M08 \\
\end{tabular} & L1 & $E B$ & $B$ & & $\checkmark$ & N/A & N/A \\
\hline $\begin{array}{l}\text { M08 } \\
\end{array}$ & L1 & WB & $A$ & $\checkmark$ & & $\mathrm{N} / \mathrm{A}$ & $\mathrm{N} / \mathrm{A}$ \\
\hline M08 & $\mathrm{L} 1$ & WB & $B$ & $\checkmark$ & & N/A & N/A \\
\hline
\end{tabular}


It should be noted that site M01, in contrast to all the other sites, is a recently built motorway. Therefore, the asphalt thickness data from the above scheme is more representative of currently achievable asphalt thickness variability compared to that from the other sites - excluding the 'thin' sites - which have received a varying degree of overlay in the past.

The average and range of Coefficient of Variation (COV) for the total asphalt and sub-base layer thickness of all sites is summarised in Table 5.3. The table also reports the values for layer thickness variability found in the literature, which refer to data from American databases. The layer thickness distribution is reported to have a normal probability distribution, see chapter 2 .

Table 5.3 Comparison between sites' coefficient of variation for the pavement layer thickness and literature review values

\begin{tabular}{l|l|l|l|l|l|l|}
\hline \multirow{2}{*}{ Layer } & \multicolumn{5}{|c|}{ Average Coefficient of Variation, COV, in \%, and typical range in brackets } \\
\cline { 2 - 8 } & All sites & M01 only & M01 to M05 & M06 to M08 & $\begin{array}{l}\text { Noureldin et al. } \\
(1994)\end{array}$ & $\begin{array}{l}\text { Aguiar et al. } \\
(2009)\end{array}$ \\
\hline $\begin{array}{l}\text { Bituminous } \\
\text { Surface }\end{array}$ & N/A & N/A & N/A & N/A & $7(3-12)$ & $7.2(3.2-18.4)$ \\
\hline $\begin{array}{l}\text { Bituminous } \\
\text { Binder Course }\end{array}$ & N/A & N/A & N/A & N/A & $10(5-15)$ & $13.8(11.7-16.0)$ \\
\hline $\begin{array}{l}\text { Bituminous Base } \\
\text { N/A }\end{array}$ & N/A & N/A & N/A & N/A & N/A \\
\hline $\begin{array}{l}\text { Total bituminous } \\
\text { pavement }\end{array}$ & $8.0(2.2-27.0)$ & $3.8(2.9-6.1)$ & $5.0(2.2-13.5)$ & $15.5(3.2-27.0)$ & N/A & N/A \\
\hline $\begin{array}{l}\text { Granular Base } \\
\text { N/A }\end{array}$ & N/A & N/A & N/A & $12(10-15)$ & $10.3(6.0-17.2)$ \\
\hline $\begin{array}{l}\text { Granular } \\
\text { Subbase }\end{array}$ & $18.5(3.2-44.2)$ & $15.9(10.5-33.8)$ & $17.9(3.2-44.2)$ & $22.6(17.0-28.6)$ & $15(10-20)$ & N/A
\end{tabular}

The following observations can be made from Table 5.1 and Table 5.3 and from Appendix A1 and Appendix A2:

- The total asphalt layer thickness probability distribution from the UK sites analysed can be assumed to be normal.

- The granular sub-base layer thickness probability distribution from the UK sites analysed can also be assumed to be normal.

- The range of values for the coefficient of variation relative to the asphalt layer thickness is small for M01 to M05 (from 2.2 to 13.5) while it is relatively large for M06 to M08 (from 3.2 to 27.0). Particularly high values are observed for M07 and M08 which are thin pavement construction. 
- The range of values for the coefficient of variation relative to the granular sub-base layer thickness is large for all sites.

- Motorway M01, representative of a recently built road with no maintenance treatment received as yet, shows a small COV for the asphalt layer compared to the other sites. However, the COV for the sub-base layer thickness is considerably higher confirming that much of the construction control process focusses on the upper (bound) layers.

- The range of values for the coefficient of variation of the sub-base layer thickness is generally wider and more variable (i.e., section to section variability) than that of the asphalt layer thickness.

- The coefficient of variation for sites overlaid in the past varies substantially from site to site, indicating good to poor construction workmanship skills.

- If extremely high section values for the COV relating to the asphalt layer are removed - such as $\mathrm{COV}_{\text {asphalt layer }}=27.0$ for section MS11 of M06 then the range of values for the coefficient of variation relating to the asphalt layer thickness for M01 to M06 varies from 2.2 to 17.0 with an average value of 5.7 .

- If extremely high section values for the COV relating to the sub-base layer is removed - such as $\mathrm{COV}_{\text {sub-base layer }}=44.2$ for section D of M04 - then the range of values for the coefficient of variation relating to the sub-base layer thickness for M01 to M06 varies from 3.2 to 33.8 with an average value of 17.1 .

- Despite the number of sites considered in the analysis being small compared to those considered in the USA, the COV for the asphalt and sub-base layer are broadly similar to those reported in the American literature, particularly if odd section values are removed as described above.

Research assumption: Total asphalt and granular sub-base layer thickness probability distribution is assumed to be normal. 


\subsubsection{Correlation between asphalt and sub- base layer thickness}

For each section of the available sites, plots of the asphalt versus sub-base layer thickness were created, see Appendix A3. Some graphs, in particular those referring to the hard shoulder or to sections that received an overlay treatment in the past, show a certain degree of correlation between the two GPR layer thickness measurements. For new built roads (M01 only) correlation appears to be low.

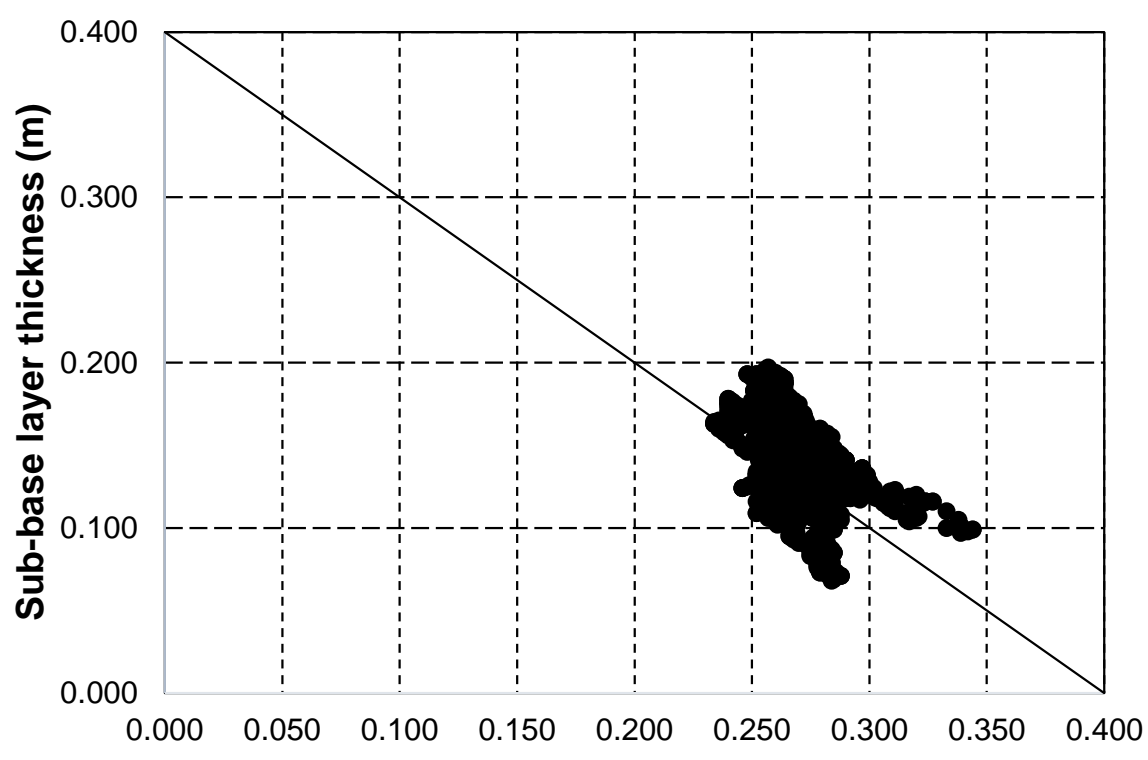

Asphalt layer thickness (m)

Figure 5.4 Asphalt versus sub-base thickness for $1 \mathrm{~km}$ section (hardshoulder) of M01

Figure 5.4 gives an example of a noticeable correlation between the asphalt and sub-base layer thickness for a $1 \mathrm{~km}$ section of the hard shoulder of motorway M01. The plot shows a negative slope, which means that when the sub-base layer is thin the asphalt layer is thick (and vice versa).

Although some degree of correlation appears to exist between the asphalt and subbase layer thickness for some sections, overall the two thickness profiles can be considered uncorrelated.

Research assumption: the asphalt and the sub-base layer thicknesses are considered uncorrelated. 


\subsubsection{Construction tolerances}

Tolerances in surface levels of pavement courses are specified in the Highways Agency Specification for Highway Works (SHW), Series 700. The standard Clause 702 contains a number of tolerances which allow the contractor to complete the construction of the pavement within a variation in thickness. The section 700 tolerances can be interpreted to suggest that the following maximum and minimum layer thicknesses could remain within a contract compliant pavement, see Table 5.4.

Table 5.4 Tolerances in surface levels of pavement courses (SHW, Series 700 )

\begin{tabular}{|l|l|}
\hline Layer & Tolerances \\
\hline Surface course & $\pm 6 \mathrm{~mm}$ \\
\hline Binder Course & $\pm 6 \mathrm{~mm}$ \\
\hline Base & $\pm 15 \mathrm{~mm}$ \\
\hline Total asphalt pavement & $-15 \mathrm{~mm}$ \\
\hline Sub-base & $+10 \mathrm{~mm}$ \\
& $-30 \mathrm{~mm}$ \\
\hline Capping & $+20 \mathrm{~mm}$ \\
& $-30 \mathrm{~mm}$ \\
\hline
\end{tabular}

Construction tolerances are already taken into account in the standards' design charts relative to the pavement thickness. Construction tolerances are particularly important when a pavement analytical design is carried out. Although design thickness proposals derived by analytical design should make allowance for construction tolerances, experience suggests that sometimes this is not the case. The analytical design method, if not conducted correctly, could lead to the design of pavement structures that are not sufficiently thick to withstand the design traffic loading. For example, if the result of an analytical design is a binder course $100 \mathrm{~mm}$ thick, the same design should be checked for a $94 \mathrm{~mm}$ binder course thickness to account for the construction tolerance expected for that layer $( \pm 6 \mathrm{~mm})$.

This study aims to see how variable the layer thickness is when a pavement is built. A review of the tolerances aims to show how well/badly built a pavement is. Sections of roads that are built thinner than the design thickness are more 
likely to be weak and to deteriorate quicker when compared to pavements built within the correct construction tolerances.

The following section explains the relationship between the standard deviation of the asphalt and subbase layer thickness and the construction tolerance, for a 3-layer pavement structure (i.e., asphalt on top of subbase on top of subgrade). The calculations assumed that the layer thickness could be approximated by the normal distribution.

The Lower Specification Limit for the total asphalt layer thickness is the designed minimum thickness allowed, below which only a small percentage of the pavement is acceptable. The upper tolerance for the total asphalt thickness is not subject to any restriction although an excessive thickness is not expected.

The percentage of the constructed asphalt layer above the designed minimum thickness is $\alpha$, where $\alpha$ is determined from the standard normal distribution tables as follows (see Figure 5.5):

$$
P\left(Z \geq \frac{L S L_{a}-\mu_{c_{a}}}{\sigma_{t_{a}}}\right)=\alpha
$$

Where:

$\mu_{c_{a}}$ is the mean thickness of the constructed asphalt layer

$L S L_{a}$ is the designed minimum thickness, i.e. the design thickness minus $15 \mathrm{~mm}$

$\sigma_{t_{a}}$ is the standard deviation of the asphalt layer.

Assuming independence between the asphalt and subbase surface profiles, the standard deviation of the asphalt thickness is estimated as follows:

$$
\sigma_{t_{a}}=\sqrt{\sigma_{s u}^{2}+\sigma_{s b}^{2}}
$$

where $\sigma_{s u}$ and $\sigma_{s b}$ are the standard deviations of the asphalt surface level departures and subbase surface level departures respectively. Surface level departures measure the difference between the constructed height of the 
pavement and the design height of the pavement, in other words measure the error of the construction process. According to Bissell (as cited in Ollis 1997), when a process is under control, statistical theory suggests that the errors are randomly and normally distributed about a mean value. Therefore, the surface level departures are assumed to be error measurements and normally distributed (Ollis 1997).

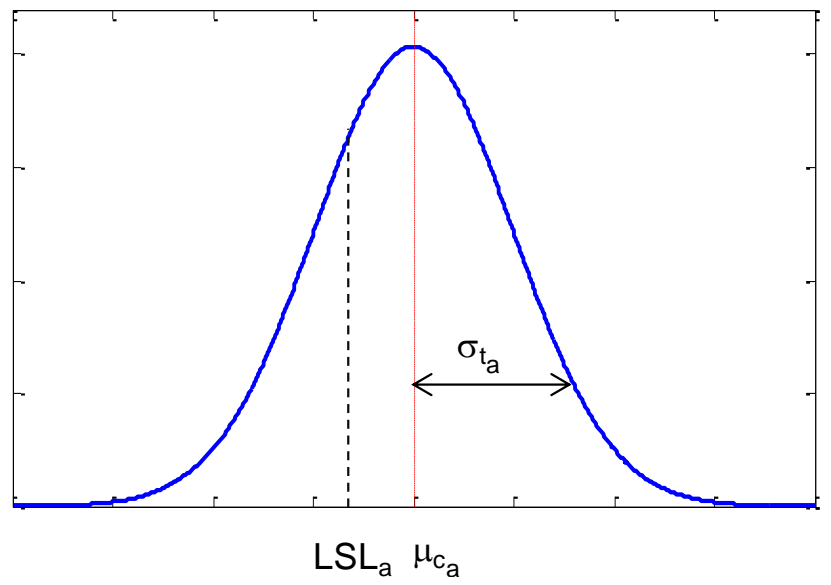

Figure 5.5 Lower tolerance range for total asphalt layer thickness

If the asphalt mean was exactly on target (i.e. the design value), the maximum value of the standard deviation, for which the probability of a thickness measurement - for the total asphalt thickness layer - being above the standards' requirement is, for example, 95\%, can be calculated with eq. (5.1) as follows:

$P\left(x \geq L_{S} a\right)=0.95$

$P\left(Z \geq \frac{L S L_{a}-\mu_{d_{a}}}{\sigma_{t_{a}}}\right)=0.95$

$P\left(Z \geq \frac{\mu_{d_{a}}-15-\mu_{d_{a}}}{\sigma_{t_{a}}}\right)=0.95$

$Z=-1.65 ;$ and $\sigma_{t_{a}}=9.11 \mathrm{~mm}$ 


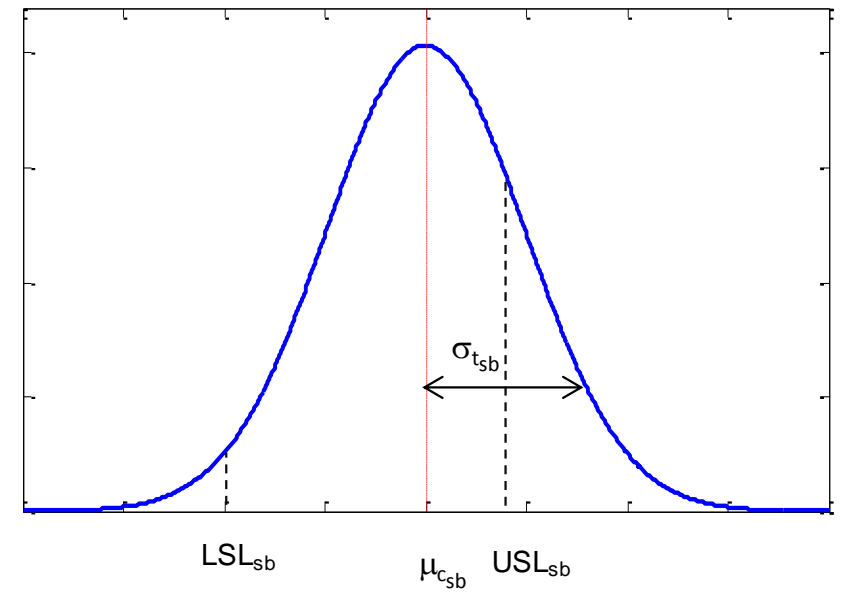

Figure 5.6 Upper and lower tolerance range for sub-base layer thickness

Similarly, the percentage of the constructed subbase layer between the upper and lower specification limits is $\alpha \%$, where $\alpha$ is determined from the standard normal distribution tables as follows (see Figure 5.6):

$$
P\left(\frac{L S L_{s b}-\mu_{c_{s b}}}{\sigma_{t_{s b}}}<Z<\frac{U S L_{s b}-\mu_{c_{s b}}}{\sigma_{t_{s b}}}\right)=\alpha
$$

Where:

$\mu_{c_{s b}}$ is the mean thickness of the constructed subbase layer

$L S L_{s b}$ is the lower specification limit for the subbase layer.

$\sigma_{t_{s b}}$ is the standard deviation of the subbase layer.

Assuming independence between the subgrade and subbase surface profiles, the standard deviation of the subbase thickness is estimated as follows:

$$
\sigma_{t_{s b}}=\sqrt{\sigma_{s g}^{2}+\sigma_{s b}^{2}}
$$

where $\sigma_{s g}$ and $\sigma_{s b}$ are the standard deviations of the subgrade surface level departures and subbase surface level departures respectively. If the subbase mean was exactly on target (i.e. the design value), the maximum value of the standard deviation, for which the probability of a thickness measurement - for the subbase thickness layer - being between the standards' requirement is, for example, $95 \%$, was calculated with eq. (5.3) as follows: 
$P\left(L_{S L s b}<x<U S L s b\right)=0.95$

$P\left(\frac{\mu_{d_{s b}}-30-\mu_{d_{s b}}}{\sigma_{d_{s b}}}<Z<\frac{\mu_{d_{s b}}+10-\mu_{d_{s b}}}{\sigma_{d_{s b}}}\right)=0.95$

After a few iterations, by selecting $\sigma=6.08 \mathrm{~mm}$, then:

$P(-4.94<Z<1.65)=0.4999+0.4505 \sim 0.95$

Appendix A3 provides an example of construction tolerances applied to the available sites. Only for motorway M01 (the only new build site available) the design thickness is known and, therefore, only for motorway M01 is it possible to see how well or badly the construction tolerances were applied. For M01 site the design thickness is $0.260 \mathrm{~m}$ for the asphalt layer and $0.150 \mathrm{~m}$ for the sub-base layer. Table 5.5 shows the results of applying eq. (5.1) and (5.3) to the sections of the M01 site. Graphs in Appendix A3 show the mean, lower and upper limits for both the design and as-built asphalt and sub-base layer thickness. It can be noted that the asphalt layer thickness is generally more controlled compared to the subbase layer thickness. All sections appear to show a large number of points relative to the subbase thickness which are well outside the permitted construction tolerances for that layer $(+10 \mathrm{~mm}-30 \mathrm{~mm})$.

Graphs relative to the other sites, which have all been overlaid in the past, are shown to illustrate the spread of the data when compared to new build sites.

Table 5.5 Review of construction tolerances for site M01, assuming $95 \%$ confidence

\begin{tabular}{|c|c|c|c|c|c|c|c|c|c|c|}
\hline \multirow[t]{2}{*}{ Lane } & \multirow{2}{*}{\begin{tabular}{|l|} 
C/way \\
Direction
\end{tabular}} & \multirow[t]{2}{*}{ Sections } & \multicolumn{3}{|c|}{ Asphalt thickness } & \multicolumn{3}{|c|}{ Sub-base thickness } & \multirow{2}{*}{$\begin{array}{l}\text { Asphalt tolerances } \\
P\left(T_{a}>0.245 m\right)\end{array}$} & \multirow{2}{*}{$\begin{array}{l}\text { Subbase tolerances } \\
\mathrm{P}\left(0.120 \mathrm{~m}<\mathrm{T}_{\mathrm{sb}}<0.160 \mathrm{~m}\right)\end{array}$} \\
\hline & & & mean $(\mathrm{m})$ & stdev $(\mathrm{m})$ & COV $(\%)$ & mean $(\mathrm{m})$ & stdev $(\mathrm{m})$ & COV $(\%)$ & & \\
\hline L1 & CW & A & 0.279 & 0.010 & 3.4 & 0.171 & 0.036 & 20.8 & $100 \%$ & $31 \%$ \\
\hline $\mathrm{L} 1$ & CW & $B$ & 0.292 & 0.015 & 5.0 & 0.112 & 0.038 & 33.8 & $100 \%$ & $31 \%$ \\
\hline $\mathrm{L} 1$ & CW & $\mathrm{D}$ & 0.268 & 0.010 & 3.5 & 0.133 & 0.015 & 11.6 & $99 \%$ & $76 \%$ \\
\hline $\mathrm{L} 1$ & $\mathrm{CW}$ & $\mathrm{E}$ & 0.274 & 0.009 & 3.1 & 0.123 & 0.016 & 12.6 & $100 \%$ & $58 \%$ \\
\hline L1 & CW & $\mathrm{F}$ & 0.274 & 0.008 & 3.0 & 0.130 & 0.016 & 12.0 & $100 \%$ & $72 \%$ \\
\hline $\mathrm{L} 1$ & $\mathrm{CW}$ & $\mathrm{G}$ & 0.276 & 0.012 & 4.2 & 0.122 & 0.029 & 24.0 & $100 \%$ & $43 \%$ \\
\hline $\mathrm{L} 1$ & $\mathrm{CW}$ & $\mathrm{H}$ & 0.266 & 0.016 & 6.1 & 0.133 & 0.020 & 14.9 & $90 \%$ & $66 \%$ \\
\hline $\mathrm{L} 1$ & ACW & $\mathrm{A}$ & 0.278 & 0.008 & 2.9 & 0.164 & 0.026 & 16.0 & $100 \%$ & $39 \%$ \\
\hline L1 & ACW & $B$ & 0.276 & 0.008 & 2.9 & 0.129 & 0.014 & 10.5 & $100 \%$ & $73 \%$ \\
\hline $\mathrm{L} 1$ & ACW & $D$ & 0.280 & 0.012 & 4.3 & 0.157 & 0.025 & 16.0 & $100 \%$ & $48 \%$ \\
\hline $\mathrm{L} 1$ & ACW & $E$ & 0.274 & 0.009 & 3.2 & 0.133 & 0.018 & 13.3 & $100 \%$ & $70 \%$ \\
\hline L1 & ACW & $\mathrm{F}$ & 0.269 & 0.011 & 4.1 & 0.129 & 0.018 & 13.6 & $98 \%$ & $65 \%$ \\
\hline L1 & ACW & $\mathrm{G}$ & 0.271 & 0.011 & 4.1 & 0.146 & 0.017 & 11.8 & $99 \%$ & $72 \%$ \\
\hline $\mathrm{L} 1$ & ACW & $\mathrm{H}$ & 0.268 & 0.011 & 3.9 & 0.132 & 0.016 & 12.2 & $99 \%$ & $73 \%$ \\
\hline
\end{tabular}




\subsection{Generation of random signals}

The research study will perform Monte Carlo simulations, by taking random samples of the input variables, and to evaluate the resulting distribution of probability of failure. In order to carry out this activity it is necessary to generate random asphalt and sub-base thickness profiles which have similar frequency characteristics to the real case study profiles.

A pavement surface displacement profile was generated from the equation given by Collop (1994) for single sided pavement surface profile spectra $\mathrm{Sz}(\gamma)$ :

$$
S_{Z}(\gamma)=c * \gamma^{-w} \quad(0<\gamma<\infty)
$$

Where $\gamma$ is the wavenumber (cycles $/ \mathrm{m}$ ), $\mathrm{w}$ is the waviness - with a typical value of 2.5 - while $\mathrm{c}$ (in $\mathrm{m}^{1 / 2} \mathrm{cycle}^{3 / 2}$ ) was estimated from:

$$
c \approx 1.6910^{-8} *(I R I)^{2}
$$

where the International Roughness Index (IRI) is used to quantify the pavement surface roughness.

For each $1 \mathrm{~km}$ section of Motorway M01 (for which surface profile measurements were available), the following procedure was adopted to generate random asphalt and sub-base layer thickness displacement histories:

- A one-dimensional random profile was generated by applying a set of random phase angles, uniformly distributed between 0 and $2 \pi$, to a series of coefficients derived from the desired direct spectral density (Cebon and Newland 1983, reported procedure below). The corresponding series of spot heights $\left\{Z_{r}^{(1)}\right\}$ at regular intervals along the section was obtained by taking the inverse discrete Fourier transform of the spectral coefficients.

$$
\left\{Z_{r}^{(1)}\right\}=\sum_{k=0}^{N-1} \sqrt{S_{k}} * e^{i\left[\theta_{k}^{(1)}+\frac{2 \pi k r}{N}\right]} \quad \mathrm{r}=0,1,2, \ldots,(\mathrm{N}-1)
$$

Where $S_{k}=\frac{2 \pi}{N \Delta} S\left(\gamma_{k}\right)$

$S\left(\gamma_{k}\right)=$ the desired spectral density, calculated with eq. (5.5) and eq. (5.6)

$\gamma_{k}=\frac{2 \pi k}{N \Delta}=$ the spatial frequency (wavenumber) 
$\Delta=$ the distance interval between successive ordinates of the surface profile; and

$\left\{\theta_{k}^{(1)}\right\}=$ a series of independent random phase angles uniformly distributed between 0 and $2 \pi$.

- With the above procedure three (uncorrelated) profiles were generated (see Figure 5.7):

- $\quad Z_{\text {su, for the pavement surface; }}$

- Zsb, for the bottom of asphalt; and

- $Z_{s g}$, for the bottom of sub-base

- The section average asphalt and sub-base layer thickness values were then added to the bottom of asphalt and bottom of sub-base profiles generated above. This is because the profiles generated with the method described above are uncorrelated random zero mean variables with Gaussian (normal) probability distribution and with a certain standard deviation.

- For motorway M01 the real IRI surface value was calculated for each section from supplied profile measurements using the ProVAL software (FHWA). The profile measurement data points (collected every $0.1 \mathrm{~m}$ ) were averaged to have the same number of data points as those from the GPR data (collected every $0.5 \mathrm{~m}$ ). The IRI values for the bottom of asphalt and sub-base profiles were instead calculated iteratively in the following way. First, a value representative of the bottom of asphalt profile which gave the best match between the real and the artificial probability distributions of the asphalt layer thickness (see next bullet point) was calculated. Once the IRI values of the pavement surface and bottom of asphalt profiles were fixed, the $I R I$ relative to the bottom of sub-base was calculated. The value that gave the best match between the real and the artificial probability distributions of the sub-base layer thickness was chosen.

- The artificially generated asphalt and sub-base (granular) layer thicknesses were calculated by subtracting the relevant profiles, i.e.:

- $h_{\text {asphalt }}=Z_{s b}-Z_{s u}$ 
- $h_{\text {granular }}=Z_{s g}-Z_{s b}$

Results of the procedure described in the previous bullet points are depicted in Figure 5.8 to Figure 5.14 for section B of the M01 motorway, Anti-Clockwise direction.

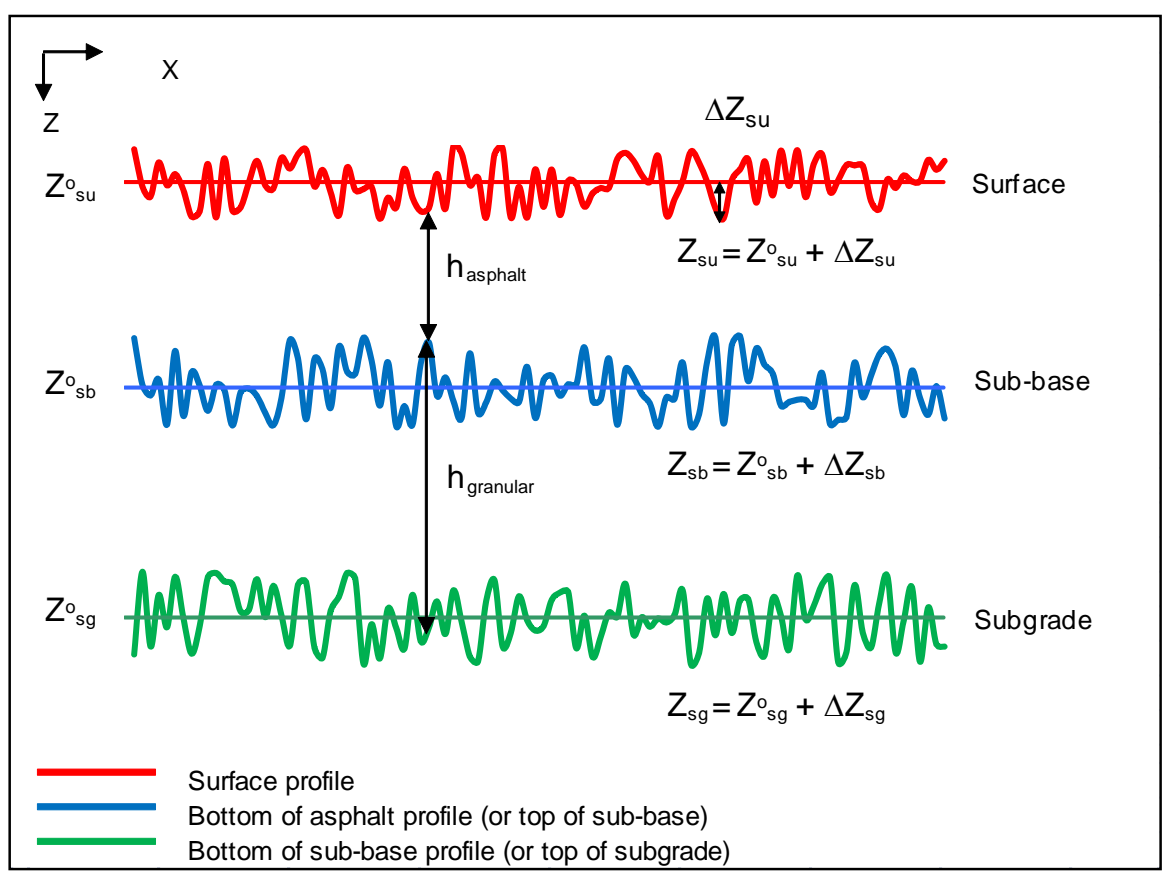

Figure 5.7 Schematic explanation of random signals generation

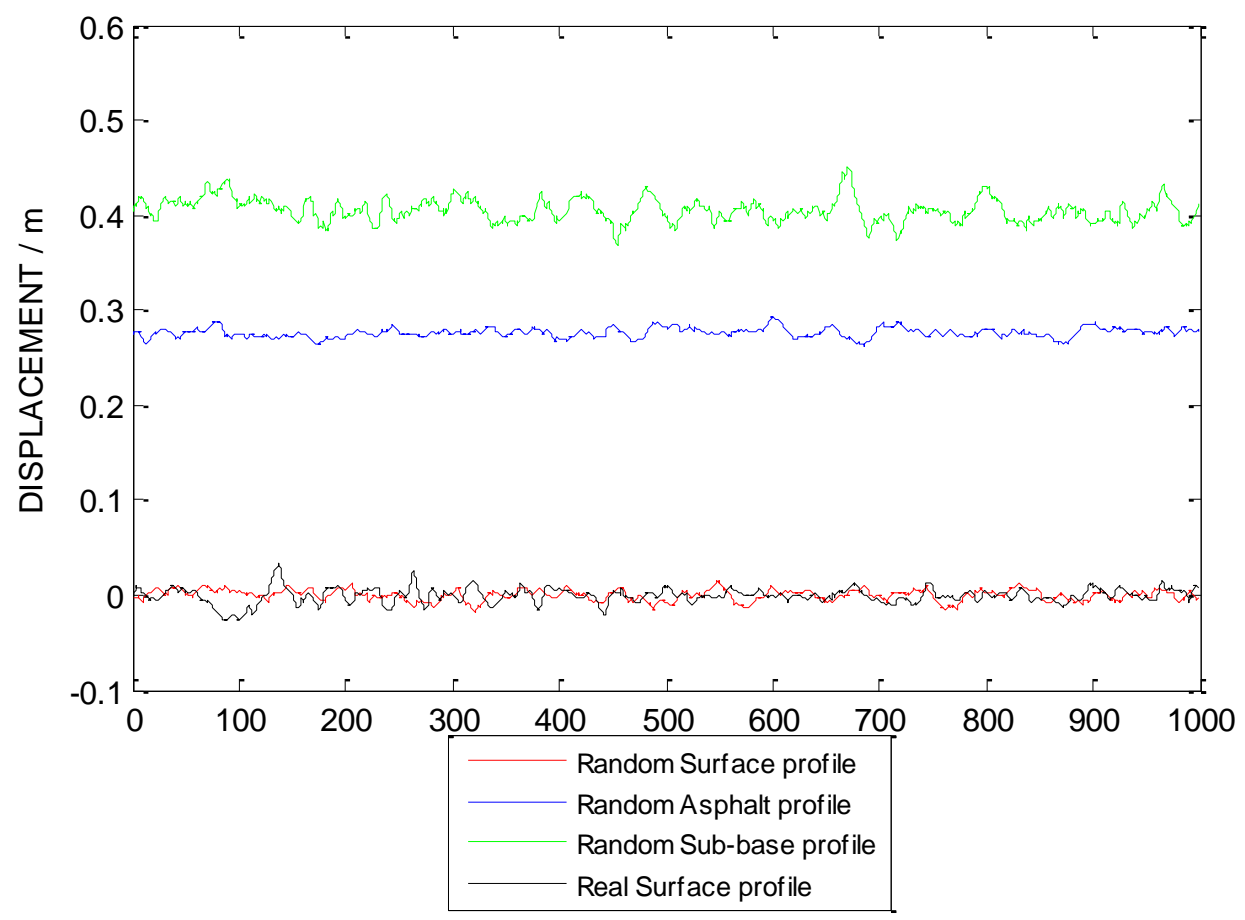

Figure 5.8 Random generated profiles 


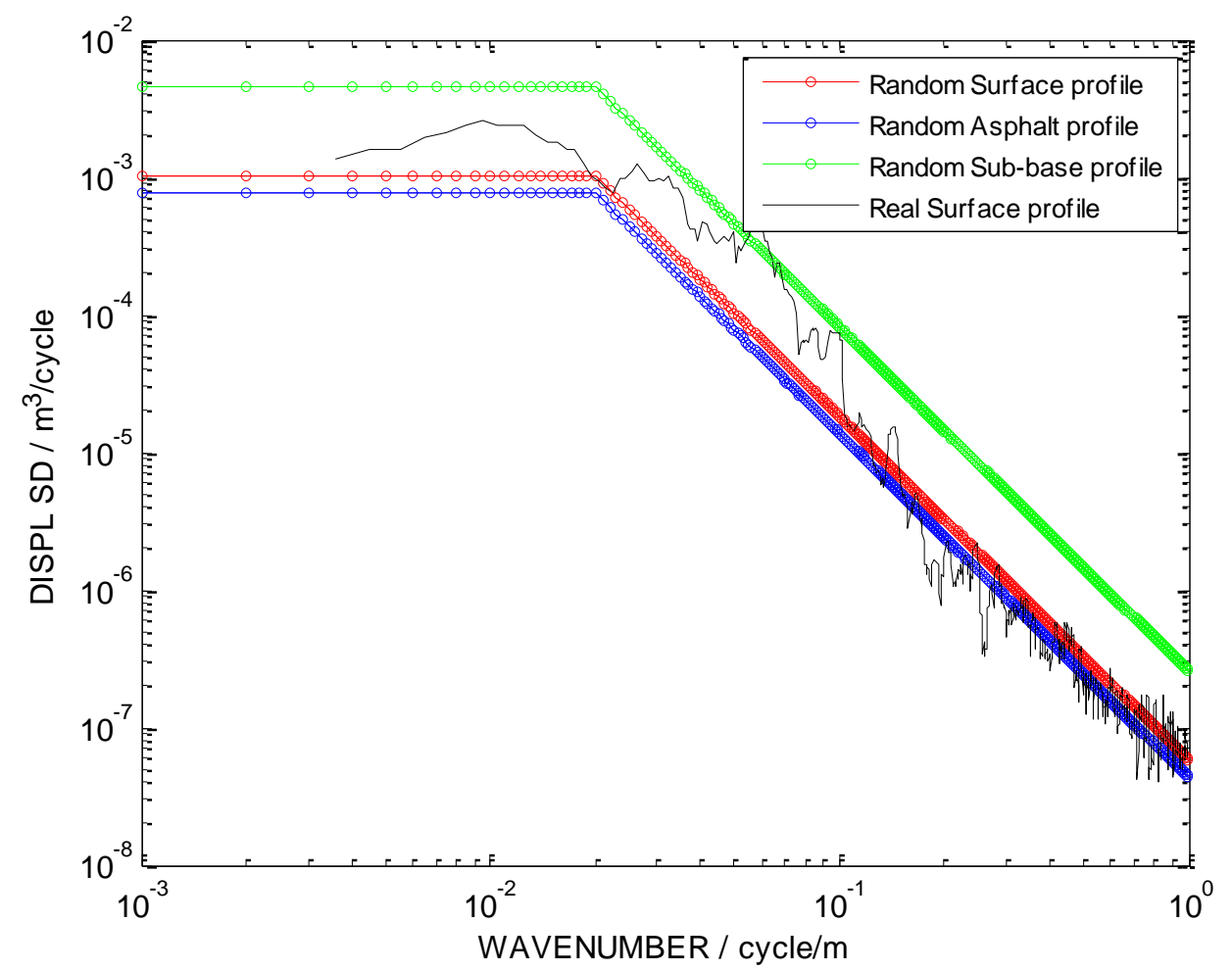

Figure 5.9 Spectral density of generated profiles and real surface profile

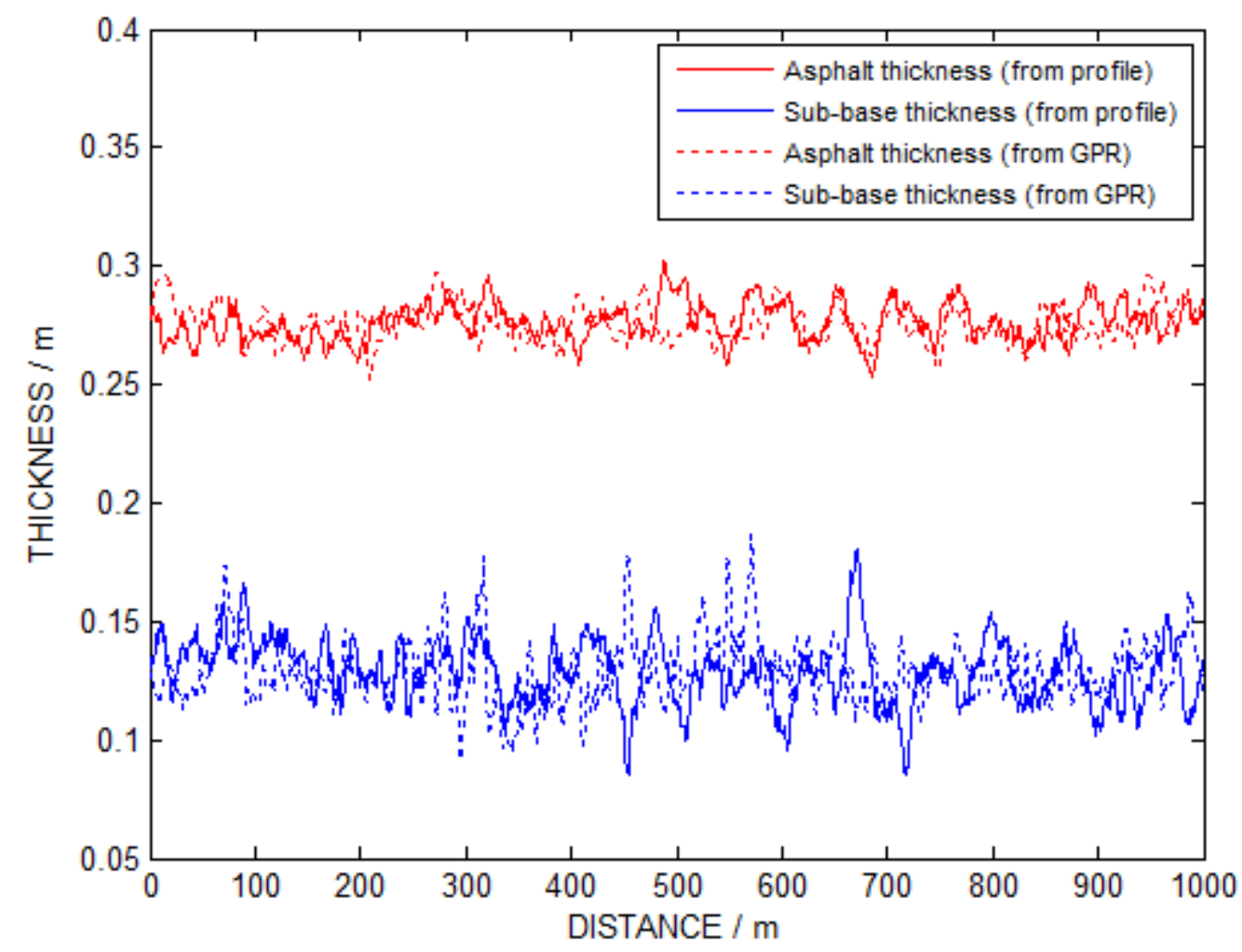

Figure 5.10 Comparison between real (from GPR data) and artificially generated (from profile) layer thicknesses 
Figure 5.11 Match between the real and the artificial probability distributions of the asphalt layer thickness
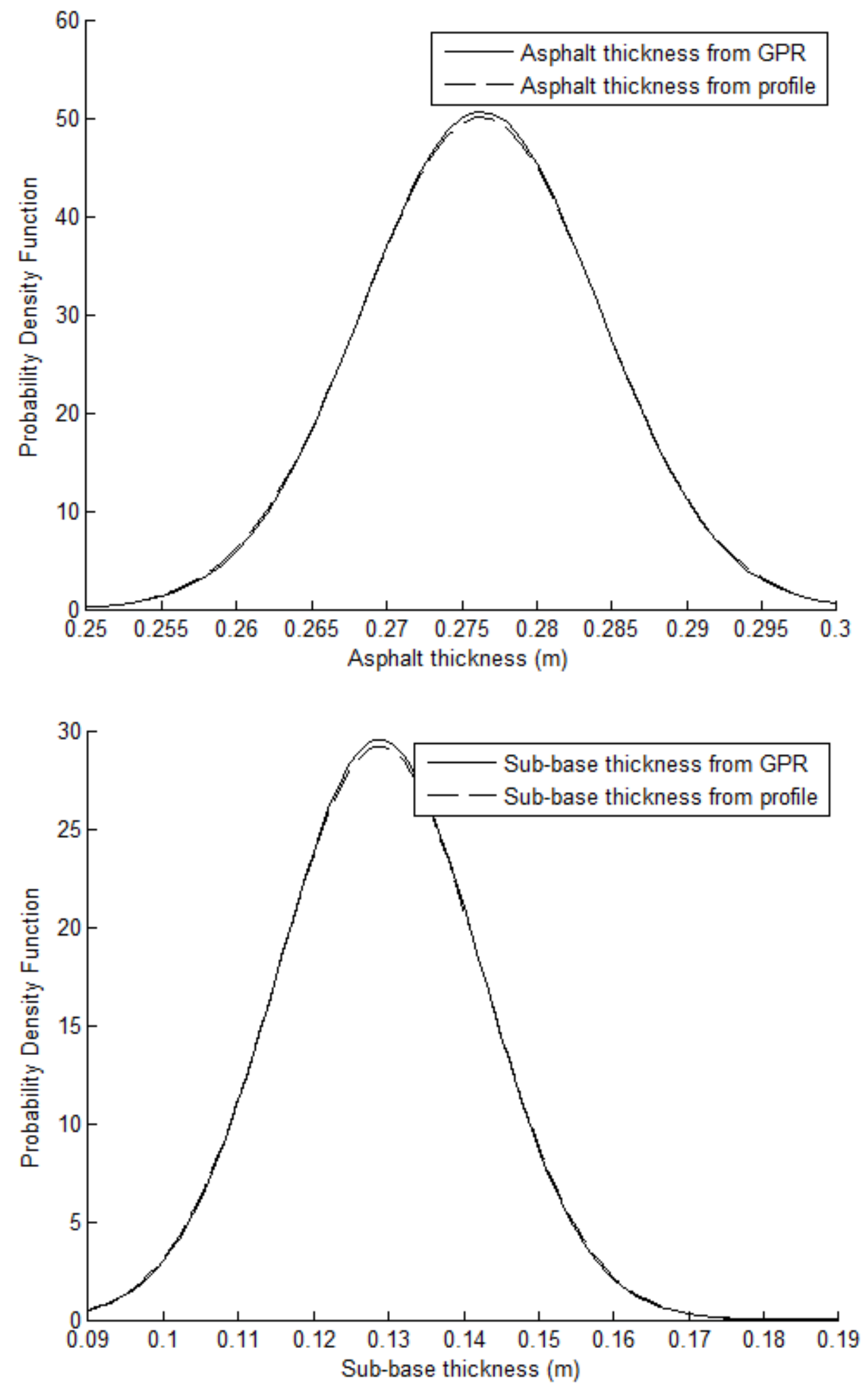

Figure 5.12 Match between the real and the artificial probability distributions of the sub-base layer thickness 


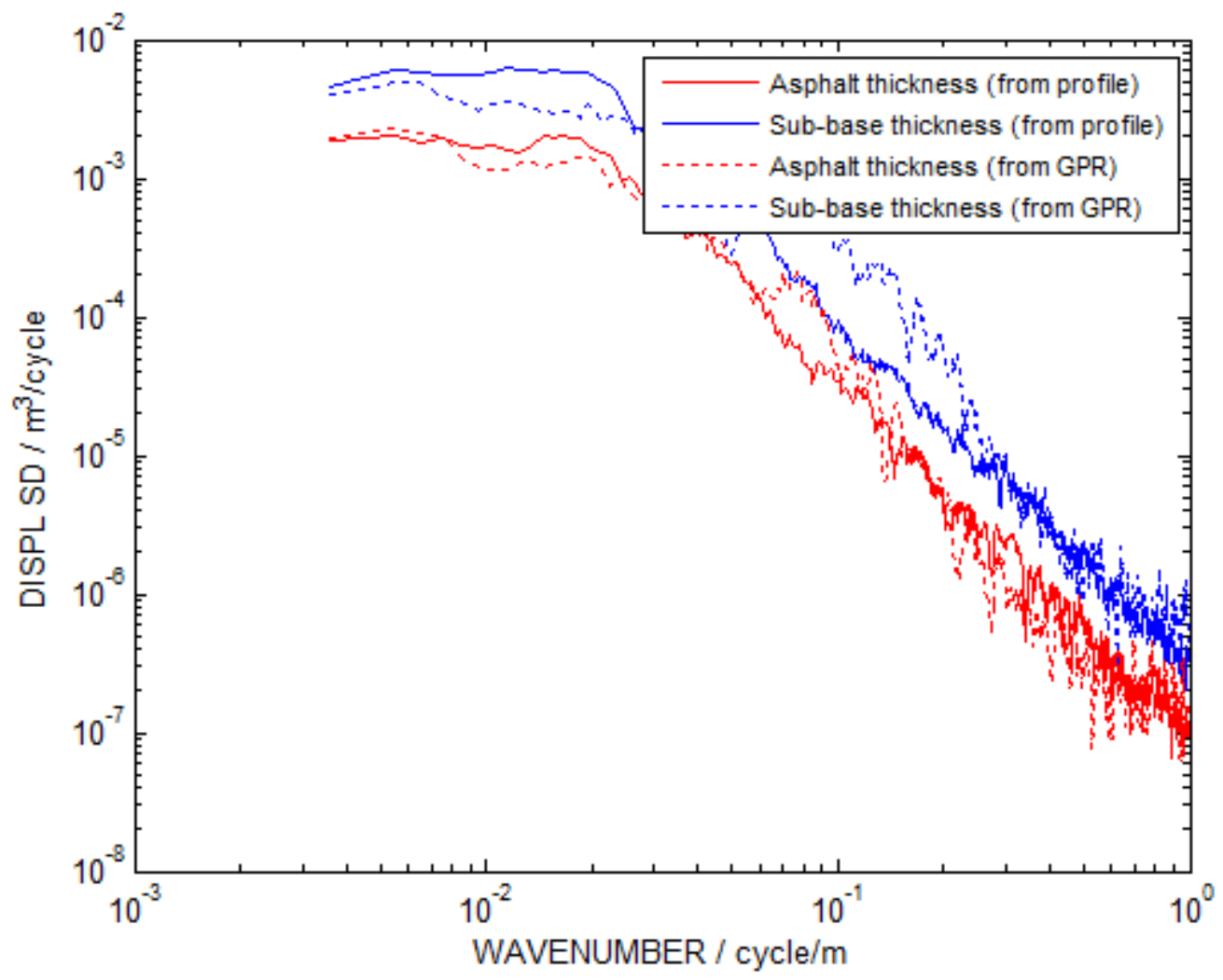

Figure 5.13 Spectral density of real and artificial layer thicknesses

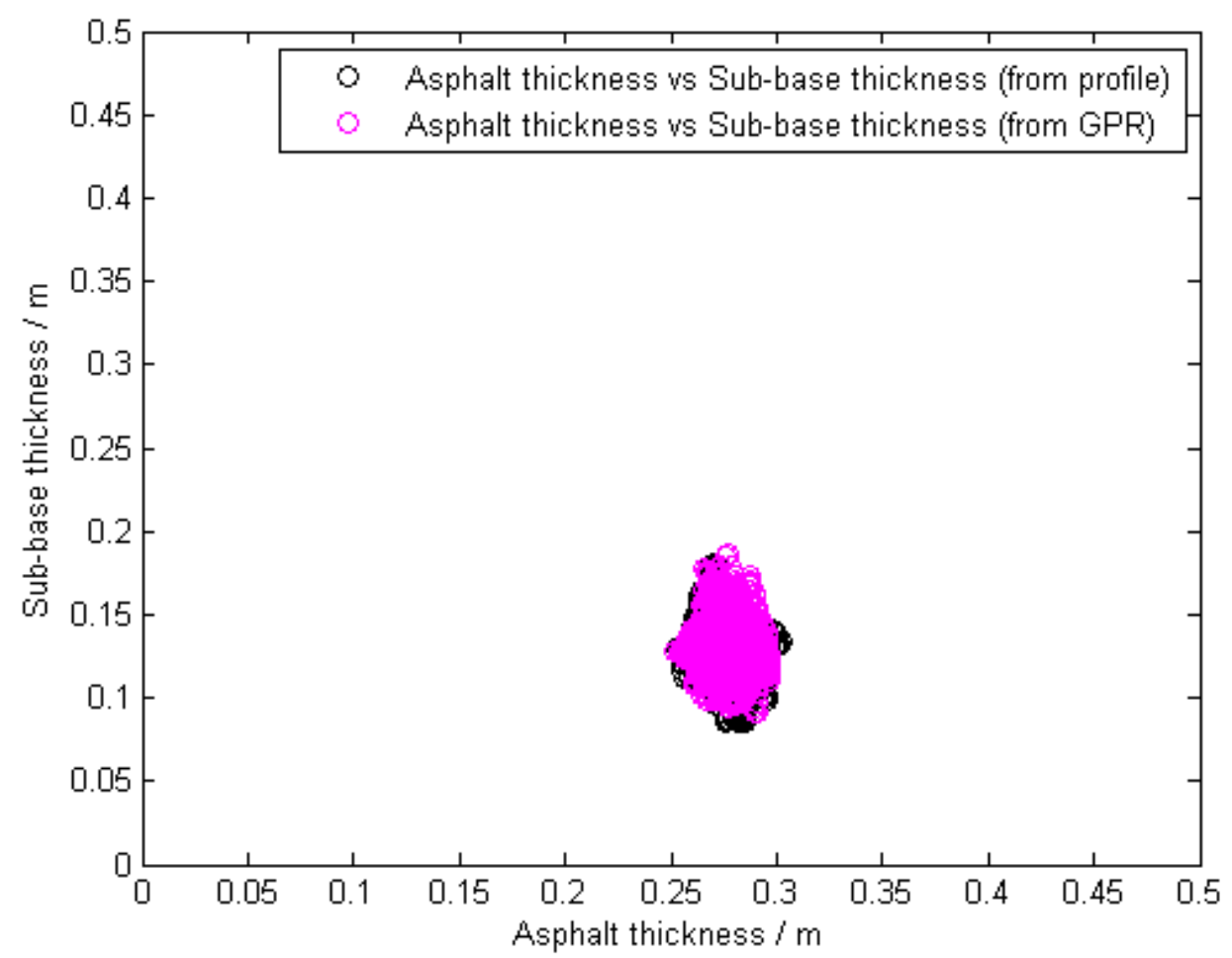

Figure 5.14 Asphalt thickness versus sub-base thickness for both real and artificial data 
A summary of the IRI values calculated by iteration for each section of motorway M01 is presented in Table 5.6.

\section{Table 5.6 Summary of IRI values calculated for each section of motorway M01}

\begin{tabular}{|c|c|c|c|c|c|c|c|}
\hline \multirow[t]{2}{*}{ Sections } & \multirow[t]{2}{*}{ Direction } & \multirow[t]{2}{*}{$\begin{array}{l}\text { From } \\
\text { MP }\end{array}$} & \multirow[t]{2}{*}{$\begin{array}{l}\text { To } \\
\text { MP }\end{array}$} & \multirow[t]{2}{*}{$\begin{array}{l}\text { Length } \\
\text { (m) }\end{array}$} & \multirow{2}{*}{\begin{tabular}{|l|} 
IRI $(\mathrm{m} / \mathrm{km})$ - \\
Measured \\
Surface Profile \\
(from profile \\
measurements)
\end{tabular}} & \multicolumn{2}{|c|}{$\begin{array}{c}\text { IRI }(\mathbf{m} / \mathbf{k m}) \text { - Calculated by } \\
\text { iteration }\end{array}$} \\
\hline & & & & & & $\begin{array}{l}\text { Bottom of } \\
\text { asphalt }\end{array}$ & $\begin{array}{l}\text { Bottom of } \\
\text { subbase }\end{array}$ \\
\hline $\mathbf{A}$ & \multirow{8}{*}{ Clockwise } & 35.0 & 36.0 & 1000 & 2.06 & 2.2 & 11.8 \\
\hline $\mathbf{B}$ & & 36.0 & 37.0 & 1000 & 2.28 & 4.5 & 9.8 \\
\hline $\mathbf{C}$ & & 37.0 & 38.0 & 1000 & Piled raft section & Piled raft section & Piled raft section \\
\hline D & & 38.3 & 39.0 & 700 & 1.46 & 2.6 & 4.5 \\
\hline $\mathbf{E}$ & & 39.0 & 40.0 & 1000 & 1.46 & 2.3 & 4.5 \\
\hline $\mathbf{F}$ & & 40.0 & 41.0 & 1000 & 1.61 & 2.2 & 4.5 \\
\hline $\mathbf{G}$ & & 41.0 & 42.0 & 1000 & 1.63 & 3.5 & 8.5 \\
\hline $\mathbf{H}$ & & 42.0 & 43.0 & 1000 & 1.85 & 4.5 & 4.8 \\
\hline $\mathbf{A}$ & \multirow{8}{*}{ Anti-Clockwise } & 35.0 & 36.0 & 1000 & 1.8 & 1.9 & 8 \\
\hline $\mathbf{B}$ & & 36.0 & 37.0 & 1000 & 1.85 & 1.6 & 3.9 \\
\hline $\mathbf{C}$ & & 37.0 & 38.0 & 1000 & Piled raft section & Piled raft section & Piled raft section \\
\hline D & & 38.4 & 39.0 & 600 & 1.82 & 3.5 & 7.5 \\
\hline $\mathbf{E}$ & & 39.0 & 40.0 & 1000 & 1.68 & 2.2 & 5 \\
\hline $\mathbf{F}$ & & 40.0 & 41.0 & 1000 & 1.58 & 3 & 3.8 \\
\hline $\mathbf{G}$ & & 41.0 & 42.0 & 1000 & 1.36 & 3.3 & 4.5 \\
\hline $\mathbf{H}$ & & 42.0 & 43.0 & 1000 & 1.49 & 3 & 4.3 \\
\hline
\end{tabular}

\subsection{Conclusions}

The importance of layer thickness variability both at design stage and during maintenance design has been discussed in this chapter. As it will be explored more in detail in chapter 6 , layer thickness variability is crucial for backcalculated moduli and remaining life before overlay.

A statistical analysis of the GPR data collected on each site has been presented in terms of mean, standard deviation, coefficient of variation and probability distribution. Results show that the probability distribution for the layer thickness for the UK sites can be considered normal and the coefficient of variation is broadly similar to those reported in the American literature.

Construction tolerances particular to UK standards have been discussed and the relationship between the standard deviation of the asphalt and subbase layer thickness and the construction tolerance, for a 3-layer pavement structure, has been shown. 
The generation of random signals for Monte Carlo simulations has been presented. The methodology uses Fourier Analysis to generate random asphalt and sub-base thickness profiles which have similar frequency characteristics to the real case study profiles.

The following research assumptions will be made in future analysis:

- The total asphalt and granular sub-base layer thickness probability distribution is assumed to be normal.

- The asphalt and the sub-base layer thicknesses are considered uncorrelated. 


\section{Statistical characterisation of asphalt stiffness modulus variability}

The stiffness of asphalt mixtures is of paramount importance in determining how well a pavement performs and is fundamental to the analysis of pavement response to traffic loading. The determination of mixture stiffness is fundamental to the evaluation and design of asphalt pavements. Mixture stiffness is necessary to evaluate the distribution of stresses and strains in asphalt pavements and has also been used to assess pavement damage. In addition, mixture stiffness is increasingly being used in pavement design and as an indicator of mixture quality. This chapter gives of an overview of common methods used in the UK to estimate asphalt stiffness and reviews the accuracy in back-calculation of pavement layer moduli.

\subsection{Background to the estimation of asphalt stiffness in the UK}

There are various laboratory tests that can be used to assess the stiffness moduli of asphaltic materials, including beam tests and uniaxial compression tests. In the UK, the assessment of asphalt mixture stiffness from in-service pavements is normally carried out by laboratory tests (ITSM tests) and field measurements (FWD surveys). This section aims to describe how asphalt stiffness can be assessed by the most popular methods.

\subsubsection{Determination the indirect tensile stiffness modulus (ITSM)}

Laboratory measurements of stiffness can be carried out in the Nottingham Asphalt Tester (NAT) or other similar type apparatus, in which the Indirect Tensile Stiffness Modulus (ITSM) test procedure is normally followed. These tests are carried out under conditions where the elastic response of the material is dominant (i.e., at low temperature or short loading times) (Widyatmoko, 2002). 
The Indirect Tensile Stiffness Modulus (ITSM) Test is a simple and rapid test method for measuring stiffness modulus of asphalt mixture which is the most important input for pavement design to obtain the structural behaviour in the road. Stiffness modulus is dependent on a number of factors such as: asphalt mixture composition, binder grade and level of compaction as well as test conditions (temperature, loading time and the stress magnitude at elevated stress level).

The principle of the ITSM test is that a cylindrical specimen is exposed to repeated sinusoidal compressive loads through the vertical diametral plane, which develops a relatively uniform tensile stress perpendicular to the direction of the applied load and along the vertical diametral plane. The resulting horizontal deformation of the specimen is measured and an assumed Poisson's ratio is used to calculate the tensile strain at the centre of the specimen.

According to EN 12697-26, stiffness modulus is calculated using measurements from the 5 load pulses using following formula:

$$
S_{m}=\frac{F *(v+0.27)}{(z * h)}
$$

Where:

$\mathrm{S}_{\mathrm{m}}=$ stiffness modulus $(\mathrm{MPa})$

$\mathrm{F}=$ applied vertical load $(\mathrm{N})$

$v=$ Poisson's ratio $(0.35)$

$\mathrm{z}=$ amplitude of the horizontal deformation $(\mathrm{mm})$

$\mathrm{h}=$ thickness of the specimen $(\mathrm{mm})$

The stiffness modulus $\left(\mathrm{S}_{\mathrm{m}}\right)$ of asphalt samples determined in the ITSM test (see Figure 6.1) and according to EN 12697-26 is usually performed under the following conditions:

- Test temperature $20 \pm 0.5^{\circ} \mathrm{C}$

- Time of loading $124 \pm 4 \mathrm{~ms}$

- Interval between pulses: $3.0 \pm 0.1 \mathrm{~s}$ 
- Number of load cycles 5

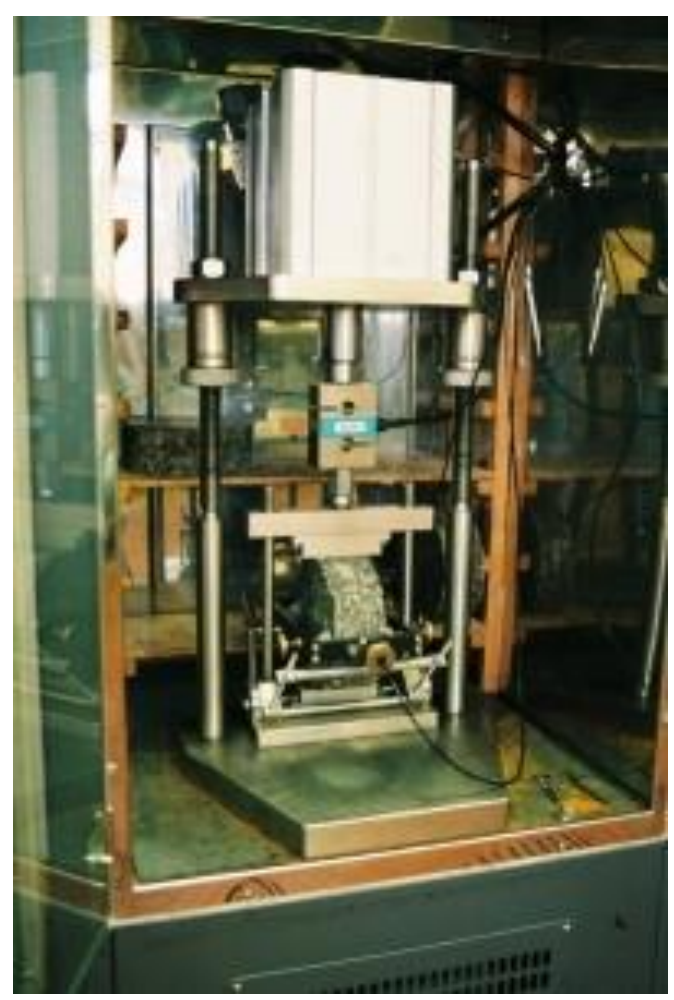

Figure 6.1 ITSM Test

\subsubsection{Determination of asphalt stiffness through FWD back analysis}

The stiffness modulus of bituminous mixtures determined using the ITSM test is generally supported by field back-analysis.

The Falling Weight Deflectometer (FWD) is a non-destructive test conducted to estimate the pavement material moduli. The FWD generates a load pulse by dropping a weight on a damped spring system mounted on a loading plate as shown in Figure 6.2. The falling mass, the spring system (rubber buffers), and drop height can each be adjusted to achieve the desired impact loading on the pavement. Vertical deflection peaks are measured at the centre of the loading plate and at multiple radial positions by a series of deflection sensors (geophones). The impulse load acting on the pavement causes a "wave front" of recoverable deformations, or deflections, that spread out from the centre of the load. Both the peak impulse load (force) and maximum vertical deflections of the "wave front" are measured at multiple radial distances from the load 
centre. These deflections, considered as a function of the applied impulse load, provide an indication of the structural stiffness of the pavement.

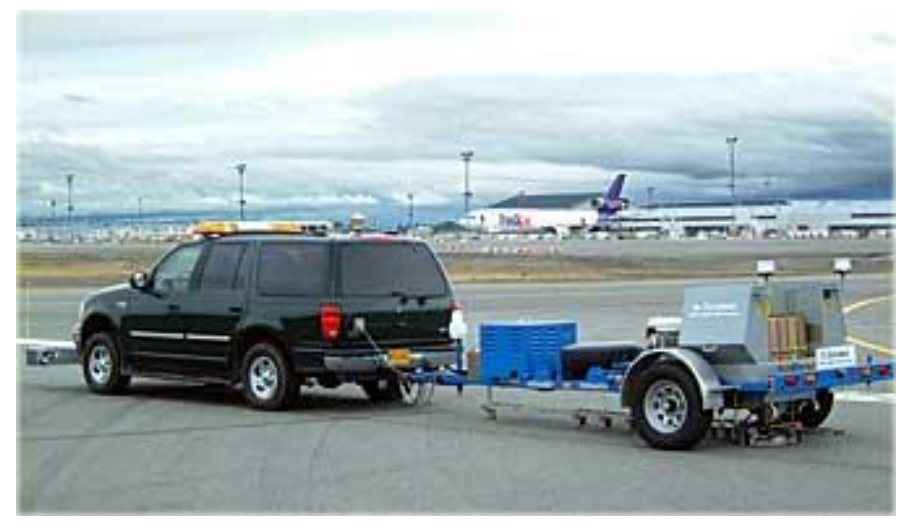

\section{Figure 6.2 Dynatest FWD machine}

Deflection measurements from the FWD are used in a 'back-calculation' procedure to determine pavement structural stiffness in terms of layer and subgrade moduli, as well as for strain analysis. Computer programs using linear elastic multi-layered analysis can be used to model the pavement structure. Essentially this analysis is based on a mathematical model of the pavement structure which predicts the surface deflection under a given applied load. An iterative procedure known as "back-analysis" is used to match the computed deflections to the measured values. The layer stiffnesses are adjusted in this process until a reasonable match is obtained (HD 29, DMRB Volume 7). One of the most used backcalculation software packages in the UK is MODULUS.

In order for the back-analysis to give reliable estimates of the in-situ asphalt stiffness modulus, it is essential to use accurate pavement layer thickness information as an input parameter to the software used for the back-analysis. Underestimating the thickness of bound layers will generally result in an overestimate of the stiffness of that layer and overestimating bound layer thickness will similarly result in an under-estimate of the stiffness of that layer. For example, a 15 per cent underestimate of the thickness of a bound layer can result in a fifty per cent overestimate of the stiffness of that layer (HD 29, DMRB Volume 7). See also section 2.6 for impact of GPR layer thickness errors on data analysis, such as in FWD backanalysis. 


\subsubsection{Comparing FWD Stiffnesses with ITSM (HD 30, DMRB Volume 7)}

Thom et al. (1997) performed an extensive study on the comparison between laboratory and in-situ determined asphalt concrete moduli. The main difference between the two devices is the loading rate. An FWD typically delivers a load pulse with a rise time of around 10-15 msecs, compared with the standard rise time used in the ITSM test in the UK of 124 msecs. As the stiffness of asphalt is loading-time dependent, the shorter pulse of the FWD results in stiffnesses greater than ITSM values. According to Van der Poel (as cited in Thom et al. 1997) the ratio between the two stiffness moduli is a factor of about 1.8. Other sources such as Clause 6.36 in HD 30/08 (reported here), suggest that, "As an approximate guide, ITSM values at $20^{\circ} \mathrm{C}$ should be multiplied by 1.5 when comparing with FWD-derived asphalt layer stiffnesses at $20^{\circ} \mathrm{C}$. However, depending on the type and age of the asphalt material this factor has been found to vary between 1.0 and 2.4. Therefore, although the ITSM values can indicate the in situ layer stiffness, core data will be needed in all cases to determine the causes of any low values."

Besides a loading rate correction, a temperature correction is also carried out. While ITSM tests are usually carried out at a controlled temperature, for example $20^{\circ} \mathrm{C}$, FWD tests are carried out at site temperature. Temperature corrections are applied to FWD back-calculated asphalt layer moduli to the standard temperature of $20^{\circ} \mathrm{C}$. Thom et al. (1997) concluded that both laboratory and in-situ determination of stiffness modulus should be used to perform a full pavement evaluation. They also stressed the importance of the corrections to be made, for both temperature and loading rate, which have to consider the true condition of the material, rather than simply applying formulae. Finally, they suggested that around 6 tests - on nominally homogenous material - will give a sufficiently good indication of the moduli applicable. 


\subsection{Accuracy in back-calculation of pavement layer moduli}

This section reports the results of the study into the effect of three major sources of errors on the back-calculated pavement layer moduli:

- Pavement thickness errors

- Random deflection errors

- Error due to the limited number of data points

It should be noted that the present research aims to assess the impact of the above errors on the back-calculated asphalt layer modulus only.

\subsubsection{Effect of pavement thickness errors on back-calculated moduli}

It is essential that accurate and reliable thickness information is obtained prior to performing a back-analysis, see section 2.6.3 for impact of GPR layer thickness errors on data analysis, such as in FWD backanalysis.

Collop (2000) presented a model for statistically correct asphalt layer stiffness moduli determined from the FWD test to account for asphalt layer thickness variations.

Equation (9) and Equation (10) given in the reference (Collop, 2000) were used to predict the FWD stiffness modulus mean error and the FWD stiffness modulus standard deviation error respectively. Both equations are reported below:

$$
\begin{aligned}
& \frac{\mu_{E_{F W D}}}{\bar{E}}=1+m *\left(\frac{h_{0}}{\bar{h}}\right)+\frac{m^{*}(m-1)}{2} *\left\{\left(\frac{\sigma_{\Delta h}}{\bar{h}}\right)^{2}+\left(\frac{h_{0}}{\bar{h}}\right)^{2}\right\}+\ldots \quad \text { Eq. (9), Collop } 2000 \\
& \frac{\sigma_{E_{F W D}}}{\sigma_{\Delta E}} \approx\left\{1+m^{2} *\left(\frac{\bar{E}}{\sigma_{\Delta E}}\right)^{2} *\left(\frac{\sigma_{\Delta h}}{\bar{h}}\right)^{2}+2 * m *\left(\frac{h_{0}}{\bar{h}}\right) *\left[1+m *(m-1) *\left(\frac{\bar{E}}{\sigma_{\Delta E}}\right)^{2} *\left(\frac{\sigma_{\Delta h}}{\bar{h}}\right)^{2}\right]+\ldots\right\} \\
& \left.+m^{*}(2 * m-1) *\left(\frac{h_{0}}{\bar{h}}\right)^{2} *\left[1+\frac{m^{*}(m-1) *(2 * m-3)}{(2 * m-1)} *\left(\frac{\bar{E}}{\sigma_{\Delta E}}\right)^{2} *\left(\frac{\sigma_{\Delta h}}{\bar{h}}\right)^{2}\right]+\ldots\right\}
\end{aligned}
$$


$\left.+m *(2 * m-1) *\left(\frac{\sigma_{\Delta h}}{\bar{h}}\right)^{2} *\left[1+\frac{m^{*}(m-1) *(m-2)}{3 *(2 * m-1)} *\left(\frac{\bar{E}}{\sigma_{\Delta E}}\right)^{2} *\left(\frac{\sigma_{\Delta h}}{\bar{h}}\right)^{2}\right] !\right\}^{\frac{1}{2}}$

Eq. (10), Collop 2000

Where:

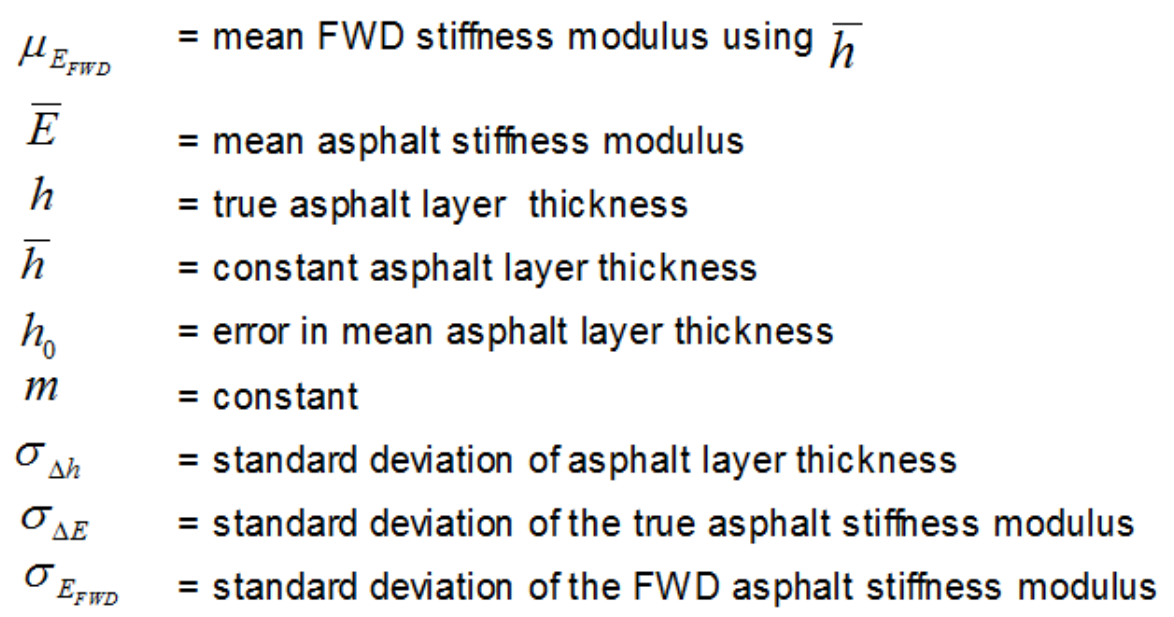

Equation (9) allows an estimate of the FWD stiffness modulus mean error in terms of the asphalt layer thickness variability about a mean level $\left(\frac{h_{0}}{\bar{h}}\right)$ and a measure of the error in the mean asphalt layer thickness $\left(\frac{\sigma_{\Delta h}}{\bar{h}}\right)$, the latter variable being the most important.

Equation (10) allows the estimate of the FWD stiffness modulus standard deviation error in terms of the asphalt layer thickness variability about a mean level $\left(\frac{\sigma_{\Delta h}}{\bar{h}}\right)$, a measure of the error in the mean asphalt layer thickness $\left(\frac{h_{0}}{\bar{h}}\right)$, and the asphalt stiffness modulus variability $\left(\frac{\sigma_{\Delta h}}{\bar{E}}\right)$.

For example, equations (9) and (10) were applied to two sections of the M01 where FWD deflection data was collected every $20 \mathrm{~m}$ and GPR data every $0.1 \mathrm{~m}$. The HA's MODULUS software was used to perform the back-analysis. The pavement was modelled as a 2-layer structure, with $260 \mathrm{~mm}$ of asphalt (i.e. the design thickness) on top of an Equivalent Foundation Modulus (EFM) (i.e. combined granular and subgrade stiffness) of $100 \mathrm{MPa}$. The true asphalt layer thickness used in the analysis is that derived from averaging the GPR measurements over the length of the section.

The results are shown in Table 6.1 and Table 6.2. 
Table 6.1 Application of equation (9) (Collop, 2000) to two sections of motorway M01

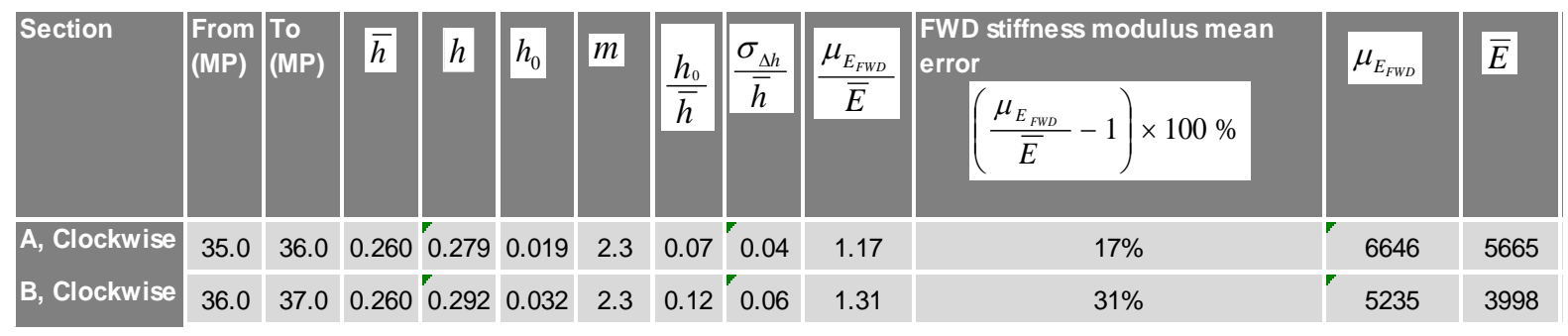

Table 6.2 Application of equation (10) (Collop, 2000) to two sections of motorway M01

\begin{tabular}{|c|c|c|c|c|c|c|c|c|c|c|c|c|}
\hline Section & $\begin{array}{l}\text { From } \\
\text { (MP) }\end{array}$ & \begin{tabular}{|l|} 
To \\
(MP)
\end{tabular} & $m$ & $\frac{h_{0}}{\bar{h}}$ & $\frac{\sigma_{\Delta h}}{\bar{h}}$ & $\sigma_{\Delta E}$ & $\frac{\sigma_{\Delta E}}{\bar{E}}$ & $\frac{\bar{E}}{\sigma_{\Delta E}}$ & $\frac{\sigma_{E_{F W D}}}{\sigma_{\Delta E}} \approx$ & $\begin{array}{l}\left(\frac{\sigma_{E_{F W D}}}{\sigma_{\Delta E}}-1\right) \times 100 \% \\
\text { FWD stifiness modulus } \\
\text { standard deviation } \\
\text { error }\end{array}$ & $\sigma_{E_{F W D}}$ & $\frac{\sigma_{E_{F W D}}}{\mu_{E_{F W D}}}$ \\
\hline , Clockwise & 35.0 & 36.0 & 2.3 & 0.07 & 0.04 & 2579 & 0.46 & 2.20 & 1.19 & $19 \%$ & 3075 & 0.46 \\
\hline B, Clockwise & 36.0 & 37.0 & 2.3 & 0.12 & 0.06 & 1839 & 0.46 & 2.17 & 1.35 & $35 \%$ & 2483 & 0.47 \\
\hline
\end{tabular}

For example, for section A of motorway M01 it can be seen that a 7\% error in the assumption of the mean asphalt layer thickness results in a FWD stiffness modulus mean error of approximately $17 \%$ and a FWD stiffness modulus standard deviation error of approximately $19 \%$.

\subsubsection{Author's revision of equation 10 in Collop (2000)}

It was noted - while performing calculations with real site data - that eq.10 in Collop (2000) sometimes returns an error value. This essentially happens when the ratio $\frac{\sigma_{E_{F W D}}}{\mu_{E_{F W D}}}$ is very small. To assess this problem the author calculated what the minimum value of the ratio $\frac{\sigma_{E_{F W D}}}{\mu_{E_{F W D}}}$ needs to be in order for the ratio $\frac{\sigma_{\Delta E}}{\bar{E}}$ to exist.

The following procedure has been adopted. Firstly, eq. 10 has been re-written expressing $\sigma_{\Delta E}$ as a function of $\sigma_{E_{F W D}}, \bar{E}, \mathrm{~m}, \frac{\sigma_{\Delta h}}{\bar{h}}$, and $\frac{h_{0}}{\bar{h}}$.

This leads to the following two equations which need to be satisfied:

$\left\{\begin{array}{lr}1+2 * m * d+\left(2 * m^{2}-m\right) * d^{2}+\left(2 * m^{2}-m\right) * c^{2}>0 & \text { (always true) } \\ \sigma_{E_{F F D}}^{2} \geq \bar{E}^{2} *\left(m^{2} * c^{2}+2 * m *\left(m^{2}-m\right) * c^{2} * d+\left(2 * m^{2}-m\right) * d^{2} * c^{2} * \frac{\left(m^{2}-m\right) *(2 * m-3)}{(2 * m-1)}+\left(2 * m^{2}-m\right) * c^{4} * \frac{\left(m^{2}-m\right) *(m-2)}{(6 * m-3)}\right.\end{array}\right.$ 
This is how the minimum value of $\sigma_{E_{F W D}}$ has been calculated, hence the ratio $\frac{\sigma_{E_{F W D}}}{\mu_{E_{F W D}}}$.

A large number of combinations has been considered (total of 20402) with:

- $\frac{\sigma_{\Delta h}}{\bar{h}}=0$ to 1 with 0.01 step increment

- $\frac{h_{0}}{\bar{h}}=0$ to 1 with 0.01 step increment

In general, for eq. 10 to exist, $\sigma_{E_{F W D}} \geq 0, \frac{\sigma_{\Delta E}}{\bar{E}}>0$ and $\{$ arg $\} \geq 0$. From Collop (2000) it appears that the minimum value for $\sigma_{\Delta E}$ is 0.01 .

An extreme situation is when $\frac{\sigma_{\Delta h}}{\bar{h}}=0$ and $\frac{h_{0}}{\bar{h}}=0$, for which $\sigma_{E_{F W D}}=\sigma_{\Delta E}>0$.

It is recommended that the minimum value for $\frac{\sigma_{E_{F W D}}}{\mu_{E_{F W D}}}$.is 0.01 .

The results of the analysis are shown in Figure 6.3 and Figure 6.4.

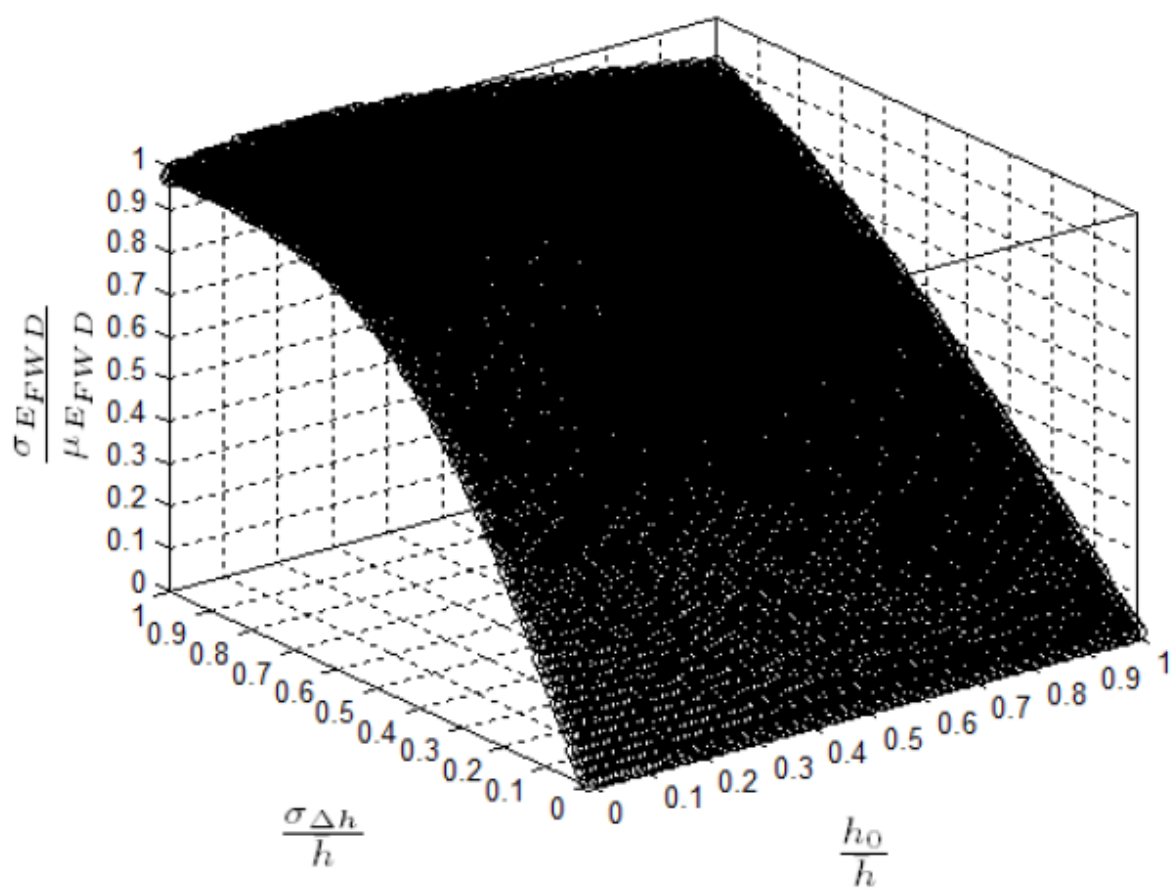

Figure 6.3 Minimum values of $\frac{\sigma_{E_{F W D}}}{\mu_{E_{F W D}}}$. for combinations of $\frac{\sigma_{\Delta h}}{\bar{h}}$ and $\frac{h_{0}}{\bar{h}}$

Figure 6.4 shows a larger view of the surface presented in Figure 6.3 in the critical area, i.e. typically when thickness variations are very low $(<=0.1)$. 
For example, for $\frac{h_{0}}{\bar{h}}=0.08$ and $\frac{\sigma_{\Delta h}}{\bar{h}}=0.01$ with real site data the ratio $\frac{\sigma_{E_{F W D}}}{\mu_{E_{F W D}}}$ was equal to 0.01 . Based on the analysis, the ratio $\frac{\sigma_{E_{F W D}}}{\mu_{E_{F W D}}}$ must be $>=0.03$. In other words, $\sigma_{E_{F W D}}$ is too small compared to what would be calculated by taking into account the layer thickness variation $\frac{\sigma_{\Delta h}}{\bar{h}}$. The true asphalt stiffness variation $\sigma_{\Delta E}$ is, therefore, very small.

It should be noted that these anomalies have been encountered only on very few instances and on short sections.

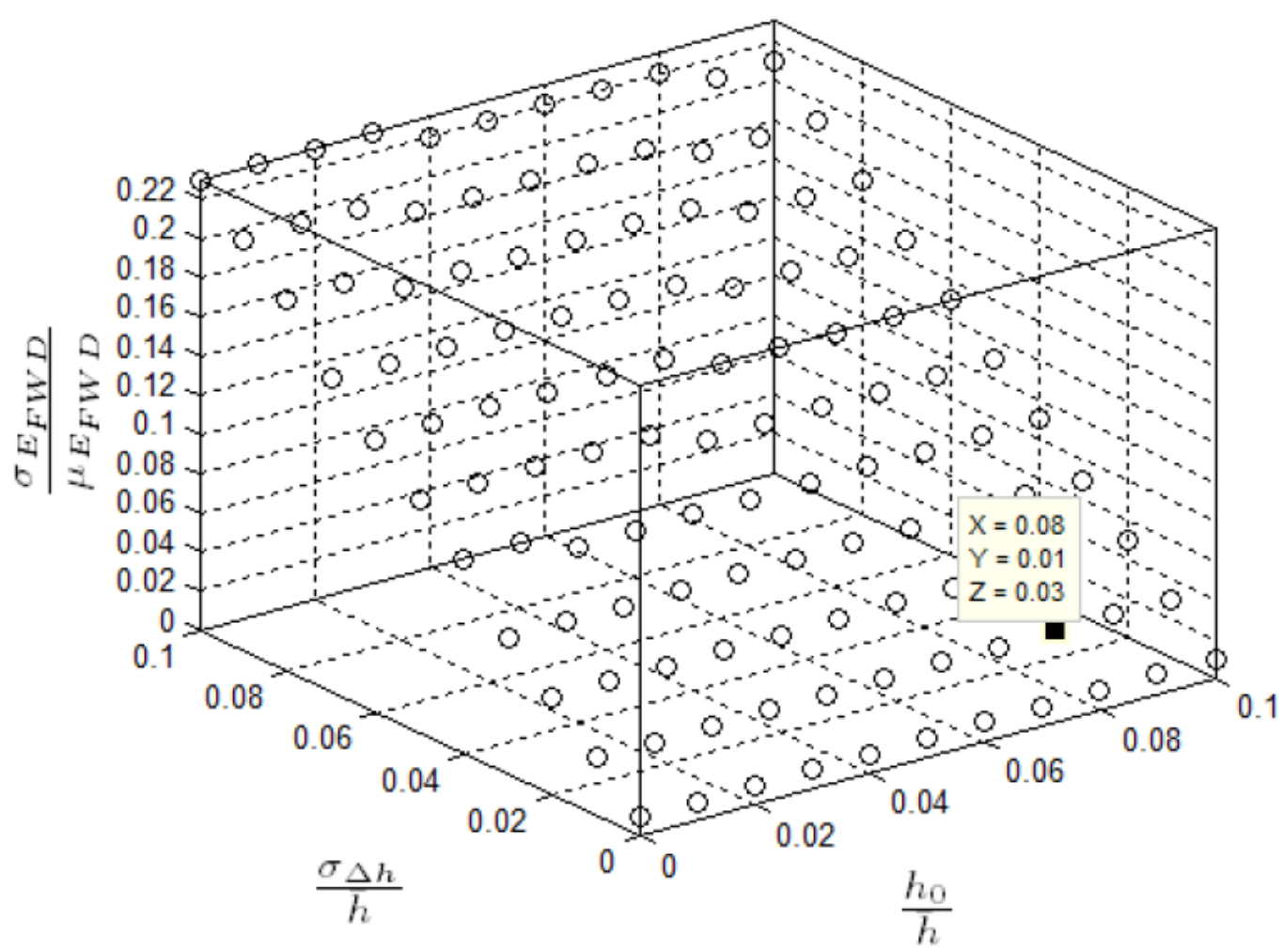

Figure 6.4 Minimum values of $\frac{\sigma_{E_{F W D}}}{\mu_{E_{F W D}}}$ when $\frac{\sigma_{\Delta h}}{\bar{h}}$ and $\frac{h_{0}}{\bar{h}}$ are $<=0.1$

\subsubsection{Effect of random errors from deflection measurements on back-calculated moduli}

The accuracy of the backcalculation procedure is affected by factors such as the inaccuracies in FWD deflection measurements, deflection data calibration and temperature variation, algorithm issues in the back-calculation procedure and inaccurate thickness input. 
Sangghaleh et al. (2013) investigated the effect of measurement errors on the back-calculation by using an improved genetic algorithm capable of backcalculating the elastic modulus and thickness simultaneously. In their analysis both a systematic and random error (i.e., both components of the measurement error, see next section) were added to the measured deflection at sensor i. For the 2-layer structure considered in the simulation - and considering the commonly used goodness of fit function represented by the root mean square - the relative error in the back-analysed moduli and coefficient of variation are:

- Back-analysed asphalt modulus: $2.54 \%$ error (from comparing the backcalculated moduli to the theoretical value without the measurement error) when only random error is considered; $0.20 \%$ error when only systematic error is considered; $2.81 \%$ error when random and systematic error are considered.

- Back-analysed subgrade modulus: $0.00 \%$ error when only random error is considered; $0.20 \%$ error when only systematic error is considered; $0.22 \%$ error when random and systematic error are considered.

- The above errors are considerably reduced when their improved genetic algorithm is employed.

\subsubsection{Sources of error in deflection measurement}

For a pulse-loading deflectometer there are three basic sources of errors in the deflection readings:

- Seating errors

- Systematic errors

- Repeatability (or random) errors.

All three of these errors must be minimised in order to yield the most accurate moduli from the deflection data. Research conducted by Irwin et al. (1989) has shown that: 
- For the FWD, deflection measurement errors due to seating effects can be minimised by making at least two drops of the mass before recording test data.

- For all deflectometers, a calibration procedure should be performed every 6 to 12 months to minimise systematic errors.

- To minimise the effect of the repeatability error on the measured deflections, multiple drops of the weight should be made from the same height at the same location.

This research study has assessed the impact of the repeatability, or random, error only on back-calculated layer moduli. Typical FWD specifications state that "Deflections shall be accurate to \pm 2 percent or \pm 2 microns, whichever is larger."

- The \pm 2 micron (standard deviation) error is a random error, independent of the magnitude of the deflection;

- The \pm 2 percent error is a systematic error.

Figure 6.5 explains the concepts of systematic and random errors. The systematic error is the difference between the mean measured value of a deflection and the absolute correct deflection value. The random error is the scatter around the mean value.

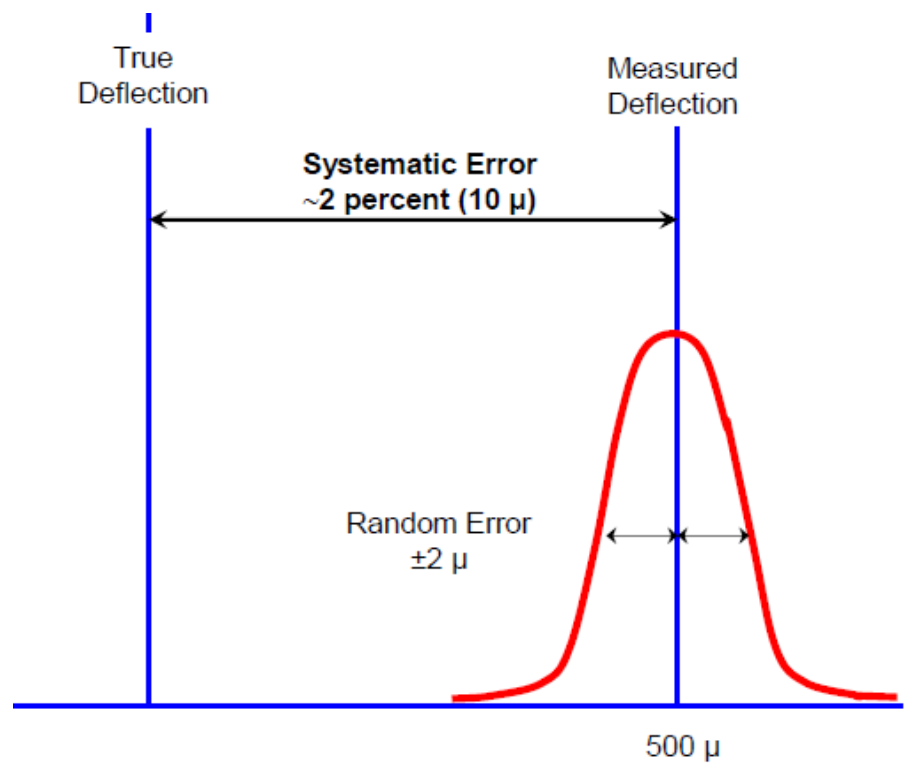

Figure 6.5 Random and systematic deflection measurements errors (figure taken from Irwin, 2009) 


\subsubsection{Methodology used for the analysis and results}

A similar exercise to that conducted by Irwin et al. (1989) was carried out to assess the impact of the random error on back-calculated layer moduli. The analysis consisted of the following steps:

1. Assumed pavement structures and calculation of deflection bowl

The pavement sections used in the analysis are shown in Table 6.3 to Table 6.5. Pavement structure 1 denotes the controlled sections where the deflection bowls were calculated with BISAR. Deflections were calculated at $0,300,600,900,1200,1500$ and $2100 \mathrm{~mm}$ geophones distances from the centre of the plate, for a $50 \mathrm{kN}$ load on a $0.300 \mathrm{~mm}$ diameter plate. Pavement structures 2 and 3 are essentially the same system. However, pavement 2 has been modelled as a 2-layer pavement system and pavement 3 as a 3layer structure. For these 2 structures real deflection bowls were used for the analysis. Section 1 can be thought of as a 'stiff' section (i.e., low deflections) while section 2 can be regarded as a 'soft' section (i.e., high deflections). The deflection bowls are shown in Table 6.6 and Table 6.7. The layer stiffnesses for pavement structures 2 and 3 are those back-calculated from the original deflections (i.e. without the effect of random errors).

Table 6.3 Pavement structure 1 (controlled sections)

\begin{tabular}{|l|l|l|l|l|l|}
\hline Section & A & B & C & D & E \\
\hline Bound Layer Thickness (m) & 0.100 & 0.200 & 0.300 & 0.400 & 0.500 \\
\hline Asphalt stiffiness (MPa) & 3100 & 3100 & 3100 & 3100 & 3100 \\
\hline Poisson's ratio for bound layer & 0.35 & 0.35 & 0.35 & 0.35 & 0.35 \\
\hline Equivalent Foundation Modulus EFM (MPa) & 100 & 100 & 100 & 100 & 100 \\
\hline Poisson's ratio for EFM & 0.45 & 0.45 & 0.45 & 0.45 & 0.45 \\
\hline
\end{tabular}

Table 6.4 Pavement structure 2 (2-layer structure)

\begin{tabular}{|l|l|l|}
\hline Section & 1 & 2 \\
\hline Bound Layer Thickness $(\mathrm{m})$ & 0.260 & 0.260 \\
\hline Asphalt stiffiness (MPa) & 8584 & 1170 \\
\hline Poisson's ratio for bound layer & 0.350 & 0.350 \\
\hline Equivalent Foundation Modulus EFM (MPa) & 256 & 149 \\
\hline Poisson's ratio for EFM & 0.45 & 0.45 \\
\hline
\end{tabular}


Table 6.5 Pavement structure 3 (3-layer structure)

\begin{tabular}{|l|l|l|}
\hline Section & 1 & 2 \\
\hline Bound Layer Thickness $(\mathrm{m})$ & 0.260 & 0.260 \\
\hline Asphalt stiffness $(\mathrm{MPa})$ & 8098 & 1527 \\
\hline Poisson's ratio for bound layer & 0.35 & 0.35 \\
\hline Sub-base thickness $(\mathrm{m})$ & 0.350 & 0.350 \\
\hline Sub-base stiffness $(\mathrm{MPa})$ & 306 & 78 \\
\hline Poisson's ratio for sub-base & 0.4 & 0.4 \\
\hline Subgrade stiffiness (MPa) & 255 & 170 \\
\hline Poisson's ratio for subgrade & 0.45 & 0.45 \\
\hline
\end{tabular}

Table 6.6 Pavement structure 1, Deflection bowl calculated with BISAR

\begin{tabular}{|c|c|c|c|c|c|c|c|c|} 
Sections & \multicolumn{7}{|c|}{ Distance from centre of plate $(\mathrm{mm})$} \\
\cline { 2 - 9 } & $\mathbf{0}$ & 300 & 600 & 900 & 1200 & 1500 & 2100 \\
\cline { 2 - 9 } & \multicolumn{7}{|c|}{ Deflections (microns) } \\
\hline A & 803 & 472 & 236 & 145 & 106 & 84 & 60 \\
\hline B & 464 & 347 & 237 & 163 & 117 & 89 & 61 \\
\hline C & 334 & 263 & 205 & 158 & 123 & 97 & 65 \\
\hline D & 267 & 210 & 175 & 144 & 119 & 98 & 68 \\
\hline E & 226 & 175 & 151 & 130 & 111 & 95 & 70 \\
\hline
\end{tabular}

Table 6.7 Pavement structures $2 \& 3$, real deflection bowl

\begin{tabular}{|c|c|c|c|c|c|c|c|}
\hline \multirow{4}{*}{ Sections } & \multicolumn{7}{|c|}{ Distance from centre of plate $(\mathrm{mm})$} \\
\hline & 0 & 300 & 600 & 900 & 1200 & 1500 & 2100 \\
\hline & \multicolumn{7}{|c|}{ Deflections (microns) } \\
\hline & do & d1 & d2 & d3 & d4 & $d 5$ & $d 6$ \\
\hline 1 & 143 & 113 & 84 & 63 & 48 & 38 & 26 \\
\hline 2 & 426 & 281 & 168 & 106 & 70 & 52 & 37 \\
\hline
\end{tabular}

2. First simulation - generation of 30 deflection bowls

A normally distributed random number generator technique - with a mean of zero and a standard deviation of 2 - was used to generate deflection bowls by adding or subtracting a random portion of the standard deviation to the theoretically calculated deflections, see Table 6.6 and Table 6.7 respectively. After algebraically adding a different random error (positive or negative) to the calculated deflection for each sensor, the result was rounded to the nearest whole micron. In the first simulation, 30 deflection bowls were generated using the randomised process described above to simulate 30 tests conducted on a hypothetical pavement. The first simulation aimed to simulate 30 tests conducted at 30 different points on a perfectly homogeneous pavement having 
the material properties and layer thicknesses given in Table 6.3 to Table 6.5. Every repetition is, therefore, made of 30 cases each. The test was repeated 5 times (referred later as 'repetition') for each section. Results of the first simulation are shown in Table 6.8 to Table 6.10, for the back-calculated asphalt modulus and in Table 6.12 to Table 6.14 for the other layers. $\bar{E}$ is the 'true' modulus, while $\mu_{E_{F W D}}$ and $\sigma_{E_{F W D}}$ are the mean layer back-calculated stiffness and standard deviation respectively.

The results of the first simulation suggest that:

- The effect of the random error on the mean back-calculated asphalt stiffness modulus is very low. The ratio $\frac{\mu_{E_{F W D}}}{\bar{E}}$ is close to 1 in almost all sections of the considered pavement structures.

- The effect of the random error on the mean back-calculated Equivalent Foundation Modulus and subgrade stiffness modulus is also negligible.

- The effect of the random error on the mean back-calculated sub-base stiffness modulus is noticeable. For pavement structure 3 (3-layer system), section 1 , the ratio $\frac{\mu_{E_{F W D}}}{\bar{E}}$ varies from $\sim 0.90$ to $\sim 1.07$ showing an error of approximately $\pm 10 \%$.

- The effect of the random error on the standard deviation of the backcalculated asphalt stiffness modulus - and assessed in terms of the coefficient of variation $\frac{\sigma_{E_{F W D}}}{\mu_{E_{F W D}}}$ is low for pavement structure 1 (controlled sections) and pavement structure 2 (2-layer system). However, the coefficient of variation was found to be as high as $15 \%$ for pavement structure 3 (3-layer system), section 1.

- The effect of the random error on the standard deviation of the backcalculated Equivalent Foundation Modulus and subgrade stiffness modulus is low.

- The effect of the random error on the standard deviation of the backcalculated sub-base stiffness modulus is noticeable. The coefficient of variation was found as high as $\sim 40 \%$ for pavement structure 3 (3-layer system), section 1 . 
Table 6.8 Effect of random deflection measurement error on backcalculated asphalt modulus on pavement structure 1 - single drop per case

\begin{tabular}{|c|c|c|c|c|c|c|}
\hline Sec & Repetition & $\frac{\mu_{E_{F W D}}}{\bar{E}}$ & $\mu_{E_{F W D}}$ & $\bar{E}$ & $\sigma_{E_{F W D}}$ & $\frac{\sigma_{E_{F W D}}}{\mu_{E_{F W D}}}$ \\
\hline \multirow[t]{5}{*}{$\mathbf{A}$} & 1 & 1.022 & 3168 & 3100 & 50 & 0.016 \\
\hline & 2 & 1.029 & 3189 & 3100 & 42 & 0.013 \\
\hline & 3 & 1.025 & 3178 & 3100 & 55 & 0.017 \\
\hline & 4 & 1.028 & 3186 & 3100 & 46 & 0.014 \\
\hline & 5 & 1.028 & 3188 & 3100 & 42 & 0.013 \\
\hline \multirow[t]{5}{*}{$\mathbf{B}$} & 1 & 0.989 & 3065 & 3100 & 55 & 0.018 \\
\hline & 2 & 0.991 & 3071 & 3100 & 43 & 0.014 \\
\hline & 3 & 0.992 & 3074 & 3100 & 57 & 0.019 \\
\hline & 4 & 0.991 & 3072 & 3100 & 51 & 0.016 \\
\hline & 5 & 0.997 & 3090 & 3100 & 55 & 0.018 \\
\hline \multirow[t]{5}{*}{ C } & 1 & 1.000 & 3100 & 3100 & 69 & 0.022 \\
\hline & 2 & 0.999 & 3096 & 3100 & 51 & 0.016 \\
\hline & 3 & 1.004 & 3112 & 3100 & 69 & 0.022 \\
\hline & 4 & 1.000 & 3100 & 3100 & 60 & 0.019 \\
\hline & 5 & 1.010 & 3130 & 3100 & 67 & 0.021 \\
\hline \multirow[t]{5}{*}{ D } & 1 & 1.006 & 3118 & 3100 & 82 & 0.026 \\
\hline & 2 & 1.003 & 3111 & 3100 & 56 & 0.018 \\
\hline & 3 & 1.008 & 3124 & 3100 & 77 & 0.025 \\
\hline & 4 & 1.003 & 3110 & 3100 & 72 & 0.023 \\
\hline & 5 & 1.017 & 3152 & 3100 & 81 & 0.026 \\
\hline \multirow[t]{5}{*}{$E$} & 1 & 1.001 & 3103 & 3100 & 90 & 0.029 \\
\hline & 2 & 0.996 & 3086 & 3100 & 65 & 0.021 \\
\hline & 3 & 1.003 & 3110 & 3100 & 84 & 0.027 \\
\hline & 4 & 0.999 & 3096 & 3100 & 78 & 0.025 \\
\hline & 5 & 1.014 & 3142 & 3100 & 92 & 0.029 \\
\hline
\end{tabular}

Table 6.9 Effect of random deflection measurement error on backcalculated asphalt modulus on pavement structure 2 - single drop per case

\begin{tabular}{|c|l|l|l|l|l|l|}
\hline Sec & Repetition & $\frac{\mu_{E_{F W D}}}{\bar{E}}$ & $\mu_{E_{F W D}}$ & $\bar{E}$ & $\sigma_{E_{F W D}}$ & $\frac{\sigma_{E_{F W D}}}{\mu_{E_{F W D}}}$ \\
\hline 1 & 1 & 1.003 & 8605 & 8584 & 458 & 0.053 \\
& 2 & 0.998 & 8563 & 8584 & 317 & 0.037 \\
& 3 & 1.004 & 8621 & 8584 & 454 & 0.053 \\
\hline & 4 & 0.999 & 8577 & 8584 & 406 & 0.047 \\
\hline 2 & 5 & 1.024 & 8787 & 8584 & 474 & 0.054 \\
\hline & 2 & 0.999 & 1169 & 1170 & 21 & 0.018 \\
& 3 & 1.001 & 1171 & 1170 & 15 & 0.013 \\
& 4 & 1.003 & 1174 & 1170 & 21 & 0.018 \\
& 5 & 1.002 & 1172 & 1170 & 18 & 0.015 \\
\hline & & 1.007 & 1178 & 1170 & 20 & 0.017 \\
\hline
\end{tabular}


Table 6.10 Effect of random deflection measurement error on backcalculated asphalt modulus on pavement structure 3 - single drop per case

\begin{tabular}{|c|c|c|c|c|c|c|}
\hline Sec & Repetition & $\frac{\mu_{E_{F W D}}}{\bar{E}}$ & $\mu_{E_{F W D}}$ & $\bar{E}$ & $\sigma_{E_{F W D}}$ & $\frac{\sigma_{E_{F W D}}}{\mu_{E_{F W D}}}$ \\
\hline \multirow[t]{5}{*}{1} & 1 & 0.989 & 8005 & 8098 & 1002 & 0.125 \\
\hline & 2 & 1.052 & 8517 & 8098 & 1164 & 0.137 \\
\hline & 3 & 1.011 & 8187 & 8098 & 1250 & 0.153 \\
\hline & 4 & 1.021 & 8271 & 8098 & 700 & 0.085 \\
\hline & 5 & 1.016 & 8228 & 8098 & 730 & 0.089 \\
\hline \multirow[t]{5}{*}{2} & 1 & 0.998 & 1525 & 1527 & 44 & 0.029 \\
\hline & 2 & 1.013 & 1547 & 1527 & 49 & 0.032 \\
\hline & 3 & 1.005 & 1534 & 1527 & 56 & 0.036 \\
\hline & 4 & 1.008 & 1540 & 1527 & 31 & 0.020 \\
\hline & 5 & 1.005 & 1535 & 1527 & 33 & 0.021 \\
\hline
\end{tabular}

Table 6.11 Effect of random deflection measurement error on backcalculated Equivalent Foundation Modulus (EFM) on pavement structure 1 - single drop per case

\begin{tabular}{|c|c|c|c|c|c|c|}
\hline Sec & Repetition & $\frac{\mu_{E_{F W D}}}{\bar{E}}$ & $\mu_{E_{F W D}}$ & $\bar{E}$ & $\sigma_{E_{F W D}}$ & $\frac{\sigma_{E_{F W D}}}{\mu_{E_{F W D}}}$ \\
\hline \multirow[t]{5}{*}{$\bar{A}$} & 1 & 1.001 & 100 & 100 & 0 & 0.004 \\
\hline & 2 & 0.999 & 100 & 100 & 0 & 0.005 \\
\hline & 3 & 1.001 & 100 & 100 & 0 & 0.004 \\
\hline & 4 & 0.999 & 100 & 100 & 0 & 0.005 \\
\hline & 5 & 1.000 & 100 & 100 & 0 & 0.004 \\
\hline \multirow[t]{5}{*}{ B } & 1 & 1.004 & 100 & 100 & 0 & 0.004 \\
\hline & 2 & 1.000 & 100 & 100 & 0 & 0.004 \\
\hline & 3 & 1.002 & 100 & 100 & 0 & 0.004 \\
\hline & 4 & 1.000 & 100 & 100 & 0 & 0.004 \\
\hline & 5 & 1.000 & 100 & 100 & 0 & 0.004 \\
\hline \multirow[t]{5}{*}{ C } & 1 & 1.004 & 100 & 100 & 0 & 0.004 \\
\hline & 2 & 0.999 & 100 & 100 & 0 & 0.005 \\
\hline & 3 & 1.002 & 100 & 100 & 0 & 0.004 \\
\hline & 4 & 1.001 & 100 & 100 & 0 & 0.004 \\
\hline & 5 & 1.001 & 100 & 100 & 0 & 0.004 \\
\hline \multirow[t]{5}{*}{ D } & 1 & 1.005 & 101 & 100 & 0 & 0.004 \\
\hline & 2 & 1.001 & 100 & 100 & 0 & 0.004 \\
\hline & 3 & 1.003 & 100 & 100 & 0 & 0.004 \\
\hline & 4 & 1.003 & 100 & 100 & 0 & 0.004 \\
\hline & 5 & 1.002 & 100 & 100 & 0 & 0.004 \\
\hline \multirow[t]{5}{*}{$\bar{E}$} & 1 & 1.003 & 100 & 100 & 0 & 0.004 \\
\hline & 2 & 1.000 & 100 & 100 & 0 & 0.004 \\
\hline & 3 & 1.001 & 100 & 100 & 0 & 0.004 \\
\hline & 4 & 1.002 & 100 & 100 & 0 & 0.004 \\
\hline & 5 & 1.000 & 100 & 100 & 0 & 0.004 \\
\hline
\end{tabular}


Table 6.12 Effect of random deflection measurement error on backcalculated EFM on pavement structure 2 - single drop per case

\begin{tabular}{|l|l|l|l|l|l|l|} 
Sec & Repetition & $\frac{\mu_{E_{F W D}}}{\bar{E}}$ & $\mu_{E_{F W D}}$ & $\bar{E}$ & $\sigma_{E_{F W D}}$ & $\frac{\sigma_{E_{F W D}}}{\mu_{E_{F W D}}}$ \\
\hline 1 & 1 & 1.003 & 257 & 256 & 5 & 0.019 \\
\hline & 2 & 0.996 & 255 & 256 & 4 & 0.015 \\
\hline & 3 & 1.001 & 256 & 256 & 5 & 0.018 \\
\hline & 4 & 1.000 & 256 & 256 & 4 & 0.015 \\
\hline & 1 & 0.997 & 255 & 256 & 4 & 0.015 \\
\hline & 2 & 1.000 & 149 & 149 & 2 & 0.011 \\
\hline & 3 & 0.996 & 148 & 149 & 1 & 0.009 \\
\hline & 4 & 0.998 & 149 & 149 & 2 & 0.010 \\
\hline & 5 & 0.998 & 149 & 149 & 1 & 0.009 \\
\hline & 0.996 & 148 & 149 & 1 & 0.008 \\
\hline
\end{tabular}

Table 6.13 Effect of random deflection measurement error on backcalculated sub-base modulus on pavement structure 3 - single drop per case

\begin{tabular}{|c|c|c|c|c|c|c|}
\hline Sec & Repetition & $\frac{\mu_{E_{F W D}}}{\bar{E}}$ & $\mu_{E_{F W D}}$ & $\bar{E}$ & $\sigma_{E_{F W D}}$ & $\frac{\sigma_{E_{F W D}}}{\mu_{E_{F W D}}}$ \\
\hline \multirow[t]{5}{*}{1} & 1 & 1.073 & 328 & 306 & 107 & 0.327 \\
\hline & 2 & 0.897 & 274 & 306 & 95 & 0.348 \\
\hline & 3 & 1.036 & 317 & 306 & 123 & 0.389 \\
\hline & 4 & 0.946 & 290 & 306 & 62 & 0.215 \\
\hline & 5 & 1.031 & 316 & 306 & 81 & 0.257 \\
\hline \multirow[t]{5}{*}{2} & 1 & 1.012 & 79 & 78 & 6 & 0.078 \\
\hline & 2 & 0.977 & 76 & 78 & 6 & 0.074 \\
\hline & 3 & 1.001 & 78 & 78 & 7 & 0.091 \\
\hline & 4 & 0.986 & 77 & 78 & 4 & 0.052 \\
\hline & 5 & 1.008 & 79 & 78 & 5 & 0.063 \\
\hline
\end{tabular}

Table 6.14 Effect of random deflection measurement error on backcalculated subgrade modulus on pavement structure 3 - single drop per case

\begin{tabular}{|c|c|c|c|c|c|c|}
\hline $\mathrm{Sec}$ & Repetition & $\frac{\mu_{E_{F W D}}}{\bar{E}}$ & $\mu_{E_{F W D}}$ & $\bar{E}$ & $\sigma_{E_{F W D}}$ & $\frac{\sigma_{E_{F W D}}}{\mu_{E_{F W D}}}$ \\
\hline \multirow[t]{5}{*}{1} & 1 & 1.008 & 257 & 255 & 15 & 0.058 \\
\hline & 2 & 1.027 & 262 & 255 & 23 & 0.086 \\
\hline & 3 & 1.017 & 259 & 255 & 20 & 0.076 \\
\hline & 4 & 1.011 & 258 & 255 & 10 & 0.038 \\
\hline & 5 & 0.998 & 254 & 255 & 11 & 0.042 \\
\hline \multirow[t]{5}{*}{2} & 1 & 1.004 & 171 & 170 & 4 & 0.025 \\
\hline & 2 & 1.007 & 171 & 170 & 4 & 0.024 \\
\hline & 3 & 1.005 & 171 & 170 & 5 & 0.028 \\
\hline & 4 & 1.006 & 171 & 170 & 3 & 0.018 \\
\hline & 5 & 1.000 & 170 & 170 & 3 & 0.019 \\
\hline
\end{tabular}


3. Second simulation - average of three drops per case

In the second simulation, sets of three successive deflection bowls (simulating three drops of the FWD at ten different points on a perfectly homogeneous pavement) were averaged. Results of the second simulation are shown in Table 6.15 to Table 6.17, for the back-calculated asphalt modulus and in Table 6.18 to Table 6.21 for the other layers.

Table 6.15 Effect of random deflection measurement error on backcalculated asphalt modulus on pavement structure 1 - average of three drops per case

\begin{tabular}{|c|c|c|c|c|c|c|}
\hline Sec & Repetition & $\frac{\mu_{E_{F W D}}}{\bar{E}}$ & $\mu_{E_{F W D}}$ & $\bar{E}$ & $\sigma_{E_{F W D}}$ & $\frac{\sigma_{E_{F W D}}}{\mu_{E_{F W D}}}$ \\
\hline \multirow[t]{5}{*}{$\bar{A}$} & 1 & 1.022 & 3167 & 3100 & 29 & 0.009 \\
\hline & 2 & 1.028 & 3188 & 3100 & 26 & 0.008 \\
\hline & 3 & 1.024 & 3176 & 3100 & 32 & 0.010 \\
\hline & 4 & 1.027 & 3185 & 3100 & 28 & 0.009 \\
\hline & 5 & 1.028 & 3187 & 3100 & 17 & 0.005 \\
\hline \multirow[t]{5}{*}{ B } & 1 & 0.990 & 3068 & 3100 & 42 & 0.014 \\
\hline & 2 & 0.991 & 3071 & 3100 & 24 & 0.008 \\
\hline & 3 & 0.993 & 3079 & 3100 & 30 & 0.010 \\
\hline & 4 & 0.990 & 3069 & 3100 & 35 & 0.011 \\
\hline & 5 & 0.997 & 3090 & 3100 & 30 & 0.010 \\
\hline \multirow[t]{5}{*}{ C } & 1 & 0.999 & 3097 & 3100 & 51 & 0.016 \\
\hline & 2 & 1.000 & 3100 & 3100 & 24 & 0.008 \\
\hline & 3 & 1.003 & 3109 & 3100 & 40 & 0.013 \\
\hline & 4 & 0.999 & 3097 & 3100 & 43 & 0.014 \\
\hline & 5 & 1.011 & 3135 & 3100 & 29 & 0.009 \\
\hline \multirow[t]{5}{*}{ D } & 1 & 1.005 & 3117 & 3100 & 61 & 0.020 \\
\hline & 2 & 1.003 & 3109 & 3100 & 25 & 0.008 \\
\hline & 3 & 1.008 & 3126 & 3100 & 45 & 0.014 \\
\hline & 4 & 1.004 & 3112 & 3100 & 50 & 0.016 \\
\hline & 5 & 1.016 & 3151 & 3100 & 38 & 0.012 \\
\hline \multirow[t]{5}{*}{$\mathbf{E}$} & 1 & 0.999 & 3097 & 3100 & 71 & 0.023 \\
\hline & 2 & 0.998 & 3093 & 3100 & 32 & 0.010 \\
\hline & 3 & 1.004 & 3112 & 3100 & 52 & 0.017 \\
\hline & 4 & 1.001 & 3103 & 3100 & 57 & 0.018 \\
\hline & 5 & 1.013 & 3141 & 3100 & 47 & 0.015 \\
\hline
\end{tabular}


Table 6.16 Effect of random deflection measurement error on backcalculated asphalt modulus on pavement structure 2 - average of three drops per case

\begin{tabular}{|c|l|l|l|l|l|l|}
\hline Sec & Repetition & $\frac{\mu_{E_{F W D}}}{\bar{E}}$ & $\mu_{E_{F W D}}$ & $\bar{E}$ & $\sigma_{E_{F W D}}$ & \\
\hline \multirow{2}{*}{1} & 1 & 1.007 & 8643 & 8584 & 349 & 0.040 \\
\hline & 2 & 0.998 & 8568 & 8584 & 146 & 0.017 \\
\hline & 3 & 1.007 & 8644 & 8584 & 249 & 0.029 \\
\hline & 4 & 0.999 & 8579 & 8584 & 279 & 0.033 \\
\hline 2 & 5 & 1.022 & 8772 & 8584 & 225 & 0.026 \\
\hline & 2 & 1.000 & 1170 & 1170 & 16 & 0.013 \\
\hline & 3 & 1.002 & 1173 & 1170 & 8 & 0.007 \\
\hline & 4 & 1.003 & 1173 & 1170 & 10 & 0.009 \\
\hline & 5 & 1.001 & 1171 & 1170 & 12 & 0.010 \\
\hline & 1 & 1.004 & 1174 & 1170 & 10 & 0.009 \\
\hline
\end{tabular}

Table 6.17 Effect of random deflection measurement error on backcalculated asphalt modulus on pavement structure 3 - average of three drops per case

\begin{tabular}{|c|c|c|c|c|c|c|}
\hline Sec & Repetition & $\frac{\mu_{E_{F W D}}}{\bar{E}}$ & $\mu_{E_{F W D}}$ & $\bar{E}$ & $\sigma_{E_{F W D}}$ & $\frac{\sigma_{E_{F W D}}}{\mu_{E_{F W D}}}$ \\
\hline \multirow[t]{5}{*}{1} & 1 & 0.990 & 8017 & 8098 & 331 & 0.041 \\
\hline & 2 & 1.036 & 8393 & 8098 & 829 & 0.099 \\
\hline & 3 & 1.013 & 8201 & 8098 & 870 & 0.106 \\
\hline & 4 & 1.024 & 8290 & 8098 & 414 & 0.050 \\
\hline & 5 & 1.011 & 8191 & 8098 & 400 & 0.049 \\
\hline \multirow[t]{5}{*}{2} & 1 & 0.999 & 1525 & 1527 & 14 & 0.009 \\
\hline & 2 & 1.011 & 1544 & 1527 & 35 & 0.023 \\
\hline & 3 & 1.005 & 1535 & 1527 & 39 & 0.025 \\
\hline & 4 & 1.008 & 1539 & 1527 & 21 & 0.014 \\
\hline & 5 & 1.002 & 1530 & 1527 & 17 & 0.011 \\
\hline
\end{tabular}


Table 6.18 Effect of random deflection measurement error on backcalculated Equivalent Foundation Modulus (EFM) on pavement structure 1 - average of three drops per case

\begin{tabular}{|c|c|c|c|c|c|c|}
\hline Sec & Repetition & $\frac{\mu_{E_{F W D}}}{\bar{E}}$ & $\mu_{E_{F W D}}$ & $\bar{E}$ & $\sigma_{E_{F W D}}$ & $\frac{\sigma_{E_{F W D}}}{\mu_{E_{F W D}}}$ \\
\hline \multirow[t]{5}{*}{ A } & 1 & 1.001 & 100 & 100 & 0 & 0.003 \\
\hline & 2 & 1.000 & 100 & 100 & 0 & 0.004 \\
\hline & 3 & 1.001 & 100 & 100 & 0 & 0.004 \\
\hline & 4 & 1.000 & 100 & 100 & 0 & 0.004 \\
\hline & 5 & 1.000 & 100 & 100 & 0 & 0.004 \\
\hline \multirow[t]{5}{*}{ B } & 1 & 1.002 & 100 & 100 & 0 & 0.004 \\
\hline & 2 & 0.999 & 100 & 100 & 0 & 0.005 \\
\hline & 3 & 1.001 & 100 & 100 & 0 & 0.004 \\
\hline & 4 & 1.000 & 100 & 100 & 0 & 0.004 \\
\hline & 5 & 1.000 & 100 & 100 & 0 & 0.004 \\
\hline \multirow[t]{5}{*}{ C } & 1 & 1.004 & 100 & 100 & 0 & 0.004 \\
\hline & 2 & 1.000 & 100 & 100 & 0 & 0.004 \\
\hline & 3 & 1.001 & 100 & 100 & 0 & 0.004 \\
\hline & 4 & 1.002 & 100 & 100 & 0 & 0.004 \\
\hline & 5 & 0.999 & 100 & 100 & 0 & 0.005 \\
\hline \multirow[t]{5}{*}{$\bar{D}$} & 1 & 1.003 & 100 & 100 & 0 & 0.004 \\
\hline & 2 & 1.001 & 100 & 100 & 0 & 0.004 \\
\hline & 3 & 1.003 & 100 & 100 & 0 & 0.004 \\
\hline & 4 & 1.003 & 100 & 100 & 0 & 0.004 \\
\hline & 5 & 1.000 & 100 & 100 & 0 & 0.004 \\
\hline \multirow[t]{5}{*}{$E$} & 1 & 1.003 & 100 & 100 & 0 & 0.004 \\
\hline & 2 & 0.999 & 100 & 100 & 0 & 0.005 \\
\hline & 3 & 1.000 & 100 & 100 & 0 & 0.004 \\
\hline & 4 & 1.001 & 100 & 100 & 0 & 0.004 \\
\hline & 5 & 0.999 & 100 & 100 & 0 & 0.005 \\
\hline
\end{tabular}

Table 6.19 Effect of random deflection measurement error on backcalculated Equivalent Foundation Modulus (EFM) on pavement structure 2 - average of three drops per case

\begin{tabular}{|c|c|c|c|c|c|c|}
\hline $\mathrm{Sec}$ & Repetition & $\frac{\mu_{E_{F W D}}}{\bar{E}}$ & $\mu_{E_{F W D}}$ & $\bar{E}$ & $\sigma_{E_{F W D}}$ & $\frac{\sigma_{E_{F W D}}}{\mu_{E_{F W D}}}$ \\
\hline \multirow[t]{5}{*}{1} & 1 & 1.002 & 257 & 256 & 4 & 0.015 \\
\hline & 2 & 0.995 & 255 & 256 & 2 & 0.008 \\
\hline & 3 & 1.000 & 256 & 256 & 2 & 0.010 \\
\hline & 4 & 1.000 & 256 & 256 & 2 & 0.009 \\
\hline & 5 & 0.996 & 255 & 256 & 2 & 0.006 \\
\hline \multirow[t]{5}{*}{2} & 1 & 0.999 & 149 & 149 & 1 & 0.009 \\
\hline & 2 & 0.995 & 148 & 149 & 1 & 0.006 \\
\hline & 3 & 0.997 & 149 & 149 & 1 & 0.007 \\
\hline & 4 & 0.998 & 149 & 149 & 1 & 0.006 \\
\hline & 5 & 0.999 & 149 & 149 & 1 & 0.004 \\
\hline
\end{tabular}


Table 6.20 Effect of random deflection measurement error on backcalculated sub-base modulus on pavement structure 3 - average of three drops per case

\begin{tabular}{|c|l|l|l|l|l|l|}
\hline Sec & Repetition & $\frac{\mu_{E_{F W D}}}{\bar{E}}$ & $\mu_{E_{F W D}}$ & $\bar{E}$ & $\sigma_{E_{F W D}}$ & $\frac{\sigma_{E_{F W D}}}{\mu_{E_{F W D}}}$ \\
\hline 1 & 1 & 1.043 & 319 & 306 & 48 & 0.151 \\
\hline & 2 & 0.916 & 280 & 306 & 71 & 0.253 \\
\hline & 3 & 1.008 & 308 & 306 & 91 & 0.294 \\
\hline & 4 & 0.933 & 286 & 306 & 40 & 0.141 \\
\hline 2 & 1 & 1.037 & 317 & 306 & 55 & 0.174 \\
\hline & 2 & 0.010 & 79 & 78 & 2 & 0.032 \\
\hline & 3 & 0.999 & 78 & 78 & 4 & 0.049 \\
& 4 & 0.986 & 77 & 78 & 3 & 0.069 \\
\hline 5 & 1.014 & 79 & 78 & 3 & 0.035 \\
\hline
\end{tabular}

Table 6.21 Effect of random deflection measurement error on backcalculated subgrade modulus on pavement structure 3 - average of three drops per case

\begin{tabular}{c|l|l|l|l|l|l|}
\hline Sec & Repetition & $\frac{\mu_{E_{F W D}}}{\bar{E}}$ & $\mu_{E_{F W D}}$ & $\bar{E}$ & $\sigma_{E_{F W D}}$ & $\frac{\sigma_{E_{F W D}}}{\mu_{E_{F W D}}}$ \\
\hline 1 & 1 & 0.997 & 254 & 255 & 8 & 0.030 \\
\hline & 2 & 1.012 & 258 & 255 & 10 & 0.040 \\
\hline & 3 & 1.007 & 257 & 255 & 14 & 0.056 \\
\hline & 4 & 1.007 & 257 & 255 & 7 & 0.027 \\
\hline & 5 & 0.995 & 254 & 255 & 6 & 0.024 \\
\hline & 1 & 1.001 & 170 & 170 & 3 & 0.015 \\
\hline & 2 & 1.004 & 171 & 170 & 3 & 0.015 \\
\hline & 4 & 1.004 & 171 & 170 & 3 & 0.020 \\
\hline & 5 & 1.005 & 171 & 170 & 2 & 0.013 \\
\hline
\end{tabular}

The results of the second simulation suggest that:

- The effect of the random deflection measurement error on the backcalculated layer modulus is generally low. The only exception is the backcalculated sub-base layer stiffness of the 3-layer pavement structure where the effect is instead noticeable.

- The standard deviations of the layer moduli decrease as the number of "drops" included in the average increases to 3 . 


\subsubsection{Conclusions from analysis of random deflection measurement error}

A summary of the effect of random deflection measurement error on backanalysed asphalt and subgrade stiffnesses is presented in Table 6.22. It can be seen that the average error due to random measurement deflection error is less than $1 \%$ on back-analysed asphalt stiffness and close to $0 \%$ on backanalysed subgrade stiffness. The results of the same analysis from Irwin et al. (1989) is also reported, see Table 6.23 and Table 6.24. It should be noted that the pavement structure included in Irwin et al.'s paper is very different from the one considered in this research analysis. The pavement structure considered in Irwin's paper consists of $\sim 75 \mathrm{~mm}$ of asphalt surface course ( $2100 \mathrm{MPa})$, $150 \mathrm{~mm}$ of unbound base (310MPa), $305 \mathrm{~mm}$ of unbound subbase (145MPa) on top of subgrade (50MPa). Irwin et al show a much wider range for the error in the back-analysed stiffnesses of the asphalt and unbound layers. The wider error range due to measurement error in Irwin's paper is probably due to the four-layer structure considered in the calculation while a two-layer structure has been used in this research analysis. It will be shown in chapter 8 that thin asphalt layers give a high mean back-analysed stiffness modulus error when compared to asphalt thickness greater than $200 \mathrm{~mm}$. The results in Table 6.23 and Table 6.24 are specific to the pavement structures considered.

\section{Table 6.22 Summary of simulation results}

\begin{tabular}{|c|c|c|c|c|c|}
\hline \multicolumn{3}{|c|}{ Error (\%) on back-calculated stiffnesses } & \multicolumn{3}{|c|}{ Error (\%) on back-calculated stiffnesses } \\
\hline \multirow{2}{*}{$\begin{array}{l}\text { 1st } \\
\text { Simulation } \\
\text { (single drop } \\
\text { per case) }\end{array}$} & $\begin{array}{l}\text { Asphalt stiffness } \\
\text { modulus }\end{array}$ & $\begin{array}{l}\text { Subgrade } \\
\text { stiffness modulus }\end{array}$ & \multirow{2}{*}{$\begin{array}{l}\text { 2nd } \\
\text { Simulation } \\
\text { (average of } \\
\text { three drops } \\
\text { per case) }\end{array}$} & $\begin{array}{l}\text { Asphalt stiffness } \\
\text { modulus }\end{array}$ & $\begin{array}{l}\text { Subgrade } \\
\text { stiffness modulus }\end{array}$ \\
\hline & $\frac{\mu_{E_{F W D}}}{\bar{E}}-1$ & $\frac{\mu_{E_{F W D}}}{\bar{E}}-1$ & & $\frac{\mu_{E_{F W D}}}{\bar{E}}-1$ & $\frac{\mu_{E_{F W D}}}{\bar{E}}-1$ \\
\hline $\min$ & $-1.14 \%$ & $-0.40 \%$ & $\min$ & $-1.02 \%$ & $-0.55 \%$ \\
\hline avg & $0.69 \%$ & $0.23 \%$ & avg & $0.66 \%$ & $0.07 \%$ \\
\hline $\max$ & $5.17 \%$ & $2.73 \%$ & $\max$ & $3.65 \%$ & $1.22 \%$ \\
\hline
\end{tabular}


Table 6.23 Summary of $1^{\text {st }}$ simulation results from Irwin et al. (1989)

\begin{tabular}{l|c|c|c|c|}
\hline \multicolumn{5}{|c|}{ Error (\%) on back-calculated stiffinesses } \\
\hline $\begin{array}{l}\text { 1st } \\
\begin{array}{l}\text { Simulation } \\
\text { (single drop } \\
\text { per case) }\end{array}\end{array}$ & $\begin{array}{l}\text { Asphalt stiffness } \\
\text { modulus }\end{array}$ & $\begin{array}{l}\text { Unbound base } \\
\text { stiffness modulus }\end{array}$ & $\begin{array}{l}\text { Unbound subbase } \\
\text { stiffiness modulus }\end{array}$ & $\begin{array}{l}\text { Subgrade } \\
\text { stiffiness modulus }\end{array}$ \\
\cline { 3 - 6 } & $\frac{\mu_{E_{F W D}}}{\bar{E}}-1$ & $\frac{\mu_{E_{F W D}}}{\bar{E}}-1$ & $\frac{\mu_{E_{F W D}}}{\bar{E}}-1$ & $\frac{\mu_{E_{F W D}}}{\bar{E}}-1$ \\
\hline $\min$ & $-34.67 \%$ & $-28.22 \%$ & $-10.95 \%$ & $-1.47 \%$ \\
\hline $\operatorname{avg}$ & $2.00 \%$ & $-0.89 \%$ & $1.43 \%$ & $-0.13 \%$ \\
\hline $\max$ & $42.00 \%$ & $33.11 \%$ & $19.05 \%$ & $2.27 \%$ \\
\hline
\end{tabular}

Table 6.24 Summary of 2nd simulation results from Irwin et al. (1989)

\begin{tabular}{l|c|c|c|c|}
\multicolumn{5}{c|}{ Error (\%) on back-calculated stiffnesses } \\
\hline $\begin{array}{l}\text { 2nd } \\
\begin{array}{l}\text { Simulation } \\
\text { (average of } \\
\text { three drops } \\
\text { per case) }\end{array}\end{array}$ & $\begin{array}{l}\text { Asphalt stiffness } \\
\text { modulus }\end{array}$ & $\begin{array}{l}\text { Unbound base } \\
\text { stiffiness modulus }\end{array}$ & $\begin{array}{l}\text { Unbound subbase } \\
\text { stiffiness modulus }\end{array}$ & $\begin{array}{l}\text { Subgrade } \\
\text { stiffiness modulus }\end{array}$ \\
\hline min & $-9.00 \%$ & $\frac{\mu_{E_{F W D}}}{\bar{E}}-1$ & $\frac{\mu_{E_{F W D}}}{\bar{E}}-1$ & $\frac{\mu_{E_{F W D}}}{\bar{E}}-1$ \\
\hline avg & $3.67 \%$ & $-24.22 \%$ & $-3.81 \%$ & $-2.53 \%$ \\
\hline $\max$ & $30.33 \%$ & $-2.89 \%$ & $2.38 \%$ & $-0.27 \%$ \\
\hline
\end{tabular}

The following conclusions can be drawn:

- The effect of random deflection measurement error can be minimised by making at least three replicate drops at each drop height and at each test point so that an average deflection bowl can be computed. In the UK it is standard practice to take the last bowl.

- If the effect of random error needs to be included in the analysis, then the following relationship will be used to calculate the standard deviation due to the combined effect of variation of layer thickness and random error in the deflection measurement:

$$
\sigma_{\Delta E}=\sqrt{\sigma_{\Delta E}^{2}+\sigma_{\text {Ran_error }}^{2}}
$$

where $\sigma_{\Delta E}$ is the standard deviation of the true asphalt stiffness modulus calculated with equation 10 (Collop, 2000) and $\sigma_{\text {Ran_error }}$ is the standard deviation of the asphalt stiffness modulus due to random deflection measurement error. 


\subsubsection{Effect of limited number of data points on back-calculated moduli}

This section examines the accuracy of the statistical properties (mean and standard deviation) calculated from the back-calculated stiffness modulus data collected using the FWD in relation to the number of samples used in the calculation.

Collop (2000) determined the confidence limits for back-calculated asphaltic material stiffness modulus from FWD tests using a variable number of measurement points using a normalised mean error and a standard deviation error calculated using the following equations:

$$
\begin{aligned}
& -\frac{\sigma_{\Delta E} * t_{n ; \alpha / 2}}{(\bar{E})^{r \text { true }} * \sqrt{N}} \leq \frac{(\bar{E})^{\text {true }}-\bar{E}}{(\bar{E})^{t r u e}}<\frac{\sigma_{\Delta E} * t_{n ; \alpha / 2}}{(\bar{E})^{\text {true }} * \sqrt{N}} \\
& 1-\sqrt{\frac{\chi_{n ; \alpha / 2}^{2}}{n}} \leq \frac{\left(\sigma_{\Delta E}\right)^{\text {true }}-\sigma_{\Delta E}}{\left(\sigma_{\Delta E}\right)^{r \text { true }}}<1-\sqrt{\frac{\chi_{n ; 1-\alpha / 2}^{2}}{n}}
\end{aligned}
$$

Eq. (13), Collop 2000

Eq. (14), Collop 2000

Where

$(\bar{E})^{\text {true }},\left(\sigma_{\Delta E}\right)^{\text {true }}$ are the values of the mean stiffness modulus and the stiffness modulus standard deviation calculated using an infinite number of data points,

$\bar{E}, \sigma_{\Delta E}$ are the values of the mean stiffness modulus and the stiffness modulus standard deviation calculated using a limited number of data points,

$N$ is the number of data points,

$n=\mathrm{N}-1$,

$\chi_{n ; \alpha}^{2}$ is the $100 \alpha$ percentage point of the $\chi^{2}$ distribution with $n$ degrees of freedom,

$t_{n ; \alpha / 2}$ is the $100 \alpha$ percentage point of the student $t$ distribution with $n$ degrees of freedom,

$\alpha$ is the level of significance (i.e. 0.05)

From the above equations it can be see that: 
- The accuracy of the mean stiffness modulus estimates depends on the ratio of estimated standard deviation to true stiffness modulus and the number of samples.

- The accuracy of the standard deviation of the layer stiffness modulus estimates depends on the number of samples.

Equations (13) and (14) (Collop, 2000) can also be used to calculate the confidence interval associated with a limited number of asphalt layer thickness measurements (i.e., from cores) by simply replacing the asphalt stiffness modulus with asphalt layer thickness:

$$
\begin{aligned}
& -\frac{\sigma_{\Delta h} * t_{n ; \alpha / 2}}{(\bar{h})^{\text {true }} * \sqrt{N}} \leq \frac{(\bar{h})^{\text {true }}-\bar{h}}{(\bar{h})^{\text {true }}}<\frac{\sigma_{\Delta h} * t_{n ; \alpha / 2}}{(\bar{h})^{\text {true }} * \sqrt{N}} \\
& 1-\sqrt{\frac{\chi_{n ; \alpha / 2}^{2}}{n}} \leq \frac{\left(\sigma_{\Delta h}\right)^{\text {true }}-\sigma_{\Delta h}}{\left(\sigma_{\Delta h}\right)^{\text {true }}}<1-\sqrt{\frac{\chi_{n: 1-\alpha / 2}^{2}}{n}}
\end{aligned}
$$

Where

$(\bar{h})^{\text {true }}=\bar{h}+h_{0},\left(\sigma_{\Delta h}\right)^{\text {true }}$ are the values of the mean asphalt layer thickness and the asphalt layer thickness standard deviation calculated using an infinite number of data points (i.e., from GPR measurements),

$\bar{h}, \sigma_{\Delta h}$ are the values of the mean asphalt layer thickness and the asphalt layer thickness standard deviation calculated using a limited number of data points (i.e. from core measurements), and the remaining terms have the same meaning described in equations (13) and (14) (Collop, 2000) above.

\subsection{ITSM tests from available sites}

ITSM tests are available for all the sites considered in this research. However, only the ITSM tests performed on motorway M01 have been analysed as the number of ITSM tests on the other sites is too low.

Despite the large number of ITSM tests available on the M01 sites, only for a few tests are the location and the layer tested known. From plotting all the available ITSM tests on the M01 site it can be seen that a lognormal probability distribution fits the data, see Figure 6.6. A tabular representation of the same 
data is presented in Table 6.25. It should be noted that the high stiffness modulus for site M01 is due to the high modulus base material containing a 15 penetration grade bitumen (known as HMB15).

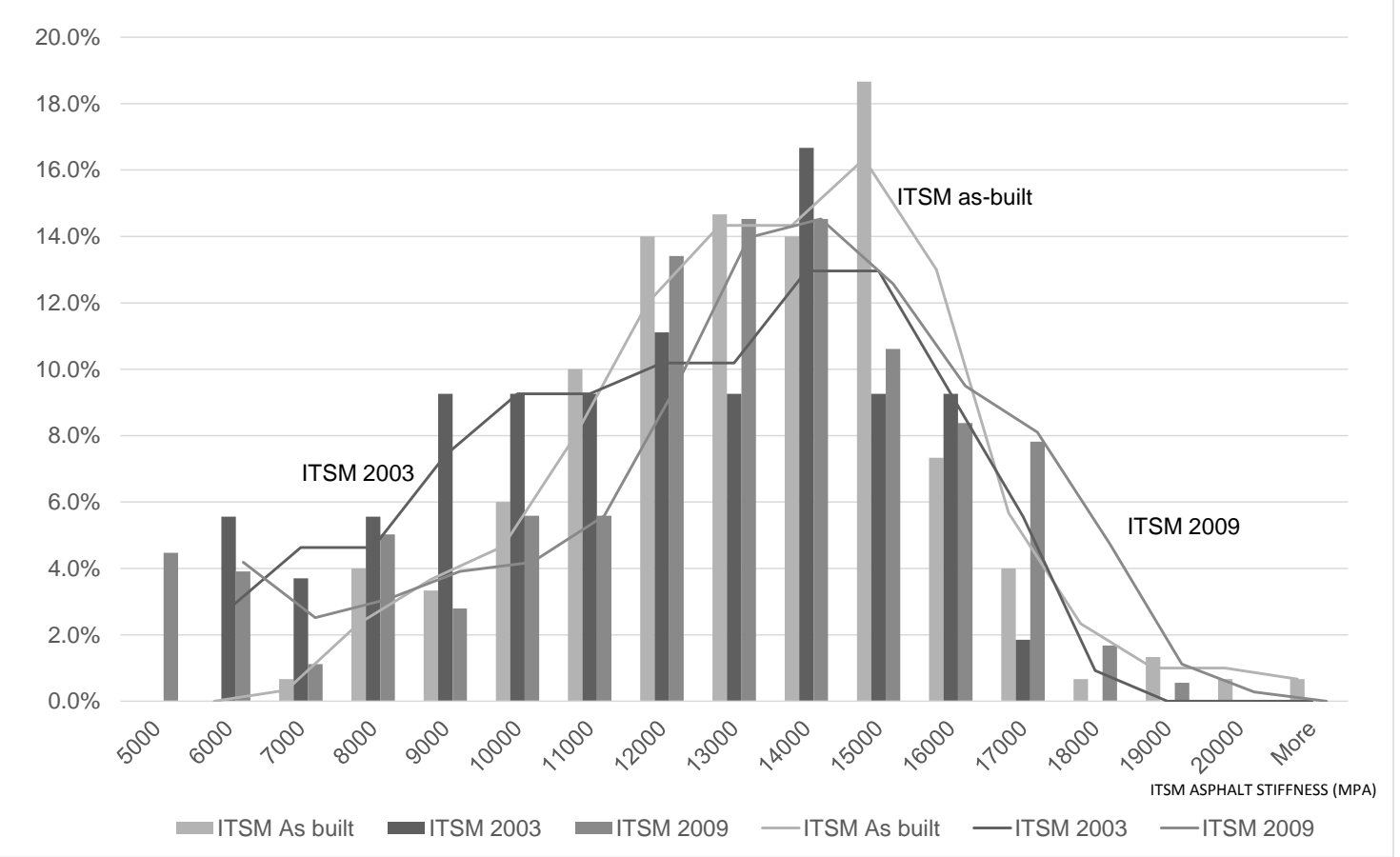

Figure 6.6 ITSM stiffness data for motorway M01

Table 6.25 Statistical properties of ITSM data from motorway M01

\begin{tabular}{|l|c|c|c|c|c|c|}
\hline Sites & $\begin{array}{l}\text { ITSM set } \\
\text { of data }\end{array}$ & Nr tests & Layers & $\begin{array}{l}\text { Mean stiffness } \\
\text { (MPa) }\end{array}$ & $\begin{array}{l}\text { Standard deviation } \\
\text { stiffiness (MPa) }\end{array}$ & COV \\
\hline \multirow{2}{*}{ M01 all } & As-built & 150 & Binder and base & 12738 & 2550 & 0.20 \\
\hline sections & 2003 & 54 & Binder and base & 11298 & 2924 & 0.26 \\
\hline & 2009 & 45 & Binder and base & 11959 & 3439 & 0.29 \\
\hline
\end{tabular}

For those few sections where the ITSM's test location was known, equations (6.5) and (6.6) were used to calculate the $95 \%$ confidence limits for the mean, standard deviation and coefficient of variation of the asphalt layer stiffness modulus, see Table 6.26 to Table 6.29. The analysis has been carried out for both the base and the binder course layers. The coefficient of variation appears, on average, to range from 10 to $30 \%$, which is in line with the results found by Noureldin et al. (1994) and Timm et al. (2000), see section 2.8. 
Table 6.26 M01 Clockwise: ITSM tests for base layer

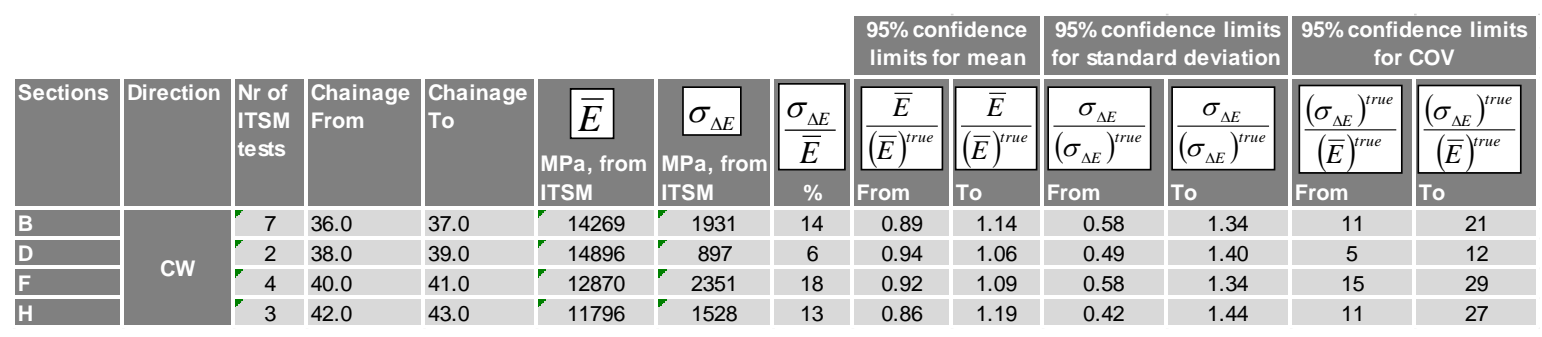

Table 6.27 M01 Anti-Clockwise: ITSM tests for base layer

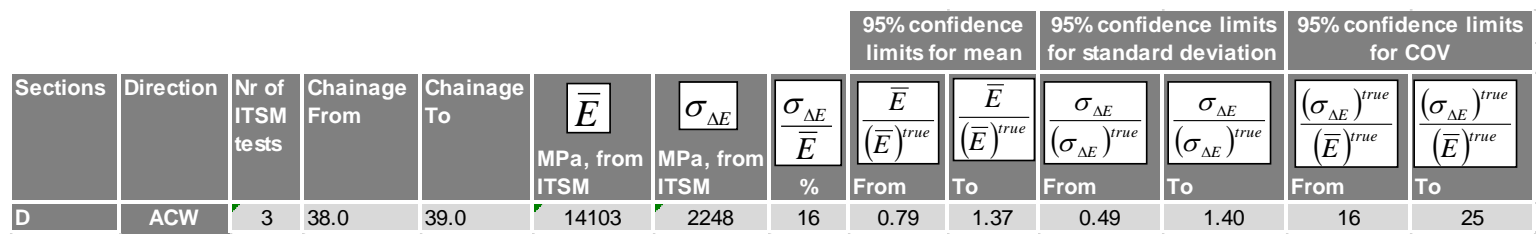

Table 6.28 M01 Clockwise: ITSM tests for binder course layer

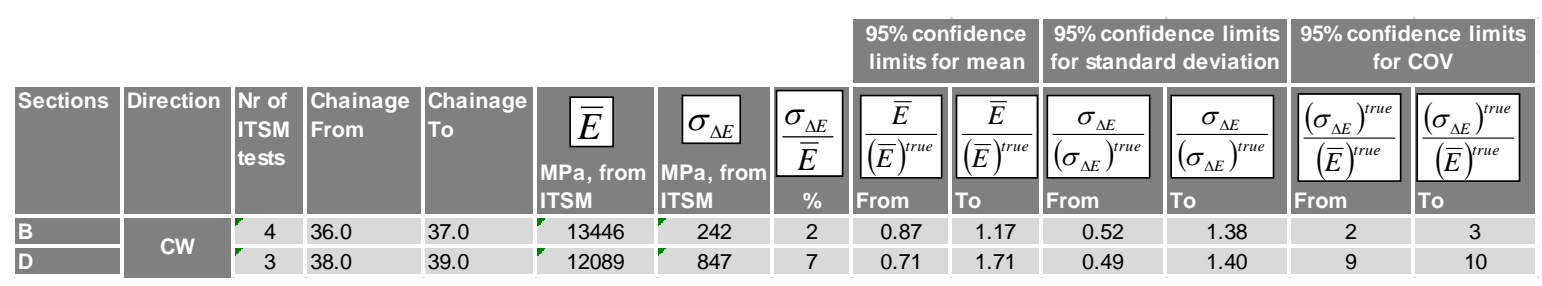

Table 6.29 M01 Anti-Clockwise: ITSM tests for binder course layer

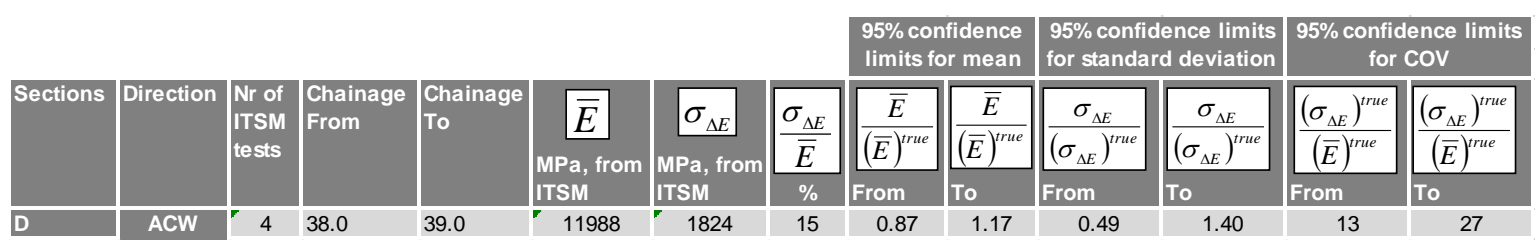

Finally, Table 6.30 shows a comparison between the standard deviation of the asphalt stiffness modulus obtained from FWD back-analysis $\left(\sigma_{E_{F W D}}\right)$, from ITSM tests $\left(\sigma_{E_{I T S M}}\right)$, and from Equation (10) $\left(\sigma_{\triangle E}\right)$ (Collop, 2000) for a $1 \mathrm{~km}$ section of motorway M01. The table shows that the 3 standard deviations are not very different from each other. However, more data would be required to make more generalised conclusions.

Table 6.30 Comparison between standard deviations of the asphalt stiffness modulus

\begin{tabular}{|l|r|r|r|r|r|}
\hline Section & $\begin{array}{l}\text { From } \\
\text { (MP) }\end{array}$ & To (MP) & $\sigma_{E_{F W D}}$ & $\sigma_{\Delta E}$ & $\sigma_{\Delta E_{\text {ITSMbas }}}$ \\
\hline B, Clockwise & 36.0 & 37.0 & 2483 & 1839 & 1931 \\
\hline
\end{tabular}




\subsection{Conclusions}

This chapter has discussed how asphalt stiffness is generally measured in the UK, i.e. by field FWD tests and by laboratory ITSM tests. The accuracy in the back-calculation of pavement layer moduli has been analysed. The impact of pavement thickness errors, deflection errors and limited number of data points has been discussed and techniques to calculate the 'true' asphalt stiffness variation have been reported.

The main observations made in this chapter are:

- Both laboratory and in-situ determination of stiffness modulus should be used to perform a full pavement evaluation.

- In order for the FWD back-analysis to give reliable estimates of the in-situ asphalt stiffness modulus, it is essential to use accurate pavement layer thickness information. For example, a 15 per cent underestimate of the thickness of a bound layer can result in a fifty per cent overestimate of the stiffness of that layer.

- The average error due to random measurement deflection error is less than $1 \%$ on back-analysed asphalt stiffness and close to $0 \%$ on back-analysed subgrade stiffness.

- The effect of random deflection measurement error can be minimised by making at least three replicate drops at each drop height.

- With both the effect of random error in the deflection measurement and the effect of variation of layer thickness included in the analysis, a relationship is proposed to calculate the adjusted standard deviation.

- The effect of limited number of data points on back-calculated moduli has shown that:

- The accuracy of the mean stiffness modulus estimates depends on the ratio of estimated standard deviation to true stiffness modulus and the number of samples.

- The accuracy of the standard deviation of the layer stiffness modulus estimates depends on the number of samples. 
- Based on the plots of ITSM tests on the M01 site, the coefficient of variation of the asphalt layer stiffness modulus appears, on average, to range from $10 \%$ to $30 \%$.

- A lognormal probability distribution was found representative of the asphalt layer stiffness modulus. 


\section{Statistical characterisation of subgrade stiffness variability}

Subgrade stiffness together with the design traffic are the main input parameters to analytical pavement design. The scope of the design, for example in terms deformation life, is to ensure that there is only limited deformation on the subgrade at the end of the design life due to stresses induced by traffic loads to the subgrade (through the road pavement). Subgrade stiffness affects not only the level of stresses in the subgrade but also the levels of stresses generated in all the overlying pavement layers.

Seasonal temperature and moisture content variations within pavement subgrade and subbase layers influence pavement load carrying capacity. Loss of support conditions (i.e., a reduction in stiffness) in these layers can occur during thawing periods and/or saturated conditions and is one of the contributors to pavement distress.

Current practice in the UK is to provide French drains at a depth of $0.6 \mathrm{~m}$ in order to ensure as far as possible that the water table is maintained at least $0.23 \mathrm{~m}$ below the level of the formation under the road (Powell et al., 1984).

Inadequate drainage can also affect the strength of the subgrade and the stability of granular foundation layers subjected to lengthy saturation. These factors are accounted for during the design stage, as will be shown later.

The first part of this chapter is an overview of different methods to estimate the subgrade stiffness and a summary of research information found to date on subgrade stiffness variability. The second part shows the results of the author's attempt to assess subgrade stiffness variability.

\subsection{Subgrade modulus for foundation design}

The subgrade is part of the foundation platform to support both the construction traffic and the design traffic.

A typical foundation includes the following elements, see Figure 7.1:

- The subgrade, referred as the natural ground (but it isn't always natural) 
- A layer of capping (optional). The provision of a capping layer may be particularly appropriate for lower strength subgrades.

- A layer of subbase (bound or unbound)

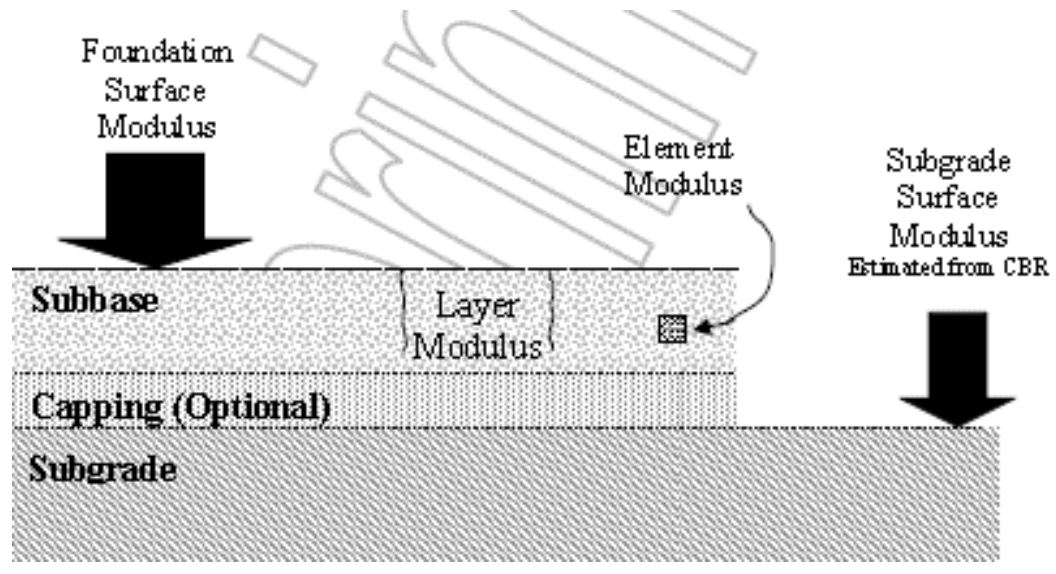

\section{Figure 7.1 Foundation layers (from Figure 2.1 in IAN 73/09 Rev 1)}

The subgrade surface modulus is an estimated value of 'Stiffness Modulus' based on subgrade CBR and used for foundation design, whereas stiffness modulus is intended as the ratio of applied stress to induced strain.

For subgrades, stiffness modulus is difficult to measure reliably and consistently, so historically California Bearing Ratio (CBR) has been used as an indirect measure.

In the UK, estimation of the likely long-term, short-term and hence Design CBR should be derived using laboratory CBR tests in accordance with BS $1377 \mathrm{Pt}$ 4 (1990).

Where it is not possible to collect material samples for assessment using laboratory CBR tests, the Suction Index Method should be used, as described in Appendix C of LR1132 (Powell et al, 1984). Table 7.1 reports Table C1 in LR 1132 with estimated values for long-term CBR depending on soil type, particularly for clay subgrades where moisture and plasticity index are significant issues. 
Table 7.1 Equilibrium suction-index CBR values (from Table $\mathrm{C} 1$ in Appendix C in LR 1132, Powell et al. 1984)

\begin{tabular}{|c|c|c|c|c|c|c|c|c|c|c|c|c|c|}
\hline \multirow[t]{4}{*}{ TYPE OF SOIL } & \multirow{4}{*}{ 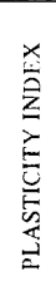 } & \multirow{2}{*}{\multicolumn{6}{|c|}{$\begin{array}{c}\text { HIGH WATER TABLE } \\
\text { CONSTRUCTION CONDITIONS: }\end{array}$}} & \multicolumn{6}{|c|}{ LOW WATER TABLE } \\
\hline & & & & & & & & \multicolumn{6}{|c|}{ CONSTRUCTION CONDITIONS: } \\
\hline & & \multicolumn{2}{|c|}{ POOR } & \multicolumn{2}{|c|}{ AVERAGE } & \multicolumn{2}{|c|}{ GOOD } & \multicolumn{2}{|c|}{ POOR } & \multicolumn{2}{|c|}{ AVERAGE } & \multicolumn{2}{|c|}{ GOOD } \\
\hline & & 站 & $\stackrel{\text { U. }}{\underline{E}}$ & 辛 & 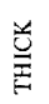 & $\underset{\Xi}{Z}$ & 总 & $\underset{I}{\underline{I}}$ & 总 & $\underset{\exists}{Z}$ & $\stackrel{\text { 总 }}{\Xi}$ & 齐 & 弟 \\
\hline HEAVY CLAY & 70 & 1.5 & 2 & 2 & 2 & 2 & 2 & 1.5 & 2 & 2 & 2 & 2 & 2.5 \\
\hline & 60 & 1.5 & 2 & 2 & 2 & 2 & 2.5 & 1.5 & 2 & 2 & 2 & 2 & 2.5 \\
\hline & so & 1.5 & 2 & 2 & 2.5 & 2 & 2.5 & 2 & 2 & 2 & 2.5 & 2 & 2.5 \\
\hline & 40 & 2 & 2.5 & 2.5 & 3 & 2.5 & 3 & 2.5 & 2.5 & 3 & 3 & 3 & 3.5 \\
\hline SILTY CLAY & 30 & 2.5 & 3.5 & 3 & 4 & 3.5 & 5 & 3 & 3.5 & 4 & 4 & 4 & 6 \\
\hline SANDY CLAY & 20 & 2.5 & 4 & 4 & 5 & 4.5 & 7 & 3 & 4 & 5 & 6 & 6 & 8 \\
\hline & 10 & 1.5 & 3.5 & 3 & 6 & 3.5 & 7 & 2.5 & 4 & 4.5 & 7 & 6 & $>8$ \\
\hline SILT * & - & 1 & 1 & 1 & 1 & 2 & 2 & 1 & 1 & 2 & 2 & 2 & 2 \\
\hline $\begin{array}{c}\text { SAND } \\
\text { (POORLY GRADED) }\end{array}$ & - & \multicolumn{6}{|c|}{$<$} & & & & & & $\Rightarrow$ \\
\hline $\begin{array}{c}\text { SAND } \\
\text { (WILLL GRADID) }\end{array}$ & - & \multicolumn{6}{|c|}{$\leftarrow$} & & & & & & \\
\hline $\begin{array}{l}\text { SANDY (GRAVEL } \\
\text { (WLLL GRADFD) }\end{array}$ & - & \multicolumn{6}{|c|}{$\leftarrow$} & & & & & & $\rightarrow$ \\
\hline
\end{tabular}

For design purposes, the Subgrade Surface Modulus must be estimated from CBR values. The Subgrade Surface Modulus used for design must be determined using the lowest value of the long term and short term CBR. The short-term, i.e. during construction, subgrade CBR strength would typically be expected to differ from the long-term, i.e. under the completed pavement, equilibrium strength.

The subgrade CBR value must be checked on site before foundation construction starts and must be equal to, or greater than, the Design CBR. The Dynamic Cone Penetrometer (DCP) method must be used to measure the in-situ subgrade strength, applicable to soft and medium fine grained subgrades.

If the in-situ CBR is found to be less than the Design CBR, then the subgrade must either be improved to the Design CBR or the foundation redesigned.

Where a subgrade has a lower CBR (less than $2 \%$ ) it is considered unsuitable support for a pavement foundation. It must therefore be permanently improved using special geotechnical measures. 


\subsection{Methods for estimating stiffness of subgrade}

There are three main methods used worldwide to estimate the subgrade stiffness:

- Laboratory tests, such as California Bearing Ratio (CBR) and Repeated Load Triaxial (RLT) tests;

- Field methods - intrusive, such as Dynamic Cone Penetrometer (DCP);

- Field methods - non-destructive such as Falling Weight Deflectometer (FWD) and Light Weight Dynamic plate (LWD).

Extensive information from several Highways Authorities across the United States regarding their preferred method for estimating the stiffness of subgrade and unbound materials for pavement design is reported in the NCHRP Synthesis 382 report (2008). The main conclusions from the report are:

- Among different laboratory methods the repeated load triaxial (RLT) test is the most preferred form of laboratory tests for repeatable and reliable moduli properties determination.

- In the field, non-destructive tools such as FWD and an intrusive method such as DCP are preferred field test methods.

- LWDs have become powerful tools for quick assessment of moduli in the field.

- The majority of the correlations with DCP are local and empirical types.

- There appears to be some variation with respect to the back-calculation programs used for determining moduli of layers (from FWD surveys).

- The report recommends the development and a universal implementation of a standardised resilient Modulus $\left(\mathrm{M}_{\mathrm{R}}\right)$ measurement approach in both laboratory and field conditions which would lead to a better and reliable moduli database. Such a database should be used to derive universal statistical correlation models for better interpretation of moduli properties of bases and subgrades. 
Similar considerations apply to the UK. An example of laboratory and field methods used in the UK is presented in the sections below. Also in the UK, correlations, such as those between FWD and LWD tests, and between LWD and DCP or plate bearing tests, are empirical and local. As a result, measured data from various materials are different. Correlations are developed from test data results which refer to a certain type of soil with a certain compaction and stress conditions. Therefore, correlations are expected to provide poor predictions when attempted on other soils.

\subsubsection{California Bearing Ratio (CBR)}

The California Bearing Ratio (CBR) test involves the insertion of a $50 \mathrm{~mm}$ diameter plunger into the ground surface at a rate of $1 \mathrm{~mm}$ per minute, whilst the load is recorded. Surcharge rings can be placed around the plunger to simulate an overburden. A laboratory version of the same test is available in which the sample tested is constrained within a $152.5 \mathrm{~mm}$ diameter mould. The load at penetrations of 2.5 and $5 \mathrm{~mm}$ is compared with the result for a standard aggregate and the ratio given as a percentage. The test is not suitable for coarse aggregates because the plunger and aggregate particles will be of similar size. The test measures neither Stiffness Modulus nor Shear Strength directly - giving a somewhat combined measure of both. It takes around half an hour on site and between 1 and 2 hours in the laboratory and there is a large body of experience of its use.

There are several variants on the CBR test: laboratory, field, with surcharge, saturated etc. The standard method used in the UK is the laboratory CBR with a surcharge to simulate the appropriate vertical overburden stress of the case being considered.

A relationship between CBR and Stiffness Modulus - derived from work on certain soils (Powell et al, 1984) - is:

$$
E=17.6{ }^{*}(\mathrm{CBR}){ }^{0.64} \mathrm{MPa}
$$

Where CBR is given as a \% value. 


\subsubsection{Dynamic Cone Penetrometer (DCP)}

The Dynamic Cone Penetrometer is applicable to soft and medium fine grained subgrades. The Dynamic Cone Penetrometer is similar to other field cone penetrometers except that it is driven into the ground under the action of a weight dropped onto an anvil. It is therefore suited to stronger and coarser materials than other penetrometers. The rate of penetration into the ground can then be related approximately to CBR by the following equation developed by the Transport Research Laboratory:

$$
\mathrm{CBR}=10\left(2.48-1.057 \times \log _{10} \mathrm{P}\right)
$$

where $P$ is the penetration rate in $\mathrm{mm}$ per blow. (NB. The accuracy of this relationship reduces for CBR values below 10 per cent.)

The Dynamic Cone Penetrometer is a device incorporating an $8 \mathrm{~kg}$ steel drop weight that falls vertically through $575 \mathrm{~mm}$ and makes contact with a relatively light steel anvil. The anvil is rigidly attached, via steel rods to a $20 \mathrm{~mm}$ diameter $60^{\circ}$ steel cone, which is thus driven vertically into the ground, see Figure 7.2.

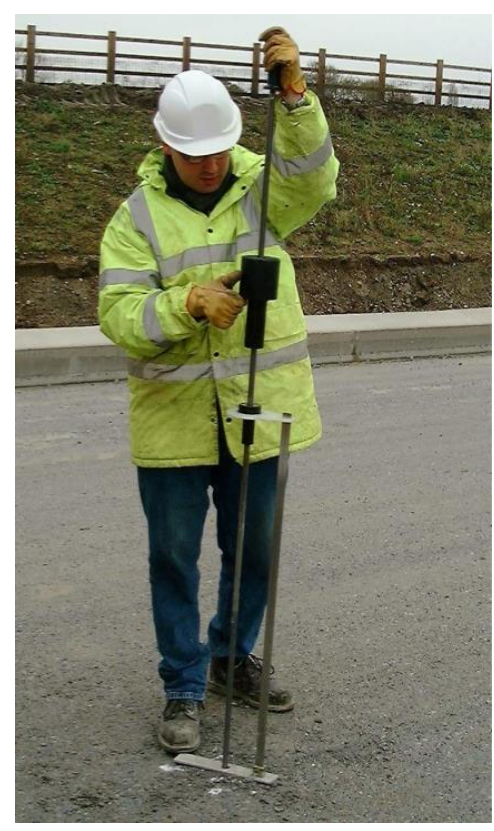

\section{Figure 7.2 Dynamic Cone Penetrometer (DCP)}

The DCP is particularly valuable for evaluating the properties of an existing pavement foundation. For example, it may also be used through many other 
materials, particularly in a composite foundation, to measure both their CBRs and layer thicknesses.

Where laboratory CBR tests have been carried out on the subgrade material, the DCP values should be calibrated to those of the laboratory tests.

\subsubsection{Plate bearing tests}

For coarser materials the Plate Bearing Test may be found appropriate for determination of subgrade CBR values. The test is described in detail in BS1377 (1990) and involves placing a circular plate on a foundation layer, see Figure 7.3.

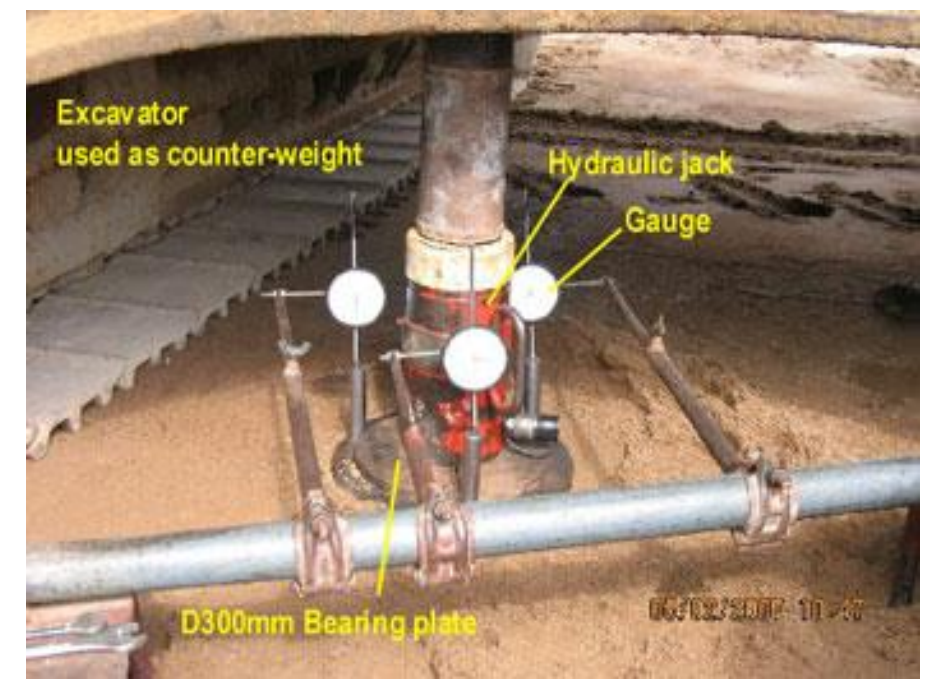

Figure 7.3 Plate bearing test (from M\&H testing Ltd website)

An approximate empirical relationship with $\mathrm{CBR}$ can be made as follows (DMRB Volume 7, IAN 73/06 Rev1):

$$
\mathrm{CBR}=6.1 * 10^{-8} \times(\mathrm{k} 762)^{1.733} \%
$$

where $\mathrm{k}_{762}$ is the Modulus of Subgrade Reaction, defined as the applied pressure under the loading platen divided by the displacement (normally $1.25 \mathrm{~mm}$ ) with a plate of $762 \mathrm{~mm}$ ( $30 \mathrm{inch}$ ) diameter. The Modulus of Subgrade Reaction for other plate sizes can be determined using an appropriate conversion factor. 


\subsubsection{Dynamic Plate testing}

When the type of soil is inappropriate for Dynamic Cone Penetrometer (DCP) testing, Dynamic Plate testing may be used, see Figure 7.4.

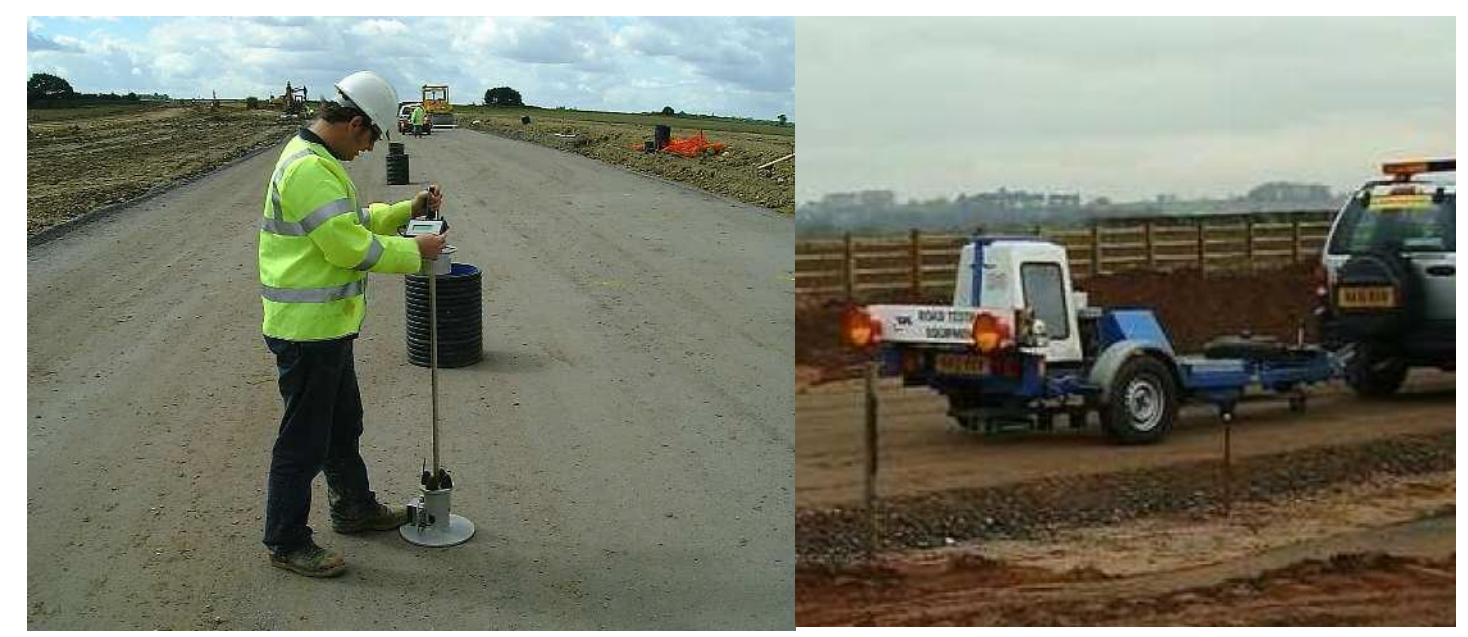

Figure 7.4 Dynamic Plate tests (LWD on the left, FWD on the right)

These tests involve dropping a weight onto a platen and measuring the deflection. Usually a damping mechanism (rubber buffers) is incorporated to control the magnitude and duration of the loading. The Falling Weight Deflectometer (FWD) measures the stress applied and the resulting deflection of the foundation at several radial positions up to 2.5 metres from the loading plate. Interpretation is generally in terms of the Stiffness Modulus of each foundation layer but is not straightforward and should be carried out by an experienced pavement engineer. If only the central deflection is used to determine a Surface Modulus for the foundation, then interpretation can be carried out as for other Dynamic Plate tests. The Lightweight Dynamic Plate (LWD) apparatus can be used for most foundation materials but care will be required for very stiff foundations, as it may be unable to deliver sufficient load to achieve a measurable deflection.

The computation of surface stiffness modulus at each point tested is based on Boussinesq elastic theory using the following formula (DMRB Volume 7, HD 29/08):

$$
E=\frac{2 *\left(1-v^{2}\right) * R * P}{D}
$$


where:

$\mathrm{E}=$ Foundation Surface Modulus (in $\mathrm{MN} / \mathrm{m}^{2}$ or $\mathrm{MPa}$ )

$v=$ Poisson's Ratio $(v$, by default, $=0.35)$

$R=$ Load Plate Radius $(R$, by default, $=150 \mathrm{~mm})$

$\mathrm{P}=$ Contact Pressure (in $\mathrm{kPa})$

$\mathrm{D}=$ Deflection under the centre of the plate (in microns)

If a lightweight test device is used, it must be correlated to an FWD which will remain the reference test method (Clause 895.5 in IAN 73/06 Rev 1).

\subsection{Spatial and seasonal variation of subgrade stiffness}

Besides variability that may result from using different testing methods, as discussed in section 7.2, variability of subgrade stiffness is also due to seasonal and spatial variations.

The heterogeneous nature of the pavement materials and non-uniform layer thicknesses lead to spatial variation which is present in all pavements and its magnitude depends to a great extent on such factors as construction quality. Spatial variation of subgrade stiffness during the pavement evaluation process is typically addressed through the selection of design values (deflections or predicted layer moduli) based on statistical variation. The use of the 85th percentile predicted layer moduli for evaluation of major roads and 50th percentile deflection values for low volume roads is common practice (Appea 2003).

It is well known the subgrade strength variability affects the long-term performance of the pavement. For example, if one area has a weaker subgrade than an adjacent area, it will be subject to larger deflections under heavy traffic loads which leads to varying deflection in the overlying pavement layers under traffic loads. The variation in the deflection along the pavement therefore leads to variations in the accumulation of damage along the pavement, both within the layers and at the pavement surface. The resulting 
damage could be permanent deformation in any of the layers, cracking of the pavement surface and eventually an uneven pavement surface.

Hossain et al. (2000) investigated the temperature and moisture effect on backcalculated layer moduli from four asphalt pavement test sections in Kansas, $150 \mathrm{~m}$ long. Most of the sites had silty clay soils as subgrade materials. The subgrade moduli were backcalculated using the elastic layer theory. They concluded that:

- For almost all sites considered, the monthly variation in subgrade moisture content was not very significant over the seasons.

- The plot of subgrade moduli versus time simulated sine-shaped forms signifying a possible temperature effect.

- Extreme test temperatures, both high and low, resulted in high variation of measured deflections and subsequently backcalculated subgrade moduli across a site. Some variabilities in backcalculated subgrade moduli can be avoided by conducting FWD tests in a moderate temperature regime.

- Coefficient of variations between $7 \%$ and $29 \%$ were observed for the backcalculated subgrade moduli, representative of seasonal and spatial variabilities.

Ali et al. (2011) examined the effect of the spatial variability in subgrade stiffness on the performance of flexible pavements (for one pavement structure). The following conclusions were drawn from the analysis results:

- The higher the variability in the subgrade stiffness, the higher is the variability in the pavement response (i.e., subgrade strain).

- The effect of variability in subgrade stiffness is more pronounced on the compressive strain at the top of the subgrade layer than on the tensile strain at the bottom of the asphalt layer. This is expected since the subgrade stiffness was varied, while the asphalt and base layer stiffnesses were kept constant.

- The effect of the spatial variability in subgrade stiffness is more pronounced on the deformation (rutting) life than on the fatigue life of flexible pavements, as predicted using the Asphalt Institute mechanistic models. 
Most research papers on evaluation of spatial and seasonal variability of subgrade stiffness deal with backcalculated subgrade layer moduli using measured deflections (Khogali and Anderson 1996, Wasniak 1999, Hossain et al. 2000, Bayomy and Salem 2005, Ali et al. 2011). Remaining field data such as moisture and density are normally used by researchers to further investigate other pavement structural factors.

The variation in the subgrade moisture content depends on the rainfall amount, the level of the ground water table and the soil type, fine or coarse, plastic or non-plastic, but the detailed dependence is very complicated according to Drumm and Meier 2003 (as cited in Bayomy and Salem 2005). Bayomy and Salem (2005) monitored subgrade modulus variation (with FWD, annually) with moisture content (with moisture sensors, monthly) on 15 FHWA (Federal Highway Administration) -LTPP (Long-Term Pavement Performance) -SMP (Seasonal Monitoring Program) sites. They concluded that:

- The variation of the subgrade modulus and moisture with time followed an inverse function, where the modulus decreased with moisture increase. This result was valid for all soils where the field moisture contents observed were above the optimum. This relationship might change if the field moisture is below optimum. In this case, an increase in the soil moisture may cause an increase in the modulus value as well.

- The data presented showed that the most sensitive soil to moisture variations was a fine silty soil, followed by clay, while coarser soils, like clayey sand, were less sensitive to moisture variations. This would indicate that the seasonal variation in the granular base layers would be minimal.

- The predicted pavement life was overestimated when disregarding the seasonal variations in any of the $A C$ (asphalt) modulus, subgrade modulus and traffic. In general, the seasonal variations in the AC (asphalt) modulus showed more severe impacts on the estimated pavement life.

- The mechanistic analysis performed using elastic layer theory in combination with the developed models to predict the pavement fatigue and rutting lives revealed that the inclusion of seasonal variation in pavement layer moduli resulted in a reduction of pavement service life of 
about $35 \%$ on average (see Figure 7.5). This indicates if an average modulus for each layer is used, instead of varied seasonal moduli values, this will result in premature failure. The configurations considered were: 1) seasonal variation in all of the layer moduli and the traffic, 2) seasonal variation in the traffic and subgrade modulus with constant AC (asphalt) modulus, 3) seasonal variation in the traffic and AC (asphalt) modulus with constant subgrade modulus, and 4) seasonal variation in the layer moduli with uniform traffic.

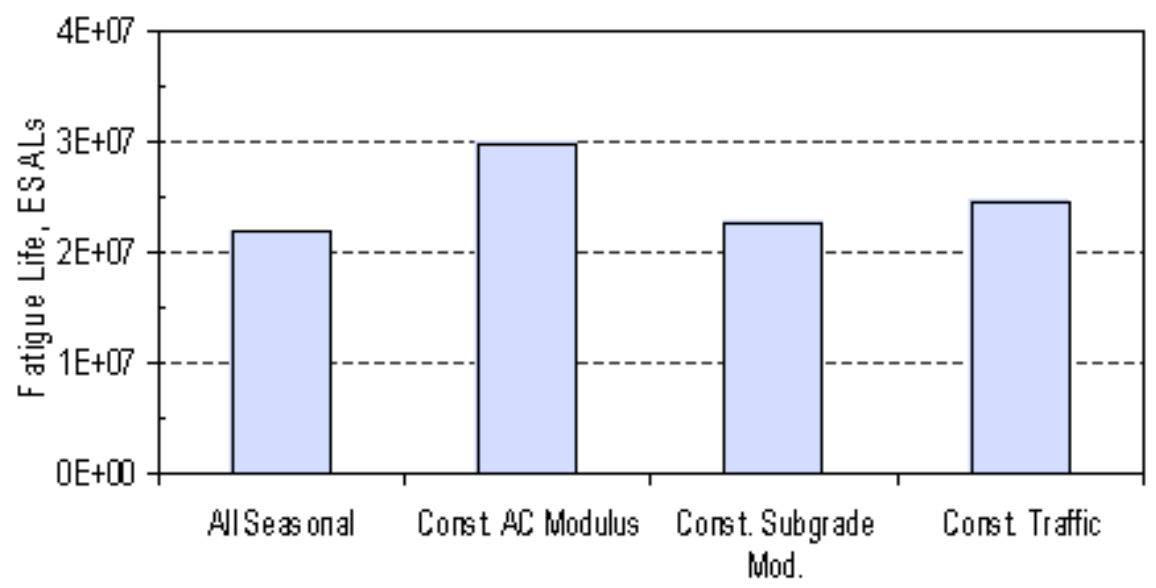

Seas onal Consideration

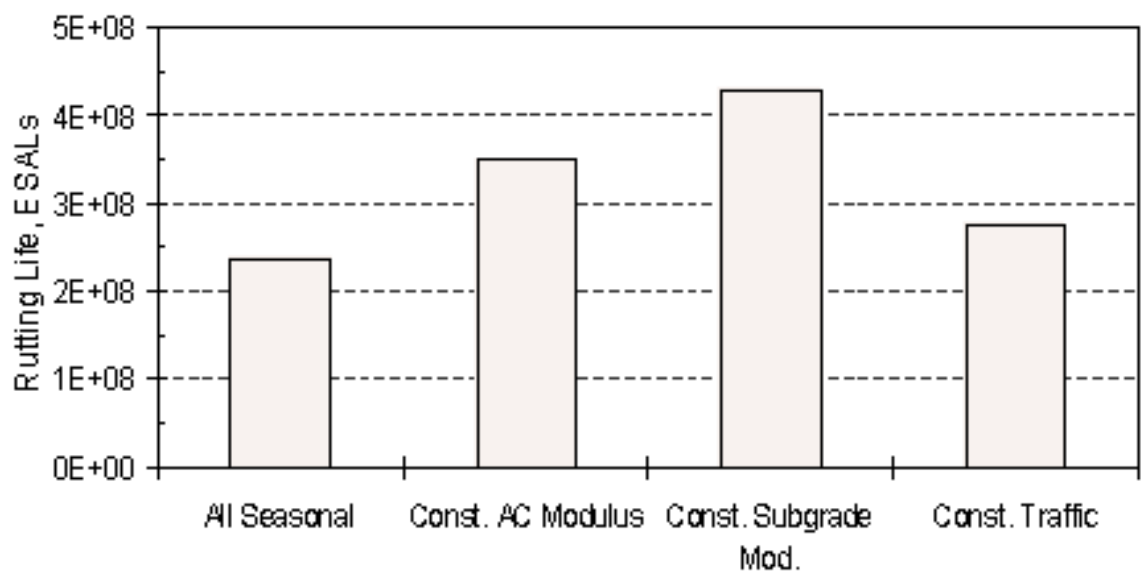

Seasonal Consideration

Figure 7.5 Estimated pavement life (in ESALs) due to fatigue and rutting failures due to seasonal variations (from Bayomy and Salem 2005) 


\subsection{Research methodology to assess subgrade stiffness variability}

To characterise the spatial variability of the subgrade stiffness for the available UK sites, FWD deflections and back-analysed layer stiffnesses were used. Despite DCP tests being available for most sites these were not included in the analysis due to the limited number of tests available.

In order to isolate the 'true' subgrade variability from other sources of variability (i.e., variations in layer thickness), a 'cusum' (cumulative sum of differences) analysis was applied to the FWD central deflection $\left(d_{1}\right)$, to the asphalt layer thickness and to the subbase layer thickness (from GPR surveys); see example for site M01 in Figure 7.6 and Figure 7.7. This statistical technique is used to determine sections with homogeneous characteristics along the length of a test section. The Cusum analysis is typically undertaken on all deflection parameters by subtracting the average deflection parameter from each individual value and then summing the results cumulatively. By plotting the Cusum against chainage, it is possible to highlight changes in pavement characteristics from changes in the gradient (slope) of the plot. In this research, whenever the slope of the cusum plots for either the d1 deflections or the asphalt layer thickness or the subbase layer thickness changed, a new homogeneous section was considered to begin. As a result, a high number of homogenous sections were derived from the cusum analysis. For example, for site M01, a total of 31 homogeneous sections were obtained, most of them less than $100 \mathrm{~m}$ long. While at least 10 thickness values from GPR surveys were available every $100 \mathrm{~m}$ for each site, only 5 deflection values (i.e. FWD deflections collected every $20 \mathrm{~m}$ ) existed. Therefore, sections that were too short to give statistically meaningful data were discarded. Sections with at least 8-10 points were considered, i.e. approximately $200 \mathrm{~m}$ minimum length. For example, for site M01 only 2 out of 31 sections were selected because of the criteria above.

Once the homogeneous sections were identified for each site, a back-analysis was carried out with the asphalt and subbase layer thicknesses derived from the GPR data. For those sections where no GPR subbase thickness was available (i.e. sites M07 and M08), a 300mm layer thickness was used, which 
is typically expected for a subgrade CBR of 3\%. FWD surveys were not available for sites M02 and M03 while for site M06 the homogeneous sections were too short. The back-analysis was carried out with the pavement modelled firstly as a 3-layer structure (i.e., asphalt layer on top of subbase layer and subgrade) and secondly as a 2-layer structure (i.e., asphalt as the top layer and the sub-base combined with the subgrade to form the second layer). The results of the back-analysis for the selected homogenous sections derived from the cusum analysis of all sites is presented in Table 7.2.

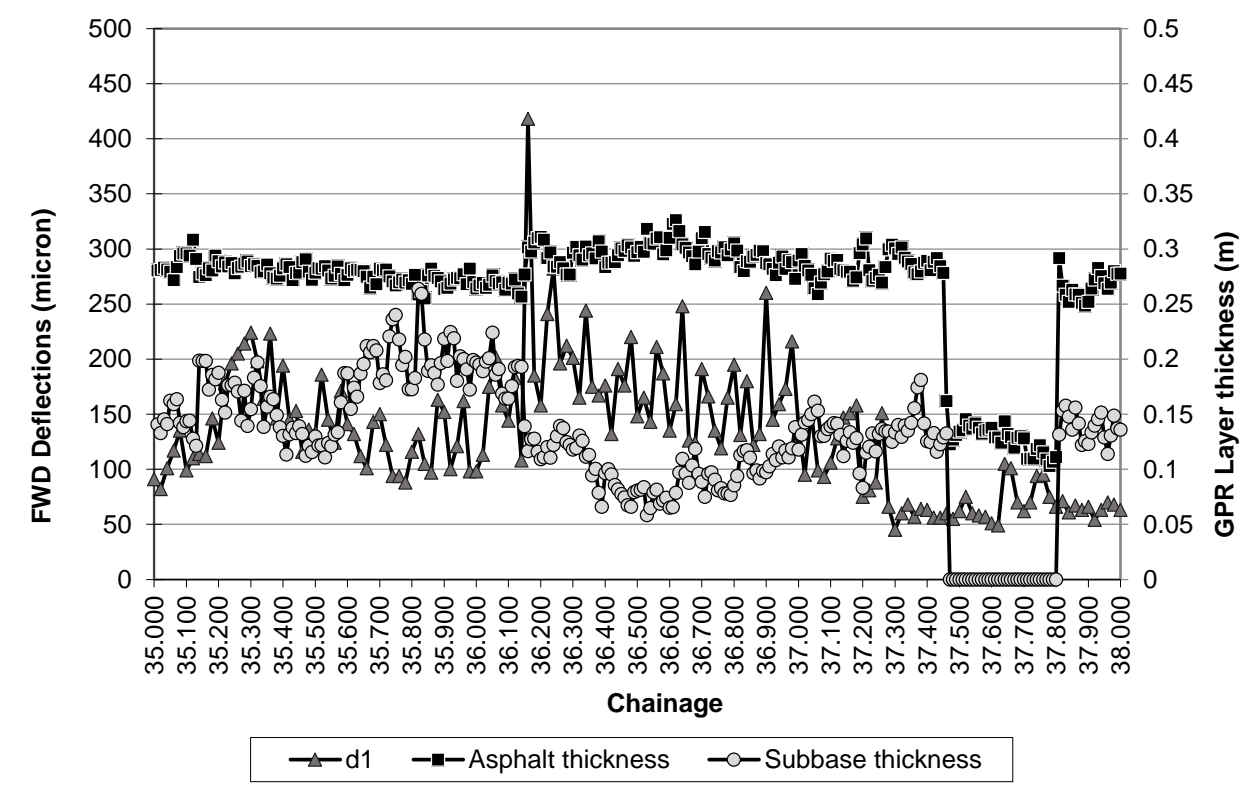

Figure 7.6 FWD and GPR plot for site M01

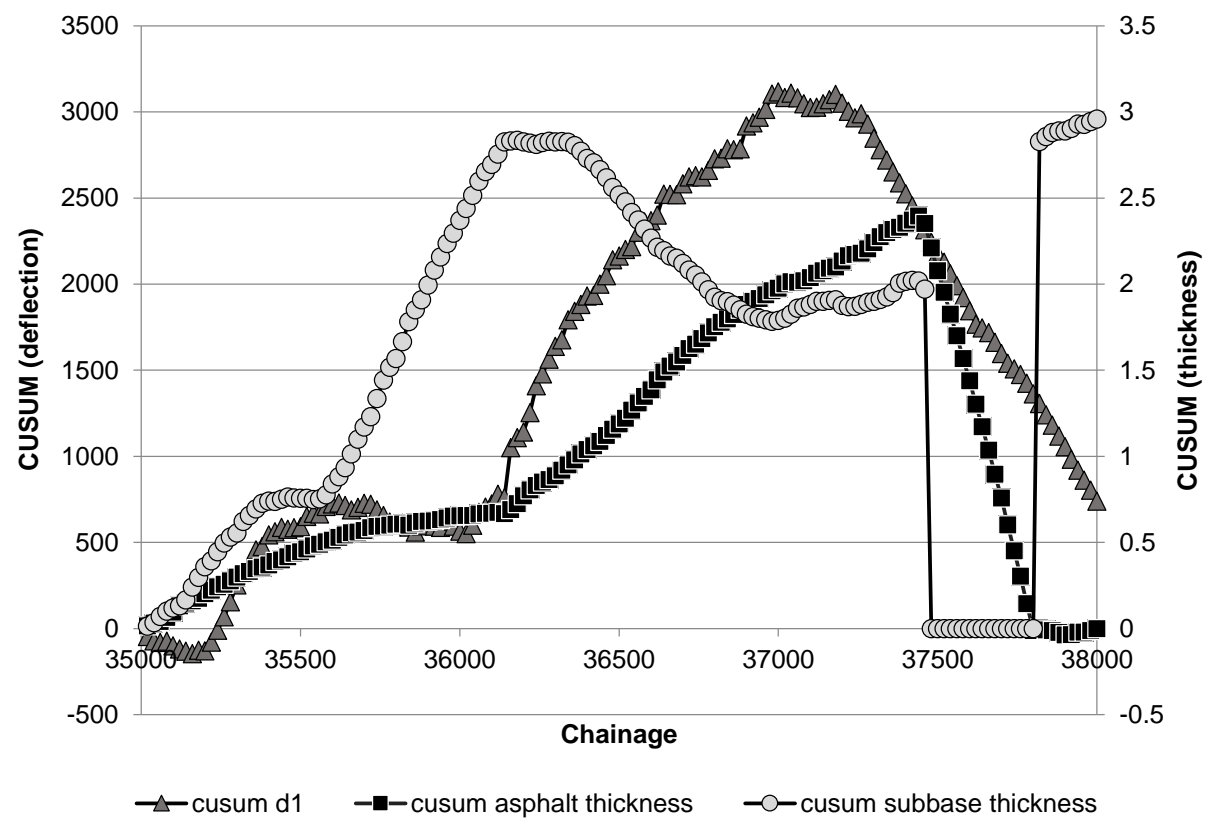

Figure 7.7 Example of Cusum analysis for site M01 
Table 7.2 Subgrade stiffness variability from FWD back-analysis for all sites (for both three- and two-layer pavement structure model)

\begin{tabular}{|c|c|c|c|c|c|c|c|c|c|c|c|}
\hline \multirow[t]{3}{*}{ Length $(m)$} & \multirow[t]{3}{*}{ Site } & \multirow[t]{3}{*}{ Section } & \multirow[t]{3}{*}{ From } & \multirow[t]{3}{*}{ To } & \multicolumn{6}{|c|}{ Back-calculated subgrade stiffness (MPa) } & \multirow{3}{*}{$\begin{array}{l}\text { Probability } \\
\text { distribution }\end{array}$} \\
\hline & & & & & \multicolumn{3}{|c|}{$\begin{array}{l}\text { Pavement modelled as } \\
\text { 3-L structure }\end{array}$} & \multicolumn{3}{|c|}{$\begin{array}{l}\text { Pavement modelled as } \\
\text { 2-L structure }\end{array}$} & \\
\hline & & & & & $\mu$ & $\bar{\sigma}$ & $\begin{array}{l}\text { COV } \\
(\%)\end{array}$ & $\bar{\mu}$ & $\bar{\sigma}$ & $\begin{array}{l}\text { COV } \\
(\%)\end{array}$ & \\
\hline 220 & M01 & 3 & 35160 & 35380 & 227 & 68 & 29.8 & 201 & 38 & 18.9 & Normal \\
\hline 320 & M01 & 16 & 36300 & 36620 & 199 & 34 & 17.0 & 183 & 20 & 11.2 & Lognormal \\
\hline 200 & M04 & 1 & 0 & 200 & 113 & 66 & 58.1 & 173 & 189 & 109.6 & Lognormal \\
\hline 375 & M04 & 10 & 875 & 1250 & 191 & 33 & 17.0 & 172 & 13 & 7.8 & Lognormal \\
\hline 250 & M04 & 20 & 1975 & 2225 & 257 & 21 & 8.1 & 238 & 44 & 18.5 & Lognormal \\
\hline 275 & M04 & 30 & 2675 & 2950 & 265 & 30 & 11.2 & 222 & 24 & 10.9 & Normal \\
\hline 200 & M04 & 37 & 3325 & 3525 & 182 & 42 & 22.9 & 160 & 23 & 14.3 & Lognormal \\
\hline 370 & M05 ACW & 10 & 460 & 830 & 184 & 32 & 17.4 & 197 & 34 & 17.4 & Normal \\
\hline 550 & M05 ACW & 11 & 830 & 1380 & 157 & 13 & 8.4 & 169 & 14 & 8.5 & Normal \\
\hline 250 & M05 CW & 4 & 350 & 600 & 134 & 24 & 17.7 & 121 & 19 & 15.7 & Lognormal \\
\hline 240 & M05 CW & 42 & 2270 & 2510 & 201 & 45 & 22.5 & 199 & 34 & 17.1 & Lognormal \\
\hline 340 & M07 NB & 2 & 100 & 440 & 137 & 41 & 29.6 & 131 & 41 & 31.0 & Lognormal \\
\hline 200 & M07 NB & 3 & 440 & 640 & 110 & 18 & 16.5 & 110 & 14 & 13.1 & Lognormal \\
\hline 620 & M07 NB & 4 & 640 & 1260 & 157 & 32 & 20.3 & 150 & 13 & 9.0 & Lognormal \\
\hline 340 & M07 NB & 5 & 1260 & 1600 & 187 & 44 & 23.3 & 151 & 23 & 15.6 & Lognormal \\
\hline 200 & M07 NB & 6 & 1600 & 1800 & 189 & 46 & 24.2 & 149 & 20 & 13.6 & Normal \\
\hline 180 & M07 SB & 3 & 100 & 280 & 176 & 37 & 20.9 & 161 & 28 & 17.6 & Lognormal \\
\hline 560 & M07 SB & 7 & 720 & 1280 & 156 & 19 & 12.0 & 150 & 19 & 12.8 & Lognormal \\
\hline 220 & M07 SB & 8 & 1280 & 1500 & 196 & 49 & 25.0 & 165 & 24 & 14.6 & Lognormal \\
\hline 300 & M07 SB & 12 & 1640 & 1940 & 210 & 51 & 24.4 & 149 & 21 & 14.2 & Lognormal \\
\hline 300 & M08 EB & 4 & 180 & 480 & 186 & 193 & 104.0 & 166 & 150 & 90.2 & Lognormal \\
\hline 180 & M08 EB & 6 & 640 & 820 & 339 & 308 & 90.8 & 287 & 239 & 83.1 & Lognormal \\
\hline 240 & M08 WB & 2 & 100 & 340 & 373 & 300 & 80.5 & 257 & 167 & 65.1 & Lognormal \\
\hline \multirow[t]{5}{*}{400} & M08 WB & 5 & 920 & 1320 & 144 & 186 & 129.1 & 124 & 154 & 123.6 & Lognormal \\
\hline & & & & Avg & \multicolumn{2}{|c|}{ M01 to M08 } & 56.4 & \multicolumn{2}{|c|}{ M01 to M08 } & 49.7 & \multirow{4}{*}{ Lognormal } \\
\hline & & & & Avg & \multicolumn{2}{|c|}{ M01 to M07* } & 20.5 & \multicolumn{2}{|c|}{ M01 to M07* } & 15.8 & \\
\hline & & & & Min & \multicolumn{2}{|c|}{ M01 to M07* } & 8.1 & \multicolumn{2}{|c|}{$\mathrm{M} 01$ to $\mathrm{M} 07^{*}$} & 7.8 & \\
\hline & & & & Max & \multicolumn{2}{|c|}{$\mathrm{M} 01$ to $\mathrm{M} 07^{\star}$} & 29.8 & \multicolumn{2}{|c|}{$\mathrm{M} 01$ to $\mathrm{M}^{*} 7^{*}$} & 31.0 & \\
\hline
\end{tabular}

The table shows the back-analysed subgrade stiffness in MPa for each homogeneous section in terms of mean $(\mu)$, standard deviation $(\sigma)$ and coefficient of variation (COV, \%) and for both the three- and two-layer pavement structure models used in the back-analysis. The table also shows the probability distribution that best fits the data. This has been achieved in Matlab by displaying first a quantile-quantile (Q_Q) plot of the section stiffness values versus theoretical quantiles from a normal and lognormal distribution and second by choosing the distribution which gives the plot closer to linear. Quantiles as well as percentiles focus on positions in the sorted list of data. 
Quantiles are the decimal version of a percentile. For example, the 50th percentile is the same as the 0.50 quantile.

The following observations are made:

- The high coefficients of variation particularly for site M08 are probably influenced by the thin asphalt layer.

- For the three-layer pavement structure model, the coefficient of variation of the back-analysed subgrade stiffness modulus for almost all sites varied from a minimum of $8 \%$ to a maximum of $30 \%$, with an average value of $20 \%$.

- For the two-layer pavement structure model, the coefficient of variation of the back-analysed subgrade stiffness modulus for almost all sites varied from a minimum of $8 \%$ to a maximum of $30 \%$, with an average value of $15 \%$.

- A lognormal probability distribution is found in most cases to be representative of the subgrade stiffness.

- The coefficient of variation of the back-analysed subgrade stiffness modulus derived by modelling the pavement as a two-layer structure was in most cases lower than the corresponding value of the three-layer structure.

The difference in back-analysis results depending on number of layers used for the pavement structure will be explored in more detail in Chapter 8.

\subsection{Sensitivity analysis}

A sensitivity analysis was carried out at this point on each homogeneous section to assess which parameter(s) have the greater influence on the pavement life. While the focus was on the effect of subgrade stiffness modulus variability on pavement life, variations in asphalt and subbase layer thicknesses and moduli were employed to get a comparative view of the importance of the subgrade.

The basic model was a three-layer pavement structure (asphalt layer with thickness $T_{a}$ and stiffness $E_{a}$, overlying a subbase layer with thickness $T_{s b}$ and 
stiffness $E_{s b}$ on top of subgrade with stiffness $E_{s g}$ ). The analysis consisted in calculating with BISAR, for each homogeneous section, the asphalt and subgrade strains for each of the following cases:

- Base case where all parameters $\left(E_{a}, T_{a}, E_{s b}, T_{s b}\right.$, and $\left.E_{s g}\right)$ are constant, i.e. with their mean values $(\mu)$;

- The 15th percentile of the parameter of interest is considered, taken from real data, while others are constant.

- The 85th percentile of the parameter of interest is considered, taken from real data, while others are constant.

- A total for 11 pavement structures were considered for each homogeneous section.

- The fatigue and deformation lives were then estimated from the calculated asphalt and subgrade strains with the equations contained in LR 1132 (Powell et al. 1984), also reported in section 4.5.3.

It is noted that for a normal probability distribution, the $15^{\text {th }}$ percentile is approximately equal to the mean minus one standard deviation. Similarly, the $85^{\text {th }}$ percentile is approximately equal to the mean plus one standard deviation. As the lognormal distribution is a probability distribution whose logarithm has a normal distribution, the $15^{\text {th }}$ and $85^{\text {th }}$ percentiles can be calculated as above but by using instead the mean and standard deviation of the logarithms.

While percentiles from real data were used for $T_{a}, E_{s b}, T_{s b}$, and $E_{s g}$, for $E_{a} a$ different procedure was employed. Corrections were made to each section's back-analysed stiffness value to account for the section's asphalt layer thickness variability (see Collop 2000 and Chapter 6). For the asphalt stiffness values, the $15^{\text {th }}$ and $85^{\text {th }}$ percentiles were calculated from the mean and standard deviation of the logarithms with the following procedure:

- Once the section's back-analysed asphalt stiffness mean $\left(\mu_{E_{F W D}}\right)$ and standard deviation $\left(\sigma_{E_{F W D}}\right)$ were calculated, Equation (9) and Equation (10) given in the reference (Collop, 2000) were used to predict the FWD stiffness modulus mean error and the FWD stiffness modulus standard deviation error respectively. The errors are termed $\varepsilon_{\text {mean }}$ and $\varepsilon_{\text {sd }}$ here for 
simplicity. The true section's asphalt stiffness mean $(\bar{E})$ and standard deviation $\left(\sigma_{\Delta E}\right)$ were then calculated (i.e $\bar{E}=\mu_{E_{F W D}} * \varepsilon_{\text {mean }}$ and $\sigma_{\Delta E}=$ $\left.\sigma_{E_{F W D}} * \varepsilon_{S d}\right)$.

- The mean of the logarithms of the individual (and corrected for asphalt thickness variability) asphalt stiffness values was calculated, which is $m=$ average $\left[\ln \left(E_{F W D_{i}} * \varepsilon_{\text {mean }}\right)\right]$, averaged over $\mathrm{n}$ number of data points, and where $E_{F W D_{i}}$ is the individual (uncorrected) asphalt stiffness value.

- The standard deviation of the logarithms of the individual asphalt stiffness values was calculated (and corrected for asphalt thickness variability), which is $s d=\operatorname{stdev}\left[\ln \left(E_{F W D_{i}}\right)\right] * \varepsilon_{s d}$.

- The $15^{\text {th }}$ percentile was calculated as $e^{(m-s d)}$ and the $85^{\text {th }}$ percentile was calculated as $e^{(m+s d)}$.

The ratio of the calculated fatigue $\left(\mathrm{N}_{\mathrm{f}}\right)$ and deformation life $\left(\mathrm{N}_{\mathrm{d}}\right)$ over the corresponding base case was calculated for each scenario. The mean and standard deviation, the $15^{\text {th }} \& 85^{\text {th }}$ percentiles of the design input parameters for all homogeneous sections are shown in Table 7.3. The results of the analysis are shown in Table 7.4 to Table 7.8. Despite the high uncertainty of the subbase stiffness and subgrade stiffness observed in some sites, for the purpose of this sensitivity analysis all sections were included.

A summary of the analysis for all sites, based on average values, is presented in Table 7.9 in tabular format and in Figure 7.8 in graphical format. 
Table 7.3 Mean \& standard deviation, 15th \& 85th percentiles of the design input parameters for all homogeneous sections

\begin{tabular}{|c|c|c|c|c|c|c|c|c|c|c|c|c|c|c|c|c|c|c|c|c|c|}
\hline \multirow[t]{2}{*}{ Site } & \multirow[t]{2}{*}{ Section } & \multicolumn{4}{|c|}{ Asphalt thickness } & \multicolumn{4}{|c|}{ Asphalt stiffness } & \multicolumn{4}{|c|}{ Subbase thickness } & \multicolumn{4}{|c|}{ Subbase stiffness } & \multicolumn{4}{|c|}{ Subgrade stiffness } \\
\hline & & $\begin{array}{l}\text { Mean } \\
(\mathrm{m})\end{array}$ & $\begin{array}{l}\text { Stdev } \\
(\mathrm{m})\end{array}$ & $\begin{array}{l}15 \text { th } \\
\text { perc }\end{array}$ & $\begin{array}{l}\text { 85th } \\
\text { perc }\end{array}$ & $\begin{array}{l}\text { Mean } \\
(\mathrm{MPa})\end{array}$ & $\begin{array}{l}\text { Stdev } \\
(\mathrm{MPa})\end{array}$ & $\begin{array}{l}15 \text { th } \\
\text { perc }\end{array}$ & $\begin{array}{l}\text { 85th } \\
\text { perc }\end{array}$ & \begin{tabular}{|l} 
Mean \\
$(\mathrm{m})$
\end{tabular} & \begin{tabular}{|l|} 
Stdev \\
$(\mathrm{m})$
\end{tabular} & $\begin{array}{l}15 \text { th } \\
\text { perc }\end{array}$ & $\begin{array}{l}\text { 85th } \\
\text { perc }\end{array}$ & $\begin{array}{l}\text { Mean } \\
(\mathrm{MPa})\end{array}$ & $\begin{array}{l}\text { Stdev } \\
\text { (MPa) }\end{array}$ & $\begin{array}{l}15 \text { th } \\
\text { perc }\end{array}$ & $\begin{array}{l}\text { 85th } \\
\text { perc }\end{array}$ & $\begin{array}{l}\text { Mean } \\
(\mathrm{MPa})\end{array}$ & $\begin{array}{l}\text { Stdev } \\
\text { (MPa) }\end{array}$ & $\begin{array}{l}\text { 15th } \\
\text { perc }\end{array}$ & $\begin{array}{l}\text { 85th } \\
\text { perc }\end{array}$ \\
\hline M01 & 3 & 0.284 & 0.004 & 0.280 & 0.286 & 4060 & 1423 & 2709 & 5440 & 0.171 & 0.016 & 0.157 & 0.187 & 345 & 398 & 42 & 872 & 227 & 68 & 155 & 311 \\
\hline M01 & 16 & 0.298 & .007 & 0.291 & 0.304 & 4731 & 716 & 3127 & 6340 & .088 & 0.020 & 0.070 & 0.115 & 83 & 59 & 28 & 158 & 199 & 34 & 163 & 236 \\
\hline M04 & 1 & 0.251 & .043 & 0.218 & 0.304 & 6406 & 570 & 4088 & 9833 & .166 & .045 & .121 & 0.204 & 504 & 479 & 74 & 925 & 113 & 66 & 48 & 136 \\
\hline M04 & 10 & 0.452 & 0.016 & 0.440 & 0.471 & 8105 & 1366 & 6729 & 9448 & 0.205 & 0.022 & 0.173 & 0.223 & 95 & 66 & 28 & 165 & 191 & 33 & 169 & 218 \\
\hline M04 & 20 & 0.449 & 0.010 & 0.438 & 0.456 & 7671 & 1016 & 6696 & 8637 & 0.257 & 0.031 & 0.229 & 0.286 & 401 & 379 & 29 & 907 & 257 & 21 & 241 & 275 \\
\hline M04 & 30 & 0.437 & 0.013 & 0.423 & 0.450 & 7875 & 1225 & 6685 & 9047 & 0.156 & 0.039 & 0.126 & 0.192 & 77 & 93 & 29 & 160 & 265 & 30 & 234 & 299 \\
\hline M04 & 37 & 0.443 & 0.008 & 0.437 & 0.453 & 7404 & 1114 & 6411 & 8396 & 0.147 & 0.013 & 0.130 & 0.160 & 60 & 45 & 28 & 103 & 182 & 42 & 148 & 210 \\
\hline M05 ACW & 0 & 0.302 & 0.015 & 0.287 & 0.319 & 5780 & 1100 & 4680 & 6811 & 0.218 & 0.006 & 0.213 & 0.225 & 934 & 183 & 945 & 1000 & 184 & 32 & 135 & 212 \\
\hline M05 ACW & 11 & 0 & 1 & 0.307 & 1 & 5631 & 7 & 2 & 6418 & 6 & 05 & & 0.220 & & 185 & 695 & 00 & 157 & & 46 & 68 \\
\hline M05 CW & 4 & 0.333 & 0.013 & 0.316 & 0.343 & 2894 & 401 & 2488 & 3282 & 0.195 & 0.047 & 0.150 & 0.248 & 71 & 55 & 28 & 100 & 134 & 24 & 109 & 163 \\
\hline M05 CW & 42 & 0.340 & 0.014 & 0.324 & 0.351 & 8106 & 2014 & 5996 & 10182 & 0.159 & 0.036 & 0.125 & 0.209 & 748 & 406 & 98 & 1000 & 201 & 45 & 168 & 238 \\
\hline M07 NB & 2 & 0.143 & 0.018 & 0.124 & 0.166 & 2725 & 2261 & 1122 & 3957 & 0.300 & 0.064 & 0.236 & 0.364 & 145 & 105 & 69 & 210 & 137 & 41 & 86 & 167 \\
\hline M07 NB & 3 & 0 & 0.020 & 0.101 & 0.136 & 1 & 1334 & 1227 & 4638 & 00 & 0.063 & 7 & 0.363 & 101 & 4 & 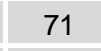 & 32 & 110 & 10 & 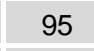 & 128 \\
\hline M07 NB & 4 & 0.191 & 0.025 & 0.170 & 0.215 & 7837 & 2256 & 4467 & 10394 & 0.300 & 0.063 & 0.237 & 0.363 & 227 & 216 & & 392 & 157 & 32 & 137 & 166 \\
\hline M07 NB & 5 & 0.255 & 0.019 & 0.244 & 0.271 & 5657 & 1813 & 3585 & 7639 & 0.300 & 0.065 & 0.235 & 0.365 & 57 & 23 & 35 & 68 & 187 & 44 & 152 & 245 \\
\hline M07 NB & 6 & 0.230 & 0.008 & 0.222 & 0.236 & 3346 & 848 & 2517 & 4176 & 0.300 & 0.062 & 0.238 & 0.362 & 73 & 71 & 34 & 69 & 189 & 46 & 158 & 222 \\
\hline M07 SB & 3 & 0.148 & 0.013 & 0.136 & 0.154 & 2098 & 1161 & 921 & 3273 & 0.300 & 0.064 & 0.236 & 0.364 & 232 & 292 & 98 & 234 & 176 & 37 & 152 & 222 \\
\hline M07 SB & 7 & 0.189 & 0.011 & 0.177 & 0.198 & 9254 & 2575 & 6076 & 12405 & 0.300 & 0.060 & 0.240 & 0.360 & 159 & 124 & 03 & 252 & 156 & 19 & 139 & 171 \\
\hline M07 SB & 8 & 0.257 & 0.009 & 0.249 & 0.265 & 6201 & 2298 & 3296 & 9467 & 0.300 & 0.056 & 0.244 & 0.356 & 91 & 55 & 42 & 160 & 196 & 49 & 161 & 225 \\
\hline M07 SB & 12 & 0.235 & 0.008 & 0.227 & 0.244 & 3504 & 1134 & 2309 & 4731 & 0.300 & 0.059 & 0.241 & 0.359 & 56 & 34 & 31 & 87 & 210 & 51 & 173 & 250 \\
\hline M08 EB & 4 & 0.245 & 0.017 & 0.234 & 0.259 & 1206 & 355 & 813 & 1583 & 0.300 & 0.063 & 0.237 & 0.363 & 121 & 91 & 30 & 198 & 186 & 193 & 66 & 240 \\
\hline M08 EB & 6 & 0.255 & 0.010 & 0.244 & 0.265 & 1454 & 227 & 1214 & 1687 & 0.300 & 0.061 & 0.239 & 0.361 & 193 & 85 & 137 & 259 & 339 & 308 & 67 & 680 \\
\hline M08 WB & 2 & 0.243 & 0.026 & 0.232 & 0.259 & 4140 & 1680 & 1930 & 6283 & 0.300 & 0.061 & 0.239 & 0.361 & 83 & 40 & 44 & 115 & 373 & 300 & 89 & 556 \\
\hline M08 WB & 5 & 0.181 & 0.031 & 0.151 & 0.208 & 1331 & 408 & 882 & 1577 & 0.300 & 0.060 & 0.240 & 0.360 & 61 & 71 & 28 & 78 & 144 & 186 & 55 & 131 \\
\hline
\end{tabular}


Table 7.4 Asphalt stiffness $15^{\text {th }}$ and $85^{\text {th }}$ percentiles - Ratio of fatigue $\left(\mathrm{N}_{\mathrm{f}}\right)$ and deformation life $\left(\mathrm{N}_{\mathrm{d}}\right)$ over base case

\begin{tabular}{|c|c|c|c|c|c|c|c|c|c|c|c|}
\hline \multicolumn{5}{|c|}{$\begin{array}{l}\text { Pavement modelled as three-layer } \\
\text { structure }\end{array}$} & \multicolumn{3}{|c|}{ Asphalt stiffness } & \multicolumn{2}{|c|}{$\begin{array}{l}\text { 15th percentile Ea; life } \\
\text { ratio over base scenario }\end{array}$} & \multicolumn{2}{|c|}{$\begin{array}{l}\text { 85th percentile Ea; life } \\
\text { ratio over base scenario }\end{array}$} \\
\hline $\begin{array}{l}\text { Length } \\
\text { (m) }\end{array}$ & Site & Section & From & To & $\begin{array}{l}\text { Mean } \\
(\mathrm{MPa})\end{array}$ & $\begin{array}{l}\text { Stdev } \\
\text { (MPa) }\end{array}$ & COV (\%) & Nf1132 & Nd1132 & Nf1132 & Nd1132 \\
\hline 220 & M01 & 3 & 35160 & 35380 & 4060 & 1423 & 35.06 & 0.36 & 0.47 & 2.24 & 1.80 \\
\hline 320 & M01 & 16 & 36300 & 36620 & 4730 & 1716 & 36.28 & 0.27 & 0.38 & 2.61 & 2.01 \\
\hline 200 & M04 & 1 & 0 & 200 & 6406 & 3570 & 55.74 & 0.33 & 0.42 & 3.27 & 2.48 \\
\hline 375 & M04 & 10 & 875 & 1250 & 8105 & 1366 & 16.85 & 0.53 & 0.64 & 1.69 & 1.45 \\
\hline 250 & M04 & 20 & 1975 & 2225 & 7671 & 1016 & 13.25 & 0.67 & 0.75 & 1.44 & 1.30 \\
\hline 275 & M04 & 30 & 2675 & 2950 & 7875 & 1225 & 15.56 & 0.57 & 0.68 & 1.61 & 1.40 \\
\hline 200 & M04 & 37 & 3325 & 3525 & 7404 & 1114 & 15.05 & 0.61 & 0.70 & 1.55 & 1.35 \\
\hline 370 & M05 ACW & 10 & 460 & 830 & 5780 & 1100 & 19.04 & 0.65 & 0.70 & 1.43 & 1.34 \\
\hline 550 & M05 ACW & 11 & 830 & 1380 & 5631 & 807 & 14.34 & 0.72 & 0.76 & 1.34 & 1.27 \\
\hline 250 & M05 CW & 4 & 350 & 600 & 2894 & 401 & 13.86 & 0.61 & 0.72 & 1.51 & 1.32 \\
\hline 240 & M05 CW & 42 & 2270 & 2510 & 8106 & 2014 & 24.85 & 0.46 & 0.53 & 1.88 & 1.66 \\
\hline 340 & M07 NB & 2 & 100 & 440 & 2730 & 2261 & 82.82 & 0.14 & 0.37 & 2.62 & 1.64 \\
\hline 200 & M07 NB & 3 & 440 & 640 & 3281 & 1334 & 40.67 & 0.15 & 0.39 & 2.29 & 1.51 \\
\hline 620 & M07 NB & 4 & 640 & 1260 & 7840 & 2256 & 28.78 & 0.20 & 0.40 & 2.33 & 1.64 \\
\hline 340 & M07 NB & 5 & 1260 & 1600 & 5660 & 1813 & 32.04 & 0.21 & 0.40 & 2.80 & 1.85 \\
\hline 200 & M07 NB & 6 & 1600 & 1800 & 3350 & 848 & 25.30 & 0.40 & 0.60 & 2.04 & 1.50 \\
\hline 180 & M07 SB & 3 & 100 & 280 & 2100 & 1161 & 55.26 & 0.24 & 0.47 & 2.65 & 1.65 \\
\hline 560 & M07 SB & 7 & 720 & 1280 & 9250 & 2575 & 27.84 & 0.27 & 0.47 & 2.54 & 1.74 \\
\hline 220 & M07 SB & 8 & 1280 & 1500 & 6200 & 2298 & 37.06 & 0.13 & 0.30 & 4.16 & 2.37 \\
\hline 300 & M07 SB & 12 & 1640 & 1940 & 3504 & 1134 & 32.37 & 0.26 & 0.46 & 2.72 & 1.78 \\
\hline 300 & M08 EB & 4 & 180 & 480 & 1210 & 355 & 29.31 & 0.38 & 0.58 & 2.04 & 1.49 \\
\hline 180 & M08 EB & 6 & 640 & 820 & 1450 & 227 & 15.63 & 0.66 & 0.79 & 1.46 & 1.23 \\
\hline 240 & M08 WB & 2 & 100 & 340 & 4140 & 1680 & 40.57 & 0.09 & 0.26 & 4.00 & 2.20 \\
\hline \multirow[t]{4}{*}{400} & M08 WB & 5 & 920 & 1320 & 1331 & 408 & 30.64 & 0.32 & 0.56 & 1.63 & 1.29 \\
\hline & & & & Avg & \multicolumn{2}{|c|}{ M01 to M05 } & 26.21 & 0.50 & 0.60 & 1.80 & 1.54 \\
\hline & & & & Avg & \multicolumn{2}{|c|}{ M07 to M08 } & 40.22 & 0.23 & 0.45 & 2.46 & 1.66 \\
\hline & & & & Avg & \multicolumn{2}{|c|}{ M01 to M08 } & 32.25 & 0.33 & 0.51 & 2.13 & 1.60 \\
\hline
\end{tabular}


Table 7.5 Asphalt thickness $15^{\text {th }}$ and $85^{\text {th }}$ percentiles - Ratio of fatigue $\left(\mathrm{N}_{\mathrm{f}}\right)$ and deformation life $\left(\mathrm{N}_{\mathrm{d}}\right)$ over base case

\begin{tabular}{|c|c|c|c|c|c|c|c|c|c|c|c|}
\hline \multicolumn{5}{|c|}{$\begin{array}{l}\text { Pavement modelled as three-layer } \\
\text { structure }\end{array}$} & \multicolumn{3}{|c|}{ Asphalt thickness } & \multicolumn{2}{|c|}{$\begin{array}{l}15 \text { th percentile Ta; life } \\
\text { ratio over base scenario }\end{array}$} & \multicolumn{2}{|c|}{$\begin{array}{l}\text { 85th percentile Ta; life } \\
\text { ratio over base scenario }\end{array}$} \\
\hline $\begin{array}{l}\text { Length } \\
\text { (m) }\end{array}$ & Site & Section & From & To & $\begin{array}{l}\text { Mean } \\
(\mathrm{m})\end{array}$ & $\begin{array}{l}\text { Stdev } \\
(\mathrm{m})\end{array}$ & COV (\%) & Nf1132 & Nd1132 & Nf1132 & Nd1132 \\
\hline 220 & M01 & 3 & 35160 & 35380 & 0.284 & 0.004 & 1.48 & 0.92 & 0.92 & 1.04 & 1.04 \\
\hline 320 & M01 & 16 & 36300 & 36620 & 0.298 & 0.007 & 2.33 & 0.85 & 0.86 & 1.15 & 1.13 \\
\hline 200 & M04 & 1 & 0 & 200 & 0.251 & 0.043 & 17.14 & 0.46 & 0.45 & 3.19 & 3.15 \\
\hline 375 & M04 & 10 & 875 & 1250 & 0.452 & 0.016 & 3.48 & 0.81 & 0.85 & 1.37 & 1.30 \\
\hline 250 & M04 & 20 & 1975 & 2225 & 0.449 & 0.010 & 2.26 & 0.83 & 0.86 & 1.12 & 1.10 \\
\hline 275 & M04 & 30 & 2675 & 2950 & 0.437 & 0.013 & 2.98 & 0.78 & 0.81 & 1.25 & 11.38 \\
\hline 200 & M04 & 37 & 3325 & 3525 & 0.443 & 0.008 & 1.81 & 0.90 & 0.91 & 1.19 & 1.15 \\
\hline 370 & M05 ACW & 10 & 460 & 830 & 0.302 & 0.015 & 4.89 & 0.75 & 0.76 & 1.38 & 1.34 \\
\hline 550 & M05 ACW & 11 & 830 & 1380 & 0.319 & 0.011 & 3.42 & 0.80 & 0.81 & 1.25 & 1.23 \\
\hline 250 & M05 CW & 4 & 350 & 600 & 0.333 & 0.013 & 3.79 & 0.69 & 0.73 & 1.23 & 1.19 \\
\hline 240 & M05 CW & 42 & 2270 & 2510 & 0.340 & 0.014 & 4.06 & 0.73 & 0.74 & 1.23 & 1.22 \\
\hline 340 & M07 NB & 2 & 100 & 440 & 0.143 & 0.018 & 12.62 & 0.57 & 0.57 & 1.95 & 1.89 \\
\hline 200 & M07 NB & 3 & 440 & 640 & 0.116 & 0.020 & 17.54 & 0.63 & 0.61 & 1.84 & 1.88 \\
\hline 620 & M07 NB & 4 & 640 & 1260 & 0.191 & 0.025 & 13.10 & 0.54 & 0.55 & 1.96 & 1.87 \\
\hline 340 & M07 NB & 5 & 1260 & 1600 & 0.255 & 0.019 & 7.26 & 0.74 & 0.78 & 1.51 & 1.42 \\
\hline 200 & M07 NB & 6 & 1600 & 1800 & 0.230 & 0.008 & 3.54 & 0.80 & 0.83 & 1.18 & 1.15 \\
\hline 180 & M07 SB & 3 & 100 & 280 & 0.148 & 0.013 & 8.49 & 0.75 & 0.74 & 1.16 & 1.16 \\
\hline 560 & M07 SB & 7 & 720 & 1280 & 0.189 & 0.011 & 6.00 & 0.69 & 0.70 & 1.31 & 1.29 \\
\hline 220 & M07 SB & 8 & 1280 & 1500 & 0.257 & 0.009 & 3.52 & 0.81 & 0.84 & 1.23 & 1.19 \\
\hline 300 & M07 SB & 12 & 1640 & 1940 & 0.235 & 0.008 & 3.60 & 0.80 & 0.83 & 1.28 & 1.22 \\
\hline 300 & M08 EB & 4 & 180 & 480 & 0.245 & 0.017 & 6.78 & 0.77 & 0.82 & 1.39 & 1.28 \\
\hline 180 & M08 EB & 6 & 640 & 820 & 0.255 & 0.010 & 3.98 & 0.78 & 0.83 & 1.25 & 1.18 \\
\hline 240 & M08 WB & 2 & 100 & 340 & 0.243 & 0.026 & 10.50 & 0.74 & 0.79 & 1.52 & 1.40 \\
\hline \multirow[t]{4}{*}{400} & M08 WB & 5 & 920 & 1320 & 0.181 & 0.031 & 17.19 & 0.41 & 0.46 & 2.14 & 1.89 \\
\hline & & & & Avg & \multicolumn{2}{|c|}{ M01 to M05 } & 4.79 & 0.76 & 0.78 & 1.33 & 1.59 \\
\hline & & & & Avg & \multicolumn{2}{|c|}{ M07 to M08 } & 8.71 & 0.68 & 0.71 & 1.48 & 1.42 \\
\hline & & & & Avg & \multicolumn{2}{|c|}{ M01 to M08 } & 6.39 & 0.72 & 0.74 & 1.41 & 1.49 \\
\hline
\end{tabular}

Table 7.6 Subbase stiffness 15 th and 85th percentiles - Ratio of fatigue $\left(\mathrm{N}_{\mathrm{t}}\right)$ and deformation life $\left(\mathrm{N}_{\mathrm{d}}\right)$ over base case

\begin{tabular}{|c|c|c|c|c|c|c|c|c|c|c|c|c|c|}
\hline \multicolumn{5}{|c|}{$\begin{array}{l}\text { Pavement modelled as three-layer } \\
\text { structure }\end{array}$} & \multicolumn{3}{|c|}{ Subbase stiffness } & \multicolumn{2}{|c|}{$\begin{array}{l}\text { 15th percentile Esb; life } \\
\text { ratio over base scenario }\end{array}$} & \multicolumn{2}{|c|}{$\begin{array}{l}\text { 85th percentile Esb; life } \\
\text { ratio over base scenario }\end{array}$} & \multicolumn{2}{|c|}{ Comments } \\
\hline $\begin{array}{l}\text { Length } \\
\text { (m) }\end{array}$ & Site & Section & From & To & $\begin{array}{l}\text { Mean } \\
\text { (MPa) }\end{array}$ & $\begin{array}{l}\text { Stdev } \\
(\mathrm{MPa})\end{array}$ & COV (\%) & Nf1132 & $\overline{N d 1132}$ & Nf1132 & Nd1132 & $\frac{E_{s b_{15 \text { thperc }}}}{E_{s g}}$ & $\frac{E_{s b_{85 \text { shper }}}}{E_{s g}}$ \\
\hline 220 & M01 & 3 & 35160 & 35380 & 345 & 398 & 115.45 & 0.29 & 9.26 & 3.19 & 1.04 & 0.19 & 3.84 \\
\hline 320 & M01 & 16 & 36300 & 36620 & 83 & 59 & 71.49 & 0.70 & 4.49 & 1.25 & 0.54 & 0.14 & 0.79 \\
\hline 200 & M04 & 1 & 0 & 200 & 504 & 479 & 94.98 & 0.37 & 2.24 & 2.00 & 1.28 & 0.65 & 8.19 \\
\hline 375 & M04 & 10 & 875 & 1250 & 95 & 66 & 69.63 & 0.72 & 5.83 & 1.17 & 0.57 & 0.15 & 0.86 \\
\hline 250 & M04 & 20 & 1975 & 2225 & 401 & 379 & 94.48 & 0.36 & 25.53 & 1.92 & 0.89 & 0.11 & 3.53 \\
\hline 275 & M04 & 30 & 2675 & 2950 & 77 & 93 & 120.95 & 0.75 & 4.68 & 1.24 & 0.41 & 0.11 & 0.60 \\
\hline 200 & M04 & 37 & 3325 & 3525 & 60 & 45 & 74.84 & 0.83 & 3.02 & 1.14 & 0.53 & 0.15 & 0.57 \\
\hline 370 & M05 ACW & 10 & 460 & 830 & 934 & 183 & 19.60 & 1.02 & 1.01 & 1.11 & 1.04 & 5.14 & 5.43 \\
\hline 550 & M05 ACW & 11 & 830 & 1380 & 899 & 185 & 20.59 & 0.71 & 0.89 & 1.18 & 1.06 & 4.43 & 6.37 \\
\hline 250 & M05 CW & 4 & 350 & 600 & 71 & 55 & 77.39 & 0.67 & 3.61 & 1.19 & 0.71 & 0.21 & 0.75 \\
\hline 240 & M05 CW & 42 & 2270 & 2510 & 748 & 406 & 54.28 & 0.40 & 3.15 & 1.29 & 1.04 & 0.49 & 4.98 \\
\hline 340 & M07 NB & 2 & 100 & 440 & 145 & 105 & 72.66 & 0.41 & 1.46 & 1.77 & 1.01 & 0.50 & 1.53 \\
\hline 200 & M07 NB & 3 & 440 & 640 & 161 & 204 & 126.41 & 0.33 & 1.20 & 0.74 & 0.98 & 0.65 & 1.20 \\
\hline 620 & M07 NB & 4 & 640 & 1260 & 227 & 216 & 95.34 & 0.46 & 2.71 & 1.69 & 0.97 & 0.42 & 2.50 \\
\hline 340 & M07 NB & 5 & 1260 & 1600 & 57 & 23 & 40.42 & 0.80 & 2.12 & 1.09 & 0.78 & 0.19 & 0.36 \\
\hline 200 & M07 NB & 6 & 1600 & 1800 & 73 & 71 & 96.61 & 0.62 & 2.94 & 0.96 & 1.07 & 0.18 & 0.37 \\
\hline 180 & M07 SB & 3 & 100 & 280 & 232 & 292 & 126.07 & 0.25 & 1.21 & 1.02 & 1.00 & 0.56 & 1.33 \\
\hline 560 & M07 SB & 7 & 720 & 1280 & 159 & 124 & 78.27 & 0.61 & 2.48 & 1.39 & 0.82 & 0.40 & 1.62 \\
\hline 220 & M07 SB & 8 & 1280 & 1500 & 91 & 55 & 60.76 & 0.69 & 2.97 & 1.38 & 0.57 & 0.21 & 0.82 \\
\hline 300 & M07 SB & 12 & 1640 & 1940 & 56 & 34 & 60.97 & 0.71 & 2.49 & 1.34 & 0.57 & 0.15 & 0.41 \\
\hline 300 & M08 EB & 4 & 180 & 480 & 121 & 91 & 75.24 & 0.24 & 4.64 & 2.15 & 0.84 & 0.16 & 1.06 \\
\hline 180 & M08 EB & 6 & 640 & 820 & 193 & 85 & 43.96 & 0.61 & 1.29 & 1.63 & 0.87 & 0.40 & 0.76 \\
\hline 240 & M08 WB & 2 & 100 & 340 & 83 & 40 & 48.45 & 0.67 & 2.73 & 1.27 & 0.64 & 0.12 & 0.31 \\
\hline \multirow[t]{4}{*}{400} & M08 WB & 5 & 920 & 1320 & 61 & 71 & 116.27 & 0.47 & 2.43 & 1.34 & 0.82 & 0.19 & 0.54 \\
\hline & & & & Avg & \multicolumn{2}{|c|}{$\mathrm{M} 01$ to M05 } & 69.71 & 0.58 & 3.74 & 1.43 & 0.78 & & \\
\hline & & & & Avg & \multicolumn{2}{|c|}{$\mathrm{M} 07$ to M08 } & 104.74 & 0.50 & 2.19 & 1.32 & 0.83 & & \\
\hline & & & & Avg & \multicolumn{2}{|c|}{ M01 to M08 } & 84.11 & 0.53 & 2.80 & 1.37 & 0.80 & & \\
\hline
\end{tabular}


Table 7.7 Subbase thickness 15th and 85th percentiles - Ratio of fatigue $\left(\mathrm{N}_{\mathrm{f}}\right)$ and deformation life $\left(\mathrm{N}_{\mathrm{d}}\right)$ over base case

\begin{tabular}{|c|c|c|c|c|c|c|c|c|c|c|c|}
\hline \multicolumn{5}{|c|}{$\begin{array}{l}\text { Pavement modelled as three-layer } \\
\text { structure }\end{array}$} & \multicolumn{3}{|c|}{ Subbase thickness } & \multicolumn{2}{|c|}{$\begin{array}{l}\text { 15th percentile Tsb; life } \\
\text { ratio over base scenario }\end{array}$} & \multicolumn{2}{|c|}{$\begin{array}{l}\text { 85th percentile Tsb; life } \\
\text { ratio over base scenario }\end{array}$} \\
\hline $\begin{array}{l}\text { Length } \\
(\mathrm{m})\end{array}$ & Site & Section & From & To & $\begin{array}{l}\text { Mean } \\
(\mathrm{m})\end{array}$ & $\begin{array}{l}\text { Stdev } \\
(\mathrm{m})\end{array}$ & COV (\%) & Nf1132 & Nd1132 & Nf1132 & Nd1132 \\
\hline 220 & M01 & 3 & 35160 & 35380 & 0.171 & 0.016 & 9.49 & 0.99 & 0.87 & 1.01 & 1.17 \\
\hline 320 & M01 & 16 & 36300 & 36620 & 0.088 & 0.020 & 22.40 & 1.04 & 0.78 & 0.95 & 1.38 \\
\hline 200 & M04 & 1 & 0 & 200 & 0.166 & 0.045 & 27.15 & 0.84 & 0.67 & 1.13 & 1.40 \\
\hline 375 & M04 & 10 & 875 & 1250 & 0.205 & 0.022 & 10.97 & 1.02 & 0.81 & 0.99 & 1.12 \\
\hline 250 & M04 & 20 & 1975 & 2225 & 0.257 & 0.031 & 11.89 & 0.98 & 0.84 & 1.01 & 1.19 \\
\hline 275 & M04 & 30 & 2675 & 2950 & 0.156 & 0.039 & 25.31 & 1.04 & 0.75 & 0.96 & 1.35 \\
\hline 200 & M04 & 37 & 3325 & 3525 & 0.147 & 0.013 & 9.17 & 1.02 & 0.86 & 0.99 & 1.11 \\
\hline 370 & M05 ACW & 10 & 460 & 830 & 0.218 & 0.006 & 2.90 & 0.98 & 0.95 & 1.02 & 1.07 \\
\hline 550 & M05 ACW & 11 & 830 & 1380 & 0.216 & 0.005 & 2.25 & 0.98 & 0.96 & 1.01 & 1.04 \\
\hline 250 & M05 CW & 4 & 350 & 600 & 0.195 & 0.047 & 24.30 & 1.04 & 0.67 & 0.96 & 1.51 \\
\hline 240 & M05 CW & 42 & 2270 & 2510 & 0.159 & 0.036 & 22.70 & 0.90 & 0.76 & 1.14 & 1.48 \\
\hline 340 & M07 NB & 2 & 100 & 440 & 0.300 & 0.064 & 21.33 & 1.00 & 0.50 & 1.00 & 1.92 \\
\hline 200 & M07 NB & 3 & 440 & 640 & 0.300 & 0.063 & 21.00 & 0.96 & 0.47 & 1.03 & 2.01 \\
\hline 620 & M07 NB & 4 & 640 & 1260 & 0.300 & 0.063 & 21.00 & 0.97 & 0.60 & 1.02 & 1.61 \\
\hline 340 & M07 NB & 5 & 1260 & 1600 & 0.300 & 0.065 & 21.67 & 1.07 & 0.63 & 0.95 & 1.50 \\
\hline 200 & M07 NB & 6 & 1600 & 1800 & 0.300 & 0.062 & 20.67 & 1.06 & 0.60 & 0.95 & 1.57 \\
\hline 180 & M07 SB & 3 & 100 & 280 & 0.300 & 0.064 & 21.33 & 0.97 & 0.45 & 1.02 & 2.08 \\
\hline 560 & M07 SB & 7 & 720 & 1280 & 0.300 & 0.060 & 20.00 & 1.00 & 0.64 & 1.00 & 1.51 \\
\hline 220 & M07 SB & 8 & 1280 & 1500 & 0.300 & 0.056 & 18.67 & 1.04 & 0.68 & 0.97 & 1.43 \\
\hline 300 & M07 SB & 12 & 1640 & 1940 & 0.300 & 0.059 & 19.67 & 1.07 & 0.63 & 0.94 & 1.52 \\
\hline 300 & M08 EB & 4 & 180 & 480 & 0.300 & 0.063 & 21.00 & 1.03 & 0.52 & 0.98 & 1.80 \\
\hline 180 & M08 EB & 6 & 640 & 820 & 0.300 & 0.061 & 20.33 & 1.04 & 0.52 & 0.97 & 1.82 \\
\hline 240 & M08 WB & 2 & 100 & 340 & 0.300 & 0.061 & 20.33 & 1.09 & 0.60 & 0.93 & 1.57 \\
\hline \multirow[t]{4}{*}{400} & M08 WB & 5 & 920 & 1320 & 0.300 & 0.060 & 20.00 & 1.07 & 0.54 & 0.96 & 1.74 \\
\hline & & & & Avg & \multicolumn{2}{|c|}{ M01 to M05 } & 16.32 & 0.98 & 0.81 & 1.02 & 1.25 \\
\hline & & & & Avg & \multicolumn{2}{|c|}{ M07 to M08 } & 20.55 & 1.03 & 0.56 & 0.98 & 1.69 \\
\hline & & & & Avg & \multicolumn{2}{|c|}{ M01 to M08 } & 20.23 & 1.01 & 0.66 & 1.00 & 1.47 \\
\hline
\end{tabular}


Table 7.8 Subgrade stiffness 15th and 85th percentiles - Ratio of fatigue $\left(\mathrm{N}_{\mathrm{f}}\right)$ and deformation life $\left(\mathrm{N}_{\mathrm{d}}\right)$ over base case

\begin{tabular}{|c|c|c|c|c|c|c|c|c|c|c|c|}
\hline \multicolumn{5}{|c|}{$\begin{array}{l}\text { Pavement modelled as three-layer } \\
\text { structure }\end{array}$} & \multicolumn{3}{|c|}{ Subgrade stiffness } & \multicolumn{2}{|c|}{$\begin{array}{l}\text { 15th percentile Esg; life } \\
\text { ratio over base scenario }\end{array}$} & \multicolumn{2}{|c|}{$\begin{array}{l}\text { 85th percentile Esg; life } \\
\text { ratio over base scenario }\end{array}$} \\
\hline $\begin{array}{l}\text { Length } \\
(\mathrm{m})\end{array}$ & Site & Section & From & To & $\begin{array}{l}\text { Mean } \\
\text { (MPa) }\end{array}$ & $\begin{array}{l}\text { Stdev } \\
\text { (MPa) }\end{array}$ & COV (\%) & Nf1132 & Nd1132 & Nf1132 & Nd1132 \\
\hline 220 & M01 & 3 & 35160 & 35380 & 227 & 68 & 29.77 & 0.80 & 0.43 & 1.19 & 2.15 \\
\hline 320 & M01 & 16 & 36300 & 36620 & 199 & 34 & 17.02 & 0.89 & 0.59 & 1.10 & 1.60 \\
\hline 200 & M04 & 1 & 0 & 200 & 113 & 66 & 58.10 & 0.59 & 0.27 & 1.12 & 1.38 \\
\hline 375 & M04 & 10 & 875 & 1250 & 191 & 33 & 17.03 & 0.94 & 0.72 & 1.06 & 1.44 \\
\hline 250 & M04 & 20 & 1975 & 2225 & 257 & 21 & 8.10 & 0.97 & 0.87 & 1.04 & 1.16 \\
\hline 275 & M04 & 30 & 2675 & 2950 & 265 & 30 & 11.21 & 0.95 & 0.70 & 1.05 & 1.43 \\
\hline 200 & M04 & 37 & 3325 & 3525 & 182 & 42 & 22.90 & 0.91 & 0.57 & 1.07 & 1.49 \\
\hline 370 & M05 ACW & 10 & 460 & 830 & 184 & 32 & 17.44 & 0.85 & 0.60 & 1.08 & 1.28 \\
\hline 550 & M05 ACW & 11 & 830 & 1380 & 157 & 13 & 8.39 & 0.96 & 0.89 & 1.04 & 1.12 \\
\hline 250 & M05 CW & 4 & 350 & 600 & 134 & 24 & 17.75 & 0.91 & 0.56 & 1.09 & 1.78 \\
\hline 240 & M05 CW & 42 & 2270 & 2510 & 201 & 45 & 22.53 & 0.89 & 0.74 & 1.12 & 1.35 \\
\hline 340 & M07 NB & 2 & 100 & 440 & 137 & 41 & 29.64 & 0.88 & 0.29 & 1.05 & 1.78 \\
\hline 200 & M07 NB & 3 & 440 & 640 & 110 & 18 & 16.50 & 0.96 & 0.69 & 1.04 & 1.50 \\
\hline 620 & M07 NB & 4 & 640 & 1260 & 157 & 32 & 20.27 & 0.95 & 0.71 & 1.02 & 1.15 \\
\hline 340 & M07 NB & 5 & 1260 & 1600 & 187 & 44 & 23.30 & 0.94 & 0.50 & 1.07 & 2.52 \\
\hline 200 & M07 NB & 6 & 1600 & 1800 & 189 & 46 & 24.18 & 0.95 & 0.56 & 1.04 & 1.72 \\
\hline 180 & M07 SB & 3 & 100 & 280 & 176 & 37 & 20.90 & 0.97 & 0.68 & 1.04 & 1.91 \\
\hline 560 & M07 SB & 7 & 720 & 1280 & 156 & 19 & 12.03 & 0.96 & 0.73 & 1.03 & 1.29 \\
\hline 220 & M07 SB & 8 & 1280 & 1500 & 196 & 49 & 25.03 & 0.94 & 0.54 & 1.04 & 1.55 \\
\hline 300 & M07 SB & 12 & 1640 & 1940 & 210 & 51 & 24.37 & 0.96 & 0.51 & 1.03 & 1.84 \\
\hline 300 & M08 EB & 4 & 180 & 480 & 186 & 193 & 103.97 & 0.75 & 0.06 & 1.05 & 2.25 \\
\hline 180 & M08 EB & 6 & 640 & 820 & 339 & 308 & 90.80 & 0.66 & 0.01 & 1.10 & 10.49 \\
\hline 240 & M08 WB & 2 & 100 & 340 & 373 & 300 & 80.54 & 0.66 & 0.01 & 1.07 & 4.25 \\
\hline \multirow[t]{4}{*}{400} & M08 WB & 5 & 920 & 1320 & 144 & 186 & 129.09 & 0.79 & 0.05 & 0.98 & 0.73 \\
\hline & & & & Avg & \multicolumn{2}{|c|}{ M01 to M05 } & 21.06 & 0.87 & 0.60 & 1.09 & 1.45 \\
\hline & & & & Avg & \multicolumn{2}{|c|}{$\mathrm{M} 07$ to M08 } & 73.27 & 0.87 & 0.22 & 1.04 & 1.97 \\
\hline & & & & Avg & \multicolumn{2}{|c|}{ M01 to M08 } & 56.36 & 0.87 & 0.35 & 1.06 & 1.71 \\
\hline
\end{tabular}

Table 7.9 Summary of Ratio of fatigue $\left(\mathrm{N}_{\mathrm{f}}\right)$ and deformation life $\left(\mathrm{N}_{\mathrm{d}}\right)$ over base case based on average results (tabular format)

\begin{tabular}{|c|c|c|c|c|c|c|}
\hline \multirow[t]{2}{*}{$\begin{array}{l}\text { Design } \\
\text { parameter }\end{array}$} & \multirow[t]{2}{*}{$\begin{array}{l}\text { Design parameter } \\
\text { description }\end{array}$} & \multirow{2}{*}{$\begin{array}{l}\text { Parameter } \\
\text { CoV (\%), } \\
\text { average of } \\
\text { all sites }\end{array}$} & \multicolumn{2}{|c|}{$\begin{array}{l}\text { 15th percentile for design } \\
\text { parameter; average life } \\
\text { ratio over base scenario }\end{array}$} & \multicolumn{2}{|c|}{$\begin{array}{l}\text { 85th percentile for design } \\
\text { parameter; average life } \\
\text { ratio over base scenario }\end{array}$} \\
\hline & & & Nf1132 & Nd1132 & Nf1132 & Nd1132 \\
\hline $\mathrm{Ea}$ & Asphalt stiffness & 32.2 & $-67 \%$ & $-49 \%$ & $113 \%$ & $60 \%$ \\
\hline $\mathrm{Ta}$ & Asphalt thickness & 6.4 & $-28 \%$ & $-26 \%$ & $41 \%$ & $49 \%$ \\
\hline Esb & Subbase stiffness & 84.1 & $-47 \%$ & $180 \%$ & $37 \%$ & $-20 \%$ \\
\hline Tsb & Subbase thickness & 20.2 & $1 \%$ & $-34 \%$ & $0 \%$ & $47 \%$ \\
\hline Esg & Subgrade stiffness & 56.4 & $-13 \%$ & $-65 \%$ & $6 \%$ & $71 \%$ \\
\hline
\end{tabular}




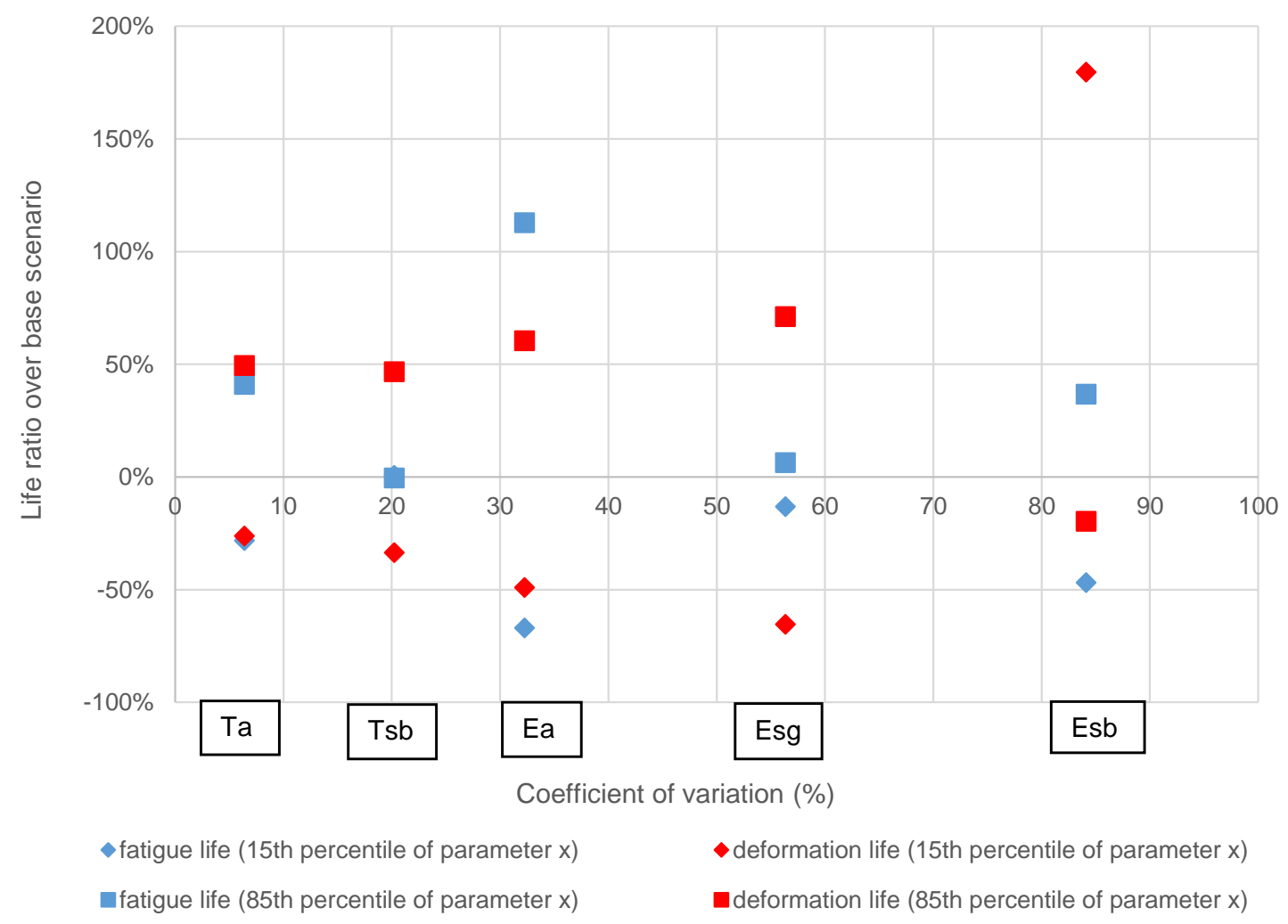

Figure 7.8 Summary of Ratio of fatigue (Nf) and deformation life $\left(\mathrm{N}_{\mathrm{d}}\right)$ over base case based on average results (graphical format)

The following observations can be made from the tables and from the graph above (i.e., by looking at the spread of the life ratio over the base scenario resulting from the $15^{\text {th }}$ and $85^{\text {th }}$ percentiles of the selected pavement input parameters):

- The parameters with the greatest influence on the variability of predicted fatigue performance are the asphalt stiffness modulus $\left(E_{a}\right)$ and thickness $(\mathrm{Ta})$.

- Fatigue life is affected by changes in the asphalt surface layer thickness $\left(T_{a}\right)$ while it is unaffected by changes in the granular subbase layer thickness $\left(T_{s b}\right)$.

- The parameters with the greatest influence on the variability of predicted deformation (rutting) performance are the granular subbase thickness $\left(T_{s b}\right)$, the asphalt thickness $\left(T_{a}\right)$, and the stiffness of the subgrade $\left(E_{s g}\right)$. 
- The observations above do not include any variation in traffic and they are in line with the results of other research findings as discussed in Section 2.9.1.

- The stiffness of the subbase $\left(\mathrm{E}_{\mathrm{sb}}\right)$ also appears to influence the variability of predicted deformation (rutting) performance. However, it is noted that the section's average backcalculated subbase stiffness (see Table 7.6) is in most cases low (less than 150MPa) and lower than the backcalculated subgrade modulus. This is true also for the ratio of the $15^{\text {th }}$ and $85^{\text {th }}$ percentiles of the subbase stiffness over the subgrade stiffness. For a typical flexible pavement with a well performing foundation, it is expected that the subbase stiffness is at least $150 \mathrm{MPa}$ and/or about 2 to 3 times the subgrade stiffness. In the very few cases where this condition is satisfied the subbase stiffness has little influence on the variability of predicted deformation (rutting) performance.

- In connection with the previous observation, it is noted that the goodness of fit has been monitored but not included in the analysis.

\subsection{Conclusions}

An overview of different methods to estimate the subgrade stiffness, spatial and seasonal variations of subgrade stiffness and the results of the author's attempt to assess the subgrade stiffness variability have been discussed in this chapter. The main findings are:

- California Bearing Ratio (CBR) and Repeated Load Triaxial (RLT) tests, Dynamic Cone Penetrometer (DCP), Falling Weight Deflectometer (FWD) and Light Weight Dynamic plate (LWD) are the main methods used worldwide to estimate the subgrade stiffness. Empirical and local correlations are used between the tests methods above with consequent variability in test results depending on the particular method used.

- Based on a 'cusum' analysis on the available sites, the coefficient of variation of the back-analysed subgrade stiffness modulus for almost all sites varied from a minimum of $8 \%$ to a maximum of $30 \%$, with an average 
value of $20 \%$. The high uncertainty sometimes observed in the data is probably due to the small sample size derived from the cusum analysis.

- A lognormal probability distribution was found in most cases to be representative of the subgrade stiffness.

- Based on a sensitivity analysis of design input parameter variability on the available sites, it was found that the stiffness of the subgrade $\left(E_{s g}\right)$ has a great influence on the variability of predicted deformation (rutting) performance together with the granular subbase thickness $\left(T_{s b}\right)$ and the asphalt thickness $\left(T_{a}\right)$. The effect of the variability in subgrade stiffness is less pronounced with respect to the fatigue life of flexible pavements. 


\section{Proposed model to predict the pavement design life}

The calculation of stresses and strains (response models) and performance models have been discussed in chapter 4. Odemark's Method of Equivalent Thicknesses (MET) and Shell's specialist software "BISAR" have been chosen to calculate the stresses and strains for various pavement structures. This chapter starts with the assessment of the difference between the two methods in the calculation of response and then explores the effect on pavement performance represented by the fatigue and deformation lives.

BISAR is one amongst many available multi-layer computer programs used to calculate stresses and strains in pavements, but the main limitation is that only a maximum of 10 pavement structures can be analysed at a time and all input parameters need to be inserted manually by the user. The MET methodology on the other hand is not a precise method from a mathematical point of view, but it can give an instant prediction of the strains and stresses in pavement layers. It should be considered as a simple and efficient method for practical purposes, for example in Pavement Management Systems or in simulation of pavement deterioration, where stresses and strains must be calculated large numbers of times (Pearson, 2011).

This chapter presents the results of a sensitivity analysis carried out to compare the values of the strains (asphalt tensile strain and compressive strain at the top of the subgrade) and fatigue and deformation lives obtained with the MET methodology with those calculated with the BISAR software. The results of a linear regression analysis are presented which can be used to predict the values of strains calculated with BISAR from those obtained with the MET methodology and with a desired level of confidence. A similar analysis is applied to the prediction models (fatigue and deformation life) and an alternative model is also discussed to improve the results of the MET methodology, particularly for the calculation of asphalt strains and fatigue life. It should be stressed that the proposed method is meant to be a practical tool to assess the (relative) effects of pavement design input variability on output performance. The ultimate interest of the research is not in the absolute values 
of fatigue and deformation lives (i.e., accuracy of the proposed model) but rather in their variations, expressed for example by the pavement life's coefficient of variation and probability distribution.

The chapter also explores the effect of different pavement models used in backcalculation procedures, in particular the three-layer vs two-layer structure, on the backanalysed layer stiffnesses and on the fatigue and deformation lives.

\subsection{Sensitivity Analysis}

A sensitivity analysis has been carried out to compare the values of the strains obtained with the MET method with those calculated with the BISAR software and to assess the impact of this difference in the prediction models (fatigue and deformation life). The analysis aimed to investigate in detail the difference between the two methods and to compare it with the $\pm 10 \%$ value commonly quoted in the literature for a given pavement structure (Ullidtz, 1987). The strains considered are the tensile strain at the bottom of the asphalt layer and the compressive strain at the top of the subgrade. BISAR was chosen because it is widely used for analytical pavement designs in the UK. Despite the reasonable accuracy of the response model predicted by BISAR, the software cannot be practically used in Pavement Management Systems or in simulation of pavement deterioration. A simplified approach to the calculation of the pavement strains and stresses such as that offered by the MET method is believed to be best suited for these purposes.

In order to assess the difference between the MET and the BISAR calculated values of the asphalt and subgrade strains with a certain level of confidence, a large number of 3-layer pavement structures were considered (i.e. asphalt layer on top of subbase layer and subgrade). The range of values considered for the input variables is presented in Table 8.1. 


\section{Table 8.1 Pavement structures considered in the analysis}

\begin{tabular}{|l|l|l|}
\hline Variable name & Variable description & Range of values \\
\hline $\mathrm{x}_{1}\left(\mathrm{E}_{\mathrm{a}}\right)$ & Asphalt stiffness $(\mathrm{MPa})$ & 2500 to $7000 \mathrm{MPa}$ \\
\hline $\mathrm{x}_{2}\left(\mathrm{~T}_{\mathrm{a}}\right)$ & Asphalt thickness $(\mathrm{m})$ & 0.100 to $0.500 \mathrm{~m}$ \\
\hline $\mathrm{x}_{3}\left(\mathrm{~T}_{\mathrm{sb}}\right)$ & Subbase thickness $(\mathrm{m})$ & 0.100 to $1.000 \mathrm{~m}$ \\
\hline $\mathrm{x}_{4}\left(\mathrm{E}_{\mathrm{sb}}\right)$ & Subbase stiffness $(\mathrm{MPa})$ & 75 to $1000 \mathrm{MPa}$ \\
\hline $\mathrm{x}_{5}\left(\mathrm{E}_{\mathrm{sg}}\right)$ & Subgrade stiffness $(\mathrm{MPa})$ & 27 to $100 \mathrm{MPa}$ \\
\hline
\end{tabular}

The following design parameters were selected:

1. The values of asphalt layer thickness $(m)$ considered were: $0.100,0.150$, $0.200,0.300,0.400$, and 0.500 .

2. The values of asphalt layer stiffness (MPa) and corresponding asphalt materials considered were: 2500 (DBM125), 3100 (DBM100), 4700 (DBM50), 6200 (HDM), and 7000 (HMB35) (as recommended in HD 26, DMRB Volume 7).

3. The values of sub-base layer thickness $(\mathrm{m})$ considered were: $0.100,0.150$, $0.200,0.300,0.400,0.500,0.600,0.700,0.800,0.900$, and 1.000 .

4. The values of subbase layer stiffness (MPa) considered were: $30,50,75$, $100,150,200,300,350,400,500,750$, and 1000.

5. The values of subgrade stiffness (MPa) considered were: 15, 27, 30, 32 , $36,43,50,67,77$ and 100. The subgrade stiffnesses covered subgrade California Bearing Ratio (CBR) values ranging from 1 to $10 \%$ when calculated using the following formula (Powell et al, 1984):

$$
E_{s b}=17.6 *(C B R)^{0.64} \quad(\mathrm{MPa})
$$

where $\mathrm{CBR}$ is given as a \% value.

Both MET and BISAR asphalt and subgrade strains were calculated for all the possible combinations of values of the 5 input variables which satisfied the conditions of validity of the MET method (i.e., $E_{a} / E_{s b}>2$ and $E_{s b} / E_{s g}>2 ; h_{e, 2>a}$ and $h_{e, 3}>a$, where $h_{e, 2}$ and $h_{e, 3}$ are the transformed asphalt and subbase layer respectively), for a total of 13368 different cases out of 19024. A Poisson's ratio of 0.35 was assumed for all layers. It should be noted that within all the arbitrary combinations of layer properties considered in the analysis, some may not be representative of real pavement structures. 
A regression analysis for 3-layer modelled pavement structures was performed for both the asphalt and subgrade strains the results of which are discussed in the following sections.

As mentioned earlier, a limitation of the MET method for a 3 or more layer modelled pavement structure is that the asphalt strain is independent of the characteristics of the formation layers (i.e., subbase thickness and subgrade stiffness for a 3-layer pavement structure; subbase thickness, capping thickness, capping stiffness and subgrade stiffness for a 4-layer pavement structure). To overcome this problem, an alternative model is proposed where the pavement is modelled as a 2-layer pavement structure with the subbase and subgrade layers transformed into an Equivalent Foundation Modulus (EFM).

\subsubsection{Regression analysis - 3-layer pavement structure}

The regression analysis discussed in this section - for both strains and performance lives - is based on the application of the MET method to 3-layer pavement structures, see Figure 8.1.

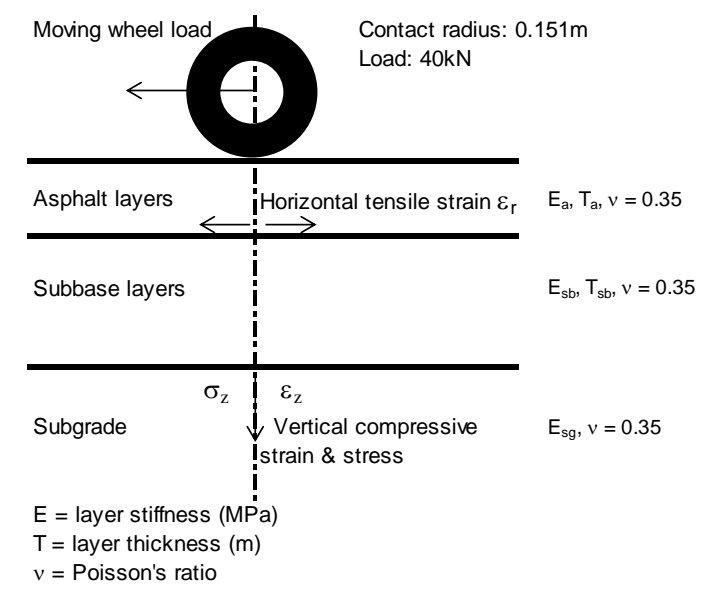

Figure 8.1 Model for 3-layer pavement structure 


\subsubsection{Model for asphalt and subgrade strains}

For every combination of pavement structures considered the plot of the asphalt tensile strains calculated with the MET method versus the asphalt tensile strains calculated with the BISAR software is illustrated in Figure 8.2. Similarly, the plot of the subgrade compressive strains calculated with the MET method versus the subgrade compressive strains calculated with the BISAR software is illustrated in Figure 8.3. The actual values of the strains have been plotted on a logarithmic scale. Both Figure 8.2 and Figure 8.3 show that an "intrinsically" linear relationship exists between the strains calculated with the MET method and those calculated with the BISAR software. The general exponential function:

$$
Y=\beta_{0} x^{\beta_{1}} \varepsilon
$$

is intrinsically linear, since it can be transformed to a straight line by a logarithmic transformation, such as:

$$
\log Y=\log \beta_{0}+\beta_{1} \log x+\log \varepsilon
$$

This transformation requires that the transformed error terms $\log \varepsilon$ are normally and independently distributed with mean 0 and variance $\sigma^{2}$. Therefore, a linear regression analysis was performed, on the log transformed variables, based on the least square method (Montgomery \& Runger, 2006).

The results of the linear regression analysis together with the plot of the $95 \%$ prediction limits are shown in Figure 8.2 and Figure 8.3. The line of best fit is also shown in all graphs.

For example, from Figure 8.2 it can be seen that, for an asphalt strain of 30 microstrain, calculated with the MET method on a 3-layer pavement structure, it is expected that:

- The best-fit predicted value of the BISAR asphalt strain is 46 microstrain

- The predicted $95 \%$ lower limit of the BISAR asphalt strain is 33 microstrain

- The predicted $95 \%$ upper limit of the BISAR asphalt strain is 65 microstrain 


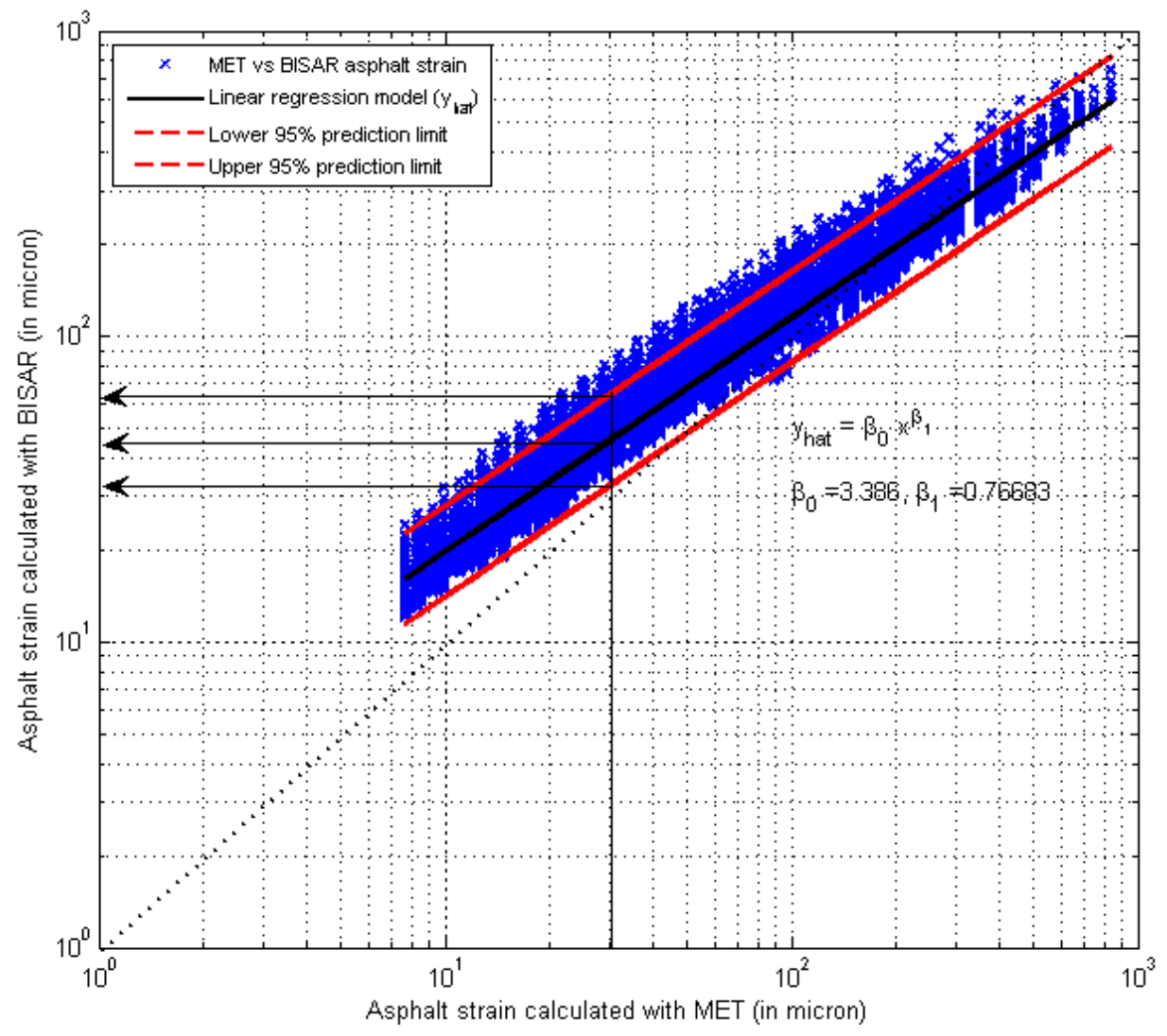

Figure 8.2 Linear regression analysis for the asphalt strain

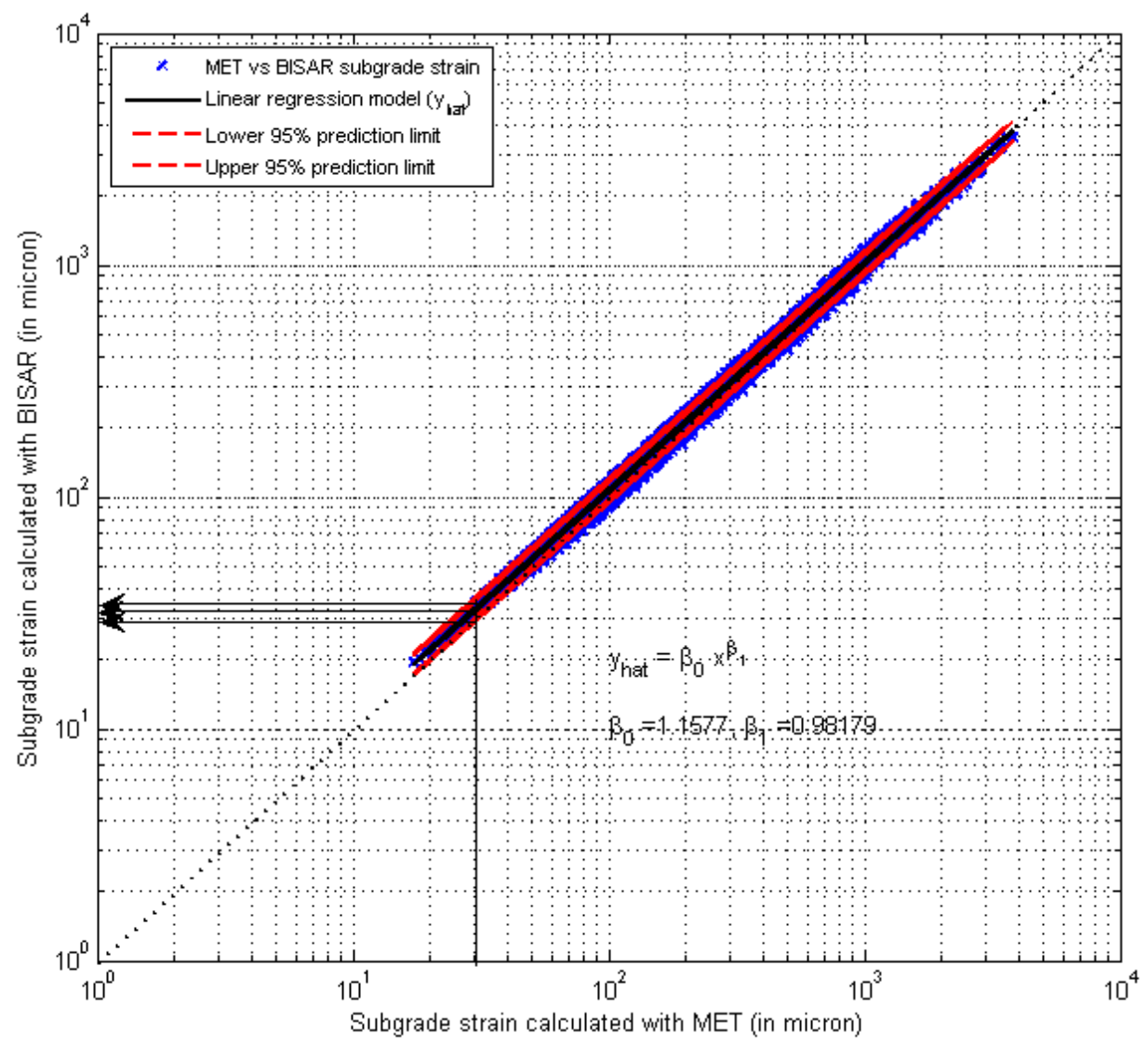

Figure 8.3 Linear regression analysis for the subgrade strain 
As anticipated, the MET method is not very accurate in the calculation of the asphalt strains because some of the formation layer properties are not taken into account.

A similar analysis can be performed for the subgrade strains for which it is noted that the fitted regression line is very close to the line of best fit. For example, from Figure 8.3 it can be seen that, for a subgrade strain of 30 microstrain, calculated with the MET method on a 3-layer pavement structure, it is expected that:

- The best-fit predicted value of the BISAR subgrade strain is 33 microstrain

- The predicted $95 \%$ lower limit of the BISAR subgrade strain is 30 microstrain

- The predicted 95\% upper limit of the BISAR subgrade strain is 36 microstrain

The prediction interval width is much narrower compared to the one referring to the asphalt strains. The width of the prediction interval gives an indication of the error of the fitted model.

This analysis has allowed the following conclusions to be reached in regard to the ratio of the MET and BISAR strains:

- The MET/BISAR asphalt strain ratio varied from 0.31 to 1.55 . In other words, the values determined using Odemark's method ranged from $-69 \%$ to $+55 \%$ of the values from the theory of elasticity (BISAR).

- The MET/BISAR subgrade strain ratio varied from 0.83 to 1.18 . In other words, the values determined using Odemark's method ranged from $-17 \%$ to $+18 \%$ of the values from the theory of elasticity (BISAR).

\subsubsection{Model for performance prediction}

The same regression analysis principles described in the previous section were applied to the fatigue and deformation life values obtained with eq. (4.13) and eq. (4.14) for all the combinations of pavement structures considered in the analysis (see section 8.1). The line of best fit is also shown in all graphs. 
The results of the linear regression analysis together with plots of the $95 \%$ prediction limits are shown in Figure 8.4 and Figure 8.5.

For example, from Figure 8.4 it can be seen that, for a calculated asphalt fatigue life of $30 \mathrm{msa}$ (million standard axles), it is expected that:

- The best-fit predicted value of the BISAR fatigue life is $15 \mathrm{msa}$

- The predicted $95 \%$ lower limit of the BISAR fatigue life is $4 \mathrm{msa}$

- The predicted $95 \%$ upper limit of the BISAR fatigue life is $60 \mathrm{msa}$

Figure 8.4 shows that, despite a positive correlation between the two methods, the prediction interval is quite wide. A similar analysis can be performed for the deformation life for which it is noted that the fitted regression line is very close to the line of best fit. For example, from Figure 8.6 it can be seen that, for a calculated deformation life of $30 \mathrm{msa}$, it is expected that:

- The best-fit predicted value of the BISAR deformation life is $25 \mathrm{msa}$

- The predicted $95 \%$ lower limit of the BISAR deformation life is $17 \mathrm{msa}$

- The predicted $95 \%$ upper limit of the BISAR deformation life is $37 \mathrm{msa}$

The prediction interval width is much narrower compared to the one referring to the asphalt fatigue life values. This is expected given the correspondingly narrower width of the subgrade strain prediction intervals. 


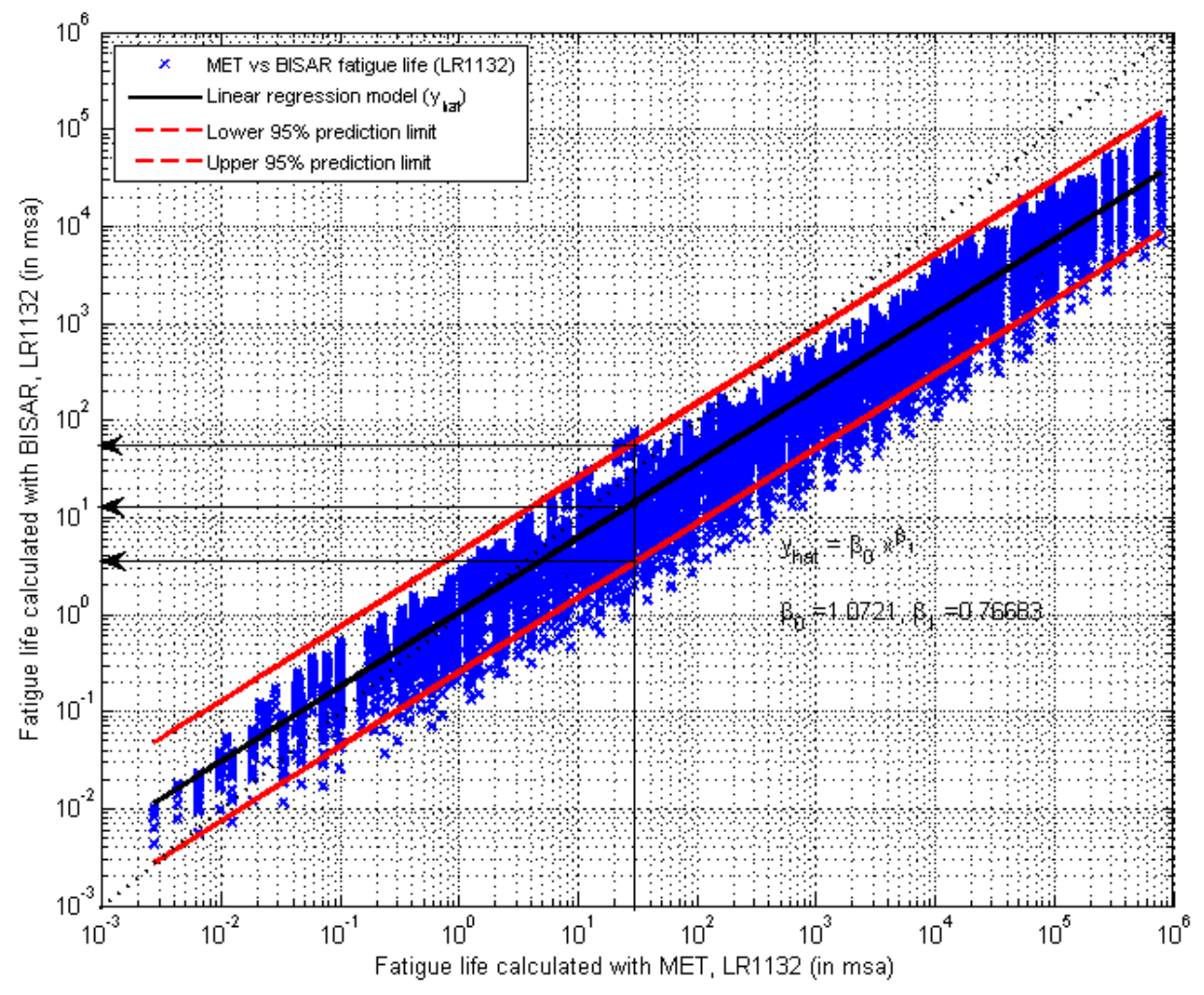

Figure 8.4 Linear regression analysis for the fatigue life model (with eq 4.13) from LR1132 report)

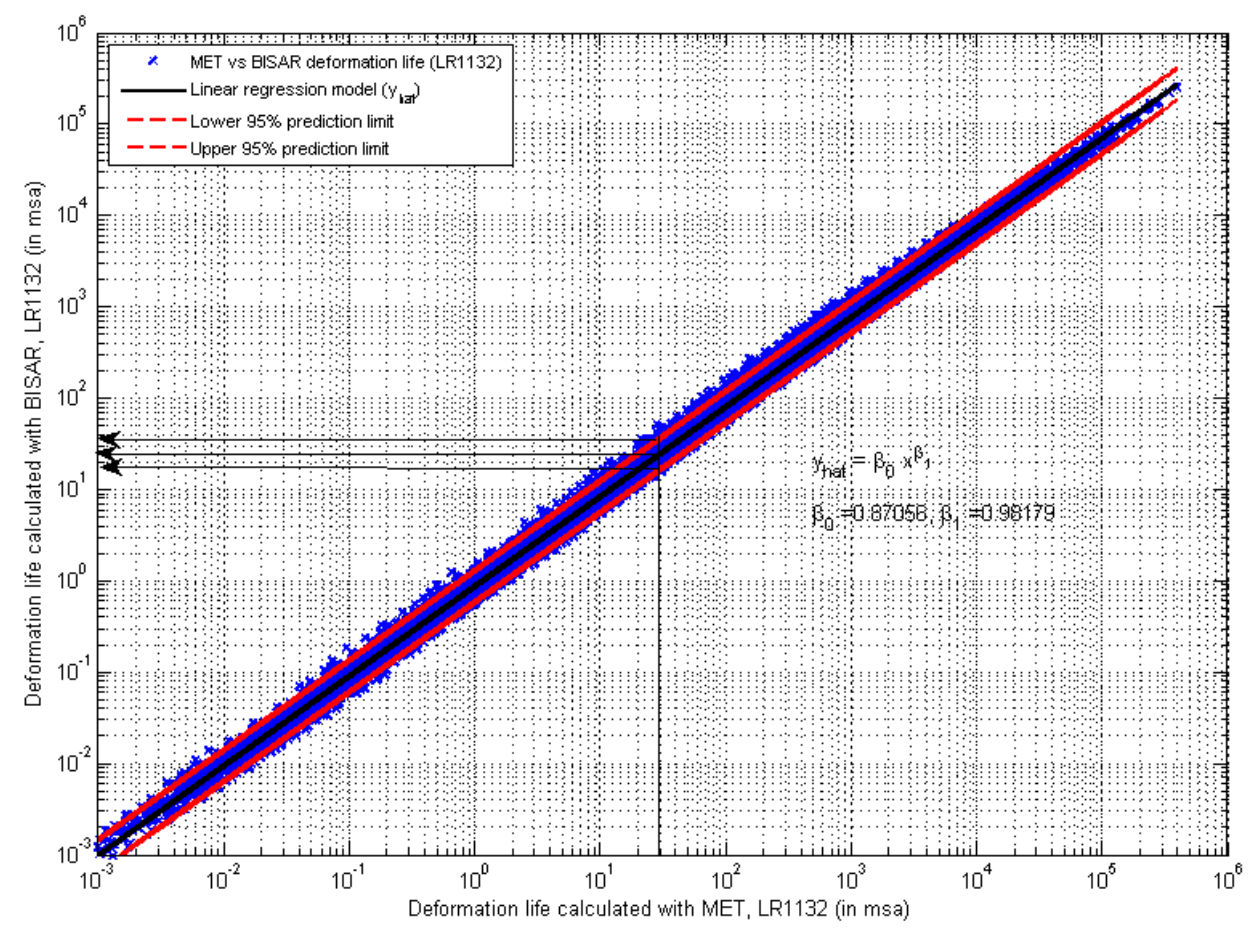

Figure 8.5 Linear regression analysis for the deformation life model (with eq. 4.14 from LR1132 report) 


\subsubsection{Regression analysis for alternative fatigue model - 2-layer pavement structure}

An alternative model is proposed that represents an improvement to the Method of Equivalent Thickness for the calculation of asphalt strains and fatigue life for flexible pavements. The aim of the proposed analysis was to overcome the limitations of the MET approach by developing a model that accounted for the contribution of the formation layers in the calculation of the asphalt strain. The proposed method transforms a 3-layer pavement structure (i.e. asphalt + subbase + subgrade) into a 2-layer structure (i.e. asphalt + Equivalent Foundation Modulus, EFM), see Figure 8.6, to which structure the MET method is applied for the calculation of the asphalt strain. The main challenge was to find a suitable model for the Equivalent Foundation Modulus that together with the overlaying asphalt layer would give the same asphalt strain, under the same wheel load, as that of the original 3-layer pavement structure calculated with BISAR.
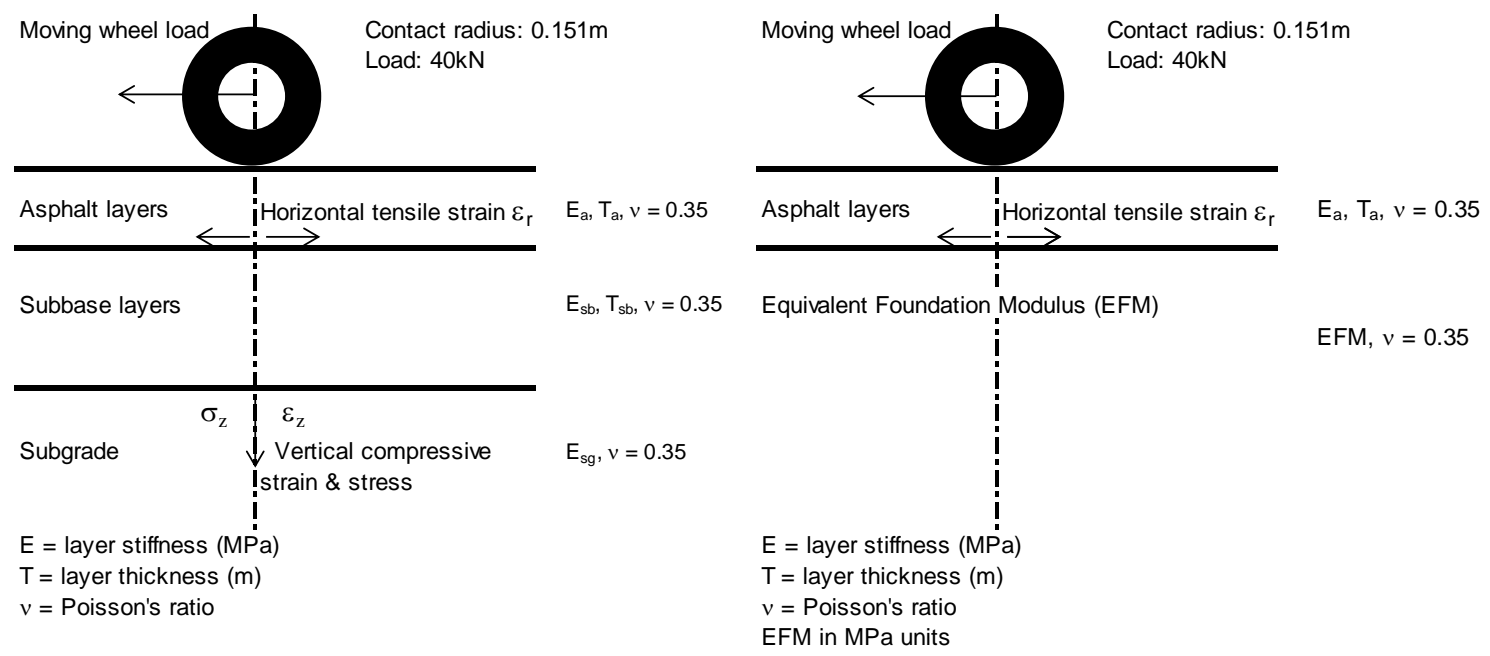

Figure 8.6 Model for 3-layer pavement structure (left) and 2-layer pavement structure (right) used in the alternative model

A first attempt to model the EFM foundation was to use the well-known concept of "Equivalent Surface Foundation Modulus" (ESFM) which is the modulus of a uniform elastic foundation that would give the same deflection, do, under the same wheel load, as that of the actual road structure. The calculation of the ESFM was made using a simplified method devised by Ullidtz and Peattie 
(1980) that transforms a multi-layered elastic structure into an equivalent semiinfinite space, by assuming a single value of Poisson's ratio for all the layers, see eq. (8.3).

$$
E S F M=\frac{2\left(1-v^{2}\right) \sigma_{0} a}{d_{0}}
$$

where ESFM is the equivalent surface foundation modulus (MPa), $\sigma_{0}$ is the applied stress (MPa), $v$ is a common Poisson's ratio for all the layers, $a$ is the radius of loaded area $(\mathrm{mm})$, and $d_{0}$ is the total deflection of the surface of a structure $(\mathrm{mm})$. However, the results obtained from the application of this simplified model were poor and it was, therefore, abandoned.

The next stage of the analysis was to calculate directly - with a simple computer routine in Matlab - what the Equivalent Foundation Modulus value should be for each 2-layer pavement structure (referred to subsequently as "EFM_actual") in order to give the same asphalt strain, under the same wheel load, as that of the original 3-layer pavement structure calculated before with BISAR. This is schematically explained in Figure 8.7.

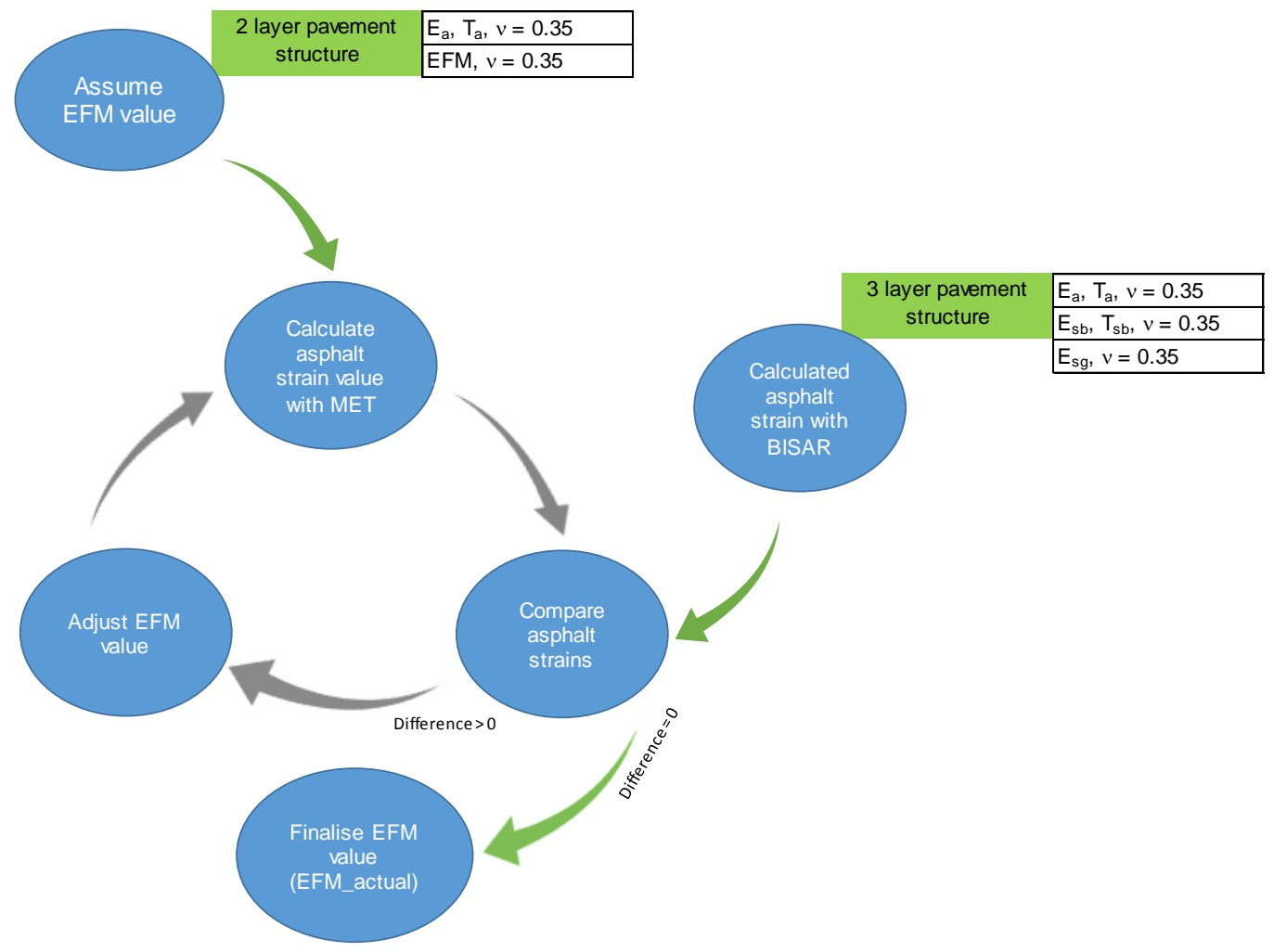

Figure 8.7 Schematic diagram representing the process of finding EFM_actual 
The final stage of the analysis was to fit a relationship between the actual EFM calculated in Matlab (EFM_actual) and the foundation layer parameters (i.e. subbase thickness and stiffness, subgrade stiffness). The following relationship was used first to combine the foundation layers of the original 3layer pavement structure into an Equivalent Foundation Modulus of the derived 2-layer pavement structure:

$$
E F I_{-} \text {combined }=\frac{h_{\text {subbas }} E_{\text {subbase }}+a_{a} E_{\text {subgrade }}}{a_{a}+h_{\text {subbase }}} \quad(\mathrm{MPa})
$$

where $h$ is the layer thickness $(m), E$ is the layer stiffness (MPa) and the coefficient $a_{a}$ was taken as being equal to the asphalt layer thickness $T_{a}(m)$. A plot of the EFM calculated with eq. (8.4) above (EFM_combined) versus the actual EFM (EFM_actual) is shown in Figure 8.8, which still shows a relatively poor correlation between the two variables. To reduce the scatter of the results, it was therefore decided to include the asphalt layer stiffness $\left(E_{a}\right)$ in the final calculation of EFM. The purpose was to express the actual EFM as a function of both the foundation layers (i.e. EFM_combined) and the asphalt layer stiffness $\left(E_{a}\right)$. Through the help of the DataFit curve fitting (nonlinear regression) software developed by Oakdale Engineering, the following empirical equation was found to give satisfactory results:

$$
E F M_{-} \text {calculated }=a+\frac{b}{x_{1}}+c * x_{2}+\frac{d}{x_{1}^{2}}+e^{*} x_{2}^{2}+f * \frac{x_{2}}{x_{1}} \quad(\mathrm{MPa})
$$

where $x_{1}$ is the asphalt stiffness $E_{a}(\mathrm{MPa})$, and $x_{2}$ is the EFM_combined of the foundation layers, in $\mathrm{MPa}$, calculated with eq. (8.5). The coefficients $a, b, c, d$, $e$, and $f$ are expressed as functions of the asphalt layer thickness $T_{a}(m)$. These were found with the following procedure:

The regression eq.(8.5) was applied to the set of data (i.e., EFM_combined and $E_{a}$ ) corresponding to a certain asphalt thickness group (i.e. $T_{a}=0.1 \mathrm{~m}$, $0.150 \mathrm{~m}$, etc). Therefore, regression coefficients for each asphalt thickness group were found, see Table 8.2. 
Table 8.2 Regression coefficients of eq. (8.5) for each asphalt thickness group

\begin{tabular}{l|l|l|l|l|l|l|}
\hline $\begin{array}{l}\text { Reg. } \\
\text { Coef }\end{array}$ & Ta=0.1m & Ta=0.15m & Ta=0.2m & Ta=0.3m & Ta=0.4m & Ta=0.5m \\
\hline a & 238.892617803 & 181.339596680 & 139.906903197 & 139.224354676 & 143.953204247 & 141.935547273 \\
\hline b & -946475.97944 & -668181.15197 & -489101.95708 & -498607.50105 & -538071.25860 & -535510.05425 \\
\hline c & 1.3603043574 & 1.0932459761 & 0.6335543295 & 0.0232151587 & -0.2238739334 & -0.3200034834 \\
\hline d & 1231293543.97 & 793752731.63 & 470925847.05 & 385931159.33 & 430823513.98 & 435548188.02 \\
\hline e & 0.00004193055 & 0.00022438727 & 0.00049635456 & 0.00078198610 & 0.00088296556 & 0.00091767814 \\
\hline f & -144.71522176 & 271.61920344 & 985.39688240 & 1874.59268950 & 2163.01838485 & 2220.14890993 \\
\hline
\end{tabular}

- The next step was to express the regression coefficients found above as a function of the asphalt layer thickness $\left(\mathrm{T}_{\mathrm{a}}\right)$. Once again, through the help of the DataFit software developed, the following empirical equation was found to give satisfactory results:

Re gression_coeff (i.e, a $)=A+\frac{B}{x}+\frac{C}{x^{2}}+\frac{D}{x^{3}}+\frac{E}{x^{4}}$

Where $\mathrm{x}$ is the asphalt layer thickness ( $T_{\mathrm{a}}$ in $\mathrm{m}$ ) and the coefficients $A$ to $E$ have the values shown in

Table 8.3.

Table 8.3 Regression coefficients of eq. (8.5)

\begin{tabular}{|l|l|l|l|l|l|l|}
\hline $\begin{array}{l}\text { Reg. } \\
\text { Coef }\end{array}$ & $\begin{array}{l}\text { Model; } \mathrm{x}=\text { asphalt } \\
\text { thickness }(\mathrm{Ta})\end{array}$ & $\mathrm{B}$ & $\mathrm{C}$ & $\mathrm{D}$ \\
\hline a & $\mathrm{a}=\mathrm{A}+\mathrm{B} / \mathrm{x}+\mathrm{C} / \mathrm{x}^{2}+\mathrm{D} / \mathrm{x}^{3}+\mathrm{E} / \mathrm{x}^{4}$ & 26.606967401 & 129.912891954 & -49.319548592 & 7.301332943 & -0.345622247 \\
\hline $\mathrm{b}$ & $\mathrm{b}=\mathrm{A}+\mathrm{B} / \mathrm{x}+\mathrm{C} / \mathrm{x}^{2}+\mathrm{D} / \mathrm{x}^{3}+\mathrm{E} / \mathrm{x}^{4}$ & -87667.529214 & -535073.852860 & 213149.524856 & -32034.952778 & 1521.194882 \\
\hline c & $\mathrm{c}=\mathrm{A}+\mathrm{B} / \mathrm{x}+\mathrm{C} / \mathrm{x}^{2}+\mathrm{D} / \mathrm{x}^{3}+\mathrm{E} / \mathrm{x}^{4}$ & 0.1691763061 & -0.7465396808 & 0.3210524112 & -0.0378270353 & 0.0014378311 \\
\hline $\mathrm{d}$ & $\mathrm{d}=\mathrm{A}+\mathrm{B} / \mathrm{x}+\mathrm{C} / \mathrm{x}^{2}+\mathrm{D} / \mathrm{x}^{3}+\mathrm{E} / \mathrm{x}^{4}$ & 201197630.36 & 361553695.08 & -176829148.36 & 30981138.47 & -1588370.81 \\
\hline e & $\mathrm{e}=\mathrm{A}+\mathrm{B} / \mathrm{x}+\mathrm{C} / \mathrm{x}^{2}+\mathrm{D} / \mathrm{x}^{3}+\mathrm{E} / \mathrm{x}^{4}$ & 0.000715335 & 0.000284491 & -0.000112130 & 0.000010891 & -0.000000320 \\
\hline $\mathrm{f}$ & $\mathrm{f}=\mathrm{A}+\mathrm{B} / \mathrm{x}+\mathrm{C} / \mathrm{x}^{2}+\mathrm{D} / \mathrm{x}^{3}+\mathrm{E} / \mathrm{x}^{4}$ & 668.2107947 & 1779.5723425 & -634.9457631 & 71.8146871 & -2.6928747 \\
\hline
\end{tabular}

The improved results derived by implementing equations (8.4), (8.5) and (8.6) in the modelling of the Equivalent Foundation Modulus for the transformed 2layer pavement structure can be seen in Figure 8.9. 


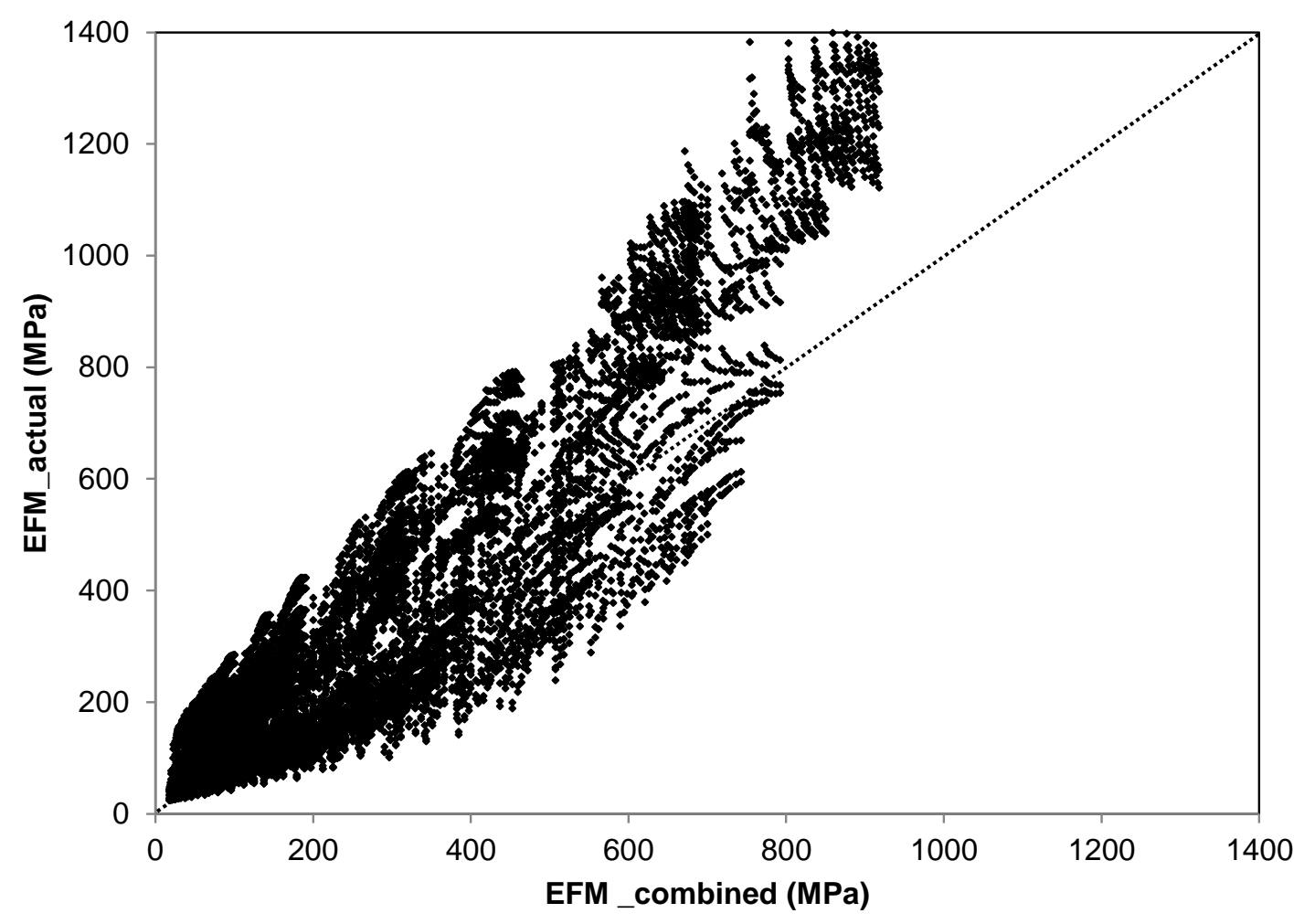

Figure 8.8 EFM calculated with eq. (8.4) versus actual EFM (calculated with Matlab)

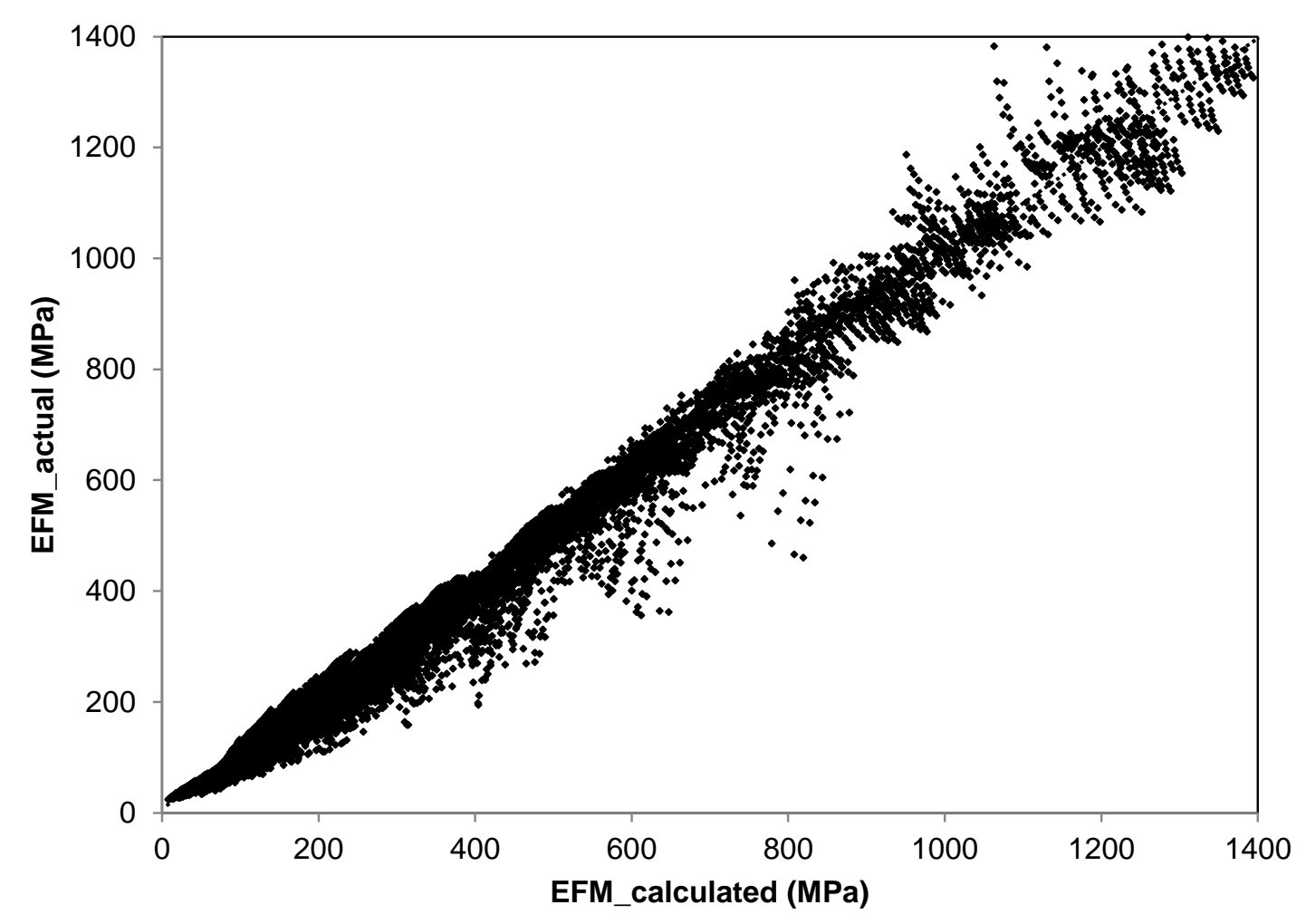

Figure 8.9 EFM calculated with (8.5) versus actual EFM (calculated with Matlab) 


\subsubsection{Model for asphalt strains}

In order to implement the proposed model for the calculation of asphalt strains, the original 3-layer pavement structure must first be transformed into a 2-layer pavement structure by applying (8.4), (8.5) and (8.6). The MET method is then applied to the derived 2-layer pavement structure to calculate the strain at the bottom of the asphalt layer. After applying the conditions of validity of the MET method to the transformed 2-layer structure (i.e., Ea / EFM_calculated $>2$ and $\mathrm{h}_{\mathrm{e}, 2}>\mathrm{a}$ ) a total of 18597 out of the original 19024 combinations were considered.

The plot of the asphalt tensile strains calculated with the MET method on the derived 2-layer pavement structure versus the asphalt tensile strains calculated with the BISAR software on the original 3-layer pavement structure is illustrated in Figure 8.10. Figure 8.10 shows a clear improvement on the original model shown in Figure 8.2.

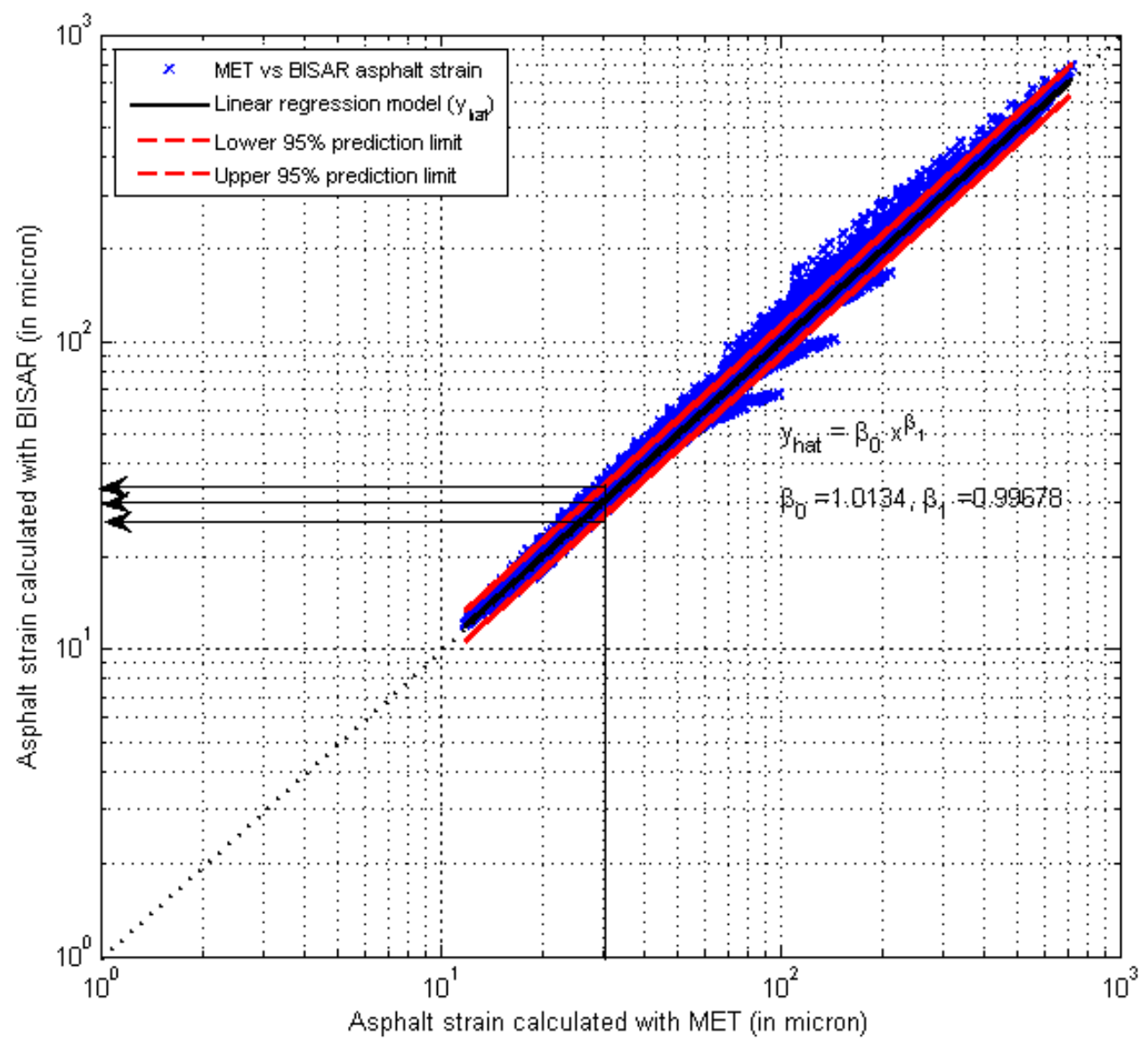

Figure 8.10 Linear regression analysis for the asphalt strain with proposed model 
The results of the linear regression analysis together with a plot of the $95 \%$ prediction limits and the line of best fit are shown in Figure 8.10. For example, from Figure 8.10 it can be seen that, for an asphalt strain of 30 microstrain, calculated with the MET method on the 2-layer pavement structure, it is expected that:

- The best-fit predicted value of the BISAR asphalt strain is 30 microstrain

- The predicted $95 \%$ lower limit of the BISAR asphalt strain is 27 microstrain

- The predicted $95 \%$ upper limit of the BISAR asphalt strain is 33 microstrain It can be seen that the proposed model offers a much better estimate of the asphalt tensile strain values when compared to the uncorrected MET approach, $93 \%$ of predictions falling within $10 \%$ of the BISAR-derived strain (compared to $14 \%$ for the uncorrected MET approach).

\subsubsection{Model for performance prediction}

The fatigue life was calculated for the asphalt strains derived using the alternative model. The results of the linear regression analysis together with a plot of the $95 \%$ prediction limits are shown in Figure 8.11, together with the line of best fit. 


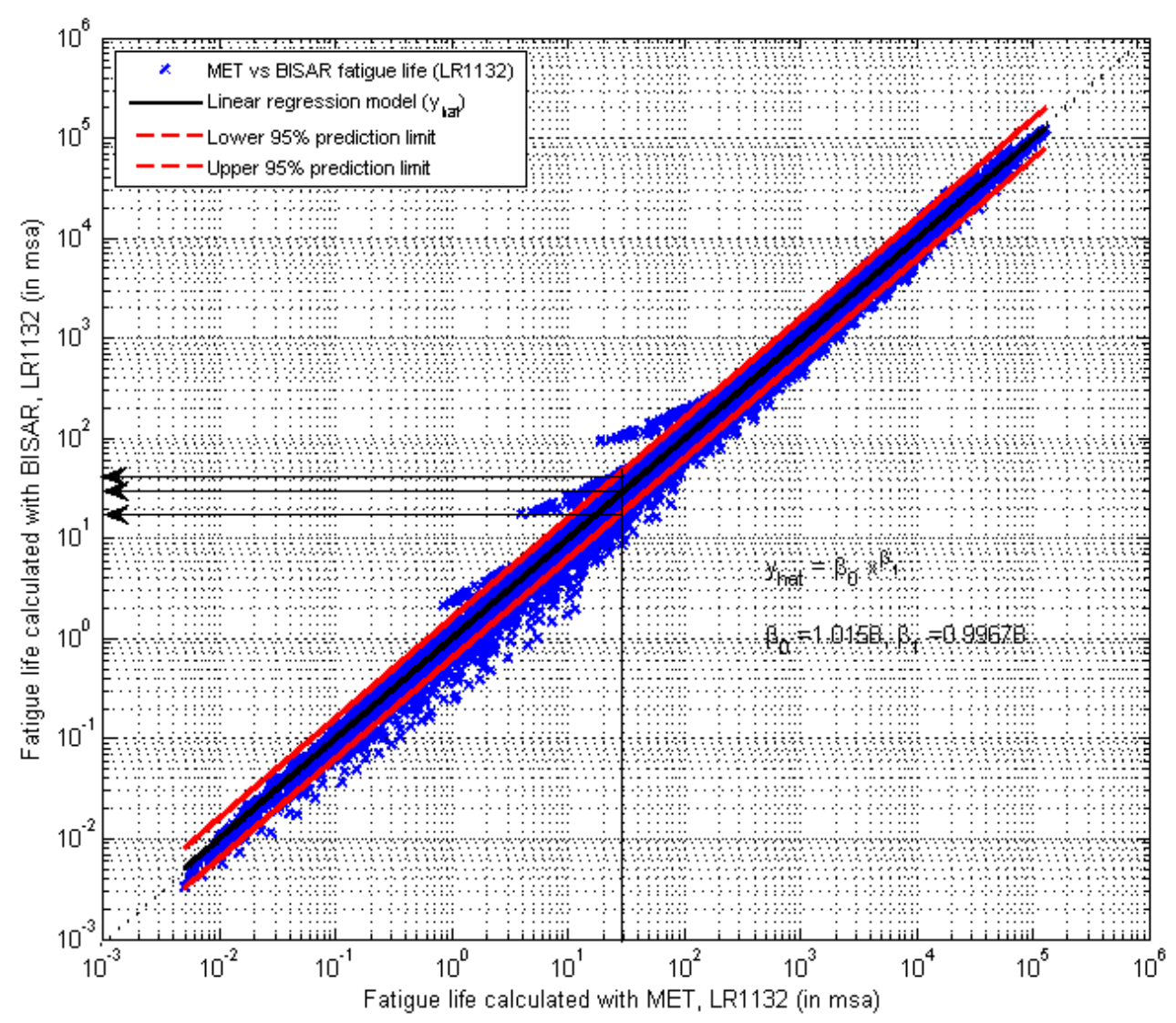

Figure 8.11 Linear regression analysis for the fatigue life with proposed model

For example, from Figure 8.11 it can be seen that, for a calculated asphalt fatigue life of $30 \mathrm{msa}$, it is expected that:

- The best-fit predicted value of the BISAR fatigue life is $31 \mathrm{msa}$

- $\quad$ The predicted $95 \%$ lower limit of the BISAR fatigue life is $21 \mathrm{msa}$

- $\quad$ The predicted $95 \%$ upper limit of the BISAR fatigue life is $45 \mathrm{msa}$

It can be seen that the proposed model offers a much better estimate of the mean asphalt fatigue life when compared to the traditional 3-layer pavement structure model in Figure 8.4. The width of the prediction interval is also greatly reduced. Therefore, this fatigue model will be used in this research.

\subsubsection{Regression analysis for alternative deformation model - 3-layer pavement structure}

This section discusses an alternative deformation model developed in a similar way to that presented in sections 8.1.1.1 and 8.1.1.2 but which accepts a 
'relaxation' in one of the MET conditions. The proposed relaxation is for the ratio of subbase stiffness over subgrade stiffness to be greater than or equal to 1 while keeping all other conditions of validity of the MET method (i.e., $E_{a} / E_{s b}>2$ and $E_{s b} / E_{s g}>=1 ; h_{e, 2}>a$ and $h_{e, 3}>a$, where $h_{e, 2}$ and $h_{e, 3}$ are the transformed asphalt and subbase layer respectively). The reason for introducing this alternative model lies in the observation that the ratios of subbase stiffness to subgrade stiffness resulting from backanalysis from real site data (see next chapter) was in many cases lower than 2. Therefore, in order not to discard important information about real site variability an alternative model is suggested for both the calculation of subgrade strains and deformation life.

The alternative model for subgrade strain is shown in Figure 8.12 while the alternative model for deformation life is shown in Figure 8.13. The following observations are made:

- The MET/BISAR subgrade strain ratio with the alternative model in Figure 8.12 varies from 0.83 to 1.40 which is not very different from the values reported in section 8.1.1.1 (i.e., 0.83 to 1.18 ).

- The MET/BISAR deformation life ratio with the alternative model in Figure 8.13 varies from 0.27 to 2.05 which is also not very different from the values obtained using the model discussed in 8.1.1.2 (i.e., 0.52 to 2.05).

- Following the above bullet points, because the variability introduced by the alternative model is not excessive, it is considered acceptable to use the alternative model for the calculation of subgrade strain and deformation life. Therefore, the alternative deformation model will be used in this research. 


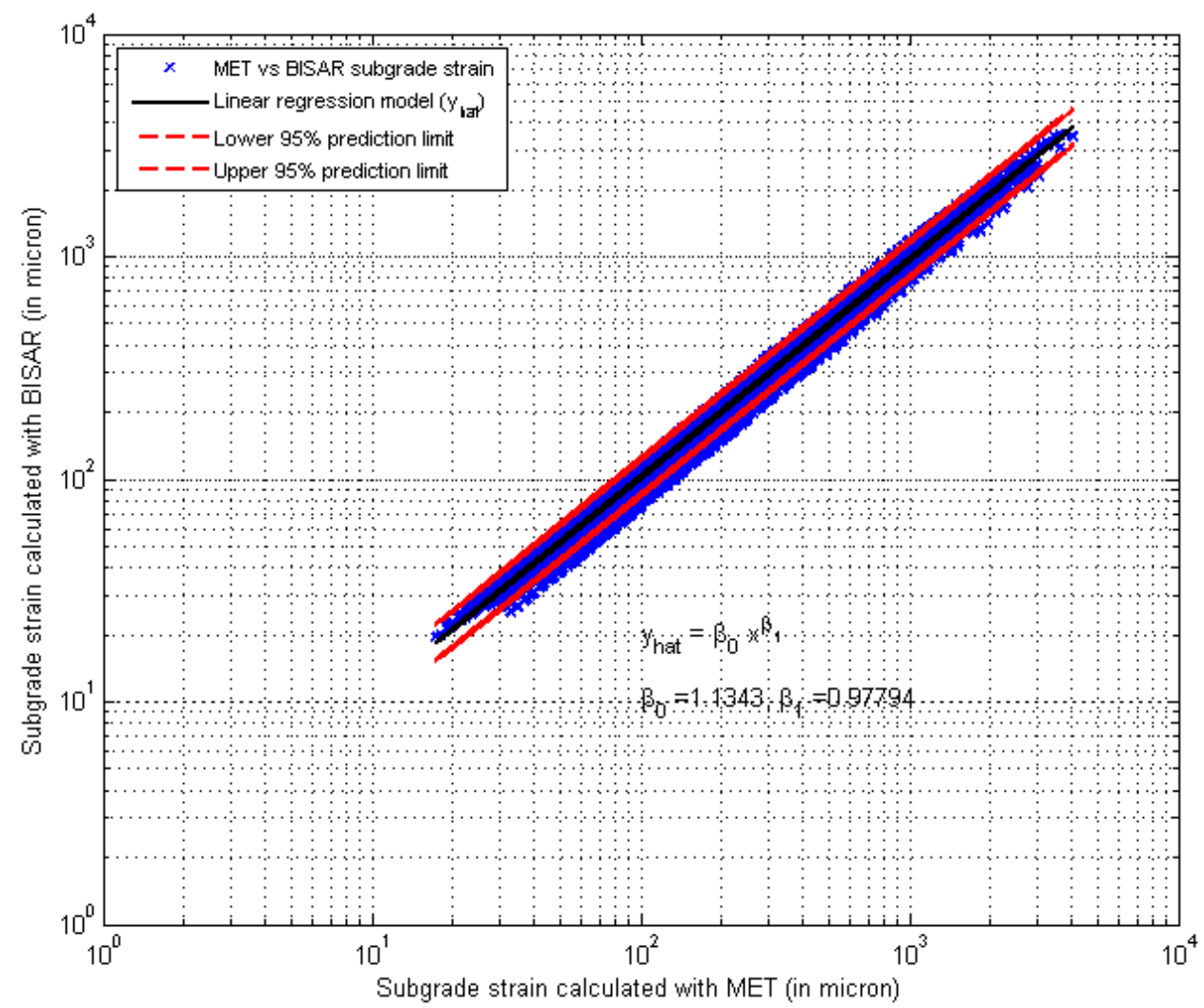

Figure 8.12 Linear regression analysis for the subgrade strain with alternative model

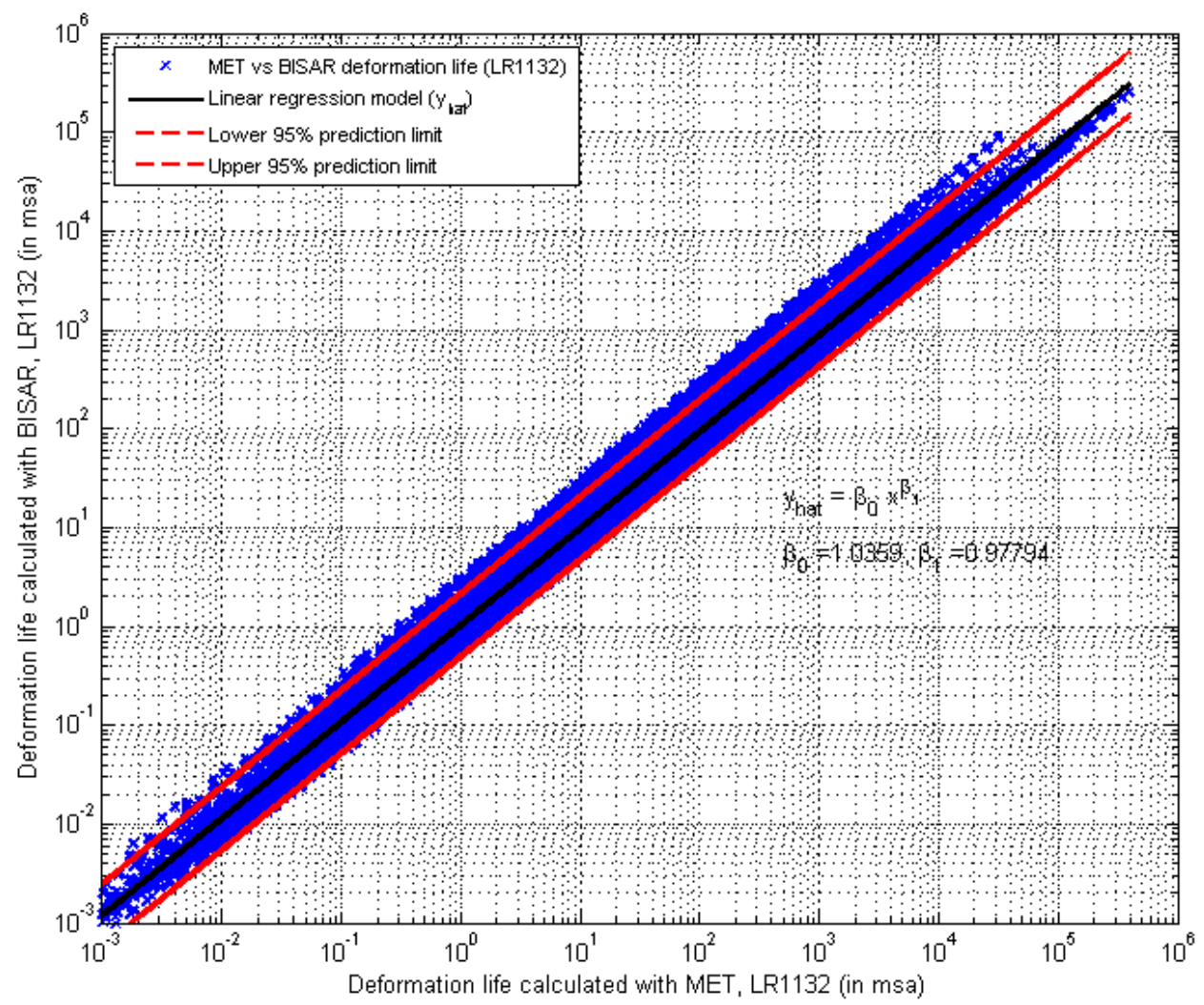

Figure 8.13 Linear regression analysis for the deformation life with alternative model 


\subsubsection{Model correction}

This section explores the characteristics of the proposed models - the fatigue and deformation models - in Figure 8.11 and Figure 8.13 - in terms of the ratio of the fatigue and deformation life (with LR1132 equations) predicted by the models (denoted here as $\mathrm{Nf}_{\text {model }}$ and $\mathrm{Nd} \mathrm{d}_{\text {model }}$ ) over the life calculated with BISAR (denoted here as $\mathrm{Nf}_{\mathrm{BISAR}}$ and NdBISAR), for different ranges of asphalt and subbase thicknesses, see Table 8.4 and Table 8.5.

Table 8.4 and Table 8.5 show, for each range of asphalt and subbase thicknesses and for those points satisfying the model conditions (see section 8.1.2.1 and 8.1), the following parameters:

- the $15^{\text {th }}, 50^{\text {th }}$ and $85^{\text {th }}$ percentile calculated from the actual ratio values (i.e.

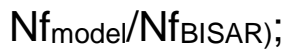

- the mean and standard deviation, and then the derived $15^{\text {th }}$ and $85^{\text {th }}$ percentile assuming a normal distribution for the ratio values;

- the mean and standard deviation, and then the derived $15^{\text {th }}$ and $85^{\text {th }}$ percentile assuming a lognormal distribution for the ratio values. The above parameters were calculated by estimating first the same parameters for the corresponding normal distribution (i.e. $\ln \left(\mathrm{Nf}_{\text {model }} / \mathrm{Nf}_{\mathrm{BISAR}}\right)$ ).

By looking at the shaded cells at the bottom of Table 8.4 and Table 8.5, which represent the overall values of the parameters in the bullet points above for the whole range of thicknesses considered, the following observations can be made:

- The ratio of the life - both fatigue and deformation with LR1132 equationspredicted by the model over the life calculated with BISAR can be approximated by a normal probability distribution. This is because the mean and percentiles calculated with the assumptions of normal probability distribution are not very different from those calculated from the percentiles of the ratios.

- For asphalt thicknesses up to $0.5 \mathrm{~m}$ and subbase thicknesses up to $1 \mathrm{~m}$ (i.e., all combinations considered in the model), the overall mean and standard deviation for the ratio of fatigue life predicted by the proposed model over 
the life calculated with BISAR (i.e., $\mathrm{Nf}_{\text {model }} / \mathrm{Nf}_{\mathrm{BISAR}}$ ) are 1.03 and 0.30 respectively.

- For asphalt thicknesses up to $0.5 \mathrm{~m}$ and subbase thicknesses up to $0.5 \mathrm{~m}$ (i.e., representative of typical newly constructed pavements), the overall mean and standard deviation for the ratio of fatigue life predicted by the proposed model over the life calculated with BISAR (i.e., $\mathrm{Nf}_{\text {model }} / \mathrm{Nf}_{\mathrm{BISAR}}$ ) are 1.09 and 0.35 .respectively.

- For asphalt thicknesses up to $0.5 \mathrm{~m}$ and subbase thicknesses up to $1 \mathrm{~m}$, the overall mean and standard deviation for the ratio of deformation life predicted by the proposed model over the life calculated with BISAR (i.e., $\mathrm{Nd}$ model/ $/ \mathrm{Nd}_{\mathrm{BISAR}}$ ) are 1.06 and 0.33 respectively.

- For asphalt thicknesses up to $0.5 \mathrm{~m}$ and subbase thicknesses up to $0.5 \mathrm{~m}$, the overall mean and standard deviation for the ratio of deformation life predicted by the proposed model over the life calculated with BISAR (i.e., $\mathrm{Nd}$ model/NdBISAR) are 1.07 and 0.34 respectively.

To account for the mean and standard deviation of the model bias (or of the proportional error), some corrections could be applied to the predicted (with model) life mean (i.e., $\mu \mathrm{Nf}$ ) and standard deviation values (i.e., $\sigma \mathrm{Nf}$ ) (both in msa). For example, considering the ratio parameters discussed above for asphalt thicknesses up to $0.5 \mathrm{~m}$ and subbase thicknesses up to $0.5 \mathrm{~m}$, the correction for the mean value is:

$$
\mu_{N_{f}}(\text { corrected })=\frac{\mu_{N_{f}}}{1.09}
$$

As for the correction of the standard deviation, the "real" coefficient of variation of the error needs to be calculated first:

$$
\operatorname{COV}_{\text {real }}=\sqrt{\left[\left(\frac{\sigma_{N_{f}}}{\mu_{N_{f}}}\right)^{2}-\left(\frac{0.35}{1.09}\right)^{2}\right]}
$$

The correction for the standard deviation is then:

$$
\sigma_{N_{f}}(\text { corrected })=\mu_{N_{f}}(\text { corrected }) * C O V_{\text {real }}
$$


As the error is not really random, in order to quantify systematic error of the design procedure it is recommended that only the correction to the mean is applied. The ratio $1 / 1.09$ (or 0.917 ) is often called 'professional' factor, defined as a representative ratio of the measured to predicted pavement performance, (also used by Kim, 2006). This factor represents uncertainties of the assumptions and simplifications used in the proposed design model. 
Table 8.4 Ratio of fatigue life calculated with model over fatigue life calculated with BISAR

\begin{tabular}{|c|c|c|c|c|c|}
\hline \multirow{3}{*}{\begin{tabular}{|l|} 
Asphalt \\
thickness \\
$(\mathrm{m})$
\end{tabular}} & \multirow{3}{*}{$\begin{array}{l}\begin{array}{l}\text { Subbase } \\
\text { thickness } \\
(\mathrm{m})\end{array}\end{array}$} & \multirow{2}{*}{\multicolumn{4}{|c|}{$\frac{\text { FROM PERCENTILES }}{\mathrm{Nf}_{\text {model }} / \mathrm{NI}_{\text {BISAR }} \text { (LR1132) }}$}} \\
\hline & & & & & \\
\hline & & $\begin{array}{l}15 \text { th } \\
\text { per }\end{array}$ & $\begin{array}{l}50 \text { th } \\
\text { per }\end{array}$ & $\begin{array}{l}85 \text { th } \\
\text { perc }\end{array}$ & $\begin{array}{l}\text { Nr pts thai } \\
\text { satisfy } \\
\text { model } \\
\text { conds }\end{array}$ \\
\hline 0.100 & 0.100 & 1.00 & 1.41 & 2.59 & 371 \\
\hline 0.100 & 0.150 & 1.09 & 1.31 & 1.67 & 169 \\
\hline 0.100 & 0.200 & 0.94 & 1.09 & 1.38 & 352 \\
\hline 0.100 & 0.300 & 0.87 & 0.94 & 1.09 & 357 \\
\hline 0.100 & 0.400 & 0.83 & 0.88 & 1.02 & 346 \\
\hline 0.100 & 0.500 & 0.80 & 0.87 & 1.01 & 338 \\
\hline 0.100 & 0.600 & 0.80 & 0.88 & 1.00 & 204 \\
\hline 0.100 & 0.700 & 0.80 & 0.89 & 1.01 & 195 \\
\hline 0.100 & 0.800 & 0.80 & 0.89 & 1.02 & 188 \\
\hline 0.100 & 0.900 & 0.81 & 0.90 & 1.04 & 188 \\
\hline 0.100 & 1.000 & 0.81 & 0.91 & 1.06 & 188 \\
\hline 0.150 & 0.100 & 0.99 & 1.24 & 1.93 & 225 \\
\hline 0.150 & 0.150 & 1.07 & 1.29 & 1.64 & 185 \\
\hline 0.150 & 0.200 & 0.93 & 1.07 & 1.30 & 225 \\
\hline 0.150 & 0.300 & 0.89 & 0.98 & 1.09 & 225 \\
\hline 0.150 & 0.400 & 0.87 & 0.94 & 1.01 & 225 \\
\hline 0.150 & 0.500 & 0.87 & 0.92 & 0.99 & 225 \\
\hline 0.150 & 0.600 & 0.86 & 0.91 & 1.00 & 225 \\
\hline 0.150 & 0.700 & 0.86 & 0.93 & 1.01 & 225 \\
\hline 0.150 & 0.800 & 0.86 & 0.94 & 1.05 & 225 \\
\hline 0.150 & 0.900 & 0.86 & 0.94 & 1.08 & 223 \\
\hline 0.150 & 1.000 & 0.85 & 0.95 & 1.10 & 220 \\
\hline 0.200 & 0.100 & 0.93 & 1.16 & 1.81 & 387 \\
\hline 0.200 & 0.150 & 1.03 & 1.23 & 1.53 & 185 \\
\hline 0.200 & 0.200 & 0.91 & 1.06 & 1.44 & 385 \\
\hline 0.200 & 0.300 & 0.88 & 1.00 & 1.19 & 389 \\
\hline 0.200 & 0.400 & 0.87 & 0.95 & 1.05 & 386 \\
\hline 0.200 & 0.500 & 0.87 & 0.93 & 1.00 & 389 \\
\hline 0.200 & 0.600 & 0.86 & 0.90 & 0.97 & 240 \\
\hline 0.200 & 0.700 & 0.84 & 0.90 & 0.99 & 240 \\
\hline 0.200 & 0.800 & 0.83 & 0.91 & 1.01 & 240 \\
\hline 0.200 & 0.900 & 0.83 & 0.91 & 1.05 & 240 \\
\hline 0.200 & 1.000 & 0.82 & 0.92 & 1.09 & 239 \\
\hline 0.300 & 0.100 & 0.86 & 1.08 & 1.59 & 387 \\
\hline 0.300 & 0.150 & 0.96 & 1.14 & 1.40 & 185 \\
\hline 0.300 & 0.200 & 0.86 & 1.05 & 1.40 & 387 \\
\hline 0.300 & 0.300 & 0.85 & 1.01 & 1.25 & 395 \\
\hline 0.300 & 0.400 & 0.84 & 0.98 & 1.14 & 381 \\
\hline 0.300 & 0.500 & 0.84 & 0.96 & 1.09 & 381 \\
\hline 0.300 & 0.600 & 0.83 & 0.92 & 1.01 & 240 \\
\hline 0.300 & 0.700 & 0.82 & 0.92 & 1.01 & 240 \\
\hline 0.300 & 0.800 & 0.81 & 0.91 & 1.02 & 240 \\
\hline 0.300 & 0.900 & 0.80 & 0.91 & 1.05 & 240 \\
\hline 0.300 & 1.000 & 0.79 & 0.91 & 1.08 & 240 \\
\hline 0.400 & 0.100 & 0.80 & 1.04 & 1.44 & 387 \\
\hline 0.400 & 0.150 & 0.89 & 1.08 & 1.33 & 185 \\
\hline 0.400 & 0.200 & 0.82 & 1.03 & 1.40 & 385 \\
\hline 0.400 & 0.300 & 0.81 & 1.02 & 1.32 & 387 \\
\hline 0.400 & 0.400 & 0.81 & 0.99 & 1.23 & 384 \\
\hline 0.400 & 0.500 & 0.81 & 0.96 & 1.17 & 386 \\
\hline 0.400 & 0.600 & 0.80 & 0.94 & 1.08 & 240 \\
\hline 0.400 & 0.700 & 0.79 & 0.92 & 1.04 & 240 \\
\hline 0.400 & 0.800 & 0.78 & 0.91 & 1.04 & 240 \\
\hline 0.400 & 0.900 & 0.78 & 0.92 & 1.04 & 240 \\
\hline 0.400 & 1.000 & 0.76 & 0.92 & 1.06 & 240 \\
\hline 0.500 & 0.100 & 0.77 & 1.02 & 1.44 & 384 \\
\hline 0.500 & 0.150 & 0.85 & 1.05 & 1.27 & 185 \\
\hline 0.500 & 0.200 & 0.79 & 1.01 & 1.41 & 394 \\
\hline 0.500 & 0.300 & 0.79 & 1.00 & 1.36 & 391 \\
\hline 0.500 & 0.400 & 0.79 & 1.00 & 1.27 & 383 \\
\hline 0.500 & 0.500 & 0.79 & 0.97 & 1.22 & 386 \\
\hline 0.500 & 0.600 & 0.78 & 0.94 & 1.10 & 240 \\
\hline 0.500 & 0.700 & 0.78 & 0.93 & 1.09 & 240 \\
\hline 0.500 & 0.800 & 0.77 & 0.92 & 1.06 & 240 \\
\hline 0.500 & 0.900 & 0.76 & 0.91 & 1.05 & 240 \\
\hline 0.500 & 1.000 & 0.76 & 0.91 & 1.05 & 240 \\
\hline 0 to $0.5 \mathrm{~m}$ & 0 to $1 \mathrm{~m}$ & 0.83 & 0.96 & 1.22 & 18597 \\
\hline 0 to $0.5 \mathrm{~m}$ & 0 to $0.5 \mathrm{~m}$ & 0.85 & 1.01 & 1.34 & 11717 \\
\hline
\end{tabular}

\begin{tabular}{|c|c|c|c|c|c|}
\hline \multicolumn{6}{|c|}{ ASSUMING NORMAL DISTRIBUTION } \\
\hline \multicolumn{6}{|c|}{$\mathrm{Ni}_{\text {model }} / \mathrm{Ni}_{\text {BISAR }}$ (LR1 132) } \\
\hline $\begin{array}{l}15 \text { th } \\
\text { per }\end{array}$ & mean & $\begin{array}{l}\text { 85th } \\
\text { perc }\end{array}$ & stdev & cov & $\begin{array}{l}\text { Nr pts that } \\
\text { satisfy } \\
\text { model } \\
\text { conds }\end{array}$ \\
\hline 0.83 & 1.74 & 2.65 & 0.91 & 0.52 & 371 \\
\hline 1.10 & 1.37 & 1.65 & 0.28 & 0.20 & 169 \\
\hline 0.93 & 1.15 & 1.36 & 0.22 & 0.19 & 352 \\
\hline 0.85 & 0.98 & 1.11 & 0.13 & 0.14 & 357 \\
\hline 0.80 & 0.92 & 1.03 & 0.11 & 0.12 & 346 \\
\hline 0.78 & 0.90 & 1.01 & 0.11 & 0.13 & 338 \\
\hline 0.80 & 0.90 & 1.00 & 0.10 & 0.11 & 204 \\
\hline 0.80 & 0.90 & 1.00 & 0.10 & 0.11 & 195 \\
\hline 0.80 & 0.91 & 1.02 & 0.11 & 0.12 & 188 \\
\hline 0.81 & 0.92 & 1.03 & 0.11 & 0.12 & 188 \\
\hline 0.81 & 0.93 & 1.05 & 0.12 & 0.13 & 188 \\
\hline 0.92 & 1.41 & 1.91 & 0.49 & 0.35 & 225 \\
\hline 1.08 & 1.35 & 1.63 & 0.28 & 0.20 & 185 \\
\hline 0.91 & 1.11 & 1.30 & 0.20 & 0.18 & 225 \\
\hline 0.87 & 0.98 & 1.10 & 0.11 & 0.12 & 225 \\
\hline 0.86 & 0.94 & 1.02 & 0.08 & 0.09 & 225 \\
\hline 0.86 & 0.92 & 0.99 & 0.06 & 0.07 & 225 \\
\hline 0.87 & 0.93 & 0.99 & 0.06 & 0.07 & 225 \\
\hline 0.86 & 0.94 & 1.01 & 0.08 & 0.08 & 225 \\
\hline 0.86 & 0.95 & 1.05 & 0.10 & 0.10 & 225 \\
\hline 0.85 & 0.96 & 1.08 & 0.11 & 0.12 & 223 \\
\hline 0.85 & 0.98 & 1.10 & 0.13 & 0.13 & 220 \\
\hline 0.83 & 1.34 & 1.85 & 0.51 & 0.38 & 387 \\
\hline 1.03 & 1.27 & 1.51 & 0.24 & 0.19 & 185 \\
\hline 0.88 & 1.15 & 1.42 & 0.27 & 0.24 & 385 \\
\hline 0.87 & 1.03 & 1.19 & 0.16 & 0.16 & 389 \\
\hline 0.86 & 0.96 & 1.07 & 0.10 & 0.11 & 386 \\
\hline 0.85 & 0.93 & 1.01 & 0.08 & 0.09 & 389 \\
\hline 0.84 & 0.91 & 0.98 & 0.07 & 0.07 & 240 \\
\hline 0.84 & 0.91 & 0.99 & 0.08 & 0.08 & 240 \\
\hline 0.83 & 0.92 & 1.02 & 0.10 & 0.10 & 240 \\
\hline 0.82 & 0.94 & 1.05 & 0.12 & 0.13 & 240 \\
\hline 0.81 & 0.95 & 1.08 & 0.13 & 0.14 & 239 \\
\hline 0.80 & 1.19 & 1.58 & 0.39 & 0.33 & 387 \\
\hline 0.97 & 1.17 & 1.37 & 0.20 & 0.17 & 185 \\
\hline 0.83 & 1.13 & 1.42 & 0.29 & 0.26 & 387 \\
\hline 0.83 & 1.05 & 1.27 & 0.22 & 0.21 & 395 \\
\hline 0.83 & 1.00 & 1.17 & 0.17 & 0.17 & 381 \\
\hline 0.82 & 0.96 & 1.11 & 0.14 & 0.15 & 381 \\
\hline 0.81 & 0.92 & 1.04 & 0.11 & 0.12 & 240 \\
\hline 0.80 & 0.92 & 1.03 & 0.11 & 0.13 & 240 \\
\hline 0.80 & 0.92 & 1.04 & 0.12 & 0.13 & 240 \\
\hline 0.79 & 0.92 & 1.05 & 0.13 & 0.14 & 240 \\
\hline 0.79 & 0.93 & 1.07 & 0.14 & 0.15 & 240 \\
\hline 0.75 & 1.12 & 1.50 & 0.37 & 0.33 & 387 \\
\hline 0.91 & 1.10 & 1.30 & 0.20 & 0.18 & 185 \\
\hline 0.79 & 1.10 & 1.42 & 0.32 & 0.29 & 385 \\
\hline 0.79 & 1.06 & 1.33 & 0.27 & 0.26 & 387 \\
\hline 0.79 & 1.01 & 1.24 & 0.23 & 0.22 & 384 \\
\hline 0.78 & 0.98 & 1.18 & 0.20 & 0.20 & 386 \\
\hline 0.78 & 0.94 & 1.10 & 0.16 & 0.17 & 240 \\
\hline 0.77 & 0.93 & 1.08 & 0.16 & 0.17 & 240 \\
\hline 0.76 & 0.92 & 1.08 & 0.16 & 0.17 & 240 \\
\hline 0.76 & 0.92 & 1.08 & 0.16 & 0.17 & 240 \\
\hline 0.76 & 0.92 & 1.08 & 0.16 & 0.18 & 240 \\
\hline 0.73 & 1.09 & 1.46 & \begin{tabular}{|l|}
0.37 \\
\end{tabular} & 0.34 & 384 \\
\hline 0.86 & 1.06 & 1.25 & 0.20 & 0.18 & 185 \\
\hline 0.75 & 1.08 & 1.41 & 0.33 & 0.30 & 394 \\
\hline 0.76 & 1.06 & 1.36 & 0.30 & 0.28 & 391 \\
\hline 0.77 & 1.02 & 1.28 & \begin{tabular}{|l|}
0.25 \\
\end{tabular} & 0.25 & 383 \\
\hline 0.77 & 1.00 & 1.22 & 0.23 & 0.23 & 386 \\
\hline 0.76 & 0.95 & 1.13 & 0.18 & 0.20 & 240 \\
\hline 0.76 & 0.93 & 1.11 & 0.18 & 0.19 & 240 \\
\hline 0.75 & 0.92 & 1.10 & 0.17 & 0.19 & 240 \\
\hline 0.75 & 0.92 & 1.09 & 0.17 & 0.19 & 240 \\
\hline 0.74 & 0.92 & 1.09 & \begin{tabular}{|l|}
0.17 \\
\end{tabular} & 0.19 & 240 \\
\hline 0.74 & 1.03 & 1.33 & 0.30 & 0.29 & 18597 \\
\hline 0.75 & 1.09 & 1.44 & 0.35 & 0.32 & 11717 \\
\hline
\end{tabular}
$\mathrm{Ni}_{\text {model }} / \mathrm{Ni}_{\text {BISAR }}$ (LR1132)
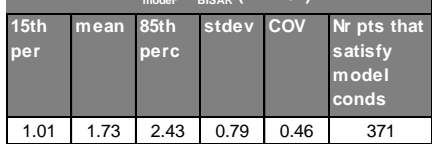

\begin{tabular}{l|l|l|l|l|l|}
\hline 1.11 & 1.37 & 1.64 & 0.27 & 0.20 & 169 \\
\hline 0.94 & 1.15 & 1.35 & 0.21 & 0.18 & 352 \\
\hline
\end{tabular}

\begin{tabular}{|l|l|l|l|l|l|}
\hline 0.94 & 1.15 & 1.35 & 0.21 & 0.18 & 352 \\
\hline 0.86 & 0.98 & 1.10 & 0.12 & 0.12 & 357 \\
\hline 0.81 & 0.92 & 1.02 & 0.11 & 0.12 & 346 \\
\hline
\end{tabular}

\begin{tabular}{|c|c|c|c|c|c|}
\hline 0.81 & 0.92 & 1.02 & 0.11 & 0.12 & 346 \\
\hline 0.79 & 0.90 & 1.00 & 0.11 & 0.12 & 338 \\
\hline
\end{tabular}

\begin{tabular}{|l|l|l|l|l|l|}
\hline 0.79 & 0.90 & 1.00 & 0.11 & 0.12 & 338 \\
\hline 0.80 & 0.90 & 0.99 & 0.10 & 0.11 & 204 \\
\hline
\end{tabular}

\begin{tabular}{l|l|l|l|l|l|l|}
\hline 0.80 & 0.90 & 0.99 & 0.10 & 0.11 & 204 \\
\hline 0.80 & 0.90 & 1.00 & 0.10 & 0.11 & \\
\hline
\end{tabular}

\begin{tabular}{|l|l|l|l|l|l|}
\hline 0.80 & 0.90 & 1.00 & 0.10 & 0.11 & 195 \\
\hline 0.81 & 0.91 & 1.01 & 0.10 & 0.11 & 188 \\
\hline 0.81 & 0.92 & 1.03 & 0.11 & 0.12 & 188 \\
\hline
\end{tabular}

\begin{tabular}{|l|l|l|l|l|l|}
\hline 0.81 & 0.91 & 1.01 & 0.10 & 0.11 & 188 \\
\hline 0.81 & 0.92 & 1.03 & 0.11 & 0.12 & 188 \\
\hline 0.82 & 0.93 & 1.04 & 0.12 & 0.12 & 188 \\
\hline
\end{tabular}

\begin{tabular}{|l|l|l|l|l|l|}
\hline 0.82 & 0.93 & 1.04 & 0.12 & 0.12 & 188 \\
\hline 0.9 & 1.41 & 1.83 & 0.44 & 0.32 & 225 \\
\hline
\end{tabular}

\begin{tabular}{|l|l|l|l|l|l|}
\hline 0.99 & 1.41 & 1.83 & 0.44 & 0.32 & 225 \\
\hline
\end{tabular}

\begin{tabular}{|l|l|l|l|l|l|l|}
\hline 1.09 & 1.35 & 1.61 & 0.27 & 0.20 & 185 \\
\hline 0.92 & 1.11 & 1.30 & 0.19 & 0.18 & 225 \\
\hline
\end{tabular}

\begin{tabular}{|l|l|l|l|l|l|}
\hline 0.92 & 1.11 & 1.30 & 0.19 & 0.18 & 225 \\
\hline 0.86 & 0.98 & 1.10 & 0.12 & 0.12 & 225 \\
\hline 0.85 & 0.94 & 1.02 & 0.08 & 0.09 & 225 \\
\hline
\end{tabular}

\begin{tabular}{|c|c|c|c|c|c|}
\hline 0.85 & 0.94 & 1.02 & 0.08 & 0.09 & 225 \\
\hline 0.86 & 0.92 & 0.9 & 0.06 & 0.07 & 225 \\
\hline
\end{tabular}

\begin{tabular}{l|l|l|l|l|l|l|}
\hline 0.86 & 0.92 & 0.99 & 0.06 & 0.07 & 225 \\
\hline
\end{tabular}

\begin{tabular}{|l|l|l|l|l|l|}
\hline 0.87 & 0.93 & 0.99 & 0.06 & 0.07 & 225 \\
\hline 0.86 & 0.94 & 1.01 & 0.07 & 0.08 & 225 \\
\hline
\end{tabular}

\begin{tabular}{|l|l|l|l|l|l|}
\hline 0.86 & 0.94 & 1.01 & 0.07 & 0.08 & 225 \\
\hline 0.86 & 0.95 & 1.05 & 0.09 & 0.10 & 225 \\
\hline 0.86 & 0.96 & 1.07 & 0.11 & 0.11 & 223 \\
\hline
\end{tabular}

\begin{tabular}{|l|l|l|l|l|l|}
\hline 0.86 & 0.96 & 1.07 & 0.11 & 0.11 & 223 \\
\hline
\end{tabular}

\begin{tabular}{|l|l|l|l|l|l|}
\hline 0.86 & 0.98 & 1.09 & 0.12 & 0.12 & 220 \\
\hline 0.91 & 1.33 & 1.75 & 0.44 & 0.33 & 387 \\
\hline
\end{tabular}

\begin{tabular}{|l|l|l|l|l|l|}
\hline 0.91 & 1.33 & 1.75 & 0.44 & 0.33 & 387 \\
\hline 1.04 & 1.27 & 1.49 & 0.23 & 0.18 & 185 \\
\hline 0.90 & 1.15 & 1.39 & 0.25 & 0.22 & 385 \\
\hline
\end{tabular}

\begin{tabular}{|l|l|l|l|l|l|}
\hline 1.04 & 1.27 & 1.49 & 0.23 & 0.18 & 185 \\
\hline 0.90 & 1.15 & 1.39 & 0.25 & 0.22 & 385 \\
\hline 0.87 & 1.03 & 1.18 & 0.16 & 0.15 & 389 \\
\hline
\end{tabular}

\begin{tabular}{|l|l|l|l|l|l|}
\hline 0.87 & 1.03 & 1.18 & 0.16 & 0.15 & 389 \\
\hline
\end{tabular}

\begin{tabular}{|l|l|l|l|l|l|}
\hline 0.86 & 0.96 & 1.06 & 0.10 & 0.11 & 386 \\
\hline
\end{tabular}

\begin{tabular}{|c|c|c|c|c|c|}
\hline 0.86 & 0.93 & 1.01 & 0.08 & 0.08 & 389 \\
\hline 0.85 & 0.91 & 0.97 & 0.06 & 0.07 & 240 \\
\hline
\end{tabular}

\begin{tabular}{|c|c|c|c|c|c|}
\hline 0.85 & 0.91 & 0.97 & 0.06 & 0.07 & 240 \\
\hline 0.84 & 0.91 & 0.9 & 0.07 & 0.08 & 240 \\
\hline
\end{tabular}

\begin{tabular}{|c|c|c|c|c|c|}
\hline 0.84 & 0.91 & 0.99 & 0.07 & 0.08 & 240 \\
\hline 0.83 & 0.92 & 1.02 & 0.09 & 0.10 & 240 \\
\hline
\end{tabular}

\begin{tabular}{|l|l|l|l|l|l|l|}
\hline 0.83 & 0.92 & 1.02 & 0.09 & 0.10 & 240 \\
\hline 0.83 & 0.94 & 1.05 & 0.11 & 0.12 & 240 \\
\hline
\end{tabular}

\begin{tabular}{|l|l|l|l|l|l|}
\hline 0.83 & 0.94 & 1.05 & 0.11 & 0.12 & 240 \\
\hline
\end{tabular}

\begin{tabular}{|l|l|l|l|l|l|}
\hline 0.82 & 0.95 & 1.07 & 0.13 & 0.14 & 239 \\
\hline 0.84 & 1.19 & 1.54 & 0.37 & 0.31 & 387 \\
\hline
\end{tabular}

\begin{tabular}{|l|l|l|l|l|l|}
\hline 0.84 & 1.19 & 1.54 & 0.37 & 0.31 & 387 \\
\hline 0.97 & 1.17 & 1.37 & 0.20 & 0.17 & 185 \\
\hline
\end{tabular}

\begin{tabular}{|l|l|l|l|l|l|}
\hline 0.85 & 1.13 & 1.40 & 0.29 & 0.26 & 387 \\
\hline 0.83 & 1.05 & 1.27 & 0.22 & 0.21 & 395 \\
\hline
\end{tabular}

\begin{tabular}{|l|l|l|l|l|l|}
\hline 0.83 & 1.05 & 1.27 & 0.22 & 0.21 & 395 \\
\hline 0.83 & 1.00 & 1.17 & 0.17 & 0.17 & 381 \\
\hline
\end{tabular}

\begin{tabular}{|c|c|c|c|c|c|}
\hline 0.83 & 1.00 & 1.17 & 0.17 & 0.17 & 381 \\
\hline 0.82 & 0.96 & 1.11 & 0.15 & 0.15 & 381 \\
\hline
\end{tabular}

\begin{tabular}{|l|l|l|l|l|l|}
\hline 0.82 & 0.96 & 1.11 & 0.15 & 0.15 & 381 \\
\hline 0.81 & 0.92 & 1.04 & 0.11 & 0.12 & \\
\hline
\end{tabular}

\begin{tabular}{|l|l|l|l|l|l|}
\hline 0.81 & 0.92 & 1.04 & 0.11 & 0.12 & 240 \\
\hline 0.80 & 0.92 & 1.03 & 0.11 & 0.12 & 240 \\
\hline
\end{tabular}

\begin{tabular}{|l|l|l|l|l|l|}
\hline 0.80 & 0.92 & 1.03 & 0.11 & 0.12 & 240 \\
\hline 0.80 & 0.92 & 1.04 & 0.12 & 0.13 & 240 \\
\hline
\end{tabular}

\begin{tabular}{|l|l|l|l|l|l|}
\hline 0.80 & 0.92 & 1.04 & 0.12 & 0.13 & 240 \\
\hline 0.79 & 0.92 & 1.05 & 0.13 & 0.14 & 240 \\
\hline
\end{tabular}

\begin{tabular}{|l|l|l|l|l|l|}
0.79 & 0.92 & 1.05 & 0.13 & 0.14 & 240 \\
\hline 0.79 & 0.93 & 1.07 & 0.14 & 0.15 & 240 \\
\hline
\end{tabular}

\begin{tabular}{|l|l|l|l|l|l|}
\hline 0.79 & 0.93 & 1.07 & 0.14 & 0.15 & 240 \\
\hline 0.76 & 1.13 & 1.49 & 0.39 & 0.34 & 387 \\
\hline 0.91 & 1.10 & 1.29 & 0.20 & 0.18 & 185 \\
\hline
\end{tabular}

\begin{tabular}{|l|l|l|l|l|l|}
0.71 & 1.10 & 1.29 & 0.20 & 0.18 & 185 \\
\hline 0.79 & 1.11 & 1.42 & 0.33 & 0.30 & 385 \\
\hline
\end{tabular}

\begin{tabular}{|l|l|l|l|l|l|l|}
\hline 0.79 & 1.11 & 1.42 & 0.33 & 0.30 & 385 \\
\hline 0.78 & 1.06 & 1.34 & 0.29 & 0.27 & 387 \\
\hline
\end{tabular}

\begin{tabular}{|l|l|l|l|l|l|}
\hline 0.78 & 1.06 & 1.34 & 0.29 & 0.27 & 387 \\
\hline 0.78 & 1.02 & 1.25 & 0.24 & 0.24 & 384 \\
\hline
\end{tabular}

\begin{tabular}{|c|c|c|c|c|c|}
\hline 0.78 & 1.02 & 1.25 & 0.24 & 0.24 & 384 \\
\hline 0.78 & 0.98 & 1.18 & 0.21 & 0.21 & 386 \\
\hline
\end{tabular}

\begin{tabular}{|l|l|l|l|l|l|}
\hline 0.78 & 0.98 & 1.18 & 0.21 & 0.21 & 386 \\
\hline 0.78 & 0.94 & 1.10 & 0.16 & 0.17 & 240 \\
\hline
\end{tabular}

\begin{tabular}{|l|l|l|l|l|l|}
\hline 0.78 & 0.94 & 1.10 & 0.16 & 0.17 & 240 \\
\hline 0.77 & 0.93 & 1.08 & 0.16 & 0.17 & 240 \\
\hline
\end{tabular}

\begin{tabular}{|l|l|l|l|l|l|}
\hline 0.77 & 0.93 & 1.08 & 0.16 & 0.17 & 240 \\
\hline 0.77 & 0.92 & 1.08 & 0.16 & 0.17 & 240 \\
\hline
\end{tabular}

\begin{tabular}{|l|l|l|l|l|l|}
\hline 0.77 & 0.92 & 1.08 & 0.16 & 0.17 & 240 \\
\hline 0.76 & 0.92 & 1.08 & 0.16 & 0.18 & 240 \\
\hline
\end{tabular}

\begin{tabular}{|l|l|l|l|l|l|}
\hline 0.76 & 0.92 & 1.08 & 0.16 & 0.18 & 240 \\
\hline 0.76 & 0.92 & 1.08 & 0.17 & 0.18 & 240 \\
\hline
\end{tabular}

\begin{tabular}{|l|l|l|l|l|l|}
\hline 0.76 & 0.92 & 1.08 & 0.17 & 0.18 & 240 \\
\hline 0.73 & 1.10 & 1.46 & 0.39 & 0.35 & 384 \\
\hline 0.87 & 1.06 & 1.25 & 0.20 & 0.19 & 185 \\
\hline
\end{tabular}

\begin{tabular}{|c|c|c|c|c|c|}
\hline 0.87 & 1.06 & 1.25 & 0.20 & 0.19 & 185 \\
\hline 0.76 & 1.09 & 1.41 & 0.34 & 0.31 & 394 \\
\hline
\end{tabular}

\begin{tabular}{|l|c|c|c|c|c|}
\hline 0.76 & 1.09 & 1.41 & 0.34 & 0.31 & 394 \\
\hline
\end{tabular}

\begin{tabular}{|l|l|l|l|l|l|}
\hline 0.76 & 1.06 & 1.36 & 0.32 & 0.30 & 391 \\
\hline 0.77 & 1.03 & 1.28 & 0.27 & 0.26 & 383 \\
\hline
\end{tabular}

\begin{tabular}{|l|l|l|l|l|l|}
\hline 0.77 & 1.03 & 1.28 & 0.27 & 0.26 & 383 \\
\hline 0.77 & 1.00 & 1.23 & 0.24 & 0.24 & 386 \\
\hline
\end{tabular}

\begin{tabular}{|l|l|l|l|l|l|}
\hline 0.77 & 1.00 & 1.23 & 0.24 & 0.24 & 386 \\
\hline 0.76 & 0.95 & 1.13 & 0.19 & 0.20 & 240 \\
\hline
\end{tabular}

\begin{tabular}{|l|l|l|l|l|l|}
0.776 & 0.95 & 1.13 & 0.19 & 0.20 & 240 \\
\hline 0.76 & 0.93 & 1.11 & 0.18 & 0.19 & 240 \\
\hline
\end{tabular}

\begin{tabular}{|l|l|l|l|l|l|}
\hline 0.76 & 0.93 & 1.11 & 0.18 & 0.19 & 240 \\
\hline 0.75 & 0.92 & 1.10 & 0.18 & 0.19 & 240 \\
\hline
\end{tabular}

\begin{tabular}{|l|l|l|l|l|l|}
0.75 & 0.92 & 1.10 & 0.18 & 0.19 & 240 \\
\hline 0.75 & 0.92 & 1.09 & 0.17 & 0.19 & 240 \\
\hline 0.74 & 0.92 & 1.09 & 0.18 & 0.19 & 240 \\
\hline
\end{tabular}

\begin{tabular}{|l|l|l|l|l|l|}
\hline 0.75 & 0.92 & 1.09 & 0.17 & 0.19 & 240 \\
\hline 0.74 & 0.92 & 1.09 & 0.18 & 0.19 & 240 \\
\hline
\end{tabular}

\begin{tabular}{|l|l|l|l|l|l|}
\hline 0.79 & 1.03 & 1.27 & 0.25 & 0.24 & 18597 \\
\hline 0.81 & 1.09 & 1.37 & 0.29 & 0.27 & 11717 \\
\hline
\end{tabular} \begin{tabular}{|l|l|l|l|l|l|}
\hline 0.79 & 1.03 & 1.27 & 0.25 & 0.24 & 18597 \\
\hline 0.81 & 1.09 & 1.37 & 0.29 & 0.27 & 11717 \\
\hline
\end{tabular} 
Table 8.5 Ratio of deformation life calculated with model over deformation life calculated with BISAR

\begin{tabular}{|c|c|c|c|c|c|}
\hline \multirow{3}{*}{\begin{tabular}{|l|} 
Asphalt \\
thickness \\
(m)
\end{tabular}} & \multirow{3}{*}{$\begin{array}{l}\begin{array}{l}\text { Subbase } \\
\text { thickness } \\
(\mathrm{m})\end{array} \\
\end{array}$} & \multirow{2}{*}{\multicolumn{4}{|c|}{$\frac{\text { FROM PERCENTILES }}{\mathrm{Nd}_{\text {mode }} / \mathrm{Nd}_{\text {BISAR }}(\text { LR1132) }}$}} \\
\hline & & & & & \\
\hline & & $\begin{array}{l}15 \text { th } \\
\text { per }\end{array}$ & \multirow{2}{*}{\begin{tabular}{|l|}
$\begin{array}{l}50 \text { th } \\
\text { per }\end{array}$ \\
0.97 \\
\end{tabular}} & \multirow{2}{*}{\begin{tabular}{|l|}
$\begin{array}{l}85 \text { th } \\
\text { perc }\end{array}$ \\
1.15 \\
\end{tabular}} & $\begin{array}{l}\text { Nr pts that } \\
\text { satisfy } \\
\text { model } \\
\text { conds }\end{array}$ \\
\hline 0.100 & 0.100 & 0.61 & & & 336 \\
\hline 0.100 & 0.150 & 1.03 & 1.22 & 1.38 & 177 \\
\hline 0.100 & 0.200 & 0.78 & 1.18 & 1.52 & 332 \\
\hline 0.100 & 0.300 & 0.82 & 1.22 & 1.60 & 344 \\
\hline 0.100 & 0.400 & 0.82 & 1.22 & 1.63 & 332 \\
\hline 0.100 & 0.500 & 0.79 & 1.18 & 1.58 & 324 \\
\hline 0.100 & 0.600 & 0.74 & 1.07 & 1.38 & 202 \\
\hline 0.100 & 0.700 & 0.73 & 1.03 & 1.32 & 202 \\
\hline 0.100 & 0.800 & 0.70 & 0.99 & 1.26 & 202 \\
\hline 0.100 & 0.900 & 0.66 & 0.95 & 1.20 & 202 \\
\hline 0.100 & 1.000 & 0.63 & 0.92 & 1.14 & 202 \\
\hline 0.150 & 0.100 & 0.58 & 0.96 & 1.12 & 205 \\
\hline 0.150 & 0.150 & 1.04 & 1.22 & 1.35 & 185 \\
\hline 0.150 & 0.200 & 0.65 & 1.14 & 1.41 & 205 \\
\hline 0.150 & 0.300 & 0.75 & 1.18 & 1.50 & 205 \\
\hline 0.150 & 0.400 & 0.79 & 1.21 & 1.52 & 205 \\
\hline 0.150 & 0.500 & 0.78 & 1.19 & 1.51 & 205 \\
\hline 0.150 & 0.600 & 0.75 & 1.14 & 1.48 & 205 \\
\hline 0.150 & 0.700 & 0.74 & 1.09 & 1.42 & 205 \\
\hline 0.150 & 0.800 & 0.73 & 1.06 & 1.37 & 205 \\
\hline 0.150 & 0.900 & 0.72 & 1.03 & 1.32 & 205 \\
\hline 0.150 & 1.000 & 0.70 & 1.00 & 1.28 & 205 \\
\hline 0.200 & 0.100 & 0.58 & 0.97 & 1.13 & 353 \\
\hline 0.200 & 0.150 & 1.00 & 1.22 & 1.33 & 185 \\
\hline 0.200 & 0.200 & 0.64 & 1.17 & 1.43 & 350 \\
\hline 0.200 & 0.300 & 0.66 & 1.23 & 1.55 & 356 \\
\hline 0.200 & 0.400 & 0.74 & 1.23 & 1.60 & 352 \\
\hline 0.200 & 0.500 & 0.78 & 1.23 & 1.62 & 351 \\
\hline 0.200 & 0.600 & 0.78 & 1.15 & 1.49 & 220 \\
\hline 0.200 & 0.700 & 0.76 & 1.11 & 1.46 & 220 \\
\hline 0.200 & 0.800 & 0.74 & 1.08 & 1.41 & 220 \\
\hline 0.200 & 0.900 & 0.72 & 1.05 & 1.37 & 220 \\
\hline 0.200 & 1.000 & 0.70 & 1.03 & 1.33 & 220 \\
\hline 0.300 & 0.100 & 0.61 & 0.94 & 1.14 & 351 \\
\hline 0.300 & 0.150 & 0.96 & 1.20 & 1.29 & 185 \\
\hline 0.300 & 0.200 & 0.63 & 1.13 & 1.34 & 353 \\
\hline 0.300 & 0.300 & 0.59 & 1.18 & 1.46 & 359 \\
\hline 0.300 & 0.400 & 0.62 & 1.22 & 1.52 & 347 \\
\hline 0.300 & 0.500 & 0.70 & 1.23 & 1.55 & 345 \\
\hline 0.300 & 0.600 & 0.70 & 1.14 & 1.46 & 220 \\
\hline 0.300 & 0.700 & 0.73 & 1.12 & 1.43 & 220 \\
\hline 0.300 & 0.800 & 0.74 & 1.11 & 1.42 & 220 \\
\hline 0.300 & 0.900 & 0.74 & 1.11 & 1.41 & 220 \\
\hline 0.300 & 1.000 & 0.73 & 1.08 & 1.41 & 220 \\
\hline 0.400 & 0.100 & 0.62 & 0.92 & 1.15 & 354 \\
\hline 0.400 & 0.150 & 0.92 & 1.15 & 1.28 & 185 \\
\hline 0.400 & 0.200 & 0.61 & 1.08 & 1.29 & 350 \\
\hline 0.400 & 0.300 & 0.59 & 1.15 & 1.39 & 353 \\
\hline 0.400 & 0.400 & 0.58 & 1.18 & 1.44 & 349 \\
\hline 0.400 & 0.500 & 0.60 & 1.20 & 1.49 & 352 \\
\hline 0.400 & 0.600 & 0.62 & 1.15 & 1.41 & 220 \\
\hline 0.400 & 0.700 & 0.64 & 1.13 & 1.42 & 220 \\
\hline 0.400 & 0.800 & 0.67 & 1.11 & 1.42 & 220 \\
\hline 0.400 & 0.900 & 0.69 & 1.08 & 1.39 & 220 \\
\hline 0.400 & 1.000 & 0.70 & 1.08 & 1.37 & 220 \\
\hline 0.500 & 0.100 & 0.62 & 0.89 & 1.12 & 352 \\
\hline 0.500 & 0.150 & 0.89 & 1.10 & 1.26 & 185 \\
\hline 0.500 & 0.200 & 0.61 & 1.02 & 1.25 & 359 \\
\hline 0.500 & 0.300 & 0.59 & 1.11 & 1.32 & 356 \\
\hline 0.500 & 0.400 & 0.58 & 1.14 & 1.38 & 349 \\
\hline 0.500 & 0.500 & 0.59 & 1.16 & 1.42 & 352 \\
\hline 0.500 & 0.600 & 0.56 & 1.14 & 1.37 & 220 \\
\hline 0.500 & 0.700 & 0.58 & 1.13 & 1.38 & 220 \\
\hline 0.500 & 0.800 & 0.60 & 1.12 & 1.37 & 220 \\
\hline 0.500 & 0.900 & 0.63 & 1.10 & 1.39 & 220 \\
\hline 0.500 & 1.000 & 0.65 & 1.08 & 1.38 & 220 \\
\hline 0 to $0.5 \mathrm{~m}$ & 0 to $1 \mathrm{~m}$ & 0.68 & 1.10 & 1.40 & 17273 \\
\hline 0 to $0.5 \mathrm{~m}$ & 0 to $0.5 \mathrm{~m}$ & 0.67 & 1.12 & 1.40 & 10838 \\
\hline
\end{tabular}
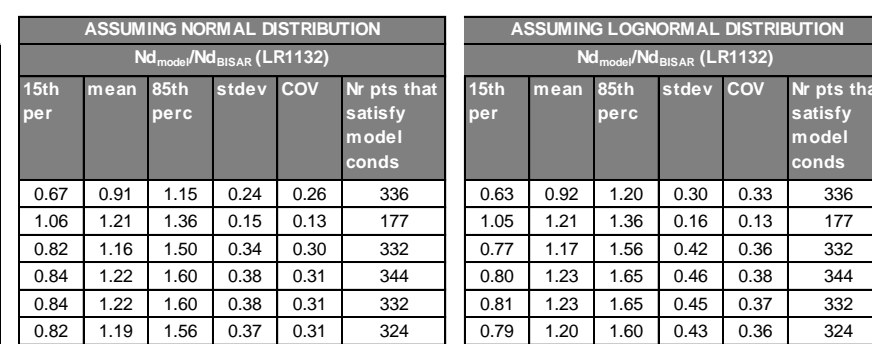

\begin{tabular}{|l|l|l|l|l|l|l|}
\hline 0.82 & 1.19 & 1.56 & 0.37 & 0.31 & 324 \\
\hline 0.77 & 1.07 & 1.37 & 0.30 & 0.28 & 202 \\
\hline
\end{tabular}

\begin{tabular}{|l|l|l|l|l|l|}
\hline 0.77 & 1.07 & 1.37 & 0.30 & 0.28 & 202 \\
\hline 0.74 & 1.02 & 1.31 & 0.29 & 0.28 & 202 \\
\hline 0.71 & 0.98 & 1.25 & 0.27 & 0.28 & 202 \\
\hline
\end{tabular}

\begin{tabular}{|l|l|l|l|l|l|}
\hline 0.74 & 1.02 & 1.31 & 0.29 & 0.28 & 202 \\
\hline 0.71 & 0.98 & 1.25 & 0.27 & 0.28 & 202 \\
\hline 0.68 & 0.93 & 1.19 & 0.26 & 0.27 & 202 \\
\hline
\end{tabular}

\begin{tabular}{l|l|l|l|l|l|}
0.68 & 0.93 & 1.19 & 0.26 & 0.27 & 202 \\
\hline 0.65 & 0.89 & 1.14 & 0.24 & 0.27 & 202 \\
\hline
\end{tabular}

\begin{tabular}{|l|l|l|l|l|l|}
\hline 0.65 & 0.89 & 1.14 & 0.24 & 0.27 & 202 \\
\hline
\end{tabular}

\begin{tabular}{|l|l|l|l|l|l|}
\hline 0.64 & 0.88 & 1.12 & 0.24 & 0.27 & 205 \\
\hline 1.04 & 1.20 & 1.35 & 0.15 & 0.13 & 185 \\
\hline 0.75 & 1.07 & 1.40 & 0.33 & 0.30 & 205 \\
\hline
\end{tabular}

\begin{tabular}{|l|l|l|l|l|l|}
\hline 1.04 & 1.20 & 1.35 & 0.15 & 0.13 & 185 \\
\hline 0.75 & 1.07 & 1.40 & 0.33 & 0.30 & 205 \\
\hline 0.79 & 1.14 & 1.49 & 0.35 & 0.31 & 205 \\
\hline
\end{tabular}

\begin{tabular}{l|l|l|l|l|l|l|}
\hline 0.79 & 1.14 & 1.49 & 0.35 & 0.31 & 205 \\
\hline
\end{tabular}

\begin{tabular}{|l|l|l|l|l|l|}
\hline 0.81 & 1.16 & 1.51 & 0.35 & 0.30 & 205 \\
\hline 0.81 & 1.15 & 1.49 & 0.34 & 0.30 & 205 \\
\hline
\end{tabular}

\begin{tabular}{|l|l|l|l|l|l|}
\hline 0.81 & 1.15 & 1.49 & 0.34 & 0.30 & 205 \\
\hline 0.79 & 1.13 & 1.46 & 0.33 & 0.29 & 205 \\
\hline
\end{tabular}

\begin{tabular}{|l|l|l|l|l|l|}
\hline 0.79 & 1.13 & 1.46 & 0.33 & 0.29 & 205 \\
\hline 0.78 & 1.10 & 1.42 & 0.32 & 0.29 & 205 \\
\hline 0.76 & 1.06 & 1.37 & 0.31 & 0.29 & 205 \\
\hline
\end{tabular}

\begin{tabular}{|l|l|l|l|l|l|}
\hline 0.76 & 1.06 & 1.37 & 0.31 & 0.29 & 205 \\
\hline 0.74 & 1.03 & 1.32 & 0.29 & 0.28 & 20 \\
\hline
\end{tabular}

\begin{tabular}{|c|c|c|c|c|c|}
\hline 0.74 & 1.03 & 1.32 & 0.29 & 0.28 & 205 \\
\hline 0.72 & 1.00 & 1.27 & 0.28 & 0.28 & 205 \\
\hline
\end{tabular}

\begin{tabular}{|l|l|l|l|l|l|}
\hline 0.72 & 1.00 & 1.27 & 0.28 & 0.28 & 205 \\
\hline 0.65 & 0.89 & 1.14 & 0.25 & 0.27 & 353 \\
\hline 1.03 & 1.18 & 1.33 & 0.15 & 0.13 & 185 \\
\hline
\end{tabular}

\begin{tabular}{|c|c|c|c|c|c|}
\hline 0.65 & 0.89 & 1.14 & 0.25 & 0.27 & 353 \\
\hline 1.03 & 1.18 & 1.33 & 0.15 & 0.13 & 185 \\
\hline 0.74 & 1.08 & 1.42 & 0.34 & 0.32 & 350 \\
\hline
\end{tabular}

\begin{tabular}{|l|l|l|l|l|l|}
\hline 0.74 & 1.08 & 1.42 & 0.34 & 0.32 & 350 \\
\hline 0.76 & 1.15 & 1.53 & 0.38 & 0.33 & 356 \\
\hline
\end{tabular}

\begin{tabular}{l|l|l|l|l|l|l|}
\hline 0.76 & 1.15 & 1.53 & 0.38 & 0.33 & 356 \\
\hline 0.79 & 1.19 & 1.59 & 0.40 & 0.33 & 352 \\
\hline
\end{tabular}

\begin{tabular}{|l|l|l|l|l|l|}
\hline 0.79 & 1.19 & 1.59 & 0.40 & 0.33 & 352 \\
\hline 0.80 & 1.20 & 1.60 & 0.40 & 0.33 & 351 \\
\hline 0.78 & 1.12 & 1.46 & 0.34 & 0.31 & 220 \\
\hline
\end{tabular}

\begin{tabular}{|l|l|l|l|l|l|}
\hline 0.80 & 1.20 & 1.60 & 0.40 & 0.33 & 351 \\
\hline 0.78 & 1.12 & 1.46 & 0.34 & 0.31 & 220 \\
\hline 0.77 & 1.10 & 1.43 & 0.33 & 0.30 & 220 \\
\hline
\end{tabular}

\begin{tabular}{|l|l|l|l|l|l|}
\hline 0.77 & 1.10 & 1.43 & 0.33 & 0.30 & 220 \\
\hline 0.76 & 1.08 & 1.40 & 0.32 & 0.30 & 220 \\
\hline
\end{tabular}

\begin{tabular}{|l|l|l|l|l|l|}
\hline 0.76 & 1.08 & 1.40 & 0.32 & 0.30 & 220 \\
\hline 0.75 & 1.06 & 1.37 & 0.31 & 0.29 & 220 \\
\hline
\end{tabular}

\begin{tabular}{l|l|l|l|l|l|l|}
\hline 0.75 & 1.06 & 1.37 & 0.31 & 0.29 & 220 \\
\hline 0.73 & 1.03 & 1.33 & 0.30 & 0.29 & 220 \\
\hline
\end{tabular}

\begin{tabular}{|l|l|l|l|l|l|}
\hline 0.73 & 1.03 & 1.33 & 0.30 & 0.29 & 220 \\
\hline 0.66 & 0.90 & 1.14 & 0.24 & 0.26 & 351 \\
\hline 1.00 & 1.15 & 1.31 & 0.16 & 0.14 & \\
\hline
\end{tabular}

\begin{tabular}{l|l|l|l|l|l}
0.1 .15 & 1.31 & 0.16 & 0.14 & 185 \\
\hline 0.71 & 1.03 & 1.35 & 0.32 & 0.31 & 353 \\
\hline
\end{tabular}

\begin{tabular}{l|l|l|l|l|l|}
\hline 0.71 & 1.03 & 1.35 & 0.32 & 0.31 & 353 \\
\hline 0.72 & 1.08 & 1.45 & 0.37 & 0.34 & 359 \\
\hline
\end{tabular}

\begin{tabular}{|l|l|l|l|l|l|}
\hline 0.72 & 1.08 & 1.45 & 0.37 & 0.34 & 359 \\
\hline 0.75 & 1.13 & 1.51 & 0.38 & 0.34 & 347 \\
\hline 0.77 & 1.15 & 1.54 & 0.39 & 0.33 & 345 \\
\hline
\end{tabular}

\begin{tabular}{|l|l|l|l|l|l|}
\hline 0.75 & 1.13 & 1.51 & 0.38 & 0.34 & 347 \\
\hline 0.77 & 1.15 & 1.54 & 0.39 & 0.33 & 345 \\
\hline 0.74 & 1.09 & 1.45 & 0.35 & 0.32 & 220 \\
\hline
\end{tabular}

\begin{tabular}{|l|l|l|l|l|l|}
\hline 0.77 & 1.15 & 1.54 & 0.39 & 0.33 & 345 \\
\hline 0.74 & 1.09 & 1.45 & 0.35 & 0.32 & 220 \\
\hline 0.74 & 1.09 & 1.43 & 0.35 & 0.32 & 220 \\
\hline
\end{tabular}

\begin{tabular}{|l|l|l|l|l|l|}
\hline 0.74 & 1.09 & 1.43 & 0.35 & 0.32 & 220 \\
\hline 0.74 & 1.08 & 1.42 & 0.34 & 0.31 & 220 \\
\hline
\end{tabular}

\begin{tabular}{|l|l|l|l|l|l|}
\hline 0.74 & 1.08 & 1.42 & 0.34 & 0.31 & 220 \\
\hline 0.74 & 1.07 & 1.40 & 0.33 & 0.31 & 220 \\
\hline 0.73 & 1.05 & 1.38 & 0.32 & 0.31 & \\
\hline
\end{tabular}

\begin{tabular}{l|l|l|l|l|l|l|}
\hline 0.74 & 1.07 & 1.40 & 0.33 & 0.31 & 220 \\
\hline 0.73 & 1.05 & 1.38 & 0.32 & 0.31 & 220 \\
\hline 0.66 & 0.89 & 1.12 & 0.23 & 0.26 & 354 \\
\hline
\end{tabular}

\begin{tabular}{|l|l|l|l|l|l|}
\hline 0.66 & 0.89 & 1.12 & 0.23 & 0.26 & 354 \\
\hline 0.96 & 1.12 & 1.27 & 0.16 & 0.14 & 185 \\
\hline
\end{tabular}

\begin{tabular}{|l|l|l|l|l|l|}
\hline 0.96 & 1.12 & 1.27 & 0.16 & 0.14 & 185 \\
\hline 0.70 & 1.00 & 1.30 & 0.30 & 0.30 & 350 \\
\hline
\end{tabular}

\begin{tabular}{|l|l|l|l|l|l|}
\hline 0.70 & 1.00 & 1.30 & 0.30 & 0.30 & 350 \\
\hline 0.70 & 1.04 & 1.39 & 0.34 & 0.33 & 353 \\
\hline 0.71 & 1.07 & 1.44 & 0.36 & 0.34 & 349 \\
\hline
\end{tabular}

\begin{tabular}{|l|l|l|l|l|l|}
\hline 0.70 & 1.04 & 1.39 & 0.34 & 0.33 & 353 \\
\hline 0.71 & 1.07 & 1.44 & 0.36 & 0.34 & 349 \\
\hline 0.72 & 1.10 & 1.47 & 0.37 & 0.34 & 352 \\
\hline
\end{tabular}

\begin{tabular}{|l|l|l|l|l|l|l}
\hline 0.72 & 1.10 & 1.47 & 0.37 & 0.34 & 352 \\
\hline
\end{tabular}

\begin{tabular}{|l|l|l|l|l|l|}
\hline 0.71 & 1.06 & 1.41 & 0.35 & 0.33 & 220 \\
\hline 0.71 & 1.06 & 1.41 & 0.35 & 0.33 & 220 \\
\hline
\end{tabular}

\begin{tabular}{|l|l|l|l|l|l|}
\hline 0.71 & 1.06 & 1.41 & 0.35 & 0.33 & 220 \\
\hline 0.71 & 1.06 & 1.40 & 0.34 & 0.32 & 220 \\
\hline 0.71 & 1.05 & 1.39 & 0.34 & 0.32 & 220 \\
\hline
\end{tabular}

\begin{tabular}{|l|l|l|l|l|l|l|}
\hline 0.71 & 1.05 & 1.39 & 0.34 & 0.32 & 220 \\
\hline 0.71 & 1.04 & 1.37 & 0.33 & 0.32 & 220 \\
\hline
\end{tabular}

\begin{tabular}{|l|l|l|l|l|l|}
\hline 0.71 & 1.04 & 1.37 & 0.33 & 0.32 & 220 \\
\hline 0.66 & 0.88 & 1.09 & 0.22 & 0.25 & 352 \\
\hline
\end{tabular}

\begin{tabular}{|l|l|l|l|l|l|}
\hline 0.66 & 0.88 & 1.09 & 0.22 & 0.25 & 352 \\
\hline 0.93 & 1.09 & 1.24 & 0.15 & 0.14 & \\
\hline
\end{tabular}

\begin{tabular}{l|l|l|l|l|l|l}
\hline 0.93 & 1.09 & 1.24 & 0.15 & 0.14 & 185 \\
\hline 0.67 & 0.96 & 1.24 & 0.28 & 0.29 & 359 \\
\hline
\end{tabular}

\begin{tabular}{l|l|l|l|l|l|}
\hline 0.67 & 0.96 & 1.24 & 0.28 & 0.29 & 359 \\
\hline 0.68 & 1.00 & 1.33 & 0.32 & 0.32 & 356 \\
\hline
\end{tabular}

\begin{tabular}{l|l|l|l|l|l}
\hline 0.69 & 1.03 & 1.38 & 0.34 & 0.33 & 349 \\
\hline 0.70 & 1.06 & 1.4 & 0.35 & 0.34 & 352 \\
\hline
\end{tabular}

\begin{tabular}{|c|c|c|c|c|c|}
\hline 0.70 & 1.06 & 1.41 & 0.35 & 0.34 & 352 \\
\hline
\end{tabular}

\begin{tabular}{l|l|l|l|l|l|}
\hline 0.69 & 1.03 & 1.38 & 0.35 & 0.34 & 220 \\
\hline 0.69 & 1.03 & 1.38 & 0.35 & 0.33 & 220 \\
\hline
\end{tabular}

\begin{tabular}{|l|l|l|l|l|l|l|}
\hline 0.69 & 1.03 & 1.38 & 0.35 & 0.33 & 220 \\
\hline 0.69 & 1.03 & 1.37 & 0.34 & 0.33 & 220 \\
\hline
\end{tabular}

\begin{tabular}{|l|l|l|l|l|l|}
\hline 0.69 & 1.03 & 1.37 & 0.34 & 0.33 & 220 \\
\hline 0.69 & 1.02 & 1.36 & 0.33 & 0.33 & 220 \\
\hline
\end{tabular}

\begin{tabular}{|l|l|l|l|l|l|}
\hline 0.69 & 1.02 & 1.36 & 0.33 & 0.33 & 220 \\
\hline
\end{tabular}

\begin{tabular}{|l|l|l|l|l|l|}
\hline 0.73 & 1.06 & 1.40 & 0.33 & 0.31 & 17273 \\
\hline 0.73 & 1.07 & 1.41 & 0.34 & 0.32 & 10838 \\
\hline
\end{tabular}

\begin{tabular}{|l|l|l|l|l|l|}
\hline 0.73 & 1.06 & 1.40 & 0.33 & 0.31 & 17273 \\
\hline 0.73 & 1.07 & 1.41 & 0.34 & 0.32 & 10838 \\
\hline
\end{tabular}

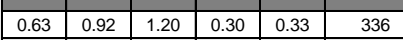

\begin{tabular}{|l|l|l|l|l|l|}
\hline 1.05 & 0.92 & 1.20 & 0.30 & 0.33 & 336 \\
\hline 0.77 & 1.17 & 1.36 & 0.16 & 0.13 & 177 \\
\hline
\end{tabular}

\begin{tabular}{|l|l|l|l|l|l|}
\hline 1.05 & 1.21 & 1.36 & 0.16 & 0.13 & 177 \\
\hline 0.77 & 1.17 & 1.56 & 0.42 & 0.36 & 332 \\
\hline 0.80 & 1.23 & 1.65 & 0.46 & 0.38 & 344 \\
\hline
\end{tabular}

\begin{tabular}{|l|l|l|l|l|l|}
\hline 0.80 & 1.23 & 1.65 & 0.46 & 0.38 & 344 \\
\hline 0.81 & 1.23 & 1.65 & 0.45 & 0.37 & 332 \\
\hline
\end{tabular}

\begin{tabular}{|l|l|l|l|l|l|l|}
\hline 0.79 & 1.20 & 1.60 & 0.43 & 0.36 & 324 \\
\hline 0.74 & 1.08 & 1.41 & 0.35 & 0.33 & 202 \\
\hline
\end{tabular}

\begin{tabular}{|l|l|l|l|l|l|}
\hline 0.74 & 1.08 & 1.41 & 0.35 & 0.33 & 202 \\
\hline 0.71 & 1.03 & 1.34 & 0.33 & 0.32 & \\
\hline
\end{tabular}

\begin{tabular}{|l|l|l|l|l|l|}
\hline 0.71 & 1.03 & 1.34 & 0.33 & 0.32 & 202 \\
\hline 0.68 & 0.98 & 1.28 & 0.32 & 0.32 & 202 \\
\hline 0.65 & 0.94 & 1.22 & 0.30 & 0.32 & 202 \\
\hline
\end{tabular}

\begin{tabular}{|c|c|c|c|c|c|}
\hline 0.65 & 0.94 & 1.22 & 0.30 & 0.32 & 202 \\
\hline 0.63 & 0.90 & 1.17 & 0.29 & 0.32 & 202 \\
\hline
\end{tabular}

\begin{tabular}{l|l|l|l|l|l|}
\hline 0.63 & 0.90 & 1.17 & 0.29 & 0.32 & 202 \\
\hline 0.61 & 0.89 & 1.17 & 0.30 & 0.33 & \\
\hline
\end{tabular}

\begin{tabular}{|l|l|l|l|l|l|}
\hline 0.61 & 0.89 & 1.17 & 0.30 & 0.33 & 205 \\
\hline 1.04 & 1.20 & 1.35 & 0.16 & 0.13 & 185 \\
\hline 0.70 & 1.08 & 1.47 & 0.42 & 0.38 & 25 \\
\hline
\end{tabular}

\begin{tabular}{|l|l|l|l|l|l|}
\hline 0.70 & 1.08 & 1.47 & 0.42 & 0.38 & 205 \\
\hline 0.74 & 1.15 & 1.56 & 0.45 & 0.39 & 205 \\
\hline
\end{tabular}

\begin{tabular}{|l|l|l|l|l|l|}
\hline 0.74 & 1.15 & 1.56 & 0.45 & 0.39 & 205 \\
\hline 0.76 & 1.17 & 1.57 & 0.44 & 0.38 & 205 \\
\hline
\end{tabular}

\begin{tabular}{|l|l|l|l|l|l|}
\hline 0.76 & 1.17 & 1.57 & 0.44 & 0.38 & 205 \\
\hline 0.76 & 1.16 & 1.55 & 0.42 & 0.37 & 205 \\
\hline
\end{tabular}

\begin{tabular}{|l|l|l|l|l|l|}
\hline 0.76 & 1.16 & 1.55 & 0.42 & 0.37 & 205 \\
\hline 0.74 & 1.10 & 1.51 & 0.40 & 0.36 & 205 \\
\hline
\end{tabular}

\begin{tabular}{|l|l|l|l|l|l|}
\hline 0.74 & 1.10 & 1.46 & 0.38 & 0.35 & 205 \\
\hline 0.73 & 1.07 & 1.41 & 0.36 & 0.34 & 205 \\
\hline
\end{tabular}

\begin{tabular}{l|l|l|l|l|l|}
0.74 & 1.07 & 1.46 & 0.38 & 0.35 & 205 \\
\hline 0.71 & 1.04 & 1.41 & 0.36 & 0.34 & 205 \\
\hline
\end{tabular}

\begin{tabular}{|l|l|l|l|l|l|}
\hline 0.69 & 1.00 & 1.31 & 0.33 & 0.33 & 205 \\
\hline 0.61 & 0.90 & 1.18 & 0.30 & 0.34 & 353 \\
\hline
\end{tabular}

\begin{tabular}{|c|c|c|c|c|c|}
\hline 0.61 & 0.90 & 1.18 & 0.30 & 0.34 & 353 \\
\hline 1.02 & 1.18 & 1.35 & 0.17 & 0.14 & \\
\hline
\end{tabular}

\begin{tabular}{|c|c|c|c|c|c|}
\hline 1.02 & 1.18 & 1.35 & 0.17 & 0.14 & 185 \\
\hline 0.68 & 1.10 & 1.51 & 0.45 & 0.41 & 350 \\
\hline
\end{tabular}

\begin{tabular}{|l|l|l|l|l|l|l|}
\hline 0.70 & 1.17 & 1.62 & 0.51 & 0.44 & 356 \\
\hline 0.73 & 1.21 & 1.68 & 0.52 & 0.43 & 352 \\
\hline
\end{tabular}

\begin{tabular}{|l|l|l|l|l|l|}
\hline 0.73 & 1.21 & 1.68 & 0.52 & 0.43 & 352 \\
\hline 0.75 & 1.21 & 1.68 & 0.51 & 0.42 & 351 \\
\hline
\end{tabular}

\begin{tabular}{|l|l|l|l|l|l|}
\hline 0.75 & 1.21 & 1.68 & 0.51 & 0.42 & 351 \\
\hline 0.73 & 1.13 & 1.52 & 0.42 & 0.38 & 220 \\
\hline 0.73 & 1.11 & 1.49 & 0.41 & 0.37 & 220 \\
\hline
\end{tabular}

\begin{tabular}{|l|l|l|l|l|l|}
\hline 0.73 & 1.11 & 1.49 & 0.41 & 0.37 & 220 \\
\hline 0.72 & 1.09 & 1.45 & 0.39 & 0.36 & 220 \\
\hline
\end{tabular}

\begin{tabular}{|l|l|l|l|l|l|}
\hline 0.72 & 1.09 & 1.45 & 0.39 & 0.36 & 220 \\
\hline 0.71 & 1.06 & 1.41 & 0.37 & 0.35 & 220 \\
\hline
\end{tabular}

\begin{tabular}{|l|l|l|l|l|l|}
\hline 0.71 & 1.06 & 1.41 & 0.37 & 0.35 & 220 \\
\hline 0.70 & 1.04 & 1.37 & 0.36 & 0.34 & 220 \\
\hline 0.64 & 0.90 & 1.17 & 0.28 & 0.31 & 351 \\
\hline
\end{tabular}

\begin{tabular}{|l|l|l|l|l|l|}
\hline 0.70 & 1.04 & 1.37 & 0.36 & 0.34 & 220 \\
\hline 0.64 & 0.90 & 1.17 & 0.28 & 0.31 & 351 \\
\hline 0.98 & 1.15 & 1.32 & 0.17 & 0.15 & 185 \\
\hline
\end{tabular}

\begin{tabular}{|l|l|l|l|l|l|}
\hline 0.98 & 1.15 & 1.32 & 0.17 & 0.15 & 185 \\
\hline 0.66 & 1.04 & 1.42 & 0.41 & 0.39 & 353 \\
\hline
\end{tabular}

\begin{tabular}{|l|l|l|l|l|l|}
\hline 0.66 & 1.04 & 1.42 & 0.41 & 0.39 & 353 \\
\hline 0.65 & 1.10 & 1.54 & 0.49 & 0.45 & 359 \\
\hline
\end{tabular}

\begin{tabular}{|l|l|l|l|l|l|}
\hline 0.65 & 1.10 & 1.54 & 0.49 & 0.45 & 359 \\
\hline 0.68 & 1.15 & 1.61 & 0.51 & 0.45 & 347 \\
\hline 0.70 & 1.17 & 1.64 & 0.52 & 0.45 & 345 \\
\hline
\end{tabular}

\begin{tabular}{|l|l|l|l|l|l|}
\hline 0.70 & 1.17 & 1.64 & 0.52 & 0.45 & 345 \\
\hline 0.69 & 1.11 & 1.52 & 0.46 & 0.41 & 220 \\
\hline
\end{tabular}

\begin{tabular}{|l|l|l|l|l|l|}
\hline 0.69 & 1.11 & 1.52 & 0.46 & 0.41 & 220 \\
\hline 0.69 & 1.10 & 1.50 & 0.44 & 0.40 & 220 \\
\hline
\end{tabular}

\begin{tabular}{|l|l|l|l|l|l|}
\hline 0.69 & 1.10 & 1.50 & 0.44 & 0.40 & 220 \\
\hline 0.69 & 1.09 & 1.48 & 0.43 & 0.39 & 220 \\
\hline
\end{tabular}

\begin{tabular}{|l|l|l|l|l|l|}
\hline 0.69 & 1.09 & 1.48 & 0.43 & 0.39 & 220 \\
\hline 0.69 & 1.08 & 1.46 & 0.42 & 0.39 & 220 \\
\hline
\end{tabular}

\begin{tabular}{|l|l|l|l|l|l|}
\hline 0.69 & 1.08 & 1.46 & 0.42 & 0.39 & 220 \\
\hline 0.69 & 1.06 & 1.43 & 0.40 & 0.38 & 220 \\
\hline
\end{tabular}

\begin{tabular}{|l|l|l|l|l|l|}
\hline 0.69 & 1.06 & 1.43 & 0.40 & 0.38 & 220 \\
\hline 0.65 & 0.89 & 1.14 & 0.26 & 0.29 & 354 \\
\hline
\end{tabular}

\begin{tabular}{|l|l|l|l|l|l|}
\hline 0.65 & 0.89 & 1.14 & 0.26 & 0.29 & 354 \\
\hline 0.95 & 1.12 & 1.28 & 0.17 & 0.15 & 185 \\
\hline
\end{tabular}

\begin{tabular}{|l|l|l|l|l|l|}
\hline 0.95 & 1.12 & 1.28 & 0.17 & 0.15 & 185 \\
\hline 0.65 & 1.00 & 1.35 & 0.38 & 0.38 & 350 \\
\hline
\end{tabular}

\begin{tabular}{|l|l|l|l|l|l|}
\hline 0.65 & 1.00 & 1.35 & 0.38 & 0.38 & 350 \\
\hline 0.64 & 1.05 & 1.46 & 0.45 & 0.43 & 353 \\
\hline
\end{tabular}

\begin{tabular}{|l|l|l|l|l|l|}
\hline 0.64 & 1.05 & 1.46 & 0.45 & 0.43 & 353 \\
\hline 0.65 & 1.09 & 1.53 & 0.49 & 0.45 & 349 \\
\hline
\end{tabular}

\begin{tabular}{|l|l|l|l|l|l|}
\hline 0.65 & 1.09 & 1.53 & 0.49 & 0.45 & 349 \\
\hline 0.65 & 1.11 & 1.57 & 0.51 & 0.46 & 352 \\
\hline
\end{tabular}

\begin{tabular}{|l|l|l|l|l|l|}
\hline 0.65 & 1.11 & 1.57 & 0.51 & 0.46 & 352 \\
\hline 0.65 & 1.08 & 1.50 & 0.47 & 0.43 & 220 \\
\hline
\end{tabular}

\begin{tabular}{|l|l|l|l|l|l|}
\hline 0.65 & 1.08 & 1.50 & 0.47 & 0.43 & 220 \\
\hline 0.66 & 1.07 & 1.49 & 0.46 & 0.43 & 220 \\
\hline
\end{tabular}

\begin{tabular}{l|l|l|l|l|l|}
\hline 0.66 & 1.07 & 1.47 & 0.45 & 0.42 & 220 \\
\hline 0.66 & 1.06 & 1.46 & 0.44 & 0.41 & 220 \\
\hline
\end{tabular}

\begin{tabular}{|c|c|c|c|c|c|}
\hline 0.66 & 1.06 & 1.46 & 0.44 & 0.41 & 220 \\
\hline 0.66 & 1.05 & 1.44 & 0.43 & 0.40 & 220 \\
\hline
\end{tabular}

\begin{tabular}{|l|l|l|l|l|l|}
\hline 0.66 & 1.05 & 1.44 & 0.43 & 0.40 & 220 \\
\hline 0.65 & 0.88 & 1.11 & 0.24 & 0.27 & 352 \\
\hline
\end{tabular}

\begin{tabular}{|c|c|c|c|c|c|}
\hline 0.65 & 0.88 & 1.11 & 0.24 & 0.27 & 352 \\
\hline 0.92 & 1.09 & 1.25 & 0.16 & 0.15 & \\
\hline
\end{tabular}

\begin{tabular}{|l|l|l|l|l|l|}
\hline 0.92 & 1.09 & 1.25 & 0.16 & 0.15 & 185 \\
\hline 0.04 & 0.96 & 1.28 & 0.34 & 0.36 & 359 \\
\hline
\end{tabular}

\begin{tabular}{|l|l|l|l|l|l|}
\hline 0.92 & 1.09 & 1.25 & 0.16 & 0.15 & 185 \\
\hline 0.64 & 0.96 & 1.28 & 0.34 & 0.36 & 359 \\
\hline 0.63 & 1.01 & 1.39 & 0.42 & 0.41 & 356 \\
\hline
\end{tabular}

\begin{tabular}{|l|l|l|l|l|l|}
\hline 0.63 & 1.01 & 1.39 & 0.42 & 0.41 & 356 \\
\hline 0.63 & 1.05 & 1.46 & 0.46 & 0.44 & 349 \\
\hline
\end{tabular}

\begin{tabular}{l|l|l|l|l|l|l|}
0.63 & 1.05 & 1.46 & 0.46 & 0.44 & 349 \\
\hline 0.64 & 1.07 & 1.51 & 0.48 & 0.45 & 352 \\
\hline
\end{tabular}

\begin{tabular}{|l|l|l|l|l|l|}
\hline 0.64 & 1.07 & 1.51 & 0.48 & 0.45 & 352 \\
\hline 0.63 & 1.05 & 1.46 & 0.46 & 0.44 & 220 \\
\hline 0.63 & 1.05 & 1.46 & 0.46 & 0.44 & 220 \\
\hline
\end{tabular}

\begin{tabular}{|c|c|c|c|c|c|}
\hline 0.63 & 1.05 & 1.46 & 0.46 & 0.44 & 220 \\
\hline 0.63 & 1.05 & 1.46 & 0.46 & 0.44 & 220 \\
\hline 0.63 & 1.04 & 1.45 & 0.45 & 0.43 & 220 \\
\hline
\end{tabular}

\begin{tabular}{|l|l|l|l|l|l|}
\hline 0.63 & 1.04 & 1.45 & 0.45 & 0.43 & 220 \\
\hline 0.63 & 1.04 & 1.44 & 0.45 & 0.43 & 220 \\
\hline
\end{tabular}

\begin{tabular}{|l|l|l|l|l|l|}
\hline 0.63 & 1.04 & 1.44 & 0.45 & 0.43 & 220 \\
\hline 0.64 & 1.04 & 1.43 & 0.44 & 0.42 & 220 \\
\hline
\end{tabular}

\begin{tabular}{|l|l|l|l|l|l|}
\hline 0.63 & 1.04 & 1.44 & 0.45 & 0.43 & 220 \\
\hline 0.64 & 1.04 & 1.43 & 0.44 & 0.42 & 220 \\
\hline
\end{tabular}

\begin{tabular}{|l|l|l|l|l|l|}
\hline 0.69 & 1.07 & 1.45 & 0.42 & 0.39 & 17273 \\
\hline 0.69 & 1.08 & 1.47 & 0.42 & 0.39 & 10838 \\
\hline
\end{tabular} 


\subsection{Assessment of pavement structure modelling in backanalysis procedures}

When back-calculation analysis is carried out, the pavement model is typically modelled as a 3-layer structure (i.e., asphalt on top of subbase and subgrade). However, a 2-layer structure could also be employed (i.e., asphalt on top of 'equivalent' foundation that accounts for the contribution of both the subbase and subgrade layers). This sections aims to investigate the effect of the modelling structure (i.e., 3-layer versus 2-layer model) used in backcalculation procedures in the assessment of both the layers' stiffness and of the ultimate performance life of the pavement.

It should be noted that goodness of fit considerations were not taken into account when considering the back-analysed stiffness values.

The following methodology was adopted for this activity:

1. A selected number of pavement structures were considered (see Table 8.6) and deflections at $0.000,0.300,0.600,0.900,1.200,1.500$ and $2.100 \mathrm{~m}$ distance were calculated with BISAR under a vertical load of $50 \mathrm{kN}$, a load radius of $0.150 \mathrm{~m}$ and a Poisson's ratio $(v)$ of 0.35 for all layers.

\section{Table 8.6 Calculations of deflections with BISAR}

\begin{tabular}{|c|c|c|c|c|c|c|c|c|c|c|}
\hline \multirow{2}{*}{$v_{a}=0.35$} & Asphalt thickness (m) & 0.200 & 0.300 & & & & & & & \\
\hline & Asphalt stiffness (MPa) & 2500 & 3100 & 4700 & 6200 & 7000 & & & & \\
\hline \multirow{2}{*}{$v_{s b}=0.35$} & Subbase thickness (m) & 0.100 & 0.200 & 0.300 & 0.400 & 0.500 & & & & \\
\hline & Subbase stiffness (MPa) & 30 & 50 & 75 & 100 & 150 & 200 & 300 & 400 & 500 \\
\hline$v_{s g}=0.35$ & Subgrade stiffiness (MPa) & 15 & 30 & 50 & 100 & & & & & \\
\hline
\end{tabular}

Deflections calculated with BISAR at:
\begin{tabular}{|l|l|l|l|l|l|l|l|} 
Distance $(\mathrm{m})$ & $\mathrm{d})$ & $\mathrm{d} 3$ & $\mathrm{~d} 4$ & $\mathrm{~d} 5$ & $\mathrm{~d} 6$ & $\mathrm{~d} 7$ \\
\hline
\end{tabular}

2. The layer stiffness was back-calculated with MODULUS, for both 3-layer and 2-layer pavement structures, with the range of layer stiffness shown in Table 8.7. 
Table 8.7 Layer stiffness range used in back-calculation analysis with MODULUS

\begin{tabular}{|l|l|c|c|c|}
\hline \multicolumn{2}{|l|}{ Back-analysis with Modulus } & Min & Max & $v$ \\
\hline Layer 1 & Asphalt stiffiness (MPa) & 50 & 50000 & 0.35 \\
\hline Layer 2 & Subbase stiffiness (MPa) & 28 & 1000 & 0.35 \\
\hline Layer 3 & Subgrade stiffiness (MPa) & 7 & & 0.35 \\
\hline
\end{tabular}

3. Asphalt and subgrade strains were then calculated with BISAR for all combinations of values considered (under a vertical load of $40 \mathrm{kN}$, a load radius of $0.151 \mathrm{~m}$ and a Poisson's ratio $(v)$ of 0.35 for all layers) for the following set of data:

- The original set of data (3-layer).

- The set of data with the layer stiffness back-calculated by using a 3layer model structure.

- The set of data with the layer stiffness back-calculated by using a 2layer model structure.

4. In the forward analysis with BISAR strains were calculated at the same depths for both the 3-layer and 2-layer model structures, see example in Table 8.8.

Table 8.8 Example of back-calculation results for 3-layer and 2-layer model structures with MODULUS and location of asphalt and subgrade strains in subsequent forward analysis with BISAR

\begin{tabular}{|l|c|c|c|}
\hline $\begin{array}{l}\text { Layers \& Strains } \\
\text { Position }\end{array}$ & Original structure & $\begin{array}{c}\text { 3L structure (from } \\
\text { backanalysis) }\end{array}$ & $\begin{array}{c}\text { 2L structure (from } \\
\text { backanalysis) }\end{array}$ \\
\cline { 2 - 5 } & $\mathrm{T}(\mathrm{m})$ and $\mathrm{E}(\mathrm{MPa})$ & $\mathrm{T}(\mathrm{m})$ and $\mathrm{E}(\mathrm{MPa})$ & $\mathrm{T}(\mathrm{m})$ and $\mathrm{E}(\mathrm{MPa})$ \\
\hline Asphalt & $0.100,2500$ & $0.100,2040$ & $0.100,2890$ \\
\hline Subbase & $0.100,30$ & $0.100,75$ & $0.100,15$ \\
\hline Subgrade & $\infty, 15$ & $\infty, 15$ & $\infty, 15$ \\
\hline$\varepsilon_{\mathrm{r}}$ calculated at: & $0.099 \mathrm{~m}$ & $0.099 \mathrm{~m}$ & $0.099 \mathrm{~m}$ \\
\hline$\varepsilon_{\mathrm{z}}$ calculated at: & $0.201 \mathrm{~m}$ & $0.201 \mathrm{~m}$ & $0.201 \mathrm{~m}$ \\
\hline
\end{tabular}

$T=$ thickness $(m)$

$\mathrm{E}=$ stiffness $(\mathrm{MPa})$

The effect of the model structure on layer stiffness is summarised in Table 8.9 and Table 8.10. The tables shows the ratio of the layer back-analysed stiffness 
for the modelled pavement structure over the original 'true' value (for example, $E_{\text {asphalt-backanalysed/ }}$ Easphalt-true).

\section{Table 8.9 Effect of 3-layer model structure on stiffness}

\begin{tabular}{|c|c|c|c|c|c|c|c|c|c|c|}
\hline \multirow{3}{*}{\begin{tabular}{|c|} 
Asphalt \\
thickness \\
$(\mathrm{m})$
\end{tabular}} & \multirow{3}{*}{$\begin{array}{c}\text { Subbase } \\
\text { thickness } \\
(\mathrm{m})\end{array}$} & \multicolumn{9}{|c|}{ 3L model for back-analysis } \\
\hline & & \multicolumn{3}{|c|}{$\mathrm{E}_{\text {asphalt-backanalysed }} / \mathrm{E}_{\text {asphalt:true }}$} & \multicolumn{3}{|c|}{$\mathrm{E}_{\text {subbase-backanalysed }} / \mathrm{E}_{\text {subbase-itue }}$} & \multicolumn{3}{|c|}{$\mathrm{E}_{\text {subgrade-backanalysed }} / \mathrm{E}_{\text {subgrade-frue }}$} \\
\hline & & 15th per & 50th per & 85th perc & 15th per & mean & 85th perc & 15th per & mean & 85th perc \\
\hline 0.200 & 0.100 & 0.83 & 0.96 & 1.07 & 0.65 & 1.20 & 4.05 & 1.00 & 1.00 & 1.00 \\
\hline 0.200 & 0.200 & 0.92 & 0.99 & 1.12 & 0.60 & 1.00 & 1.34 & 1.00 & 1.00 & 1.00 \\
\hline 0.200 & 0.300 & 0.96 & 0.99 & 1.04 & 0.93 & 1.00 & 1.10 & 1.00 & 1.00 & 1.00 \\
\hline 0.200 & 0.400 & 0.97 & 0.99 & 1.03 & 0.93 & 1.00 & 1.02 & 1.00 & 1.00 & 1.00 \\
\hline 0.200 & 0.500 & 0.98 & 1.00 & 1.03 & 0.93 & 1.00 & 1.00 & 1.00 & 1.00 & 1.00 \\
\hline 0.300 & 0.100 & 0.91 & 0.99 & 1.08 & 0.33 & 1.15 & 4.63 & 1.00 & 1.00 & 1.00 \\
\hline 0.300 & 0.200 & 0.85 & 1.00 & 1.12 & 0.53 & 1.00 & 2.91 & 1.00 & 1.00 & 1.00 \\
\hline 0.300 & 0.300 & 0.93 & 1.00 & 1.10 & 0.60 & 1.00 & 1.60 & 1.00 & 1.00 & 1.00 \\
\hline 0.300 & 0.400 & 0.94 & 1.01 & 1.08 & 0.83 & 1.00 & 1.33 & 1.00 & 1.00 & 1.00 \\
\hline 0.300 & 0.500 & 0.96 & 1.01 & 1.07 & 0.80 & 1.00 & 1.17 & 1.00 & 1.00 & 1.00 \\
\hline 0.2 to $0.3 \mathrm{~m}$ & 0 to $0.5 \mathrm{~m}$ & 0.92 & 1.00 & 1.07 & 0.74 & 1.00 & 1.60 & 1.00 & 1.00 & 1.00 \\
\hline
\end{tabular}

\section{Table 8.10 Effect of 2-layer model structure on stiffness}

\begin{tabular}{|c|c|c|c|c|c|c|c|c|c|c|}
\hline \multirow{3}{*}{$\begin{array}{c}\text { Asphalt } \\
\text { thickness } \\
(\mathrm{m})\end{array}$} & \multirow{3}{*}{$\begin{array}{c}\text { Subbase } \\
\text { thickness } \\
(\mathrm{m})\end{array}$} & \multicolumn{9}{|c|}{ 2L model for back-analysis } \\
\hline & & \multicolumn{3}{|c|}{$E_{\text {asphalt-backanalysed }} / E_{\text {asphalt-true }}$} & \multicolumn{3}{|c|}{$E_{\text {subbase-backanalysed }} / E_{\text {subbase-true }}$} & \multicolumn{3}{|c|}{$E_{\text {subgrade-backanalysed }} / E_{\text {subgrade-frue }}$} \\
\hline & & 15th per & 50 th per & 85th perc & 15th per & mean & 85th perc & 15th per & mean & 85th perc \\
\hline 0.200 & 0.100 & 0.99 & 1.09 & 1.27 & 0.08 & 0.28 & 1.00 & 1.00 & 1.00 & 1.00 \\
\hline 0.200 & 0.200 & 0.99 & 1.24 & 1.70 & 0.10 & 0.29 & 1.00 & 1.00 & 1.00 & 1.33 \\
\hline 0.200 & 0.300 & 0.99 & 1.39 & 2.27 & 0.10 & 0.29 & 1.00 & 1.00 & 1.00 & 1.33 \\
\hline 0.200 & 0.400 & 0.99 & 1.55 & 2.81 & 0.10 & 0.30 & 1.00 & 1.00 & 1.15 & 1.33 \\
\hline 0.200 & 0.500 & 0.99 & 1.69 & 3.26 & 0.10 & 0.30 & 1.00 & 1.00 & 1.20 & 1.33 \\
\hline 0.300 & 0.100 & 1.00 & 1.05 & 1.14 & 0.08 & 0.28 & 1.00 & 1.00 & 1.00 & 1.00 \\
\hline 0.300 & 0.200 & 1.00 & 1.12 & 1.34 & 0.10 & 0.29 & 1.00 & 1.00 & 1.00 & 1.10 \\
\hline 0.300 & 0.300 & 1.00 & 1.20 & 1.57 & 0.10 & 0.29 & 1.00 & 1.00 & 1.00 & 1.33 \\
\hline 0.300 & 0.400 & 1.00 & 1.27 & 1.80 & 0.10 & 0.30 & 1.00 & 1.00 & 1.10 & 1.33 \\
\hline 0.300 & 0.500 & 1.00 & 1.33 & 2.04 & 0.10 & 0.30 & 1.00 & 1.00 & 1.20 & 1.33 \\
\hline 0.2 to $0.3 \mathrm{~m}$ & 0 to $0.5 \mathrm{~m}$ & 0.99 & 1.20 & 1.90 & 0.10 & 0.30 & 1.00 & 1.00 & 1.00 & 1.33 \\
\hline
\end{tabular}

The following observations are made:

- For the selected range of pavement structures considered in the analysis (i.e., asphalt thickness from $0.2 \mathrm{~m}$ to $0.3 \mathrm{~m}$ and subbase thickness from $0.1 \mathrm{~m}$ to $0.5 \mathrm{~m}$ ), the ratio of layer back-analysed stiffness over the original 'true' value for the 3-layer model structure has the following values: 
- The $15^{\text {th }}$ percentile is 0.92 , the $50^{\text {th }}$ percentile is 1.00 and the $85^{\text {th }}$ percentile is 1.07 for the asphalt layer.

- The $15^{\text {th }}$ percentile is 0.74 , the $50^{\text {th }}$ percentile is 1.00 and the $85^{\text {th }}$ percentile is 1.60 for the subbase layer.

- The $15^{\text {th }}$ percentile is 1.00 , the $50^{\text {th }}$ percentile is 1.00 and the $85^{\text {th }}$ percentile is 1.00 for the subgrade layer.

- For the selected range of pavement structures considered in the analysis, the ratio of layer back-analysed stiffness over the original 'true' value for the 2-layer model structure has the following values:

- The $15^{\text {th }}$ percentile is 0.99 , the $50^{\text {th }}$ percentile is 1.20 and the $85^{\text {th }}$ percentile is 1.90 for the asphalt layer.

- The $15^{\text {th }}$ percentile is 0.10 , the $50^{\text {th }}$ percentile is 0.30 and the $85^{\text {th }}$ percentile is 1.00 for the subbase layer.

- The $15^{\text {th }}$ percentile is 1.00 , the $50^{\text {th }}$ percentile is 1.00 and the $85^{\text {th }}$ percentile is 1.33 for the subgrade layer.

- The results suggest that the 3-layer model is the preferred model for the estimation of back-analysed layer stiffnesses and confirms that the backanalysis with MODULUS and the forward analysis with BISAR are in good agreement.

- The back-analysed layer stiffnesses are adversely affected when subbase layer thickness is below $200 \mathrm{~mm}$. As generally a minimum thickness of $150 \mathrm{~mm}$ is recommended in the UK standards for the subbase layer, the results for thicknesses lower than $200 \mathrm{~mm}$ can be discarded.

The effect of the model structure on pavement performance is summarised in Table 8.1 and Table 8.12. The tables shows the ratio of the performance life calculated with BISAR for the modelled pavement structure over the performance life calculated with BISAR for the original 3-layer pavement

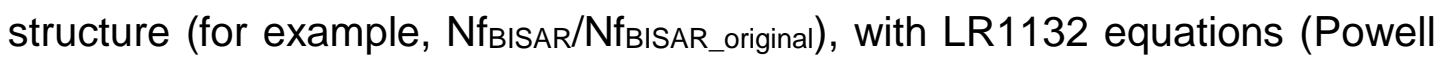
et al., 1984). The following observations are made:

- For the selected range of pavement structures considered in the analysis (i.e., asphalt thickness from $0.2 \mathrm{~m}$ to $0.3 \mathrm{~m}$ and subbase thickness from $0.1 \mathrm{~m}$ to $0.5 \mathrm{~m}$ ), the ratio of the performance life calculated with BISAR for the 3- 
layer pavement structure over the performance life calculated with BISAR for the original 3-layer pavement structure has the following values:

- The $15^{\text {th }}$ percentile is 0.93 , the $50^{\text {th }}$ percentile is 0.99 and the $85^{\text {th }}$ percentile is 1.05 for the fatigue life.

- The $15^{\text {th }}$ percentile is 0.82 , the $50^{\text {th }}$ percentile is 0.99 and the $85^{\text {th }}$ percentile is 1.07 for the deformation life.

- For the selected range of pavement structures considered in the analysis (i.e., asphalt thickness from $0.2 \mathrm{~m}$ to $0.3 \mathrm{~m}$ and subbase thickness from $0.1 \mathrm{~m}$ to $0.5 \mathrm{~m}$ ), the ratio of the performance life calculated with BISAR for the 2layer pavement structure over the performance life calculated with BISAR for the original 3-layer pavement structure has the following values:

- The $15^{\text {th }}$ percentile is 0.92 , the $50^{\text {th }}$ percentile is 1.11 and the $85^{\text {th }}$ percentile is 2.00 for the fatigue life.

- The $15^{\text {th }}$ percentile is 0.98 , the $50^{\text {th }}$ percentile is 2.47 and the $85^{\text {th }}$ percentile is 4.57 for the deformation life.

- The results suggest that the 3-layer model is definitely the preferred model for the calculation of the performance life. However, if the designer is interested in the fatigue performance only of the pavement, then the 2-layer model could be employed.

Table 8.11 Effect of 3-layer model structure on fatigue and deformation life with LR1132 equations

\begin{tabular}{|c|c|c|c|c|c|c|c|}
\hline \multirow{3}{*}{\begin{tabular}{|l|} 
Asphalt \\
thickness \\
$(\mathrm{m})$
\end{tabular}} & \multirow{3}{*}{$\begin{array}{l}\text { Subbase } \\
\text { thickness } \\
(\mathrm{m})\end{array}$} & \multicolumn{6}{|c|}{ FROM PERCENTILES } \\
\hline & & \multicolumn{3}{|c|}{$\mathrm{Nf}_{\text {BISAR }} / \mathrm{NI}_{\text {BISAR_original }}$ (LR1132) } & \multicolumn{3}{|c|}{$\mathrm{Nd}_{\text {BISAR }} / \mathrm{Nd}_{\text {BISAR_original }}$ (LR1132) } \\
\hline & & 15 th per & 50th per & $\begin{array}{l}\text { 85th } \\
\text { perc }\end{array}$ & 15th per & 50th per & $\begin{array}{l}\text { 85th } \\
\text { perc }\end{array}$ \\
\hline 0.200 & 0.100 & 0.90 & 0.98 & 1.06 & 0.62 & 0.93 & 1.03 \\
\hline 0.200 & 0.200 & 0.91 & 0.96 & 1.01 & 0.84 & 0.98 & 1.09 \\
\hline 0.200 & 0.300 & 0.92 & 0.97 & 1.02 & 0.92 & 0.98 & 1.02 \\
\hline 0.200 & 0.400 & 0.93 & 0.97 & 1.04 & 0.96 & 0.99 & 1.03 \\
\hline 0.200 & 0.500 & 0.94 & 0.98 & 1.04 & 0.96 & 0.98 & 1.02 \\
\hline 0.300 & 0.100 & 0.90 & 1.01 & 1.15 & 0.67 & 0.99 & 1.47 \\
\hline 0.300 & 0.200 & 0.95 & 1.01 & 1.05 & 0.71 & 0.99 & 1.56 \\
\hline 0.300 & 0.300 & 0.96 & 1.00 & 1.05 & 0.85 & 1.00 & 1.13 \\
\hline 0.300 & 0.400 & 0.95 & 1.01 & 1.06 & 0.83 & 1.00 & 1.08 \\
\hline 0.300 & 0.500 & 0.95 & 1.01 & 1.08 & 0.95 & 1.00 & 1.11 \\
\hline 0.2 to $0.3 \mathrm{~m}$ & 0 to $0.5 \mathrm{~m}$ & 0.93 & 0.99 & 1.05 & 0.82 & 0.99 & 1.07 \\
\hline
\end{tabular}


Table 8.12 Effect of 2-layer model structure on fatigue and deformation life with LR1132 equations

\begin{tabular}{|c|c|c|c|c|c|c|c|}
\hline \multirow{3}{*}{$\begin{array}{l}\text { Asphalt } \\
\text { thickness } \\
(\mathrm{m})\end{array}$} & \multirow{3}{*}{$\begin{array}{l}\text { Subbase } \\
\text { thickness } \\
(\mathrm{m})\end{array}$} & \multicolumn{6}{|c|}{ FROM PERCENTILES } \\
\hline & & \multicolumn{3}{|c|}{$\mathrm{Ni}_{\text {BISAR }} / \mathrm{Ni}_{\text {BISAR_original }}(\mathrm{LR1132})$} & \multicolumn{3}{|c|}{$\mathrm{Nd}_{\text {BISAR }} / \mathrm{Nd}_{\text {BISAR_original }}(\mathrm{LR1132})$} \\
\hline & & 15 th per & 50 th per & $\begin{array}{l}\text { 85th } \\
\text { perc }\end{array}$ & 15th per & 50th per & $\begin{array}{l}\text { 85th } \\
\text { perc }\end{array}$ \\
\hline 0.200 & 0.100 & 0.86 & 0.95 & 0.99 & 0.97 & 2.12 & 2.57 \\
\hline 0.200 & 0.200 & 0.96 & 1.17 & 1.42 & 0.97 & 2.83 & 4.62 \\
\hline 0.200 & 0.300 & 0.96 & 1.57 & 2.31 & 0.97 & 3.35 & 5.72 \\
\hline 0.200 & 0.400 & 0.96 & 2.00 & 3.90 & 0.98 & 3.63 & 5.84 \\
\hline 0.200 & 0.500 & 0.96 & 2.50 & 5.85 & 0.98 & 3.66 & 5.62 \\
\hline 0.300 & 0.100 & 0.83 & 0.96 & 1.00 & 1.00 & 1.91 & 2.24 \\
\hline 0.300 & 0.200 & 0.92 & 1.01 & 1.04 & 1.00 & 2.43 & 3.14 \\
\hline 0.300 & 0.300 & 0.99 & 1.11 & 1.32 & 1.00 & 2.84 & 4.43 \\
\hline 0.300 & 0.400 & 1.00 & 1.29 & 1.61 & 1.00 & 3.02 & 4.58 \\
\hline 0.300 & 0.500 & 1.00 & 1.42 & 2.08 & 1.00 & 3.23 & 4.56 \\
\hline 0.2 to $0.3 \mathrm{~m}$ & 0 to $0.5 \mathrm{~m}$ & 0.92 & 1.11 & 2.00 & 0.98 & 2.47 & 4.57 \\
\hline
\end{tabular}

\subsection{Summary and Conclusions}

A large number of computer programs have been developed for calculating stresses, strains and displacements in layered elastic systems, such as the BISAR software developed by Shell. BISAR is believed to provide reasonably accurate values of stresses and strains in pavement response models but the main limitation is that only a maximum of 10 pavement structures can be analysed at a time and all input parameters need to be inserted manually by the user. Consequently, the software is not suitable for obtaining stresses and strains for a large number of pavement structures.

A simplified method for calculating stresses, strains and displacements in layered elastic systems is represented by Odemark's Method of Equivalent Thicknesses (MET).

Values of asphalt and subgrade strains were calculated with both the Method of Equivalent Thicknesses (MET) method and the BISAR software for a large number of flexible pavements (3-layer systems). The analysis showed that, while subgrade strains were predicted acceptably well, differences greater than $\pm 10 \%$ exist between the two methods in the case of the asphalt strains. 
To overcome some limitations of the MET method and, therefore, to reduce the differences between the two methods an alternative model was proposed, for the calculation of the asphalt strains. An alternative model for the fatigue life was also introduced.

This involves the calculation of an Equivalent Foundation Modulus (EFM), which in this analysis represents the contribution of both the foundation layers and the asphalt layer. A linear regression analysis has been presented which allows the prediction of values of asphalt strain and fatigue life calculated with BISAR from those obtained with the MET methodology and with a relatively high level of confidence. Approximately $93 \%$ of predicted strains were found to lie within $10 \%$ of the BISAR-derived values. A 'professional' factor is also discussed which represents uncertainties of the assumptions and simplifications used in the proposed design model.

Because of variability in subbase stiffness values found from real site data, an alternative deformation model was also introduced which accepts a relaxation in one of the MET conditions. The proposed relaxation is for the ratio of subbase stiffness over subgrade stiffness to be greater than or equal to 1 while keeping all other conditions of validity of the MET method. The alternative deformation model was demonstrated not to introduce a significant difference in variability when compared to the model where all the original MET conditions are satisfied (i.e., the one which only accepts $E_{\text {subbase }} / E_{\text {subgrade }}>2$ ).

Finally, a comparison of results for selected pavement structures resulting from both 3-layer and 2-layer models employed in back-calculation analysis, has suggested that the preferred pavement model, for more reliable results in both back-analysed layer stiffnesses and performance lives (fatigue and deformation), is a 3-layer structure. 


\section{$9 \quad$ Monte Carlo simulation and design life probability distributions}

To predict pavement performance in terms of fatigue and deformation life - by means of a forecasting model such as the one discussed in chapter 8 - some assumptions must be made related to each pavement input variable. As discussed in previous chapters, these variables are far from being certain. Probably the most popular method to estimate pavement performance life to account for uncertainty of input variables - and hence to calculate the probability of failure of a pavement structure - is to perform the simulation technique called Monte Carlo analysis. The simulation basically consists of random sampling from probability distributions of the input variables to obtain approximate solutions to problems. In this approach, uncertain inputs are modelled with probability distributions (such as the normal distribution), and random values for those inputs are generated by drawing from those distributions. The input values are then used in a predictive model to generate an outcome. The process is repeated many times (typically thousands or tens of thousands of times), resulting in a distribution of outcomes that can be used to answer questions of a probabilistic nature to determine behaviour, to analyse risk and more (IBM 2012).

The use of the Monte Carlo Simulation (MSC) technique to evaluate the variation in predicted performance based on the variability of a set of input parameters for a specified pavement design section is recommended by many authors (Graves and Mahboub, 2007; Darter et al. 2005; Zhang and Piepmeyer, 2005; Timm et al., 2000). The use of this technique in the context of reliability analysis in pavement design has been discussed in chapter 3 .

The purpose of this chapter is to show how the MCS technique could be used to predict output distributions of both fatigue and deformation performance, by treating data input of pavement design parameters as random variables.

Finally, to support the outcome of the MCS the Method of Moments (MM), also fully described in chapter 3 , is employed to assess performance variability. 


\subsection{Steps of Monte Carlo simulation for pavement performance analysis}

This section describes how the simulation has been carried out in this study together with assumptions made. The steps of the analysis, see Figure 9.1, are:

1. Input pavement structure and input variables

The input pavement structure is a 3-layer model. The input variables are: asphalt thickness $\left(T_{\mathrm{a}}\right)$, granular subbase thickness $\left(T_{\mathrm{sb}}\right)$, asphalt stiffness modulus $\left(E_{a}\right)$, stiffness of the subbase $\left(E_{s b}\right)$, and the stiffness of the subgrade $\left(E_{s g}\right)$. These variables have been chosen as having the most influence on the pavement fatigue and deformation life, see chapter 7. Poisson's ratio for all layers is assumed to be 0.35 .

2. Assign input probability distribution

A normal probability distribution is selected for layer thickness ( $T_{a}$ and $T_{s b}$ ) while a lognormal probability distribution is selected for layer stiffness $\left(E_{a}, E_{s b}\right.$, and $E_{s g}$ ). The chosen probability distribution for each input is based on the findings of this research and is supported by the literature review (see Table 2.1 in chapter 2$)$.

3. Generate random input variables

A sample from the input variables is generated (i.e., $\mathrm{N}$ random numbers for each of the random variables will give $N$ sets of random numbers, each set representing a realisation of the problem). A sample $\mathrm{N}$ of 1000 points from probability distributions of the inputs has been considered. The generation of random numbers for layer stiffness is easily performed in Matlab. Also in Matlab - based on the Fourier Analysis technique described in chapter 5 random asphalt and sub-base thickness profiles are generated which have similar frequency characteristics to the real case study profiles (i.e., same mean, standard deviation and probability distribution).

4. Calculate response variables (fatigue and deformation life) 
The improved Method of Equivalent Thickness model, discussed in chapter 8, is used to estimate the fatigue and deformation life for each generated pavement structure.

It involves first the calculation of an Equivalent Foundation Modulus (EFM), then a linear regression model is used to predict values of fatigue and deformation life calculated with BISAR from those obtained with the MET methodology. Alternatively, BISAR could have been used to compute the values of fatigue and deformation life. This would results in an increased computational cost, but would lead to a smaller coefficient of variation of the results, as described in Section 8.1.4.

\section{Generate output probability distribution}

The model is processed for the sample size of $N$ points generating $N$ values for fatigue and deformation life. During a simulation, the values of the input variables are generated randomly according to specified probability distributions as discussed in previous steps. Only one iteration run of a particular Monte Carlo simulation is needed (i.e., repeated random sampling has not been done) because this is considered sufficient for the purpose of this analysis. The focus of the research is to assess variability of pavement performance rather than its absolute values. The results of the model (the fatigue and deformation life) for each run are computed and stored away for statistical analysis (mean, percentiles etc). The expectation or mean value represents the (probability-weighted) average value of the results while the standard deviation of these results is then a measure of the spread of the results around the mean value and is known as the uncertainty. 


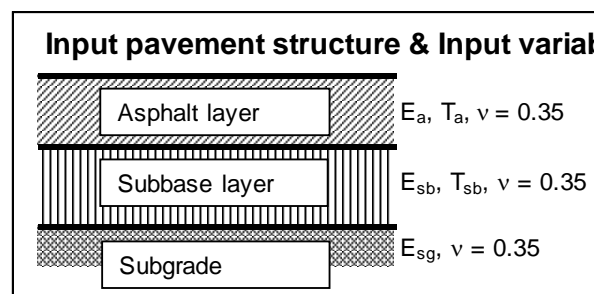

$E_{a}, E_{s b}, E_{s g}=$ asphalt, subbase and subgrade layer stiffness ( $\left.M P a\right)$

$\mathrm{T}_{\mathrm{a}}, \mathrm{T}_{\mathrm{sb}}=$ asphalt and subbase layer thickness $(\mathrm{m})$

\section{Assign Input probability distribution}

Lognormal probability distribution for layer stiffness $\left(E_{a}, E_{s b}, E_{s g}\right)$ Normal probability distribution for layer thickness $\left(T_{a}, T_{s b}\right)$
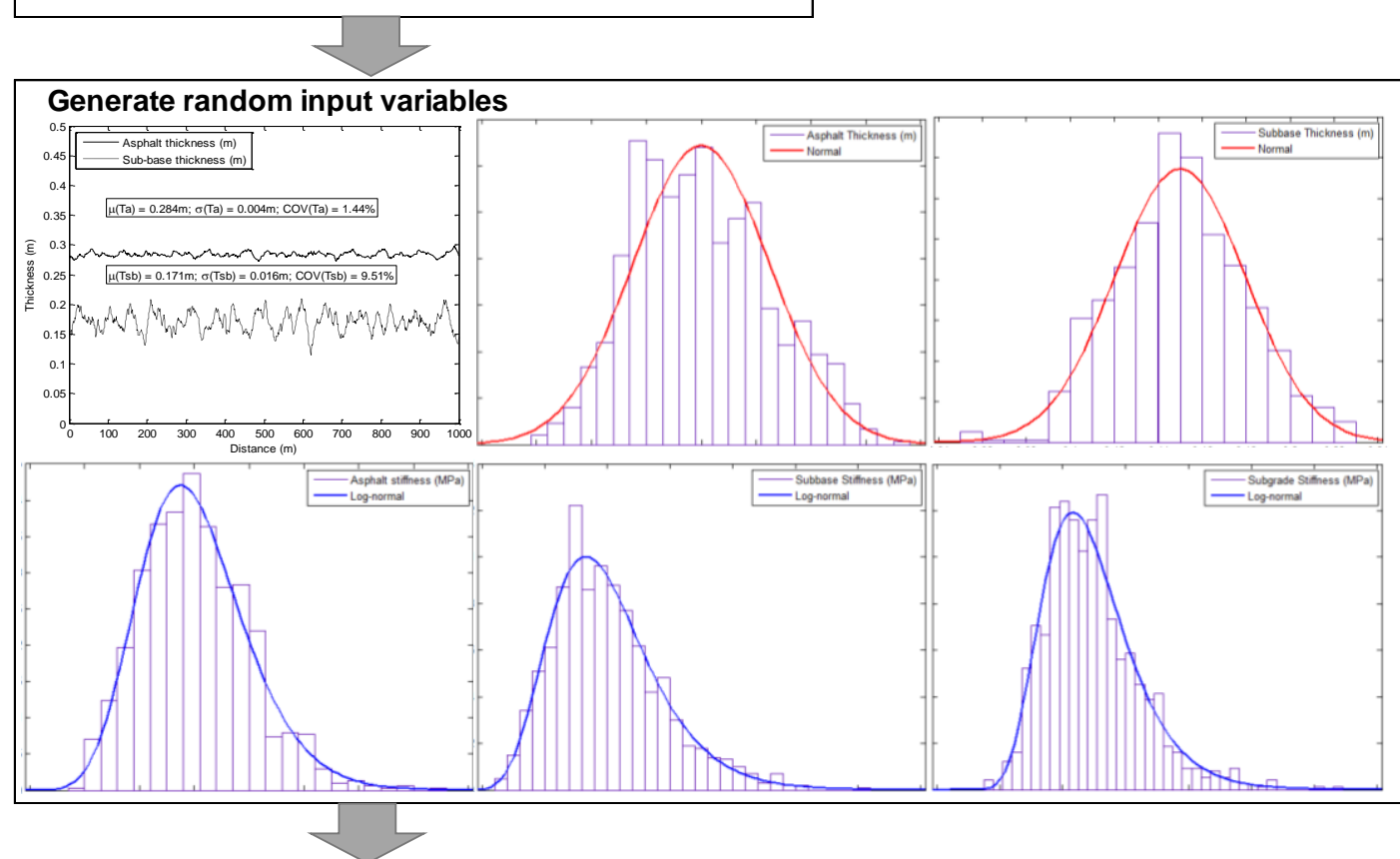

Calculate response variables (fatigue and deformation life)

Using improved MET discussed in chapter 8

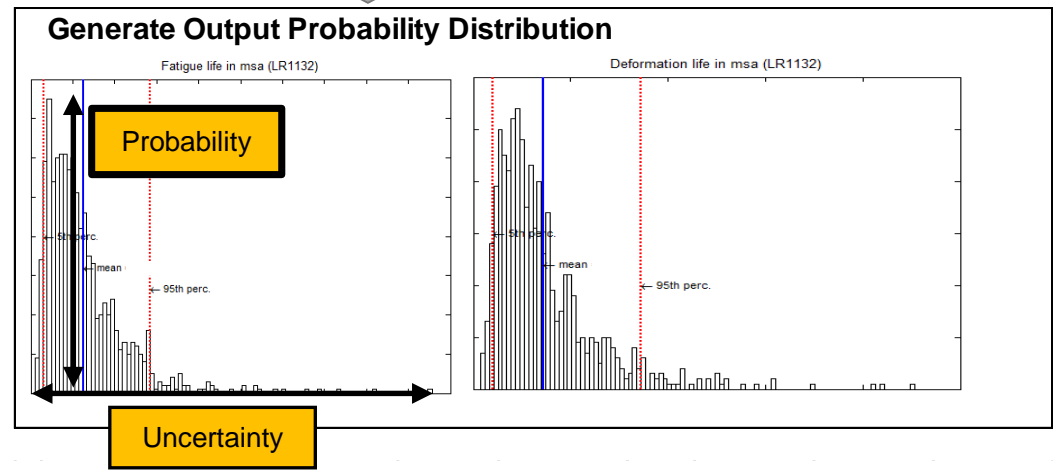

Figure 9.1 Steps of Monte Carlo simulation for pavement performance analysis 


\subsubsection{Artificial adjustments to subbase stiffness $\left(E_{s b}\right)$}

The subbase stiffness variation obtained from FWD backanalysis of the homogeneous sections in Table 7.3 (see also Table 9.1 and Table 9.2) was noted to be high compared to published values from available literature (see Table 2.1 in chapter 2). The high uncertainty in the data is probably due to the small sample size derived from the cusum analysis, which was used to identify the homogeneous sections for each site (see paragraph 7.4). Therefore, the available subbase stiffness variation was not considered representative of real subbase stiffness variations.

The following adjustments were made:

- While the back-calculated mean subbase stiffness values were retained, a fixed coefficient of variation (COV) of 30\% (arbitrary chosen but within the range available from literature, see Table 2.1 in chapter 2) was introduced to derive the standard deviation. The adjusted subbase stiffness values were used to generate a subbase stiffness probability distribution and to calculate performance life. This is referred to henceforth as the "first adjustment' to the subbase stiffness.

- A 'second adjustment' was made to the subbase stiffness to overcome the problem of empty plots for deformation life. Because the condition $E_{s b} / E_{s g}$ $\geq 1$ (ratio of subbase stiffness over subgrade stiffness to be greater or equal to 1 - needed for use of MET) from the randomly generated values (with the parameters of the first adjustment) was never satisfied for some sections - with resulting deformation life values being excluded from the analysis - an algorithm was created to force the subbase stiffness to be equal to the subgrade stiffness whenever the condition $E_{s b} / E_{s b}<1$ was encountered.

To summarise, the set of subbase stiffness data resulting from the first adjustment (i.e. imposed COV of $30 \%$ ) was used for fatigue life calculations while the set of subbase stiffness data resulting from the second adjustment (i.e. imposed $E_{s b}=E_{s g}$ when $E_{s b} / E_{s b}<1$ ) was used for deformation life 
calculations. The input data used for the simulation for each variable and section is summarised in Table 9.1 and Table 9.2.

\section{Table 9.1 Input variables for simulation}

\begin{tabular}{|c|c|c|c|c|c|c|c|c|c|c|c|c|c|c|c|c|}
\hline \multicolumn{5}{|c|}{ Pavement site sections } & \multicolumn{4}{|c|}{ Asphalt thickness (m) } & \multicolumn{4}{|c|}{ Subbase thickness (m) } & \multicolumn{4}{|c|}{$\begin{array}{c}\text { Back-calculated \& corrected } \\
\text { asphalt stiffness (MPa) }\end{array}$} \\
\hline $\begin{array}{l}\text { Length } \\
\text { (m) }\end{array}$ & Site & Section & From & To & $\mu$ & $\sigma$ & COV & $\begin{array}{l}\text { COV } \\
(\%)\end{array}$ & $\mu$ & $\sigma$ & COV & $\begin{array}{l}\text { COV } \\
(\%)\end{array}$ & $\mu$ & $\sigma$ & COV & $\begin{array}{l}\text { COV } \\
(\%)\end{array}$ \\
\hline 220 & M01 & 3 & 35160 & 35380 & 0.284 & 0.004 & 0.015 & 1.48 & 0.171 & 0.016 & 0.095 & 9.49 & 4060 & 1423 & 0.351 & 35.06 \\
\hline 320 & M01 & 16 & 36300 & 36620 & 0.298 & 0.007 & 0.023 & 2.33 & 0.088 & 0.020 & 0.224 & 22.40 & 4730 & 1716 & .363 & 36.28 \\
\hline 200 & M04 & 1 & 0 & 200 & 0.251 & 0.043 & 0.171 & 17.14 & 0.166 & 0.045 & 0.272 & 27.15 & 6406 & 3570 & 0.557 & 55.74 \\
\hline 375 & M04 & 10 & 875 & 1250 & 0.452 & 0.016 & 0.035 & 3.48 & 0.205 & 0.022 & 0.110 & 10.97 & 8105 & 1366 & 0.169 & 16.85 \\
\hline 250 & M04 & 20 & 1975 & 2225 & 0.449 & 0.010 & 0.023 & 2.26 & 0.257 & 0.031 & 0.119 & 11.89 & 7671 & 1016 & 0.132 & 13.25 \\
\hline 275 & M04 & 30 & 2675 & 2950 & 0.437 & 0.013 & 0.030 & 2.98 & 0.156 & 0.039 & 0.253 & 25.31 & 7875 & 1225 & 0.156 & 15.56 \\
\hline 200 & M04 & 37 & 3325 & 3525 & 0.443 & 0.008 & 0.018 & 1.81 & 0.147 & 0.013 & 0.092 & 9.17 & 7404 & 1114 & 0.150 & 15.05 \\
\hline 370 & M05 ACW & 10 & 460 & 830 & 0.302 & 0.015 & 0.049 & 4.89 & 0.218 & 0.006 & 0.029 & 2.90 & 5780 & 1100 & 0.190 & 19.04 \\
\hline 550 & M05 ACW & 11 & 830 & 1380 & 0.319 & 0.011 & 0.034 & 3.42 & 0.216 & 0.005 & 0.022 & 2.25 & 5631 & 807 & 0.143 & 14.34 \\
\hline 250 & M05 CW & 4 & 350 & 600 & 0.333 & 0.013 & 0.038 & 3.79 & 0.195 & 0.047 & 0.243 & 24.30 & 2894 & 401 & 0.139 & 13.86 \\
\hline 240 & M05 CW & 42 & 2270 & 2510 & 0.340 & 0.014 & 0.041 & 4.06 & 0.159 & 0.036 & 0.227 & 22.70 & 8106 & 2014 & 0.249 & 24.85 \\
\hline 340 & M07 NB & 2 & 100 & 440 & 0.143 & 0.018 & 0.126 & 12.62 & 0.300 & 0.064 & 0.213 & 21.33 & 2730 & 2261 & 0.828 & 82.82 \\
\hline 200 & M07 NB & 3 & 440 & 640 & 0.116 & 0.020 & 0.175 & 17.54 & 0.300 & 0.063 & 0.210 & 21.00 & 3281 & 1334 & 0.407 & 40.67 \\
\hline 620 & M07 NB & 4 & 640 & 1260 & 0.191 & 0.025 & 0.131 & 13.10 & 0.300 & 0.063 & 0.210 & 21.00 & 7840 & 2256 & 0.288 & 28.78 \\
\hline 340 & M07 NB & 5 & 1260 & 1600 & 0.255 & 0.019 & 0.073 & 7.26 & 0.300 & 0.065 & 0.217 & 21.67 & 5660 & 1813 & 0.320 & 32.04 \\
\hline 200 & M07 NB & 6 & 1600 & 1800 & 0.230 & 0.008 & 0.035 & 3.54 & 0.300 & 0.062 & 0.207 & 20.67 & 3350 & 848 & 0.253 & 25.30 \\
\hline 180 & M07 SB & 3 & 100 & 280 & 0.148 & 0.013 & 0.085 & 8.49 & 0.300 & 0.064 & 0.213 & 21.33 & 2100 & 1161 & 0.553 & 55.26 \\
\hline 560 & M07 SB & 7 & 720 & 1280 & 0.189 & 0.011 & 0.060 & 6.00 & 0.300 & 0.060 & 0.200 & 20.00 & 9250 & 2575 & 0.278 & 27.84 \\
\hline 220 & M07 SB & 8 & 1280 & 1500 & 0.257 & 0.009 & 0.035 & 3.52 & 0.300 & 0.056 & 0.187 & 18.67 & 6200 & 2298 & 0.371 & 37.06 \\
\hline 300 & M07 SB & 12 & 1640 & 1940 & 0.235 & 0.008 & 0.036 & 3.60 & 0.300 & 0.059 & 0.197 & 19.67 & 3504 & 1134 & 0.324 & 32.37 \\
\hline 300 & M08 EB & 4 & 180 & 480 & 0.245 & 0.017 & 0.068 & 6.78 & 0.300 & 0.063 & 0.210 & 21.00 & 1210 & 355 & .293 & 29.31 \\
\hline 180 & M08 EB & 6 & 640 & 820 & 0.255 & 0.010 & 0.040 & 3.98 & 0.300 & 0.061 & 0.203 & 20.33 & 1450 & 227 & .156 & 15.63 \\
\hline 240 & M08 WB & 2 & 100 & 340 & 0.243 & 0.026 & 0.105 & 10.50 & 0.300 & 0.061 & 0.203 & 20.33 & 4140 & 1680 & 0.406 & 40.57 \\
\hline 400 & M08 WB & 5 & 920 & 1320 & 0.181 & 0.031 & 0.172 & 17.19 & 0.300 & 0.060 & 0.200 & 20.00 & 1331 & 408 & 0.306 & 30.64 \\
\hline
\end{tabular}


Table 9.2 Input variables for simulation - adjustments to subbase stiffness

\begin{tabular}{|c|c|c|c|c|c|c|c|c|c|c|c|c|c|c|c|c|c|c|c|c|}
\hline \multicolumn{5}{|c|}{ Pavement site sections } & \multicolumn{4}{|c|}{$\begin{array}{l}\text { Original back-calculated } \\
\text { subbase stiffness (MPa) }\end{array}$} & \multicolumn{4}{|c|}{$\begin{array}{l}\text { 1. First adjustment to } \\
\text { subbase stiffness (COV=30\%) } \\
\text { for fatigue life calcs }\end{array}$} & \multicolumn{4}{|c|}{$\begin{array}{l}\text { 2. Second adjustment to } \\
\text { subbase stiffness (Esb=Esg } \\
\text { when Esb/Esg<1) for } \\
\text { deformation life calcs }\end{array}$} & \multicolumn{4}{|c|}{$\begin{array}{c}\text { Back-calculated } \\
\text { subgrade stiffness (MPa) }\end{array}$} \\
\hline $\begin{array}{l}\text { Length } \\
\text { (m) }\end{array}$ & Site & Section & From & To & $\bar{\mu}$ & $\bar{\sigma}$ & COV & $\begin{array}{l}\text { COV } \\
(\%)\end{array}$ & $\bar{\mu}$ & $\bar{\sigma}$ & COV & $\begin{array}{l}\text { COV } \\
(\%)\end{array}$ & $\bar{\mu}$ & $\bar{\sigma}$ & cov & $\begin{array}{l}\text { COV } \\
(\%)\end{array}$ & $\bar{\mu}$ & $\bar{\sigma}$ & cov & $\begin{array}{l}\text { COV } \\
(\%)\end{array}$ \\
\hline 220 & M01 & 3 & 35160 & 35380 & 345 & 398 & 1.154 & 115.45 & 345 & 104 & 000 & 30.00 & 353 & 99 & 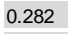 & 28.18 & 27 & 68 & 0.298 & 29.77 \\
\hline 320 & M01 & 16 & 36300 & 36620 & 83 & 59 & 0.715 & 71.49 & 83 & 25 & .300 & 30.00 & 200 & 35 & 0.174 & 17.39 & 199 & 34 & .170 & 17.02 \\
\hline 200 & M04 & 1 & 0 & 200 & 504 & 479 & 0.950 & 94.98 & 504 & 151 & 0.300 & 30.00 & 499 & 147 & 0.295 & 29.47 & 113 & 66 & 0.581 & 58.10 \\
\hline 375 & M04 & 10 & 875 & 1250 & 95 & 66 & 0.696 & 69.63 & 95 & 29 & 0.300 & 30.00 & 191 & 33 & 0.172 & 17.17 & 191 & 33 & 0.170 & 17.03 \\
\hline 250 & M04 & 20 & 1975 & 2225 & 401 & 379 & 0.945 & 94.48 & 401 & 120 & 0.300 & 30.00 & 398 & 109 & 0.274 & 27.39 & 257 & 21 & 0.081 & 8.10 \\
\hline 275 & M04 & 30 & 2675 & 2950 & 77 & 93 & 1.209 & 120.95 & 77 & 23 & 0.300 & 30.00 & 263 & 30 & 0.116 & 11.57 & 265 & 30 & 0.112 & 11.21 \\
\hline 200 & M04 & 37 & 3325 & 3525 & 60 & 45 & 748 & 74.84 & 60 & 18 & 300 & 30.00 & 180 & 42 & 0.231 & 23.06 & 182 & 42 & 0.229 & 22.90 \\
\hline 370 & M05 ACW & 10 & 460 & 830 & 934 & 183 & 0.196 & 19.60 & 934 & 280 & 0.300 & 30.00 & 920 & 283 & 0.308 & 30.78 & 184 & 32 & 0.174 & 17.44 \\
\hline 550 & M05 ACW & 11 & 830 & 1380 & 899 & 185 & 0.206 & 20.59 & 899 & 270 & 0.300 & 30.00 & 893 & 260 & 0.292 & 29.15 & 157 & 13 & 0.084 & 8.39 \\
\hline 250 & M05 C & 4 & 350 & 600 & 71 & 55 & 774 & 77.39 & 71 & 2 & 0.300 & 30.00 & 133 & 24 & 0.181 & 18.14 & 134 & 24 & 0.177 & 17.75 \\
\hline 240 & M05 CW & 42 & 2270 & 2510 & 748 & 406 & 0.543 & 54.28 & 748 & 224 & 0.300 & 30.00 & 747 & 219 & 0.292 & 29.25 & 201 & 45 & 0.225 & 22.53 \\
\hline 340 & M07 NB & 2 & 100 & 440 & 145 & 105 & 0.727 & 72.66 & 145 & 44 & 0.300 & 30.00 & 165 & 40 & 0.242 & 24.16 & 137 & 41 & 0.296 & 29.64 \\
\hline 200 & M07 NB & 3 & 440 & 640 & 161 & 204 & 1.264 & 126.41 & 161 & 48 & .300 & 30.00 & 164 & 47 & 0.285 & 28.48 & 110 & 18 & 0.165 & 16.50 \\
\hline 620 & M07 NB & 4 & 640 & 1260 & 227 & 216 & 0.953 & 95.34 & 227 & 68 & 0.300 & 30.00 & 231 & 61 & 0.265 & 26.50 & 157 & 32 & 0.203 & 20.27 \\
\hline 340 & M07 NB & 5 & 1260 & 1600 & 57 & 23 & 0.404 & 40.42 & 57 & 17 & 0.300 & 30.00 & 187 & 45 & 0.239 & 23.89 & 187 & 44 & 0.233 & 23.30 \\
\hline 200 & M07 NB & 6 & 1600 & 1800 & 73 & 71 & 0.966 & 96.61 & 73 & 22 & 0.300 & 30.00 & 190 & 43 & 0.225 & 22.51 & 189 & 46 & 0.242 & 24.18 \\
\hline 180 & M07 SB & 3 & 100 & 280 & 232 & 292 & 1.261 & 126.07 & 232 & 70 & 0.300 & 30.00 & 242 & 63 & 0.262 & 26.18 & 176 & 37 & 0.209 & 20.90 \\
\hline 560 & M07 SB & 7 & 720 & 1280 & 159 & 124 & 0.783 & 78.27 & 159 & 48 & 0.300 & 30.00 & 180 & 38 & 0.210 & 20.96 & 156 & 19 & 0.120 & 12.03 \\
\hline 220 & M07 SB & 8 & 1280 & 1500 & 91 & 55 & 0.608 & 60.76 & 91 & 27 & 0.300 & 30.00 & 195 & 49 & 0.253 & 25.33 & 196 & 49 & 0.250 & 25.03 \\
\hline 300 & M07 SB & 12 & 1640 & 1940 & 56 & 34 & 0.610 & 60.97 & 56 & II & 0.300 & 30.00 & 212 & 53 & 0.250 & 25.00 & 210 & 51 & 0.244 & 24.37 \\
\hline 300 & M08 EB & 4 & 180 & 480 & 121 & 91 & 0.752 & 75.24 & 121 & 36 & 0.300 & 30.00 & 222 & 202 & 0.910 & 91.04 & 186 & 193 & 1.040 & 103.97 \\
\hline 180 & M08 EB & 6 & 640 & 820 & 193 & 85 & 0.440 & 43.96 & 193 & 58 & 0.300 & 30.00 & 368 & 265 & 0.719 & 71.92 & 339 & 308 & 0.908 & 90.80 \\
\hline 240 & M08 WB & 2 & 100 & 340 & 83 & 40 & 0.484 & 48.45 & 83 & 20 & 0.300 & 30.00 & $3 / 3$ & 303 & 0.813 & 81.28 & 373 & 300 & 0.805 & 80.54 \\
\hline 400 & M08 WB & 5 & 920 & 1320 & 61 & 71 & 1.163 & 116.27 & 61 & 18 & 0.300 & 30.00 & 156 & 174 & 1.116 & 111.58 & 144 & 186 & 1.291 & 129.09 \\
\hline
\end{tabular}

\subsection{Results of MCS for homogeneous sections}

The simulation steps described in section 9.1 have been repeated with input data from the homogeneous sections summarised in Table 9.1 and Table 9.2. Due to the small number of survey data points available for each homogeneous section, a random sample of 1000 points for each input variable was generated instead, with the mean and standard deviation values shown in Table 9.1 and Table 9.2. A tabular summary of the statistical parameters of the fatigue and deformation life (in msa) is shown in Table 9.3 and Table 9.4.

The tables also show the values of the mean and standard deviation of the calculated performance life corrected for the mean and standard deviation of the model bias (see paragraph 8.1.4). A detailed graphical representation of the results can be seen in Appendix A4, but not corrected for the model bias (except for section M03 of site M01). The probability distribution for the performance life, corrected for the model bias, are obtained by generating random numbers from the lognormal distribution with the corrected mean and 


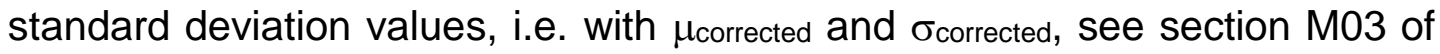
site M01 in Appendix A4.

Table 9.3 Fatigue life results from MCS

\begin{tabular}{|c|c|c|c|c|c|c|c|c|c|c|}
\hline \multicolumn{2}{|c|}{$\begin{array}{l}\text { Pavement site } \\
\text { sections }\end{array}$} & \multicolumn{6}{|c|}{ Fatigue life in msa $\left(\mathrm{Nf}_{\text {model }}(\mathrm{LR} 1132)\right)$} & \multicolumn{3}{|c|}{ Correction for model bias } \\
\hline Site & Section & $\mu$ & $\sigma$ & $\begin{array}{l}\text { COV } \\
(\%)\end{array}$ & $\begin{array}{c}5 \text { th } \\
\text { perc }\end{array}$ & $\begin{array}{l}\text { 95th } \\
\text { perc }\end{array}$ & $\begin{array}{c}\text { Nr pts that satisfy } \\
\text { model conds }\end{array}$ & $\mu_{\text {corrected }}$ & $\sigma_{\text {corrected }}$ & $\mathrm{COV}_{\text {real }}(\%)$ \\
\hline M01 & 3 & 140 & 172 & 122.70 & 28 & 409 & 1000 & 129 & 152 & 118.42 \\
\hline M01 & 16 & 144 & 158 & 109.69 & 24 & 444 & 1000 & 132 & 138 & 104.88 \\
\hline M04 & 1 & 405 & 1300 & 321.08 & 9 & 1408 & 997 & 371 & 1187 & 319.47 \\
\hline M04 & 10 & 9321 & 5684 & 60.98 & 3176 & 19239 & 1000 & 8552 & 4433 & 51.84 \\
\hline M04 & 20 & 14116 & 6444 & 45.65 & 6467 & 25439 & 1000 & 12950 & 4203 & 32.45 \\
\hline M04 & 30 & 8117 & 4072 & 50.17 & 3359 & 16640 & 1000 & 7447 & 2870 & 38.55 \\
\hline M04 & 37 & 5842 & 2881 & 49.32 & 2390 & 11241 & 1000 & 5359 & 2006 & 37.44 \\
\hline M05 ACW & 10 & 1390 & 1317 & 94.71 & 330 & 3513 & 1000 & 1275 & 1136 & 89.10 \\
\hline M05 ACW & 11 & 1389 & 996 & 71.70 & 446 & 3281 & 1000 & 1275 & 817 & 64.10 \\
\hline M05 CW & 4 & 38 & 21 & 54.09 & 15 & 78 & 1000 & 35 & 15 & 43.53 \\
\hline M05 CW & 42 & 3526 & 2789 & 79.09 & 806 & 8526 & 1000 & 3235 & 2338 & 72.28 \\
\hline M07 NB & 2 & 1 & 4 & 302.47 & 0 & 5 & 825 & 1 & 4 & 300.76 \\
\hline M07 NB & 3 & 0 & 1 & 156.06 & 0 & 2 & 962 & 0 & 1 & 152.72 \\
\hline M07 NB & 4 & 58 & 105 & 180.62 & 5 & 187 & 1000 & 53 & 95 & 177.74 \\
\hline M07 NB & 5 & 64 & 78 & 123.02 & 7 & 203 & 1000 & 58 & 69 & 118.76 \\
\hline M07 NB & 6 & 7 & 6 & 87.09 & 2 & 18 & 1000 & 6 & 5 & 80.95 \\
\hline M07 SB & 3 & 1 & 2 & 122.69 & 0 & 4 & 836 & 1 & 1 & 118.41 \\
\hline M07 SB & 7 & 48 & 49 & 102.24 & 9 & 133 & 1000 & 44 & 43 & 97.07 \\
\hline M07 SB & 8 & 102 & 157 & 154.43 & 12 & 311 & 1000 & 93 & 141 & 151.06 \\
\hline M07 SB & 12 & 10 & 12 & 112.05 & 2 & 32 & 1000 & 9 & 10 & 107.35 \\
\hline M08 EB & 4 & 3 & 4 & 130.28 & 1 & 10 & 834 & 3 & 4 & 126.26 \\
\hline M08 EB & 6 & 10 & 11 & 109.58 & 2 & 29 & 877 & 9 & 10 & 104.77 \\
\hline M08 WB & 2 & 64 & 137 & 213.60 & 2 & 263 & 999 & 59 & 124 & 211.17 \\
\hline M08 WB & 5 & 1 & 1 & 202.79 & 0 & 2 & 871 & 1 & 1 & 200.23 \\
\hline
\end{tabular}


Table 9.4 Deformation life results from MCS

\begin{tabular}{|c|c|c|c|c|c|c|c|c|c|c|}
\hline \multicolumn{2}{|c|}{$\begin{array}{l}\text { Pavement site } \\
\text { sections }\end{array}$} & \multicolumn{6}{|c|}{ Deformation life in msa ( $\mathbf{N d}_{\text {model }}(\mathbf{L} \mathbf{R 1 3 2})$ ) } & \multicolumn{3}{|c|}{ Correction for model bias } \\
\hline Site & Section & $\mu$ & $\sigma$ & $\begin{array}{l}\text { COV } \\
(\%)\end{array}$ & $\begin{array}{c}5 \text { th } \\
\text { perc }\end{array}$ & $\begin{array}{l}95 \text { th } \\
\text { perc }\end{array}$ & $\begin{array}{c}\text { Nr pts that satisfy } \\
\text { model conds }\end{array}$ & $\mu_{\text {corrected }}$ & $\sigma_{\text {corrected }}$ & $\mathrm{COV}_{\text {real }}(\%)$ \\
\hline M01 & 3 & 97 & 98 & 101.52 & 19 & 266 & 999 & 90 & 87 & 96.42 \\
\hline M01 & 16 & 61 & 57 & 93.79 & 12 & 159 & 1000 & 57 & 50 & 88.24 \\
\hline M04 & 1 & 85 & 220 & 258.45 & 2 & 310 & 999 & 80 & 204 & 256.49 \\
\hline M04 & 10 & 5552 & 3075 & 55.39 & 2058 & 11238 & 1000 & 5189 & 2354 & 45.36 \\
\hline M04 & 20 & 11130 & 4438 & 39.87 & 5534 & 19830 & 1000 & 10402 & 2505 & 24.08 \\
\hline M04 & 30 & 5416 & 2467 & 45.55 & 2411 & 10260 & 1000 & 5062 & 1652 & 32.64 \\
\hline M04 & 37 & 2812 & 1536 & 54.63 & 1048 & 5458 & 1000 & 2628 & 1168 & 44.44 \\
\hline M05 ACW & 10 & 508 & 285 & 56.07 & 179 & 1064 & 1000 & 474 & 219 & 46.19 \\
\hline M05 ACW & 11 & 478 & 187 & 39.12 & 234 & 839 & 1000 & 446 & 102 & 22.82 \\
\hline M05 CW & 4 & 44 & 25 & 57.18 & 16 & 93 & 1000 & 41 & 20 & 47.54 \\
\hline M05 CW & 42 & 1218 & 896 & 73.59 & 299 & 2969 & 1000 & 1138 & 756 & 66.37 \\
\hline M07 NB & 2 & 3 & 6 & 226.96 & 0 & 9 & 991 & 2 & 5 & 224.72 \\
\hline M07 NB & 3 & 1 & 1 & 112.41 & 0 & 3 & 997 & 1 & 1 & 107.83 \\
\hline M07 NB & 4 & 69 & 124 & 179.18 & 6 & 225 & 1000 & 65 & 114 & 176.34 \\
\hline M07 NB & 5 & 162 & 191 & 118.25 & 22 & 512 & 1000 & 151 & 172 & 113.90 \\
\hline M07 NB & 6 & 39 & 43 & 109.98 & 6 & 119 & 1000 & 36 & 38 & 105.29 \\
\hline M07 SB & 3 & 4 & 5 & 135.86 & 0 & 14 & 995 & 4 & 5 & 132.09 \\
\hline M07 SB & 7 & 50 & 38 & 76.41 & 13 & 119 & 1000 & 47 & 33 & 69.49 \\
\hline M07 SB & 8 & 204 & 215 & 105.31 & 33 & 658 & 1000 & 190 & 191 & 100.40 \\
\hline M07 SB & 12 & 56 & 52 & 92.33 & 9 & 168 & 1000 & 53 & 46 & 86.69 \\
\hline M08 EB & 4 & 20 & 109 & 558.90 & 0 & 83 & 948 & 18 & 102 & 558.00 \\
\hline M08 EB & 6 & 68 & 175 & 255.55 & 2 & 279 & 895 & 64 & 162 & 253.57 \\
\hline M08 WB & 2 & 595 & 1489 & 250.25 & 8 & 2547 & 996 & 556 & 1381 & 248.23 \\
\hline M08 WB & 5 & 4 & 15 & 357.94 & 0 & 17 & 961 & 4 & 14 & 356.53 \\
\hline
\end{tabular}

\subsection{Results of MM for homogeneous sections}

The Method of Moments (second moment method) was employed for comparison of the results obtained with the MCS.

An example of the fatigue life calculations involved is presented below for the first homogeneous section (M01 section 3). The input variables (mean and standard deviation) are:

- $\mu E a=4060 \mathrm{MPa}$ and $\sigma \mathrm{Ea}_{\mathrm{a}}=1423 \mathrm{MPa}$ for the asphalt stiffness, with lognormal distribution;

- $\mu E s b=345 \mathrm{MPa}$ and $\sigma E s b=104 \mathrm{MPa}$ for the subbase stiffness, with lognormal distribution; 
- $\mu \mu_{\mathrm{Esg}}=227 \mathrm{MPa}$ and $\sigma \mathrm{Esg}_{\mathrm{s} g}=68 \mathrm{MPa}$ for the subgrade stiffness, with lognormal distribution;

- $\mu T a=0.284 \mathrm{~m}$ and $\sigma T a=0.004 \mathrm{~m}$ for the asphalt thickness, with normal distribution;

- $\mu T s b=0.171 \mathrm{~m}$ and $\sigma T s b=0.016 \mathrm{~m}$ for the subbase thickness, with normal distribution;

The steps described in chapter 3 are:

1. The constant $\mathrm{G} \mu=\mathrm{Nf}_{\mu}=\mathrm{Nf}(\mu \mathrm{Ea}, \mu \mathrm{Ta}, \mu \mathrm{Esb}, \mu \mathrm{Tsb}, \mu \mathrm{Esg})$ (e.g. the fatigue life performance function calculated with the mean value of each input variable) was calculated to be $109.047 \mathrm{msa}$. For a five-point estimate, using the inverse Rosenblatt transformation expressed as $\mathrm{T}^{-1}\left(\mathrm{u}_{\mathrm{i}}\right)=\mathrm{F}^{-}$ ${ }^{1}\left[\Phi\left(u_{i}\right)\right]$, where $F=$ cumulative distribution of the input variable $\left(E_{a}, T_{a}, E_{s b}\right.$, $\mathrm{T}_{\mathrm{sb}}, \mathrm{E}_{\mathrm{sg}}$ ), and $\Phi=$ standard normal probability, the five estimating points can be easily transformed into original space as:

$\mathrm{T}^{-1}\left(\mathrm{u}_{\mathrm{i}}\right)=\exp \left[\lambda+\xi^{*} \mathrm{u}_{\mathrm{i}}\right]$, where $\zeta=\sqrt{\ln \left(1+\left(\frac{\sigma}{\mu}\right)^{2}\right)}, \lambda=\ln \mu-0.5 \zeta^{2}$

where $\mu$ and $\sigma$ are the mean and standard deviation of the lognormal variable, or:

$\mathrm{T}^{-1}\left(\mathrm{u}_{\mathrm{i}}\right)=\mu+\sigma^{*} \mathrm{ui}_{\mathrm{i}}$

where $\mu$ and $\sigma$ are the mean and standard deviation of the normal variable.

The Rosenblatt transformation of the five input variables are:

- $\mathrm{T}^{-1}\left(\mathrm{u}_{2}\right) \mathrm{Ea}=\exp [(\ln 4060$

$\left.\left.0.5 x\left(\ln \left(1+(1423 / 4060)^{2}\right)\right)+\operatorname{sqrt}\left(\ln \left(1+(1423 / 4060)^{2}\right)\right) \times(-2.85697)\right)\right]=1448$ $\mathrm{MPa}$, similarly $\mathrm{T}^{-1}\left(\mathrm{u}_{1-}\right) \mathrm{Ea}=2415 \mathrm{MPa}, \mathrm{T}^{-1}\left(\mathrm{u}_{0}\right) \mathrm{Ea}=3831 \mathrm{MPa}, \mathrm{T}^{-1}\left(\mathrm{u}_{1+}\right) \mathrm{Ea}=$ $6079 \mathrm{MPa}, \mathrm{T}^{-1}\left(\mathrm{U}_{2+}\right) \mathrm{Ea}=10135 \mathrm{MPa}$.

- $\mathrm{T}^{-1}\left(\mathrm{u}_{2}\right) \mathrm{Ess}=143 \mathrm{MPa}, \mathrm{T}^{-1}\left(\mathrm{u}_{1-}\right) \mathrm{Ess}=222 \mathrm{MPa}, \mathrm{T}^{-1}\left(\mathrm{u}_{0}\right)_{\mathrm{Esb}}=330 \mathrm{MPa}$, $\mathrm{T}^{-1}\left(\mathrm{u}_{1+}\right) \mathrm{Esb}=492 \mathrm{MPa}^{-1} \mathrm{~T}^{-1}\left(\mathrm{u}_{2+}\right) \mathrm{Esb}=764 \mathrm{MPa}$.

- $\mathrm{T}^{-1}\left(\mathrm{u}_{2}\right) \mathrm{Ess}_{\mathrm{E} g}=95 \mathrm{MPa}, \mathrm{T}^{-1}\left(\mathrm{u}_{1}-\right)_{\mathrm{Esg}}=147 \mathrm{MPa}, \mathrm{T}^{-1}\left(\mathrm{u}_{0}\right)_{\mathrm{Esg}}=218 \mathrm{MPa}$, $\mathrm{T}^{-1}\left(\mathrm{u}_{1+}\right) \mathrm{Esg}_{\mathrm{s}}=323 \mathrm{MPa}, \mathrm{T}^{-1}\left(\mathrm{u}_{2+}\right) \mathrm{Esg}=500 \mathrm{MPa}$. 
- $\mathrm{T}^{-1}\left(\mathrm{U}_{2}-\right) \mathrm{Ta}=0.284+0.004^{*}(-2.85697)=0.272 \mathrm{~m}, \mathrm{~T}^{-1}\left(\mathrm{u}_{1-}\right) \mathrm{Ta}=0.278 \mathrm{~m}$, $\mathrm{T}^{-1}\left(\mathrm{u}_{0}\right) \mathrm{Ta}=0.284 \mathrm{~m}, \mathrm{~T}^{-1}\left(\mathrm{u}_{1_{+}}\right) \mathrm{Ta}=0.290 \mathrm{~m}, \mathrm{~T}^{-1}\left(\mathrm{u}_{2+}\right) \mathrm{Ta}=0.296 \mathrm{~m}$.

- $\mathrm{T}^{-1}\left(\mathrm{u}_{2-}\right) \mathrm{Tsb}=0.125 \mathrm{~m}, \mathrm{~T}^{-1}\left(\mathrm{u}_{1-}\right) \mathrm{Tsb}_{\mathrm{sb}}=0.149 \mathrm{~m}, \mathrm{~T}^{-1}\left(\mathrm{u}_{0}\right) \mathrm{Tsb}=0.171 \mathrm{~m}$, $\mathrm{T}^{-1}\left(\mathrm{u}_{1+}\right) \mathrm{Tsb}=0.193 \mathrm{~m}, \mathrm{~T}^{-1}\left(\mathrm{u}_{2+}\right) \mathrm{Tsb}=0.217 \mathrm{~m}$.

2. Using the corresponding weights for a five point estimate, the first four moments of $\mathrm{Nf}_{\mathrm{var}}$ (function in which all the basic random variables are evaluated with their mean values, except the basic random variable that appears in the index of the function) are approximately:

$\mu_{N f_{E a}}=134.583 \mathrm{msa}, \sigma_{N f_{E a}}=130.276 \mathrm{msa}, \alpha_{3 N f_{E a}}=3.944, \alpha_{4 N f_{E a}}=26.209$

Similarly, the first four moments of $\mathrm{Nf}_{\mathrm{Ta}}, \mathrm{Nf}_{\mathrm{Esb}}, \mathrm{Nf}_{\mathrm{Tsb}}, \mathrm{Nf}_{\mathrm{Esg}}$ are:

$\mu_{N f_{T a}}=109.452 \mathrm{msa}, \sigma_{N f_{T a}}=9.884 \mathrm{msa}, \alpha_{3 N f_{T a}}=0.247, \alpha_{4 N f_{T a}}=3.106$

$\mu_{N f_{E s b}}=111.399 \mathrm{msa}, \sigma_{N f_{E s b}}=29.573 \mathrm{msa}, \alpha_{3 N f_{E s b}}=1.719, \alpha_{4 N f_{E s b}}=8.494$

$\mu_{N f_{T s b}}=108.993 \mathrm{msa}, \sigma_{N f_{T s b}}=1.814 \mathrm{msa}, \alpha_{3 N f_{T s b}}=-0.181, \alpha_{4 N f_{T s b}}=3.065$

$\mu_{N f_{E g g}}=111.822 \mathrm{msa}, \sigma_{N f_{E s g}}=32.380 \mathrm{msa}, \alpha_{3 N f_{E s g}}=1.784, \alpha_{4 N f_{E s g}}=8.894$

3. The first two moments of the performance function $\mathrm{Nf}$ can then be approximated to:

$$
\begin{aligned}
& \mu \mathrm{Nf}=\mu_{N f_{E a}}+\mu_{N f_{T a}}+\mu_{N f_{E s b}}+\mu_{N f_{T s b}}+\mu_{N f_{E s g}}-4 N f_{\mu}=140.060 \mathrm{msa} \\
& \sigma \mathrm{Nf}=\sqrt{\sigma_{N f_{E a}}^{2}+\sigma_{N f_{T a}}^{2}+\sigma_{N f_{E s b}}^{2}+\sigma_{N f_{T s b}}^{2}+\sigma_{N f_{E s g}}^{2}}=137.826 \mathrm{msa} \\
& \alpha_{3_{N f}}^{2}=\frac{\alpha_{3 N f_{E a}} \sigma_{N f_{E a}}^{3}+\alpha_{3 N f_{T a}} \sigma_{N f_{T a}}^{3}+\alpha_{3 N f_{E s b}} \sigma_{N f_{E s b}}^{3}+\alpha_{3 N f_{T s b}} \sigma_{N f_{T s b}}^{3}+\alpha_{3 N f_{E s g}} \sigma_{N f_{E s g}}^{3}=3.371}{\sigma_{N f}^{3}} \\
& \alpha_{4_{N f}}=\frac{s 1+6 \times(s 2+s 3)}{\sigma_{N f}^{4}}=21.557
\end{aligned}
$$

Where:

$$
\begin{aligned}
& s 1=\alpha_{4 N f_{E a}} \sigma_{N f_{E a}}^{4}+\alpha_{4 N f_{T a}} \sigma_{N f_{T a}}^{4}+\alpha_{4 N f_{E s b}} \sigma_{N f_{E s b}}^{4}+\alpha_{4 N f_{T s b}} \sigma_{N f_{T s b}}^{4}+\alpha_{4 N f_{E s g}} \sigma_{N f_{E s g}}^{4} \\
& =7565841681.2 \\
& s 2=\sigma_{N f_{E a}}^{2} \sigma_{N f_{T a}}^{2}+\sigma_{N f_{E a}}^{2} \sigma_{N f_{E s b}}^{2}+\sigma_{N f_{E a}}^{2} \sigma_{N f_{T s b}}^{2}+\sigma_{N f_{E a}}^{2} \sigma_{N f_{E s g}}^{2}+\sigma_{N f_{T a}}^{2} \sigma_{N f_{E s b}}^{2}+\sigma_{N f_{T a}}^{2} \sigma_{N f_{T s b}}^{2}+\sigma_{N f_{T a}}^{2} \sigma_{N f_{E s g}}^{2} \\
& =34539125.6 \\
& s 3=\sigma_{N f_{E s b}}^{2} \sigma_{N f_{T s b}}^{2}+\sigma_{N f_{E s b}}^{2} \sigma_{N f_{E s g}}^{2}+\sigma_{N f_{T s b}}^{2} \sigma_{N f_{E s g}}^{2}=923260.36
\end{aligned}
$$

In the calculations above only performance fatigue values satisfying the model conditions were included in the calculations. The moments of the performance 
functions and reliability indices can also be easily calculated but are not included here as the main focus of the research is to assess variability of the performance life in terms of mean, standard deviation and probability distribution. Similar calculations were performed for the deformation life. To be consistent with the approach described for the deformation life the MCS simulation, wherever the subbase stiffness was less than the subgrade stiffness (including the Rosenblatt transformation for the subbase stiffness variable) for each point estimate, the subbase stiffness for that point estimate was forced to be equal to the subgrade stiffness. A tabular summary of the statistical parameters of the fatigue and deformation life (in msa) calculated with the Method of Moments (second moment method) is shown in Table 9.5 and Table 9.6.

The tables also show the values of the mean and standard deviation of the calculated performance life corrected for the mean and standard deviation of the model bias. 
Table 9.5 Fatigue life results from MM

\begin{tabular}{|c|c|c|c|c|c|c|c|}
\hline \multicolumn{2}{|c|}{$\begin{array}{l}\text { Pavement site } \\
\text { sections }\end{array}$} & \multicolumn{3}{|c|}{$\begin{array}{l}\text { Predicted fatigue life (msa) with } \\
\text { LR1132, with model and MM }\end{array}$} & \multicolumn{3}{|c|}{ Correction for model bias } \\
\hline Site & Section & $\mu$ & $\sigma$ & $\operatorname{cov}(\%)$ & $\mu_{\text {corrected }}$ & $\left|\sigma_{\text {corrected }}\right|$ & $\mathrm{COV}_{\text {real }}(\%)$ \\
\hline M01 & 3 & 140 & 138 & 98.40 & 128 & 120 & 93.02 \\
\hline M01 & 16 & 152 & 183 & 120.85 & 139 & 162 & 116.50 \\
\hline M04 & 1 & 336 & 609 & 181.44 & 308 & 550 & 178.57 \\
\hline M04 & 10 & 9203 & 5070 & 55.10 & 8443 & 3780 & 44.77 \\
\hline M04 & 20 & 14136 & 5974 & 42.26 & 12969 & 3564 & 27.48 \\
\hline M04 & 30 & 8158 & 3897 & 47.77 & 7484 & 2647 & 35.36 \\
\hline M04 & 37 & 5751 & 2754 & 47.89 & 5276 & 1875 & 35.53 \\
\hline M05 ACW & 10 & 1313 & 944 & 71.89 & 1205 & 775 & 64.32 \\
\hline M05 ACW & 11 & 1413 & 920 & 65.08 & 1296 & 734 & 56.61 \\
\hline M05 CW & 4 & 38 & 19 & 50.39 & 35 & 13 & 38.83 \\
\hline M05 CW & 42 & 3602 & 2681 & 74.44 & 3305 & 2219 & 67.16 \\
\hline M07 NB & 2 & 1 & 4 & 344.16 & 1 & 4 & 342.66 \\
\hline M07 NB & 3 & 0 & 0 & 108.42 & 0 & 0 & 103.56 \\
\hline M07 NB & 4 & 51 & 49 & 95.95 & 47 & 42 & 90.42 \\
\hline M07 NB & 5 & 64 & 70 & 109.79 & 58 & 61 & 104.99 \\
\hline M07 NB & 6 & 7 & 6 & 80.61 & 6 & 5 & 73.94 \\
\hline M07 SB & 3 & 1 & 1 & 135.38 & 1 & 1 & 131.52 \\
\hline M07 SB & 7 & 49 & 42 & 86.39 & 45 & 36 & 80.20 \\
\hline M07 SB & 8 & 103 & 133 & 129.69 & 94 & 118 & 125.65 \\
\hline M07 SB & 12 & 10 & 11 & 108.03 & 9 & 9 & 103.14 \\
\hline M08 EB & 4 & 3 & 3 & 113.59 & 3 & 3 & 108.96 \\
\hline M08 EB & 6 & 14 & 15 & 103.45 & 13 & 13 & 98.34 \\
\hline M08 WB & 2 & 64 & 129 & 201.70 & 58 & 116 & 199.13 \\
\hline M08 WB & 5 & 0 & 0 & 108.00 & 0 & 0 & 103.12 \\
\hline
\end{tabular}


Table 9.6 Deformation life results from MM

\begin{tabular}{|c|c|c|c|c|c|c|c|}
\hline \multicolumn{2}{|c|}{$\begin{array}{l}\text { Pavement site } \\
\text { sections }\end{array}$} & $\begin{array}{l}\text { Predicted } \\
\text { fatigue life } \\
\text { (msa) with } \\
\text { LR1132, with } \\
\text { model and MM }\end{array}$ & $\begin{array}{l}\text { Correction } \\
\text { for model } \\
\text { bias }\end{array}$ & $\begin{array}{l}\text { Calculated } \\
\text { fatigue life (msa) } \\
\text { with LR1132, } \\
\text { with BISAR }\end{array}$ & $\begin{array}{l}\text { Predicted } \\
\text { deformation } \\
\text { life (msa) with } \\
\text { LR1132, with } \\
\text { model and MM }\end{array}$ & $\begin{array}{l}\text { Correction } \\
\text { for model } \\
\text { bias }\end{array}$ & $\begin{array}{l}\text { Calculated } \\
\text { deformation life } \\
\text { (msa) with } \\
\text { LR1132, with } \\
\text { BISAR }\end{array}$ \\
\hline Site & Section & $\begin{array}{l}\mu \text { (from } \\
\text { average } \\
\text { variables' }\end{array}$ & $\mu_{\text {corrected }}$ & $\begin{array}{l}\mu \text { (from average } \\
\text { variables' } \\
\text { values) }\end{array}$ & $\begin{array}{l}\mu \text { (from } \\
\text { average } \\
\text { variables' }\end{array}$ & $\mu_{\text {corrected }}$ & $\begin{array}{l}\mu \text { (from average } \\
\text { variables' } \\
\text { values) }\end{array}$ \\
\hline M01 & 3 & 109 & 100 & 111 & 82 & 77 & 133 \\
\hline M01 & 16 & 110 & 101 & 107 & 52 & 48 & 104 \\
\hline M04 & 1 & 149 & 137 & 160 & 43 & 40 & 38 \\
\hline M04 & 10 & 8244 & 7563 & 12179 & 5079 & 4746 & 12696 \\
\hline M04 & 20 & 13215 & 12124 & 19766 & 10746 & 10043 & 18000 \\
\hline M04 & 30 & 7487 & 6869 & 9731 & 5123 & 4788 & 11447 \\
\hline M04 & 37 & 5290 & 4853 & 6964 & 2586 & 2417 & 5767 \\
\hline M05 ACW & 10 & 1095 & 1004 & 1205 & 475 & 444 & 413 \\
\hline M05 ACW & 11 & 1229 & 1128 & 1355 & 471 & 440 & 392 \\
\hline M05 CW & 4 & 34 & 31 & 32 & 40 & 37 & 94 \\
\hline M05 CW & 42 & 3001 & 2753 & 3871 & 1065 & 996 & 1035 \\
\hline M07 NB & 2 & 0 & 0 & 0 & 1 & 1 & 3 \\
\hline M07 NB & 3 & 0 & 0 & 0 & 1 & 1 & 1 \\
\hline M07 NB & 4 & 35 & 33 & 45 & 43 & 40 & 78 \\
\hline M07 NB & 5 & 44 & 41 & 43 & 120 & 112 & 346 \\
\hline M07 NB & 6 & 6 & 5 & 4 & 30 & 28 & 84 \\
\hline M07 SB & 3 & 1 & 1 & 1 & 3 & 3 & 5 \\
\hline M07 SB & 7 & 39 & 36 & 54 & 40 & 38 & 115 \\
\hline M07 SB & 8 & 72 & 66 & 79 & 162 & 152 & 472 \\
\hline M07 SB & 12 & 7 & 7 & 5 & 45 & 42 & 128 \\
\hline M08 EB & 4 & 3 & 2 & 1 & 8 & 8 & 24 \\
\hline M08 EB & 6 & 11 & 10 & 2 & 54 & 50 & 180 \\
\hline M08 WB & 2 & 30 & 27 & 16 & 253 & 236 & 767 \\
\hline M08 WB & 5 & 0 & 0 & 0 & 1 & 1 & 4 \\
\hline
\end{tabular}

Finally, the fatigue and deformation life values that would derive from considering average values of each input variable, calculated both with the MM and with BISAR methods, are presented in Table 9.7. 
Table 9.7 Fatigue and deformation lives from average values of input variables

\begin{tabular}{|c|c|c|c|c|c|}
\hline \multicolumn{2}{|c|}{$\begin{array}{l}\text { Pavement site } \\
\text { sections }\end{array}$} & \multirow{2}{*}{$\begin{array}{l}\text { Predicted fatigue life } \\
\text { (msa) with LR1132, } \\
\text { with model and MM } \\
\mu \text { (from average } \\
\text { variables' values) }\end{array}$} & \multirow{2}{*}{$\begin{array}{l}\text { Calculated fatigue } \\
\text { life (msa) with } \\
\text { LR1132, with BISAR } \\
\mu \text { (from average } \\
\text { variables' values) }\end{array}$} & \multirow{2}{*}{\begin{tabular}{|l} 
Predicted deformation \\
life (msa) with LR1132, \\
with model and MM \\
$\mu$ (from average \\
variables' values)
\end{tabular}} & \multirow{2}{*}{$\begin{array}{l}\text { Calculated deformation } \\
\text { life (msa) with LR1132, } \\
\text { with BISAR } \\
\mu \text { (from average } \\
\text { variables' values) }\end{array}$} \\
\hline Site & Section & & & & \\
\hline M01 & 3 & 109 & 111 & 82 & 133 \\
\hline M01 & 16 & 110 & 107 & 52 & 104 \\
\hline M04 & 1 & 149 & 160 & 43 & 38 \\
\hline M04 & 10 & 8244 & 12179 & 5079 & 12696 \\
\hline M04 & 20 & 13215 & 19766 & 10746 & 18000 \\
\hline M04 & 30 & 7487 & 9731 & 5123 & 11447 \\
\hline M04 & 37 & 5290 & 6964 & 2586 & 5767 \\
\hline M05 ACW & 10 & 1095 & 1205 & 475 & 413 \\
\hline M05 ACW & 11 & 1229 & 1355 & 471 & 392 \\
\hline M05 CW & 4 & 34 & 32 & 40 & 94 \\
\hline M05 CW & 42 & 3001 & 3871 & 1065 & 1035 \\
\hline M07 NB & 2 & 0 & 0 & 1 & 3 \\
\hline M07 NB & 3 & 0 & 0 & 1 & 1 \\
\hline M07 NB & 4 & 35 & 45 & 43 & 78 \\
\hline M07 NB & 5 & 44 & 43 & 120 & 346 \\
\hline M07 NB & 6 & 6 & 4 & 30 & 84 \\
\hline M07 SB & 3 & 1 & 1 & 3 & 5 \\
\hline M07 SB & 7 & 39 & 54 & 40 & 115 \\
\hline M07 SB & 8 & 72 & 79 & 162 & 472 \\
\hline M07 SB & 12 & 7 & 5 & 45 & 128 \\
\hline M08 EB & 4 & 3 & 1 & 8 & 24 \\
\hline M08 EB & 6 & 11 & 2 & 54 & 180 \\
\hline M08 WB & 2 & 30 & 16 & 253 & 767 \\
\hline M08 WB & 5 & 0 & 0 & 1 & 4 \\
\hline
\end{tabular}

The following observations can be made on the resulting variability of performance life values:

- The MCS simulation results for the probability distribution of both the fatigue and deformation life (shown in Appendix A4) show that a lognormal distribution can be fitted.

- The mean fatigue and deformation life values obtained by MCS and by MM are comparable while the standard deviation values are larger for MCS. The larger variation is due to random combinations of input variables in the underlying data sets created by MCS (i.e., input variables chosen at random from a population having a large number of possible values).

- The coefficient of variation (COV) for fatigue life of all sites resulting from MCS varies from a minimum of $46 \%$ to a maximum of $321 \%$, and with an average value of $127 \%$. The corresponding COV values from MM vary 
from a minimum of $42 \%$ to a maximum of $344 \%$, and with an average value of $108 \%$.

- The coefficient of variation for deformation life of all sites resulting from MCS varies from a minimum of $39 \%$ to a maximum of $559 \%$, and with an average value of $144 \%$. The corresponding COV values from MM vary from a minimum of $38 \%$ to a maximum of $151 \%$, and with an average value of $87 \%$.

- If sites M07 and M08 are excluded (sites with thin asphalt layer), the coefficient of variation (COV) for fatigue life of sites M01 to M05 resulting from MCS varies from a minimum of $46 \%$ to a maximum of $321 \%$, and with an average value of $96 \%$. The corresponding COV values from MM vary from a minimum of $42 \%$ to a maximum of $181 \%$, and with an average value of $78 \%$.

- If sites M07 and M08 are excluded (sites with thin asphalt layer), the coefficient of variation (COV) for deformation life of sites M01 to M05 resulting from MCS varies from a minimum of $39 \%$ to a maximum of $258 \%$, and with an average value of $80 \%$. The corresponding COV values from MM vary from a minimum of $38 \%$ to a maximum of $136 \%$, and with an average value of $65 \%$.

- The above results for the coefficient of variation refer to the uncorrected values of the mean and standard deviation of the performance life. If correction for model bias is applied, the coefficient of variation is, on average, approximately $10 \%$ lower.

- The comparison between the mean fatigue life value calculated with BISAR (with mean values of the input parameters) and that calculated with MCS and/or MM shows that the BISAR approach provides the most conservative design (i.e. mean fatigue values calculated with BISAR are less than those calculated with MCS and/or MM).

- The comparison between the mean deformation life value calculated with BISAR (with mean values of the input parameters) and that calculated with MCS and/or MM shows that the MCS and/or MM approaches provide the 
most conservative design (i.e. mean deformation values calculated with MCS and/or MM are less than those calculated with BISAR).

\subsection{Effect of correlation between variables}

This section briefly explores the effect of correlation between variables on the calculated performance life. For example, let's suppose that the asphalt thickness and asphalt stiffness are negatively correlated. The degree of correlation used here was taken from Clause 5.28 in HD 29/08 (see section 2.6.3.1):

$\begin{array}{llll}\text { Real asphalt thickness }(\mathrm{m}) & 1 & 1 & \text { Real asphalt stiffness (MPa) } \\ 15 \% \text { underestimate in asphalt thickness } & 0.85 & 1.5 & 50 \% \text { overestimate in asphalt stiffness }\end{array}$

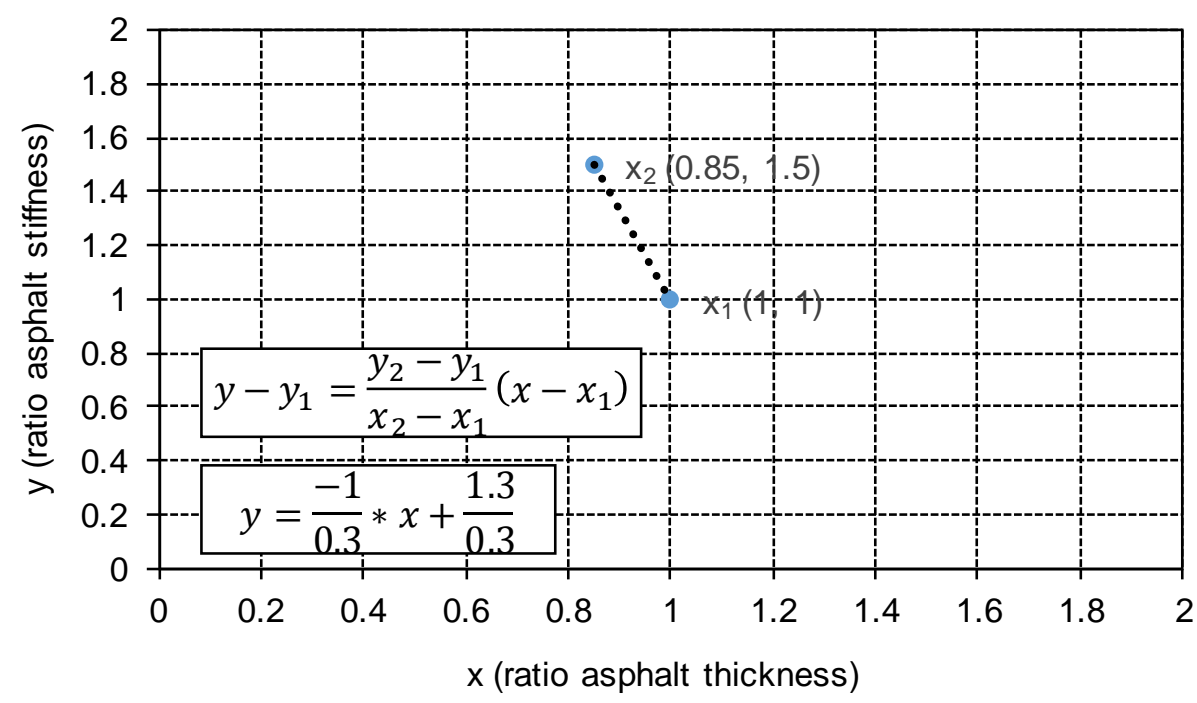

Figure 9.2 Negative correlation between variables

Figure 9.2 shows how the equation to apply to the stiffness data was calculated. For example, let's take Section 3 of site M01 and let's assume that $0.284 \mathrm{~m}$ and $4060 \mathrm{MPa}$ are the real section asphalt layer thickness and stiffness. Random values for the input parameters (relative to Section 3) were created with the procedure discussed in the previous sections. To the randomly generated values of the asphalt stiffness (i..e, uncorrelated) the equation in Figure 9.2 was then applied to simulate correlation. The results of the fatigue and deformation life for Section 3 of site M01 - for both cases of uncorrelation and correlation between asphalt thickness and asphalt stiffness - are shown in Table 9.8 and in Figure 9.3 to Figure 9.6. The results show 
that if the negative correlation is not considered (i.e., asphalt thickens and asphalt stuffiness are assumed uncorrelated), a larger than real uncertainty in performance will result.

Table 9.8 Fatigue and deformation life results for Section 3 of site M01 - effect of correlation between asphalt thickness and asphalt stiffness

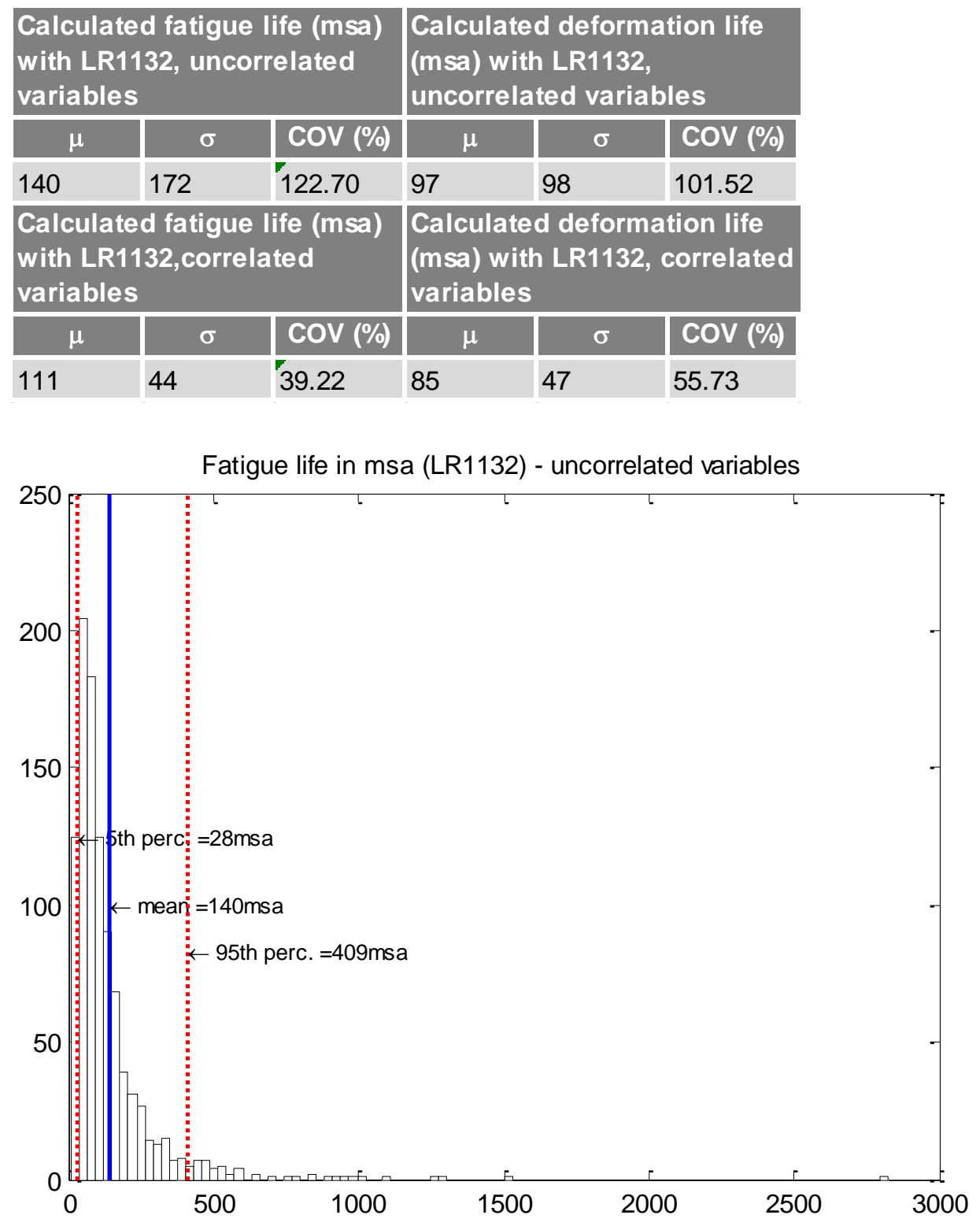

Figure 9.3 Fatigue life for Section 3 of site M01 - uncorrelated variables 


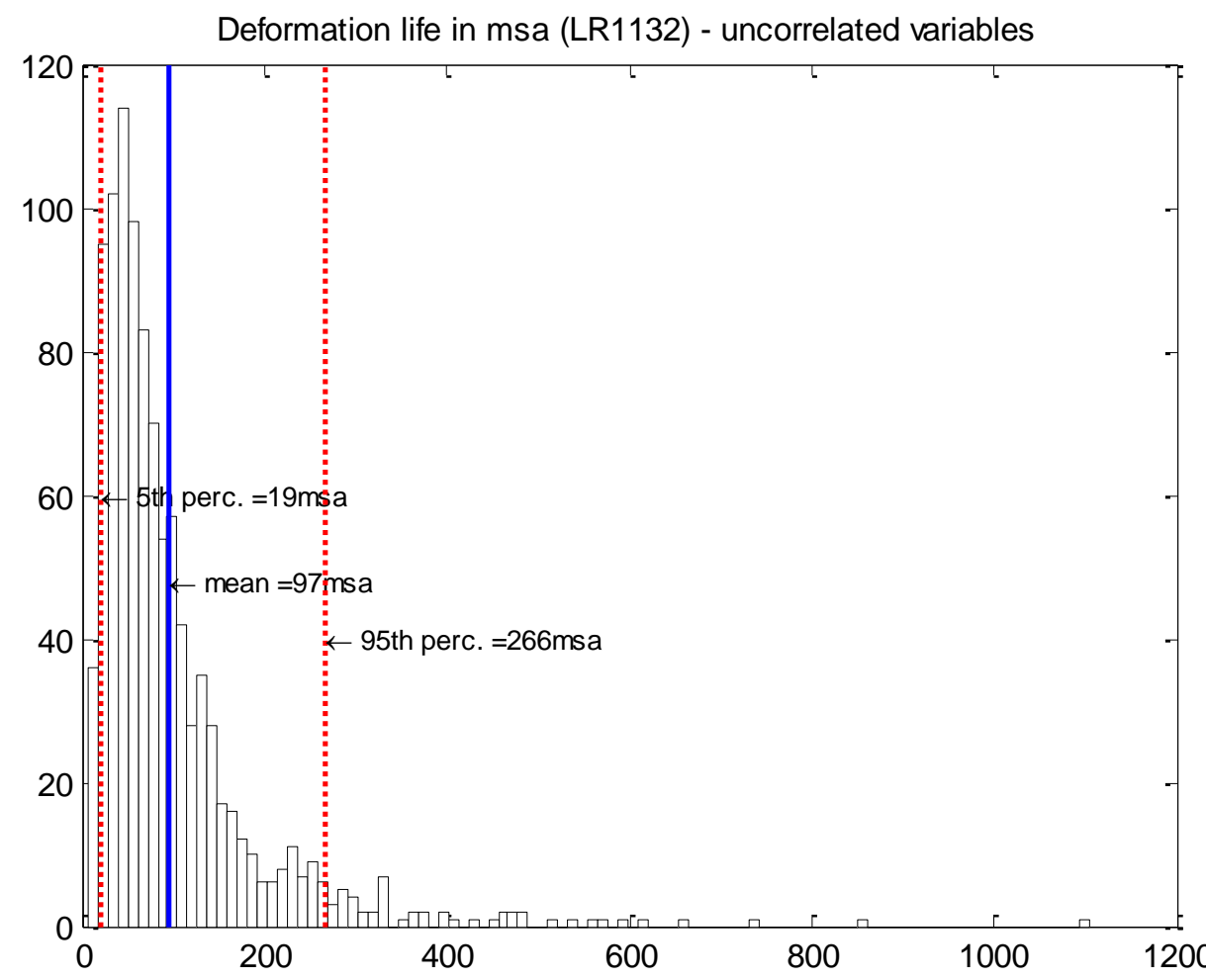

Figure 9.4 Deformation life for Section 3 of site M01 - uncorrelated variables

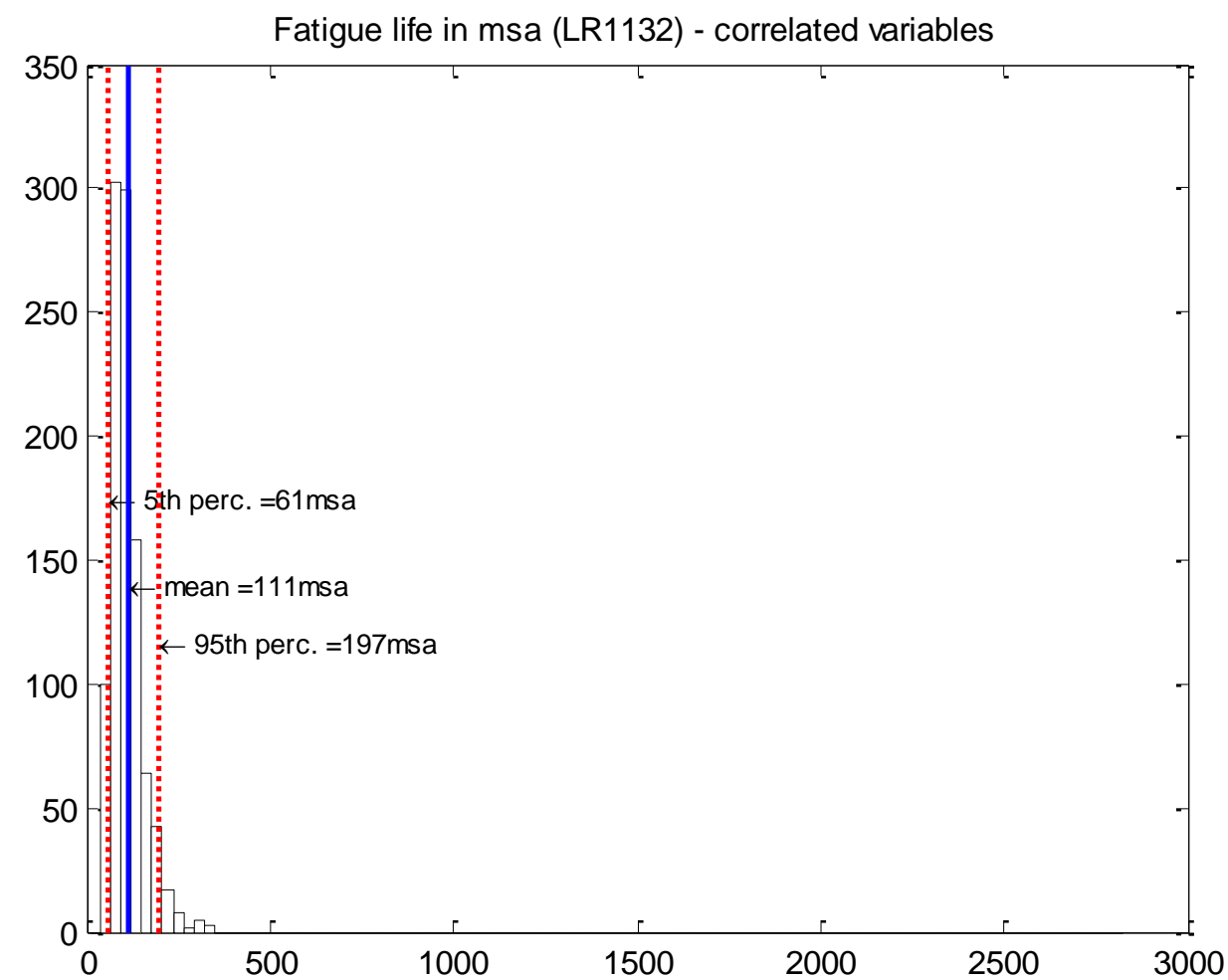

Figure 9.5 Fatigue life for Section 3 of site M01 -correlated variables 


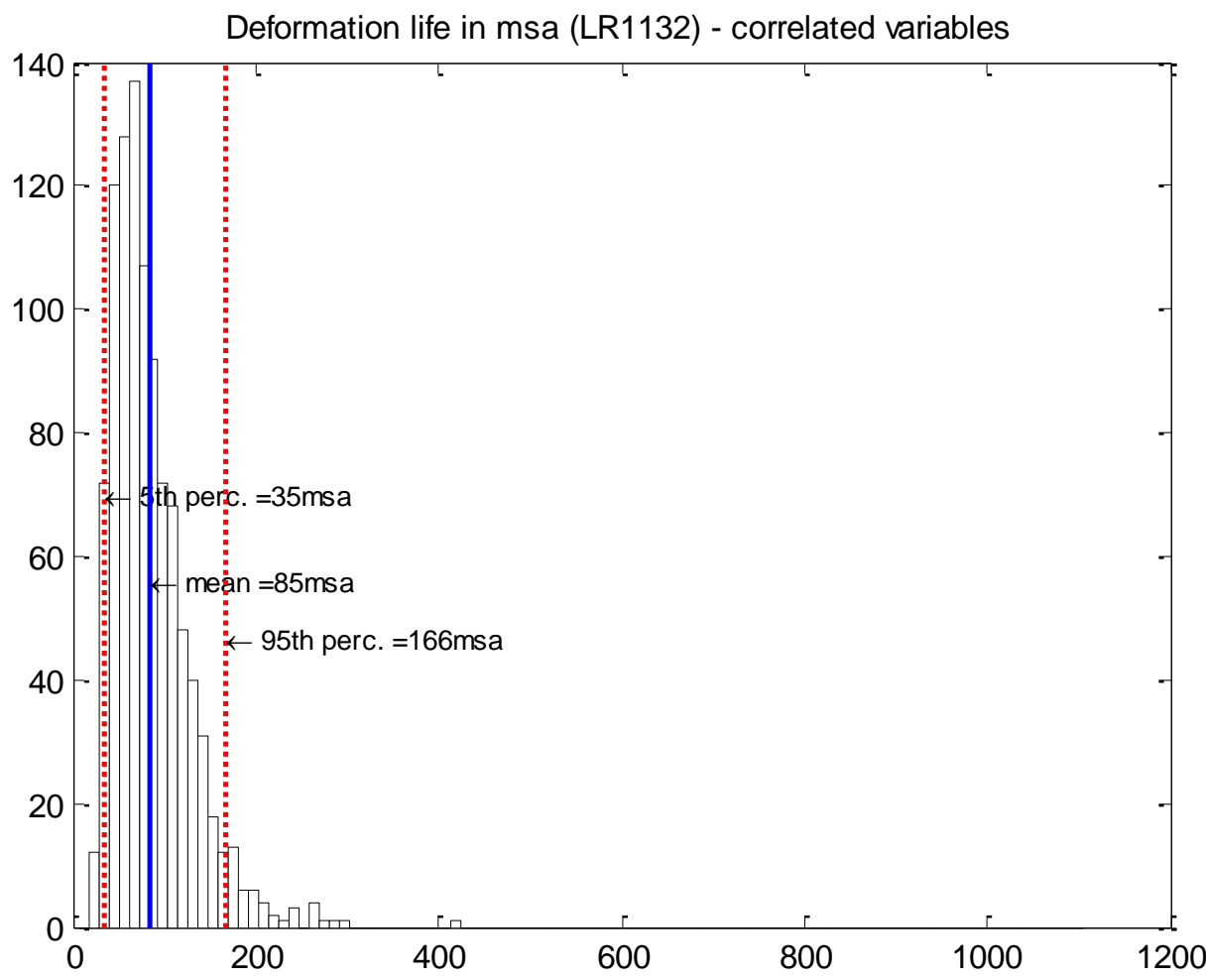

Figure 9.6 Deformation life for Section 3 of site M01 - correlated variables

\subsection{Conclusions}

This chapter has presented two methods to estimate the variability of the fatigue and deformation life of a pavement structure to account for uncertainty of input variables, namely the MCS and the MM method.

Both methods have been shown to give similar results in terms of variability as expressed by the coefficient of variation (COV). For the range of sites considered in this research - excluding the sites with thin asphalt layer - the average coefficient of variation (COV) for fatigue and deformation life (not corrected for model bias) from the two methods can be assumed to be $\sim 90 \%$ and $\sim 70 \%$ respectively. The probability distribution that best fits both fatigue and deformation lives has been shown to be lognormal.

Finally, the research has shown that when considering the mean values only of the input variables the fatigue life results are conservative compared to those obtained by considering full variability of all the input variables. However, the opposite is true for the deformation life, i.e. the mean deformation life is underestimated when variability of the input variables is not considered. 


\section{Conclusions and Recommendations}

The research has confirmed that variability of key pavement design input variables - but excluding the variability of the traffic loading - and variations due to lack-of-fit of the design models affect the pavement performance. Variations of input design parameters and errors related to the model bias have been discussed and quantified, as well as resulting variations of pavement performance (in terms of fatigue and deformation life). Variability has been described by statistical terms such as mean and standard deviation and by its probability density distribution. A useful dimensionless way of expressing the variability of a material's property was to use the ratio of the standard deviation over the mean, known as coefficient of variation (COV).

The goal of this work was to develop a 'gauge' for application for a range of circumstances, e.g. at design stage and/or any time after pavement construction. The research concentrated on flexible pavements only, i.e. asphalt layer on top of granular subbase and subgrade. What goes into the gauge is the main pavement design input parameters: the asphalt layer thickness and stiffness, the subbase layer thickness and stiffness and the subgrade stiffness. Each of the input variables is described by mean, standard deviation and probability distribution. The output is a statistical assessment of the estimated pavement performance which is not a single deterministic result that would derive by considering average values of input variables but rather a range of values and probability of any particular outcome. The proposed model to calculate the fatigue and deformation life is very fast and simple, can be included in a spreadsheet or used in a pavement management system where stresses and strains must be calculated millions of times. Both fatigue and deformation life are described by a lognormal distribution for which a statistical analysis and risk evaluation can be performed. Therefore, this method makes structural analysis of pavements a more practical and convenient tool for engineers.

Risk assessment for the design, construction and performance of pavements is essential. Risk analysis combines probability descriptions of uncertain 
variables and computer simulation to characterise risk associated with the outcome. The implications of the proposed methodology in terms of improved grasp of design risk could be significant and it could offer an attractive tool for designers and contractors to economically evaluate this risk. Given that some variability is expected with the major input design parameters (i.e., their values are not fixed), it is possible that these variations can greatly decrease the expected performance of the pavement structure being designed. Inadequate pavement performance may include premature pavement defects such as rutting and cracking, excessive localised deflections, stripping in asphalt and other surface distress types.

Therefore, understanding and assessing the risk to the pavement performance throughout its design life is of paramount importance for both design and maintenance aspects. The risks associated with variability of materials leading to potential inadequate pavement performance could be managed more effectively during the design stage. Although the risk can't be completely removed, through enhancing the pavement design, the risk can be managed.

Also, recognising the uncertainty of input variables and the uncertainty that this variability creates in the results is of paramount importance in conducting a Life-cycle cost-analysis (LCCA). Highway authorities could apply the probabilistic approach to their current life-cycle cost-analysis procedures. As discussed in Herbold (2000), then they can answer three basic questions: What can happen? How likely is it to happen? What are the consequences if it happens? It should be noted though that this research does not include the predictive modelling for pavement deterioration which is required when doing LCCA. The proposed methodology is intended to be applied to the results of any predictive modelling techniques. By adopting a probabilistic approach to LCCA, the analyst can advise the decision-maker about the risks associated with various courses of action and the probability of an outcome actually occurring. By applying this information, the decision-maker can select the best, most cost-effective solutions to provide the greatest long-term benefits.

Finally, the results of this research could form the basis for revising acceptance values of construction tolerances. This study has shown that the effect of variability on performance is important and how much effort is still needed to 
go toward achieving low variability. More specific and precise knowledge of the effect of variability could allow this property to be rationally incorporated into specifications and to form a defensible basis for price adjustment systems for contractor quality.

This chapter summarises the main conclusions from each chapter and finally presents some recommendations for future research.

\subsection{Examples of risk application of proposed research}

A couple of examples of how the proposed methodology could be used for risk analysis is presented in this section.

The input parameters for the sections of road of interest could come from real site investigation data (such as for the sections discussed here) or could be the result of research activities to assess the impact of input design parameters variability. The practical application of the research activities discussed and shown in Figure 3.9 are reproduced in Figure 10.1, which also includes reference to the relevant chapters in the thesis.

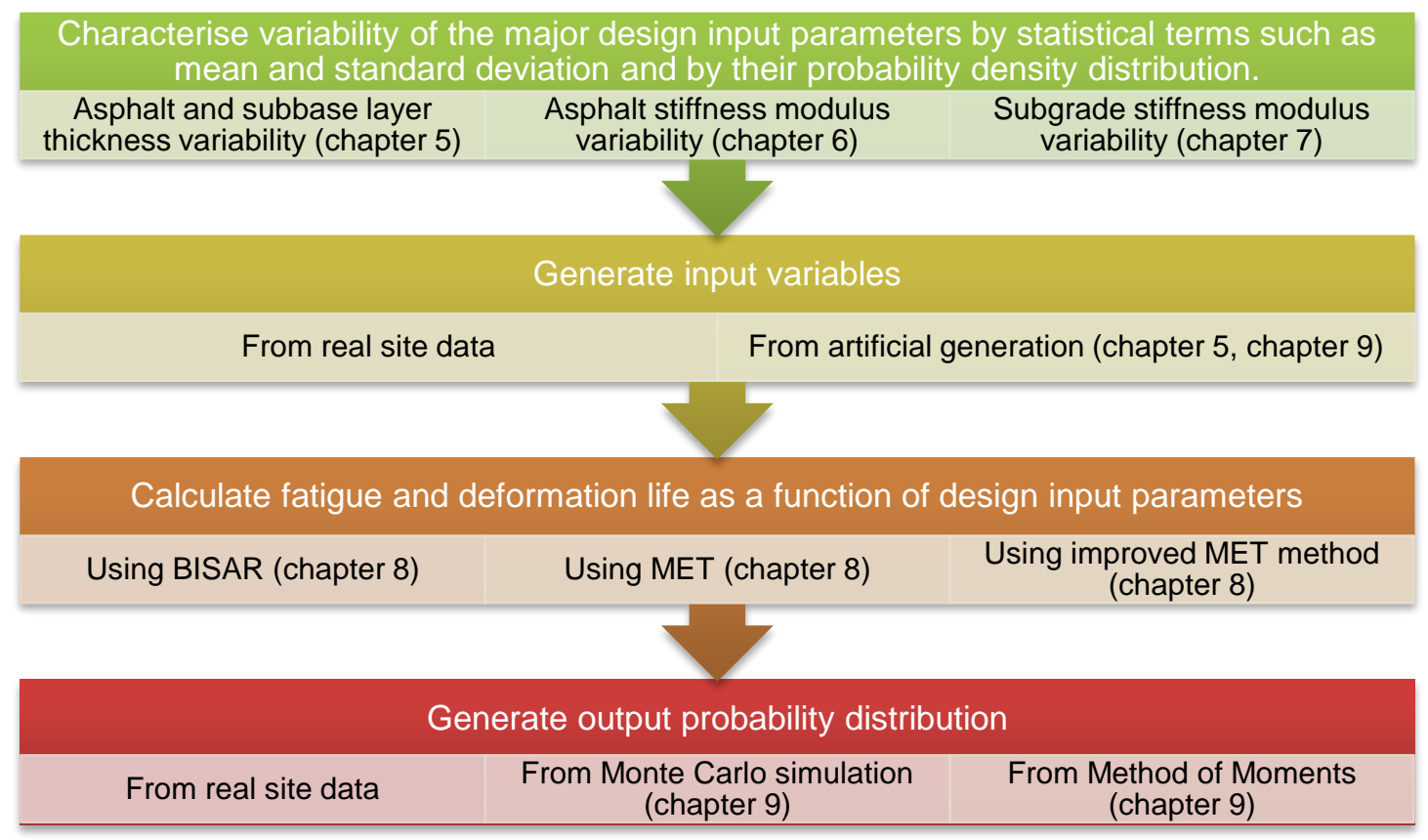

Figure 10.1 Practical application of the research activities shown in Figure 3.9 
Two different sections of a motorway, corresponding to section 4 of site M05 CW and section 6 of site M07 NB, were studied. The mean and standard deviation values of the fatigue and deformation life without model correction are obtained from Table 9.3 and Table 9.4, and reproduced in Table 10.1.

Table 10.1 Parameters of performance life for hypothetical section, for example Section 4 of site M05 CW and Section 6 of site M07 NB

\begin{tabular}{|c|c|c|c|c|c|c|c|c|c|}
\hline \multicolumn{2}{|c|}{$\begin{array}{l}\text { Pavement site } \\
\text { sections }\end{array}$} & \multirow{2}{*}{$\begin{array}{l}\text { Performance life } \\
\text { Fatigue }\end{array}$} & \multirow{2}{*}{$\mu$} & \multirow{2}{*}{$\begin{array}{l}\sigma \\
21\end{array}$} & \multirow{2}{*}{$\begin{array}{c}\text { COV (\%) } \\
0.6 \\
\end{array}$} & \multirow{2}{*}{$\begin{array}{c}\lambda=\ln \mu-0.5 \xi^{2} \\
3.5\end{array}$} & \multirow{2}{*}{$\xi=\sqrt{\ln }$} & \multirow{2}{*}{$\frac{\left(1+\left(\frac{\sigma}{\mu}\right)^{2}\right)}{0.5}$} & \multirow{2}{*}{$\begin{array}{l}\text { Probabilty distribution } \\
\text { Lognormal }\end{array}$} \\
\hline & & & & & & & & & \\
\hline IVIUS CVV & 4 & Deformation & 44 & 25 & 0.6 & 3.6 & & 0.5 & Lognormal \\
\hline \multirow{2}{*}{ M07 NB } & \multirow{2}{*}{6} & Fatigue & 7 & 6 & 0.9 & 1.7 & & 0.7 & Lognormal \\
\hline & & Deformation & 39 & 43 & 1.1 & 3.3 & & 0.9 & Lognormal \\
\hline
\end{tabular}

Both fatigue and deformation life are described by a lognormal distribution for which a statistical analysis can be performed. Because of the similarities between the normal and lognormal distributions, the calculation of the probability of an event when a random variable is lognormal is relatively easy.

Suppose the following questions need to be answered: For section 4 of site M05 CW, what is the probability that the fatigue life exceeds $20 \mathrm{msa}$ ? What is the probability that the fatigue life is less than $10 \mathrm{msa}$ ? What fatigue life value is exceeded by $99.9 \%$ of the points? What fatigue life value is not exceeded by $99.9 \%$ of the points?

The answer to the first and second problem can be calculated in the same way as for the normal variables, except that for the lognormal case, the standard variable $S$ is expressed as:

$S=\frac{\ln X-\lambda_{X}}{\zeta_{X}}$

where $X$ is a lognormal variable and $\lambda x$ and $\xi x$ are the two parameters (mean and standard deviation) of the lognormal distribution. The cumulative distribution function of the standard normal distribution $\Phi()$ can be obtained from standard tables. If follows that:

$$
\begin{aligned}
& P(X>20)=\Phi\left(\frac{\ln 20-3.5}{0.5}\right)=\Phi(-1.01)=0.843 \text { or } 84.3 \% \\
& P(X<10)=\Phi\left(\frac{\ln 10-3.5}{0.5}\right)=\Phi(-2.39)=(1-0.5-0.4916)=0.008 \text { or } 0.8 \%
\end{aligned}
$$


Similarly, the answer to the third and fourth question can be calculated thus:

$$
\begin{aligned}
& P(X>x)=\Phi\left(\frac{\ln X-3.5}{0.5}\right)=0.99 \\
& \left(\frac{\ln X-3.5}{0.5}\right)=-2.33 \rightarrow \ln X=2.34 \rightarrow X \cong 10 \mathrm{msa} \\
& P(X<x)=\Phi\left(\frac{\ln X-3.5}{0.5}\right)=0.99 \\
& \left(\frac{\ln X-3.5}{0.5}\right)=2.33 \rightarrow \ln X=4.66 \cong 106 \mathrm{msa}
\end{aligned}
$$

The same calculations were carried out for the deformation life, see results in Table 10.2.

The same approach can be used for the M07 segment, with the outcomes shown in Table 10.2. For example, the probability that the fatigue life is less

\begin{tabular}{|c|c|c|c|c|c|c|c|c|c|c|c|c|c|c|c|c|c|c|}
\hline \multirow{2}{*}{\multicolumn{2}{|c|}{$\begin{array}{l}\text { Pavement site } \\
\text { sections }\end{array}$}} & \multirow[t]{2}{*}{ Performance life } & \multirow{2}{*}{$\begin{array}{c}\mu \\
\text { (msa) }\end{array}$} & \multirow[t]{2}{*}{$\begin{array}{c}\sigma \\
\text { (msa) }\end{array}$} & \multirow[t]{2}{*}{$\begin{array}{c}\text { Cov } \\
(\%)\end{array}$} & \multirow[t]{2}{*}{$\lambda=\ln \mu-0.5 \xi^{2}$} & \multirow{2}{*}{$\xi=\sqrt{\ln (}$} & \multirow{2}{*}{$\left(1+\left(\frac{\sigma}{\mu}\right)^{2}\right)$} & \multirow[t]{2}{*}{$x$ (msa) } & \multirow[t]{2}{*}{$|P(X>x)|$} & \multirow[t]{2}{*}{$|P(X<x)|$} & \multirow[t]{2}{*}{$\begin{array}{c}\text { Desired } \\
\text { probability }\end{array}$} & \multicolumn{3}{|c|}{$P(X<x)$} & \multicolumn{3}{|c|}{$P(X>x)$} \\
\hline & & & & & & & & & & & & & \begin{tabular}{|c|}
$z$ \\
value
\end{tabular} & \begin{tabular}{|l|}
$\ln x$ \\
\end{tabular} & \begin{tabular}{c|c}
$\mathbf{x}$ \\
(msa)
\end{tabular} & \begin{tabular}{|c|}
$z$ \\
value
\end{tabular} & $\mid \ln x$ & $\begin{array}{c}\mathrm{x} \\
\text { (msa) }\end{array}$ \\
\hline \multirow{4}{*}{ M05 CW } & \multirow{4}{*}{4} & Fatigue & 38 & 21 & 0.6 & 3.5 & & 0.5 & 20 & $84.3 \%$ & $15.7 \%$ & 0.99 & 2.33 & 4.66 & 106 & -2.33 & 2.34 & 10 \\
\hline & & Fatigue & 38 & 21 & 0.6 & 3.5 & & 0.5 & 10 & $99.2 \%$ & $0.8 \%$ & 0.99 & 2.33 & 4.66 & 106 & -2.33 & 2.34 & 10 \\
\hline & & Deformation & 44 & 25 & 0.6 & 3.7 & & 0.5 & 20 & $92.1 \%$ & $7.9 \%$ & 0.99 & 2.33 & 4.86 & 129 & -2.33 & 2.54 & 13 \\
\hline & & Deformation & 44 & 25 & 0.6 & 3.7 & & 0.5 & 10 & $99.7 \%$ & $0.3 \%$ & 0.99 & 2.33 & 4.86 & 129 & -2.33 & 2.54 & 13 \\
\hline \multirow{4}{*}{ M07 NB } & \multirow{4}{*}{6} & Fatigue & 7 & 6 & 0.9 & 1.7 & & 0.7 & 2 & $92.5 \%$ & $7.5 \%$ & 0.99 & 2.33 & 3.33 & 28 & -2.33 & 0.07 & 1 \\
\hline & & Fatigue & 7 & 6 & 0.9 & 1.7 & & 0.7 & 3 & $80.5 \%$ & $19.5 \%$ & 0.99 & 2.33 & 3.33 & 28 & -2.33 & 0.07 & 1 \\
\hline & & Deformation & 39 & 43 & 1.1 & 3.3 & & 0.9 & 20 & $63.2 \%$ & $36.8 \%$ & 0.99 & 2.33 & 5.39 & 220 & -2.33 & 1.21 & 3 \\
\hline & & Deformation & 39 & 43 & 1.1 & 3.3 & & 0.9 & 10 & $86.6 \%$ & $13.4 \%$ & 0.99 & 2.33 & 5.39 & 220 & -2.33 & 1.21 & 3 \\
\hline
\end{tabular}
than $2 \mathrm{msa}$ is $7.5 \%$ and $99.9 \%$ of the points have a fatigue life less than $28 \mathrm{msa}$.

Table 10.2 Example of statistical analysis of fatigue and deformation life results for risk analysis

Therefore, the probabilistic approach offers a simple and practical tool for an expert to assess the impact of input design parameters' variability on the expected performance life for a particular site. The design risk can be calculated and economically priced.

\subsection{Summary of main conclusions}

\subsubsection{Variability in pavement design (chapter 2)}

Three basic types of variations were encountered in flexible pavement designs: variations within a design length (i.e. a section with nominally uniform pavement thickness and materials type); variations between design and actual 
values; and variations due to lack-of-fit of the design models. Quantifying and analysing variability of pavement materials and design inputs was found therefore to be fundamental in developing a probabilistic-based design that evaluates reliability.

A summary of the variability of design input parameters from published sources was presented. The results of various sensitivity analyses carried out in the past to assess the variability of each design parameter and to measure its effect on the design were (for fully flexible pavements):

- The most influential design inputs on reliability were layer properties and thickness, followed by traffic and lack-of-fit error.

- The parameters with the greatest influence on the variability of predicted fatigue performance, without considering variable loads, were AC (asphalt) modulus and thickness.

- Fatigue cracking was affected by changes in the asphalt layer thickness while it was unaffected by changes in the granular base layer thickness.

- The parameters with the greatest influence on the variability of predicted deformation (rutting) performance, without considering variable loads, were the granular base thickness, AC (asphalt) thickness, and stiffness of the subgrade.

- If the traffic axle weight variability was added the output variability for fatigue and deformation performance was significantly changed (i.e., more than doubled).

\subsubsection{Literature review on reliability in pavement design (chapter 3 )}

The review of the literature available in the UK, US, France and Australia, showed that design reliability is becoming increasingly important for consideration in pavement design procedures and whole-life cost analysis. Modern design methods were found to be moving towards a probabilistic approach to design because it is better suited to treat the variety of uncertainty, namely that related to the prediction of traffic loading and prediction of pavement performance. The variability observed in pavement performance 
was reported primarily to be derived from variability in pavement materials, layer thicknesses, climatic effects, and the distribution of pavement distress along the length of the pavement may vary as a result.

To account for input variability, different techniques are available and the Monte Carlo simulation (MCS) technique was reported by many authors as the simplest, albeit time consuming and costly, to be implemented in computer programming.

Other reliability analysis techniques, such as FORM and PEM - including a new point estimate approach called the method of moments (MM) - were also discussed and compared with the MCS technique. The moment method in particular, being very simple, requires neither iteration nor the computation of derivatives, and thus was found convenient to be applied to structural reliability analysis.

\subsubsection{Methodology (chapter 4)}

The sites used in the research were eight in total and were mainly motorways with two sites being of a thinner pavement construction. All sites had a fully flexible construction. The survey data available for these sites were: GPR, cores, FWD, DCP, ITSM and traffic data. The survey data referred to lane 1, the most heavily trafficked lane. The length of each site varied but, wherever possible, a length of $1 \mathrm{~km}$ was used.

The theory behind Fourier analysis and the Method of Equivalent Thickness (MET) were introduced in this chapter. Fourier analysis was explored to obtain information about the frequency characteristics of the GPR profile data for future artificial generation of layer thickness in Monte Carlo simulation.

The MET technique was utilised because it is very powerful when calculating stresses and strains for a large number of pavement structures, for Monte Carlo simulation. 


\subsubsection{Statistical characterisation of layer thickness variability (chapter 5)}

A statistical analysis of the GPR data collected on each site was presented in terms of mean, standard deviation, coefficient of variation and probability distribution. Results showed that the probability distribution for the layer thickness for the UK sites could be considered normal and the coefficient of variation was broadly similar to those reported in the American literature (e.g average $10 \%$ COV for the asphalt layer and $15 \%$ COV for the subbase layer).

The generation of random signals for Monte Carlo simulations was presented. The methodology used Fourier Analysis to generate random asphalt and subbase thickness profiles which had similar frequency characteristics to the real case study profiles.

For the research analysis, the total asphalt and granular sub-base layer thickness probability distribution was assumed to be normal. Also, the asphalt and the sub-base layer thicknesses were considered uncorrelated.

\subsubsection{Statistical characterisation of asphalt stiffness modulus variability (chapter 6)}

The accuracy in the back-calculation of pavement layer moduli was analysed. The impact of three major sources of errors on the back-calculated pavement layer moduli - namely pavement thickness errors, random deflection errors and limited number of data points - was discussed and a technique to calculate the 'true' asphalt stiffness variation was reported. The analysis was done on a limited number of pavement structures and the results were compared with previous research findings.

The main observations made in this chapter were:

- Both laboratory and in-situ determination of stiffness modulus should be used to perform a full pavement evaluation.

- In order for the FWD back-analysis to give reliable estimates of the in-situ asphalt stiffness modulus, it is essential to use accurate pavement layer thickness information. For example, a 15 per cent underestimate of the 
thickness of a bound layer can result in a fifty per cent overestimate of the stiffness of that layer.

- The average error due to random measurement deflection error was less than $1 \%$ on back-analysed asphalt stiffness and close to $0 \%$ on backanalysed subgrade stiffness.

- The effect of random deflection measurement error can be minimised by making at least three replicate drops at each drop height.

With both the effect of random error in the deflection measurement and the effect of variation of layer thickness included in the analysis, a relationship was proposed to calculate the adjusted standard deviation.

- The effect of limited number of data points on back-calculated moduli showed that:

- The accuracy of the mean stiffness modulus estimates depends on the ratio of estimated standard deviation to true stiffness modulus and the number of samples.

- The accuracy of the standard deviation of the layer stiffness modulus estimates depends on the number of samples.

- Based on the plots of ITSM tests on the M01 site, the coefficient of variation of the asphalt layer stiffness modulus appeared, on average, to range from $10 \%$ to $30 \%$.

- A lognormal probability distribution was found representative of the asphalt layer stiffness modulus.

\subsubsection{Statistical characterisation of subgrade stiffness modulus variability (chapter 7)}

An overview of different methods to estimate the subgrade stiffness, spatial and seasonal variations of subgrade stiffness and the results of the author's attempt to assess the subgrade stiffness variability were discussed in this chapter. The main findings were: 
- California Bearing Ratio (CBR) and Repeated Load Triaxial (RLT) tests, Dynamic Cone Penetrometer (DCP), Falling Weight Deflectometer (FWD) and Light Weight Dynamic plate (LWD) are the main methods used worldwide to estimate the subgrade stiffness. Empirical and local correlations are used between the tests methods above with consequent variability in test results depending on the particular method used.

- Based on a 'cusum' analysis on the available sites, the coefficient of variation of the back-analysed subgrade stiffness modulus for almost all sites varied from a minimum of $8 \%$ to a maximum of $30 \%$, with an average value of $20 \%$. The high uncertainty sometimes observed in the data is probably due to the small sample size derived from the cusum analysis.

- A lognormal probability distribution was found in most cases to be representative of the subgrade stiffness.

Based on a sensitivity analysis of design input parameter variability on the available sites, it was found that the stiffness of the subgrade ( $\left.E_{s g}\right)$ has a great influence on the variability of predicted deformation (rutting) performance together with the granular subbase thickness $\left(T_{s b}\right)$ and the asphalt thickness $\left(T_{a}\right)$. The effect of the variability in subgrade stiffness was found to be less pronounced with respect to the fatigue life of flexible pavements.

\subsubsection{Proposed model to predict the pavement design life (chapter 8)}

A large number of computer programs have been developed for calculating stresses, strains and displacements in layered elastic systems, such as the BISAR software developed by Shell. BISAR is believed to provide reasonably accurate values of stresses and strains in pavement response models but the main limitation is that only a maximum of 10 pavement structures can be analysed at a time and all input parameters need to be inserted manually by the user. Consequently, the software is not suitable for obtaining stresses and strains for a large number of pavement structures. 
A simplified method for calculating stresses, strains and displacements in layered elastic systems is represented by Odemark's Method of Equivalent Thicknesses (MET).

Values of asphalt and subgrade strains were calculated with both the Method of Equivalent Thicknesses (MET) and the BISAR software for a large number of flexible pavements (3-layer systems). The analysis showed that, while subgrade strains were predicted acceptably well, differences greater than $\pm 10 \%$ exist between the two methods in the case of the asphalt strains. To overcome some limitations of the MET method and, therefore, to reduce the differences between the two methods an alternative model was proposed, for the calculation of the asphalt strains. An alternative model for the fatigue life was also introduced. The proposed models provide a simple and efficient method for practical purposes, for example in Pavement Management Systems or in simulation of pavement deterioration, where stresses and strains must be calculated a large number of times.

This involved the calculation of an Equivalent Foundation Modulus (EFM), which in this analysis represented the contribution of both the foundation layers and the asphalt layer. A linear regression analysis was presented which allowed the prediction of values of asphalt strain and fatigue life calculated with BISAR from those obtained with the MET methodology and with a relatively high level of confidence. Approximately $93 \%$ of predicted strains were found to lie within $10 \%$ of the BISAR-derived values. A 'professional' factor was also discussed which represents uncertainties of the assumptions and simplifications used in the proposed design model.

Because of variability in subbase stiffness values found from real site data, an alternative deformation model was also introduced which accepts a relaxation in one of the MET conditions. The proposed relaxation was for the ratio of subbase stiffness over subgrade stiffness to be greater than or equal to 1 while keeping all other conditions of validity of the MET method. The alternative deformation model was demonstrated not to introduce a significant difference in variability when compared to the model where all the original MET conditions are satisfied (i.e., the one which only accepts $E_{\text {subbase }} / E_{\text {subgrade }}>2$ ). 
Finally, a comparison of results for selected pavement structures resulting from both 3-layer and 2-layer models employed in back-calculation analysis, suggested that the preferred pavement model, for more reliable results in both back-analysed layer stiffnesses and performance lives (fatigue and deformation), was a 3-layer structure.

\subsubsection{Monte Carlo simulation and design life probability distributions (chapter 9)}

Two methods were presented to estimate the variability of the fatigue and deformation life of a pavement structure to account for uncertainty of input variables, namely the Monte Carlo Simulation (MCS) and the Method of Moments (MM) methods.

Both methods were shown to give similar results in terms of variability as expressed by the coefficient of variation (COV). For the range of sites considered in this research - excluding the sites with thin asphalt layer - the average coefficient of variation (COV) for fatigue and deformation life (not corrected for model bias) from both methods can be assumed to be $\sim 90 \%$ and $\sim 70 \%$ respectively. The probability distribution that best fits both fatigue and deformation lives was shown to be lognormal.

Finally, the research showed that when considering the mean values only of the input variables the fatigue life results were conservative compared to those obtained by considering variability of the input variables. However, the opposite was true for the deformation life, i.e. the mean deformation life was underestimated when variability of the input variables was not considered.

\subsection{Recommendations for further work}

\subsubsection{Investigation on how design relates to in-service performance}

The research has shown how important it is to achieve low variability to ensure a satisfactory performance of a pavement structure through its design life. An under-performing pavement structure will inevitably lead to increased maintenance and rehabilitation costs at an earlier stage in the pavement 
service life and will ultimately increase the life cycle cost. Contractors should be incentivised to achieve in-service performance and at the same time not incentivised to exceed the designed in-service performance due to the limited benefits in LCC terms. To implement knowledge of the effect of variability in construction practice, variability could be rationally incorporated into specifications and form a defensible basis for price adjustment systems for contractor quality. A revision of current specification requirements is required to assess their robustness and/or weaknesses. Revised threshold values and/or different methods to assess as-built variability could be implemented. The performance specifications must, however, be realistic and achievable for the contractor.

\subsubsection{Include variability in life cycle cost analysis}

An assessment of including variability in life cycle cost analysis has not been carried out. A sensitivity analysis could be carried out to show how variability affects the life cycle cost of a pavement over a 40 year analysis period, for example. This information would help highway authorities to make more informed decisions about future level of funding for road improvement and to reduce maintenance and rehabilitations costs.

\subsubsection{Apply research to a larger number of real sites}

Only eight sites were included in this analysis. A larger sample should be included to validate the research results for possible future development into design and construction practice procedures. Each site should have sufficient survey information to make a consistent assessment of the data, including more ITSM stiffness data.

\subsubsection{Variability to include more material properties}

This research has concentrated on the assessment of variability of key pavement design input parameters, namely layer thickness and stiffness. To complete the research other important design properties should be considered 
such as traffic, bitumen content and compaction (i.e., air voids percent) to name the most critical ones. Also, the analysis should be completed to include the effect of correlation among selected pavement variables, such as between the asphalt and subbase layer thickness and/or between asphalt thickness and stiffness, on pavement life and performance correlation between variables. The research could also be extended to hydraulically bound layers, i.e. flexible composite pavements, and possibly to surface damage (fretting, potholes) as predictive methods become available. 


\section{References}

Aguiar-Moya, J.P., Banerjee, A., and Prozzi, J.A. (2009) "Sensitivity Analysis of the M-E PDG Using Measured Probability Distributions of Pavement Layer Thickness", 88th Transportation Research Board Annual Meeting.

Ali, A.W., Abbas, A.R., and Sett, K. (2011) "Effect of Spatial Variations in Subgrade Stiffness on Pavement Performance", Geo-Frontiers.

Alsherri, A. and George, K.P. (1988) "Reliability model for pavement performance", Journal of Transportation Engineering, ASCE, Vol.114, pp294-306.

AMADEUS (2000) " Advanced Models for Analytical Design of European Pavement Structures, project funded by the European commission under the transport rtd programme of the 4th framework programme".

American Association of State Highway and Transportation Officials (1993) "AASHTO Guide for Design of Pavement Structures", Washington D.C.

American Association of State Highway and Transportation Officials (2008) "Comparative Performance Measurement for Pavement Smoothness".

American Association of State Highway and Transportation Officials (2008) "Mechanistic-Empirical Pavement Design Guide", Washington D.C.

Appea, A.K. (2003) "Validation of FWD Testing Results at the Virginia Smart Road: Theoretically and by Instrument Responses", Thesis (PhD), Faculty of the Virginia Polytechnic Institute and State University.

Attoh-Okine, B.N. (1994) "Time series analysis for ground penetrating radar (GPR) asphalt thickness profile", Applied Stochastic Models and Data Analysis, Vol.10, pp153-167.

Attoh-Okine, N.O. and Roddis, W.M.K. (1994) "Pavement Thickness Variability and Its Effect on Determination of Moduli and Remaining Life", Transportation Research Record, Vol.1449, pp39-45.

Basma, A.A. and Al-Balbissi, A.H. (1989) "Probabilistic Design of Flexible and Rigid Pavements Using AASHTO Equations", Transportation Research Record, Washington D.C., TRR 1227, pp 34-43.

Bayomy, F. and Salem, H. (2005) "Monitoring and Modeling Subgrade Soil Moisture for Pavement Design and Rehabilitation in Idaho Phase III: Data Collection and Analysis", Idaho Transportation Department.

Boussinesq, J. (1883) "Application des potentials a l'etude d'equilibre et du movement des solides elastiques ", Gautier-Villars, Paris.

Briggs, R.C., Scullion, T., and Maser, K.R. (1992) "Asphalt Thickness Variation on Texas Strategic Highway Research Program Sections and Effect on Backcalculated Moduli", Transportation Research Record 1377.

BS 1377-4:1990 (1990) "Methods of test for soils for civil engineering purposes. Compaction-related tests". 
Burmister, D. M. (1943) "The Theory of Stresses and Displacements in Layered Systems and Applications to the Design of Airport Runways", Proceedings of Highway Research Board, Vol. 23, pp. 126-144.

Cebon, D and Newland, D.E. (1984) "Artificial generation of road surface topography by the Inverse FFT method", Dynamics of vehicles on roads and on tracks, Proceedings of IAVSD Symposium, pp. 29-42.

Chen, X., Lambert, J.R., Tsai, C., and Zhang, Z. (2013) "Evaluation of superheavy load movement on flexible pavements", International Journal of Pavement Engineering , Vol.14(5), pp440-448.

Chua, K.H., Der Kiureghian, A., and Monismith, C.L. (1992) "Stochastic model for pavement design", Journal of Transportation Engineering, ASCE, Vol.118, pp769-786.

Collop, A.C. (1994) "Effects of traffic and temperature on flexible pavement wear", PhD Thesis, Cambridge University Engineering Department.

Collop, A.C. (2000) "The Effect of Asphalt Layer Thickness Variations on Pavement Evaluation Using the Falling Weight Deflectometer", School of Civil Engineering, Vol.1 (4), pp247-263.

Cunagin, W.D. and Kent, P.M. (1998) "Reliability of Traffic Data", Transportation Research Record, Vol.1625, pp18-25.

Darter, M., Hudson, W. R., and Brown, J. L. (1973) "Statistical variations of flexible pavement properties and their consideration in design", Proceedings, Association of Asphalt Technologists, Vol.42, pp589-613.

Darter, M., Khazanovich, L., Yu, T., and Malleha, J. (2005) "Reliability analysis of cracking and faulting prediction included in the new Mechanistic-Empirical pavement design procedure", 84th Transportation Research Board Annual Meeting.

Ditlevesen, O.J. (1981) "Uncertainty modeling with applications to multidimensional civil engineering systems", McGraw-Hill book Co., Inc., New York, N.Y.

El-Badawy, S.M. and Kamel, M.A. (2011) "Assessment and improvement of the accuracy of the Odemark transformation method", International Journal of Advanced Engineering Sciences and Technologies, Vol.5(2), pp105-110.

EN 12697-26 (2012) "Bituminous mixtures - test methods for hot mix asphalt - Part 26: Stiffness".

Fernandes Jr, J.L., Pais, J.C., and Pereira, P.A.A. (2006) "Effects of traffic loading on Portuguese and Brazilian pavements performance", 85th Transportation Research Board Annual Meeting.

George, K.P. and Husain, S. (1986) "Thickness design for flexible pavement: a probabilistic approach", Transportation Research Record, Vol.1095, pp26-36.

Graves, R.C. and Mahboub, K.C. (2007) "Sampling based flexible pavement design reliability evaluation of the NCHRP Mechanistic Empirical 
Pavement Design Guide (MEPDG)", 86th Transportation Research Board Annual Meeting.

Haldar, A. and Mahadeva, S. (2000) "Probability, Reliability and Statistical Methods in Engineering Design", New York, John Wiley \& Sons, Inc.

Harr, M.E. (1987) "Reliability-Based Design in Civil Engineering", New York, McGraw-Hill.

Hasofer, A.M. and Lind, N.C. (1974) "An exact and invariant secondmoment code format", J. Engrg. Mech. Div., ASCE, Vol.100 (1), pp111121.

Hayward, D. (2010) "Remote control pavement", NCE article.

Herbold, K.D. (2000) "Using Monte Carlo Simulation for Pavement Cost Analysis", Public Roads Magazine, FHWA, Vol 64 No 3.

Highways Agency, Design Manual for Roads and Bridges (DMRB), "HD 25 Foundations".

Highways Agency, Design Manual for Roads and Bridges (DMRB), "HD 26 Pavement Design".

Highways Agency, Design Manual for Roads and Bridges (DMRB), "HD 29 Data for Pavement Assessment".

Highways Agency, Design Manual for Roads and Bridges (DMRB), "HD 30 Maintenance Assessment Procedure".

Highways Agency, Design Manual for Roads and Bridges (DMRB) (2009) "IAN 73/09 Rev 1 Design Guidance For Road Pavement Foundations (Draft HD25)", Stationery Office Ltd HD.

Highways Agency, Manual of Contract Documents for Highway Works (MCHW) "Volume 1 and 2".

Hong, H.P. and Somo, S. (2001) "Probabilistic assessment of pavement conditions", Canadian Journal of Civil Engineering, Vol.28, pp813-822.

Hossain, M., Romanoschi, S., and Gisi, A. J. (2000) "Seasonal and spatial variation of subgrade response", Pavement Subgrade, Unbound Materials, and Nondestructive Testing.

Huang, Y.H. (2004) "Pavement Analysis and Design", 2nd Edition, Upper Saddle River, NJ: Pearson Education, Inc.

Hudson, W.R. (1975) "State of the art in predicting pavement reliability from input variability", Report No. FAA-RD-75-207.

Hughes, C.S. (1996) "NCHRP Synthesis of Highway Practice 232:

Variability in Highway Pavement Construction", National Research Council, Washington D.C.

IBM Software Business Analytics, (2012) "Better decision making under uncertain conditions using Monte-Carlo Simulation Monte Carlo simulation and risk analysis techniques in IBM SPSS Statistics", . 
Irik, P., Hudson, W.R., and McCullough, B.F. (1987) "Application of reliability concepts to pavement design", 6th Int. Conf. of Struct. Design of Asphalt Pavements.

Irwin, L.H., Yang, W.S., and Stubstad, R.N. (1989) "Deflection Reading Accuracy and Layer Thickness Accuracy in Backcalculation of Pavement Layer Moduli", American Society for Testing and Materials.

Jiménez, L.A. and Mrawira, D. (2012) "Bayesian Regression in Pavement Deterioration Modeling: Revisiting the AASHO Road Test Rut Depth Model", Infraestructura Vial Nr 25.

Johanneck, L. and Dai, S. (2013) "Responses and performance of stabilized full depth reclaimed 1 pavements at MnROAD", 92nd Transportation Research Board Annual Meeting.

Johnson, K.L. (1985) "Contact Mechanics", Cambridge University Press, Cambridge.

Khazanovich, L. and Wang, QC. (2007) "MNLAYER - A high performance layered elastic analysis program", 86th Transportation Research Board Annual Meeting.

Khogali, W.E.I. and Anderson, K.O. (1996) "Evaluation of seasonal variability in cohesive subgrades using backcalculation", Transportation Research Record, Vol.1546, pp140-150.

Killingsworth, B.M. and Zollinger, D.G. (1995) "Sensitivity Analysis of Input Parameters for Pavement Design and Reliability", Transportation Research Record, Vol.1482, pp111-122.

Kim, H. B. (2006) "A practical probabilistic design format for flexible pavements", 85th Transportation Research Board Annual Meeting.

Kim, H. B. and Buch, N. (2003) "Reliability-based pavement design model accounting for inherent variability of design parameters", 82nd Transportation Research Board Annual Meeting.

Lagarde-Forest, D.R, Cook, A., and Fairclough, R. (2008) "Quality Assurance of GPR Pavement Investigation Surveys", 12th International Conference on Ground Penetrating Radar.

Lemer, AC and Moavenzadeh, F. (1971) "Reliability of highway pavements", Highway Research Record, Vol.362, pp1-8.

Li, N., Haas, R., and Xie, W.C. (1996) "Reliability-based processing of Markov chains for modeling pavement network deterioration", TRB , Vol.1524, pp203-213.

Li, S., Li, Y., and Metcalf, J.B. (2002) "Enhancement of Asphalt Pavement Evaluation Using Monte Carlo Simulation and Accelerated Pavement Testing", Journal of Testing and Evaluation, JTEVA, Vol.30, pp392-399.

Liu, J., Zollinger, D.G., Tayabji, S.D., and Smith, K.D. (2005) "Application of reliability concept in concrete pavement rehabilitation decision making", 84th Transportation Research Board Annual Meeting. 
Loizos, A. and Plati, C. (2007) "Accuracy of pavement thickness estimation using different ground penetrating radar analysis approaches", NDT\&E International 40.

Losa, M., Bacci, R., and Leandri, P. (2008) "A statistical model for prediction of critical strains in pavements from deflection measurements", Road Materials and Pavement Design, EATA.

Low, B.K. and Tang, W.H. (2007) "Efficient spreadsheet algorithm for First-Order Reliability Method", Journal of Engineering Mechanics, Vol.133(12), pp1378-1387.

Lu, Q., Signore, J.M., Basheer, I., Ghuzlan, K., and Ullidtz, P. (2008) "CalBack: enhancing Caltrans mechanistic-empirical design process with new backcalculation software", 87th Transportation Research Board Annual Meeting.

Maji, A. and Das, A. (2008) "Reliability considerations of bituminous pavement design by mechanistic-empirical approach", International Journal of Pavement Engineering , Vol.9(1), pp19-31.

Maser, K. and Vandre, B. (2006) "Network-level pavement structure assessment using automated processing of Ground Penetrating Radar (GPR) data", Proceedings of the 2006 Airfield and Highway Pavement Specialty Conference, Vol., pp719-728.

Maser, K.R., Holland, T.J., Roberts, R., and Popovics, J. (2013) "NDE methods for quality assurance of new pavement thickness", International Journal of Pavement Engineering.

Mateos, A. (2013) "Evaluation of the structural response of cracked pavements at Cedex test track", 92nd Transportation Research Board Annual Meeting.

Moavenzadeh, F. and Brademeyer, B. (1977) "A stochastic model for pavement performance and management", Proc., 4th Int. Conf. on Struct. Design of Asphalt Pavements.

Molenaar, A.A.A. (2007) "Lecture Notes CT 4860, Structural Pavement Design, Design of Flexible Pavements", Delft.

Montgomery, D.C. and Runger., G.C. (2007) "Applied Statistics and Probability for Engineers", 4th Edition, John Wiley \& Sons, Inc.

National Cooperative Highway Research Program, Transportation Research Board National Research Council (2004) "Part I Introduction Guide for Mechanistic-Empirical Design of New and Rehabilitated Pavement Structures", NCHRP 1-37A, TRB, Washington D.C.

National Cooperative Highway Research Program, Transportation Research Board National Research Council (2008) "Estimating stiffness of subgrade and unbound materials for pavement design", NCHRP Synthesis 382, TRB, Washington D.C.

Newland, D.E. (1975) "An Introduction to Random Vibrations, Spectral \& Wavelet Analysis", 3rd Edition, Dover Publications, Inc, Mineola, New York. 
Noureldin, A.S., Sharaf, E., Arafah, A., and Al-Sugair, F. (1994)

"Estimation of Standard Deviation of Predicted Performance of Flexible Pavements using AASHTO Model", Transportation Research Record, Vol.1449, pp46-56.

Nunn, M. E. (2004) "Development of a more versatile approach to flexible and flexible composite pavement design", TRL Report TRL615, UK.

Nunn, M. E. and Merrill, D. B., (1997) "Review of Flexible and Composite Pavement Design Methods", TRL Paper PA/3298/97, Transport Research Laboratory, Crowthorne.

Ollis, J. E. (1997), "Statistical tolerances for concrete road pavement surfaces", Master of Total Quality Management thesis, University of Wollongong.

Ozawa, Y., Maina, J.W., and Matsui, K. (2009) "Linear elastic analysis of pavement structure loaded over rectangular area", 88th Transportation Research Board Annual Meeting.

Pais, J., Pereira, P., and Picado-Santos, L. (2002) "Variability of Laboratory Fatigue Life of Asphalt Mixes Using Four Point Bending Test Results", IJP Volume 1, Vol.1, pp1-12.

Park, D-W., Fernando, E., and Leidy, J. (2005) "Evaluation of predicted pavement response using measured tire contact stresses", 84th Transportation Research Board Annual Meeting.

Pearson, D. (2011) "Mechanistic-empirical Pavement Design Principles. In: Pearson, D. Deterioration and Maintenance of Pavements.", London: ICE, Vol., pp245-251.

Pologruto, M. (2001) "A procedure for using an FWD to determine the structural layer coefficients for flexible pavement materials", 80th Transportation Research Board Annual Meeting.

Powell, W.D., Potter, J.F., Mayhew, H.C., and Nunn, M.E. (1984) "The structural design of bituminous roads", Transport and Road Research Laboratory, LR 1132, UK.

Prozzi, J.A. and Guo, R. (2007) "Reliability-based approach for using LTPP and APT test results for estimating fatigue performance", 86th Transportation Research Board Annual Meeting.

Prozzi, J.A., Gossain, V., and Manuel, L. (2005) "Reliability of pavement structures using empirical-mechanistic models", 84th Transportation Research Board Annual Meeting.

Ramirez, R.W. (1985) "The FFT Fundamentals and Concepts", PrenticeHall, Inc., Englewood Cliffs, N.J.

Retherford, J.Q. and McDonald, M. (2010) "A Study of Reliability Methods Applicable to Mechanistic-Empirical Pavement Design Method", 89th Transportation Research Board Annual Meeting.

Riggins, M., Lytton, R., and Garcia-Diaz, A. (1985) "Developing stochastic flexible pavement distress and serviceability equations", 64th Transportation Research Board Annual Meeting. 
Rondón, H.A., Reyes, F.A., Flintsch, G.W., and Mogrovejo, D.E. (2012) "Environmental effects on hot mix asphalt dynamic mechanical properties a case study in Bogota, Colombia", 91st Transportation Research Board Annual Meeting.

Selezneva, O., Jiang, Y. J., Mdadenovic, G., Aref, S., and Darter, M. (2003) "Estimation of pavement layer thickness variability for reliabilitybased design", 82nd Transportation Research Board Annual Meeting.

Shekharan, R.A. and Ostrom, B.K. (2002) "Effect of traffic load estimates on pavement design", Transportation Research Record, Vol.1809, pp105109.

Theyse, H.L. (2006) "The precision and accuracy of Mechanistic-Empirical Pavement Design", CSIR Built Environment, South Africa.

Thom, N.H., Elliott, R.C., and Cheung, L.W. (1997) "Comparisons Between Laboratory and In Situ Determined Asphalt Concrete Moduli", Eighth International Conference on Asphalt Pavements, Seattle, Washington.

Tighe, S. (2001) "Guidelines for probabilistic pavement life cycle cost analysis", TRB , Vol.1769, pp28-38.

Tighe, S., He, Z., and Haas, R. (2001) "Environmental deterioration model for flexible pavement design: an Ontario example", 80th Transportation Research Board Annual Meeting.

Timm, D.H., Newcomb, D.E., and Galambos, T.V. (2000) "Incorporation of reliability into Mechanistic-Empirical pavement design", Transportation Research Record, Vol.1730, pp73-80.

Ullidtz, P. (1987) "Pavement analysis", Elsevier.

Ullidtz, P. (1998) "Modelling flexible pavement response and performance", 1st Edition, Polyteknisk Forlag.

Ullidtz, P. and Peattie, K. R. (1980) "Pavement Analysis by Programmable Calculators", Journal of Transportation Engineering, ASCE.

Ullidtz, W. and Zhang, W. (2002) "Back-Calculation of Pavement Layer Moduli and Forward-Calculation of Stresses and Strains", Ninth International Conference on Asphalt Pavements, Copenhagen, Denmark, Vol.3, pp1-4.

Wang, K.C.P., Zaniewski, J., and Way, G. (1994) "Probabilistic behaviour of pavements", Journal of Transportation Engineering, ASCE, Vol.120, pp358-375.

Wang, S.S. and Hong, H.P. (2004) "Partial safety factors for designing and assessing flexible pavement performance", Canadian Journal of Civil Engineering, Vol.31, pp397-406.

Wasniak, D.L. (1999) "Subgrade Variability on the Ohio SHRP Test Road", Ohio Research Institute for Transportation and the Environment.

Widyatmoko, I. (2002) "Some practical aspects of performance related testing of bituminous materials", Proceedings of the 4th European 
Symposium on performance of bituminous and hydraulic materials in pavements, Bitmat 4, pp99-104.

Yin, H. (2013) "An analytical procedure for strain response prediction of flexible pavement", International Journal of Pavement Engineering , Vol.14(5), pp486-497.

Youdale, G.P., Vos, R. M., Tooma, G., and Richmond, C. J. (2003) "The Impact of risk on whole of life costing", Proceedings - Conference of the Australian Road Research Board, Vol.21, pp2825-2844.

Zaghloul, S., Helali, K., Ahmed, R., Ahmed, Z., and Jumikis, A.A. (2005) "Implementation of a reliability-based backcalculation analysis", 84th Transportation Research Board Annual Meeting.

Zhang, W. and Macdonald, R.A. (2000) "Modeling pavement response and estimating pavement performance", Pavement subgrade performance study in the Danish Road Testing Machine.

Zhang, Z. and Damnjanovic, I. (2006) "Applying method of moments to model reliability of pavements infrastructure", Journal of Transportation Engineering, ASCE, Vol.132, pp416-424.

Zhang, Z. and Piepmeyer, Z. (2005) "Reliability-based approach to optimising preventive maintenance for pavements", 84th Transportation Research Board Annual Meeting.

Zhao, Y.G. and Ono, T. (2000) "Some applications of moment method for structural reliability", 8th ASCE Specialty Conference on Probabilistic Mechanics and Structural Reliability.

Zhao, Y.G. and Ono, T. (2001) "Moment methods for structural reliability", Struct. Safety, Vol.23, pp47-75.

Zollinger, D.G. and McCullough, B.F. (1994) "Development of Weibull Reliability factors and analysis for calibration of pavement Design Models Using Filed Data", Transportation Research Record, Vol.1449, pp18-25. 
12 Appendices 


\section{A1 Mean, standard deviation and coefficient of variation for all sites}

\section{A1.1 Asphalt mean, standard deviation and coefficient of variation}

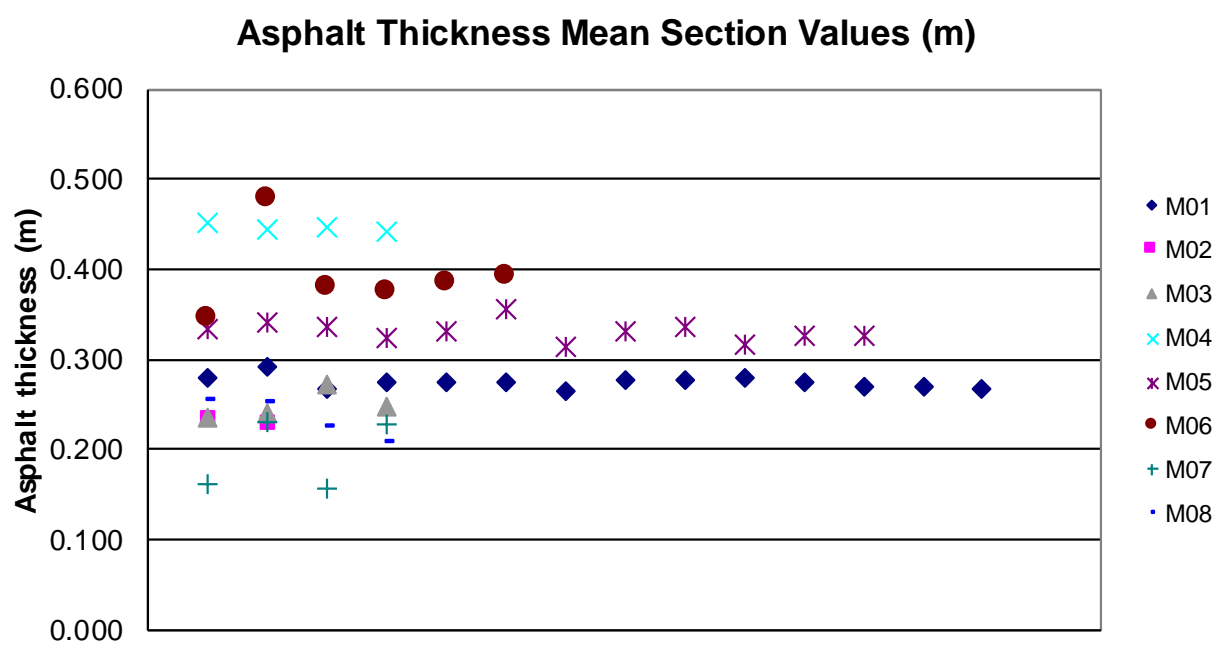

Asphalt Thickness Standard Deviation Section Values (m)

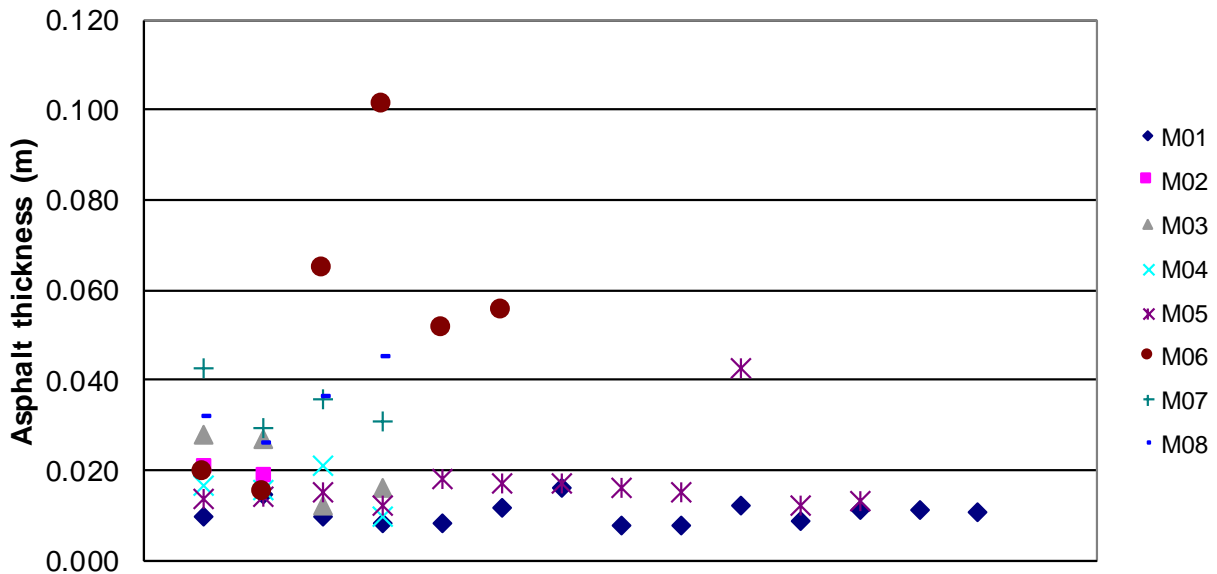

Asphalt Thickness Coefficient of Variation Section Values

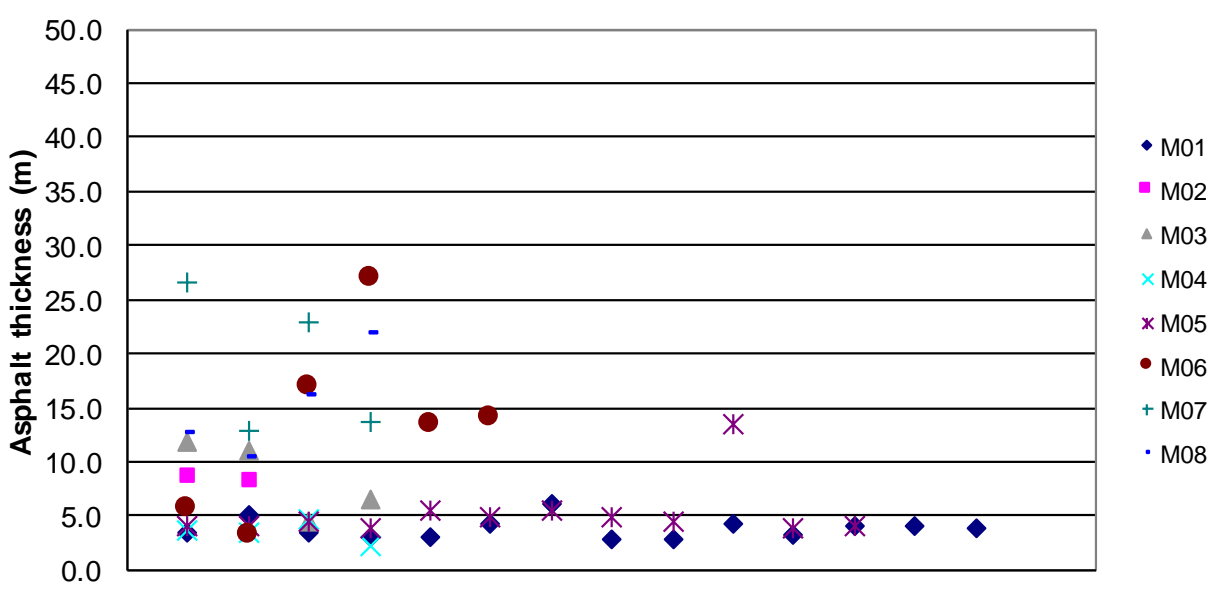




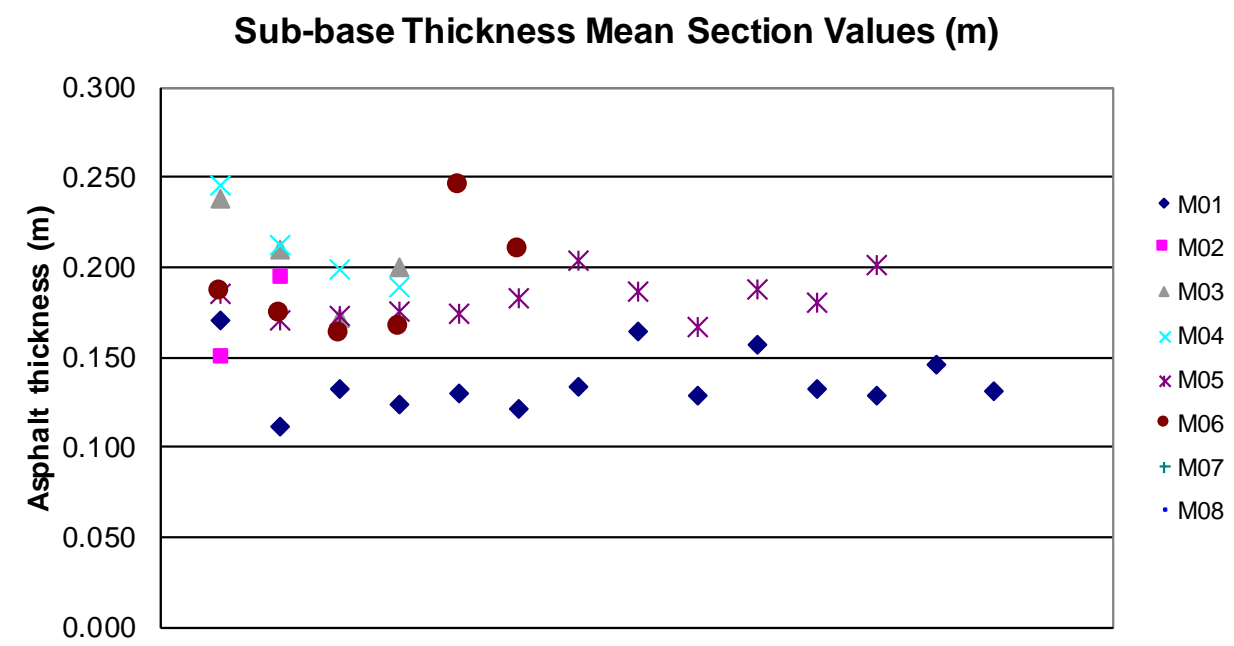

Sub-base Thickness Standard Deviation Section Values

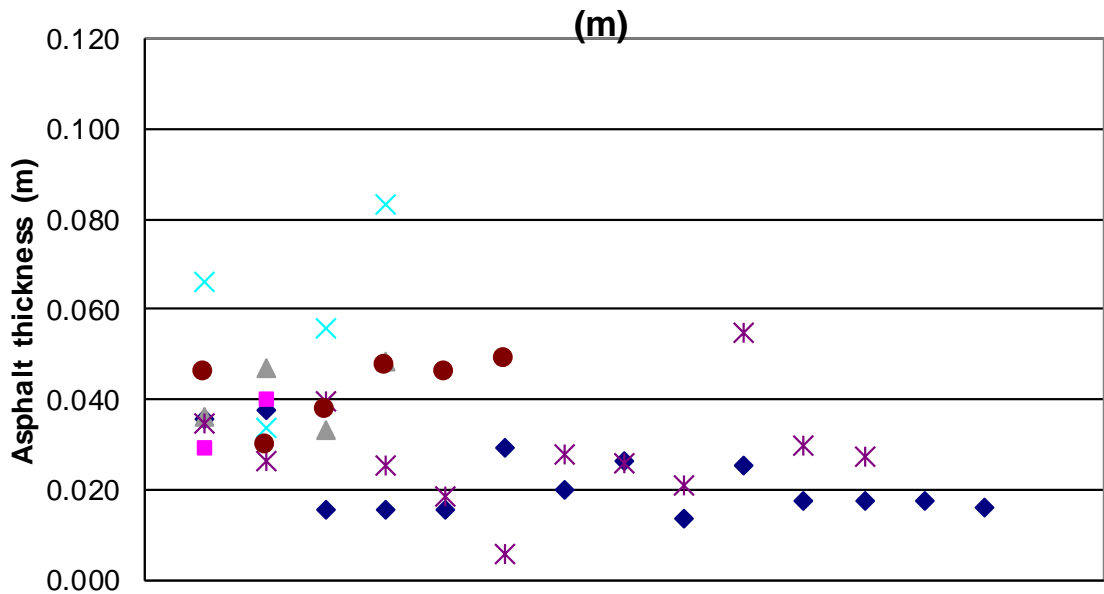

Sub-base Thickness Coefficient of Variation Section Values

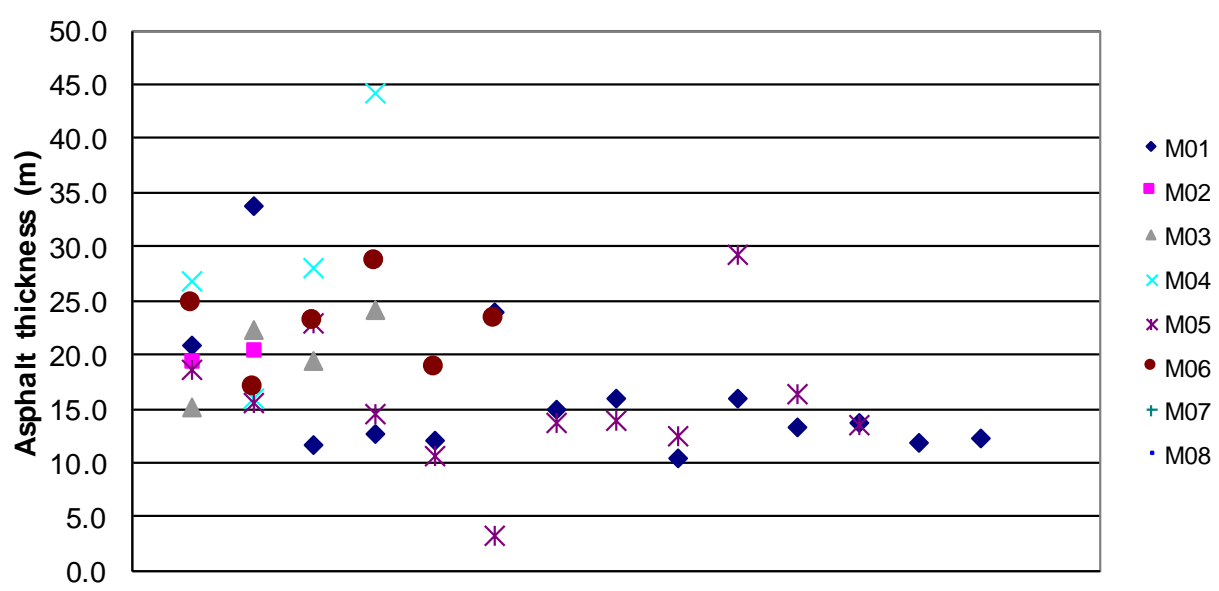


A1.3 Ratio of standard deviation over mean for asphalt and sub-base layer thickness

Standard deviation $(\sigma)$ versus mean $(m)$ asphalt thickness

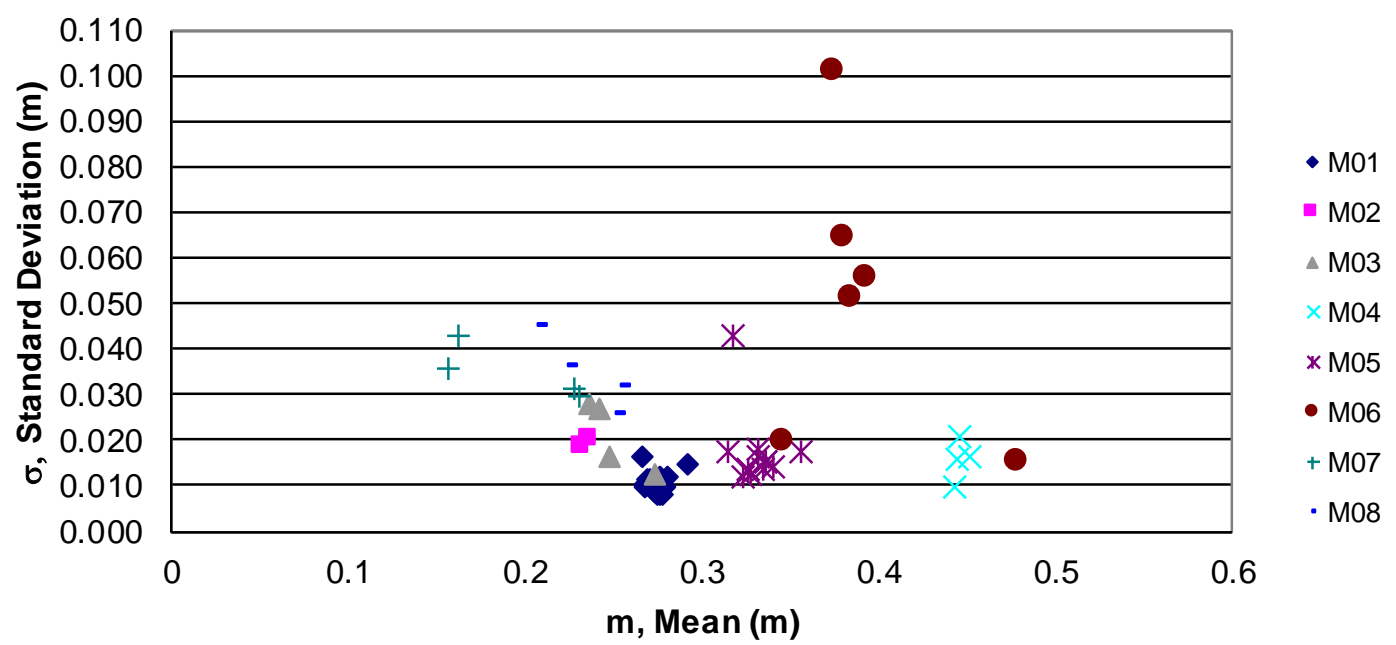

Standard deviation $(\sigma)$ versus mean $(m)$ sub-base thickness

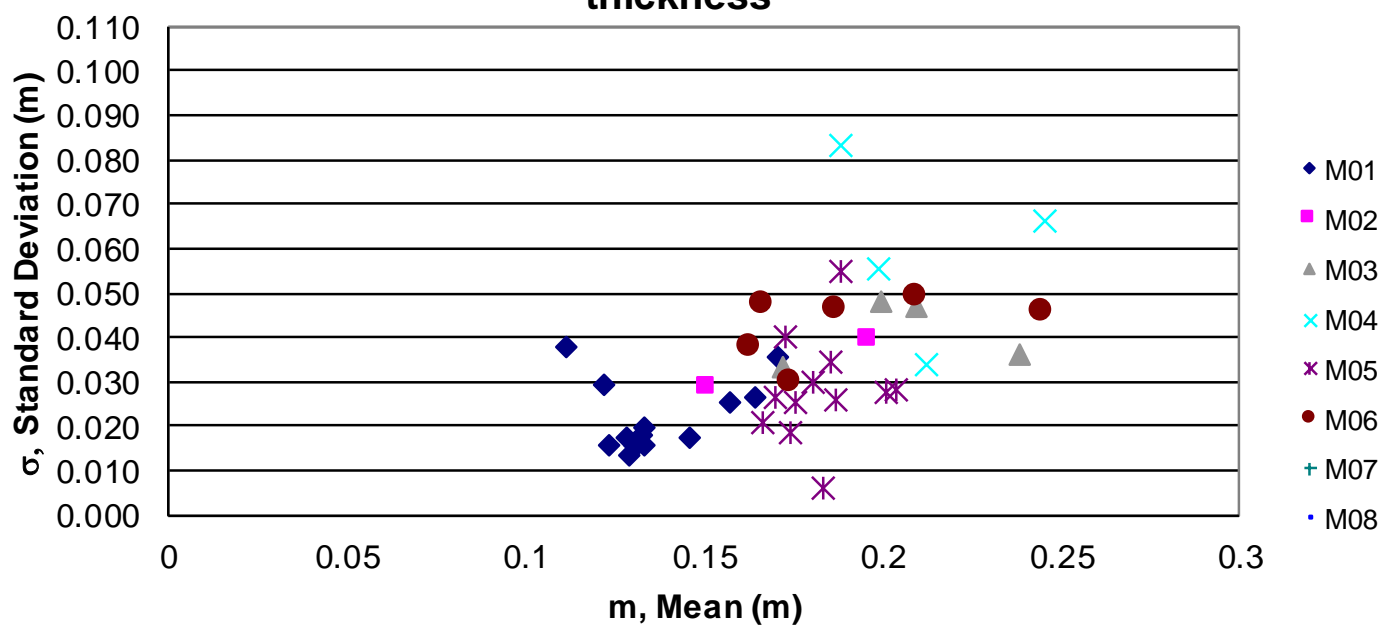




\section{A2 Layer thickness distribution from GPR surveys}

A2.1 M01
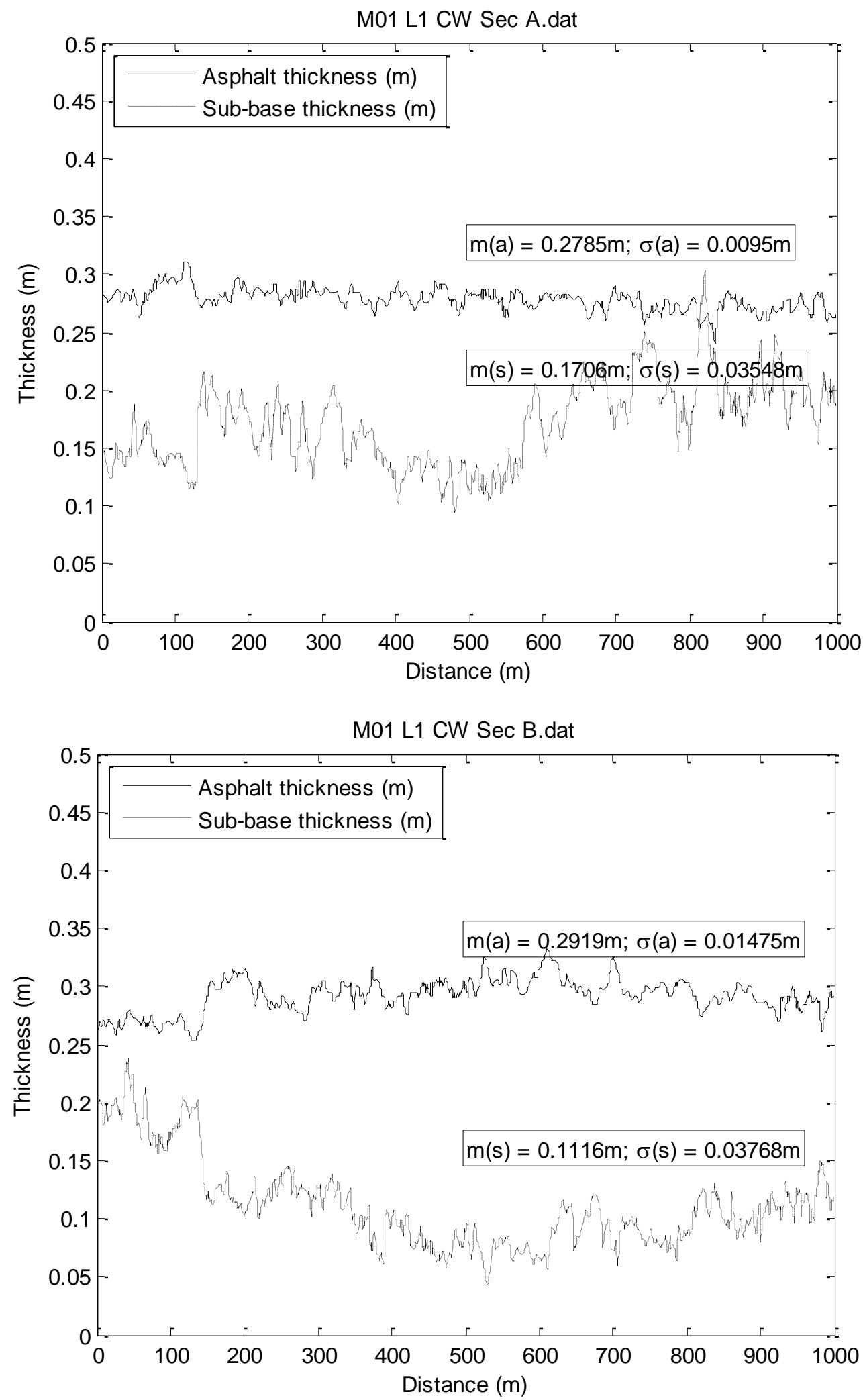

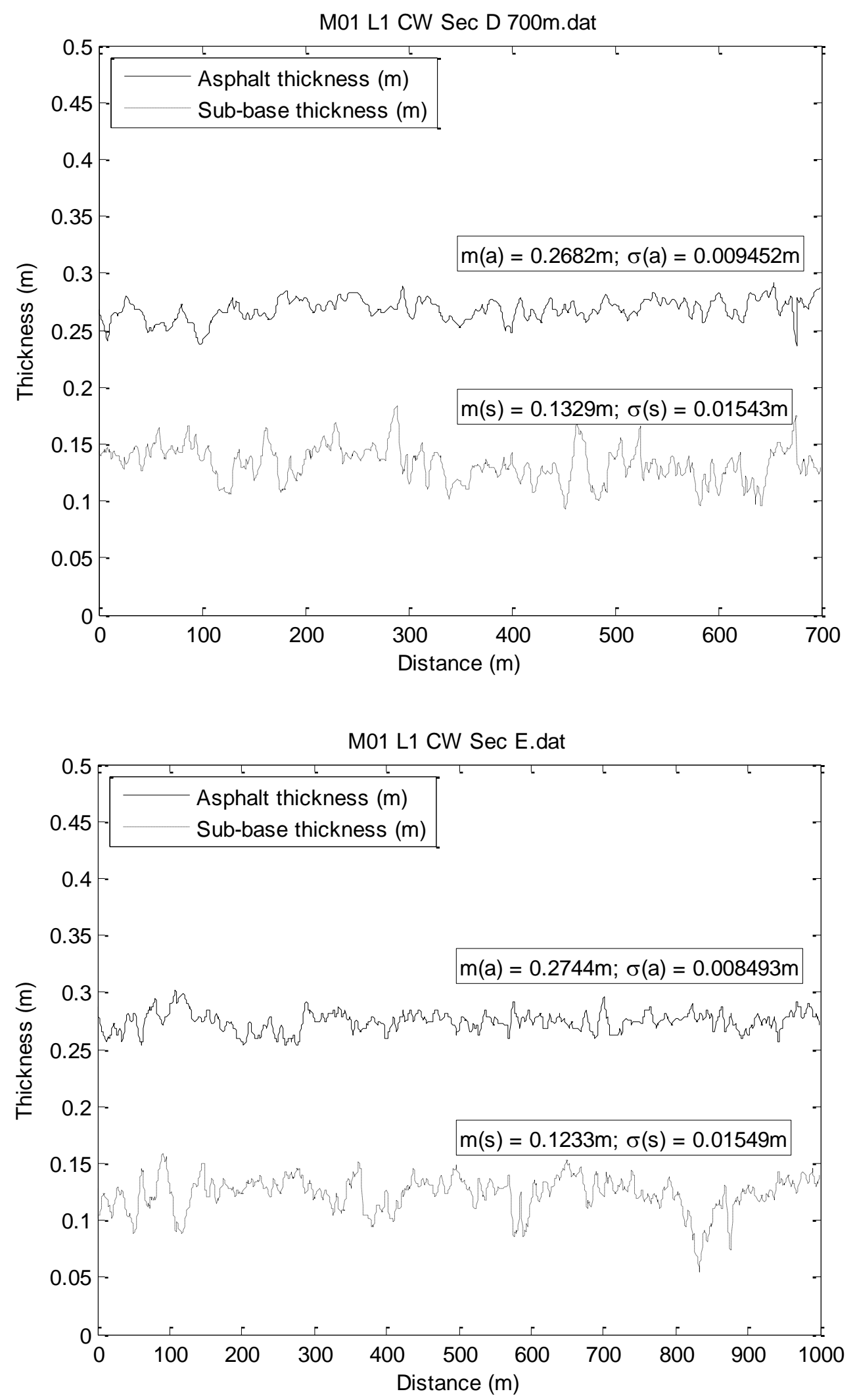

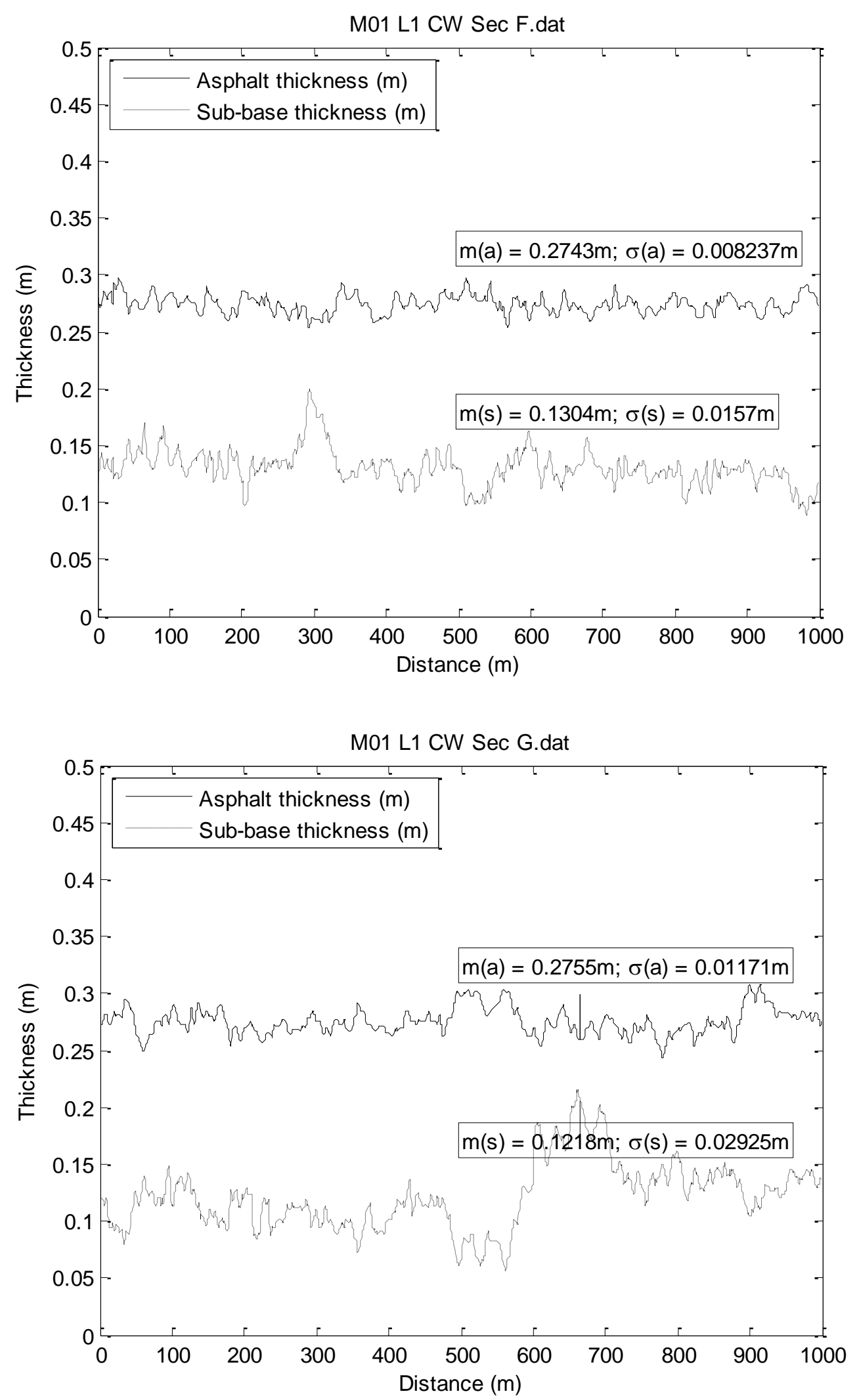
M01 L1 CW Sec H.dat
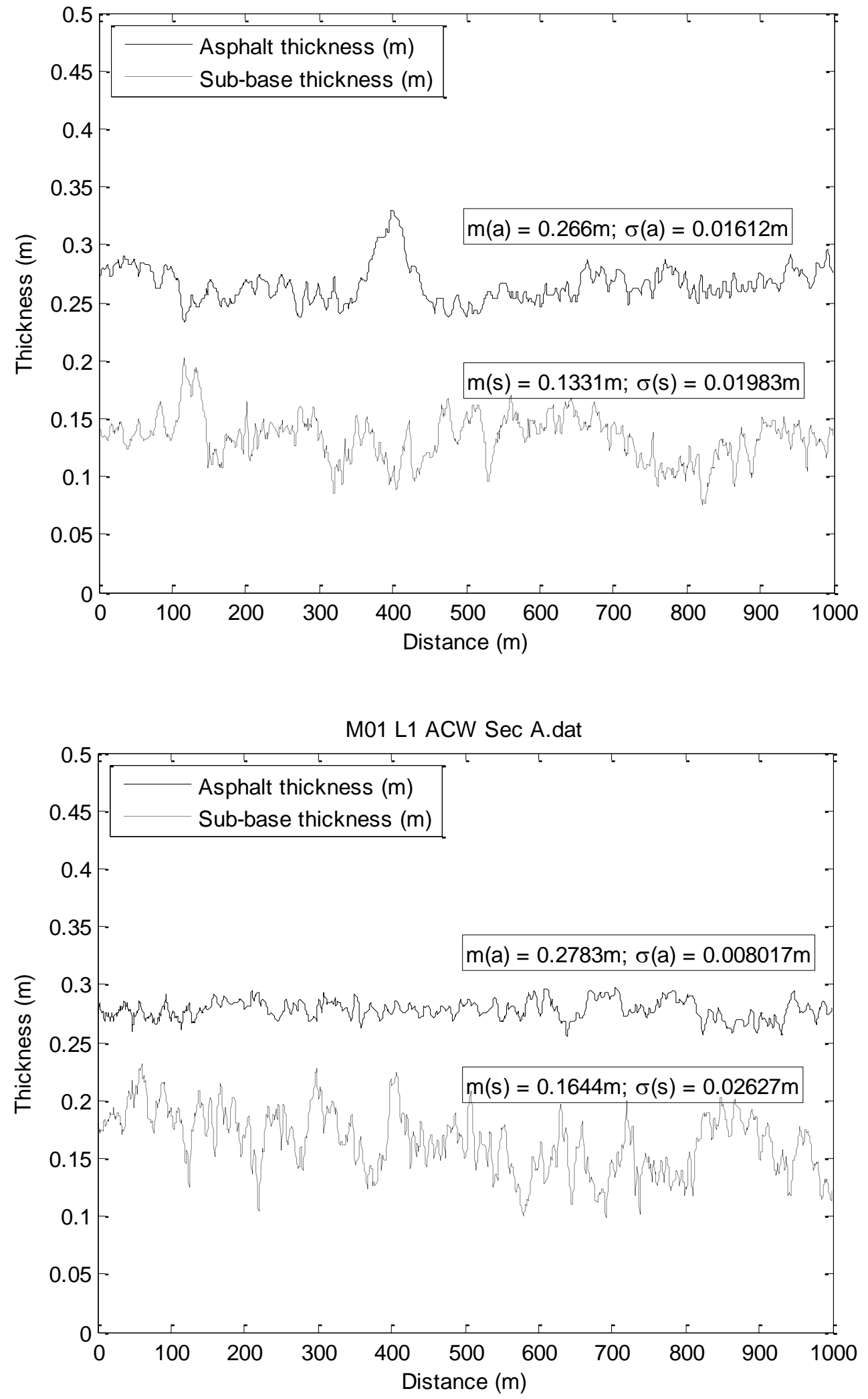

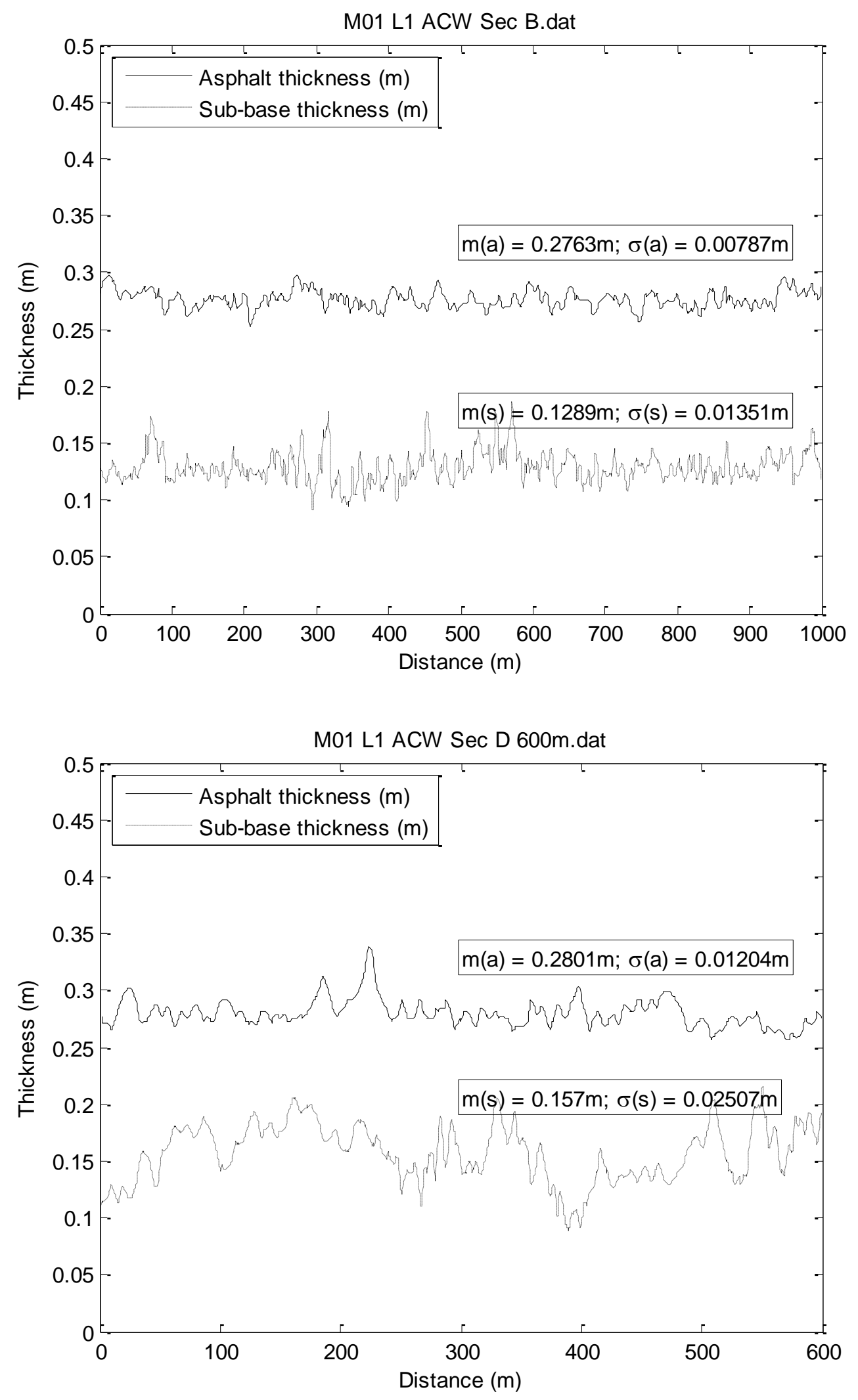

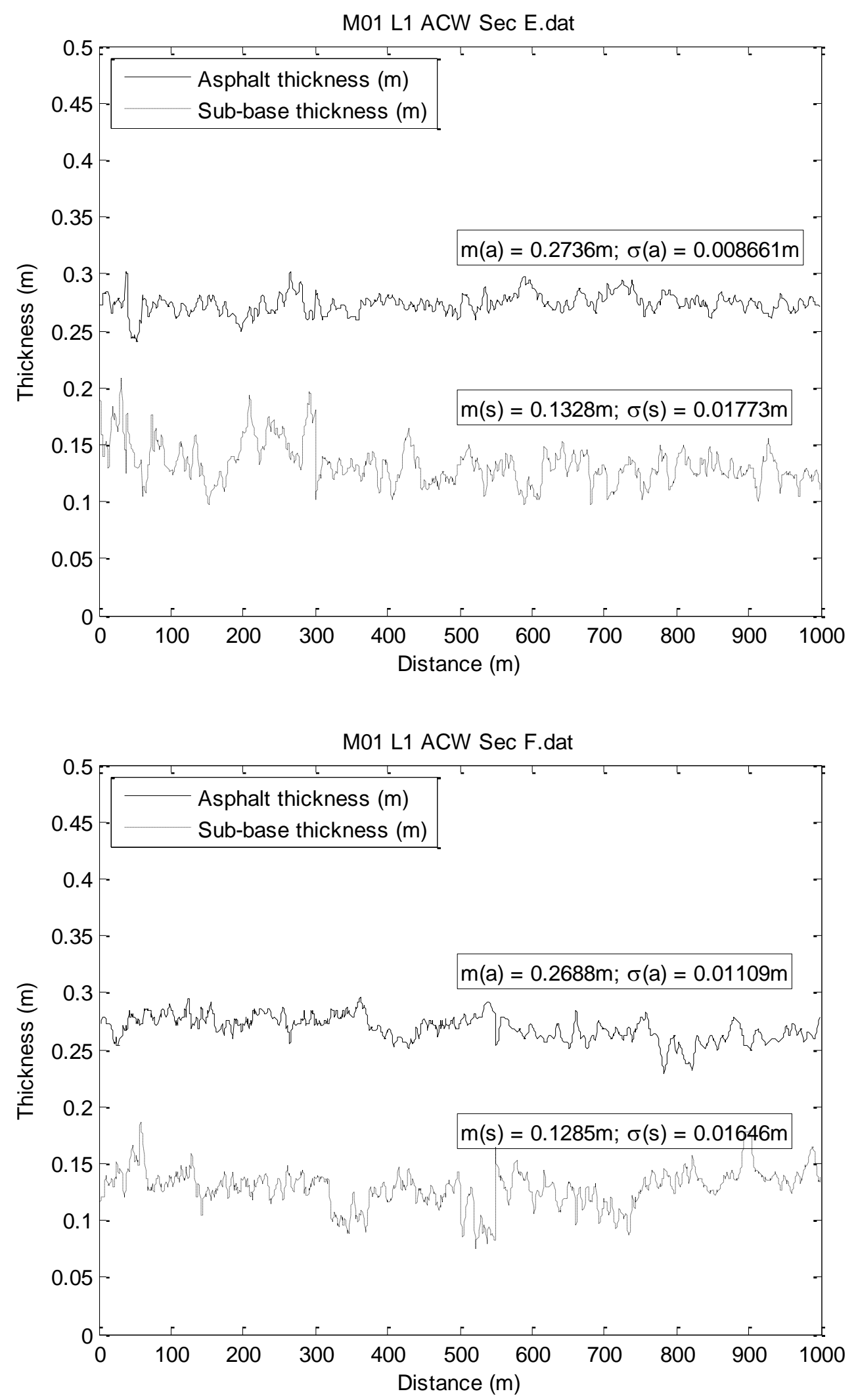

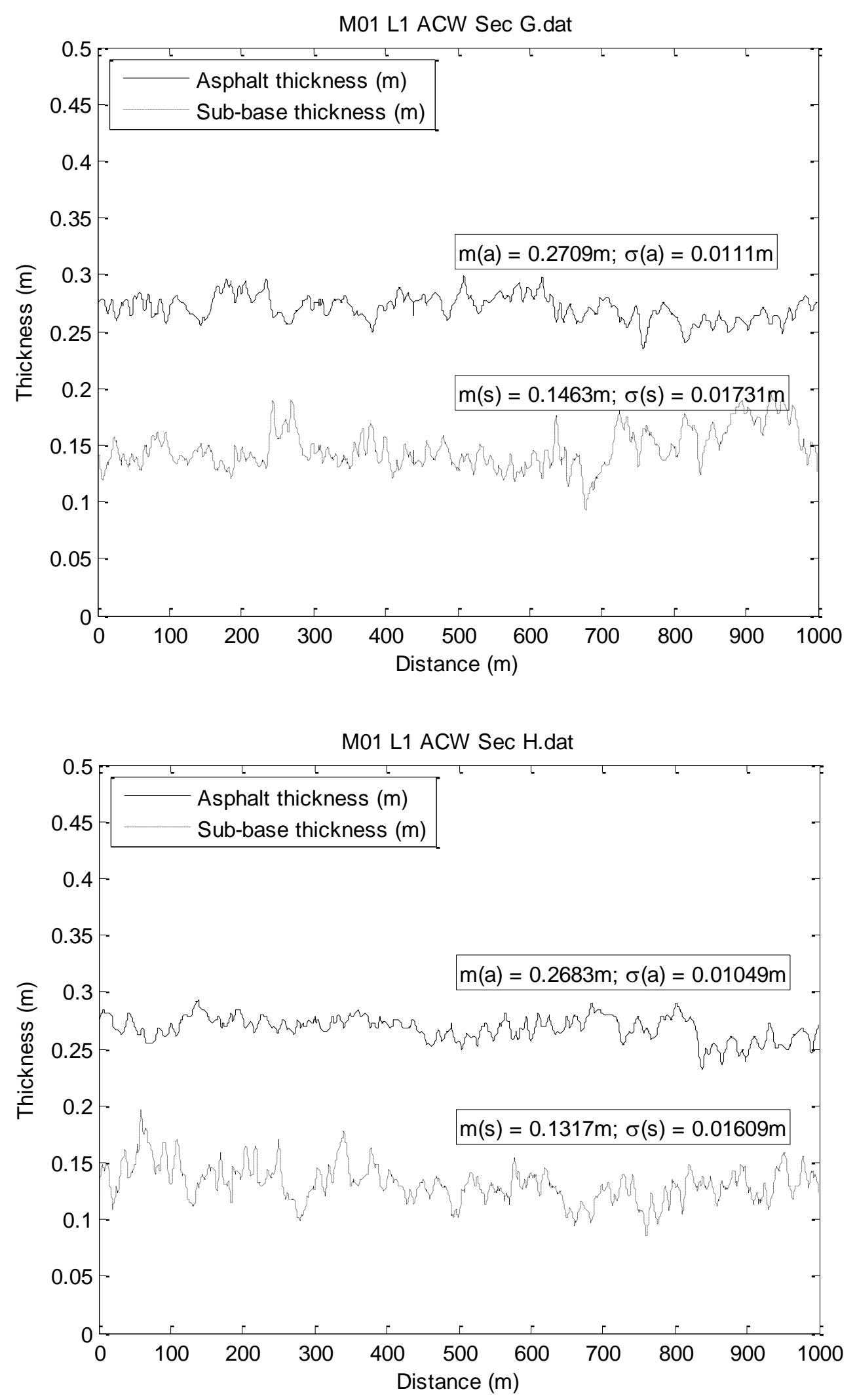

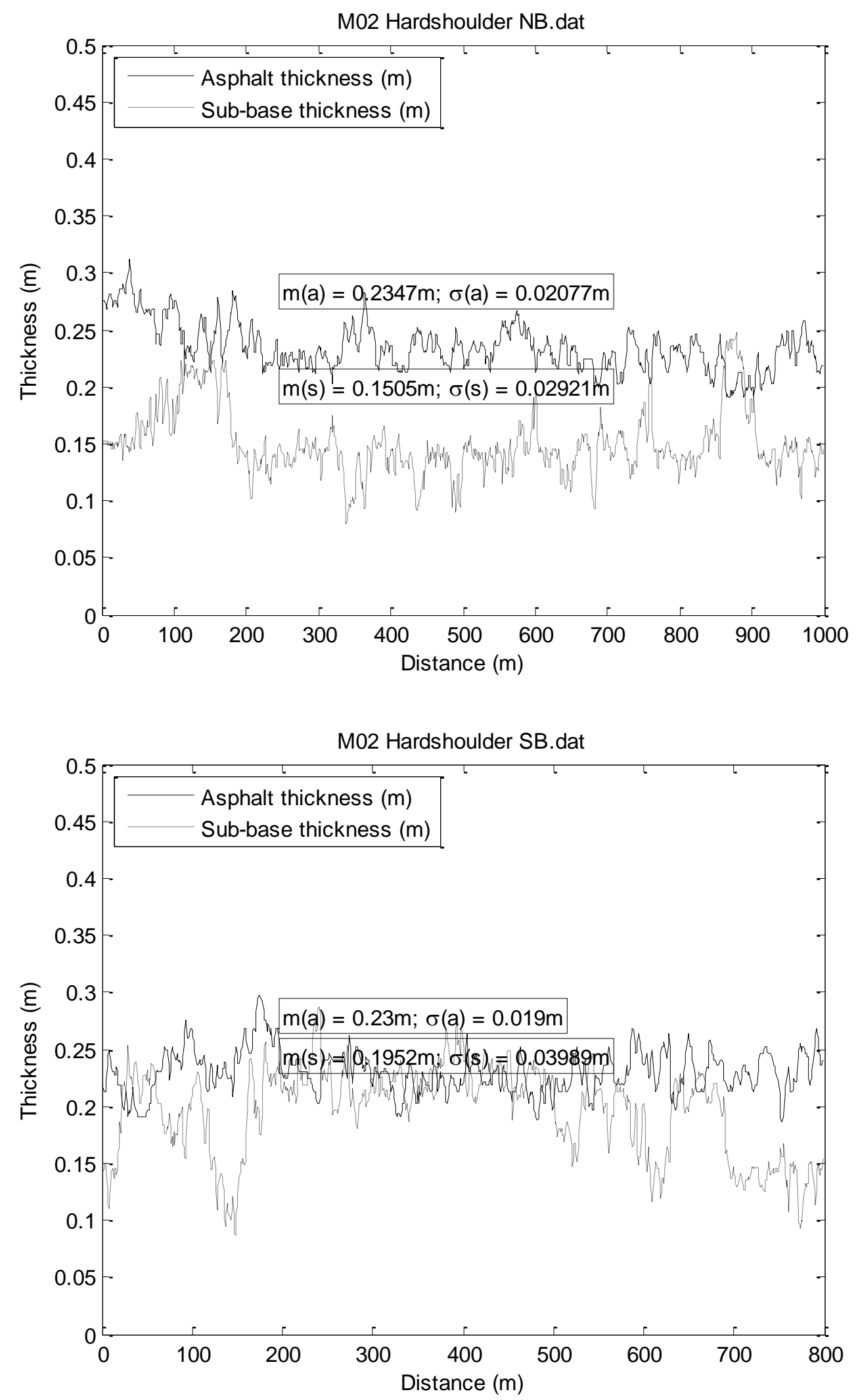

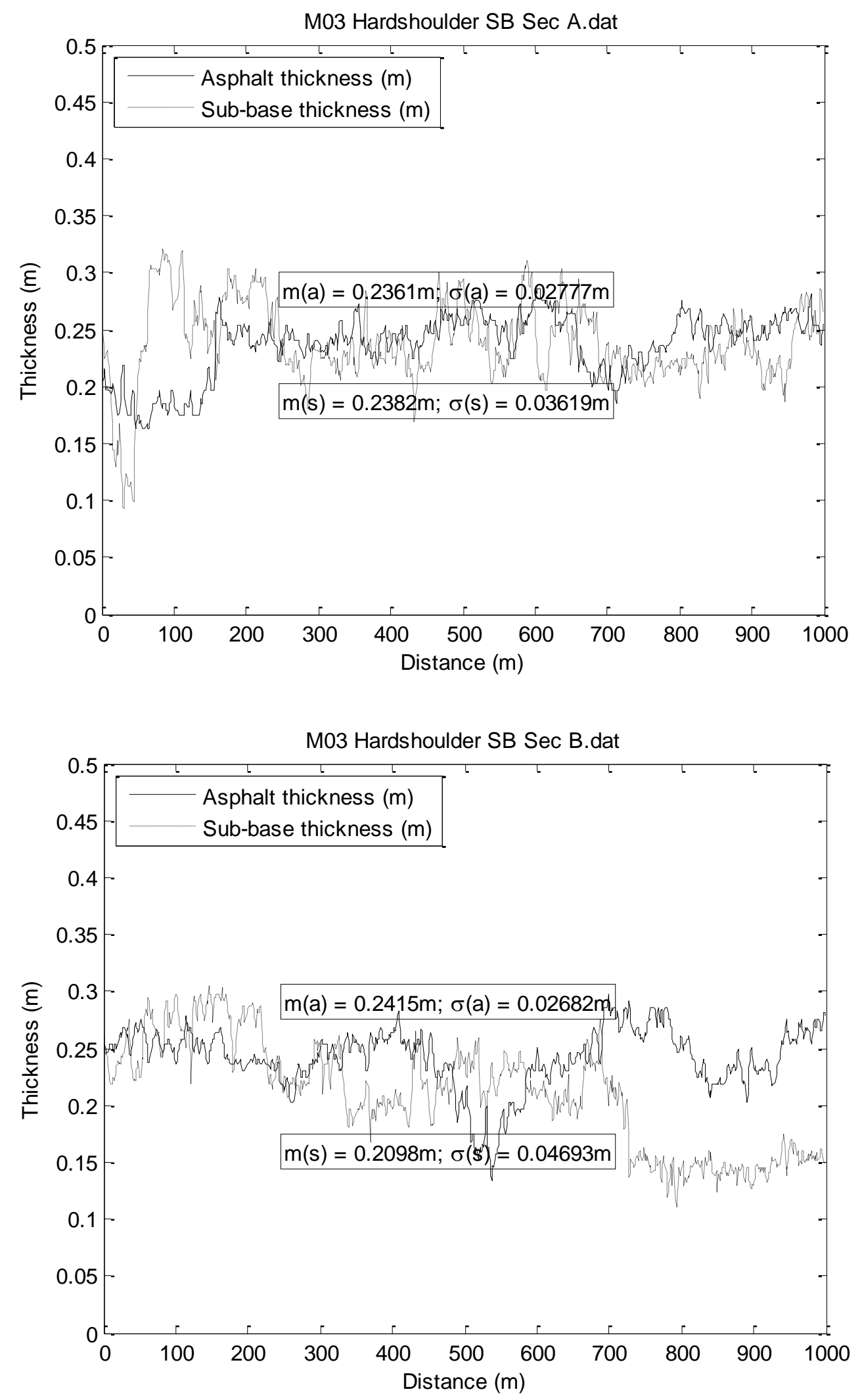

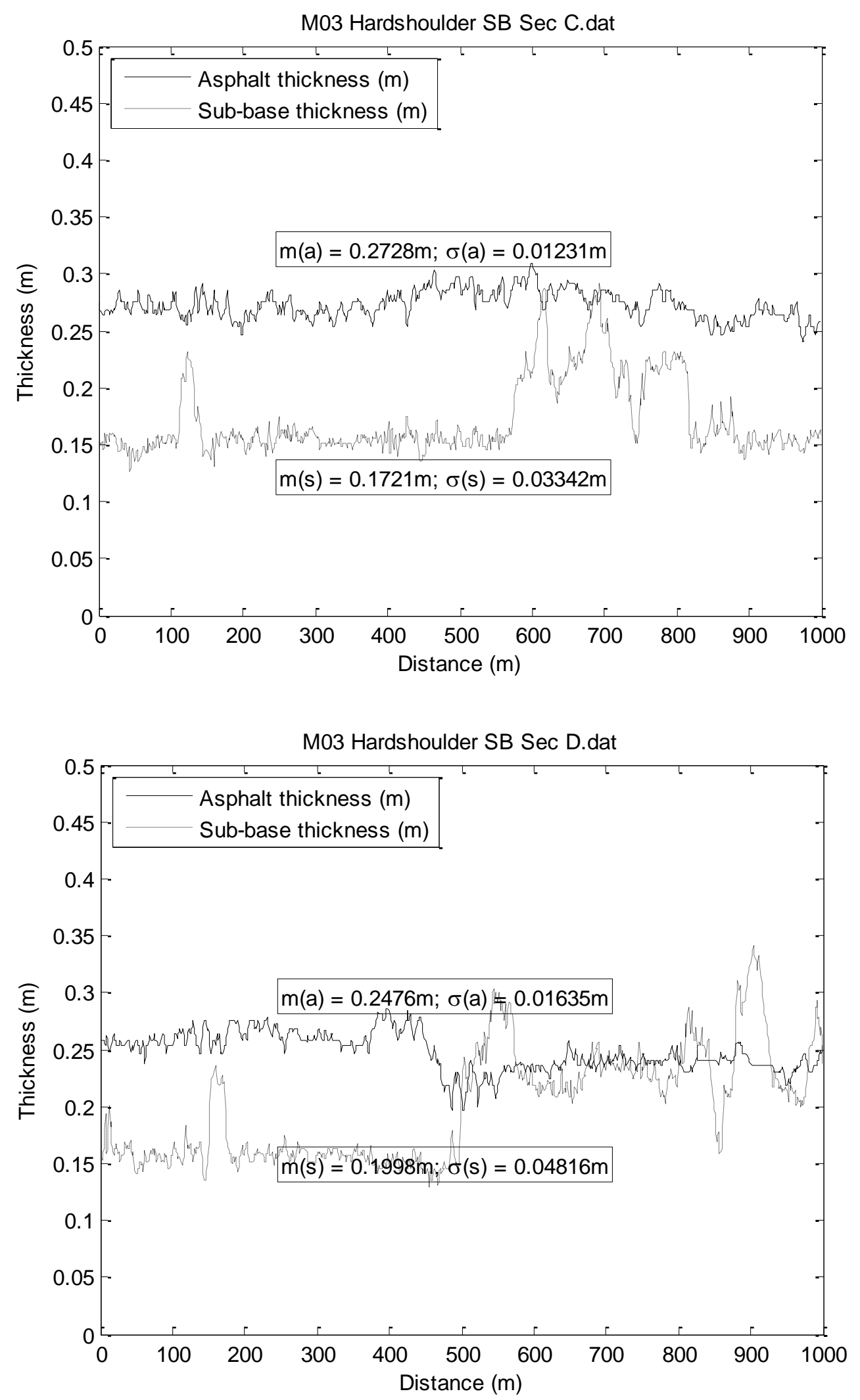

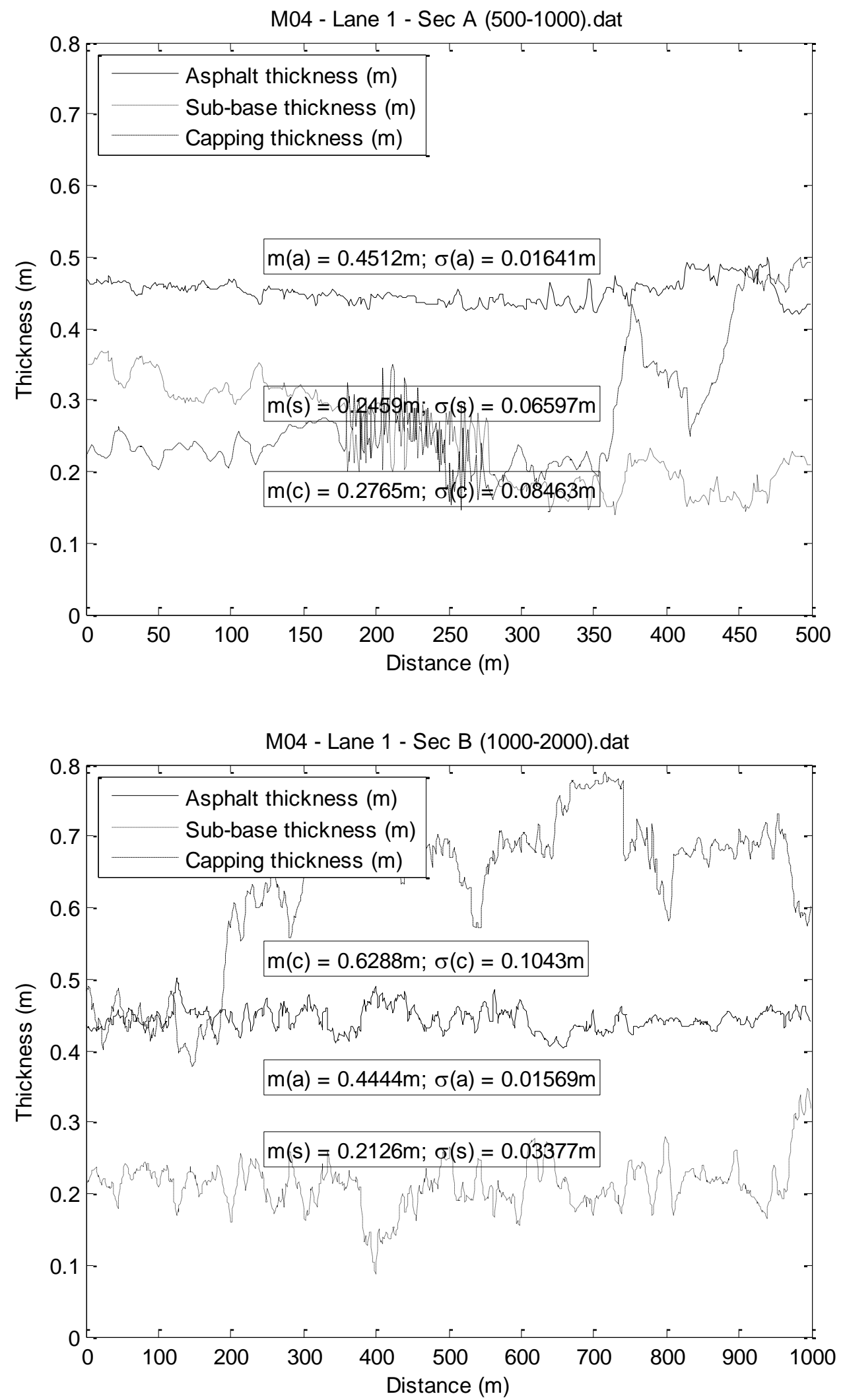

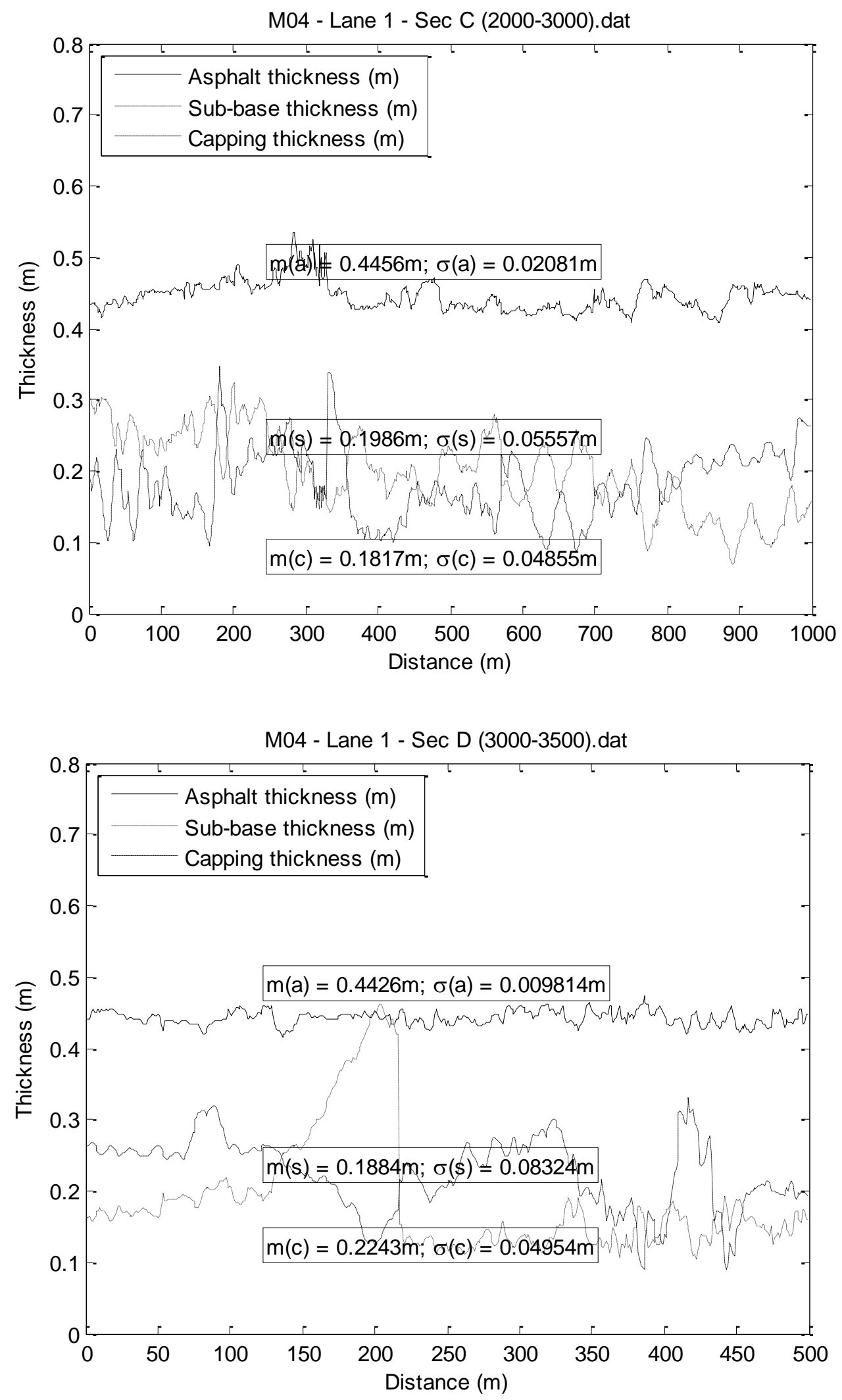

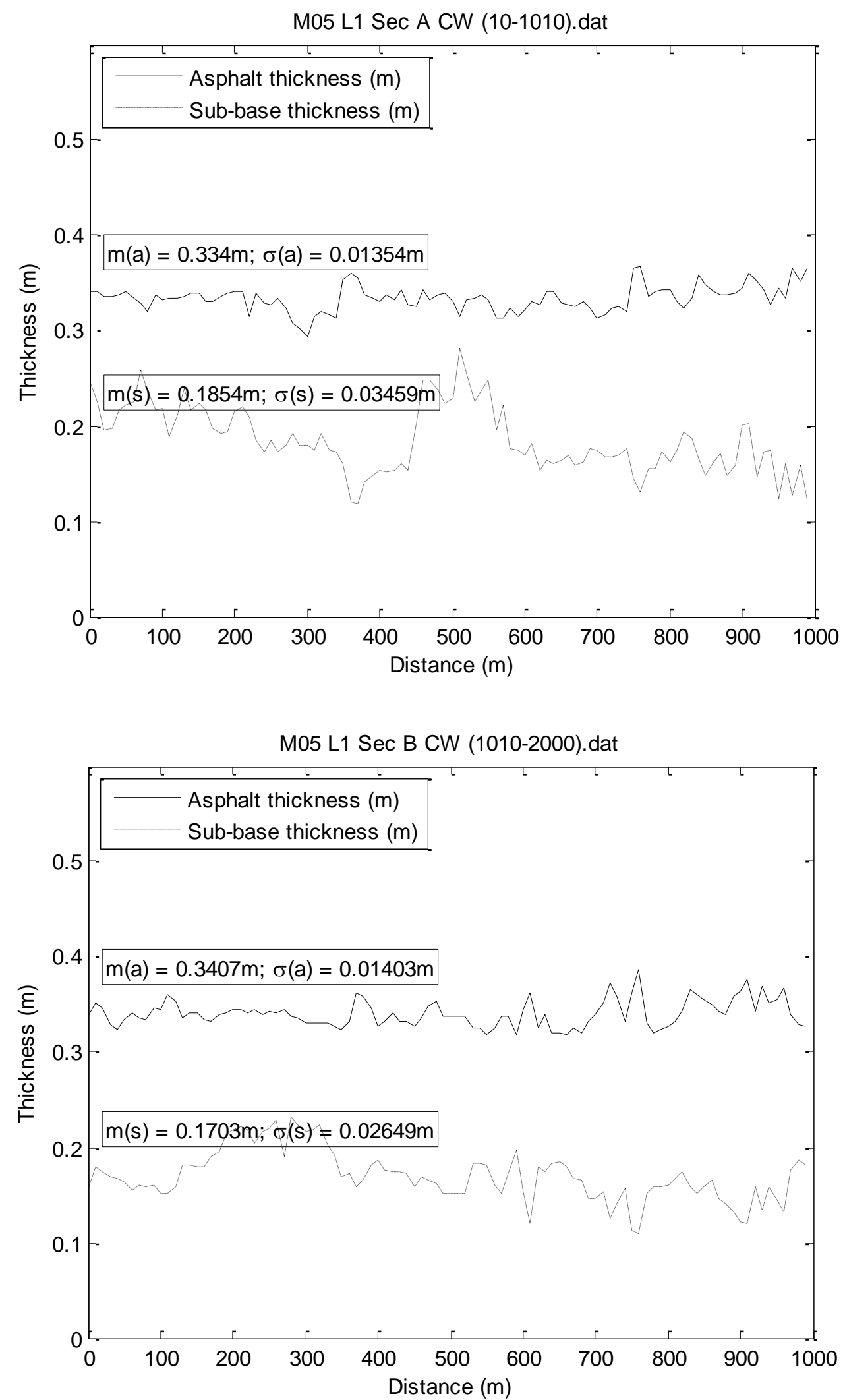

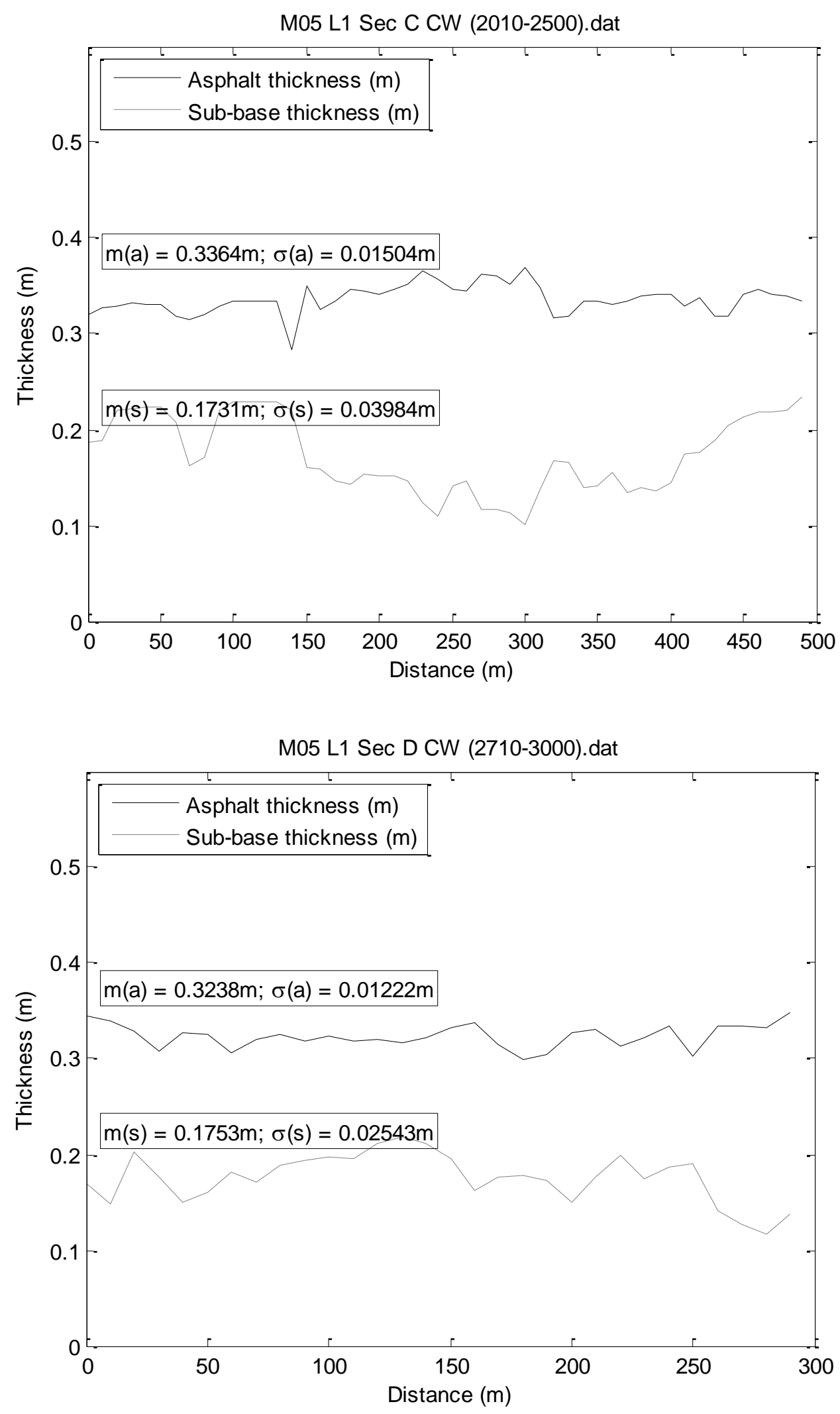

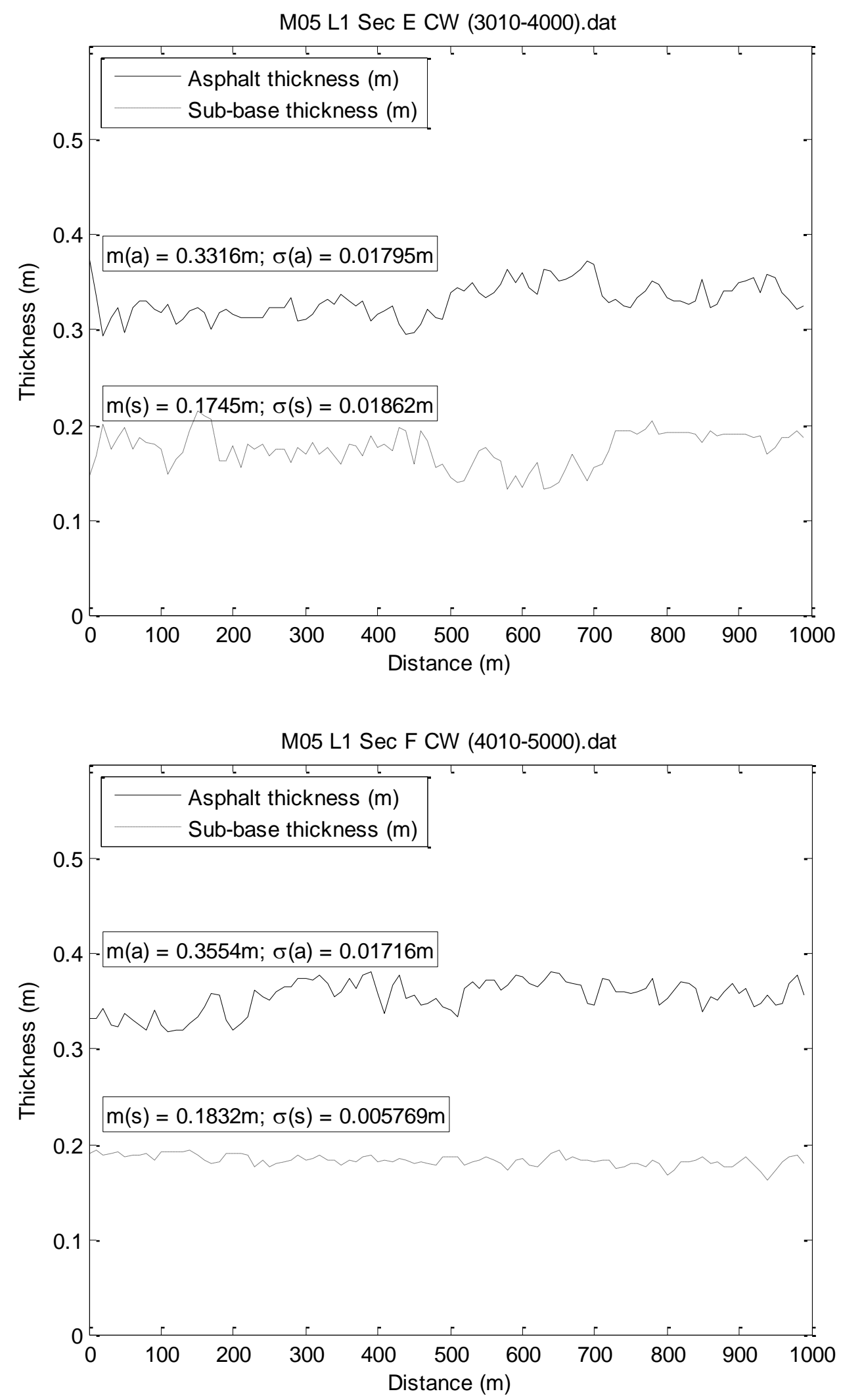

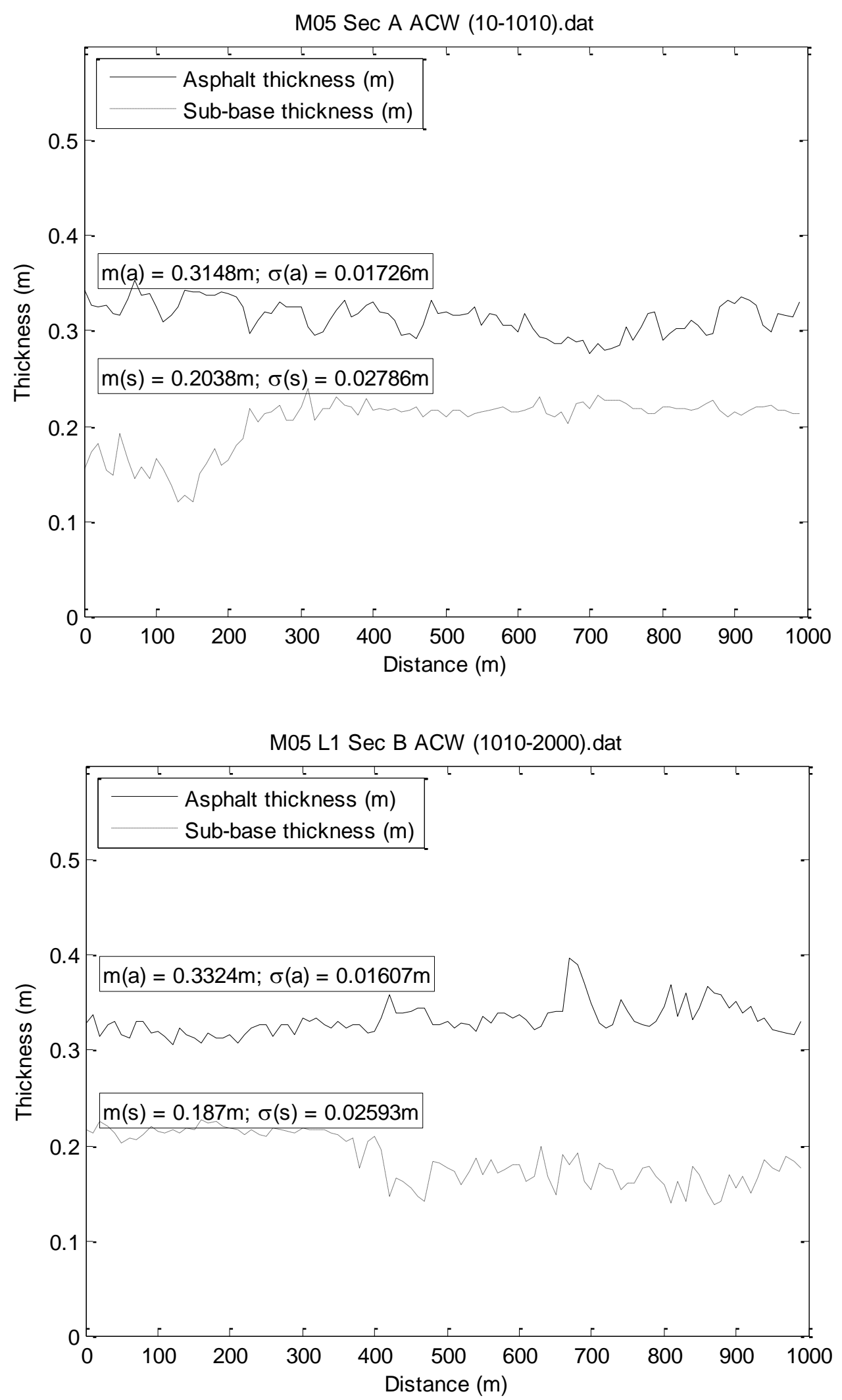

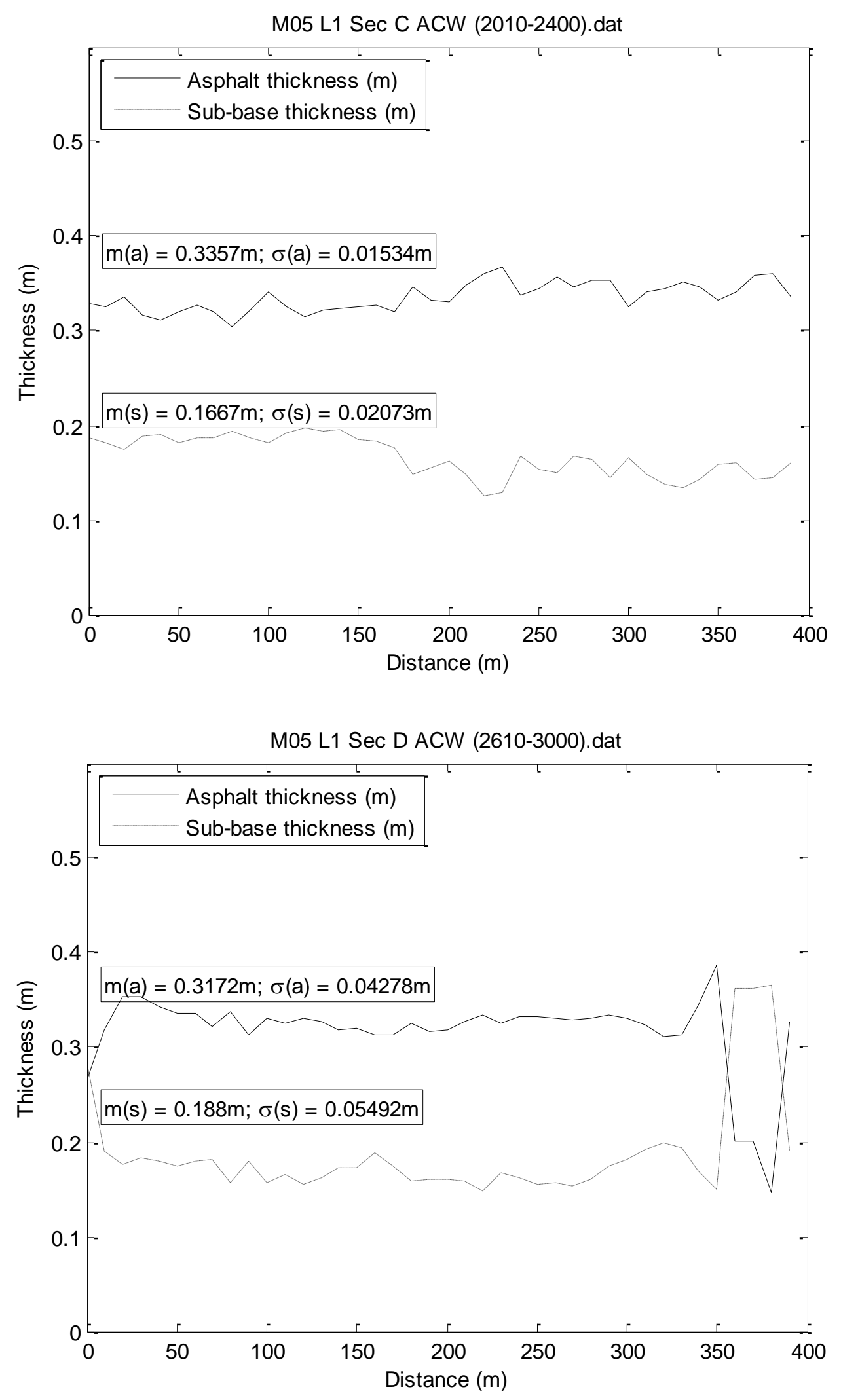

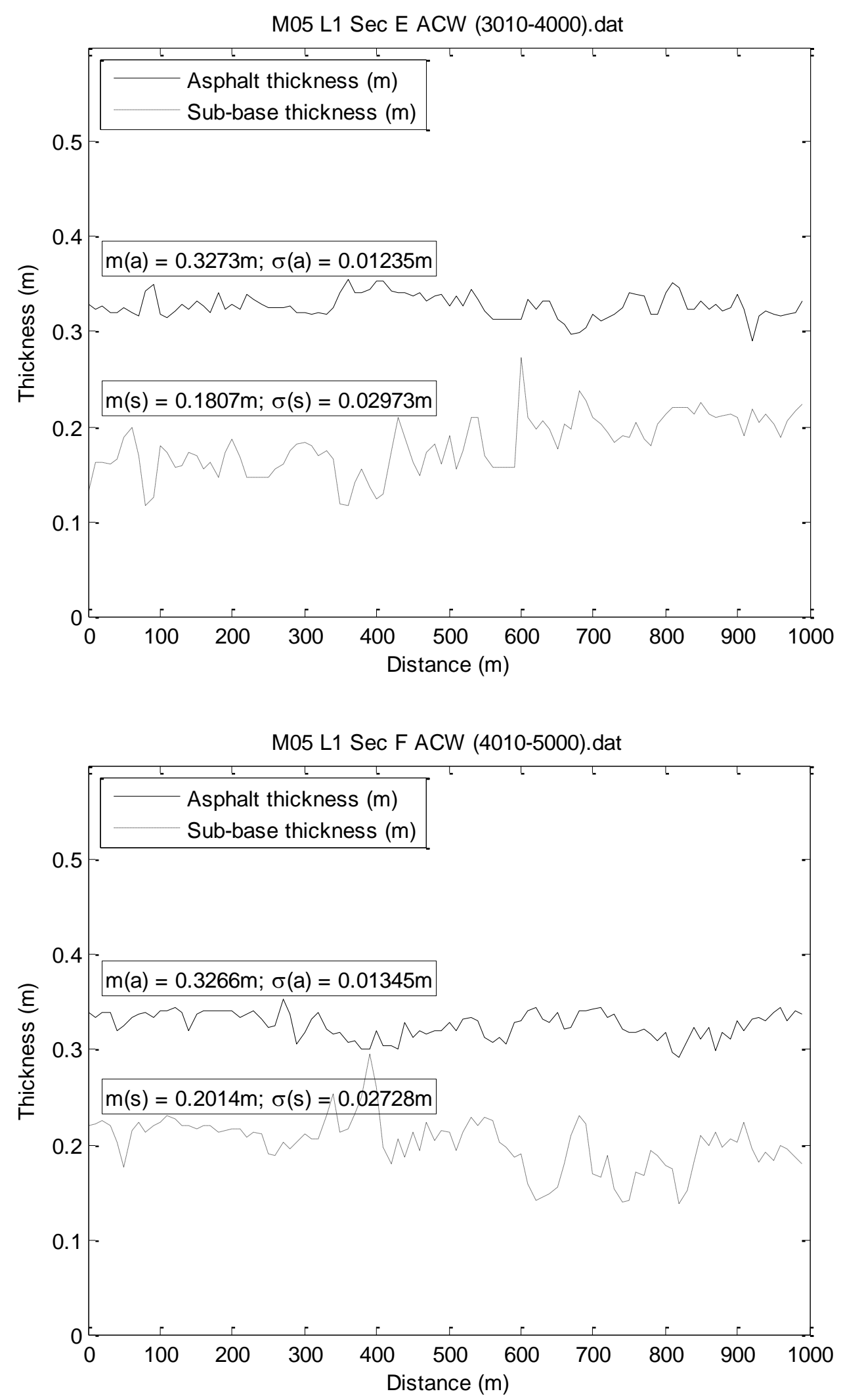

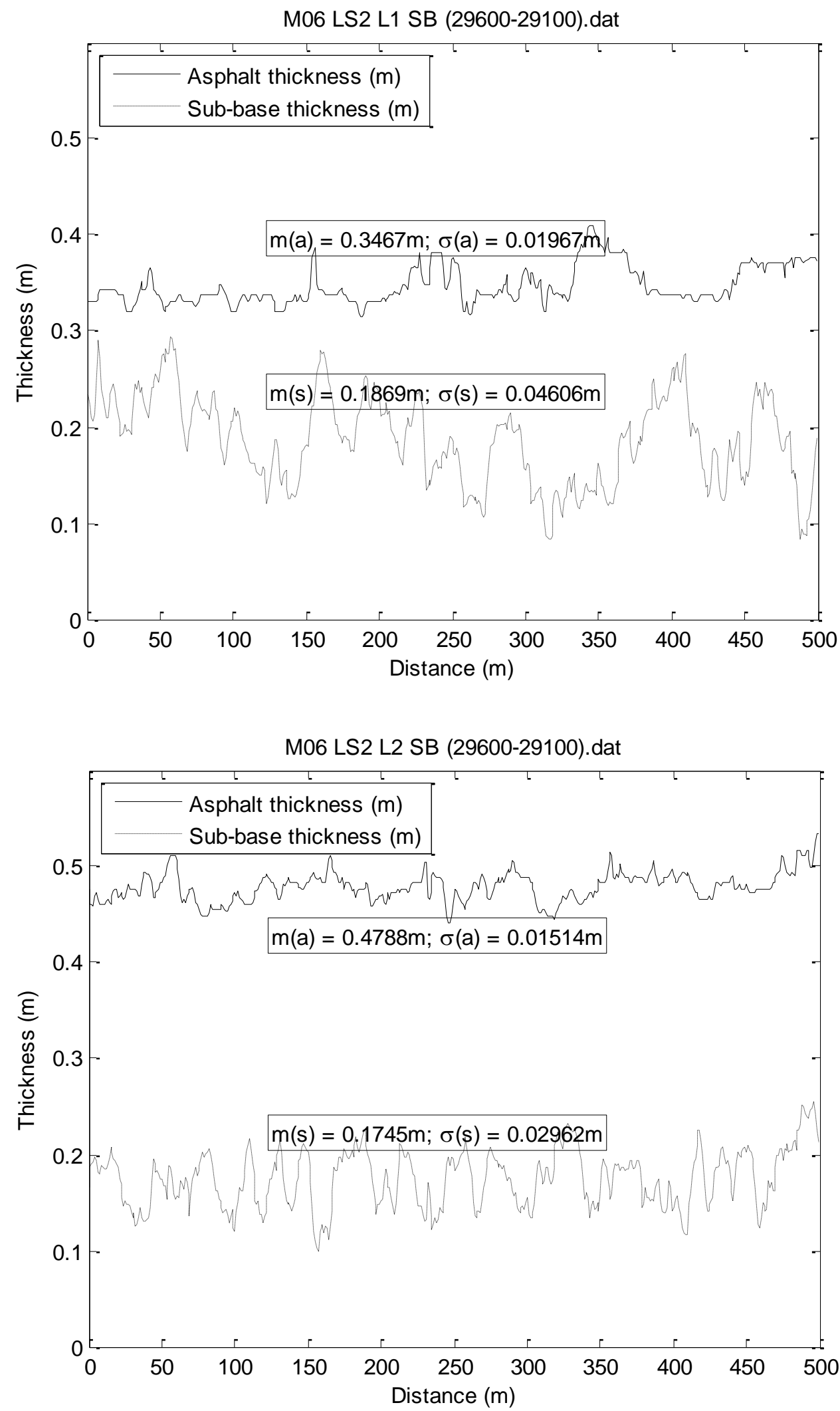

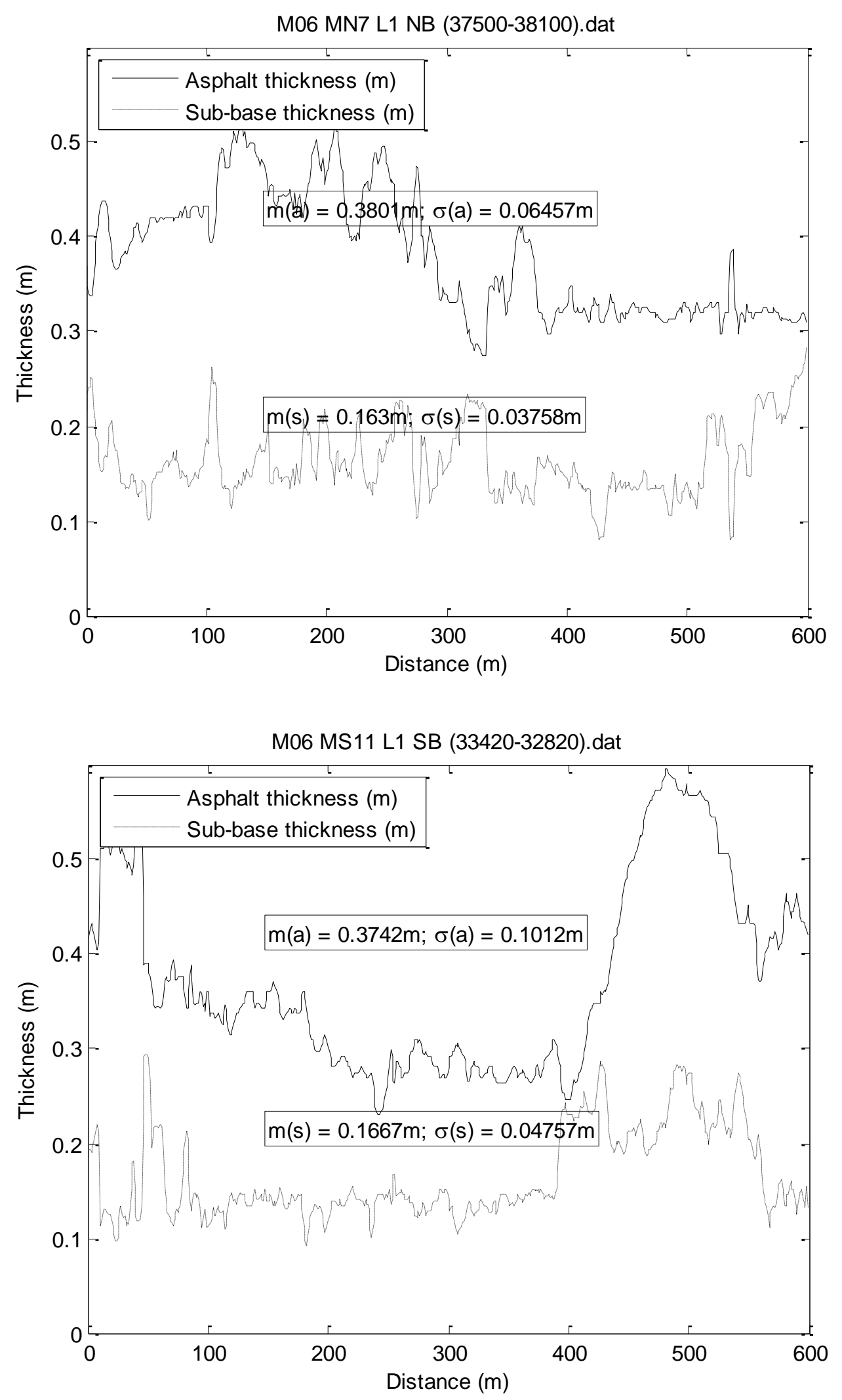

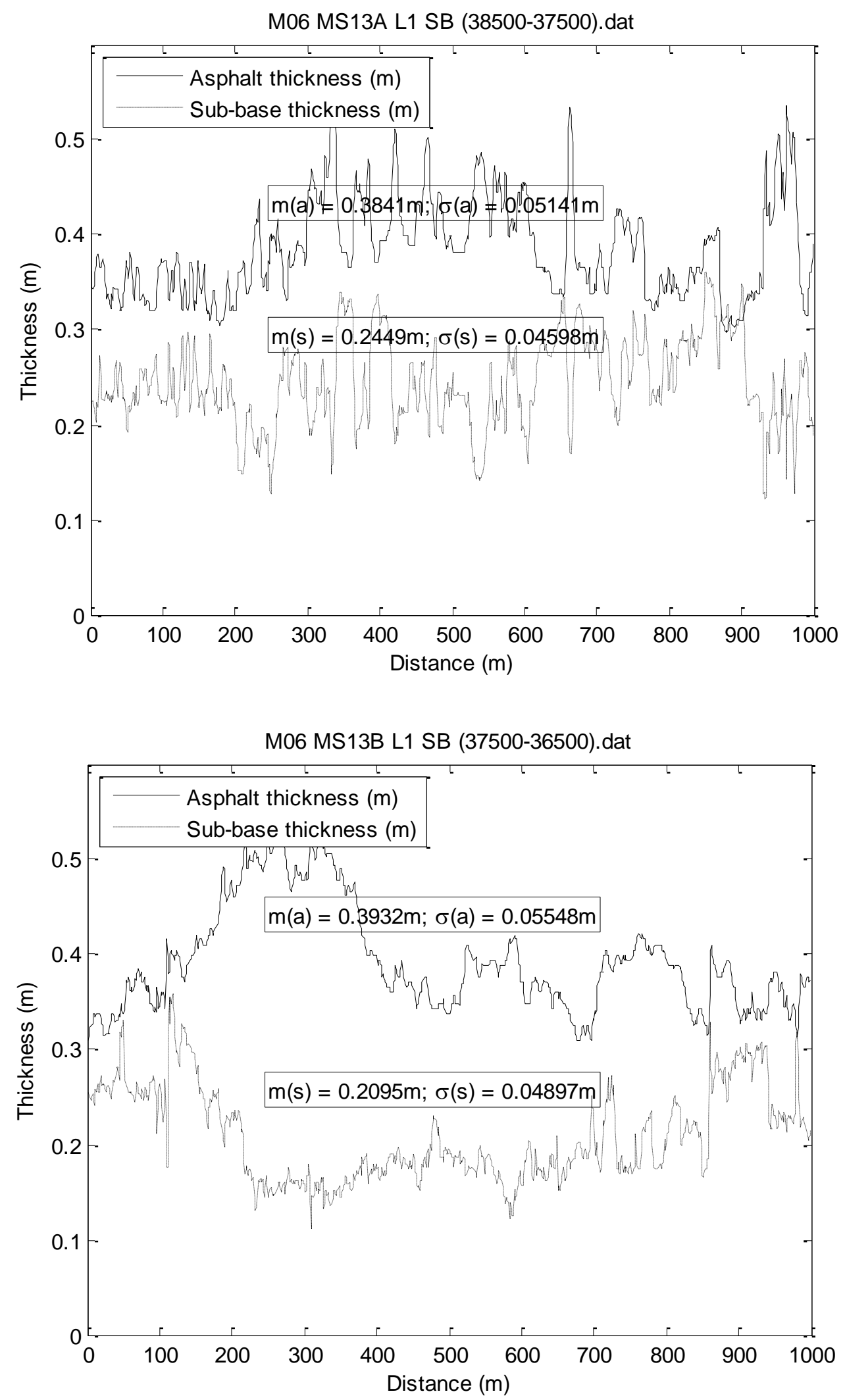

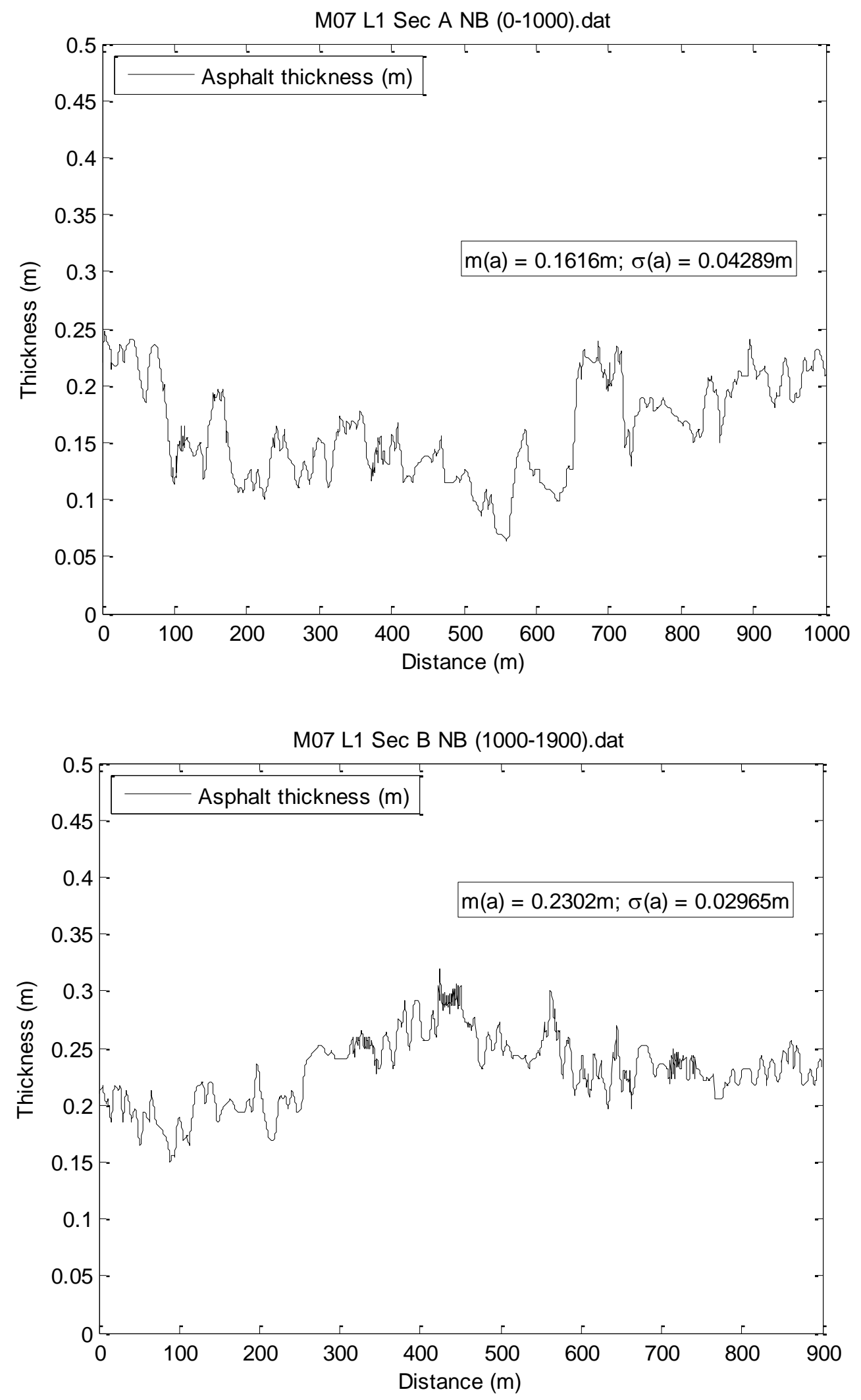

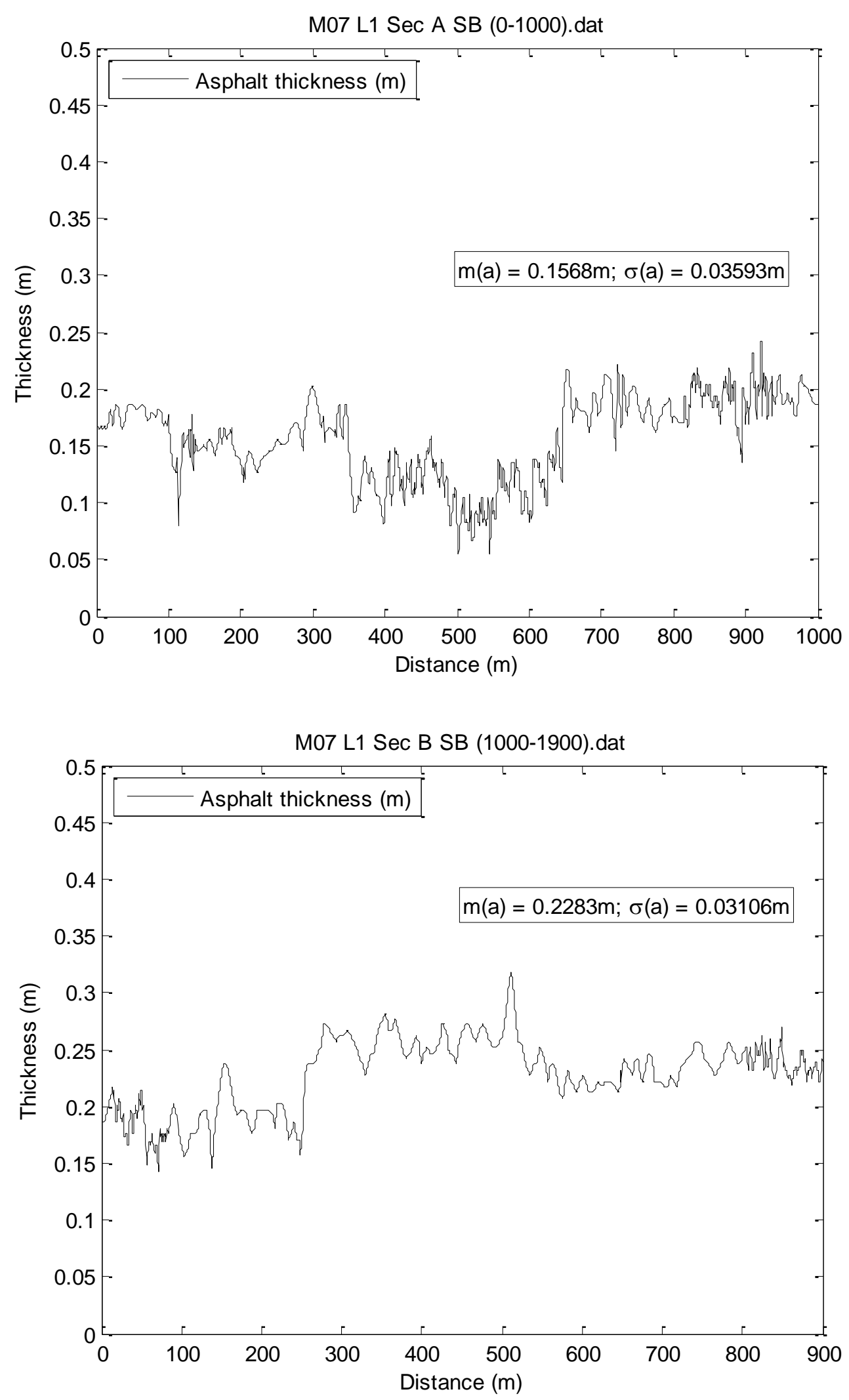

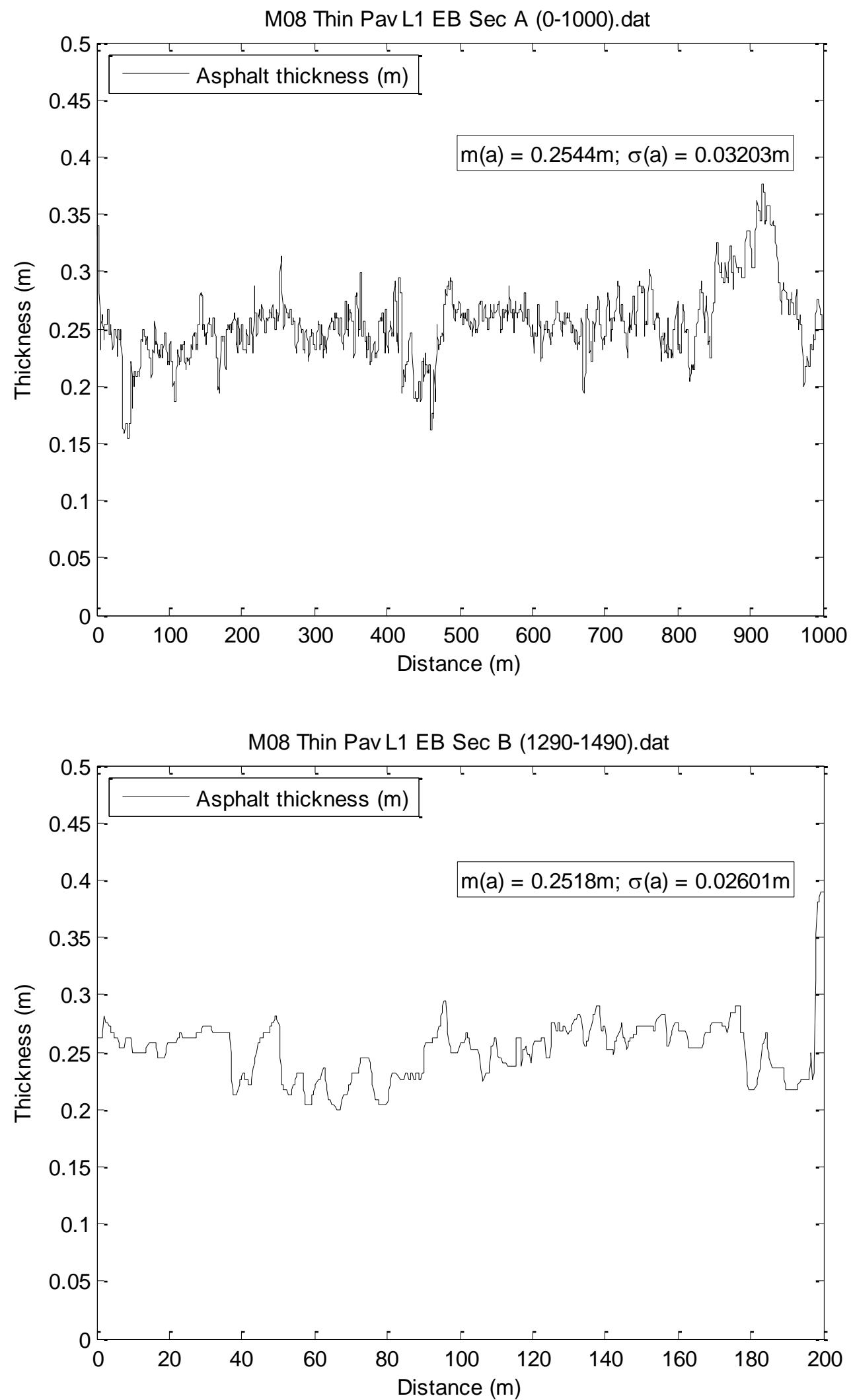

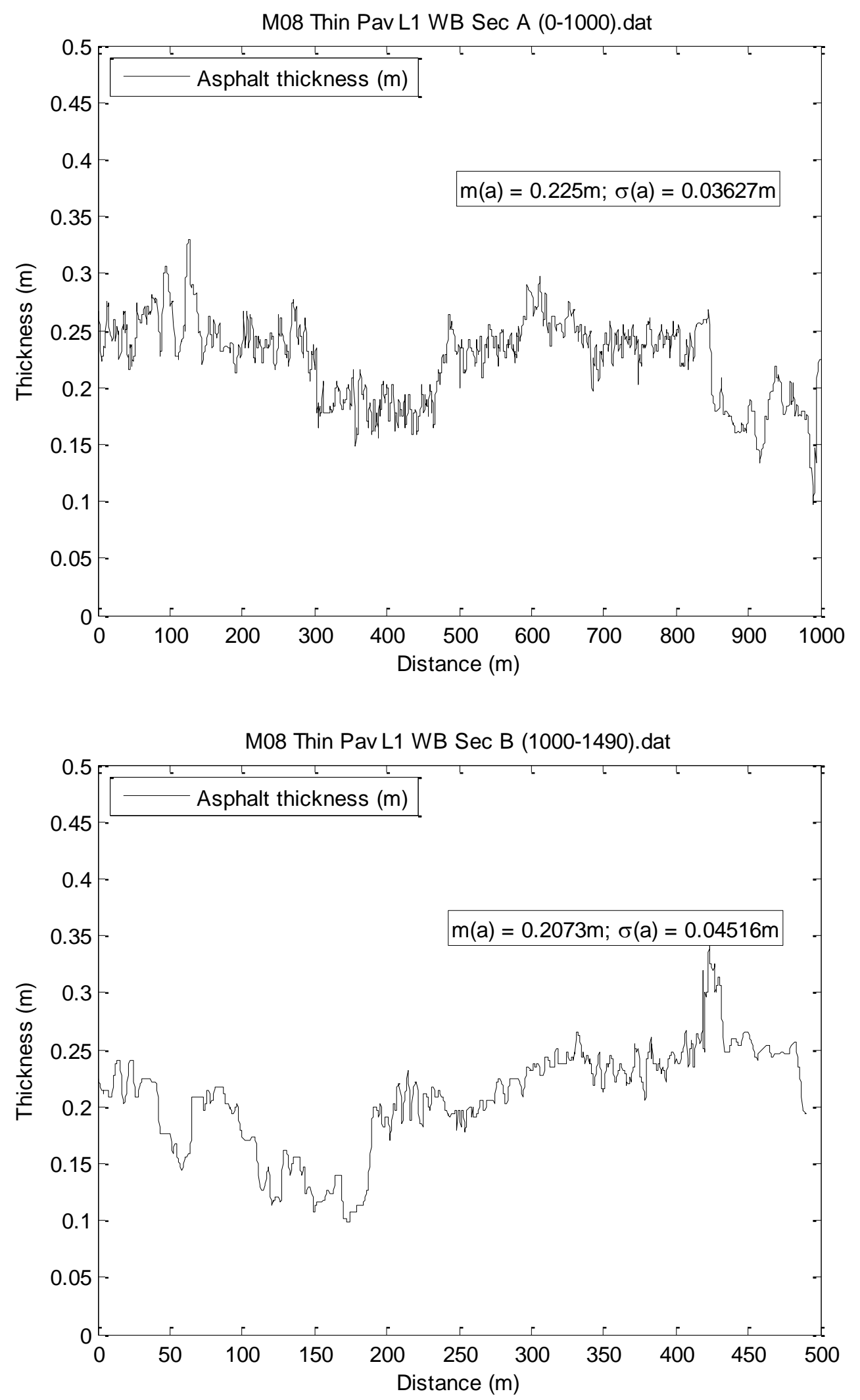


\section{A3 Construction tolerance}

A3.1 M01
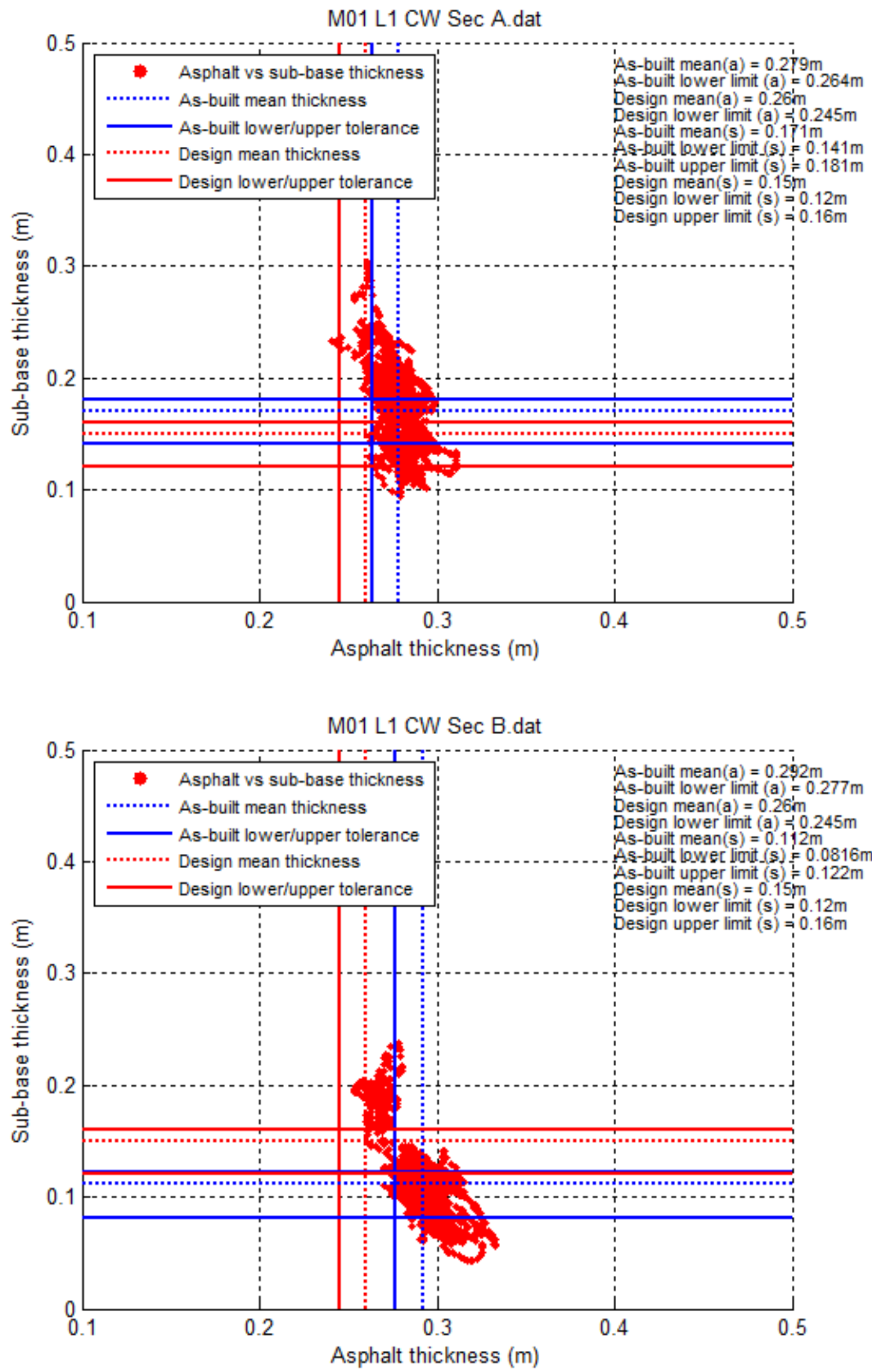


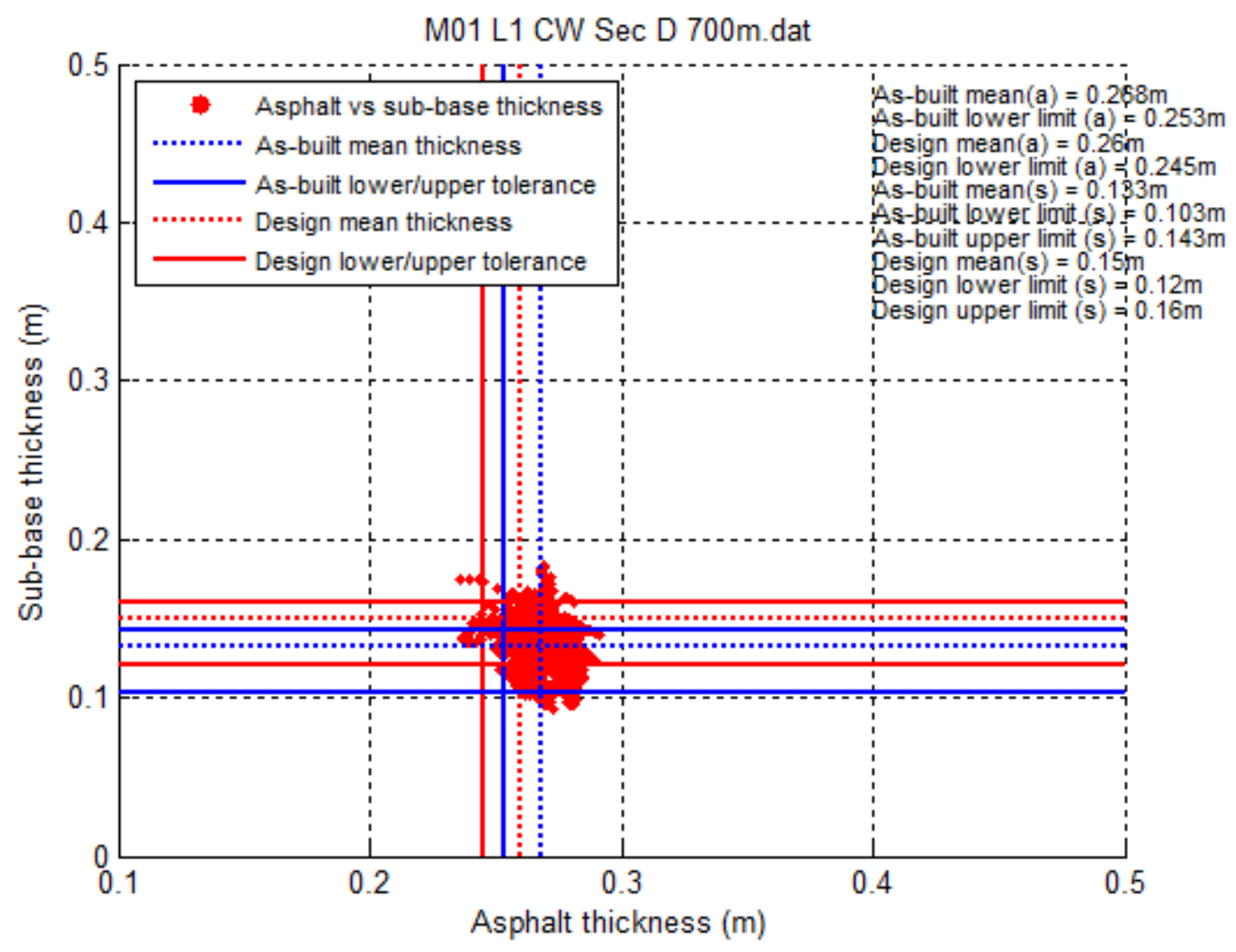

M01 L1 CW Sec E.dat

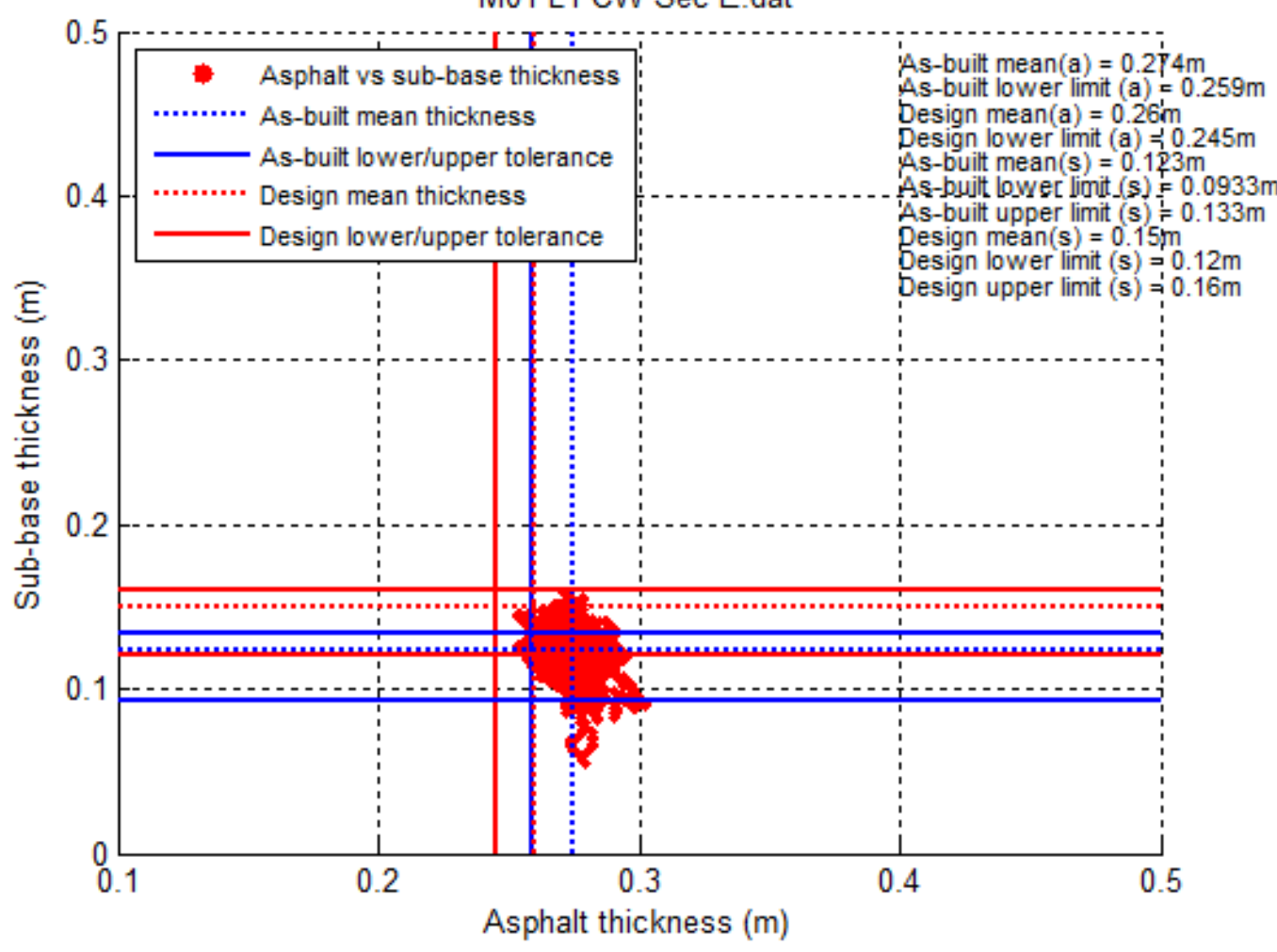




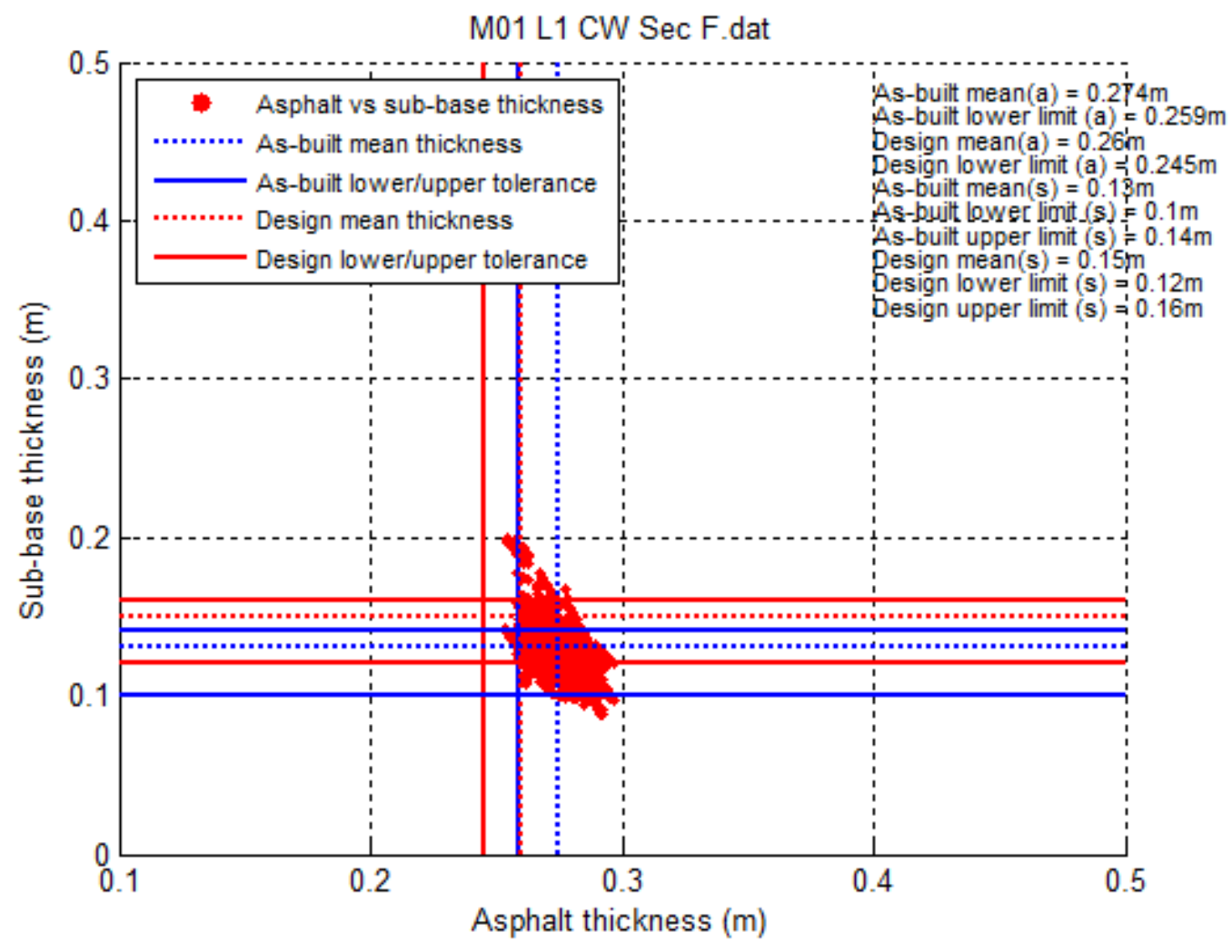

M01 L1 CW Sec G.dat

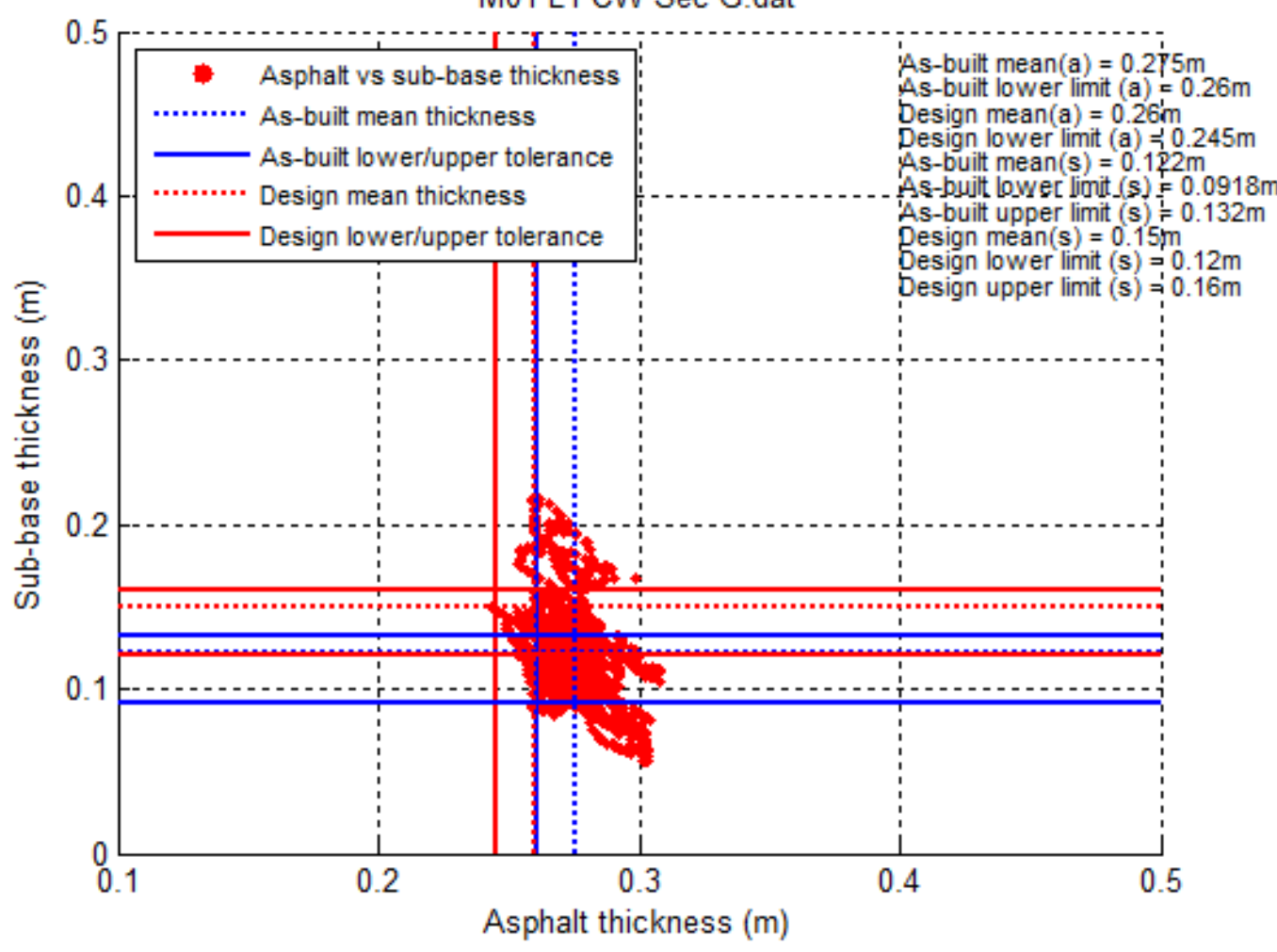



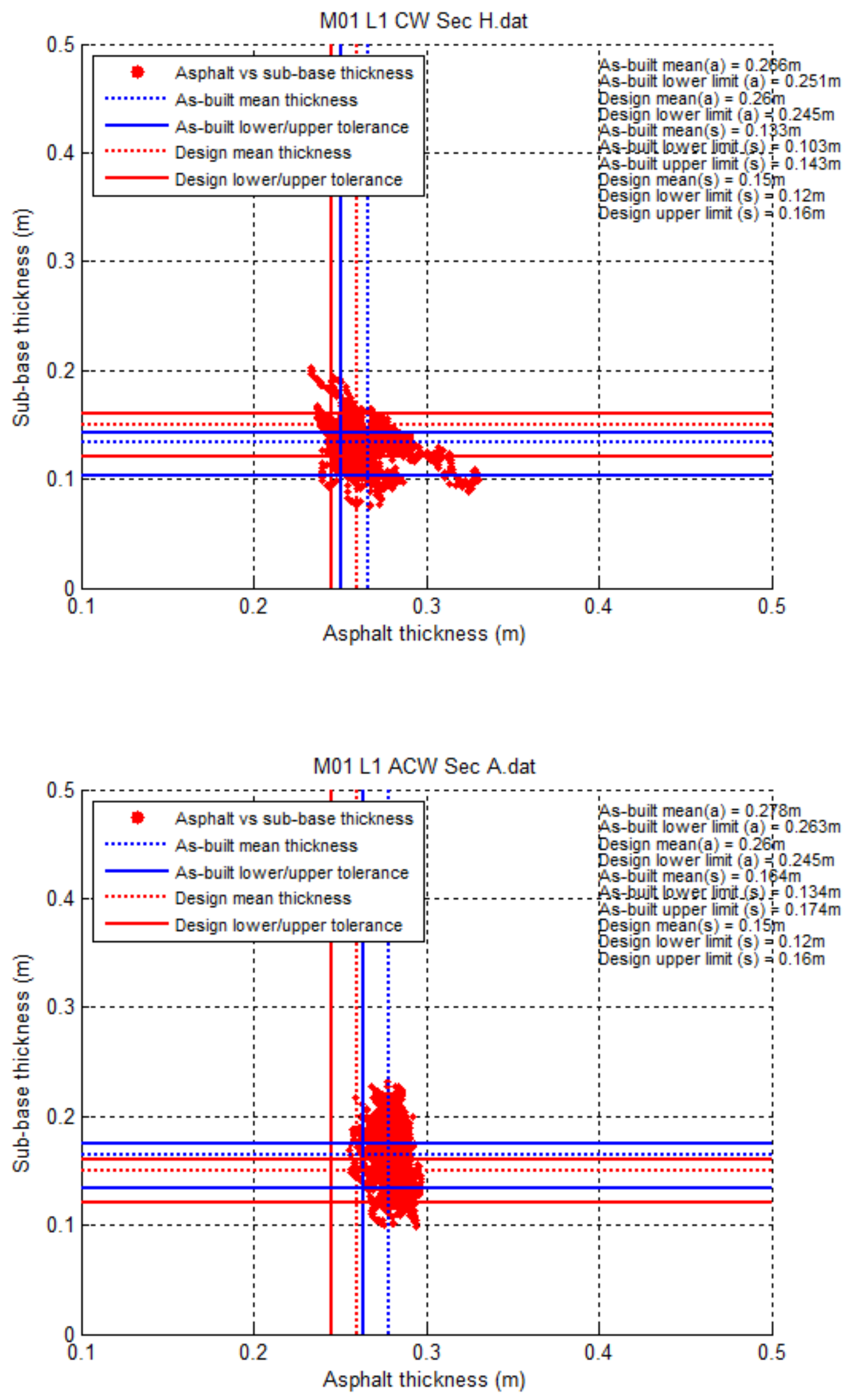

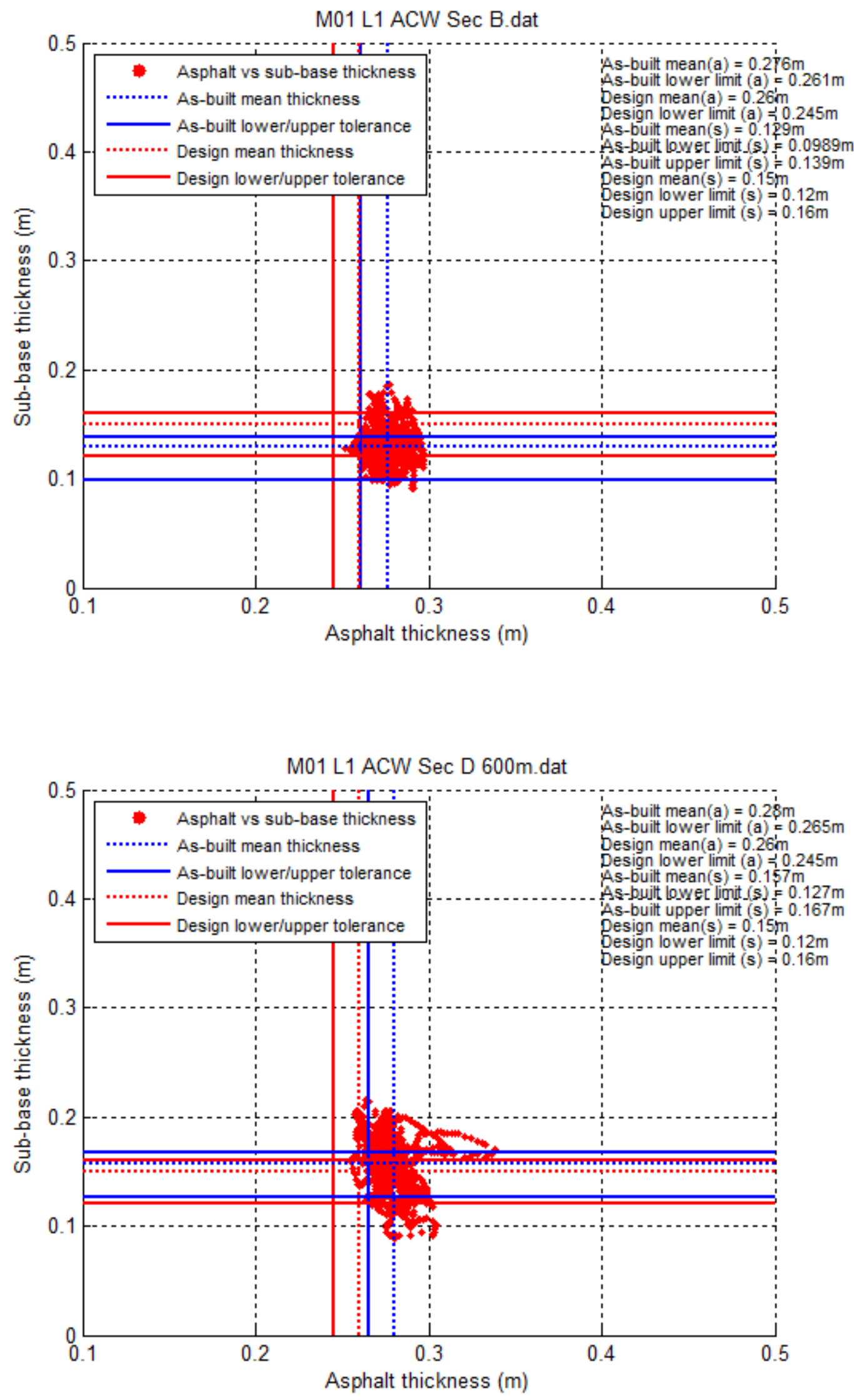

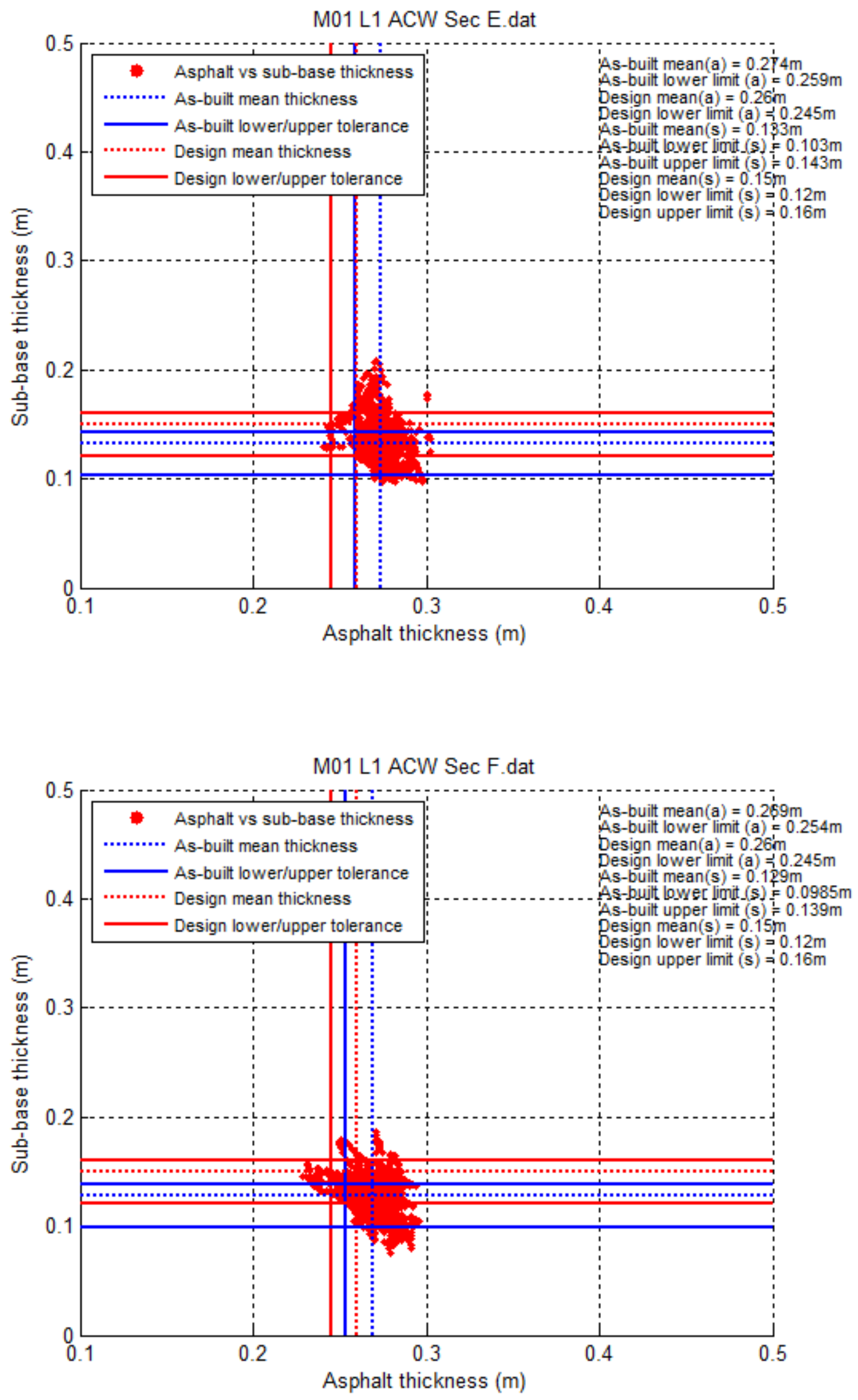

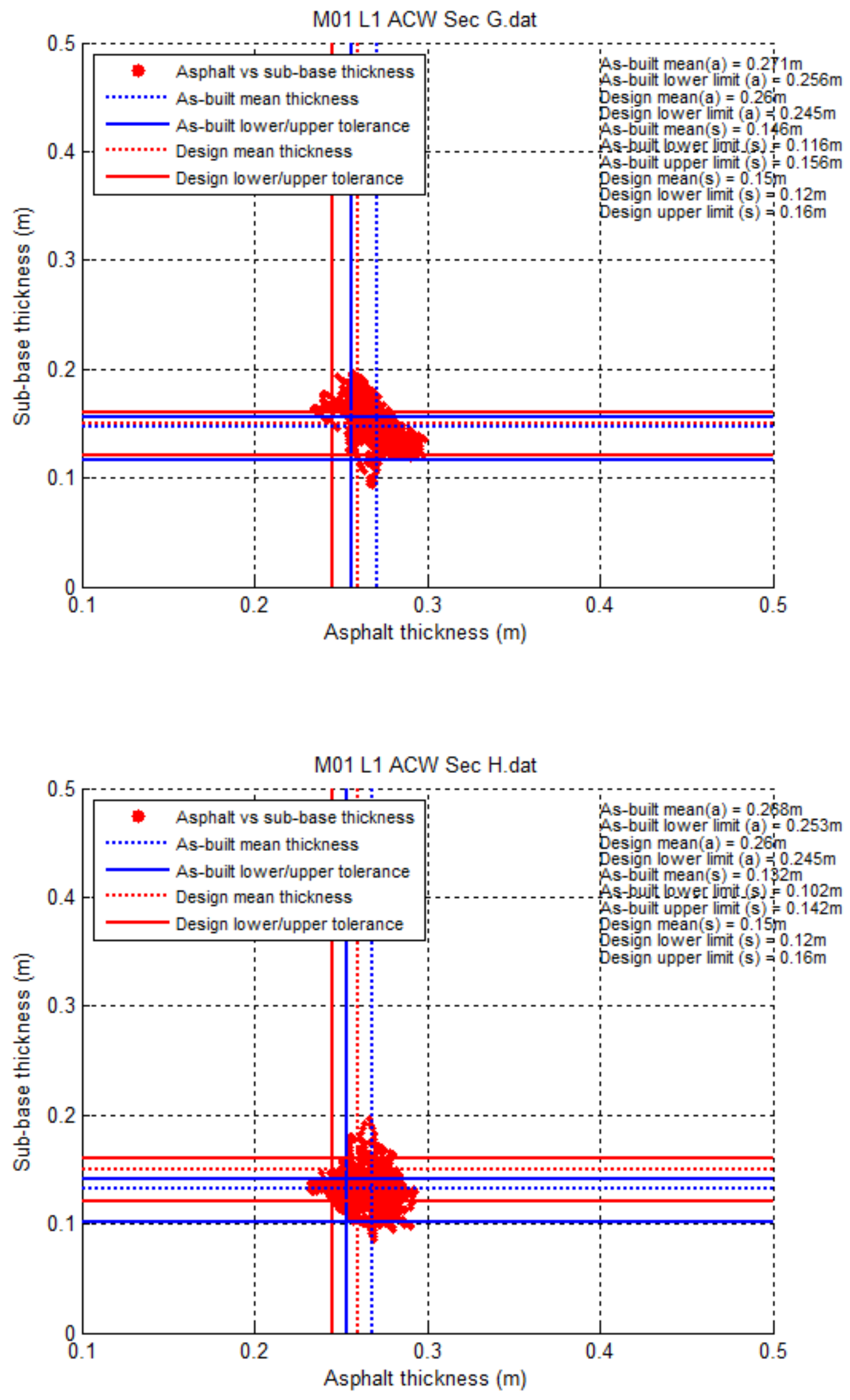

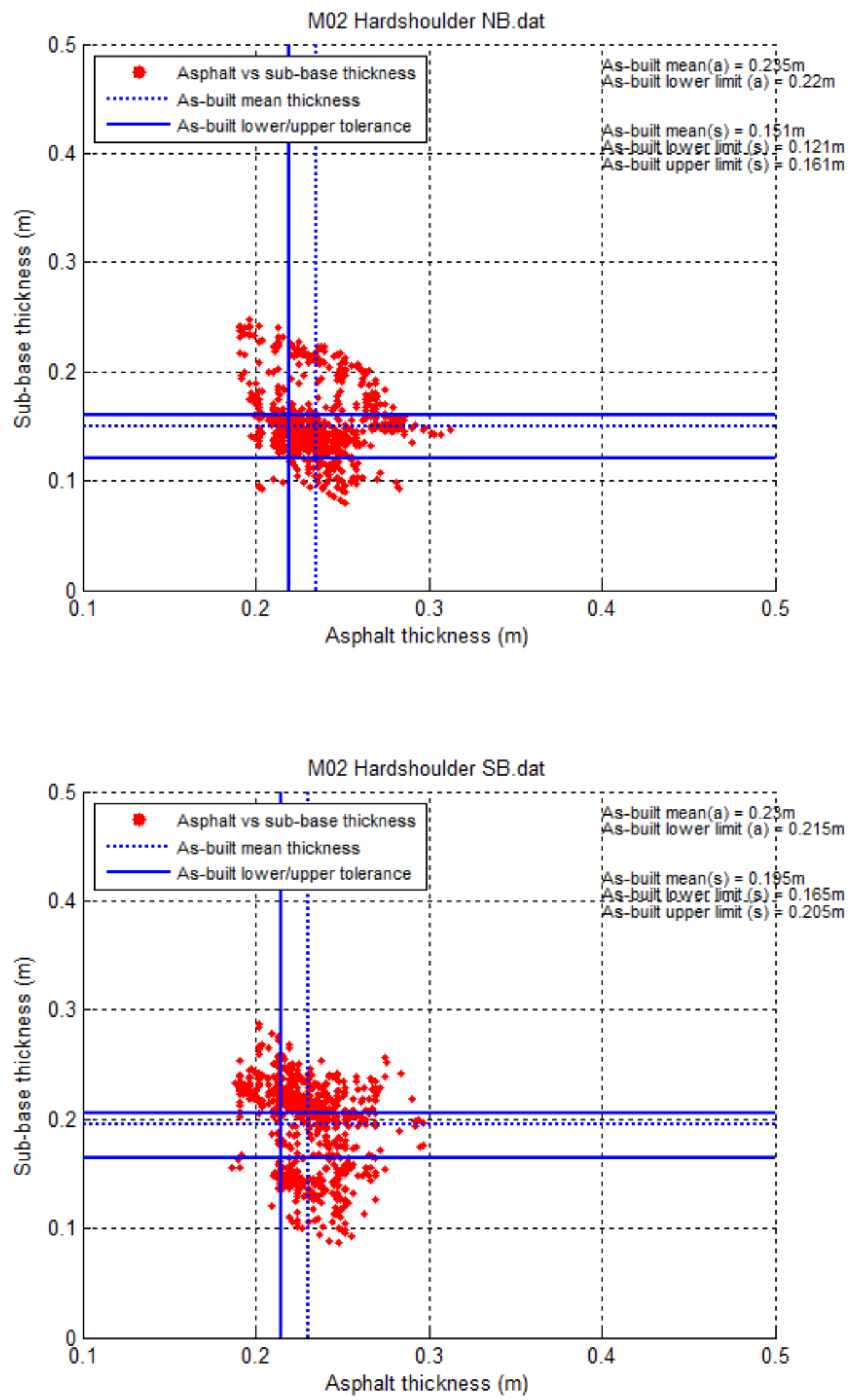

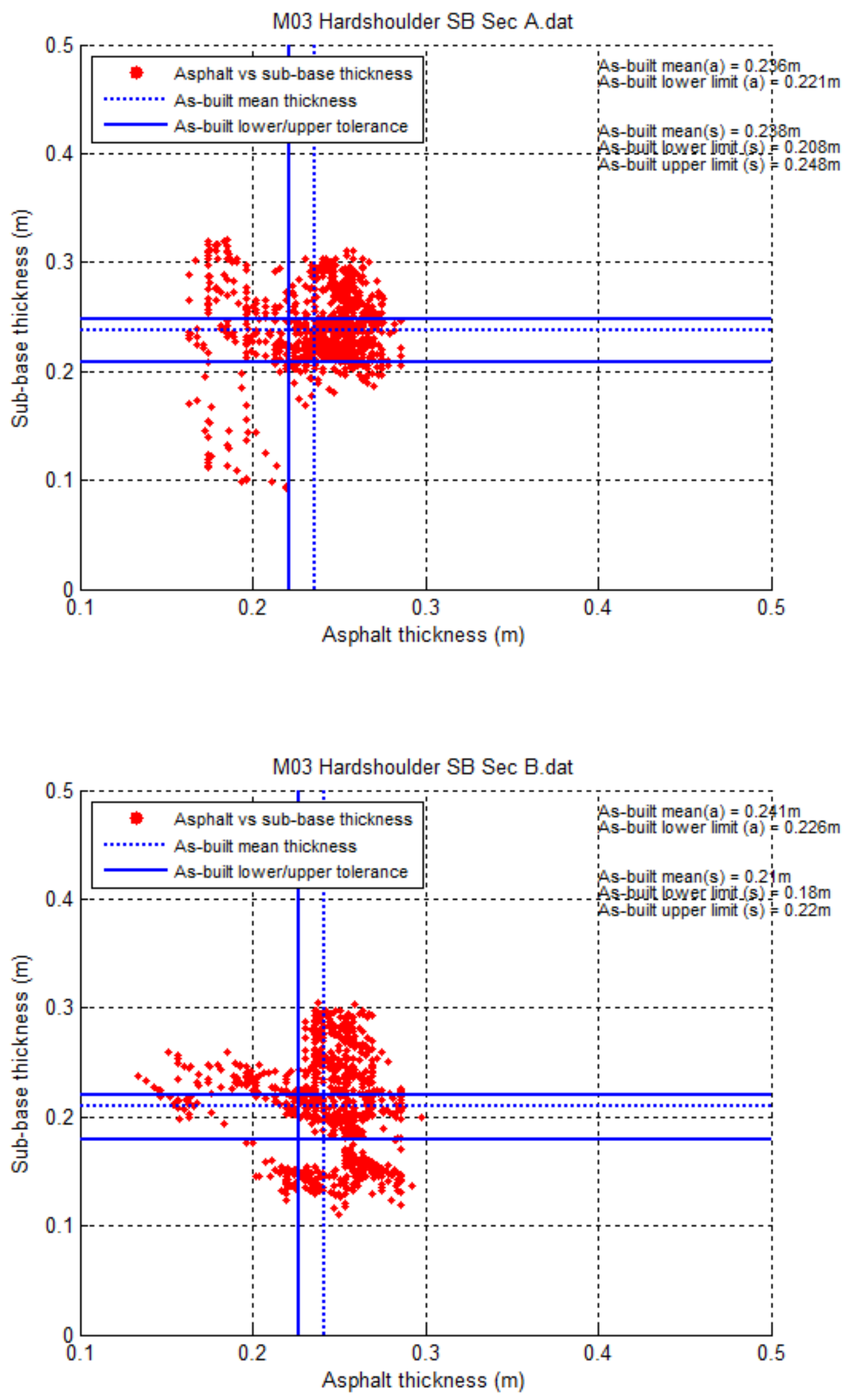

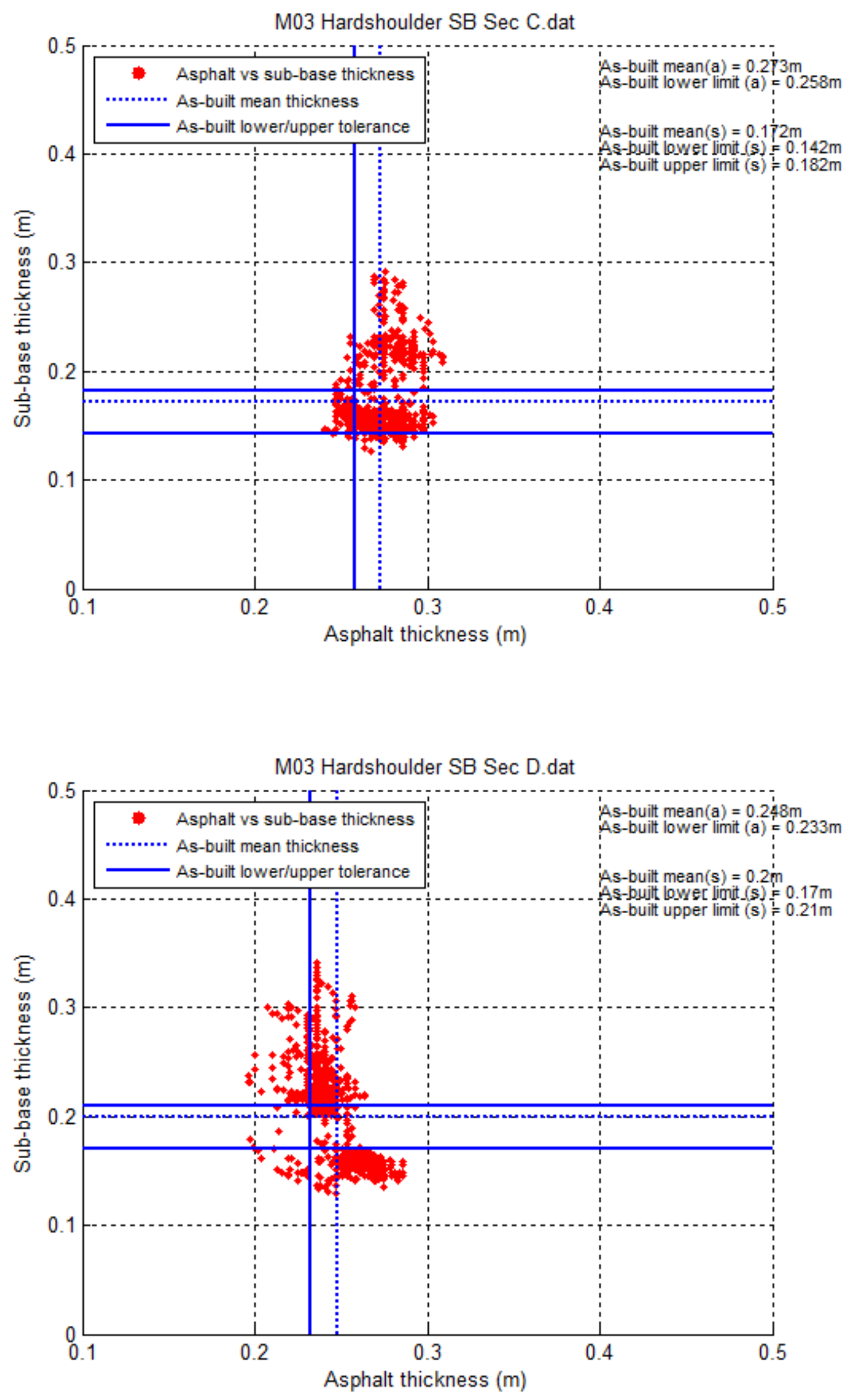

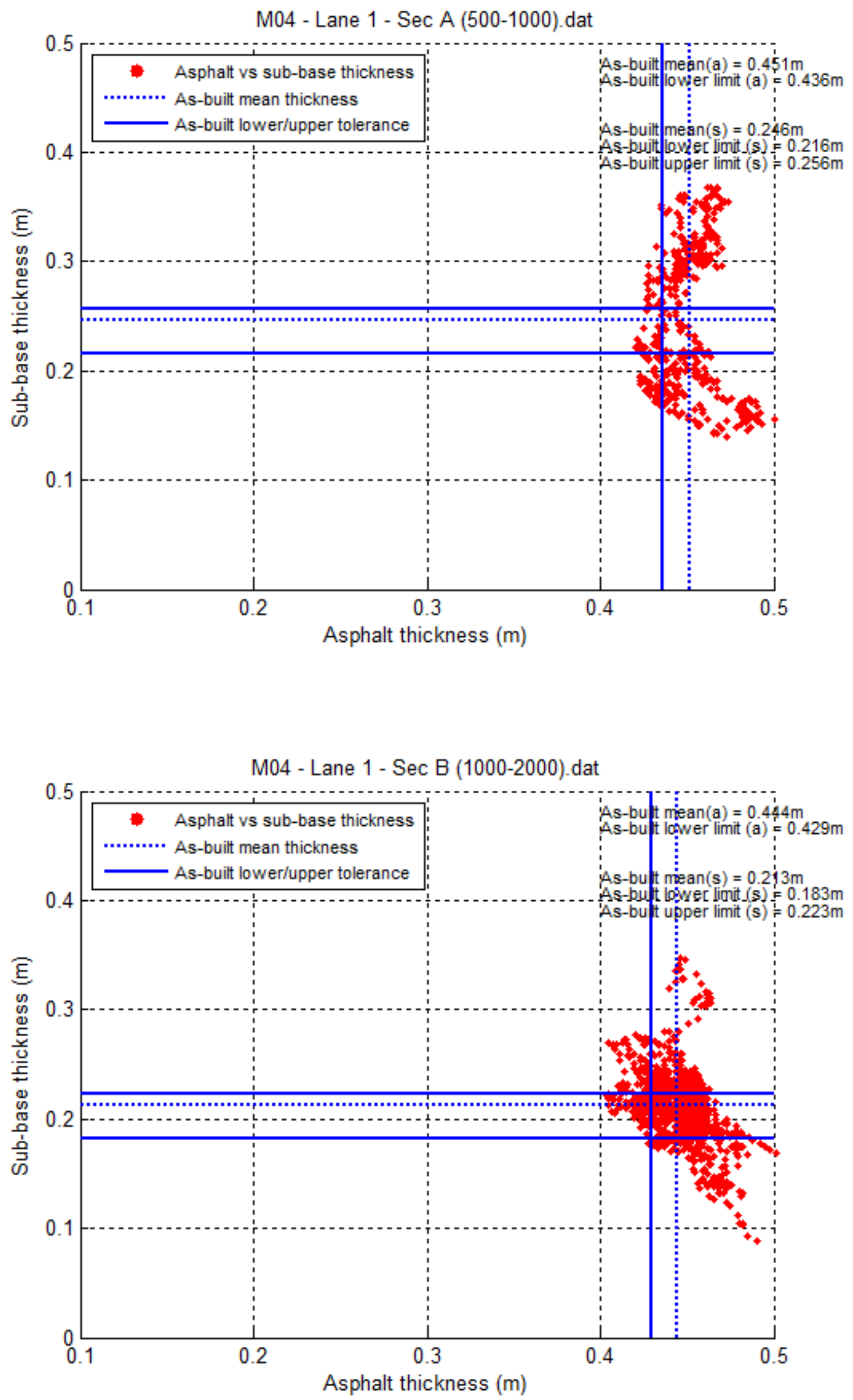

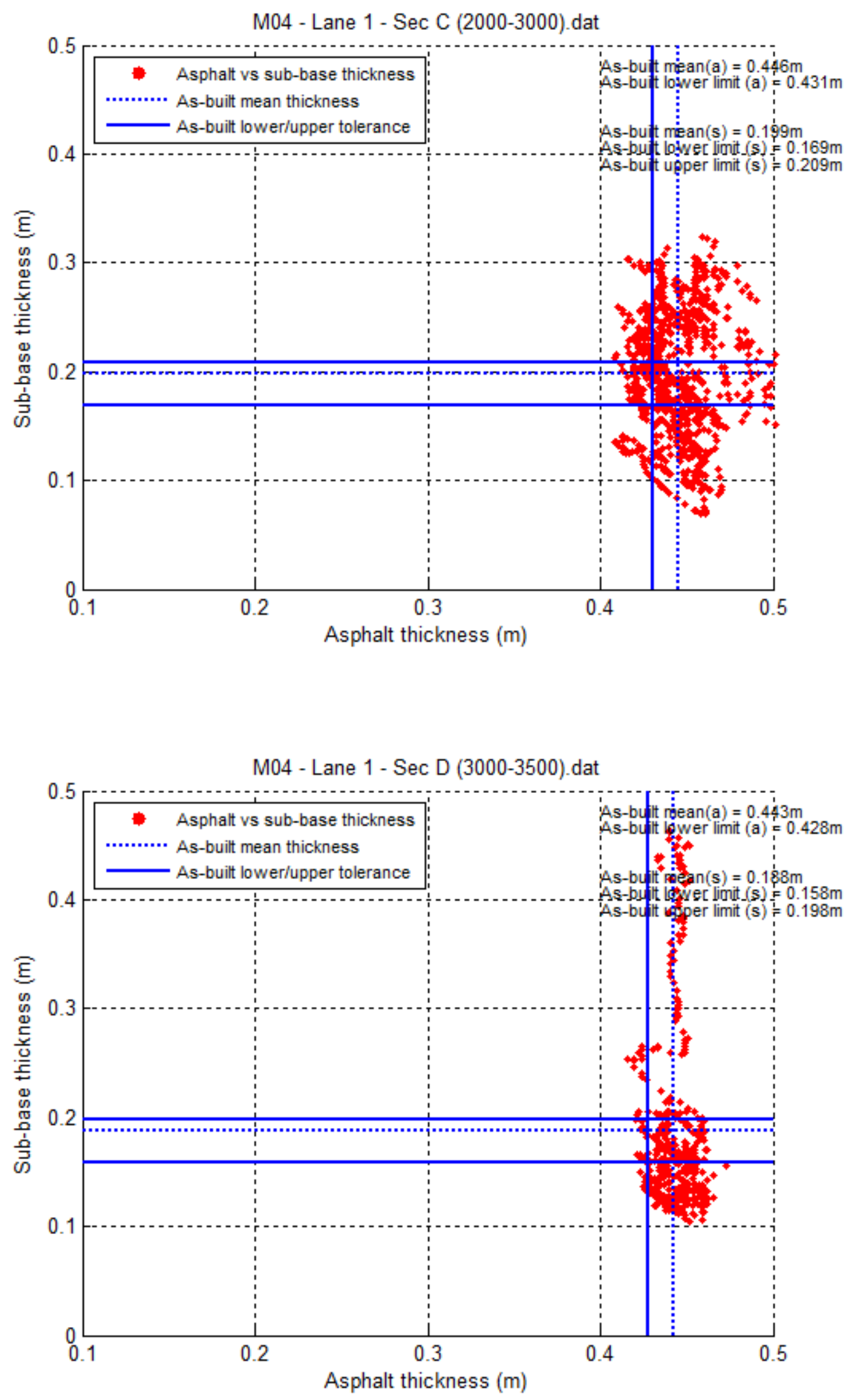

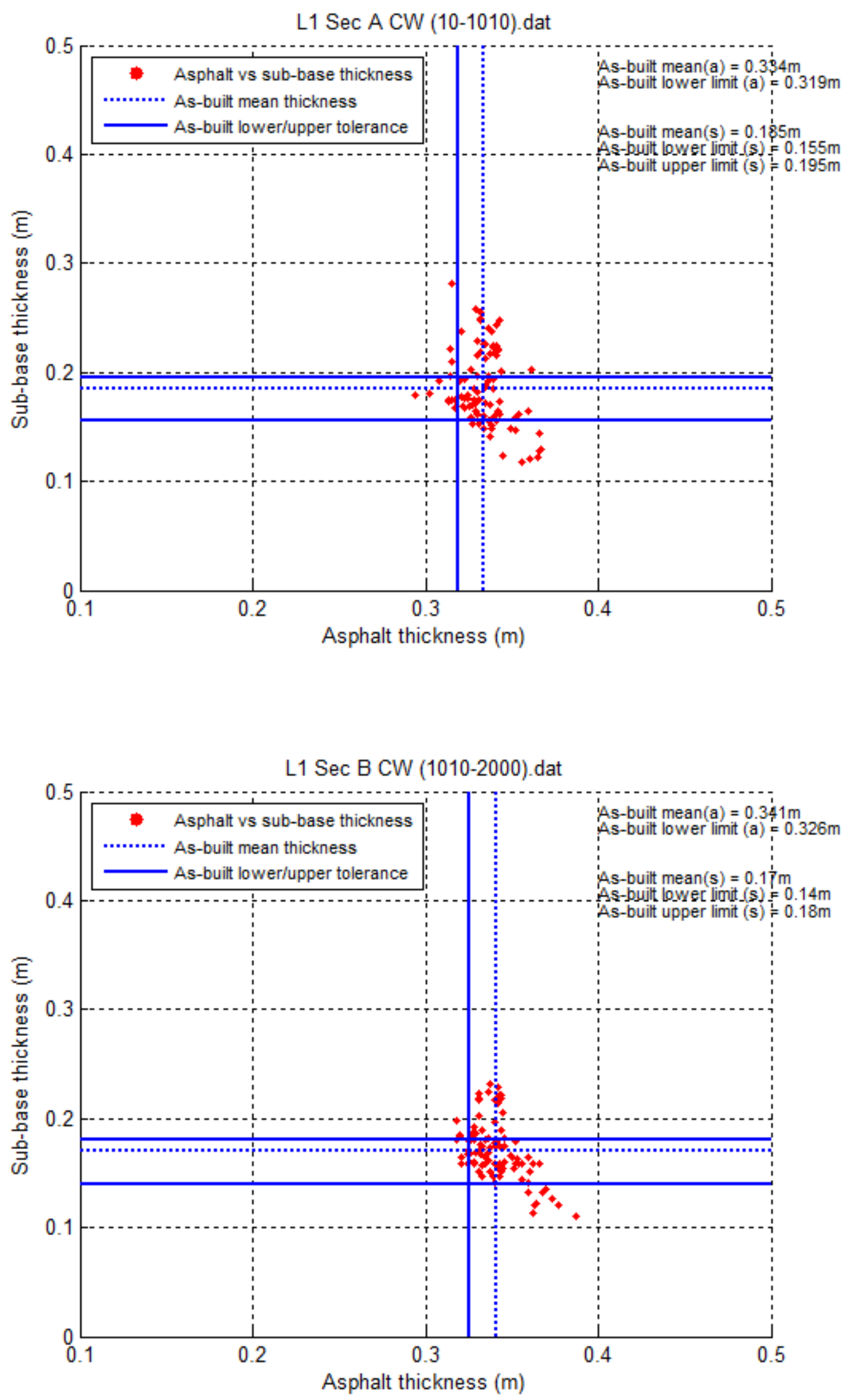
L1 Sec C CW (2010-2500).dat

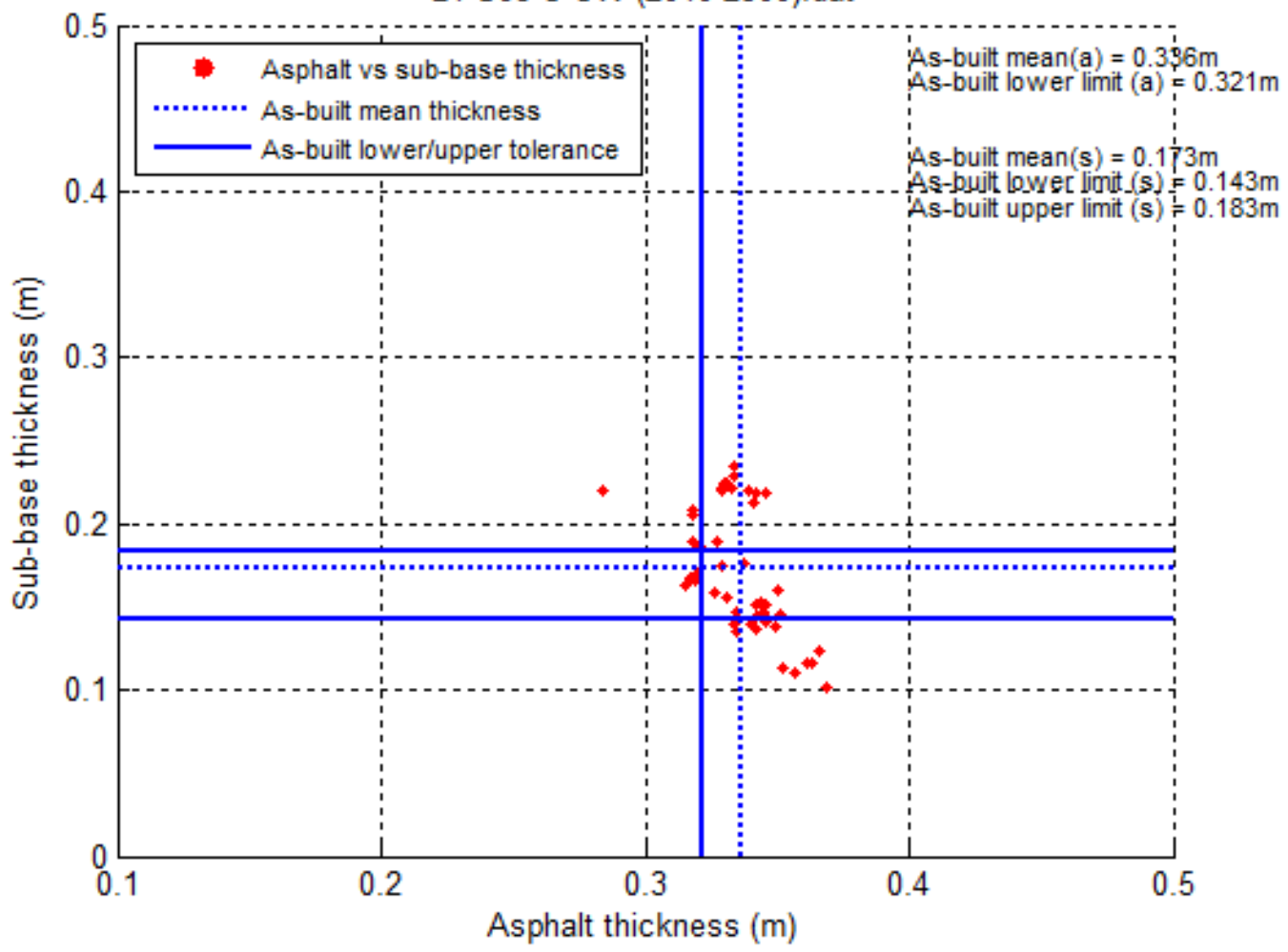

L1 Sec D CW (2710-3000).dat

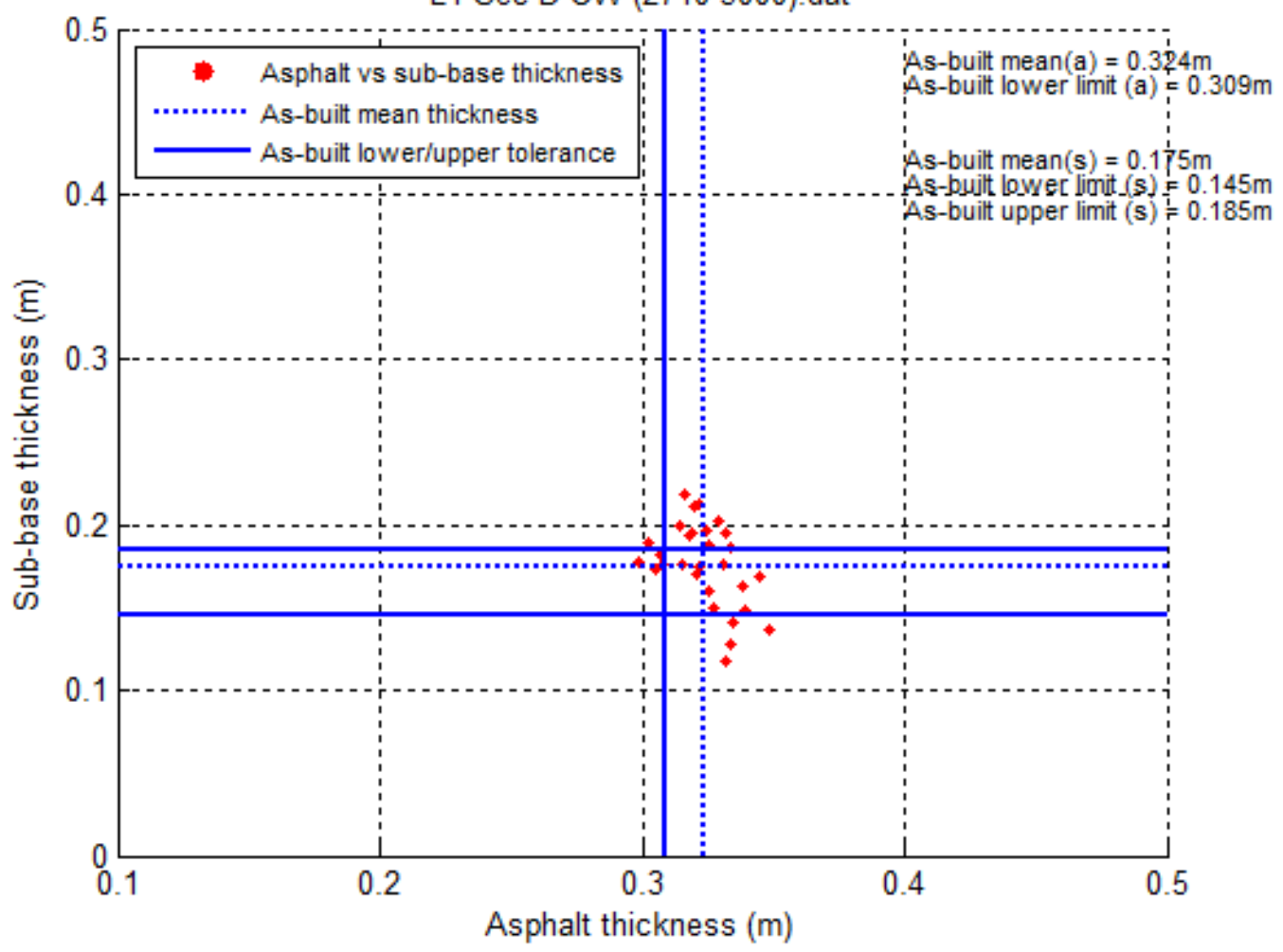



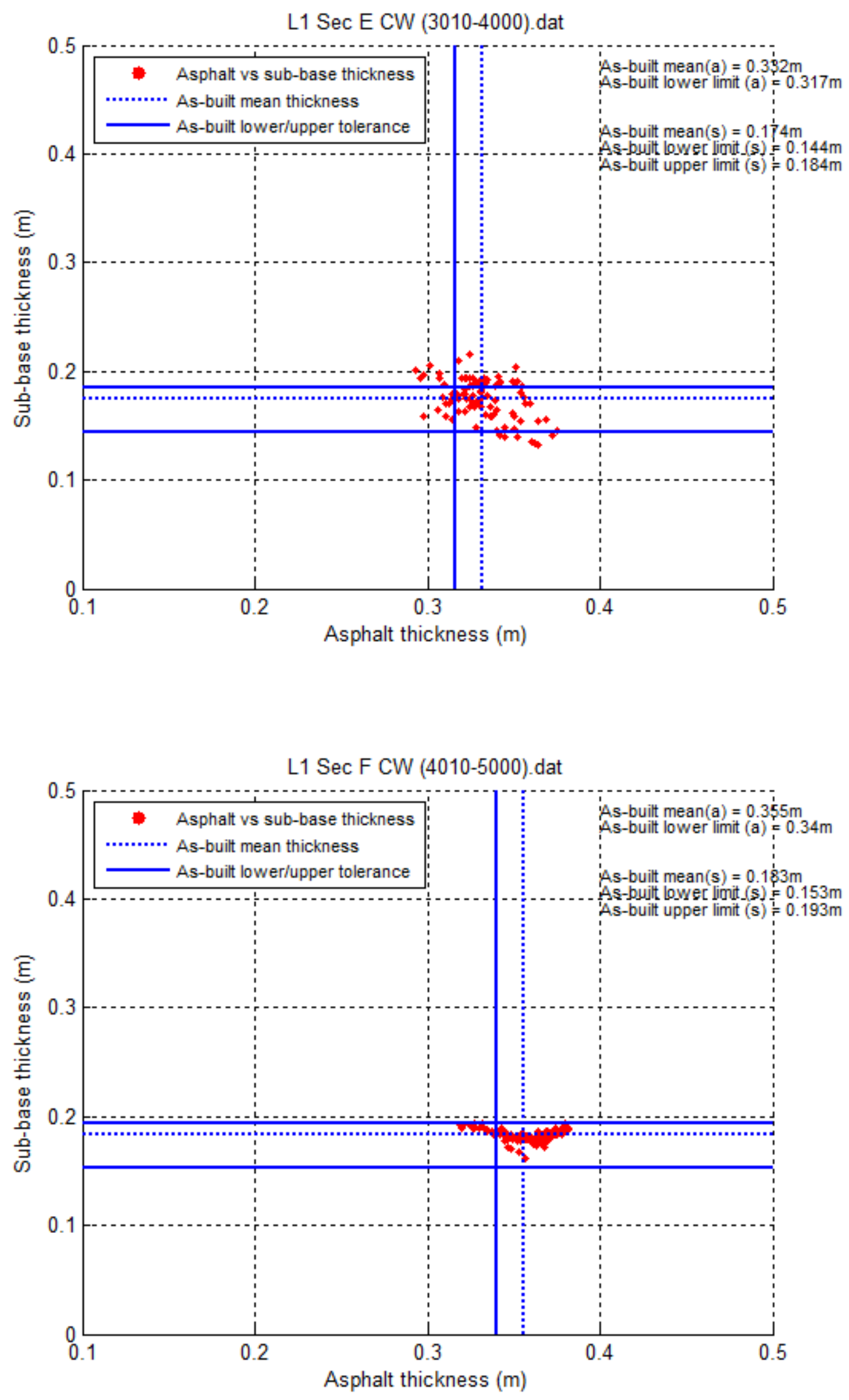


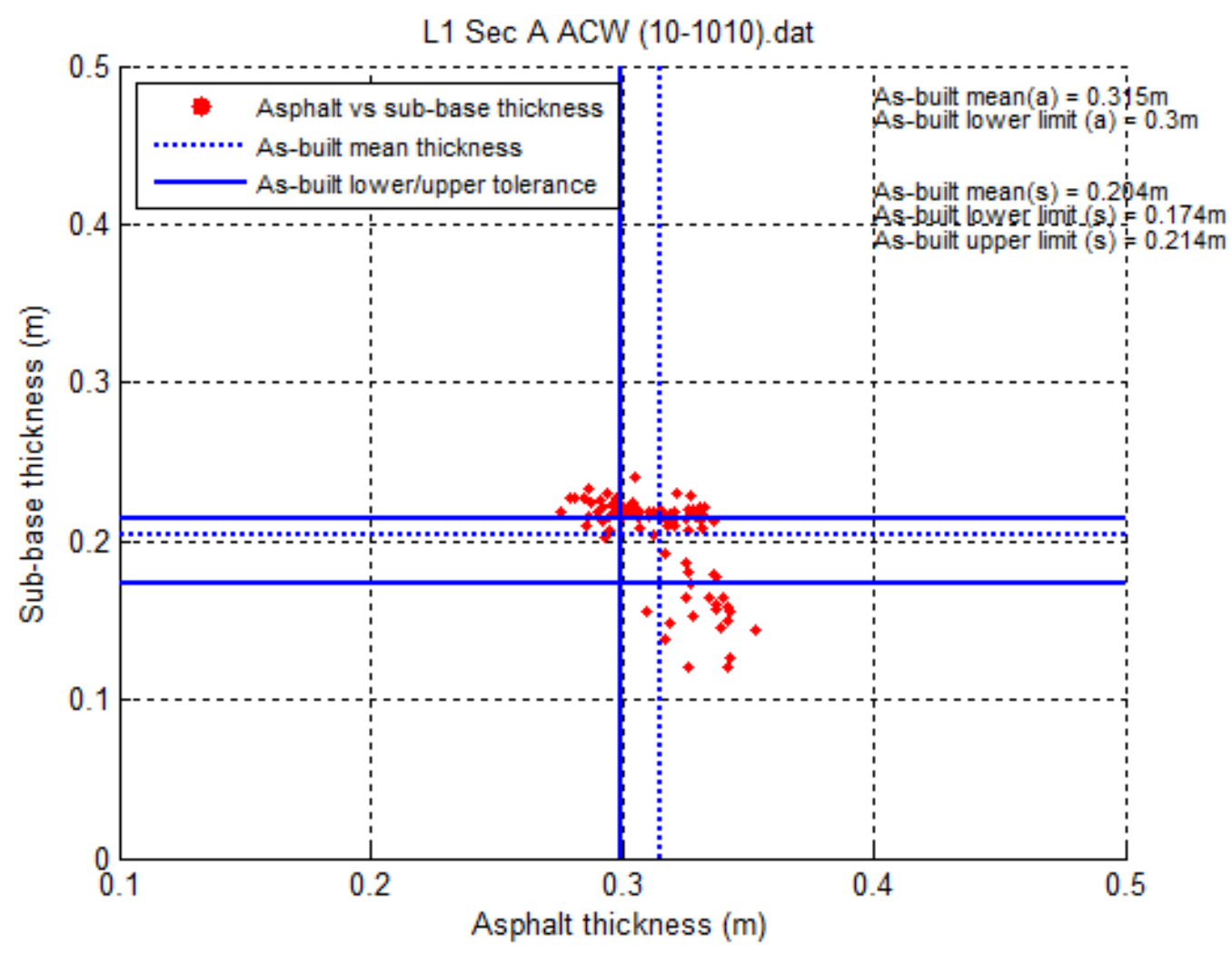

L1 Sec B ACW (1010-2000).dat

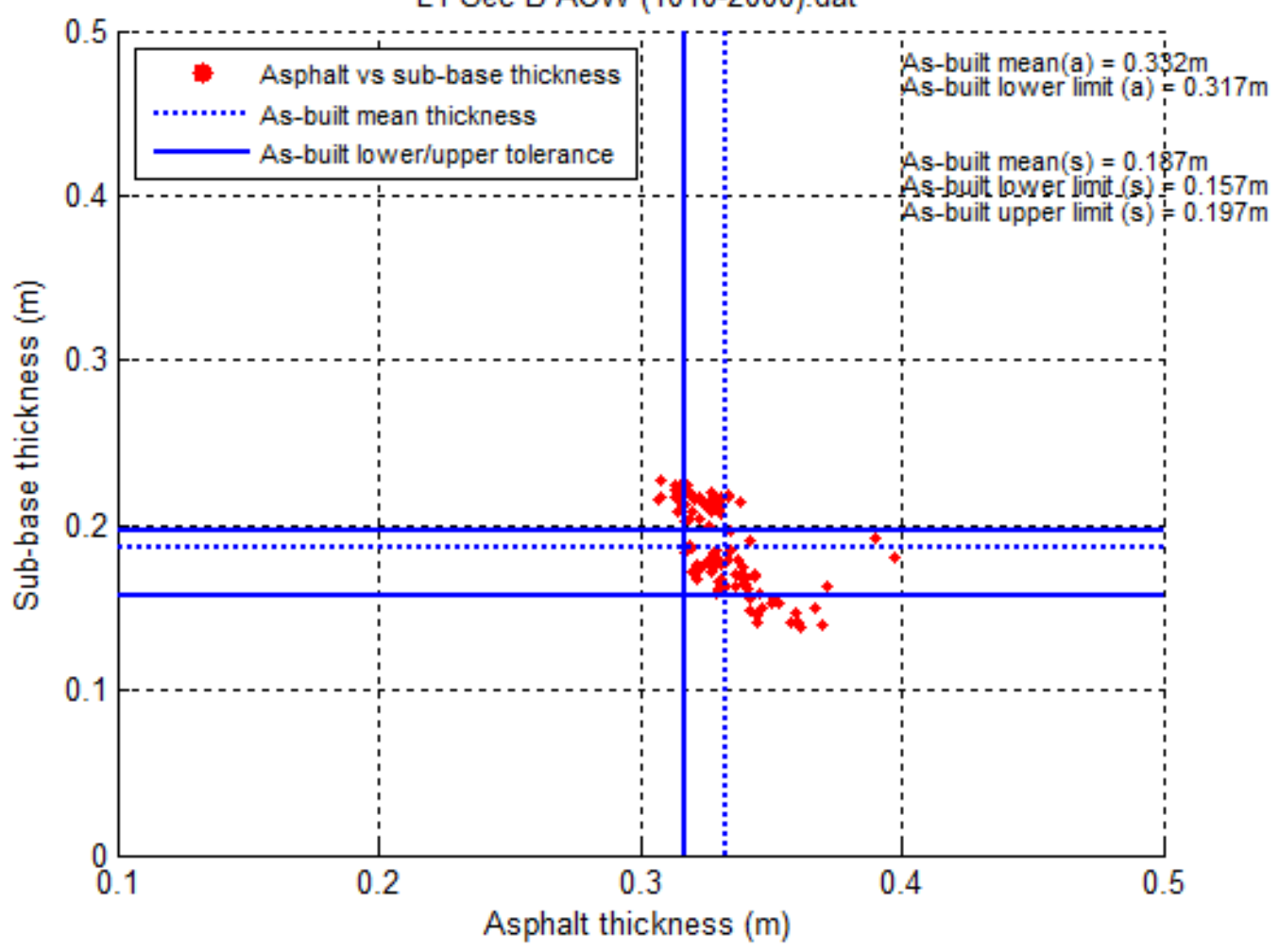




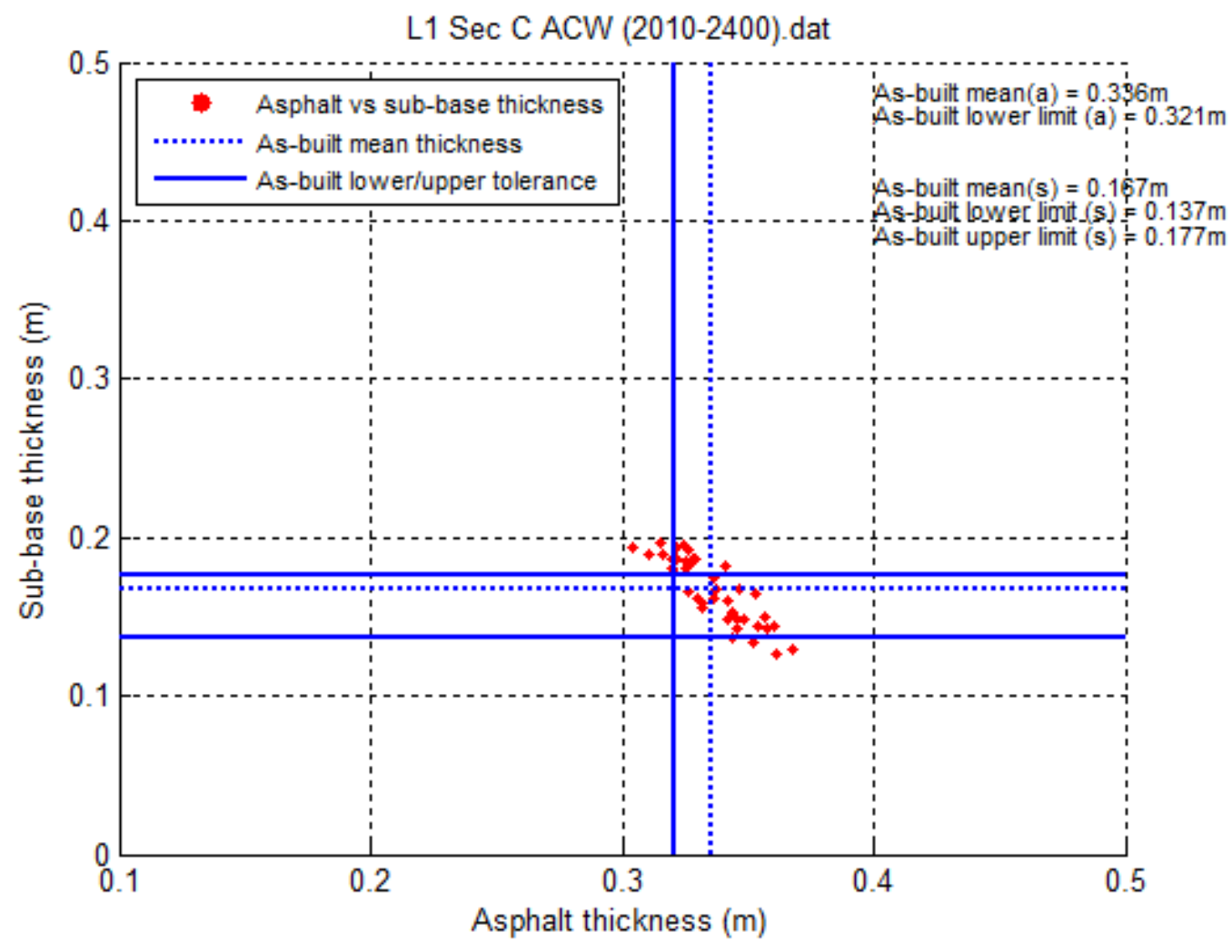

L1 Sec D ACW (2610-3000).dat

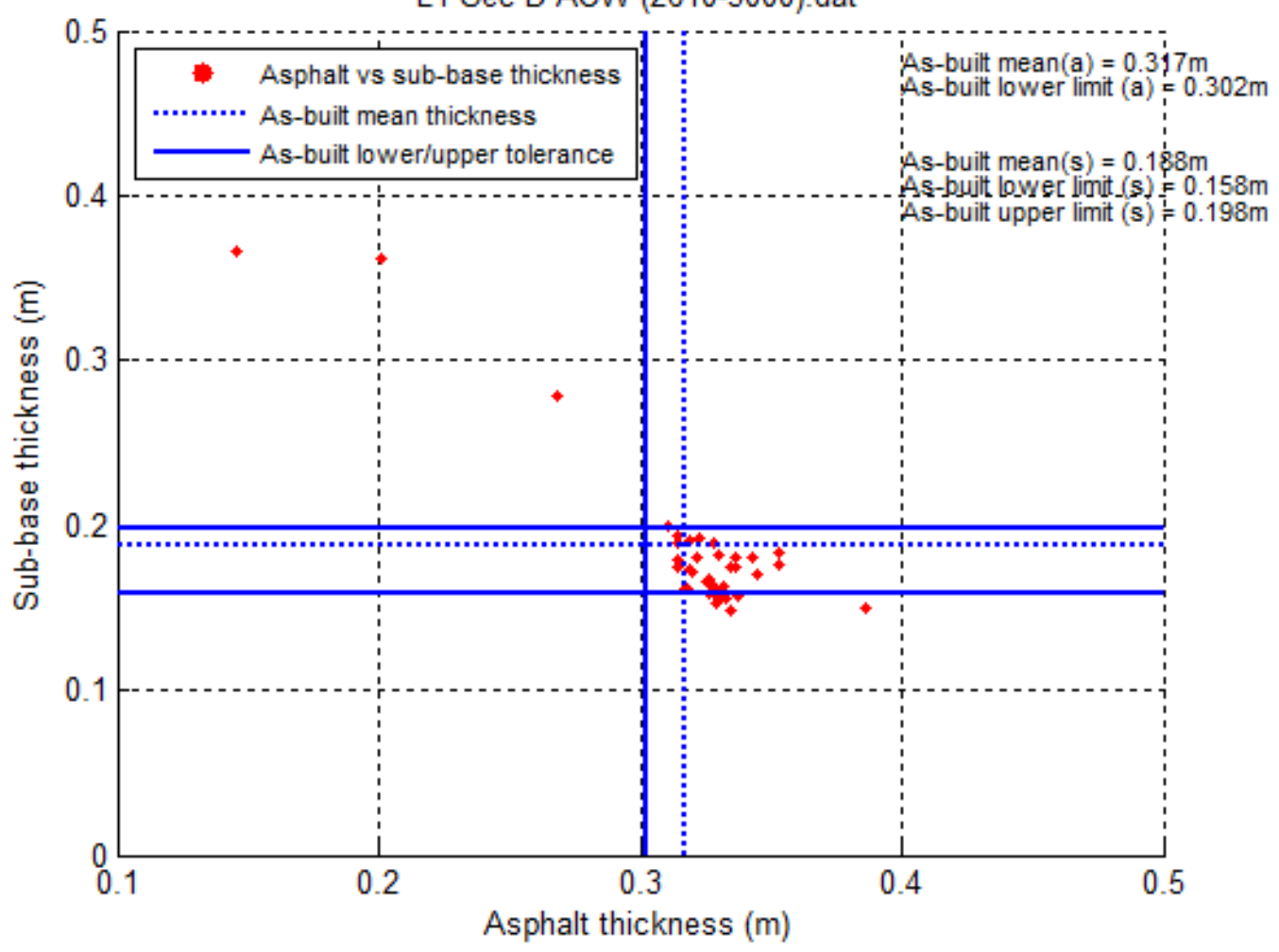




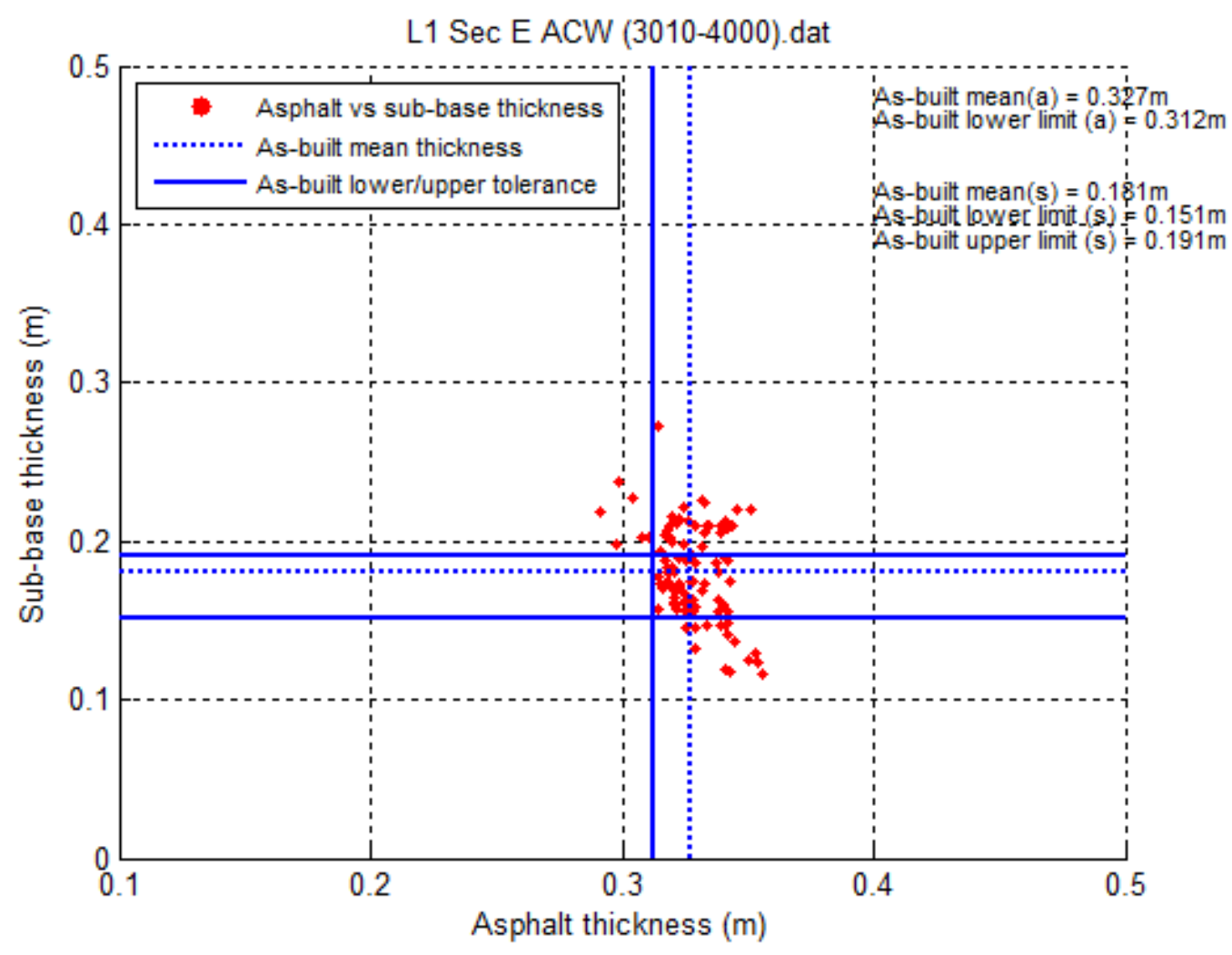

L1 Sec F ACW (4010-5000).dat

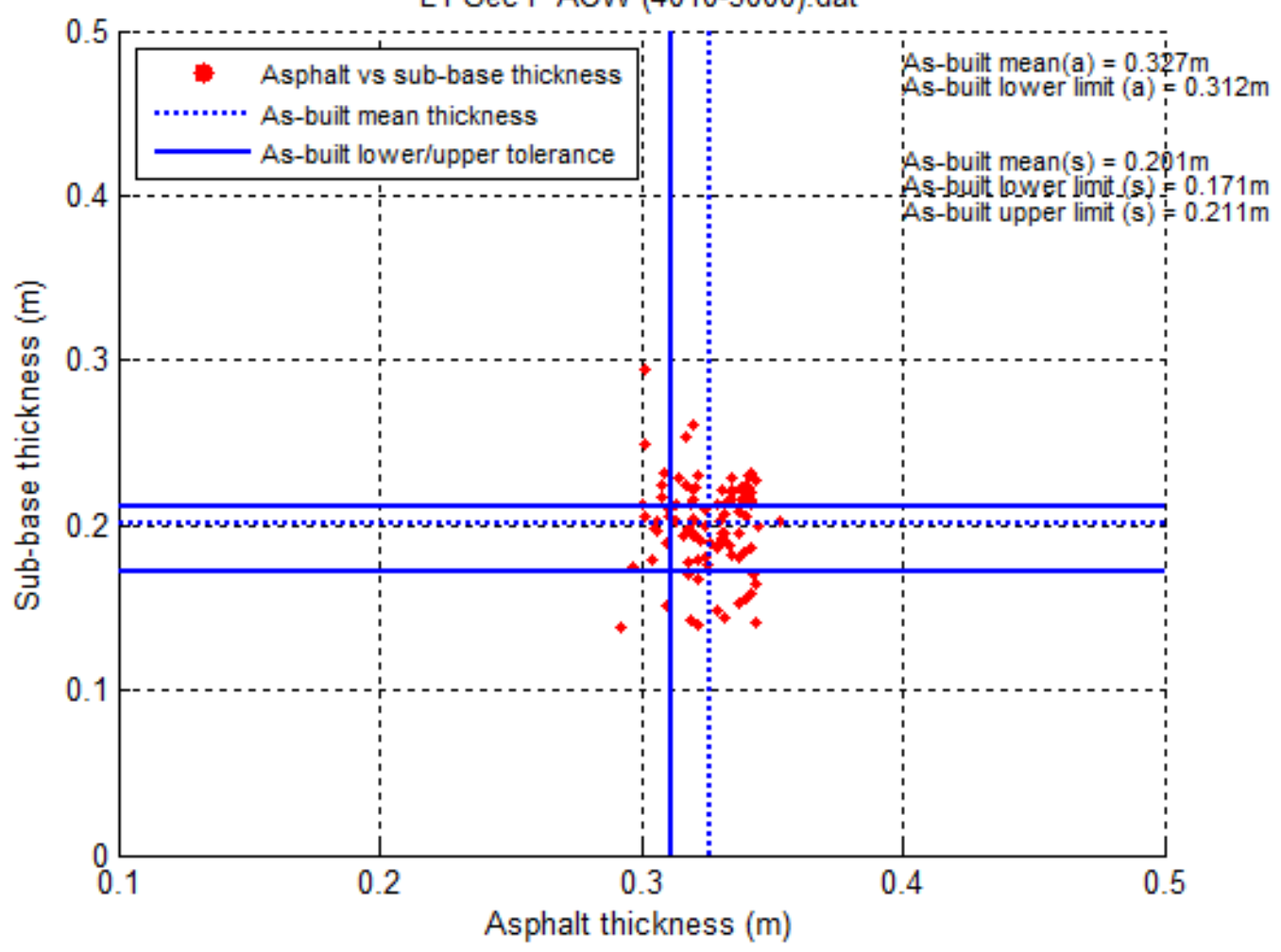



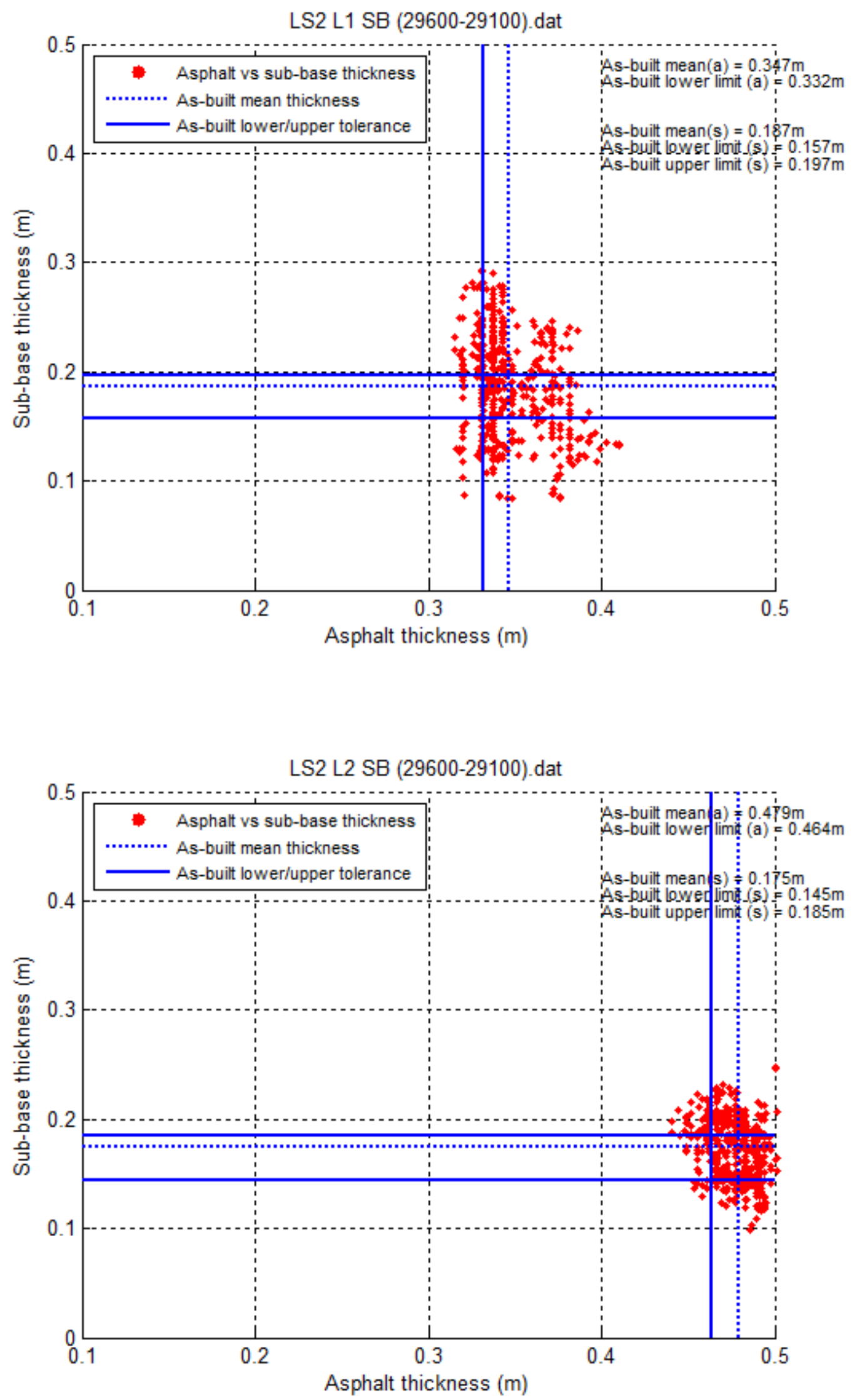

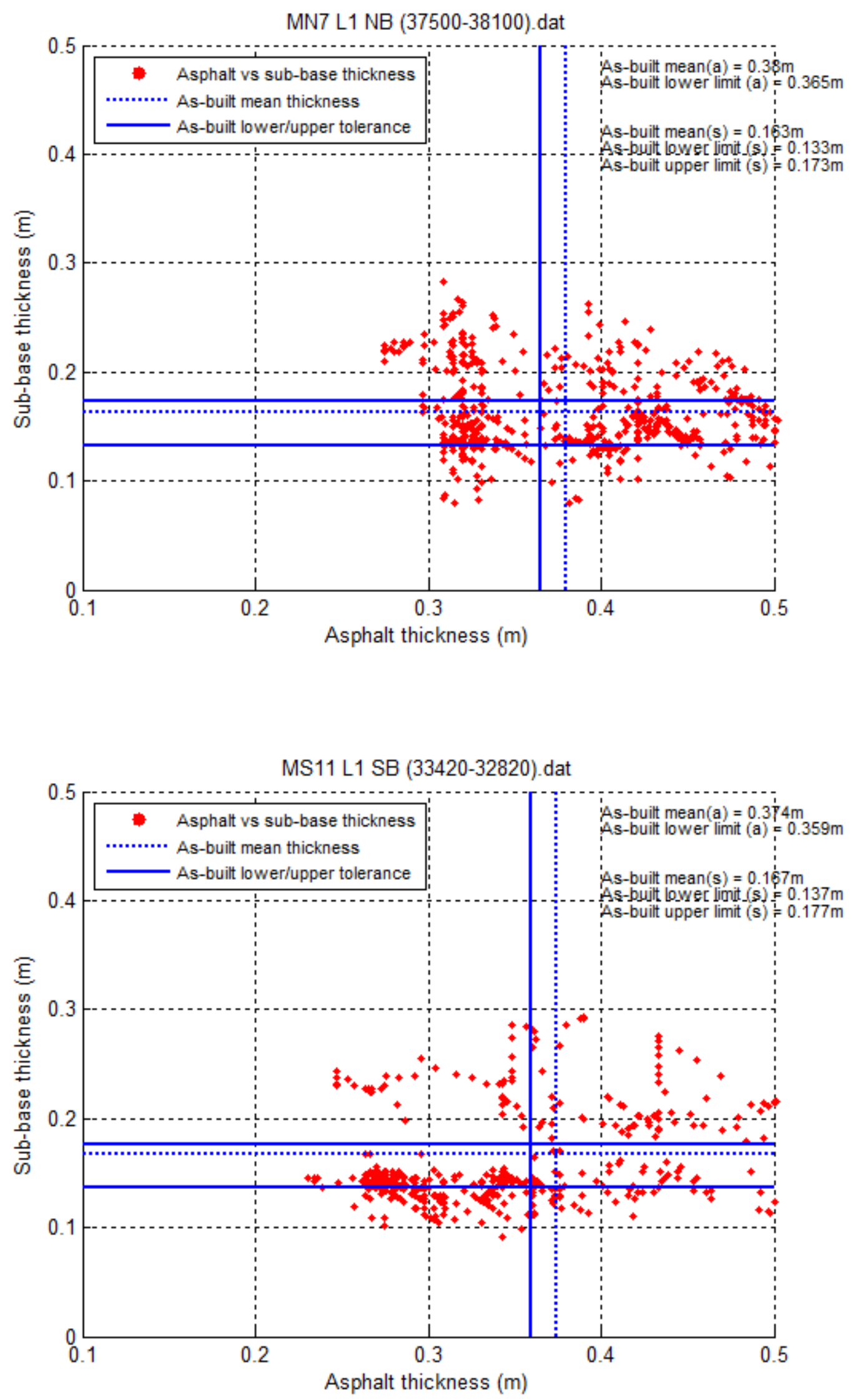

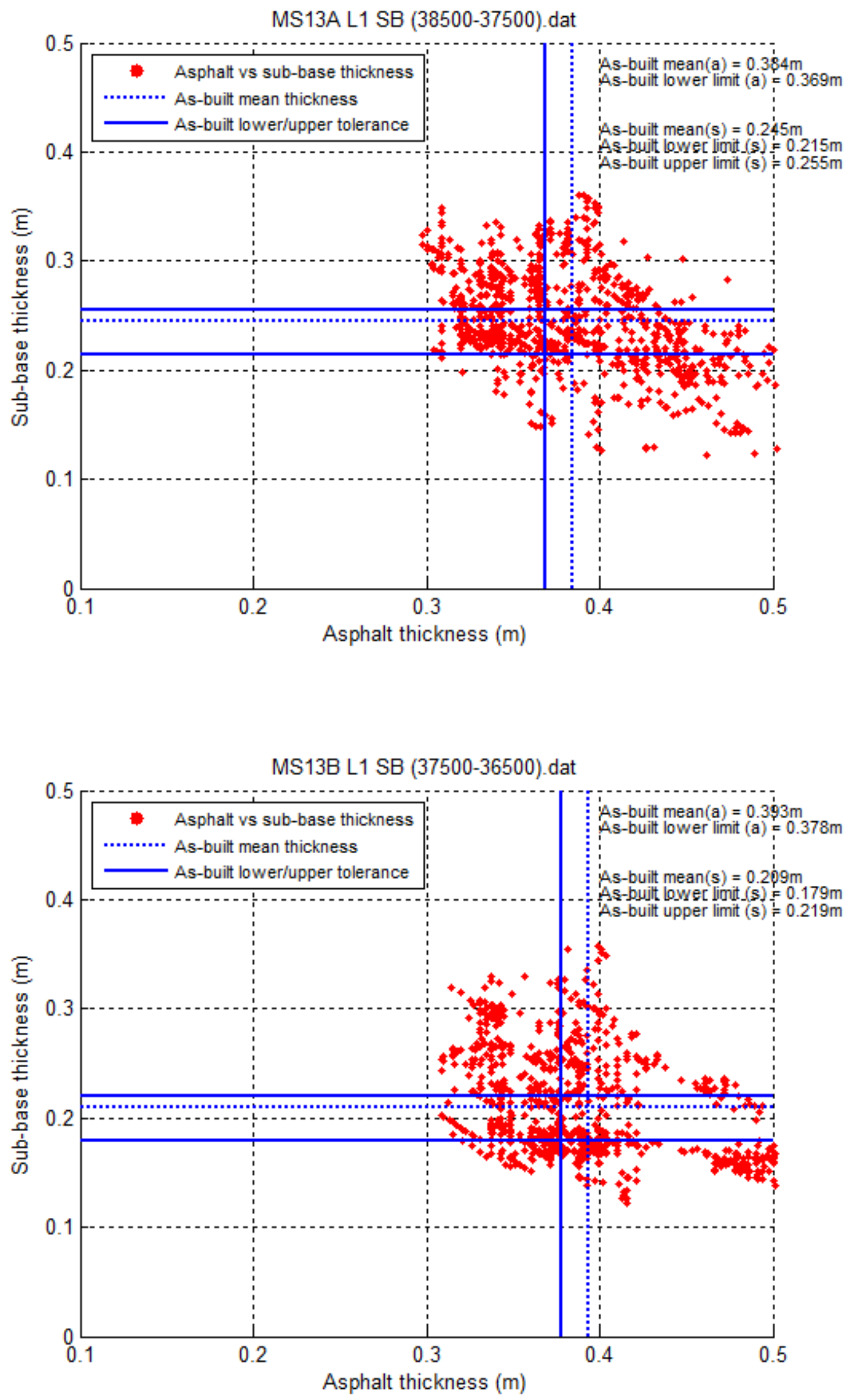


\section{A4 Monte Carlo Simulation results}

A4.1 M01

\section{A4.1.1 Section 3}
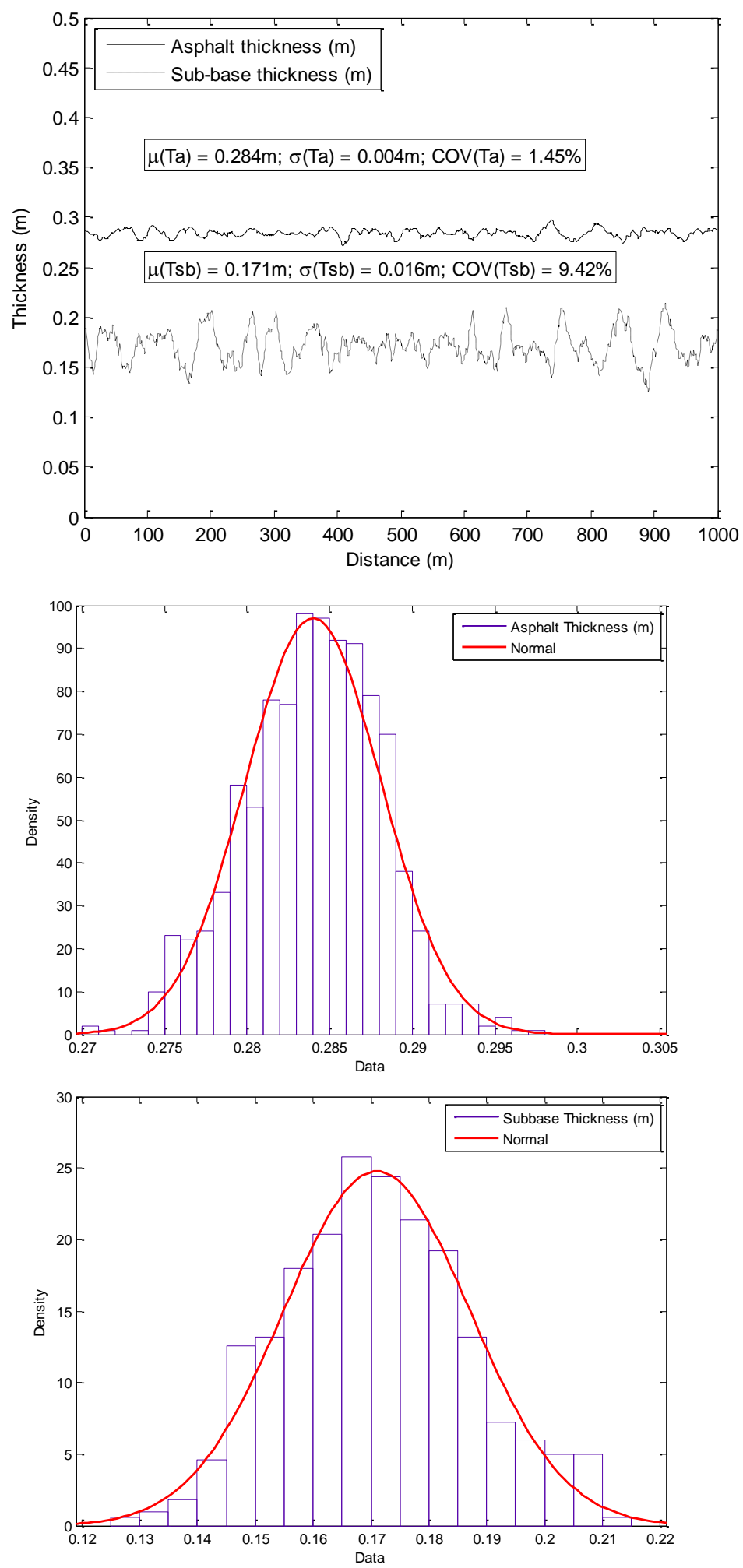

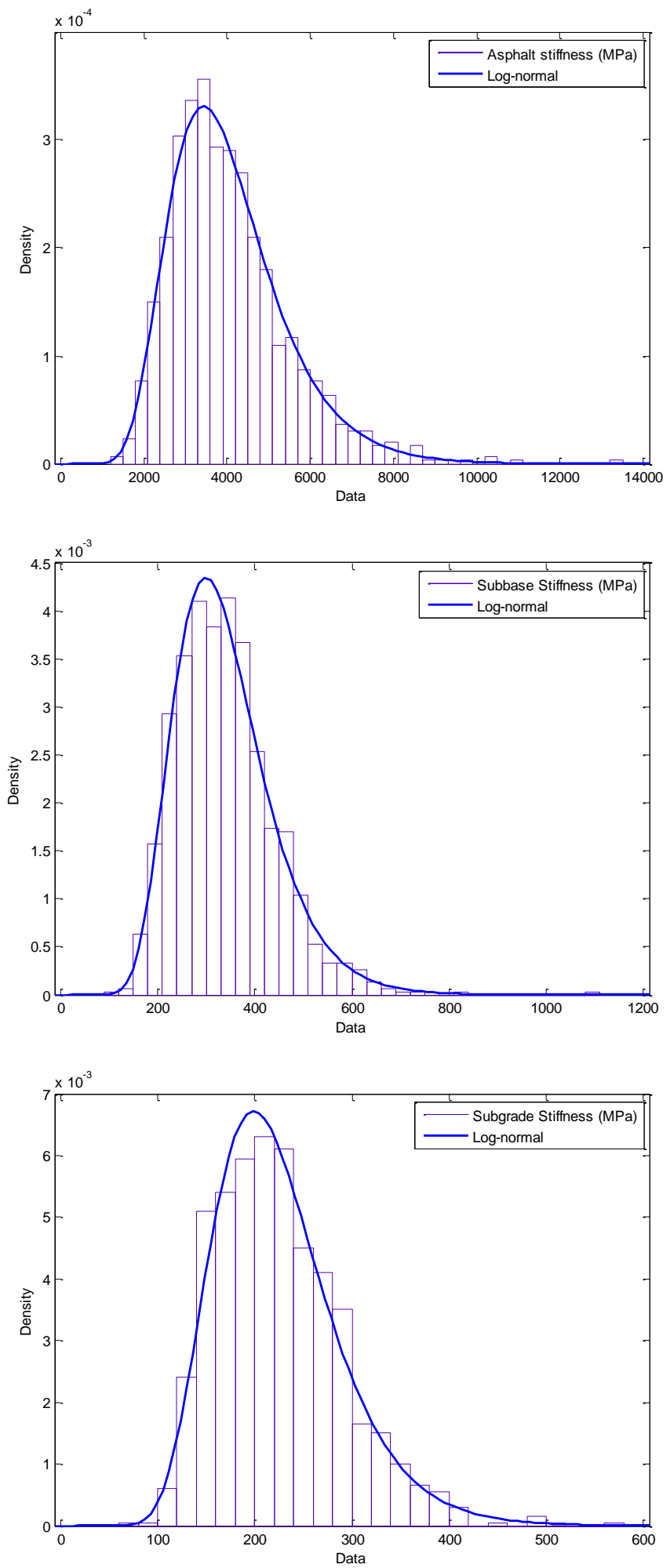

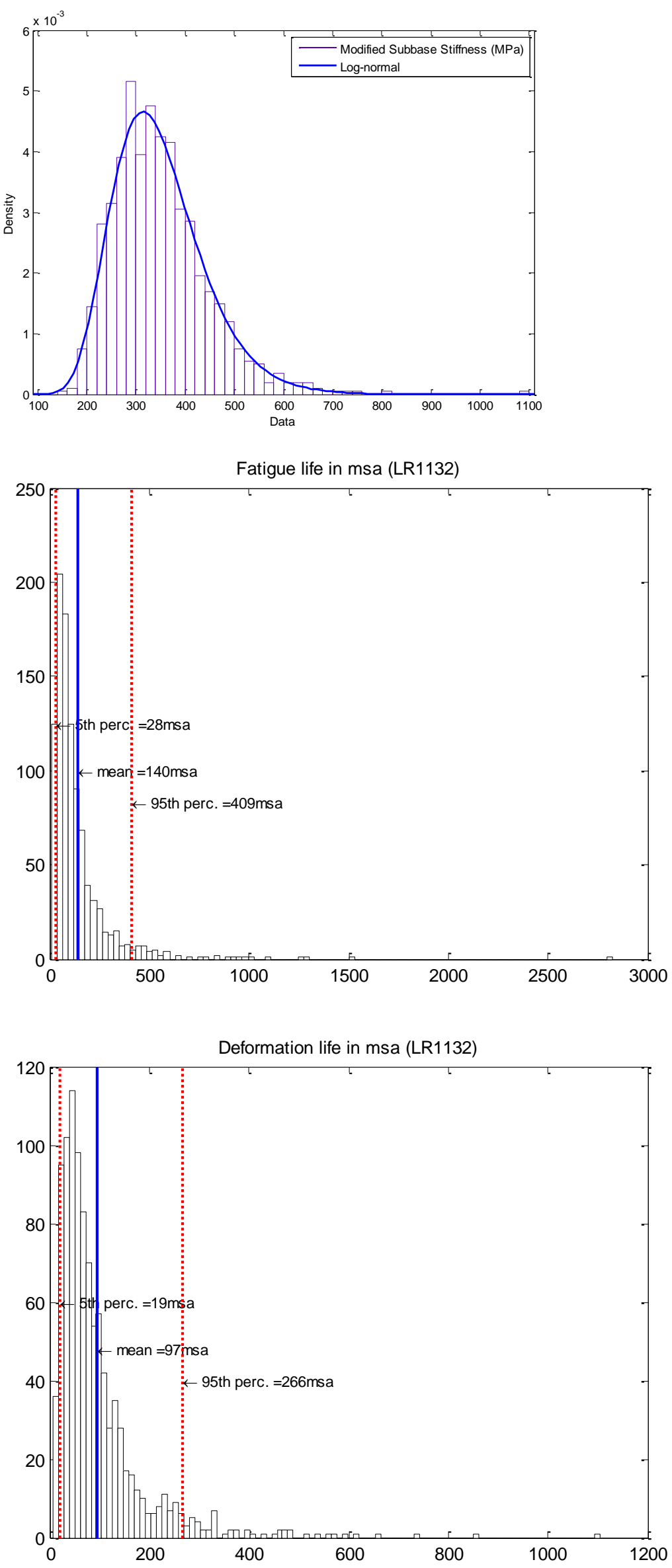

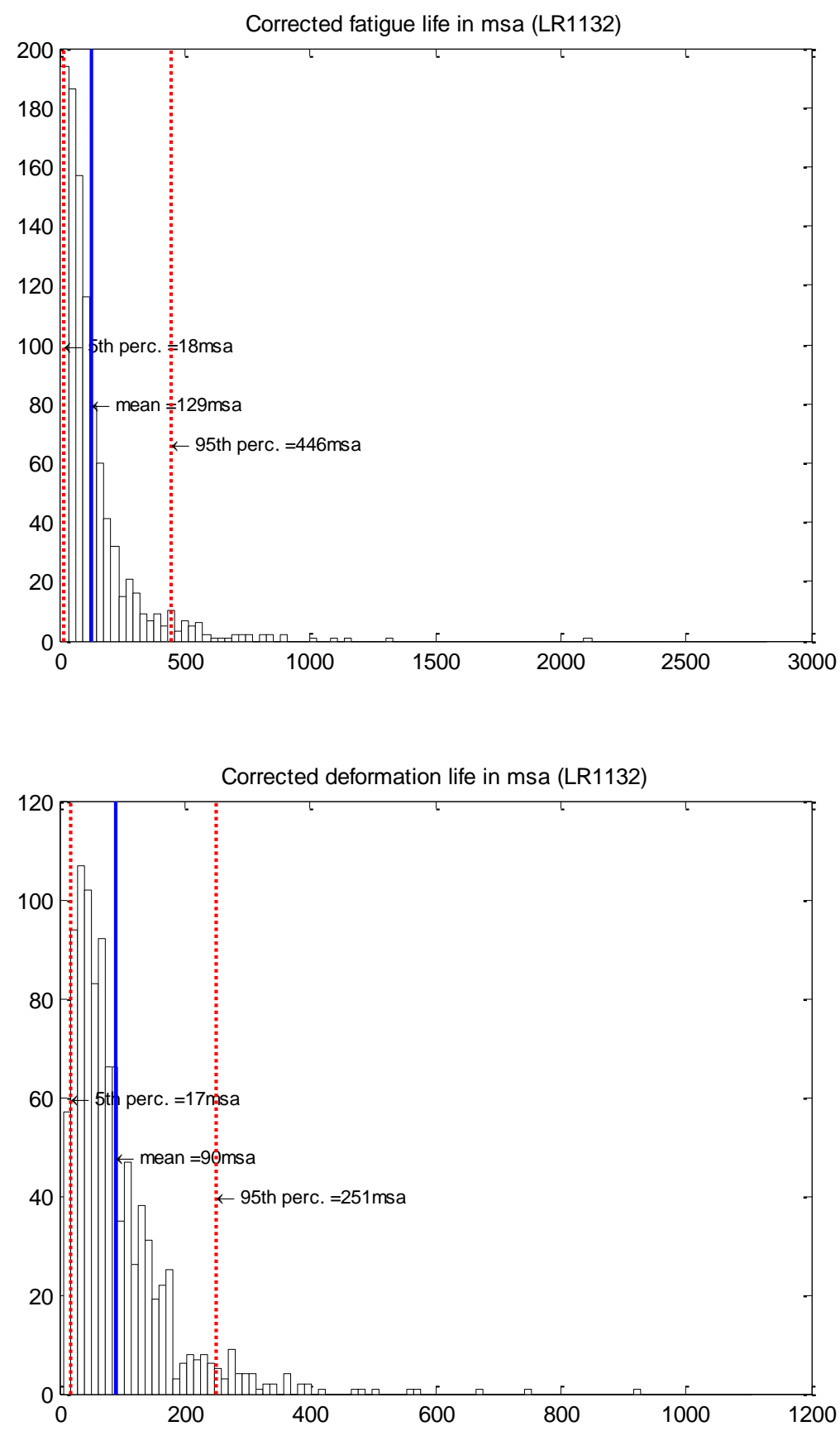


\section{A4.1.2 Section 16}
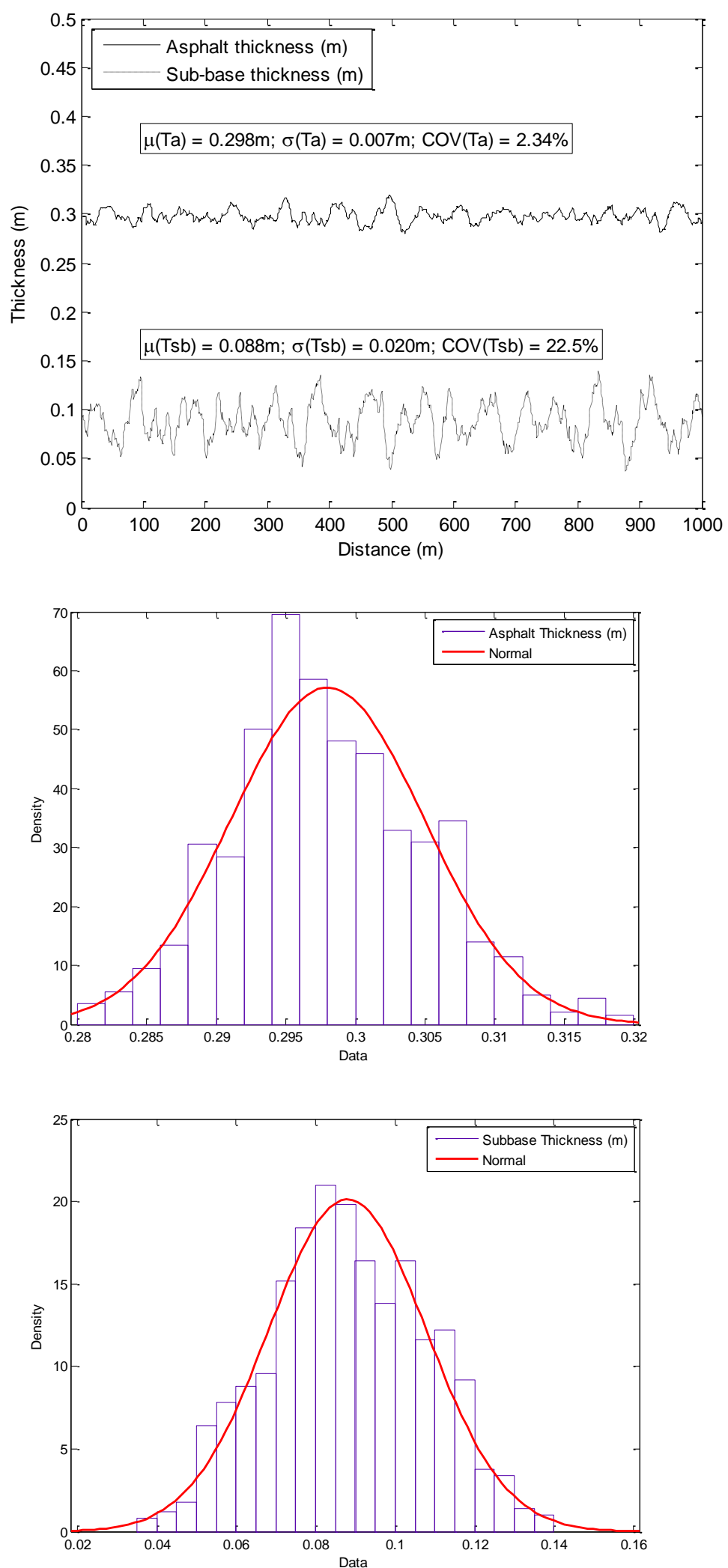

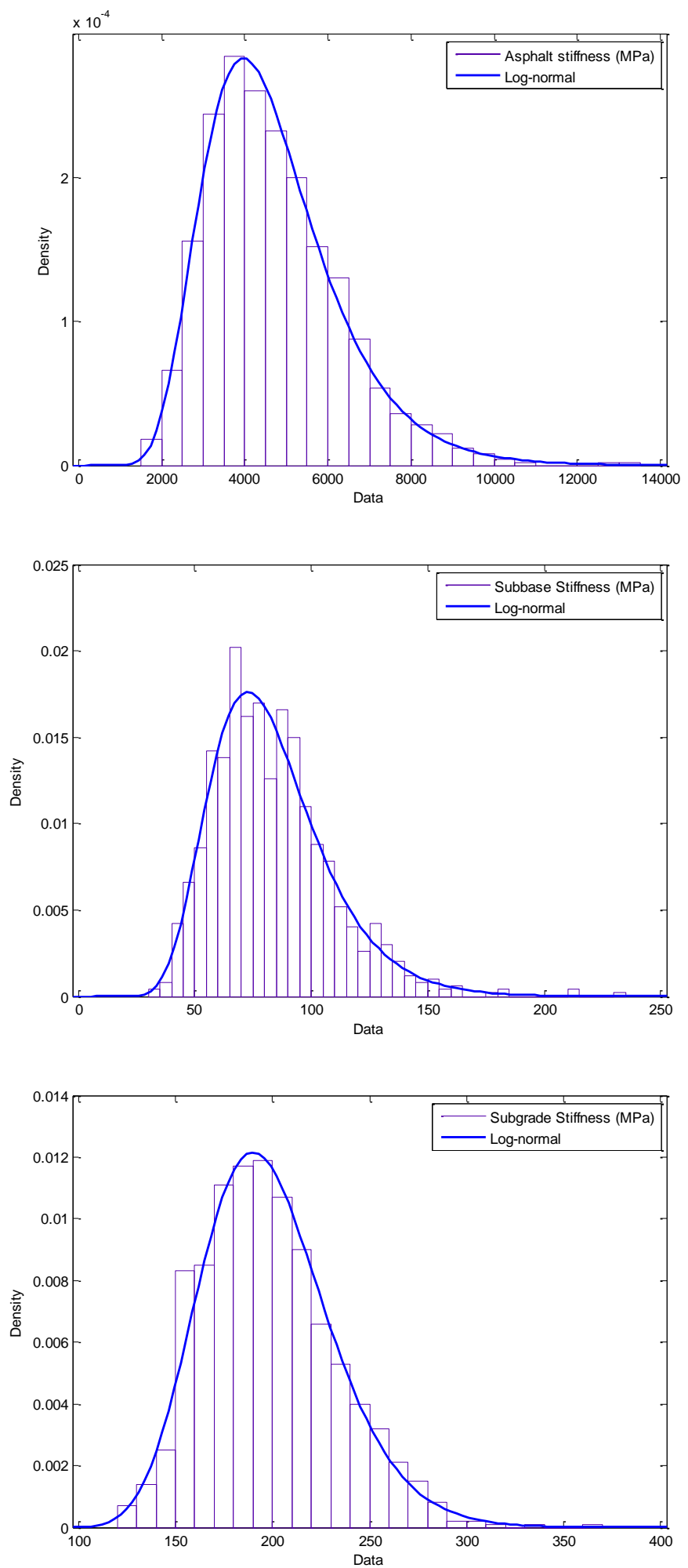

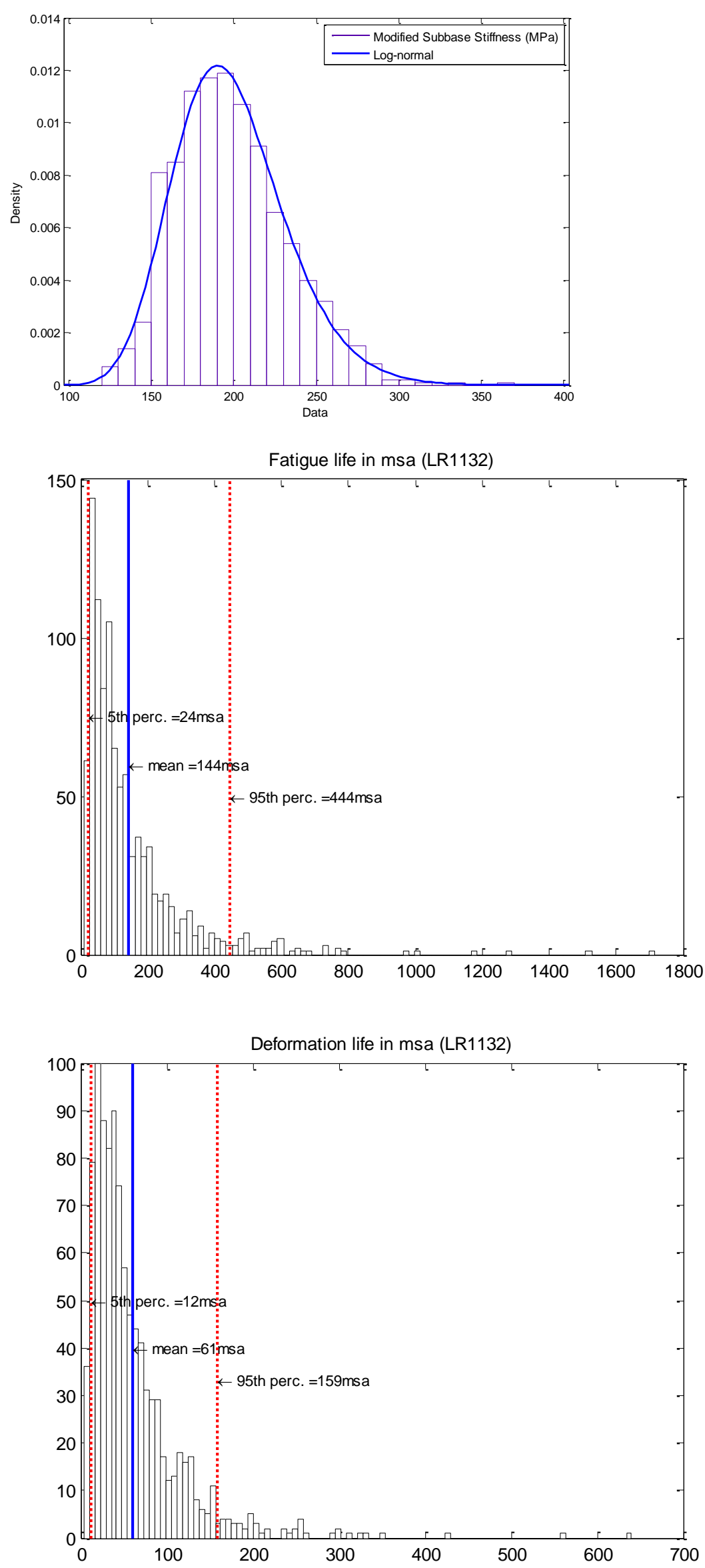


\section{A4.2 M04}

\section{A4.2.1 Section 1}
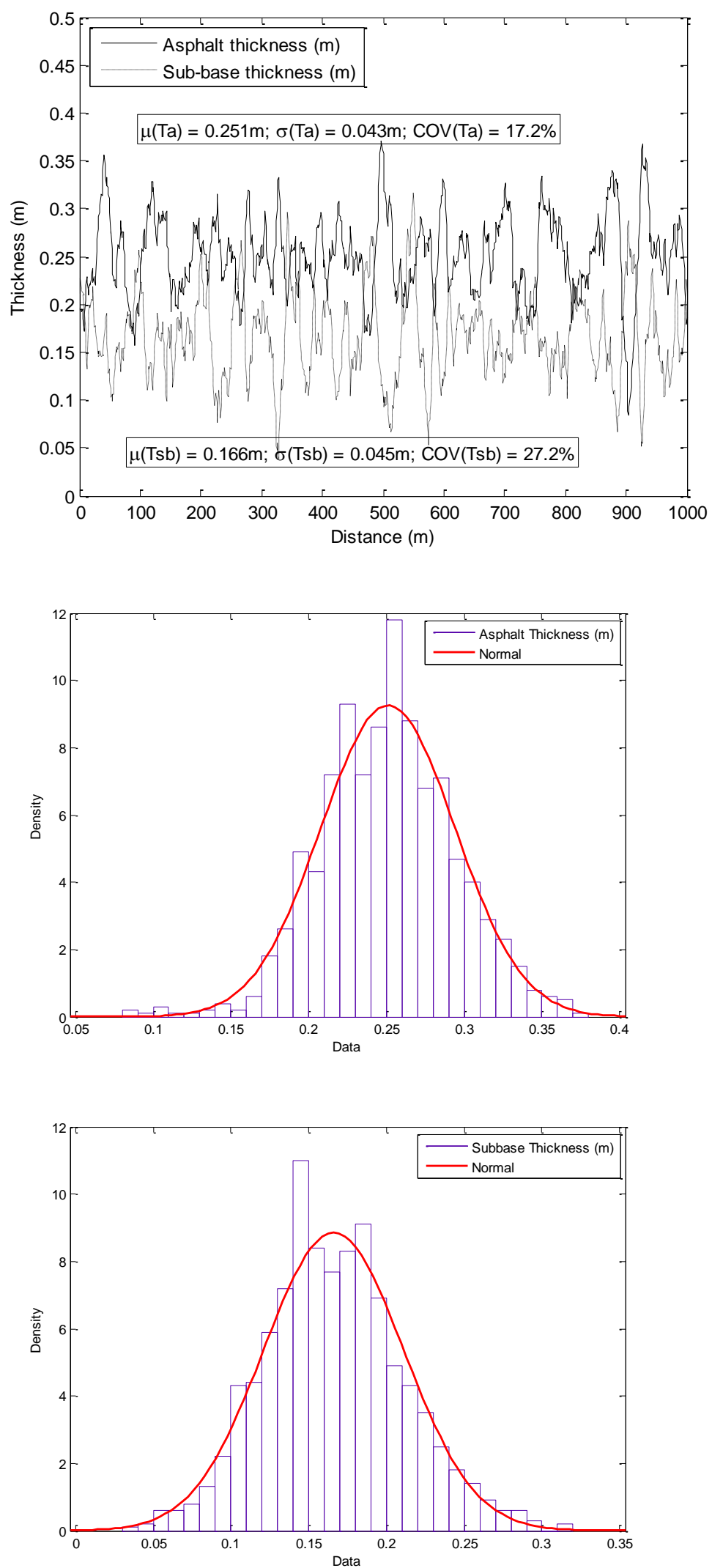

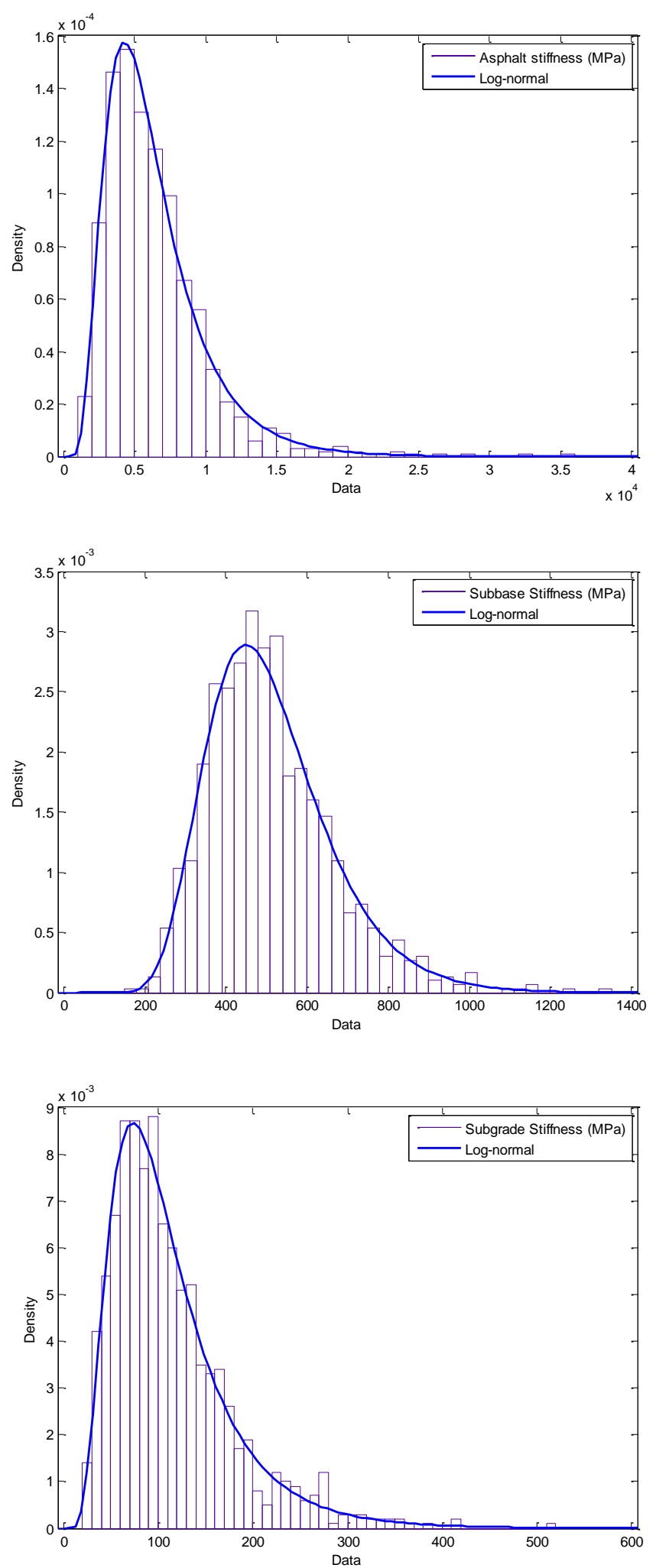

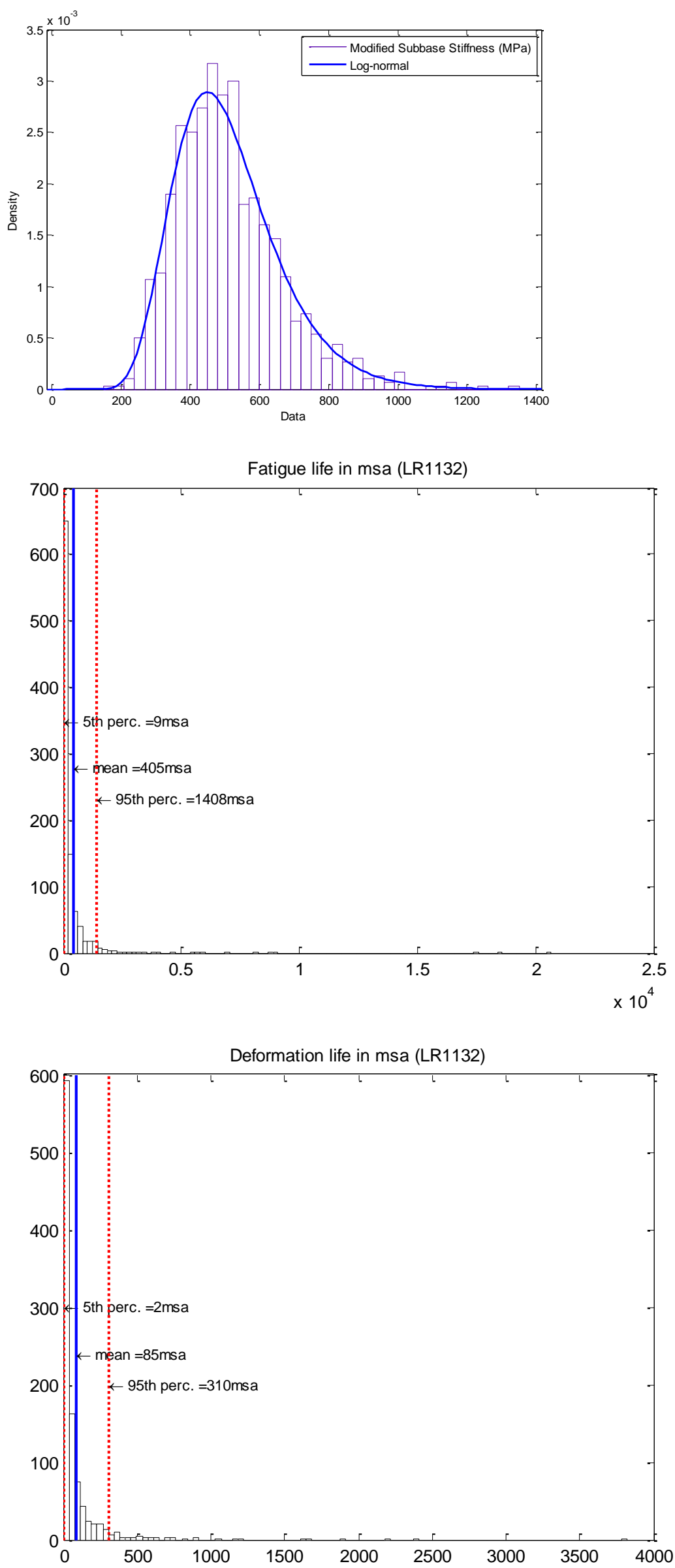
A4.2.2 Section 10
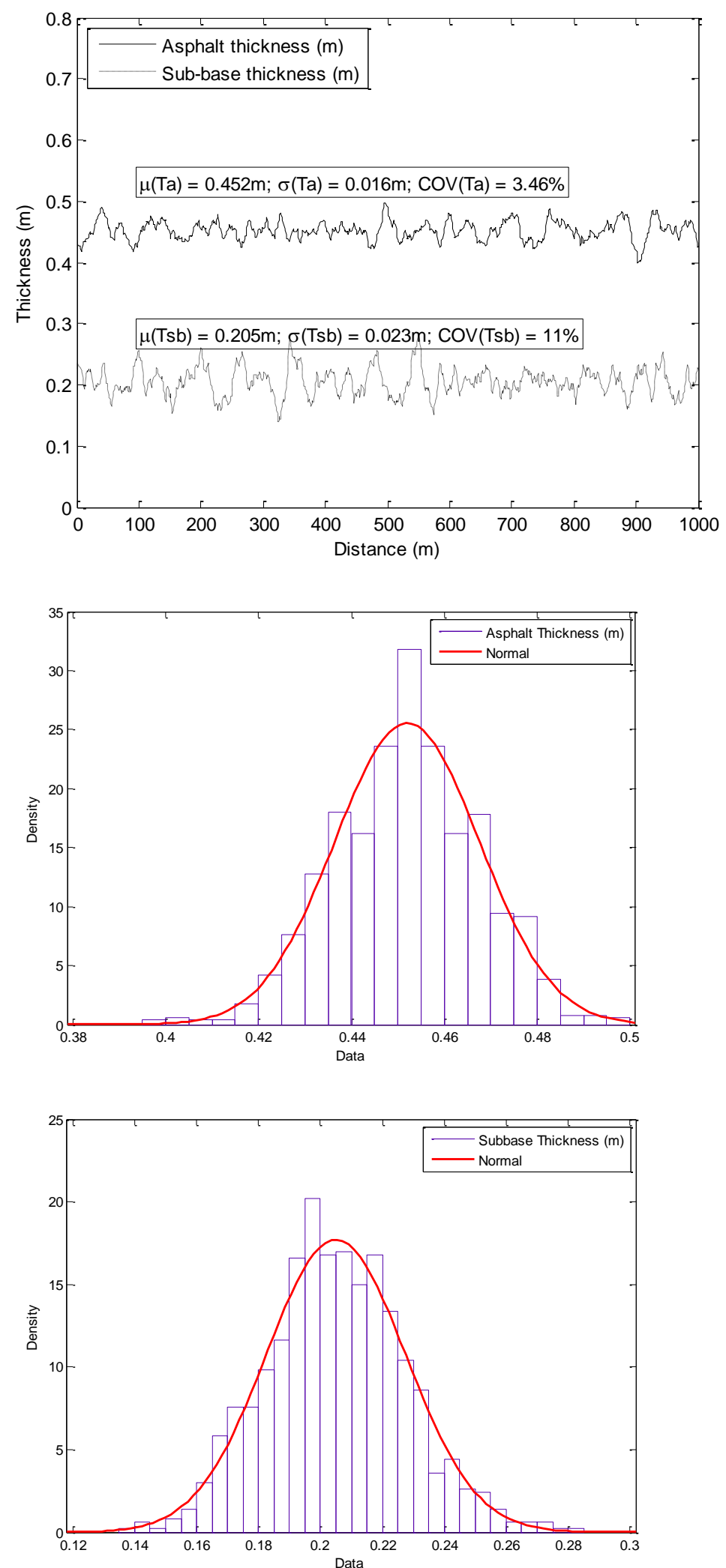

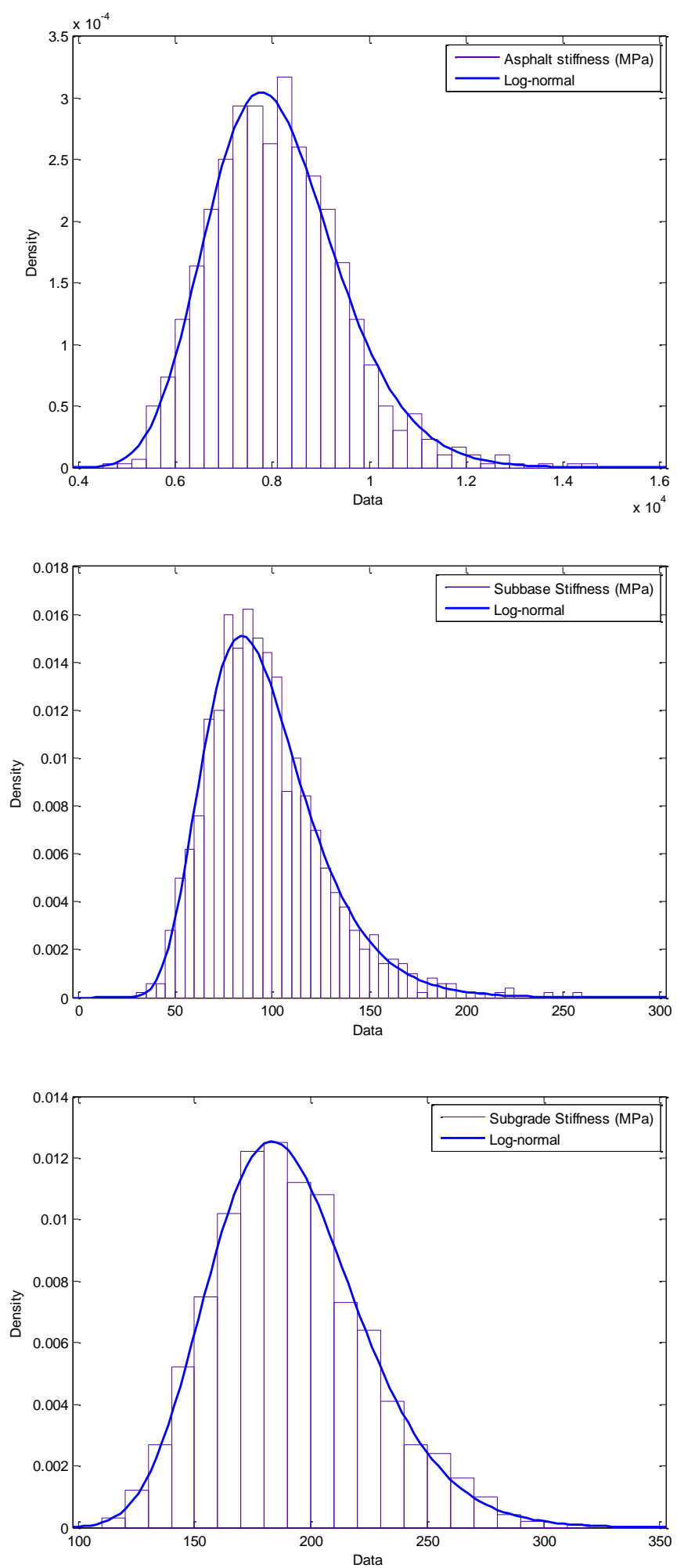

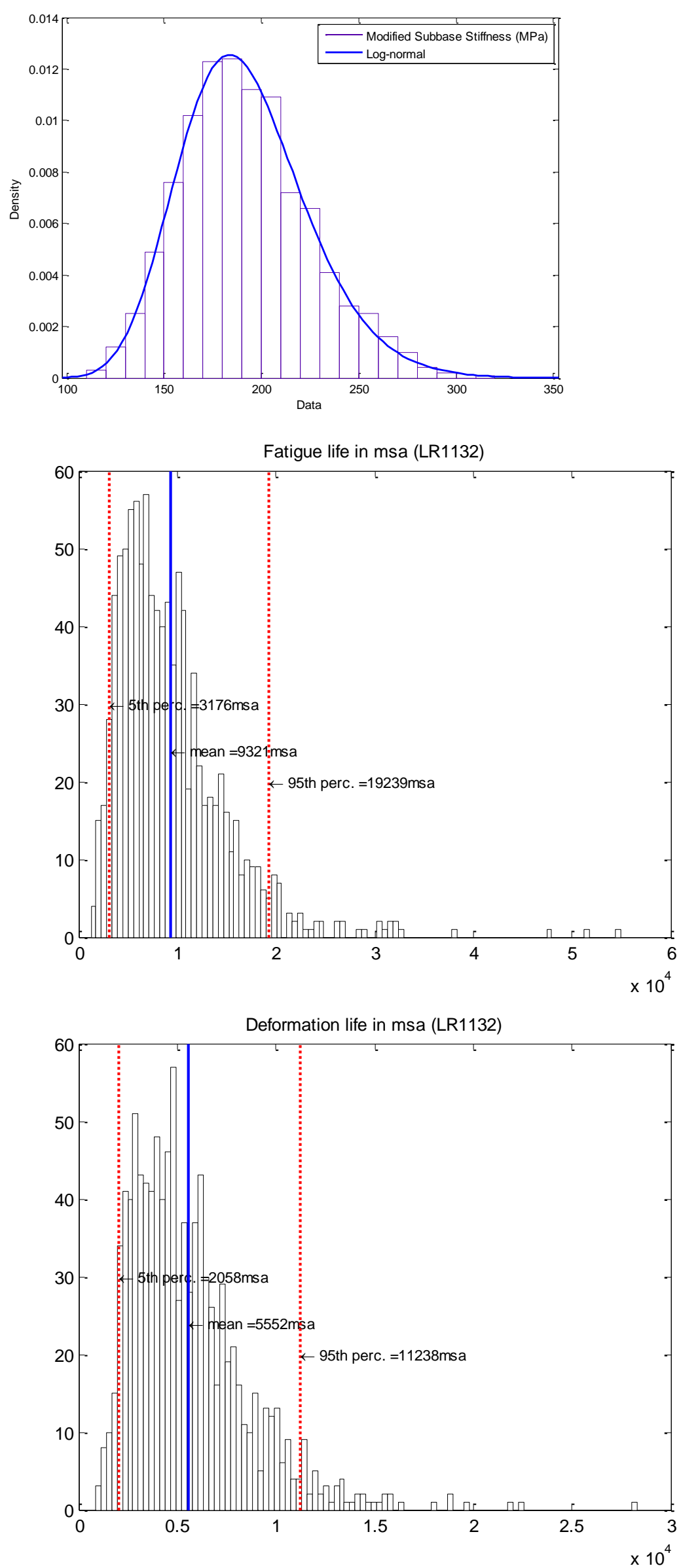
A4.2.3 Section 20
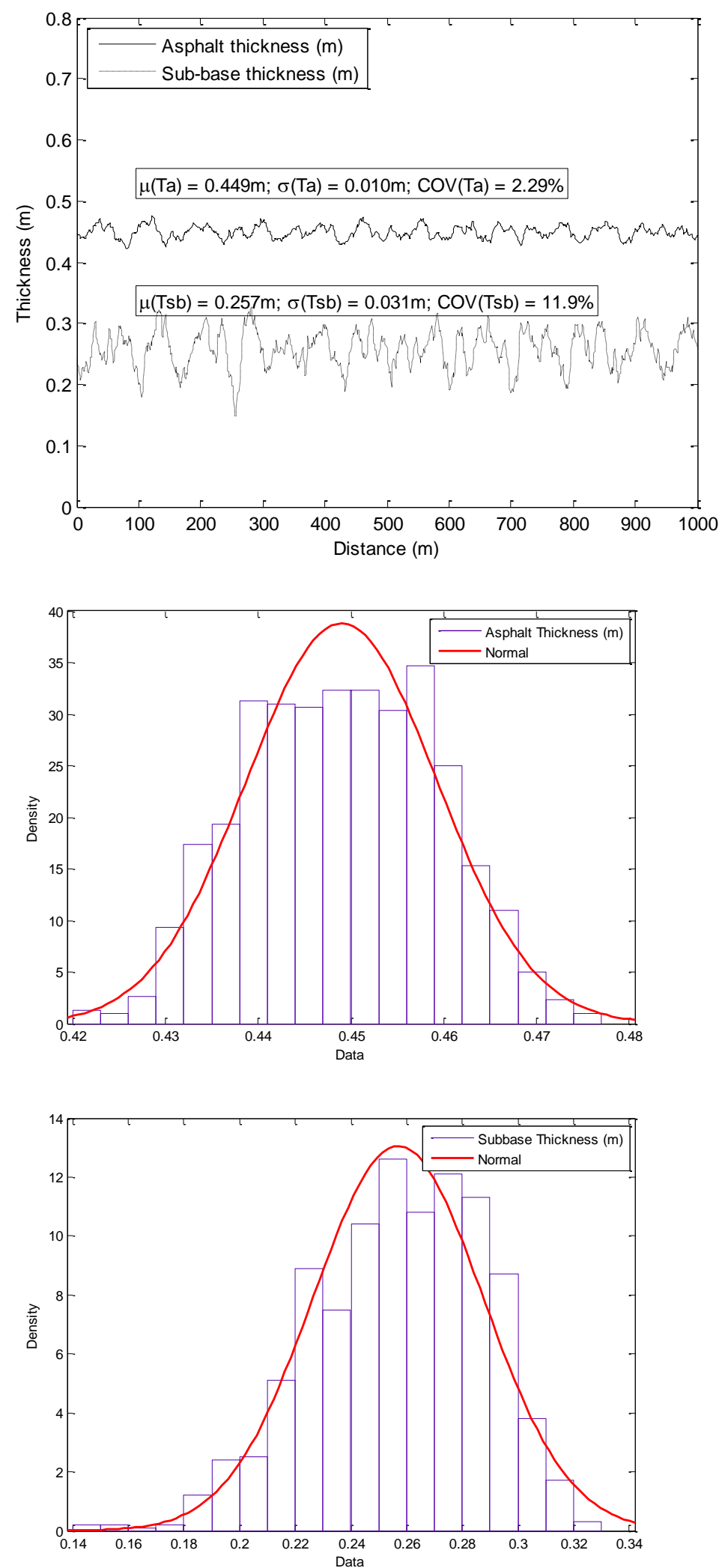

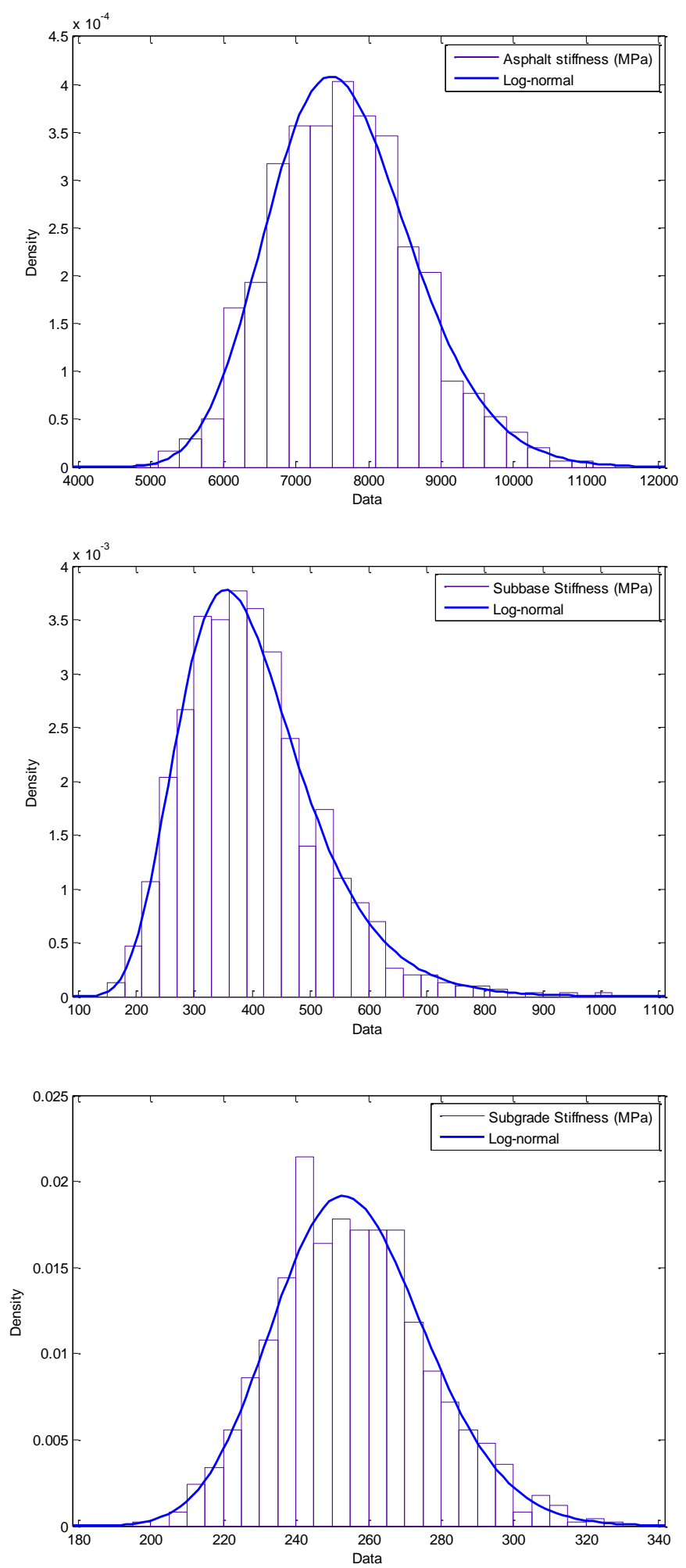

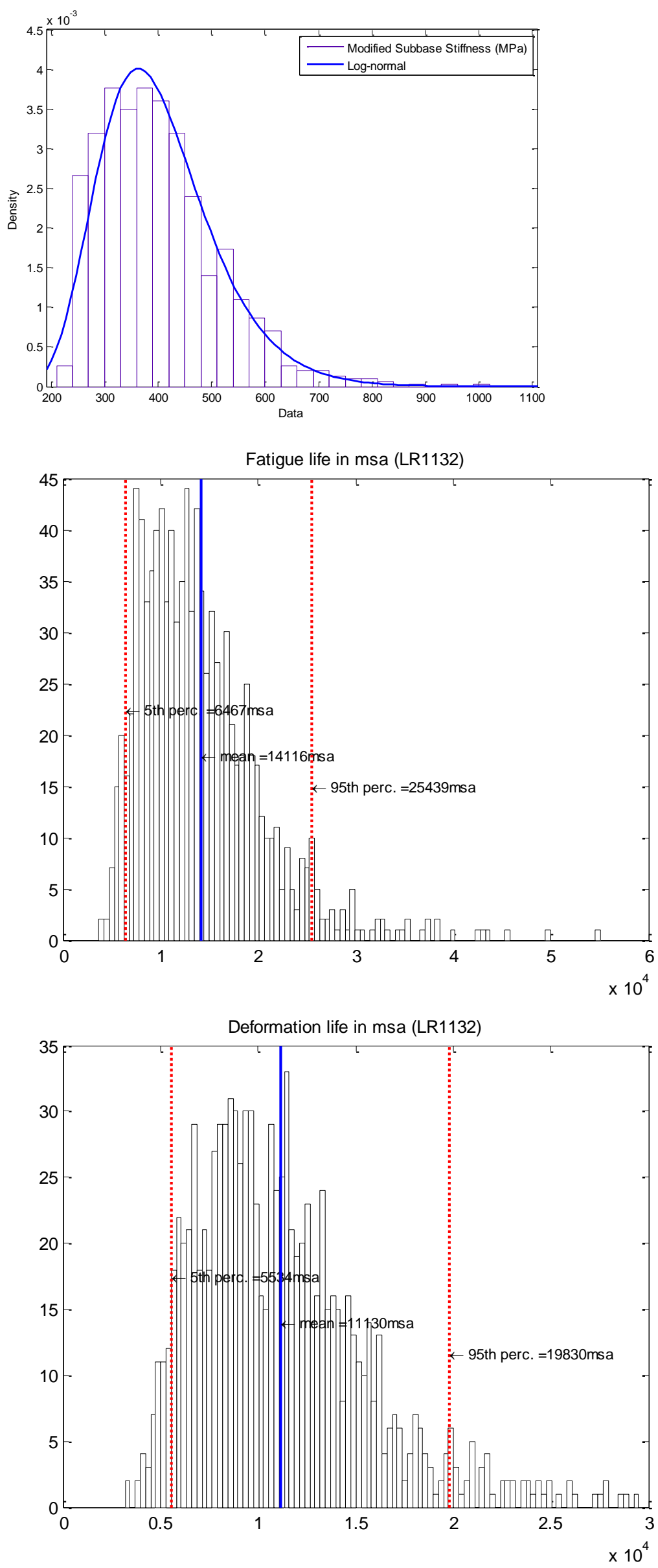
A4.2.4 Section 30
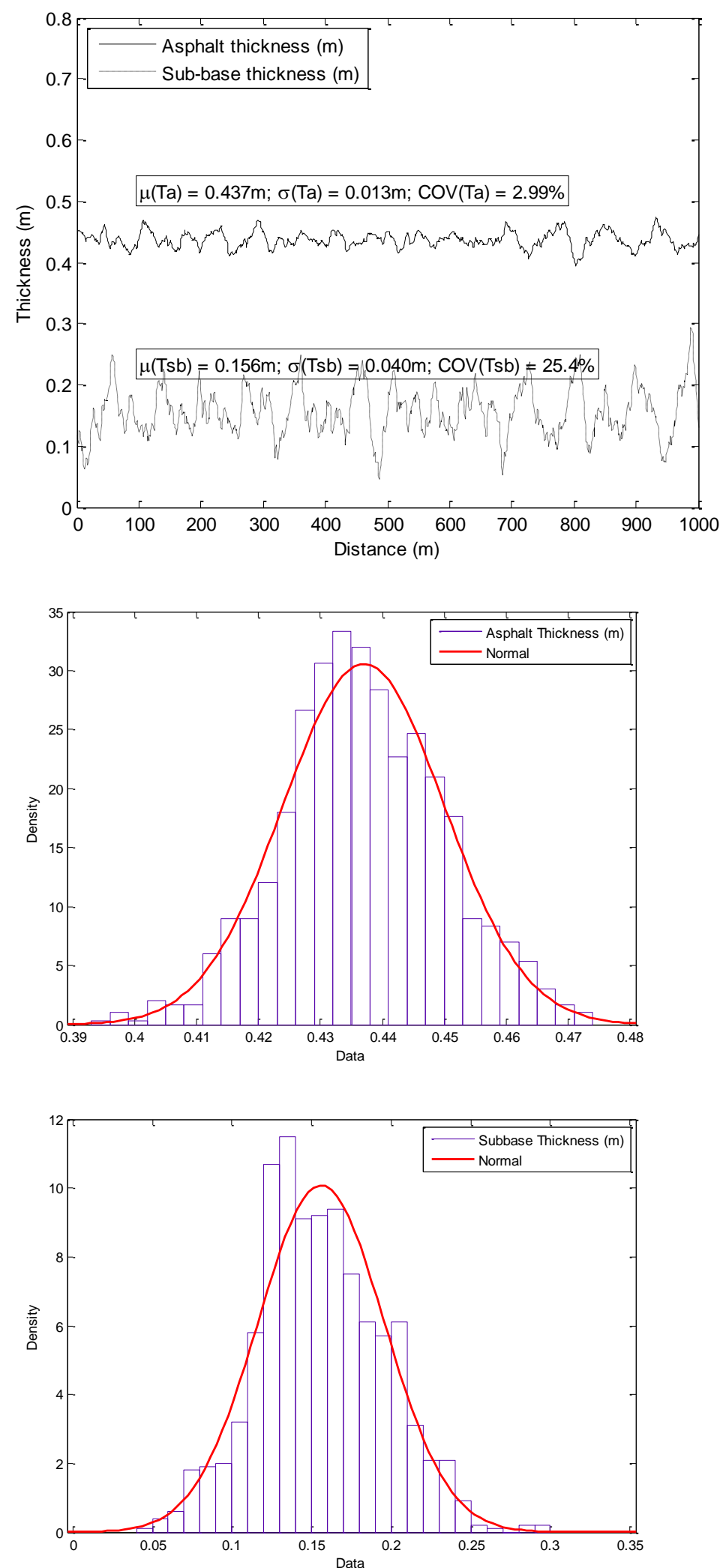

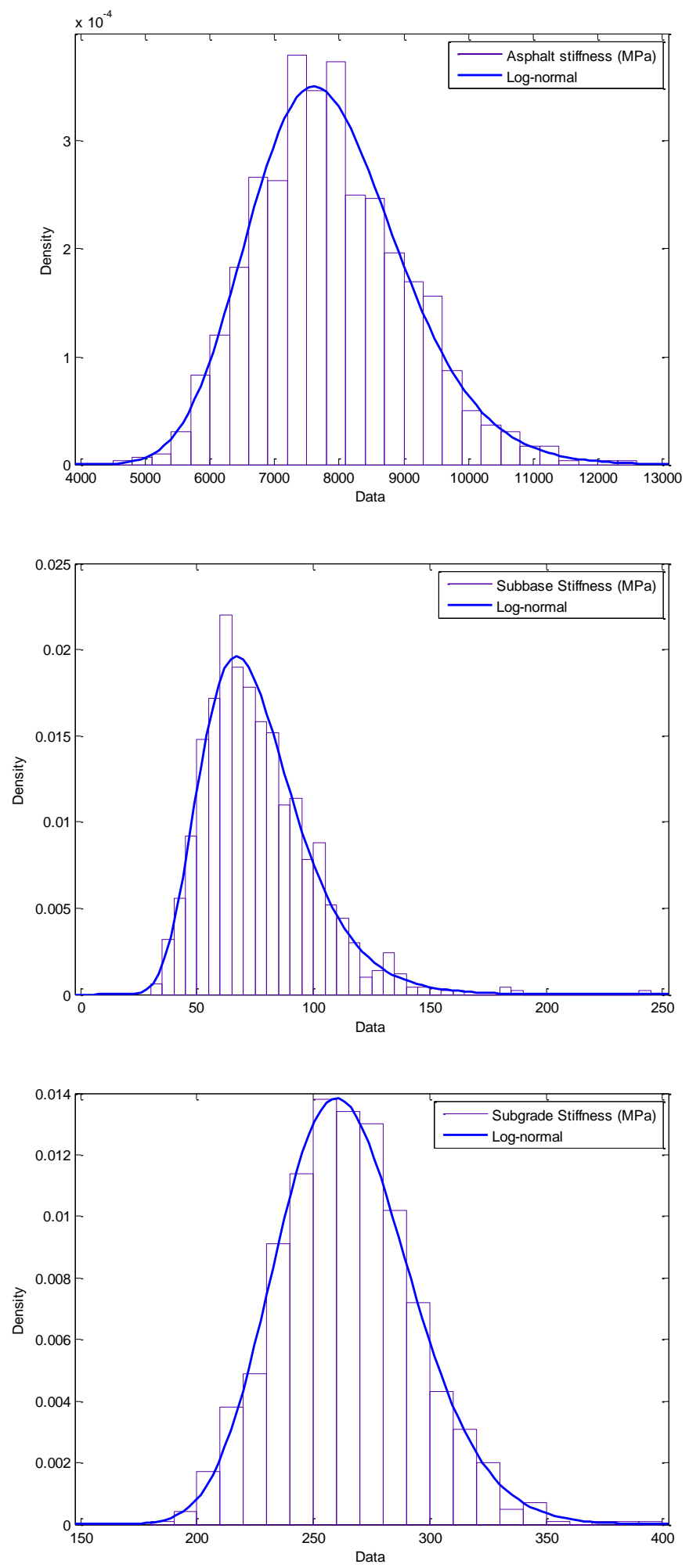

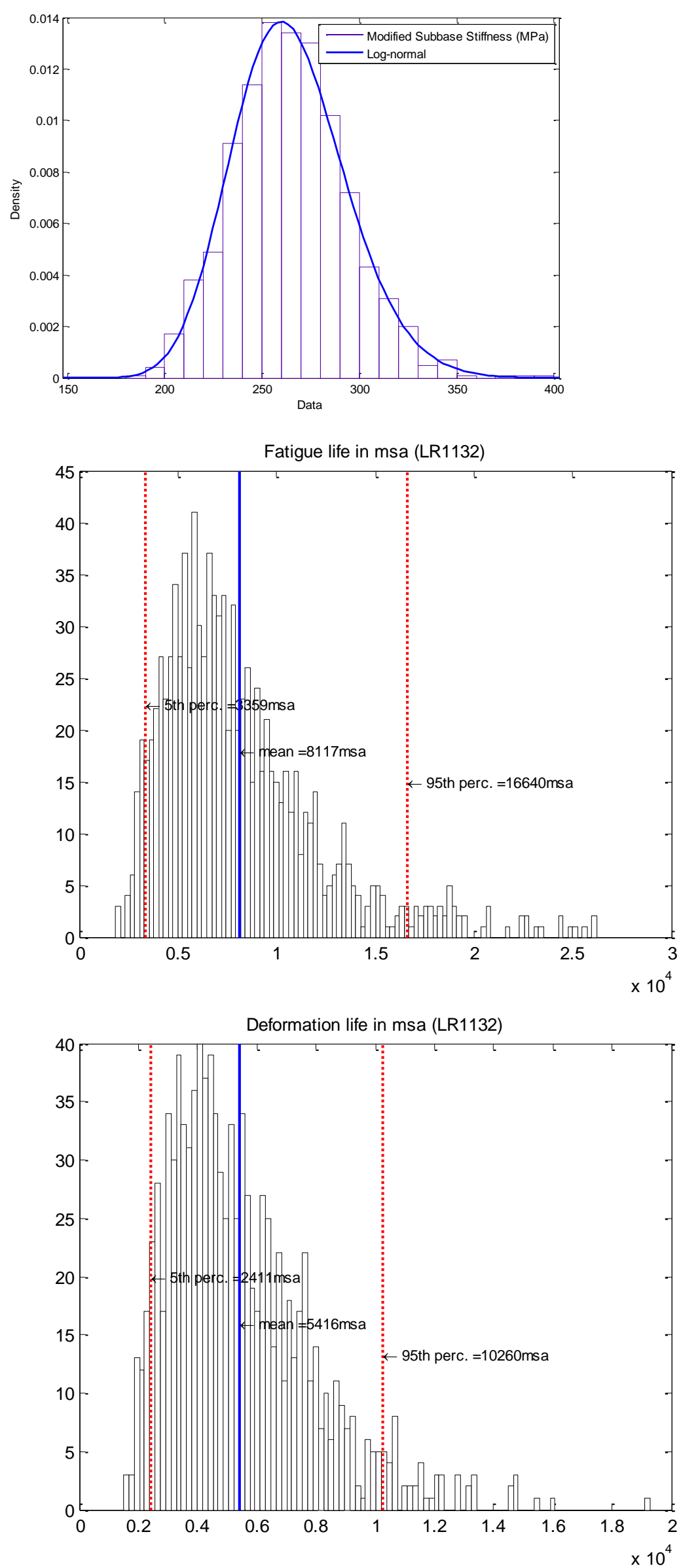
A4.2.5 Section 37
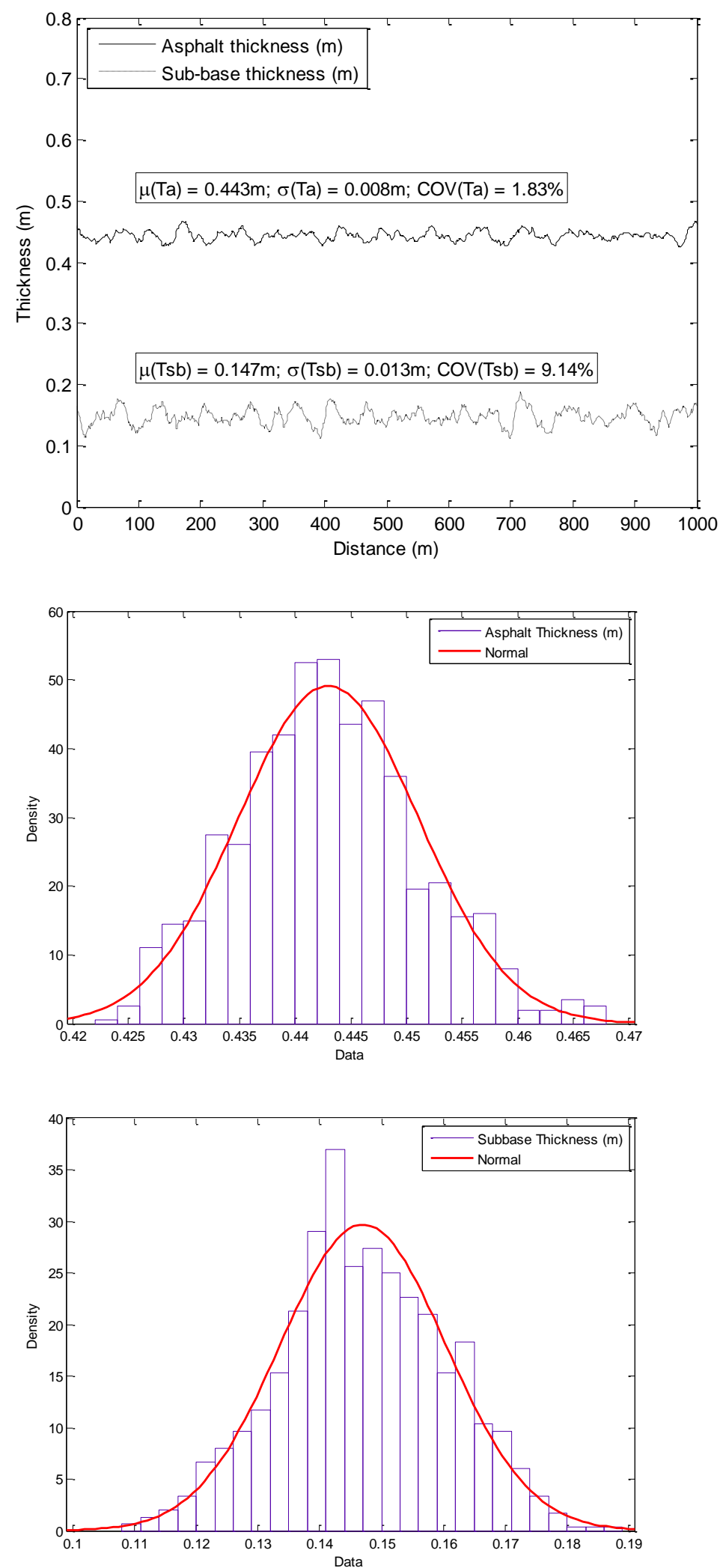

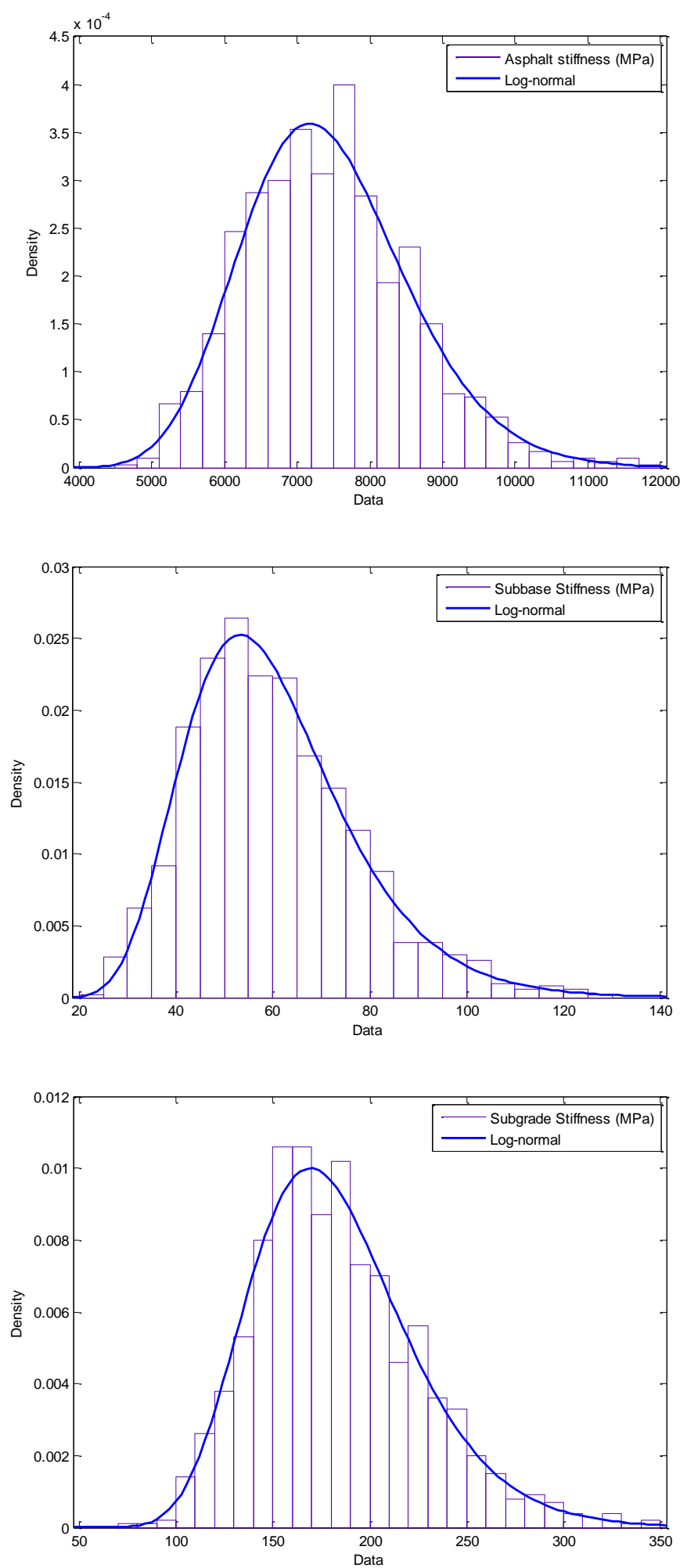

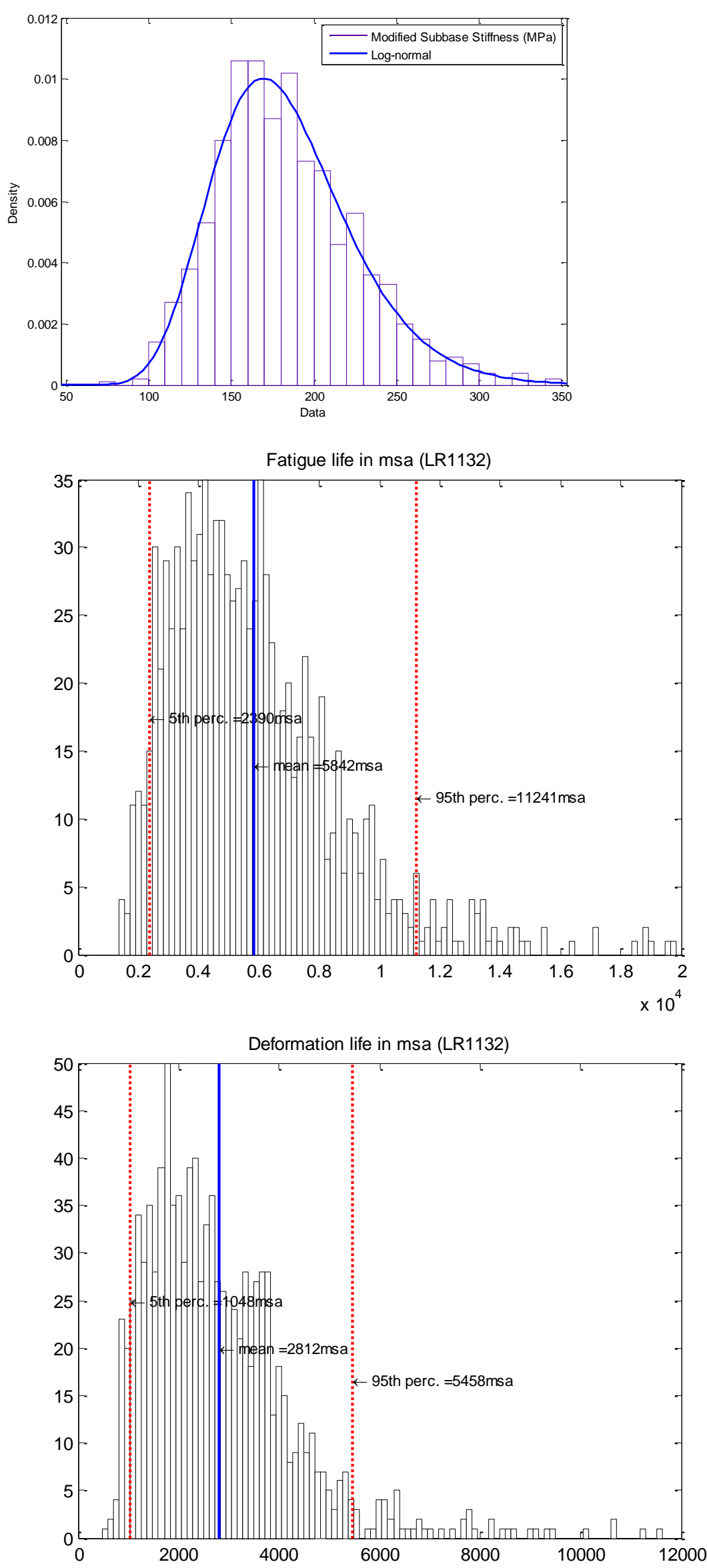


\section{A4.3 M05 ACW}

\section{A4.3.1 Section 10}
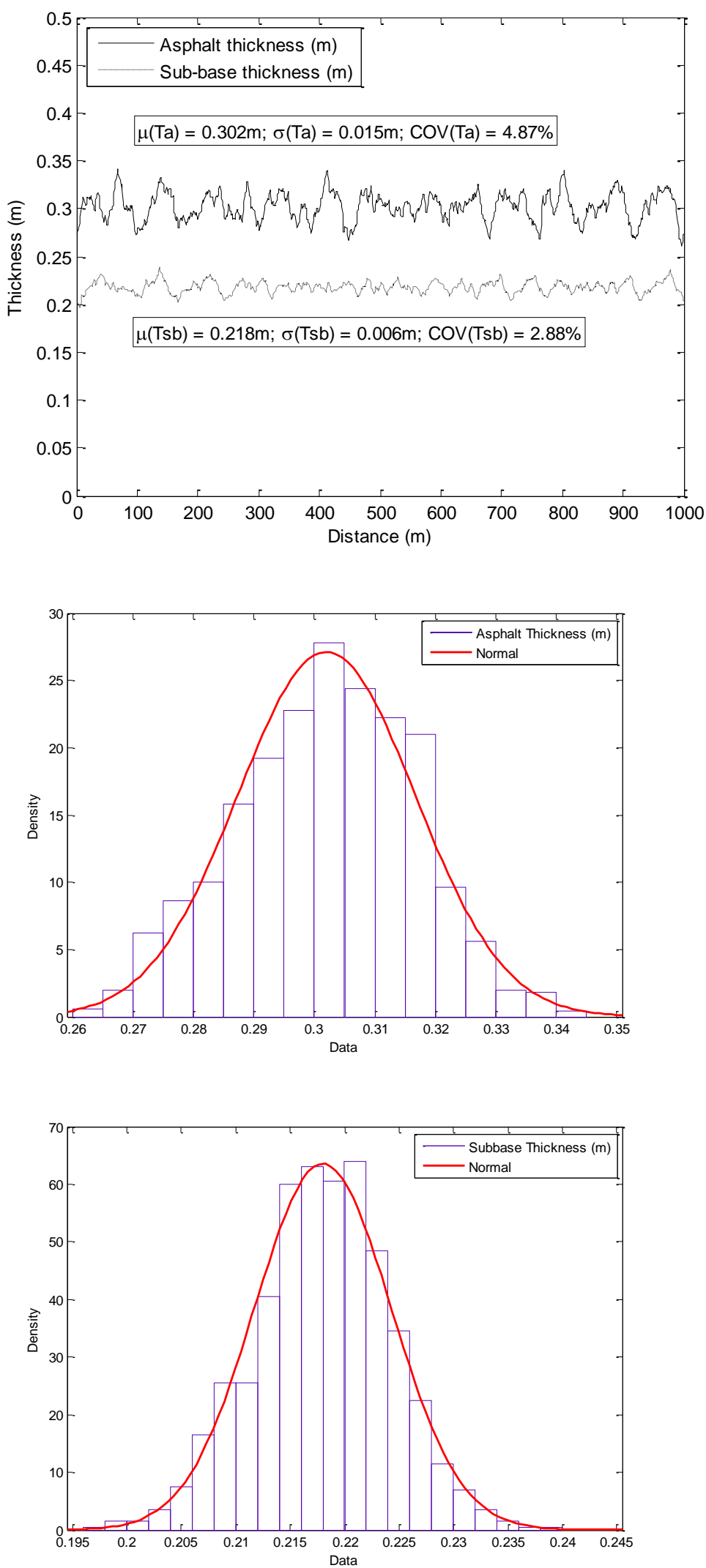

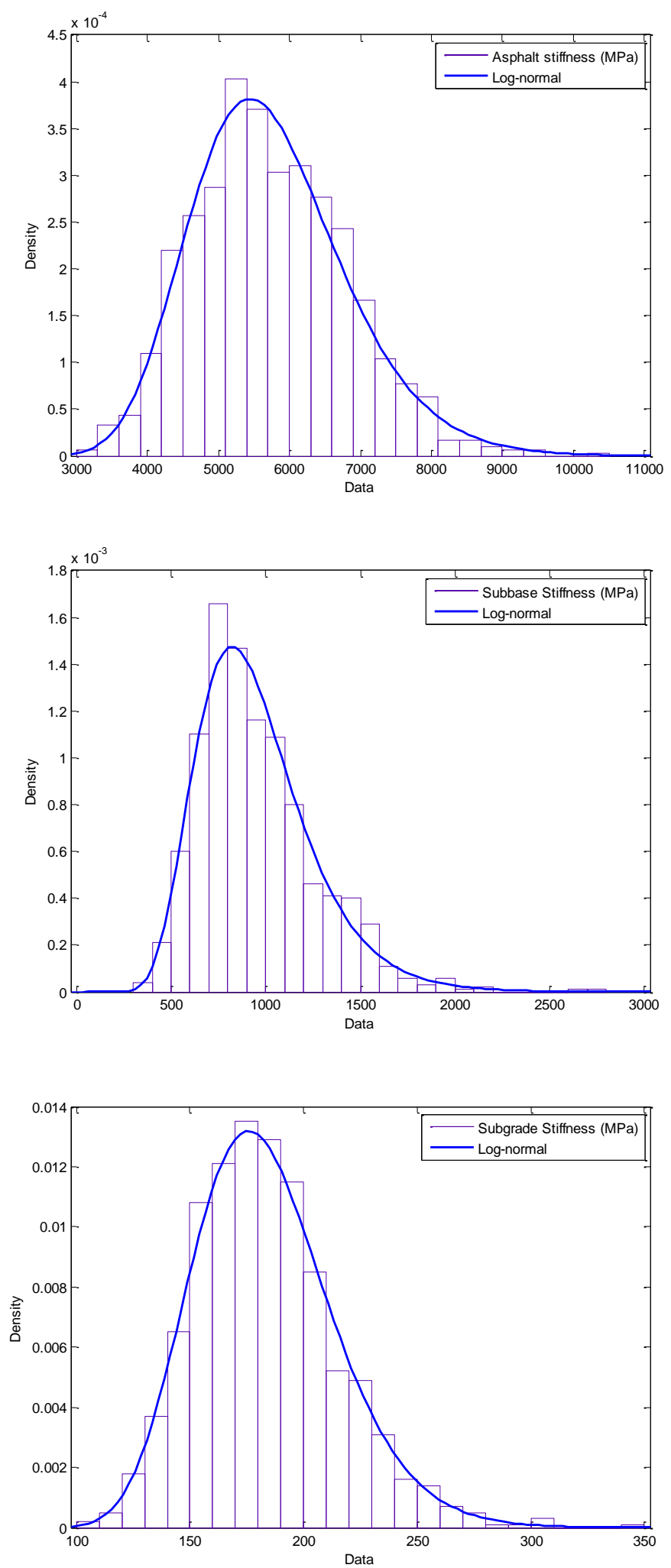


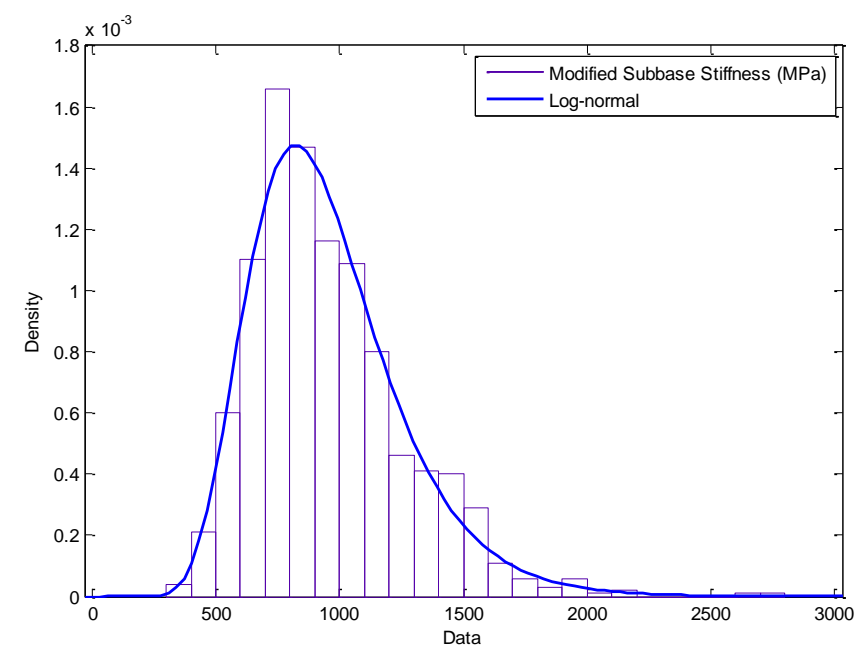

Fatigue life in msa (LR1132)
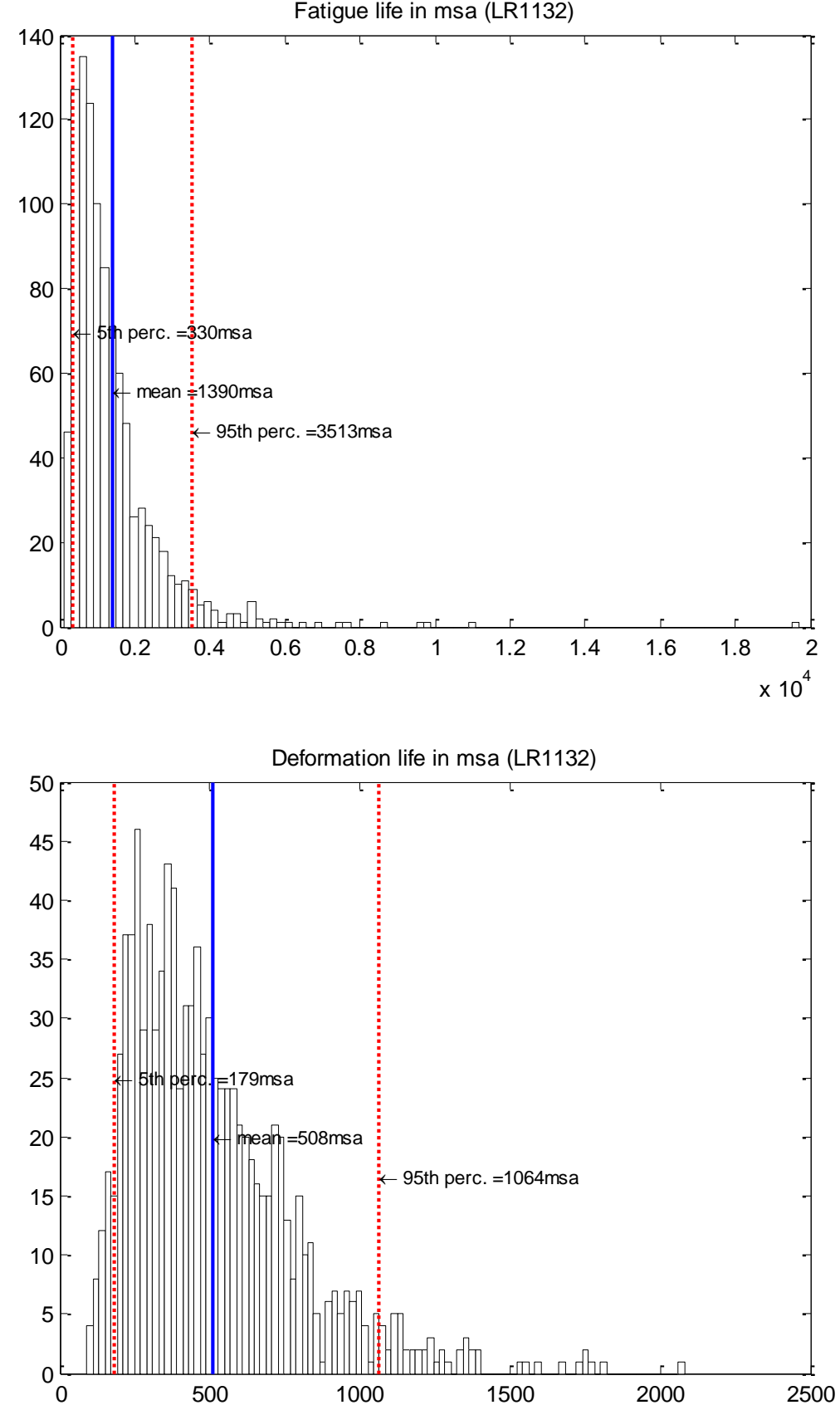
A4.3.2 Section 11
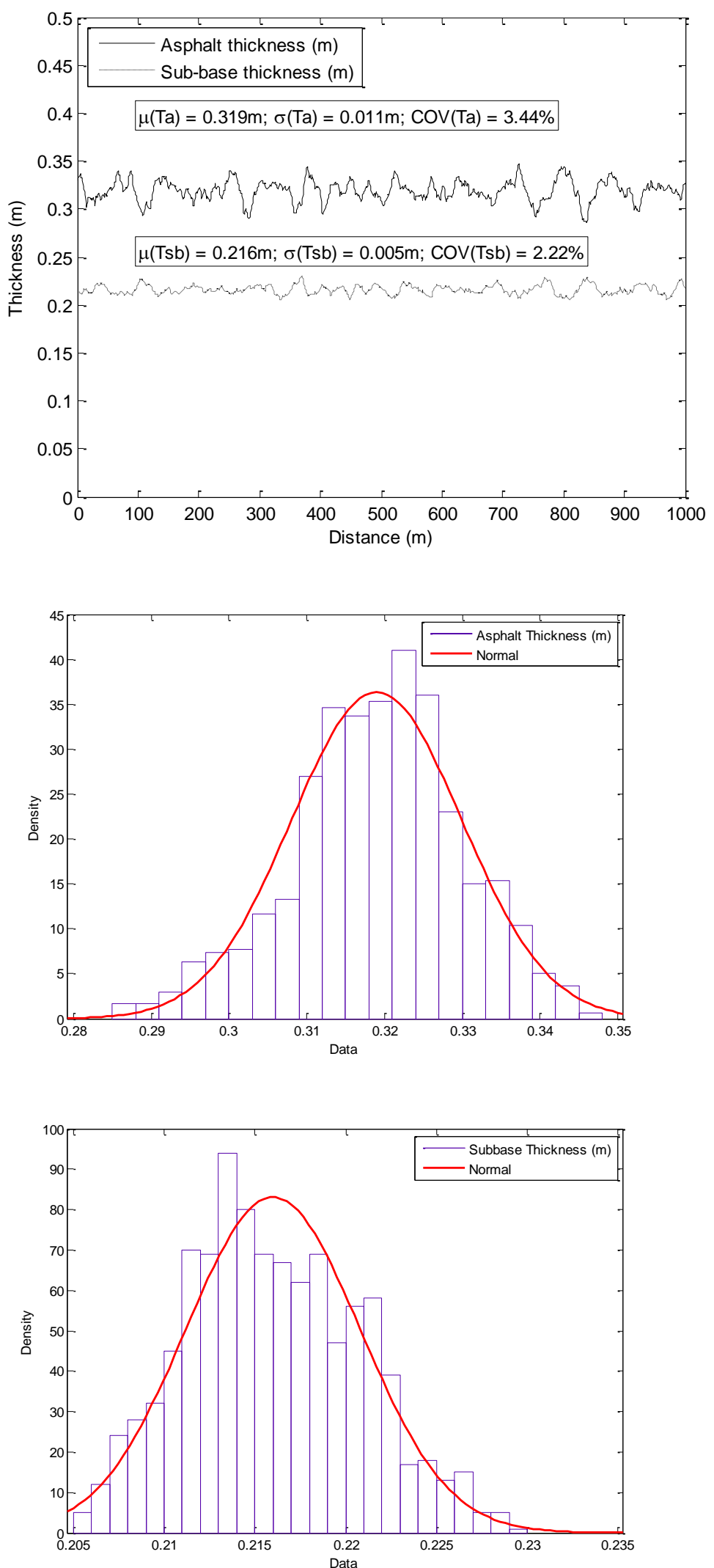

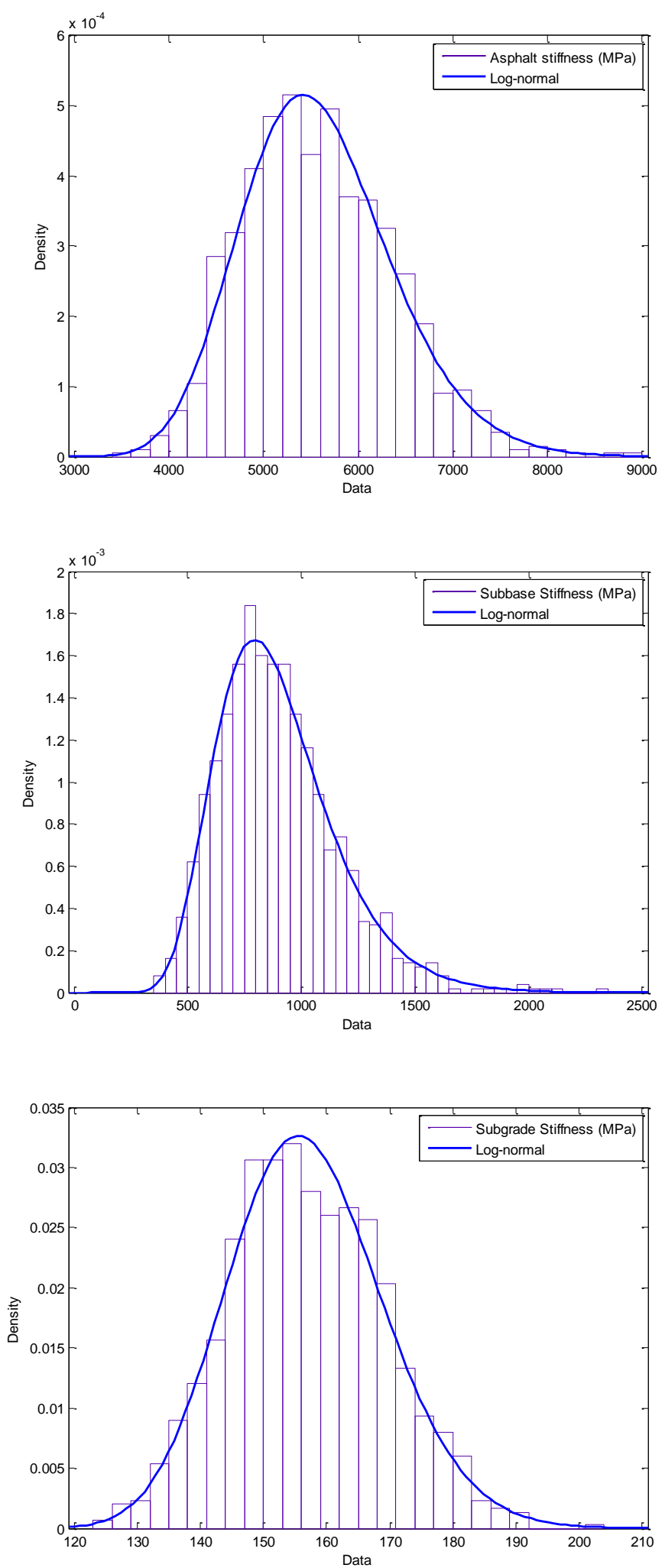

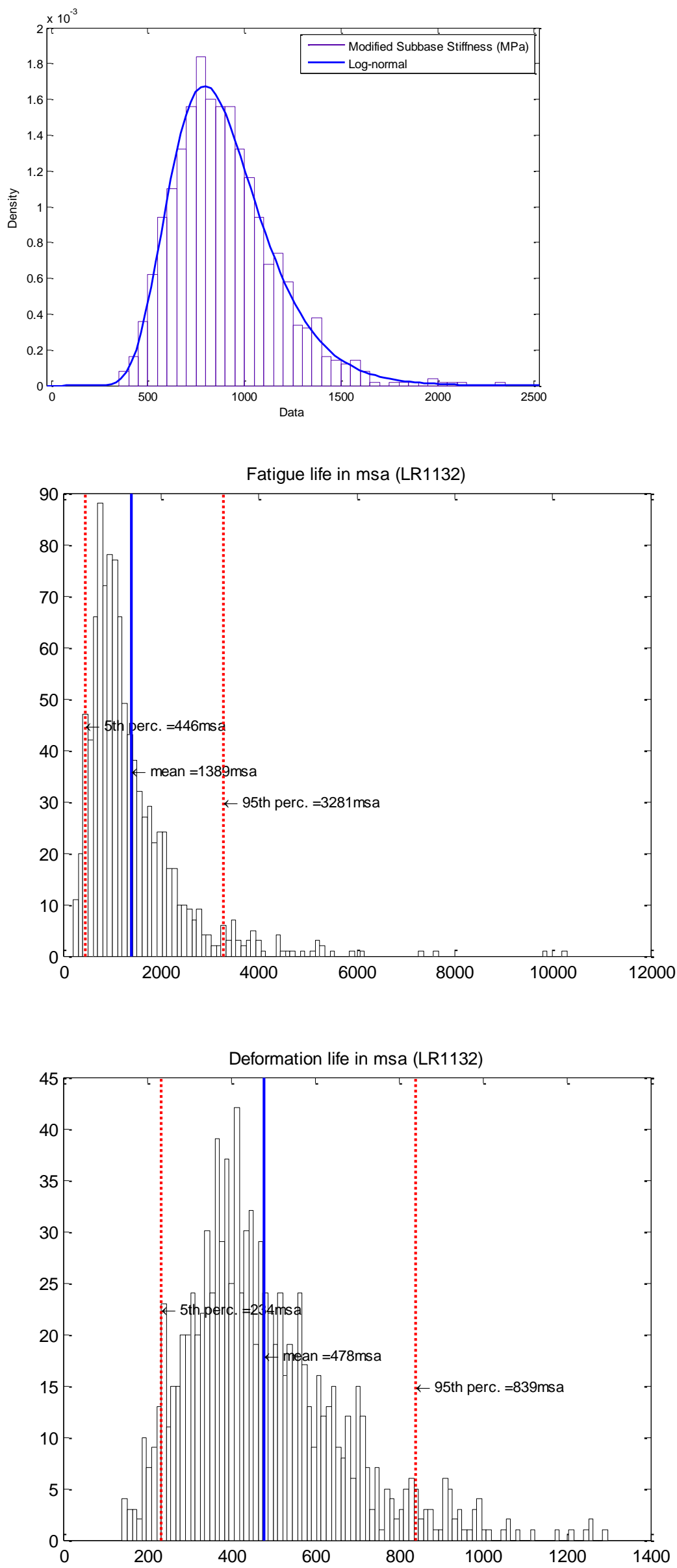


\section{A4.4.1 Section 4}
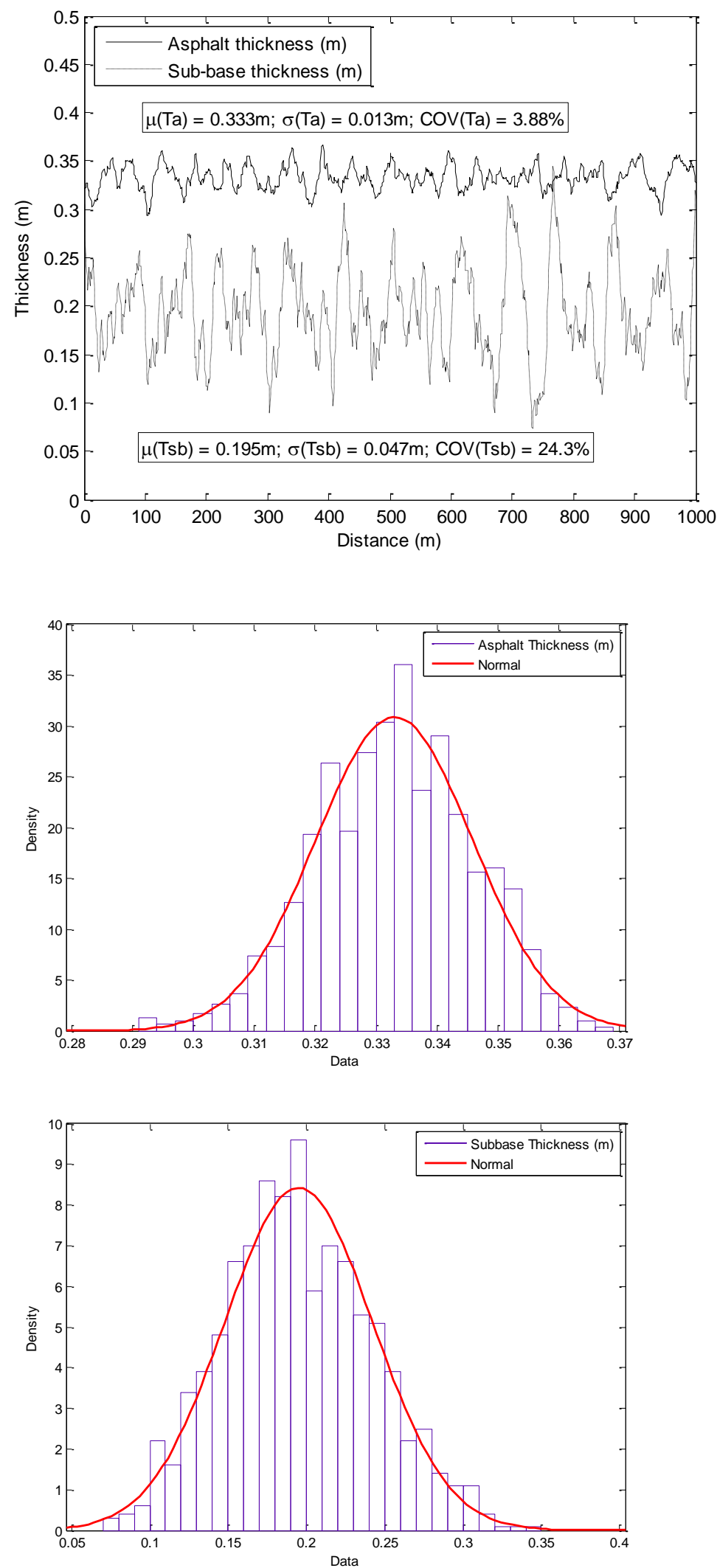

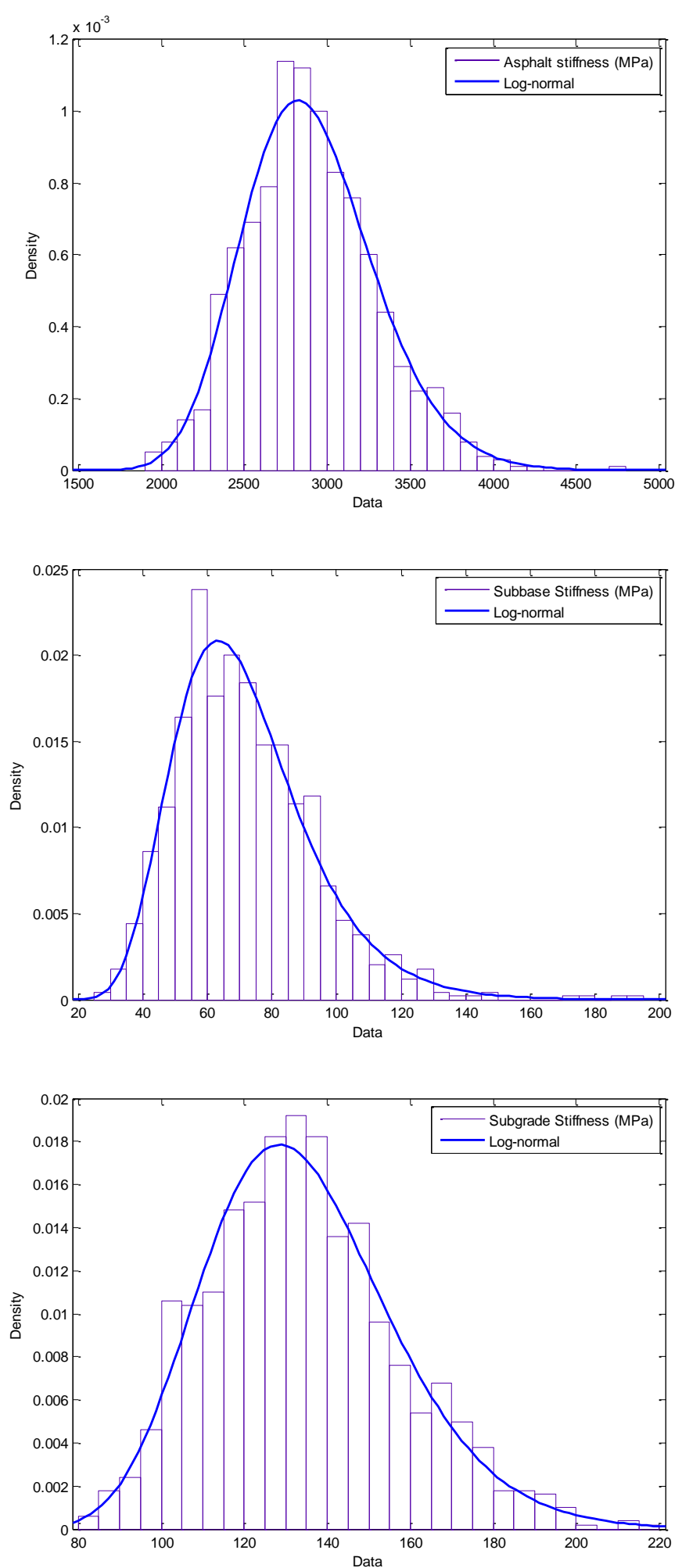

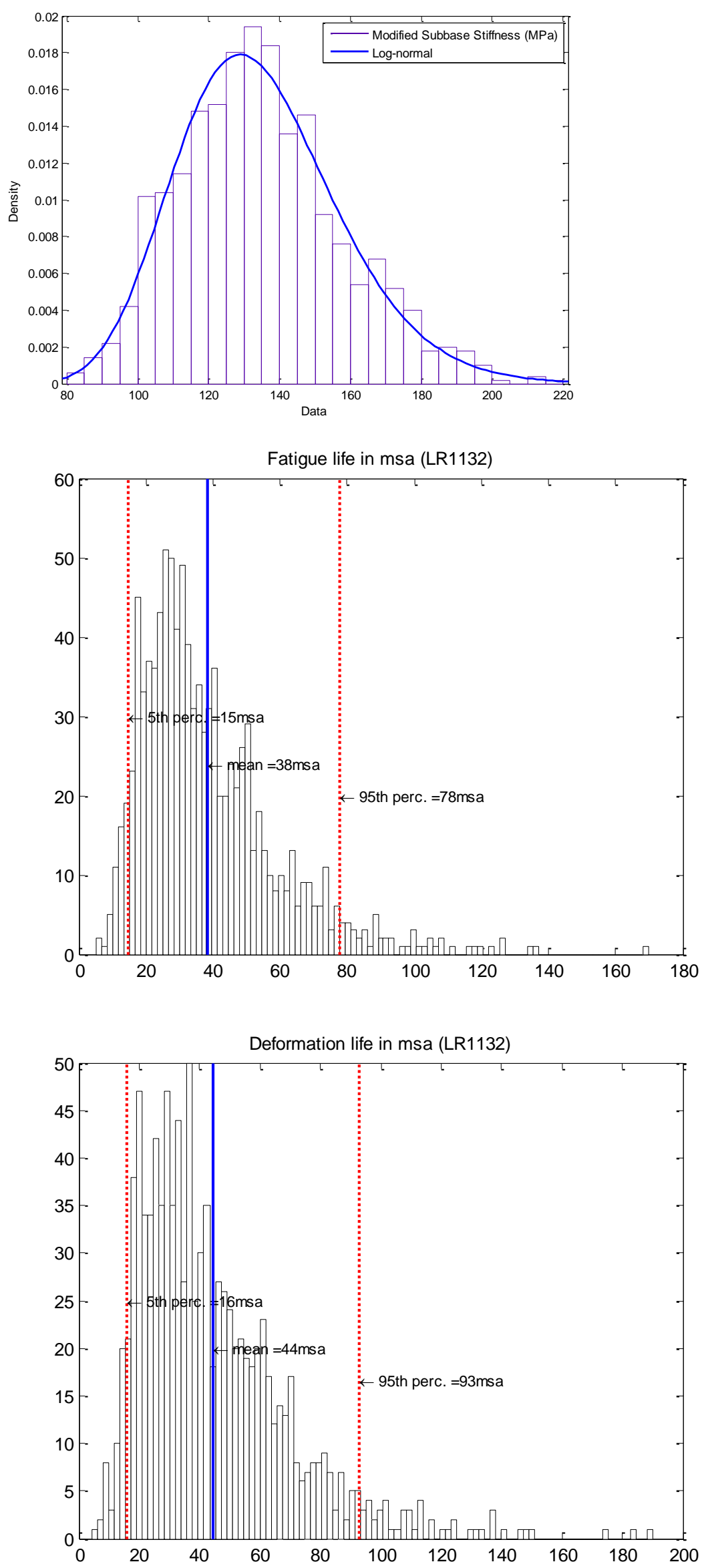
A4.4.2 Section 42
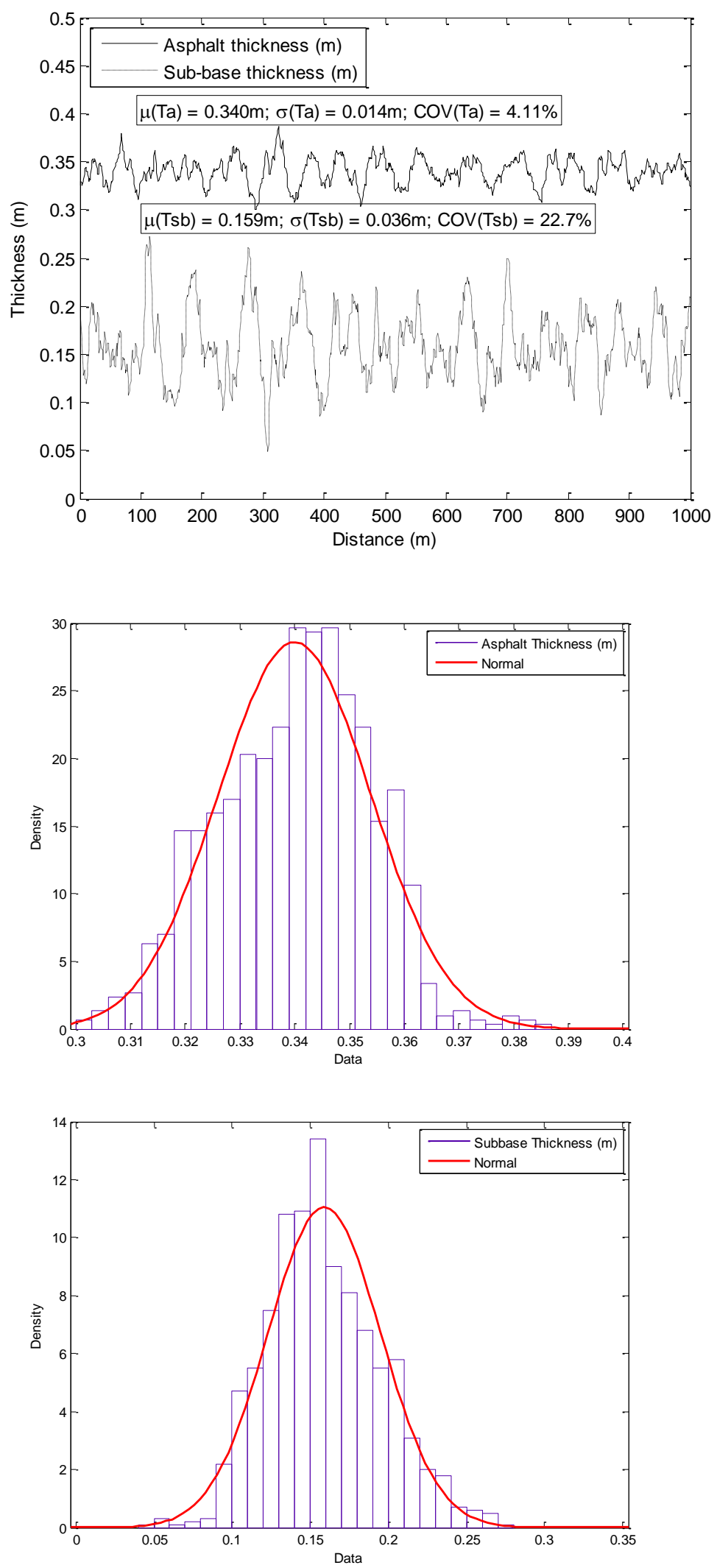

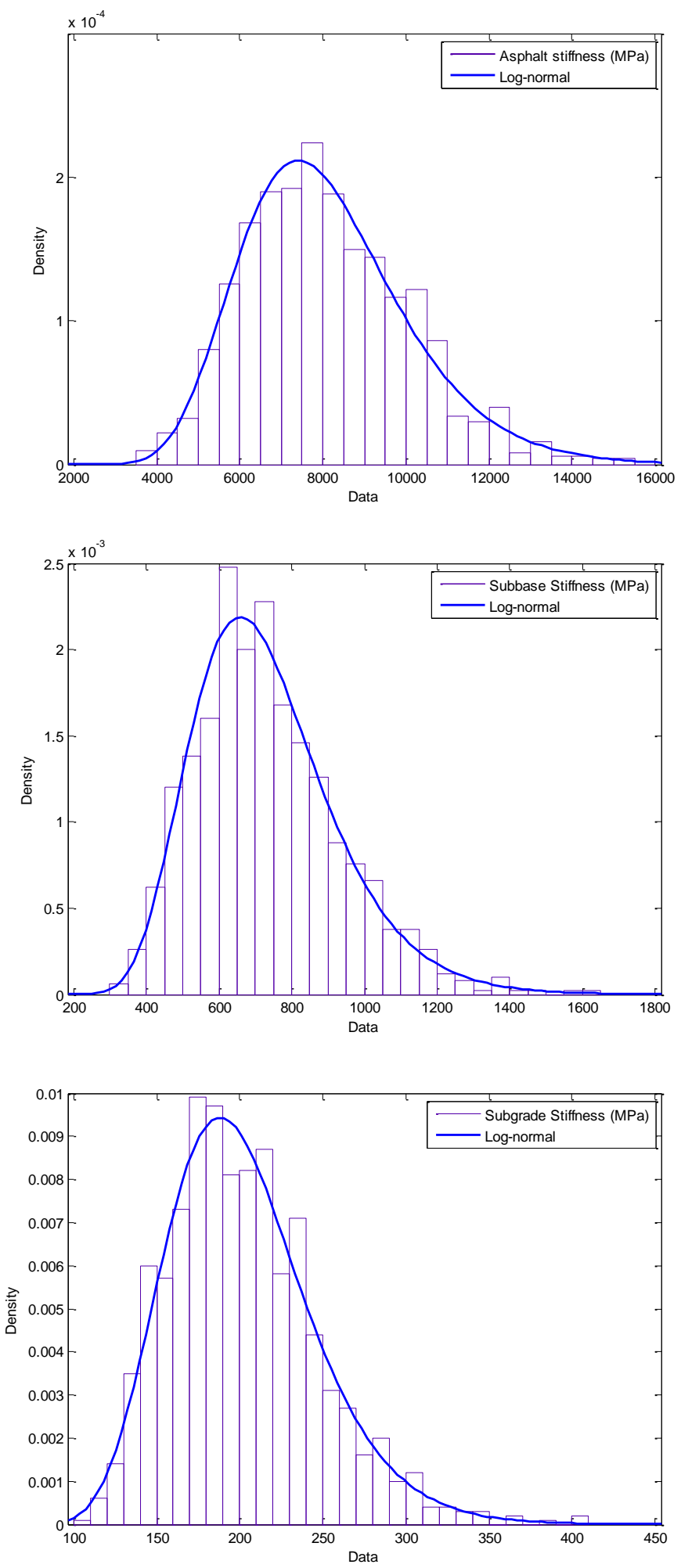

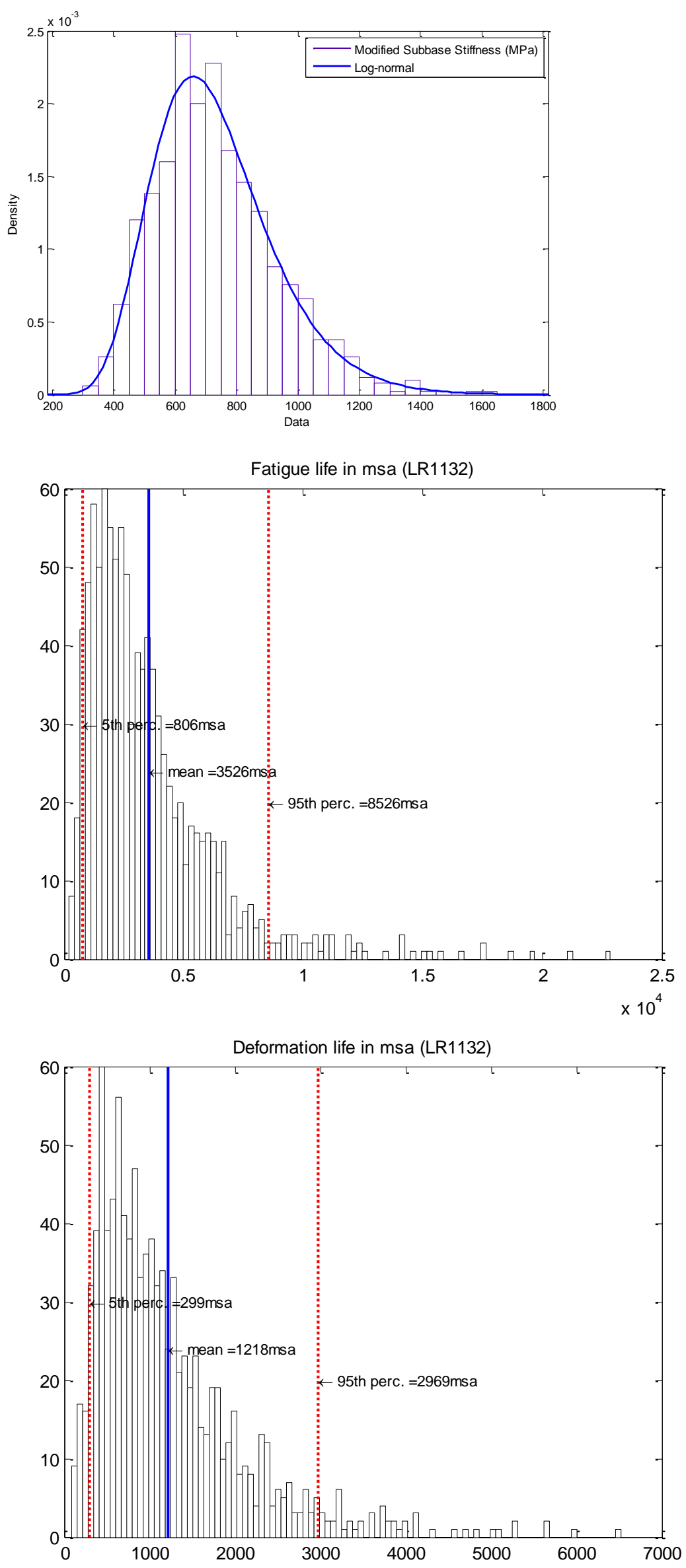


\section{A4.5.1 Section 2}
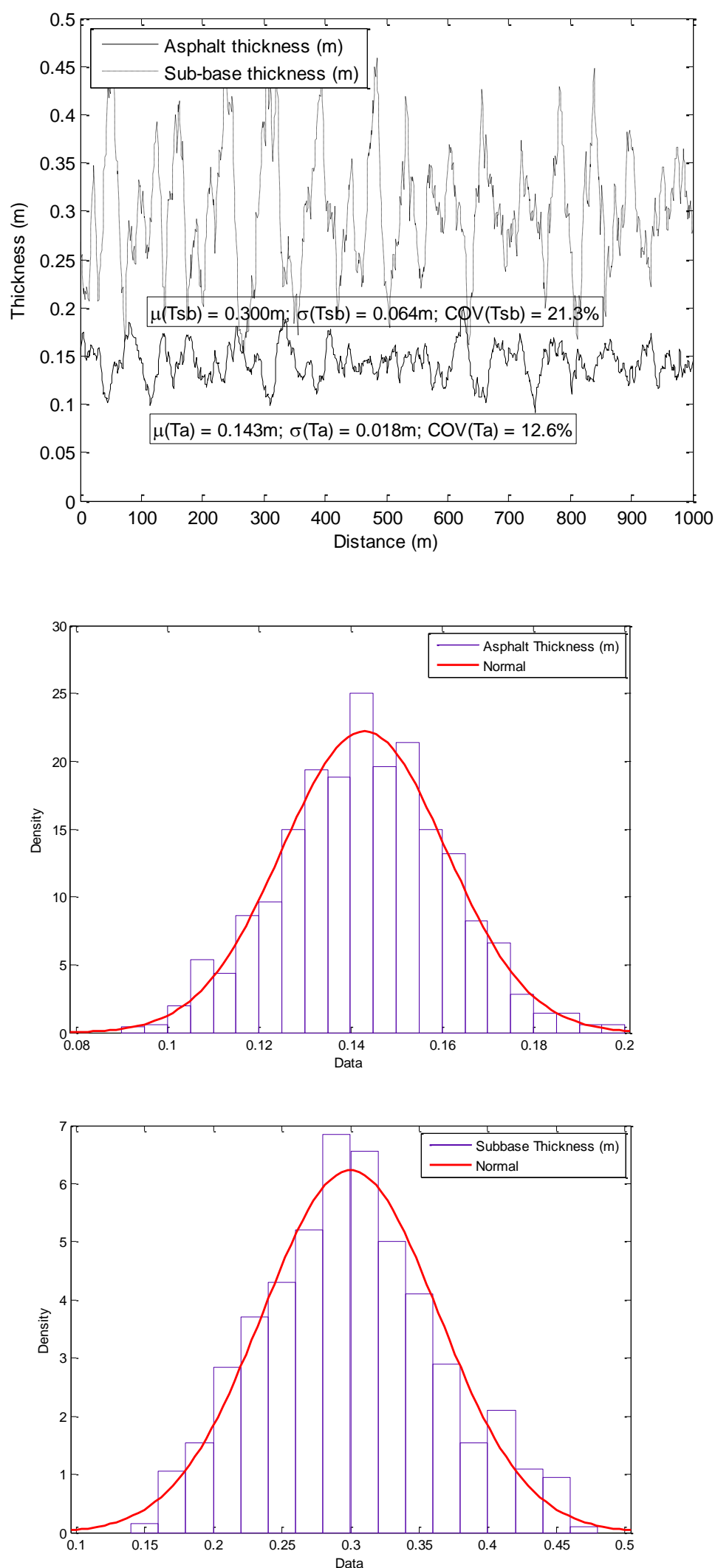

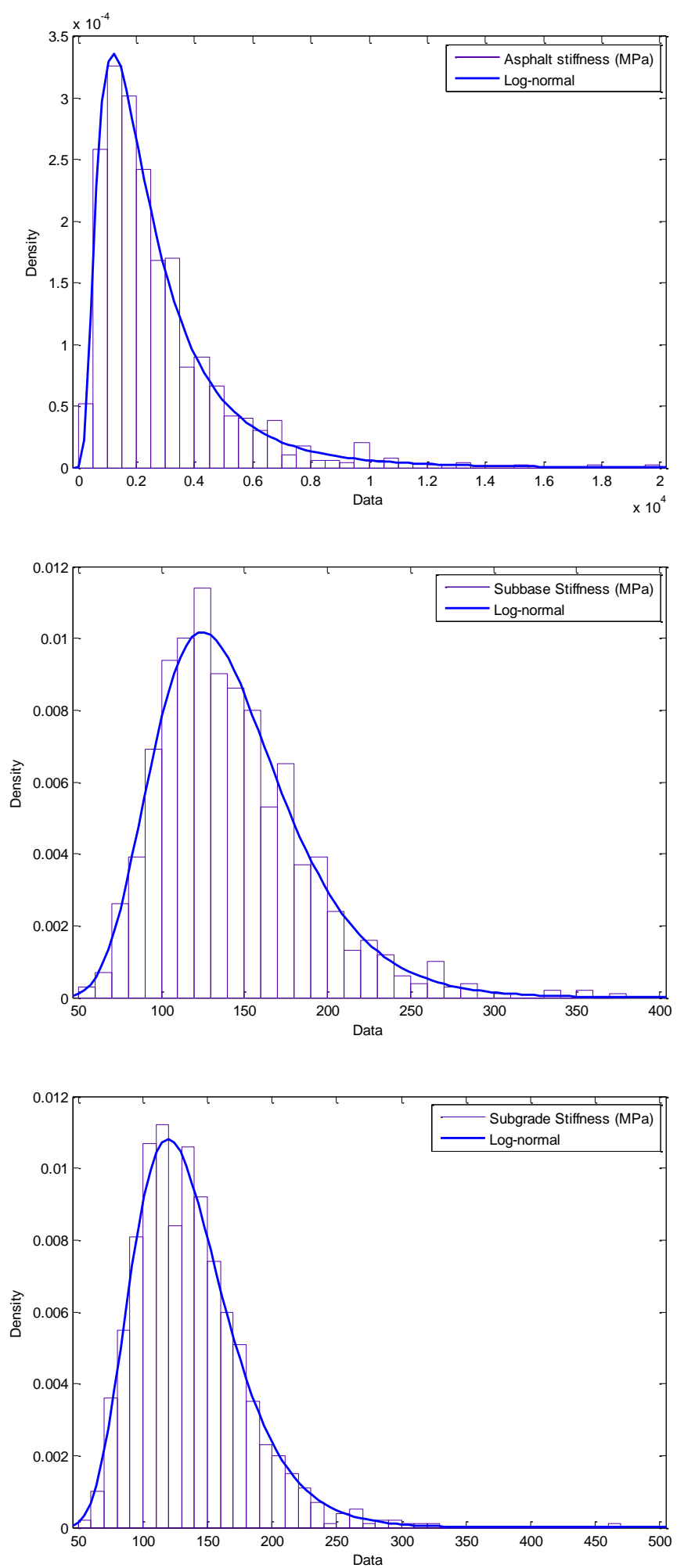

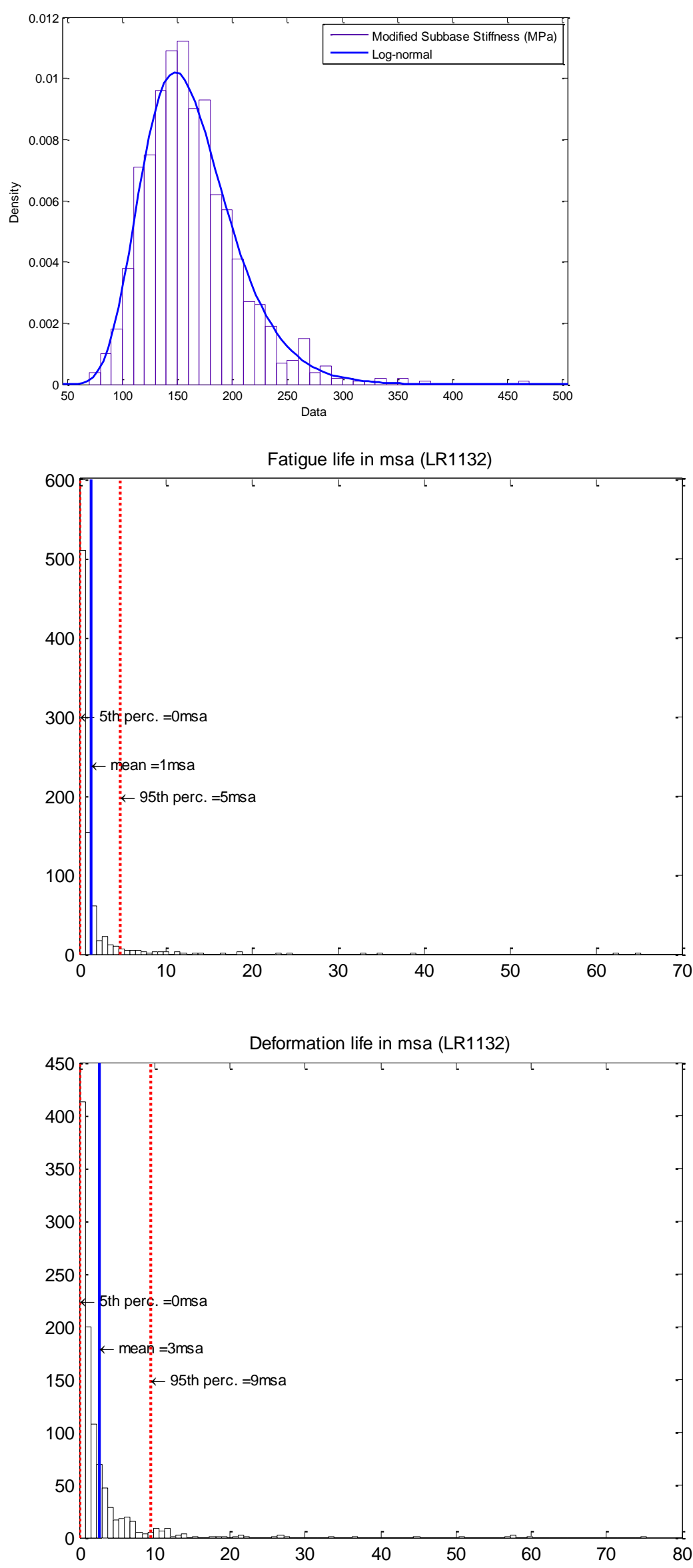


\section{A4.5.2 Section 3}
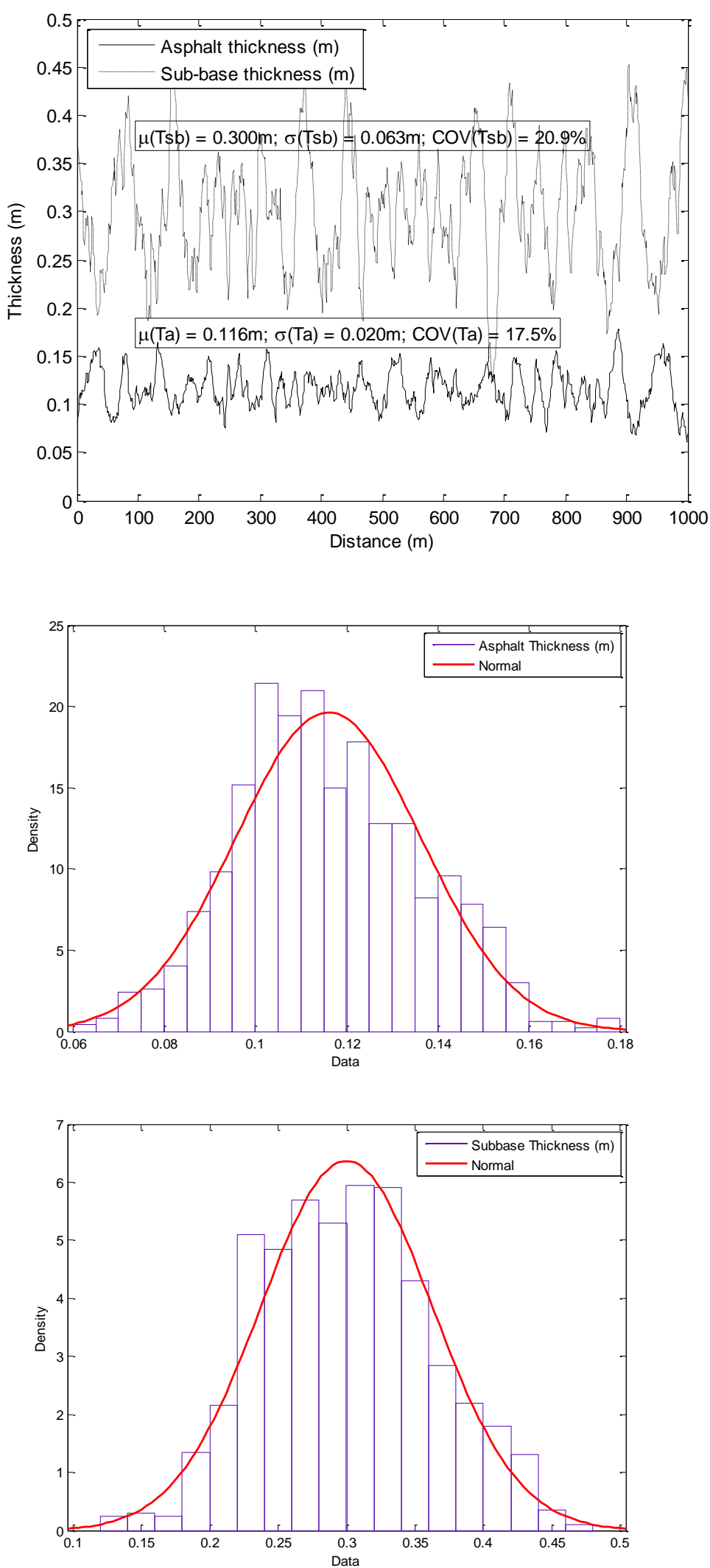

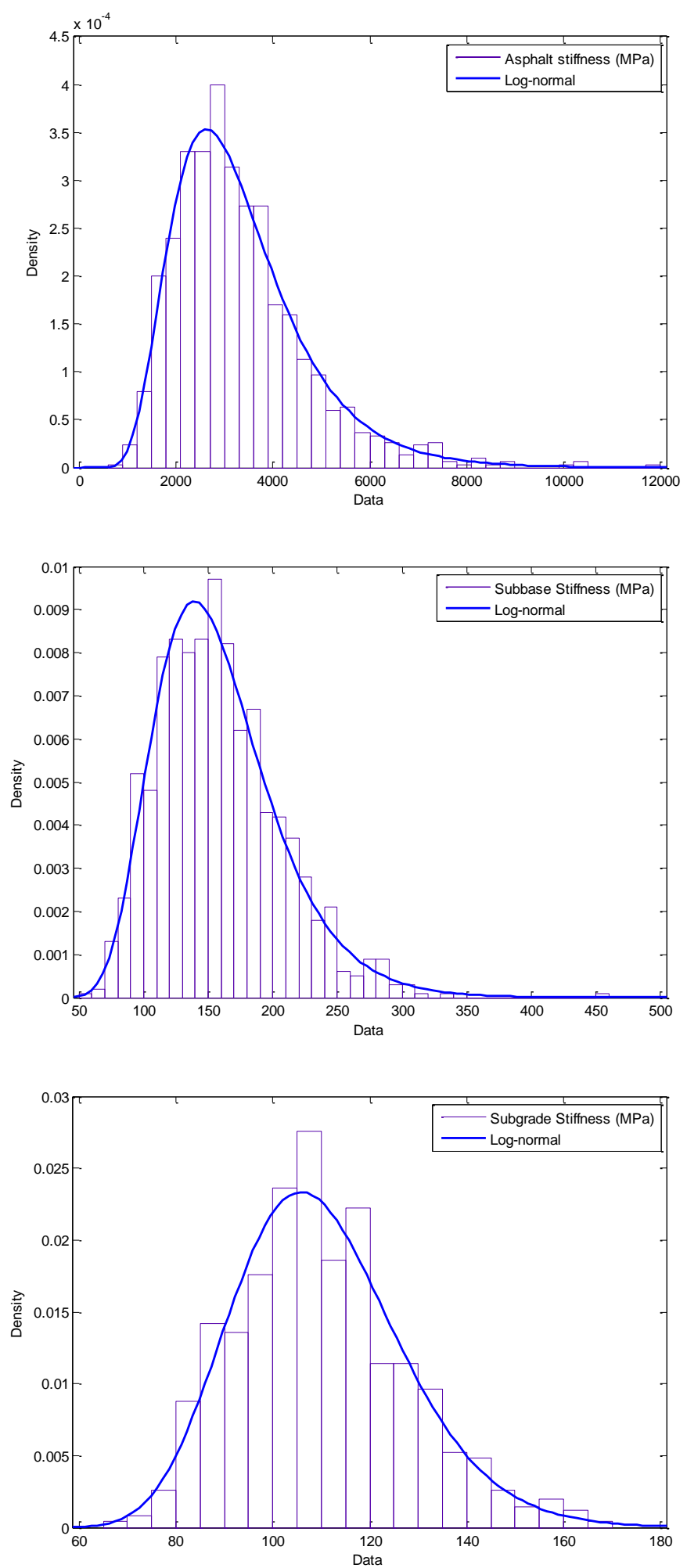

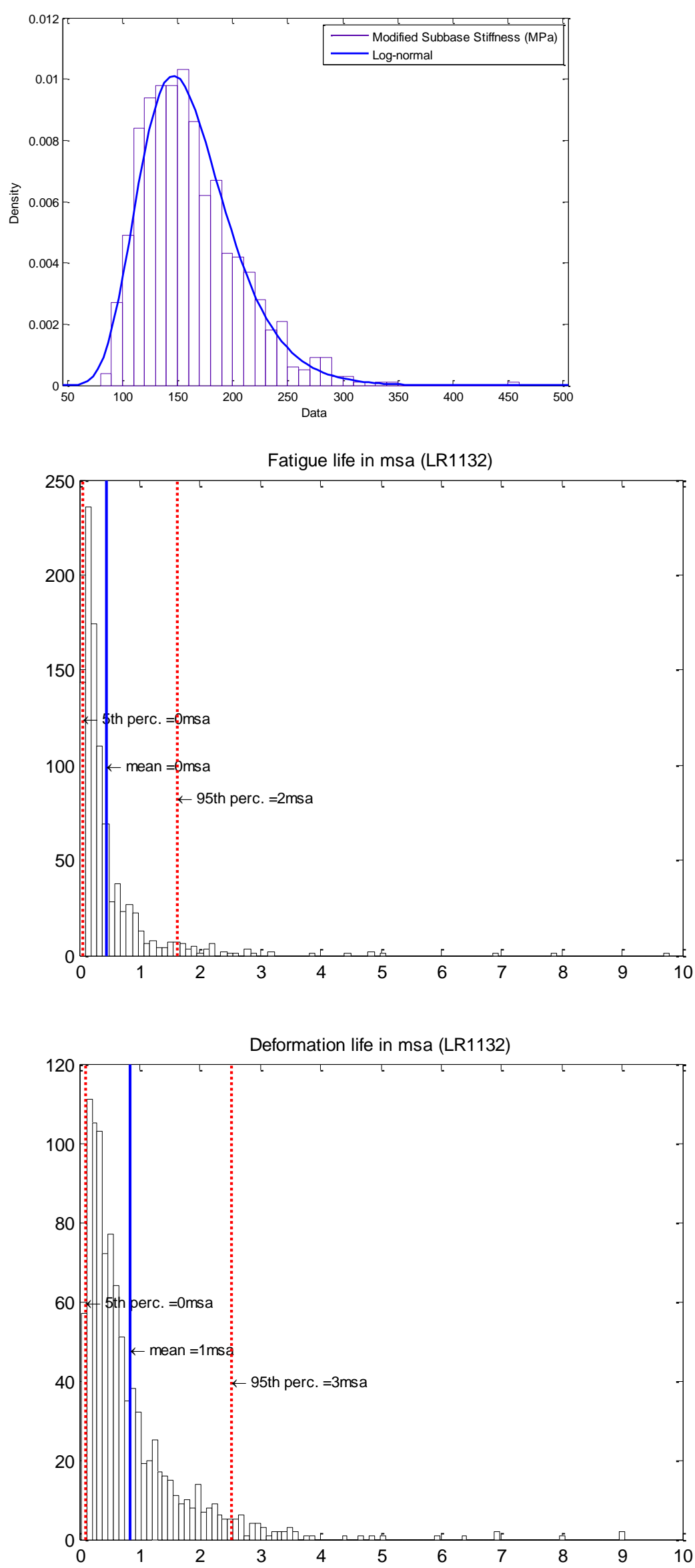


\section{A4.5.3 Section 4}
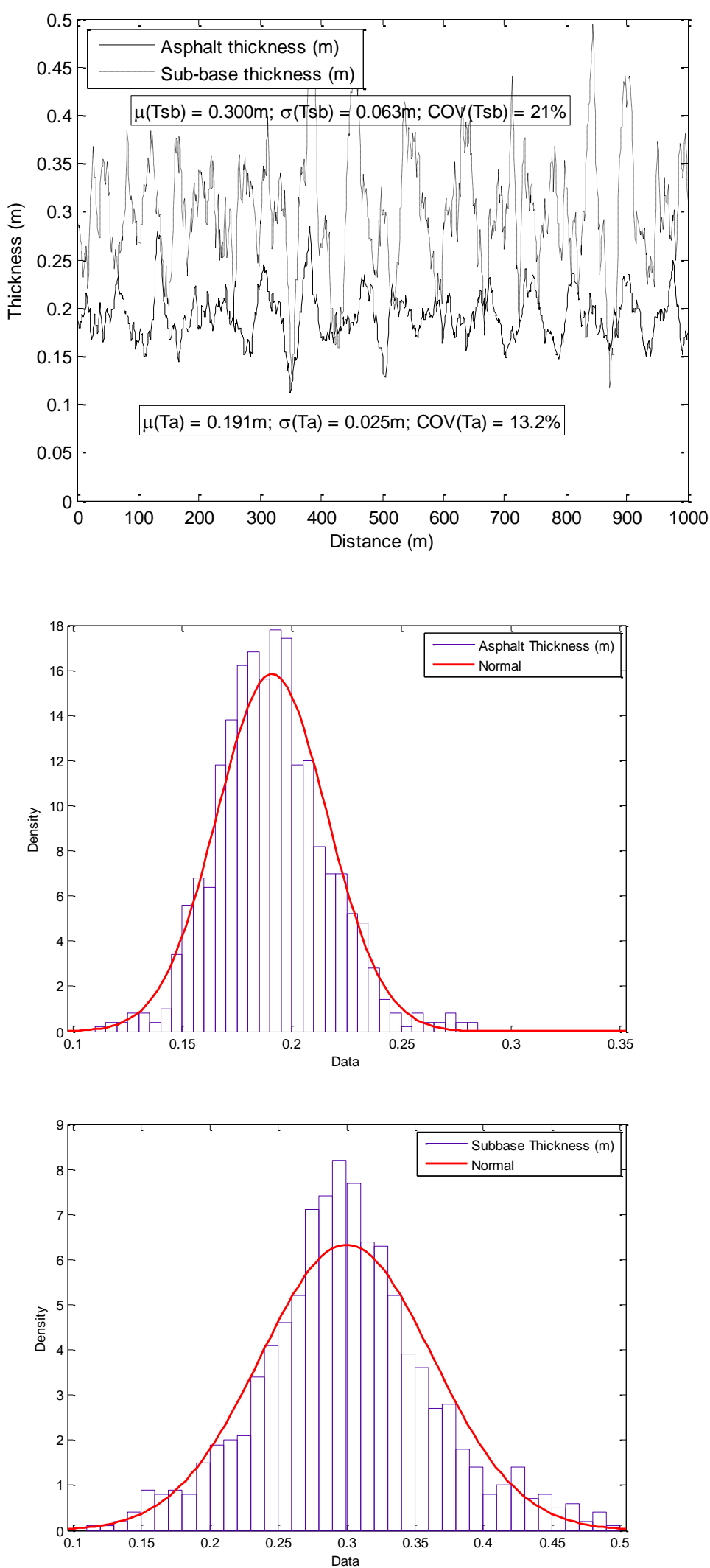

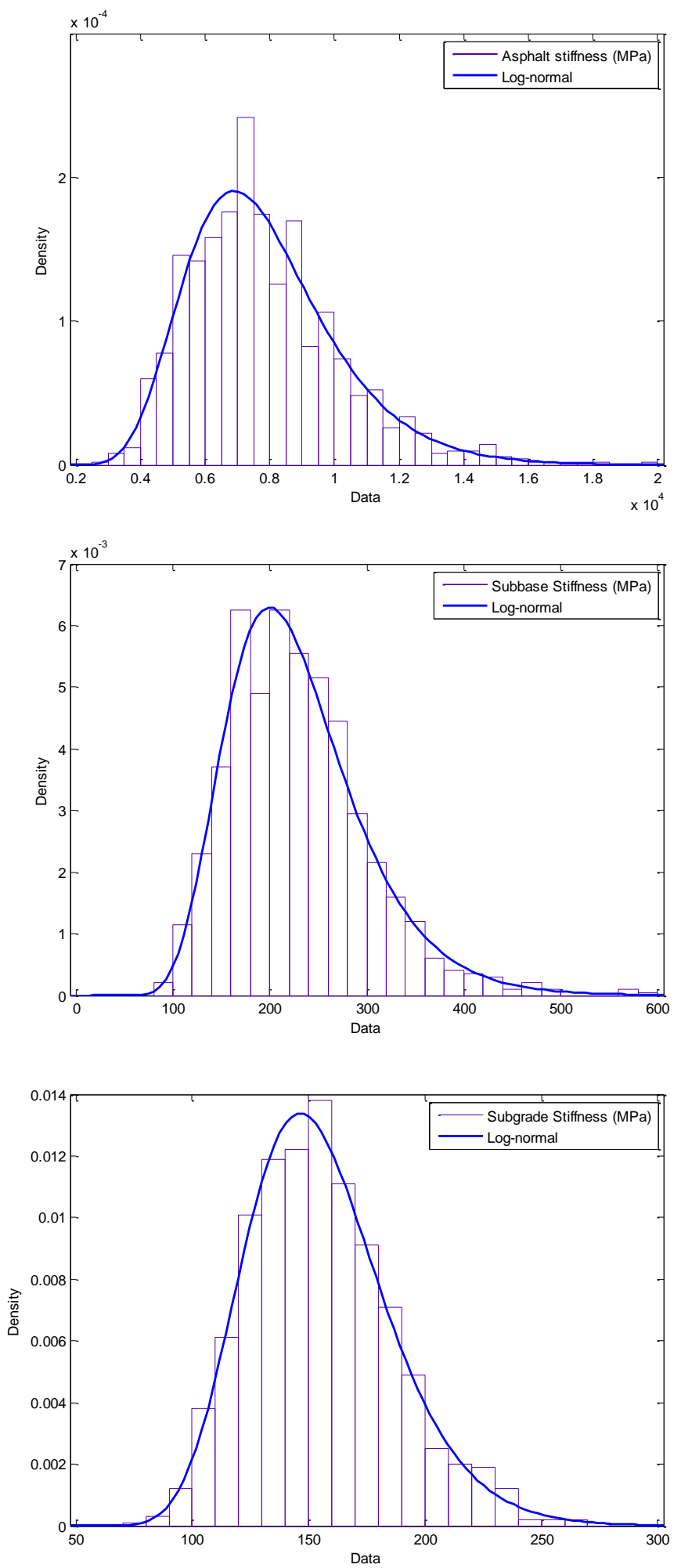

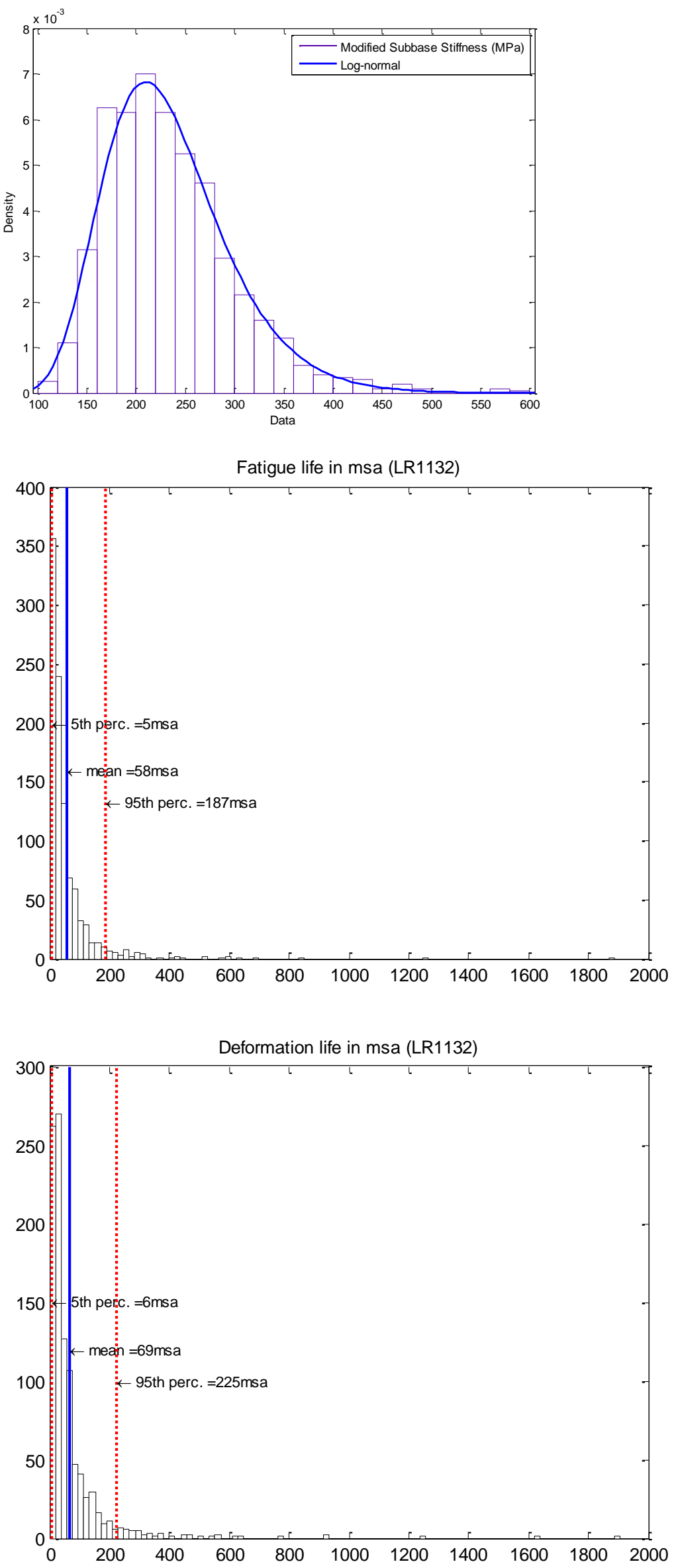
A4.5.4 Section 5
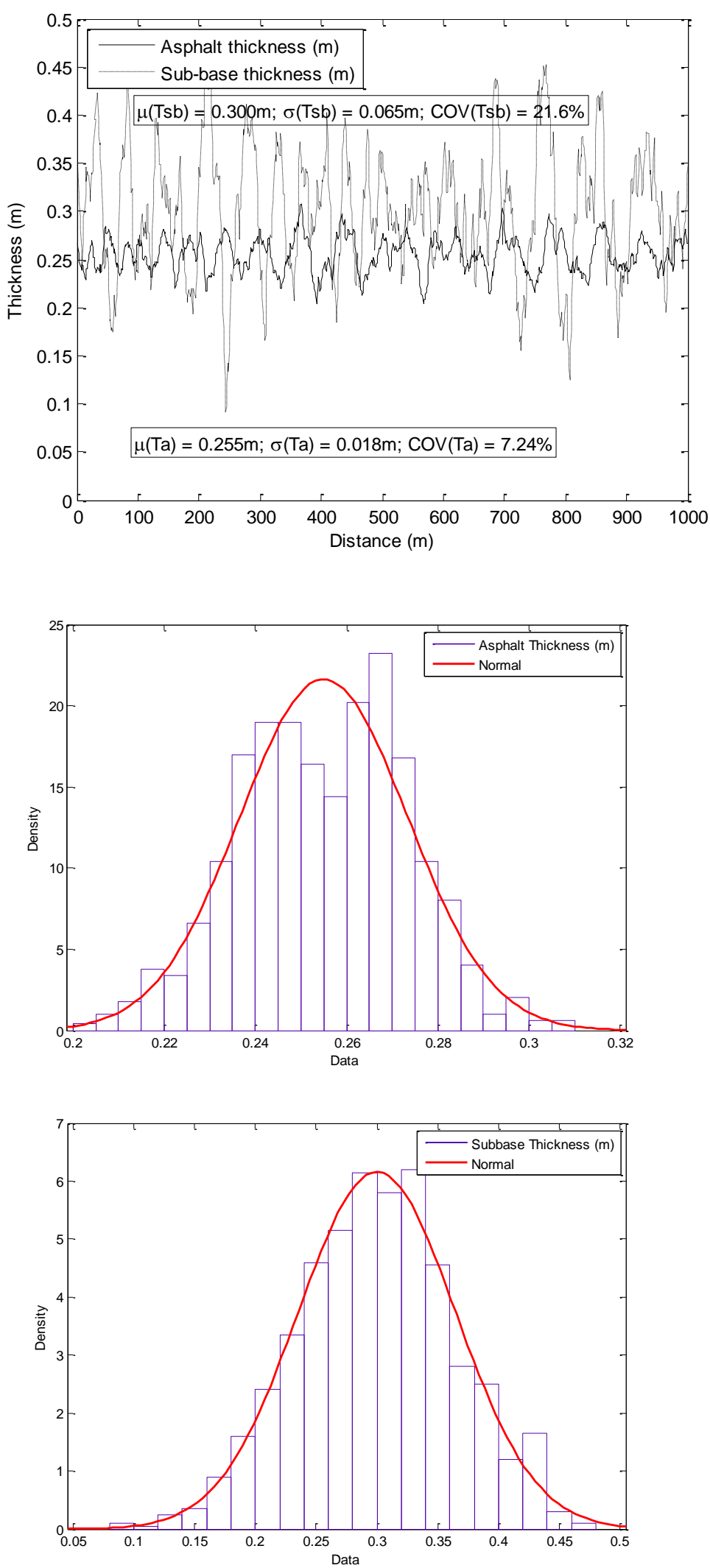

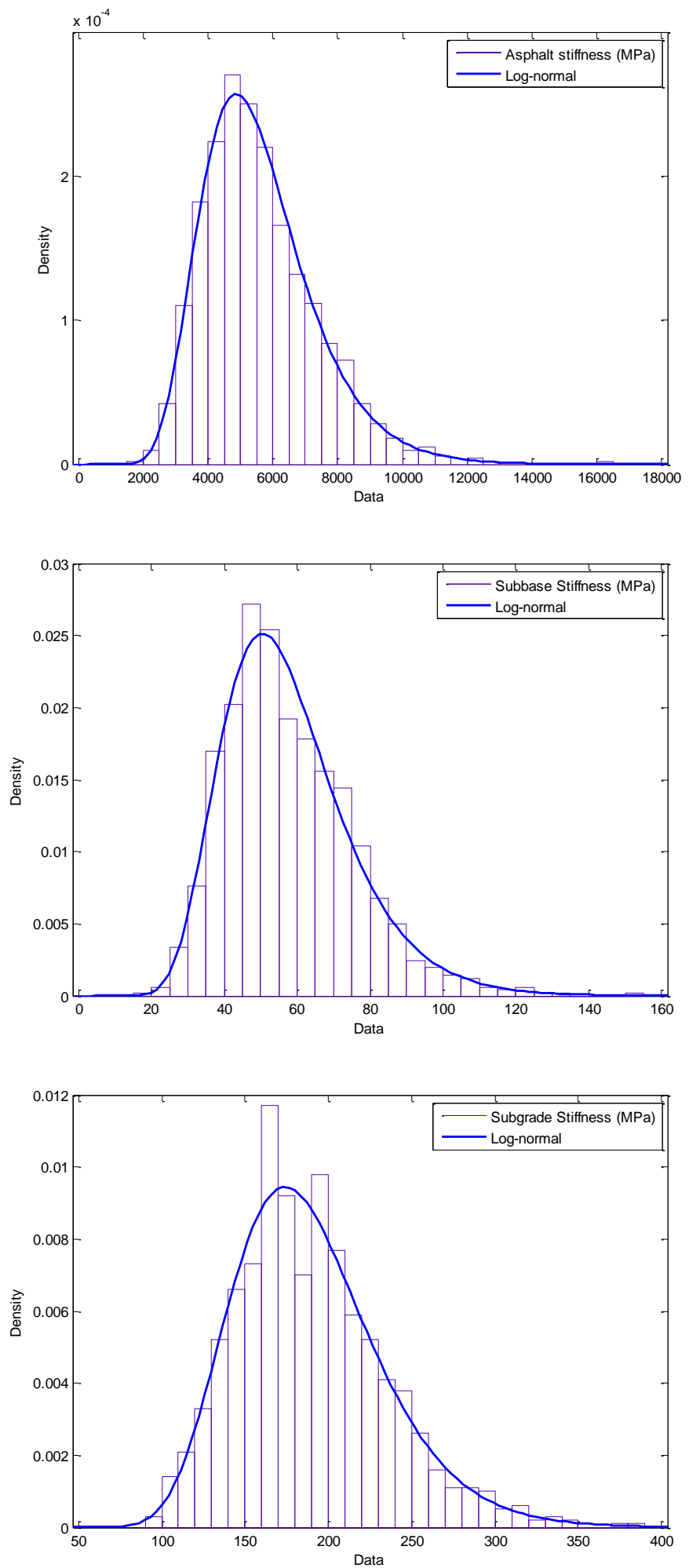

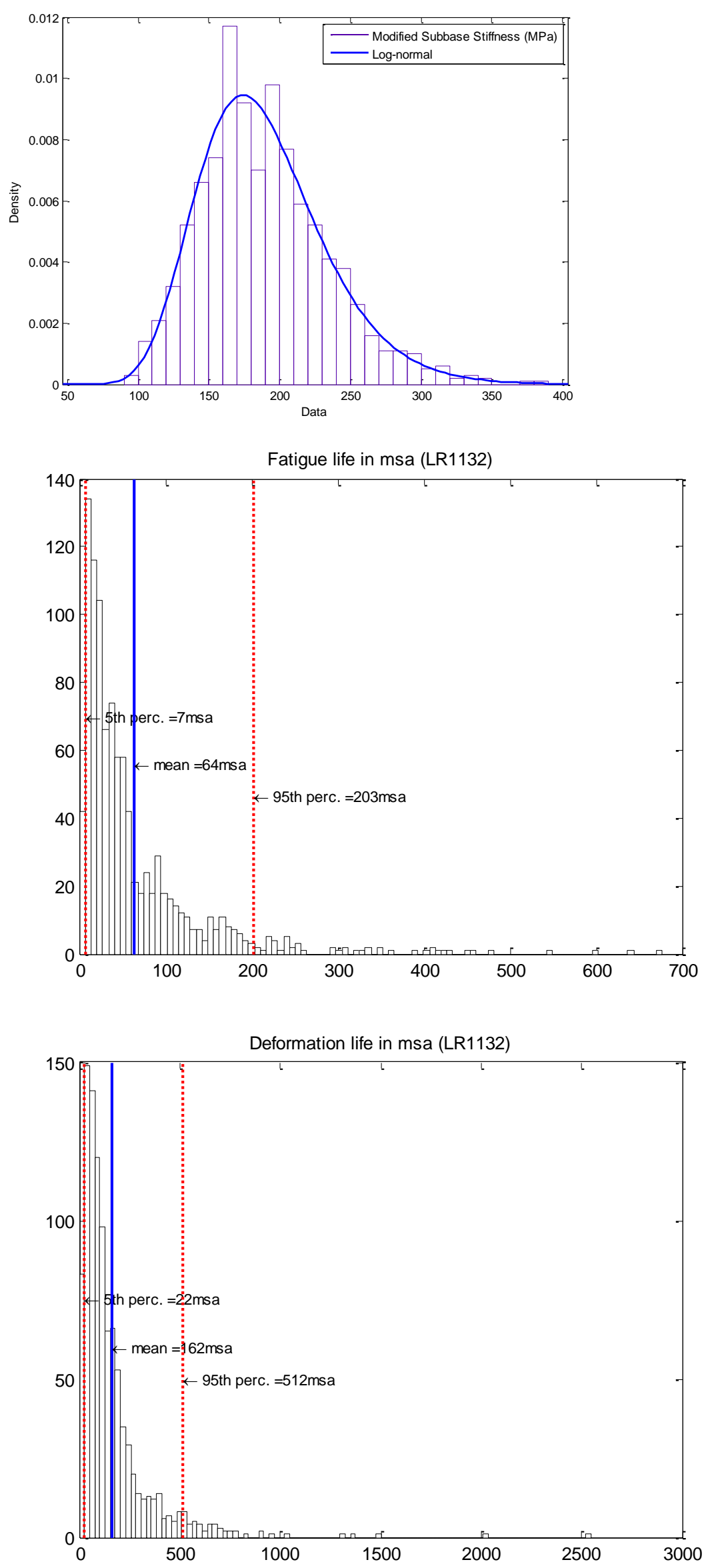


\section{A4.5.5 Section 6}
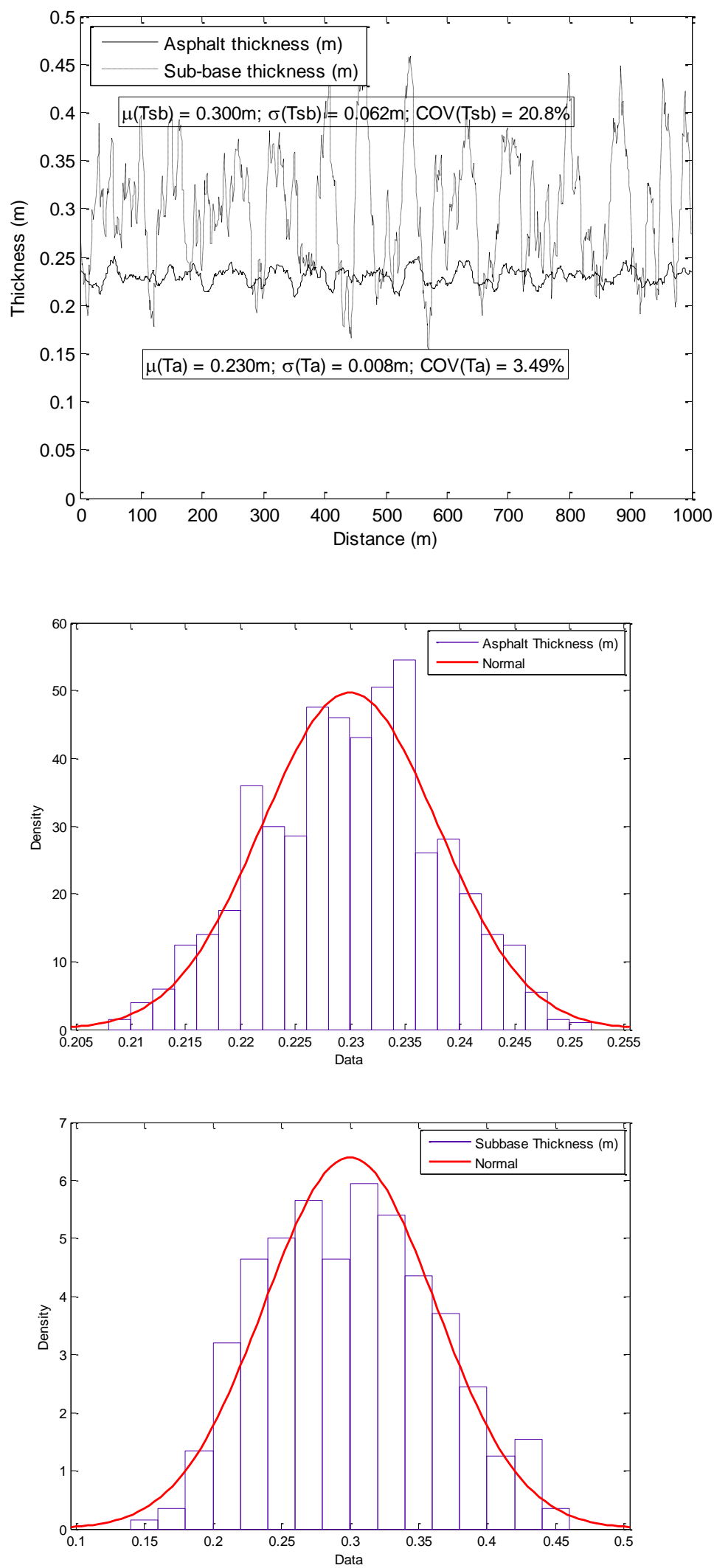

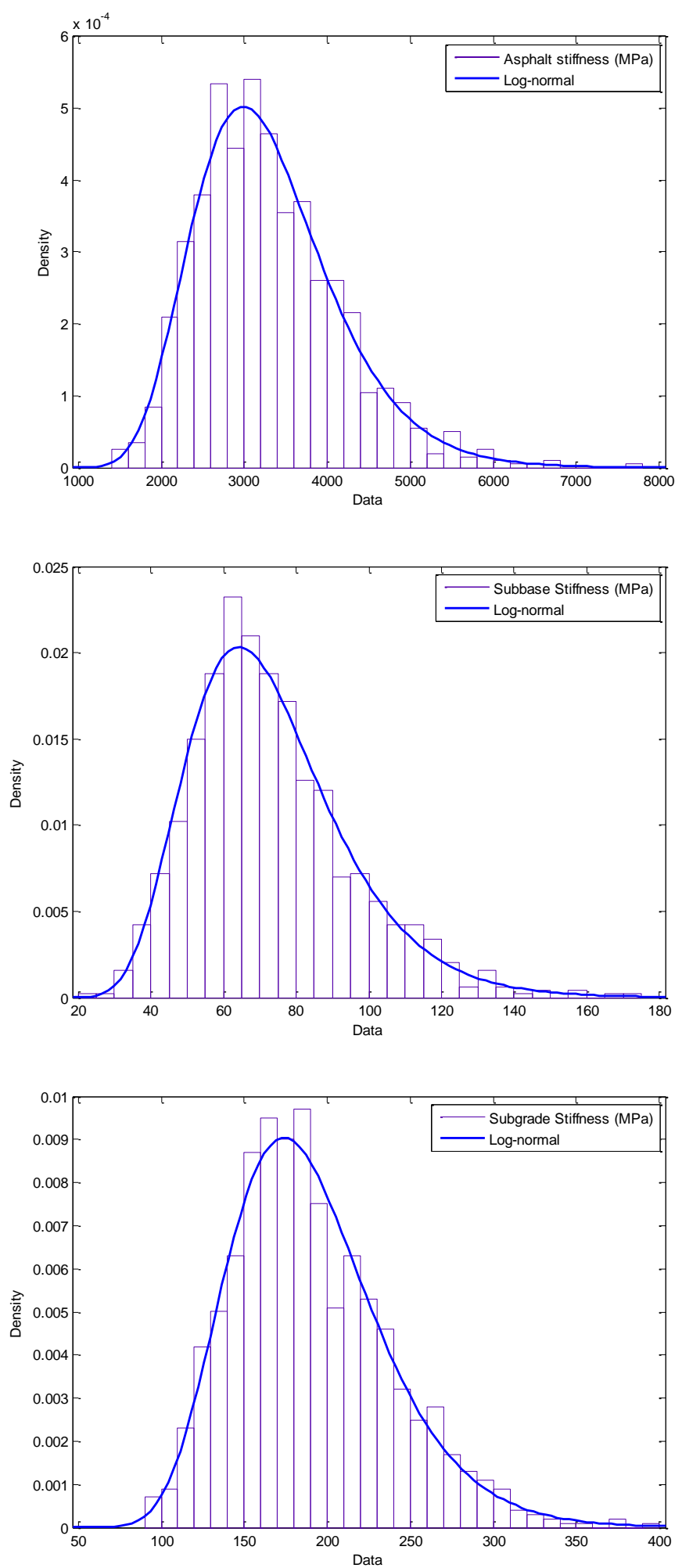

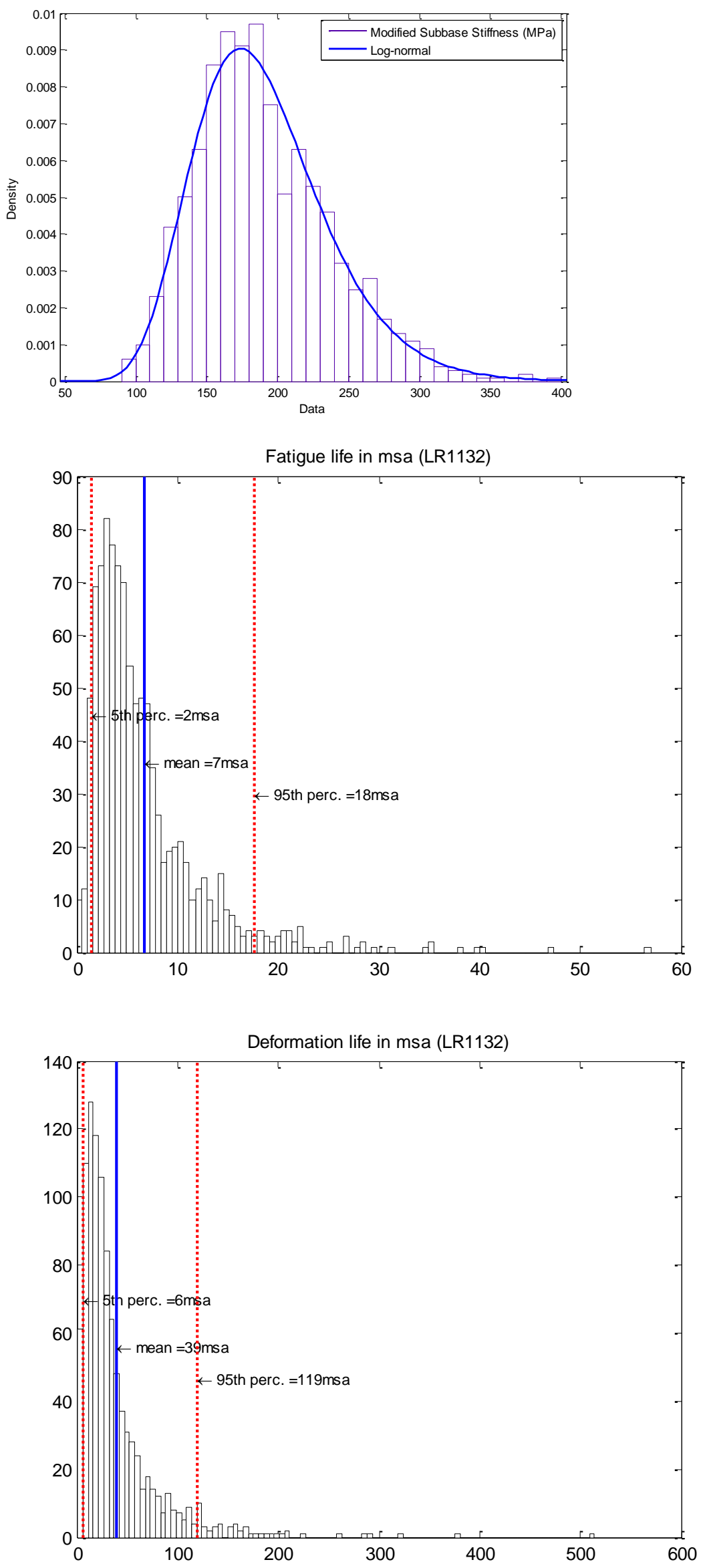


\section{A4.6.1 Section 3}
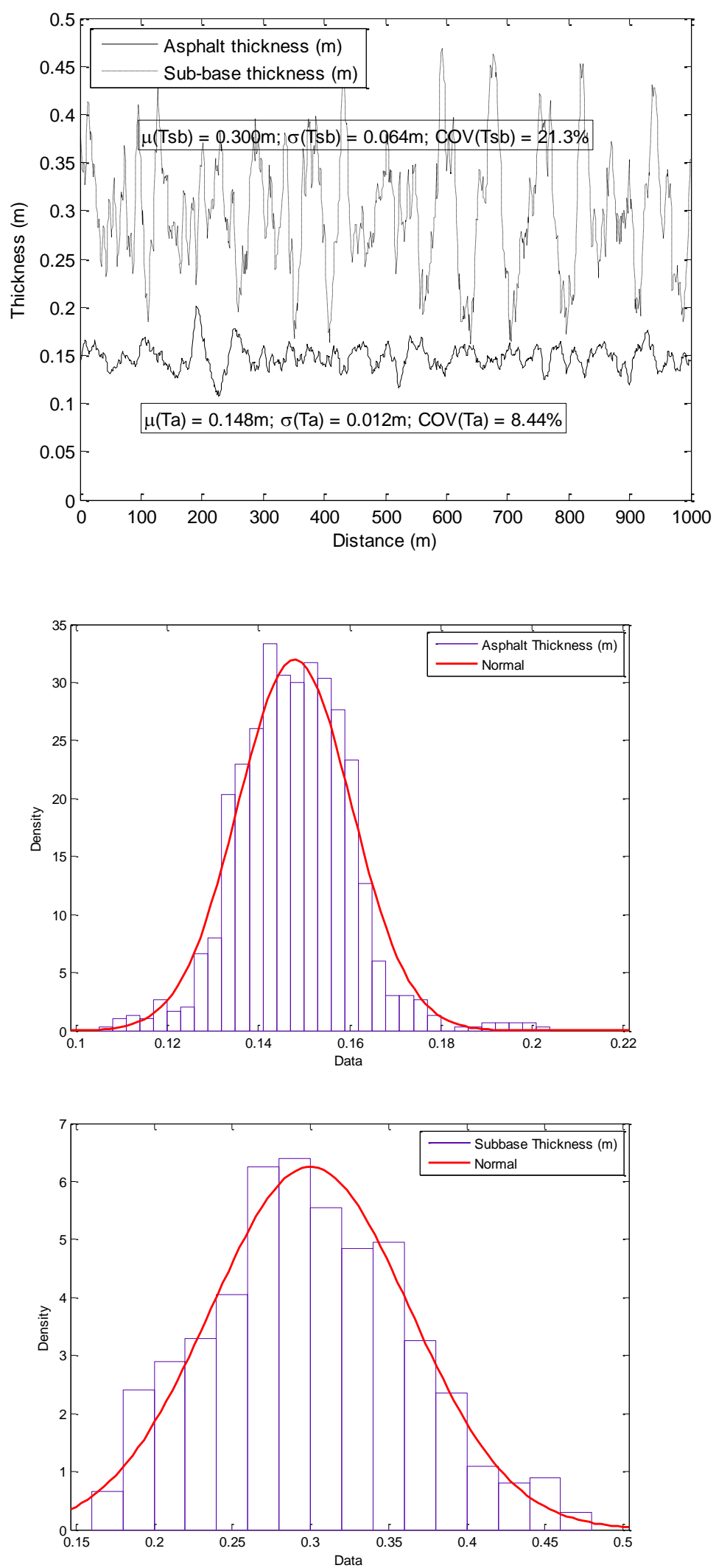

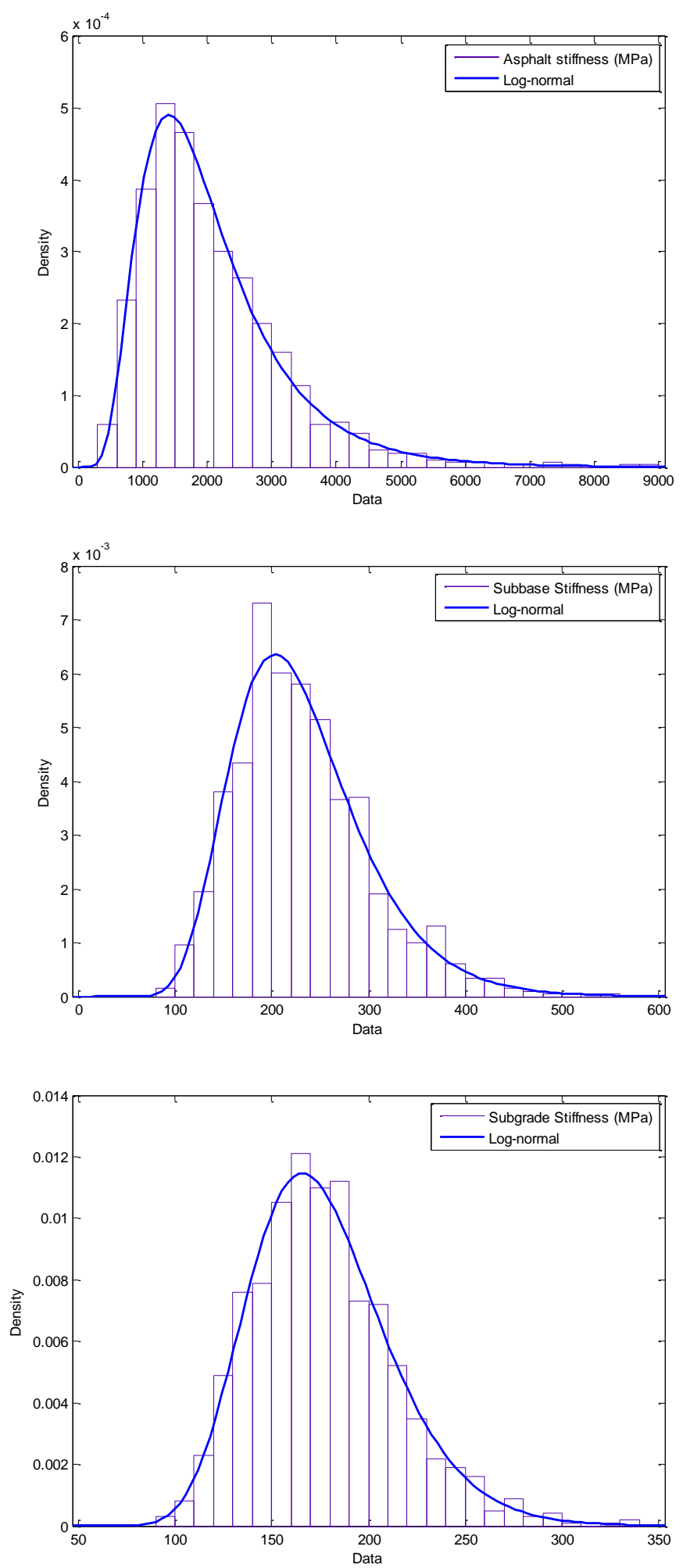

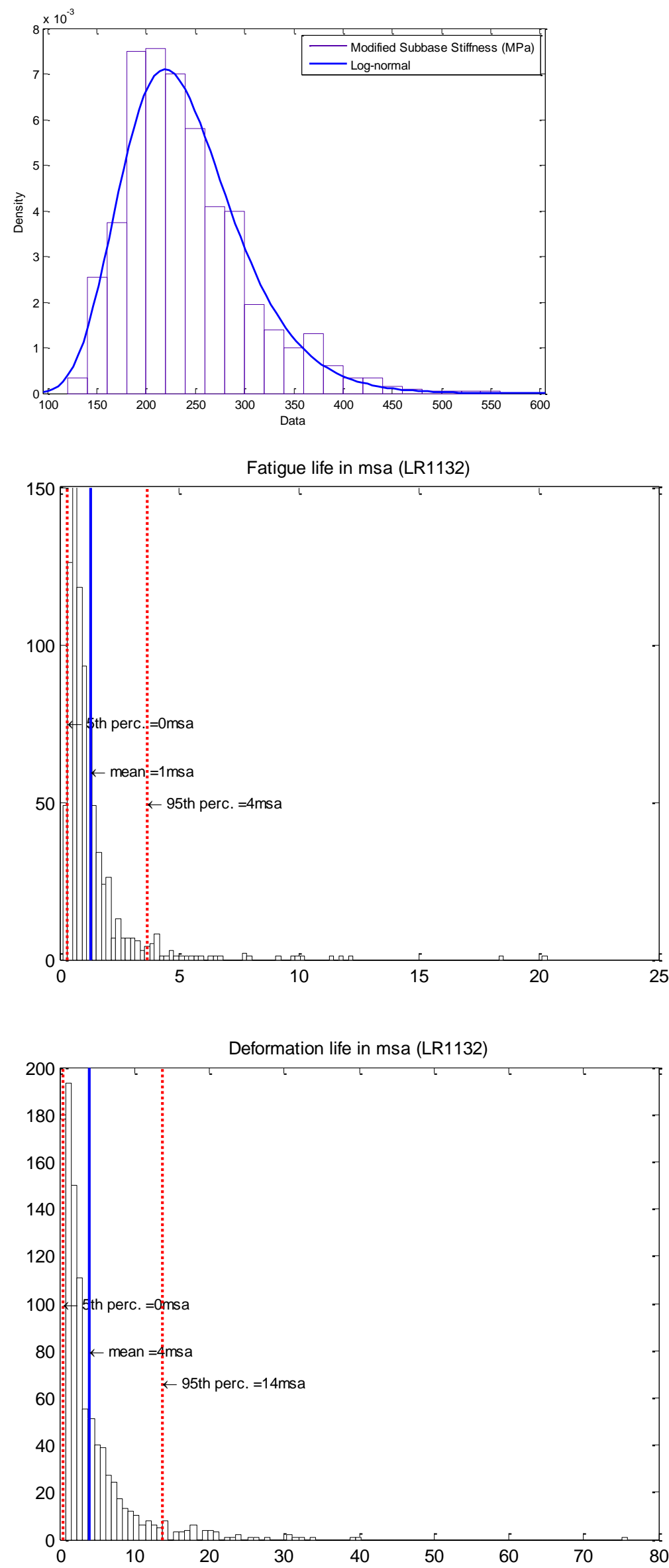


\section{A4.6.2 Section 7}
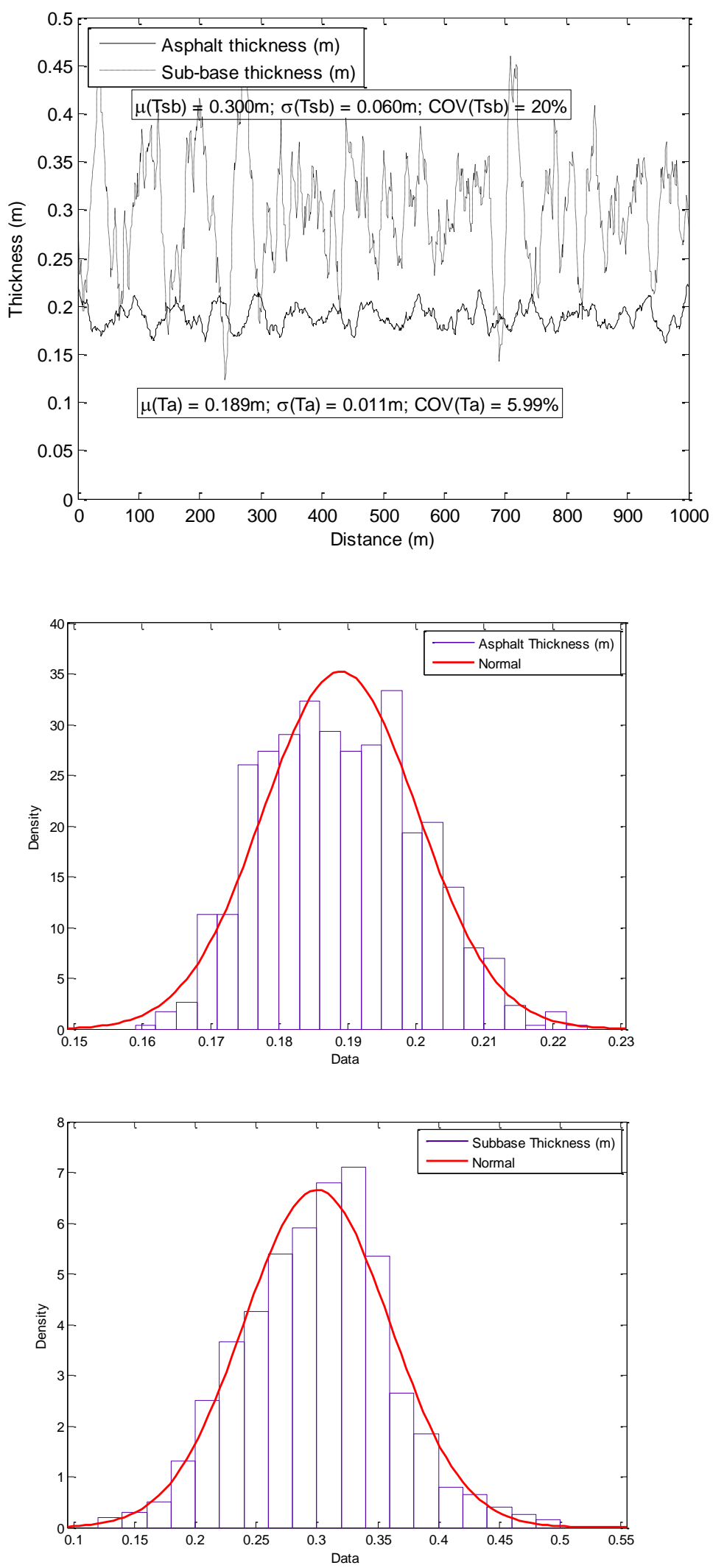

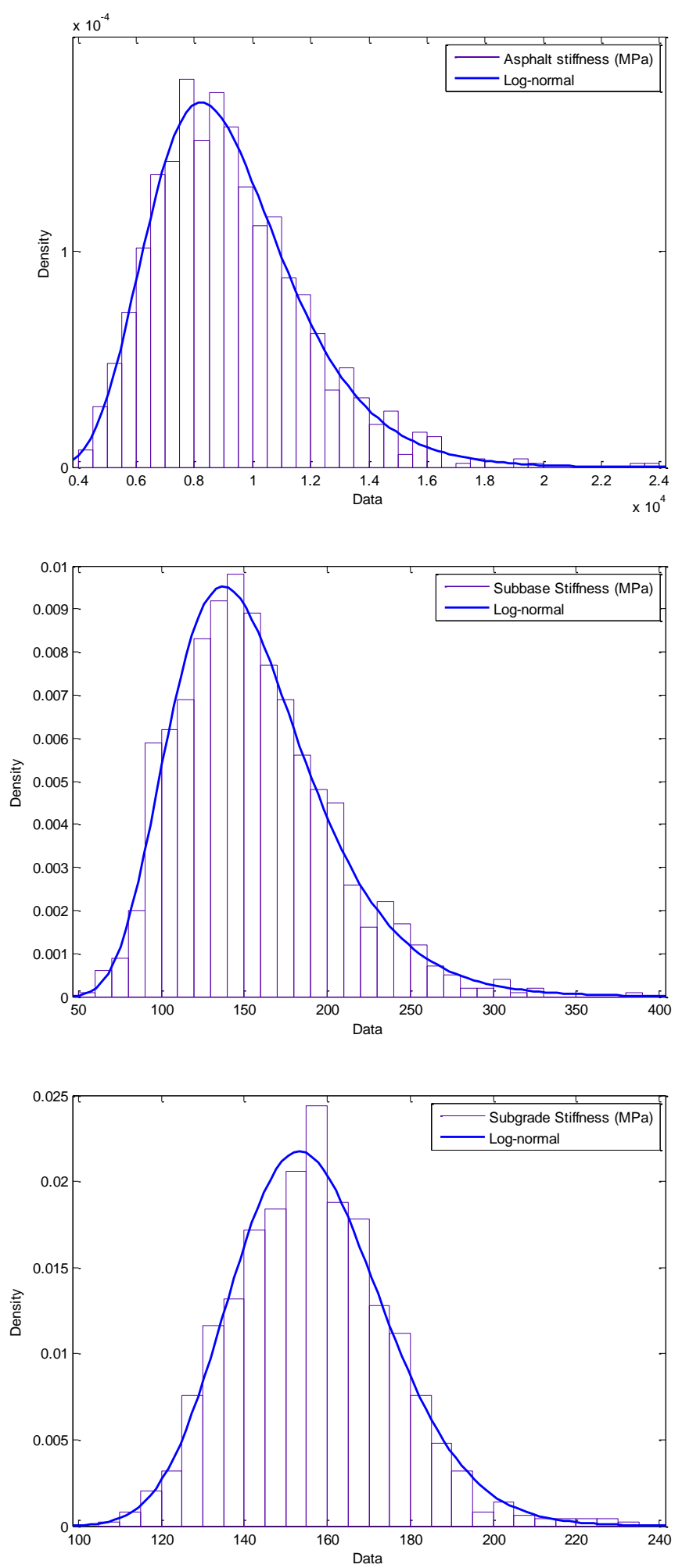

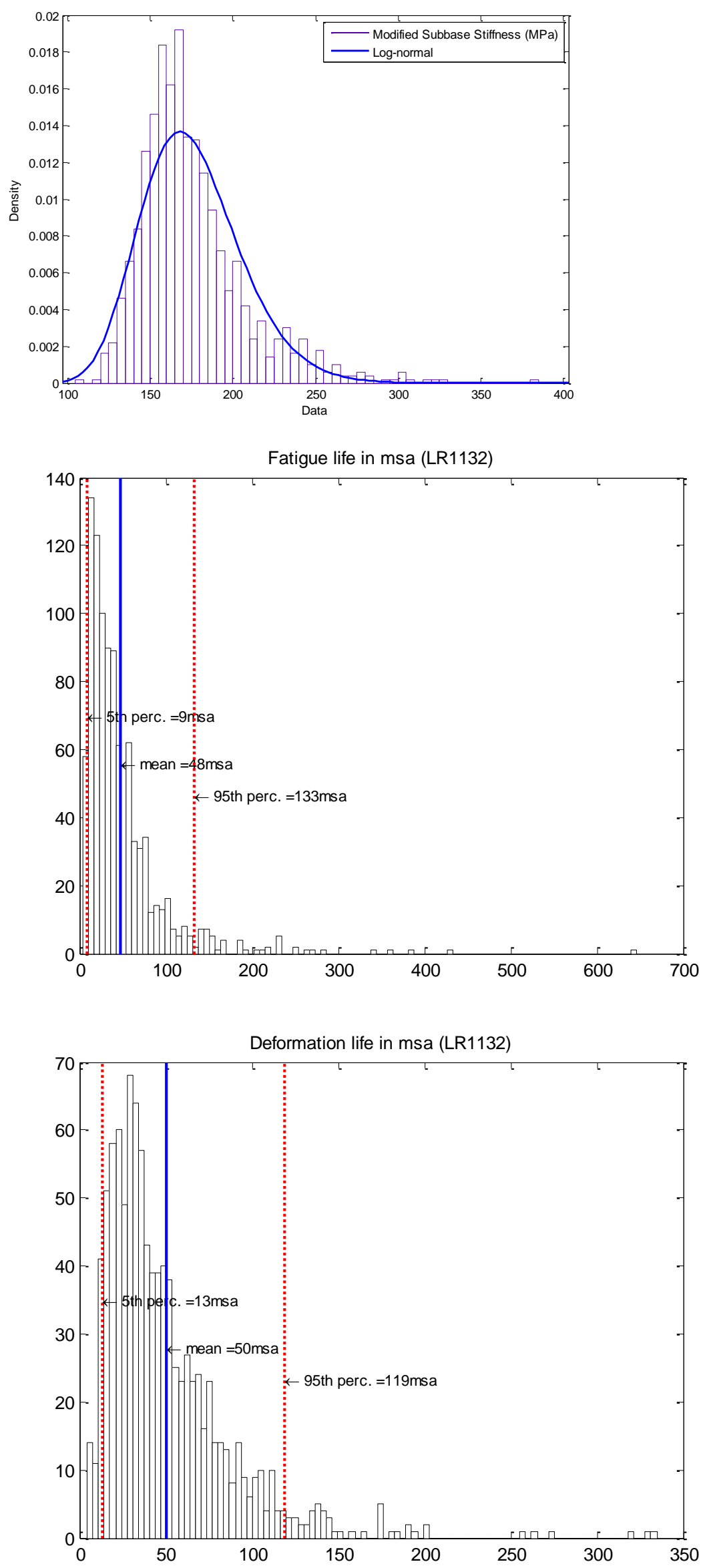


\section{A4.6.3 Section 8}
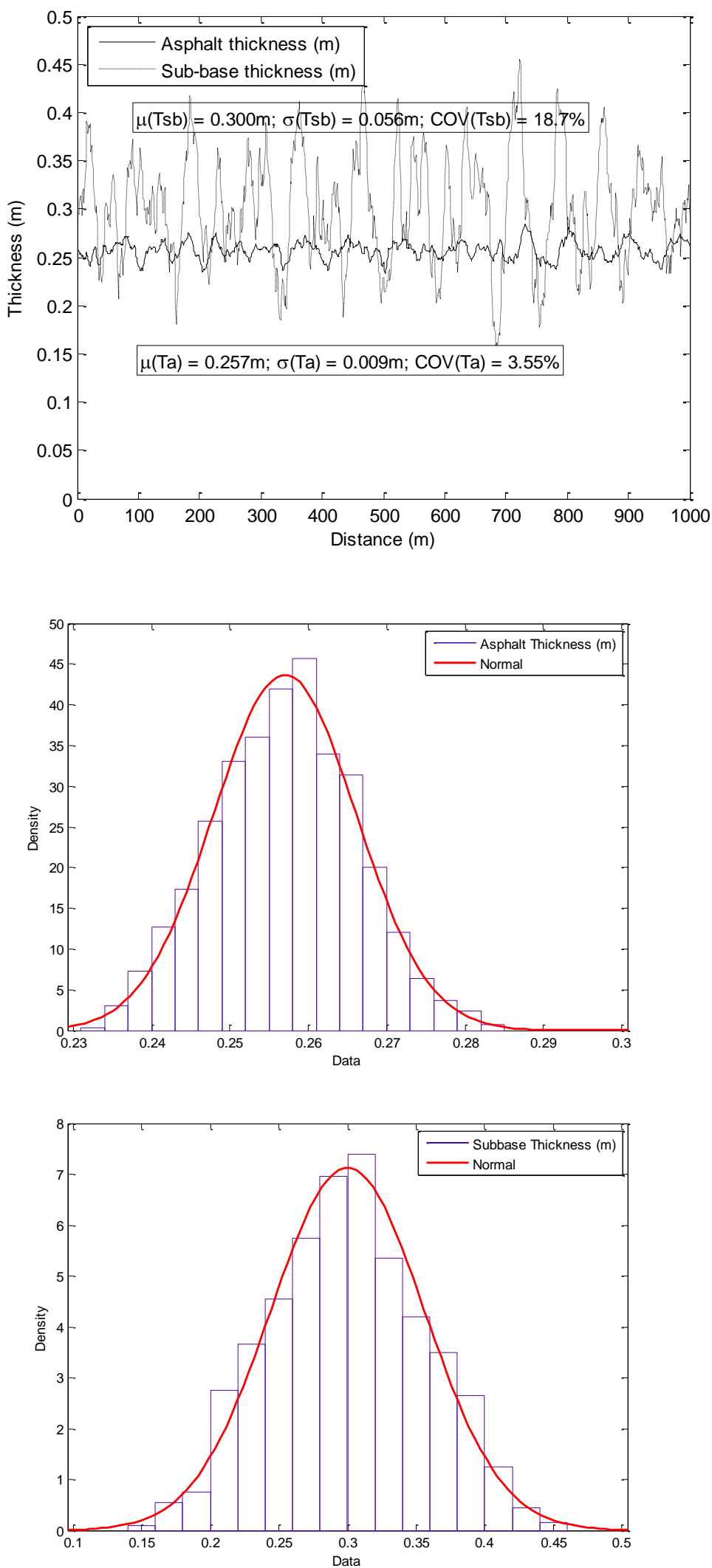

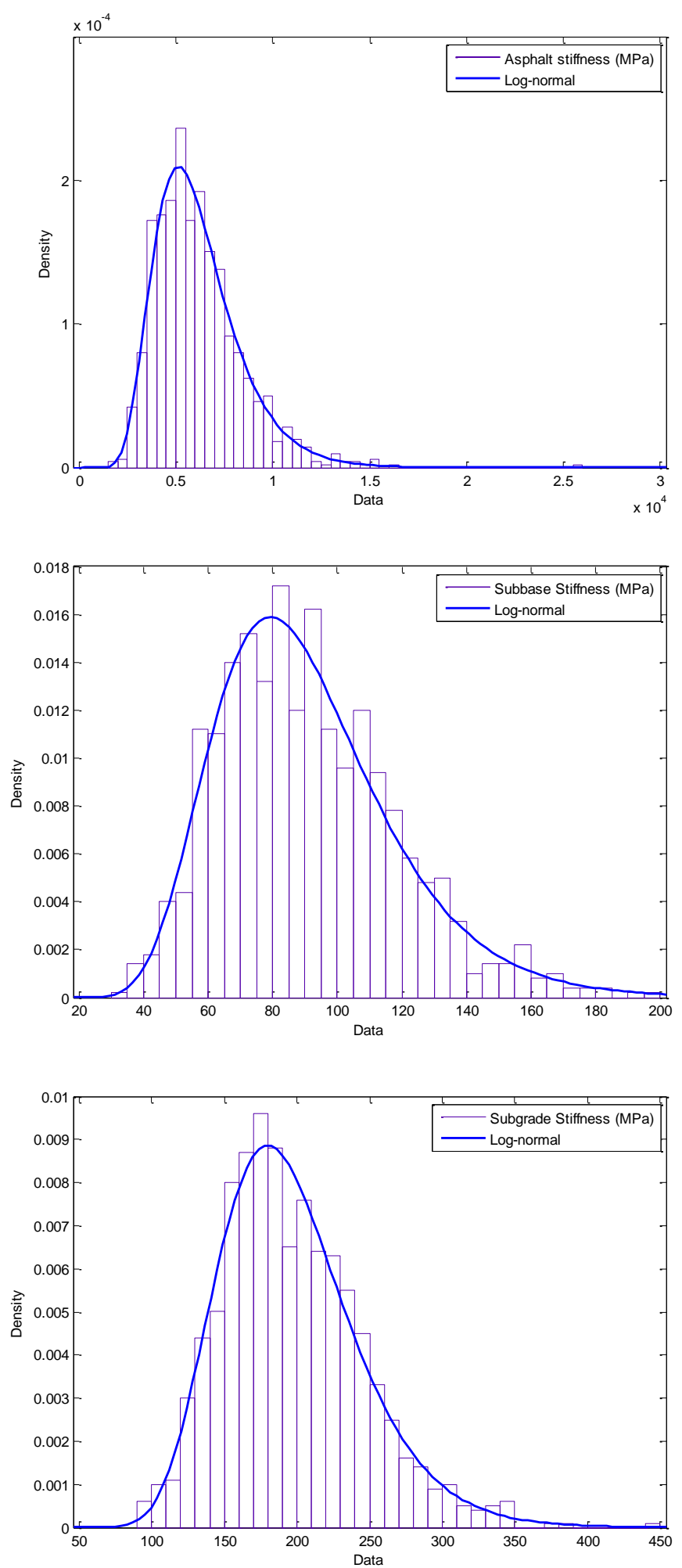

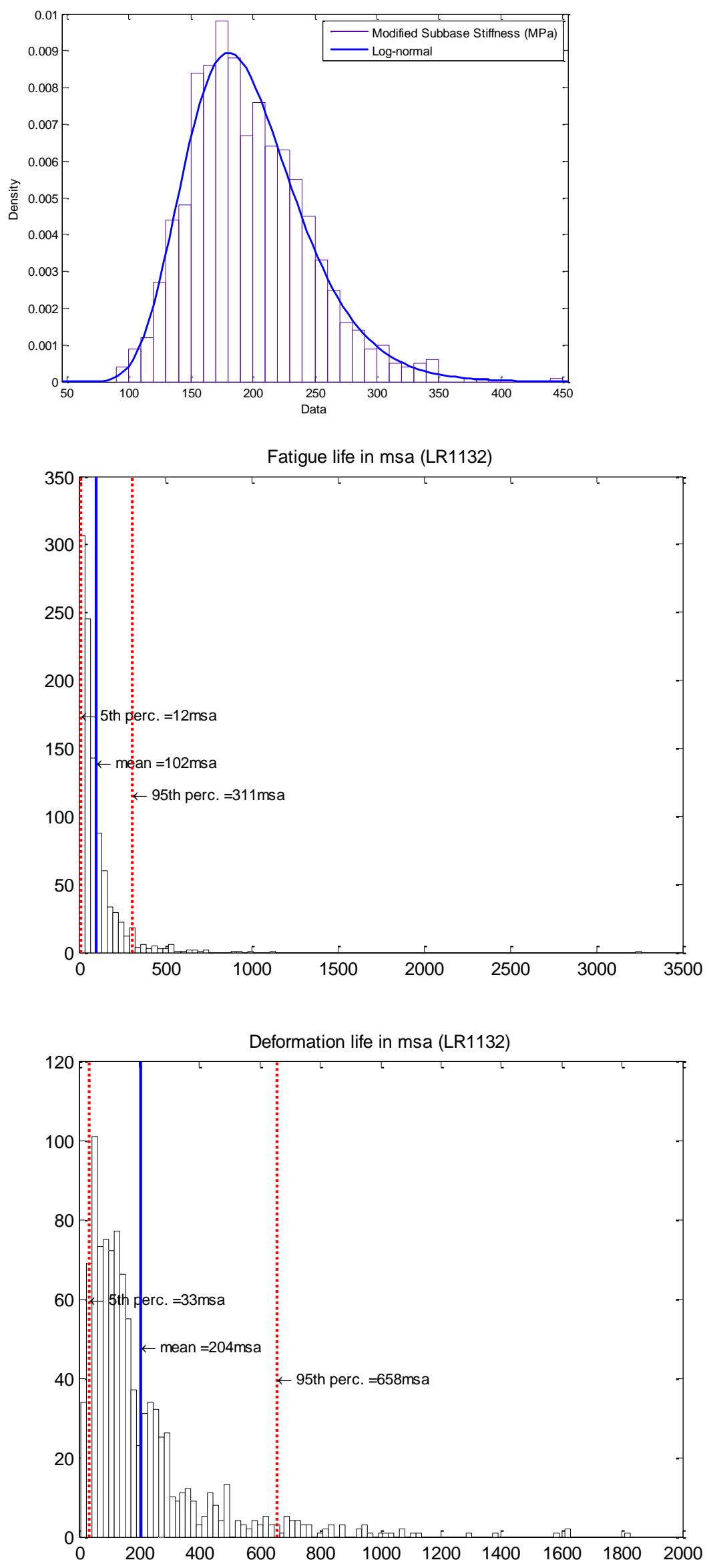
A4.6.4 Section 12
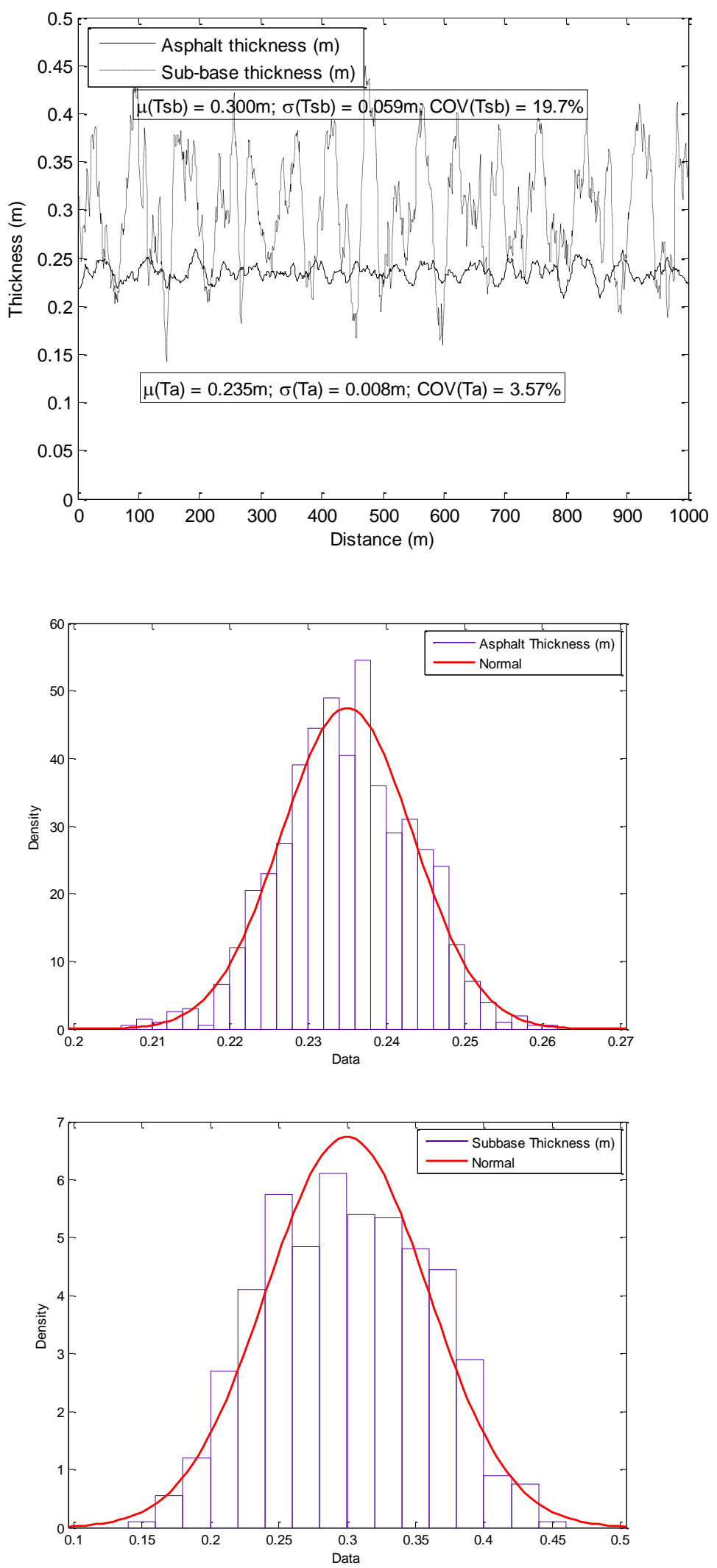

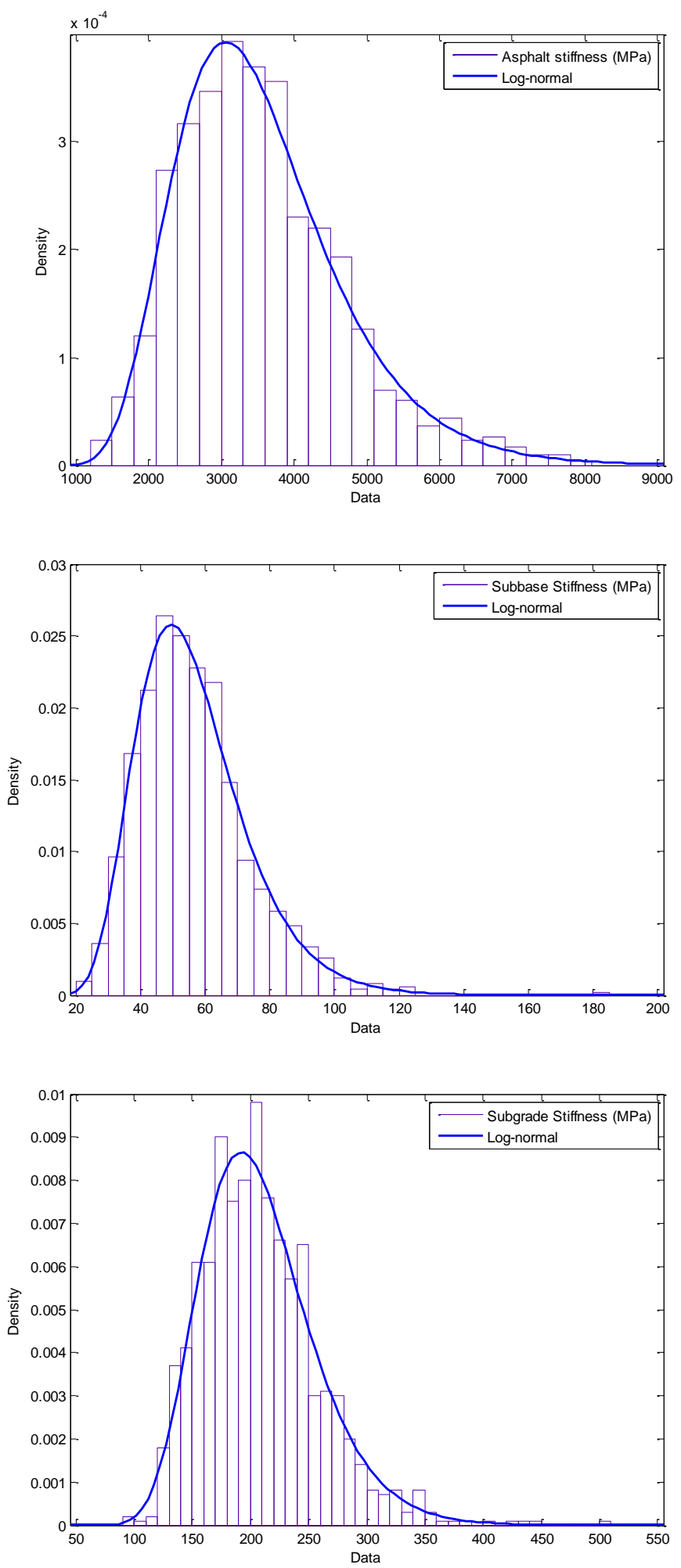

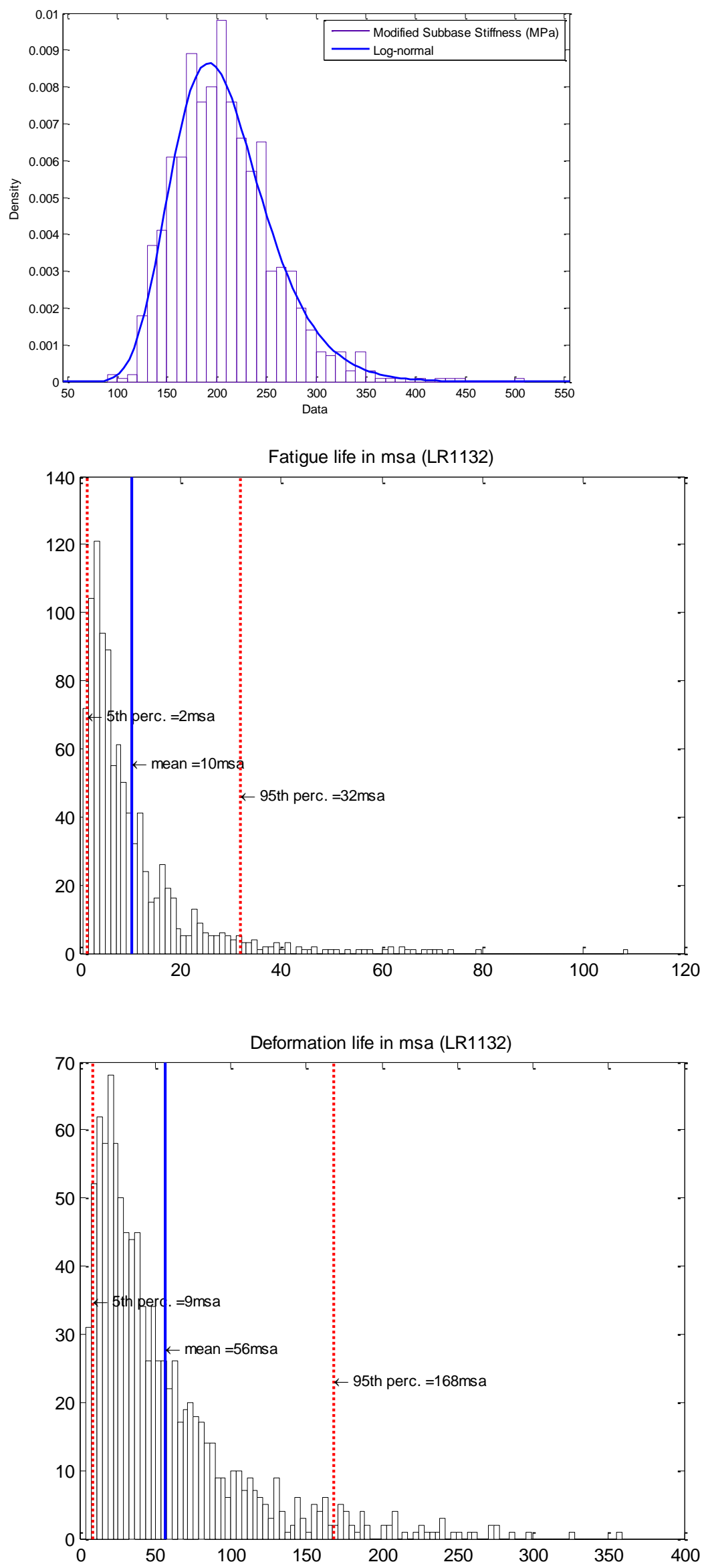


\section{A4.7 M08 EB}

\section{A4.7.1 Section 4}
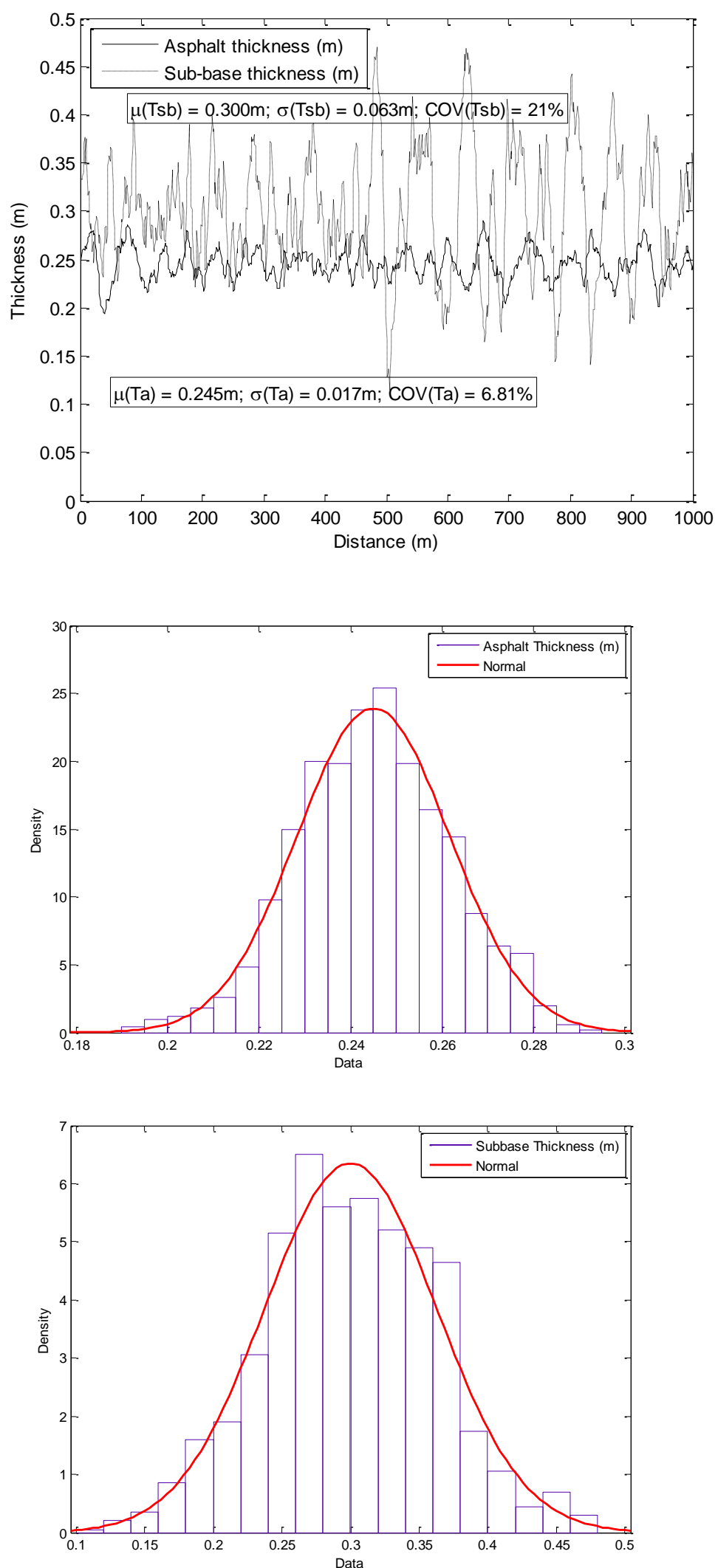

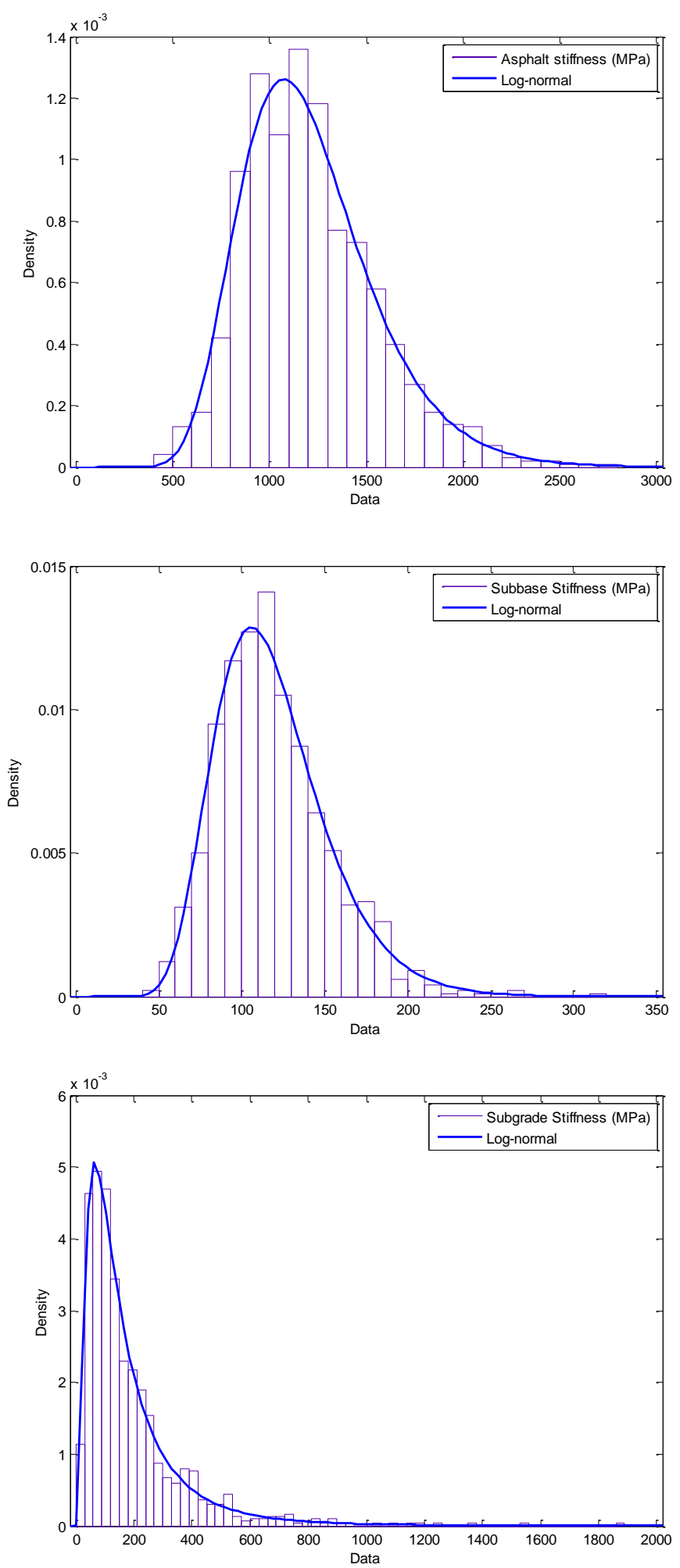

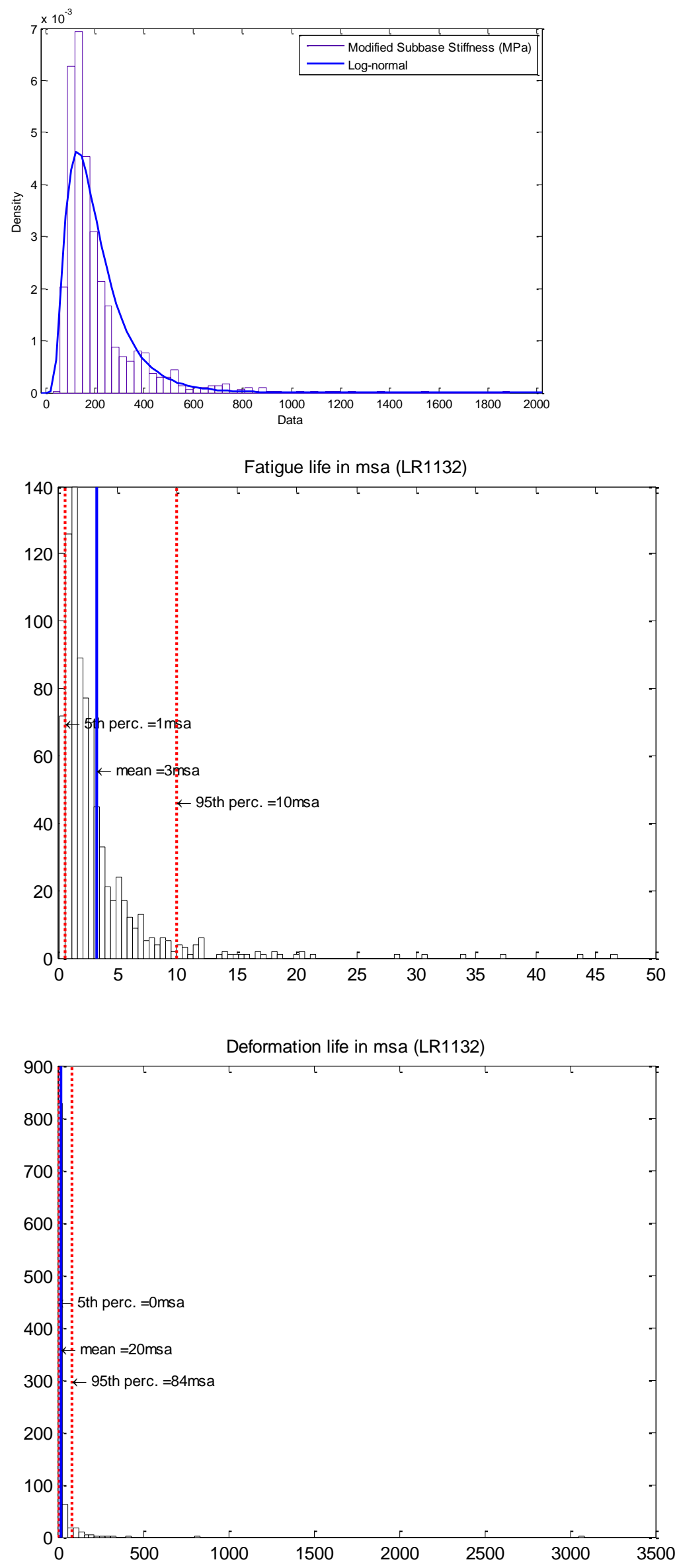


\section{A4.7.2 Section 6}
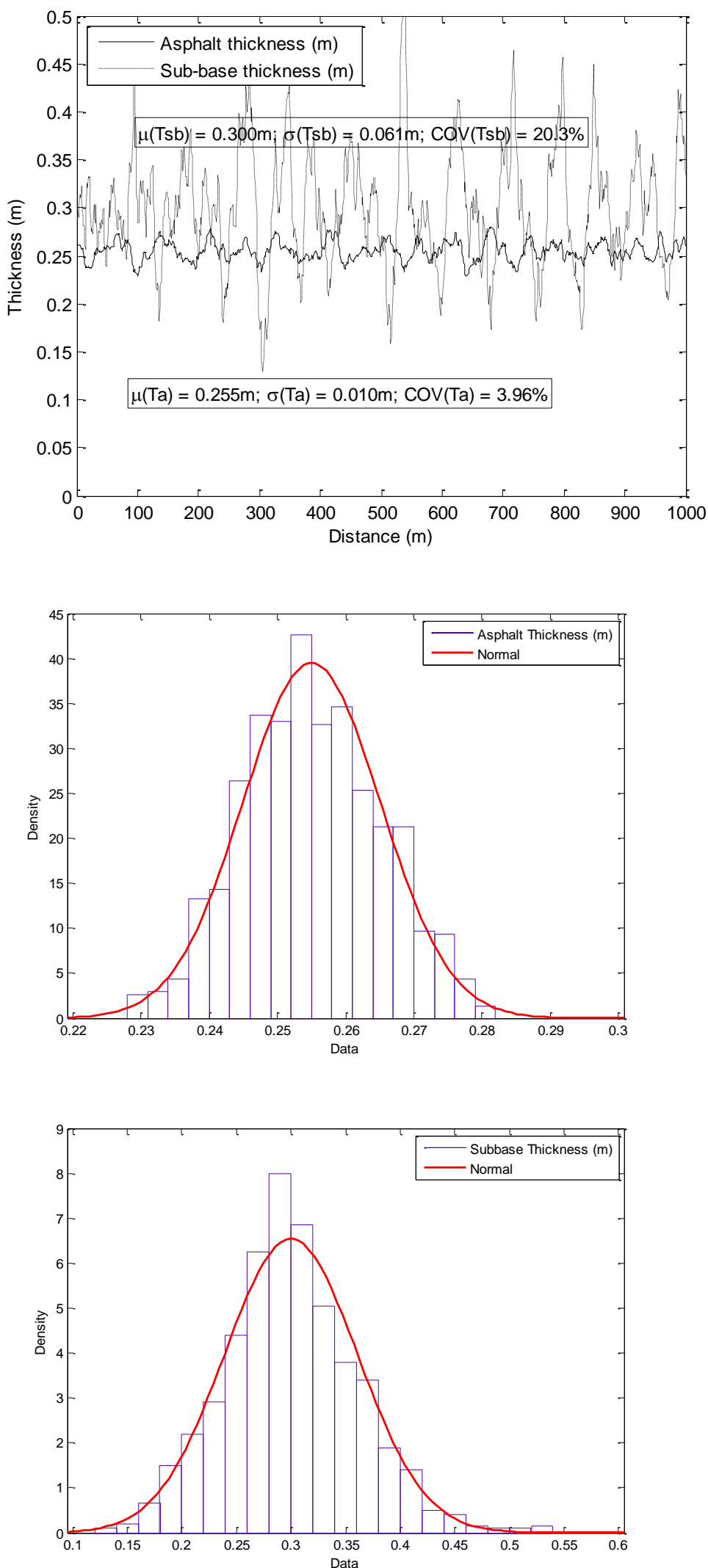

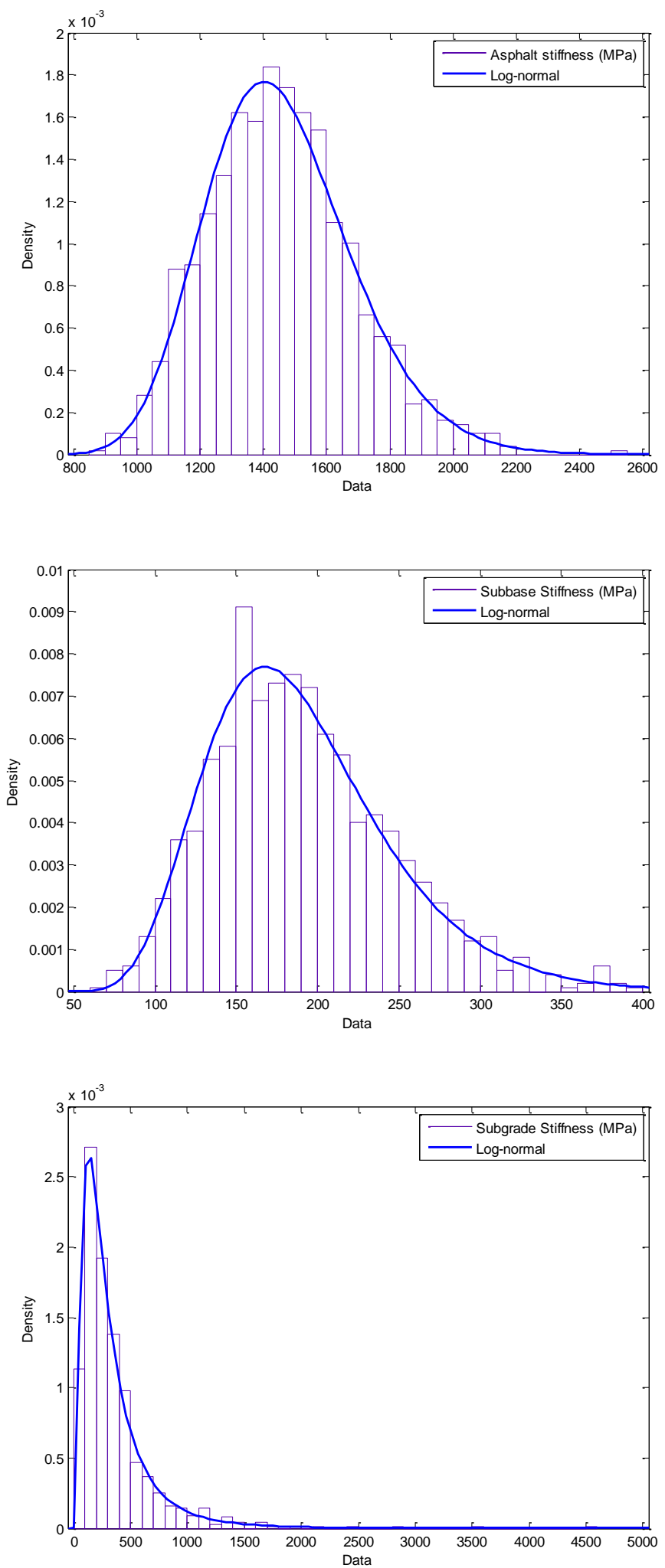

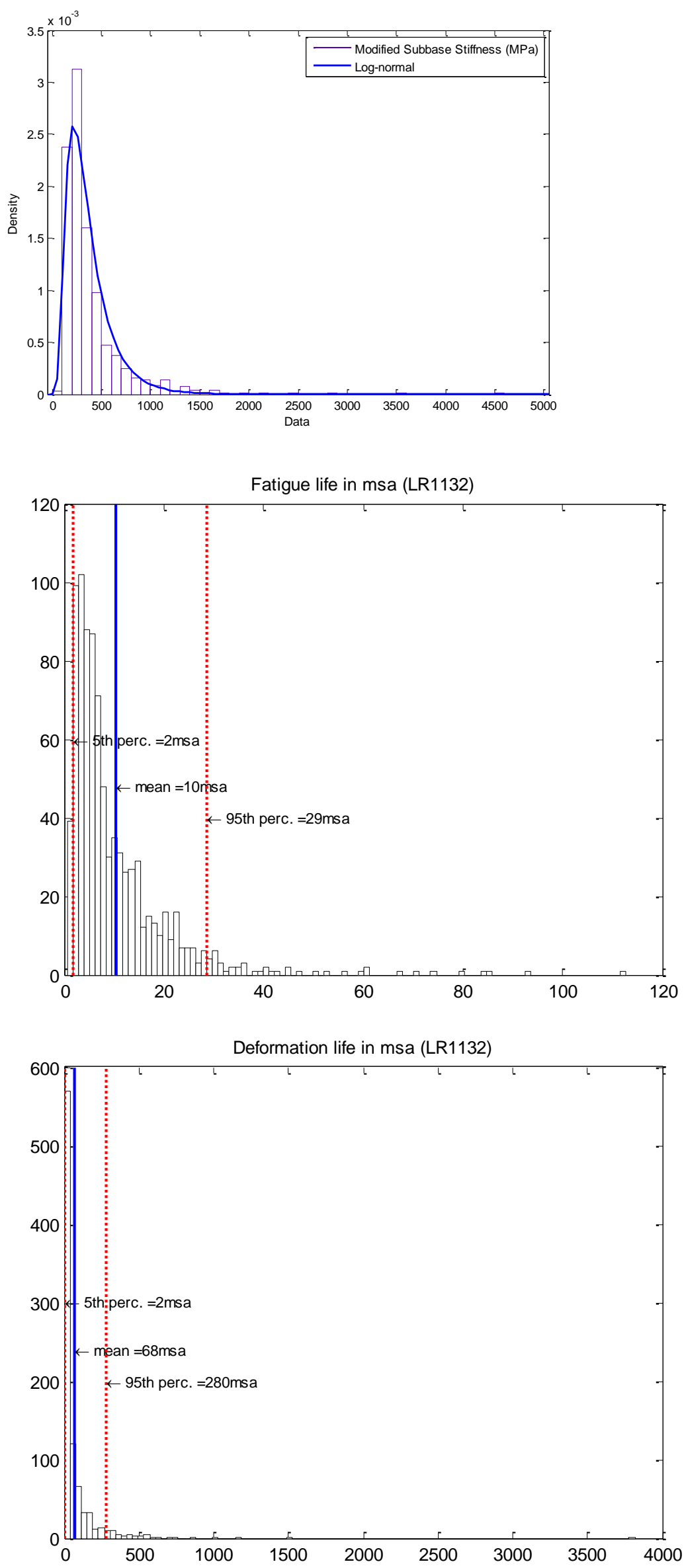


\section{A4.8.1 Section 2}
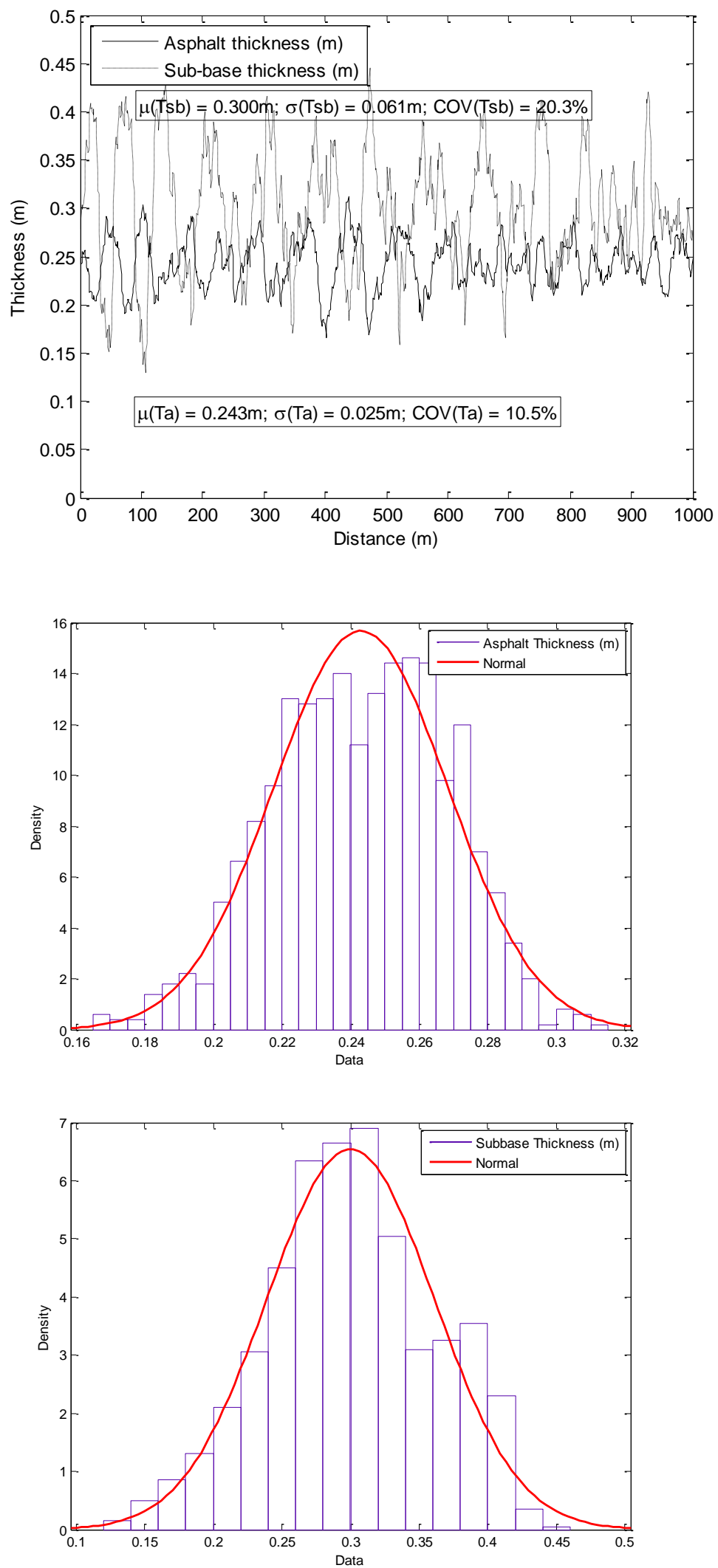

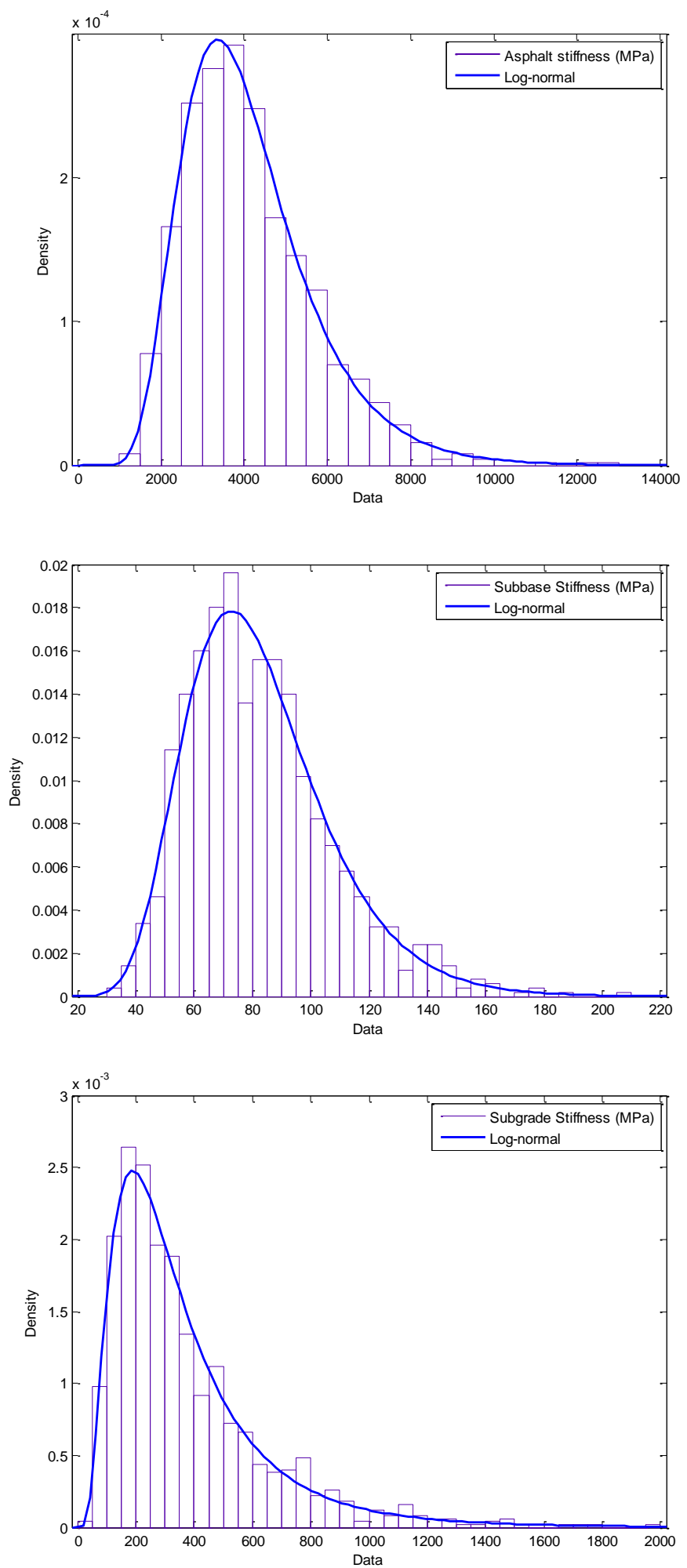

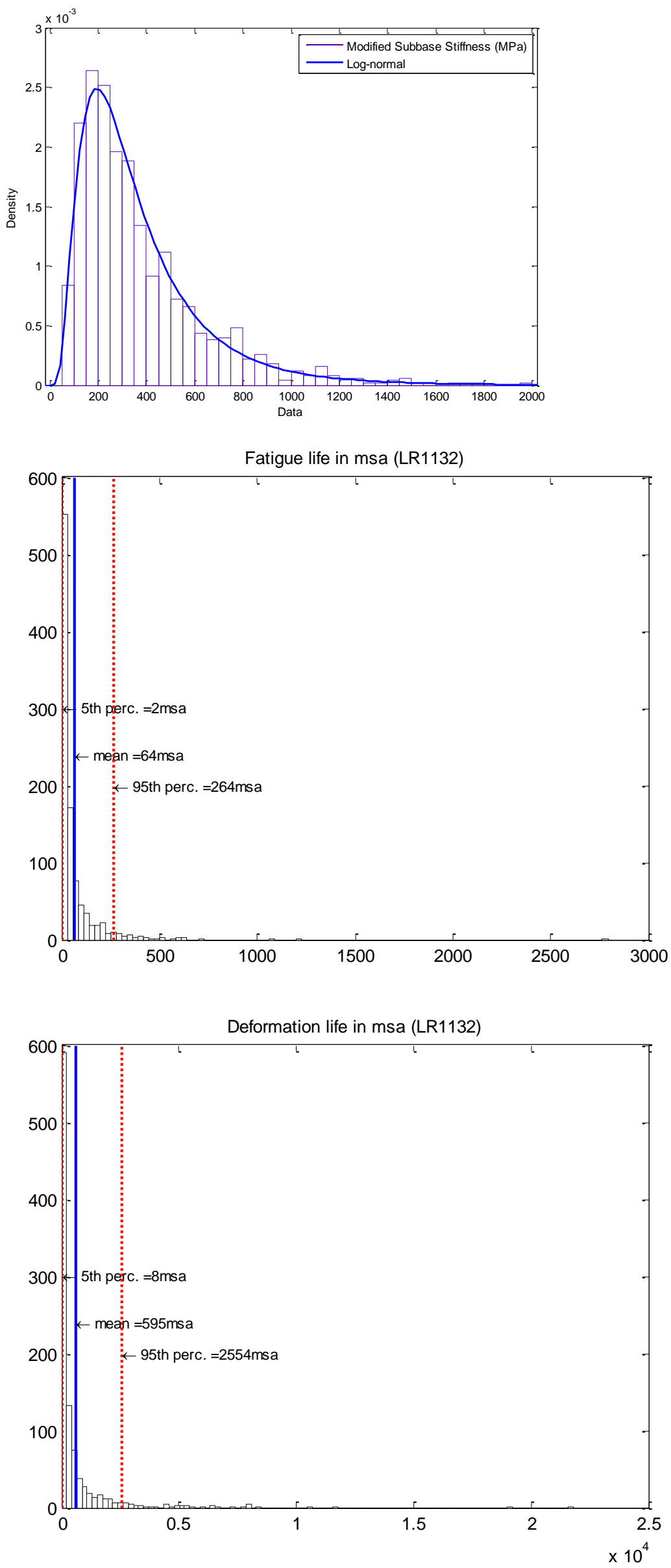


\section{A4.8.2 Section 5}
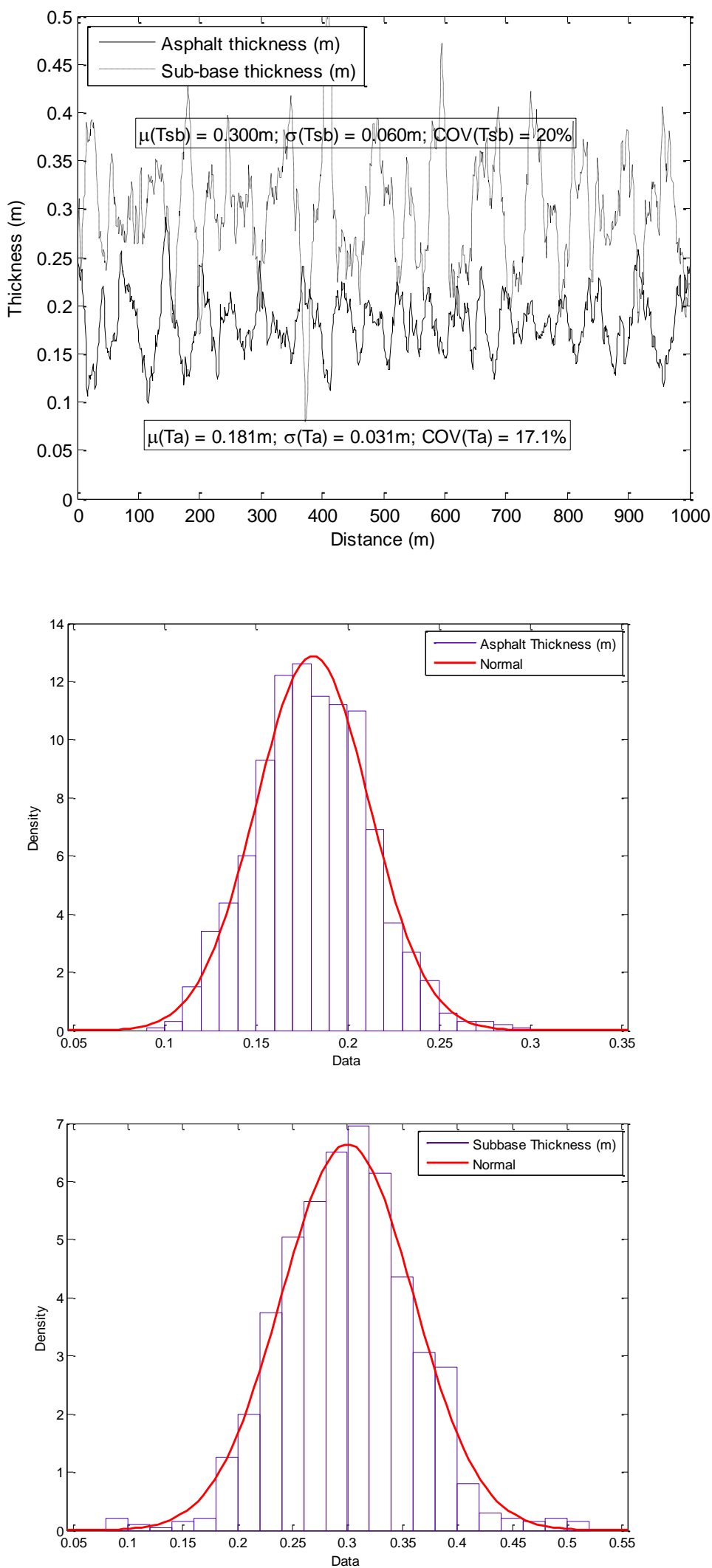

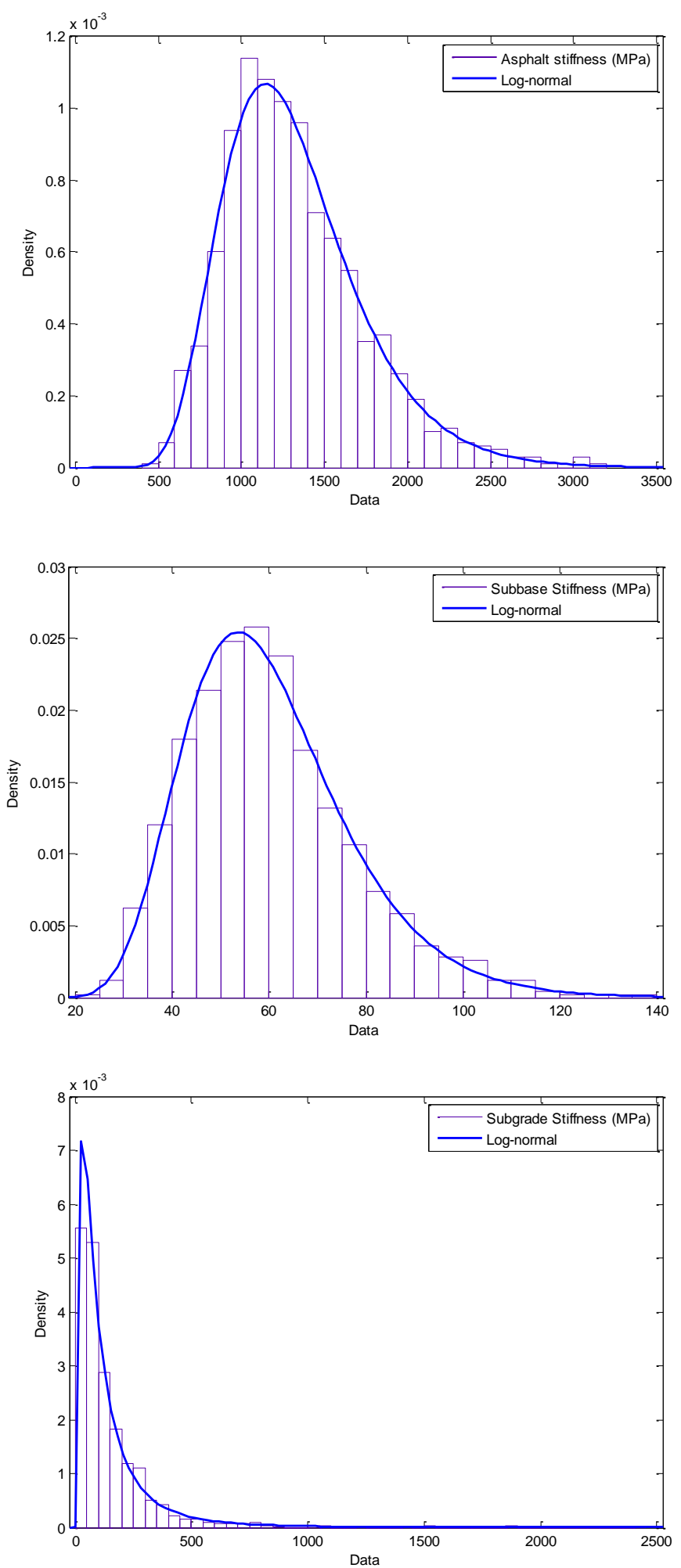

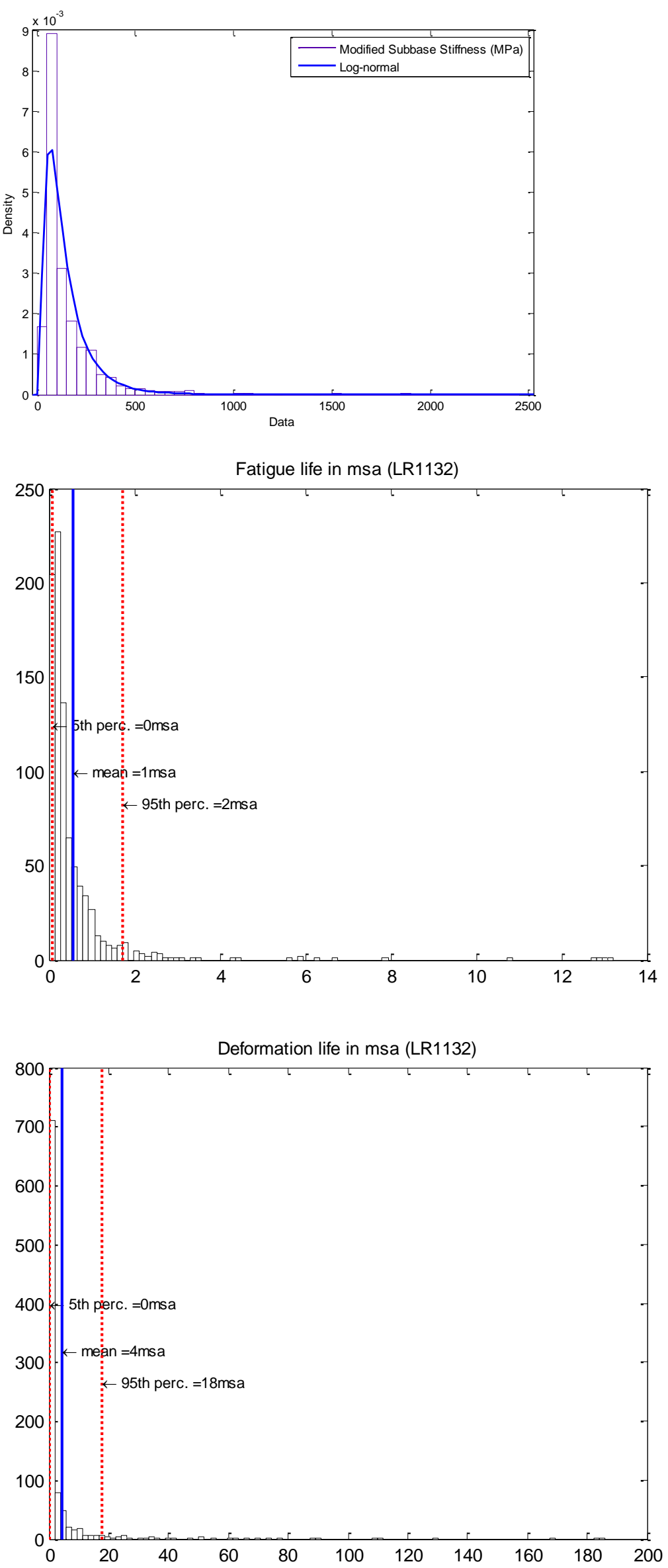